\title{
The Design, Construction and Research Application of a Differential Electrochemical Mass Spectrometer (DEMS)
}

\author{
Sean James Ashton
}

Vollständiger Abdruck der von der Fakultät für Chemie der Technischen Universität München zur Erlangung des akademischen Grades eines

\section{Doktors der Naturwissenschaften}

genehmigten Dissertation.

Vorsitzender: Univ.-Prof. H. Gasteiger, Ph.D.

Prüfer der Dissertation:

1. Univ.-Prof. Dr. U. K. Heiz

2. Univ.-Prof. M. Tromp, Ph.D.

3. Prof. Dr. M. Arenz, University of Copenhagen / Dänemark

Die Dissertation wurde am 06.06.2011 bei der Technischen Universität München eingereicht und durch die Fakultät für Chemie am 25.07.2011 angenommen. 



\section{Abstract}

Electrochemical half-cell studies on industrial electrocatalysts contribute significantly towards our understanding of fuel cell processes. However, the study of complex, often overlapping reactions using standard methods is limited to the interpretation of a single electrode current. Presented here are details of the design, construction and characterisation of a differential electrochemical mass spectrometer (DEMS) that enables the in-situ elucidation of electrode currents. The capability of the instrument is demonstrated in two studies. In the first, DEMS is used resolve the conversion of the methanol oxidation reaction to carbon dioxide on high surface area carbon (HSAC) supported Pt and PtRu catalysts, whilst the second focuses on the corrosion of industrial HSACs, separating partial and complete oxidation processes. Despite that both systems have long since been studied, new insights and understanding can be obtained using DEMS.

\section{Zusammenfassung}

Untersuchungen industrieller Elektrokatalysatoren in elektrochemischen HalbZellen tragen wesentlich zum Verständnis der Prozesse in Brennstoffzellen bei. Die Untersuchung von komplexen - sich oft überlappenden - elektrochemischen Reaktionen mittels Standardmethoden ist aber regelmäßig auf die Interpretation einer einzigen „Stom-Antwort“ begrenzt. In der vorliegenden Arbeit werden das Design, der Aufbau und die Anwendung eines differentiellen elektrochemischen Massenspektrometers (DEMS) beschrieben, welcher es ermöglicht, die einzelnen Komponenten des in der elektrochemischen Zelle fließenden Stromes zu differenzieren. Die Wirkungsweise wird anhand zweier Systeme demonstriert, die Methanol Oxidation auf Kohlenstoff geträgerten Pt und PtRu Katalysatoren sowie die Korrosion industriell eingesetzter Kohlenstoff Trägern. Für beide Systeme, welche schon seit Längerem untersucht werden, konnten mittels DEMS neue Erkenntnisse erlangt werden. 
'Electrochemistry is the science which deals with the conversion of matter to electricity; and/or electricity to matter'

Kyvstiakovsky (L.Antropov, Theoretical Electrochemistry) 1910. 


\section{Table of Contents}

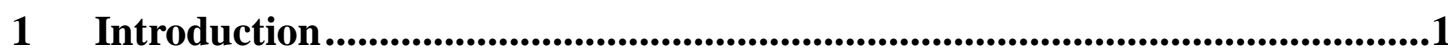

1.1 Background ......................................................................................................1

1.1.1 Fuel Cell Technology ......................................................................2

1.1.2 Electrocatalyst Development .....................................................................5

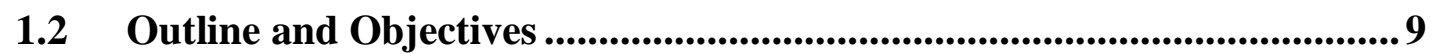

2 Differential Electrochemical Mass Spectrometry ........................................10

2.1 Principle of Operation ........................................................................................... 10

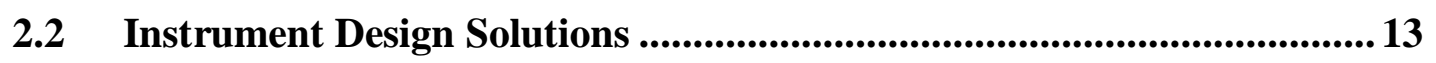

2.2.1 Electrochemical Cells ............................................................... 13

2.2.2 Membrane Interfaces ................................................................ 22

2.2.3 Vacuum Systems \& Mass Spectrometer ........................................ 25

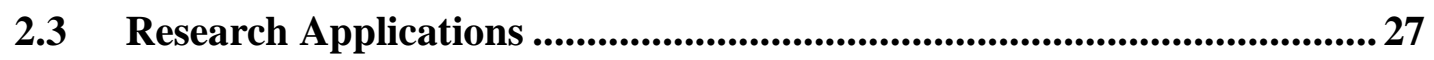

2.3.1 Radio-Isotope Labelled Experimentation ........................................ 27

2.3.2 Characterisation of Organic Adsorbates ............................................ 28

2.3.3 Study of the Electro-oxidation of Small Organic Compounds.........28

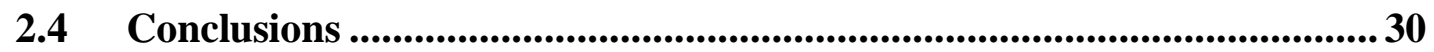

3 Design and Construction of the DEMS Instrument .....................................32

3.1 Design and Development Process ...........................................................33

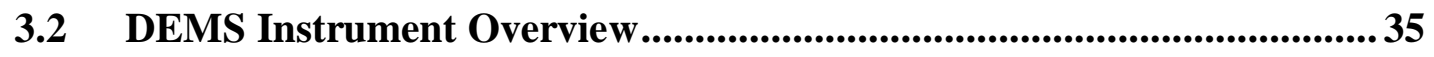

3.2.1 Principle Components ........................................................................ 37

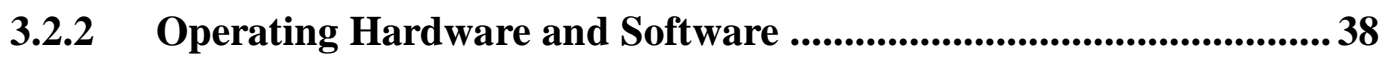

3.4 Electrochemical Half-Cell Setup .............................................................40 
3.4.1 Dual Thin-layer Flow Cell Design ...................................................42

3.5 Membrane Interface ..............................................................................48

3.6 Vacuum System Design ..................................................................52

3.6.1 Three-Stage Differential Pumping ..................................................54

3.6.2 Tubular Aperture......................................................................................55

3.7 Instrumentation, Control and Data Acquisition ......................................61

3.7.1 DEMS Measurement Setup .......................................................63

3.7.2 QMS Calibration Setup ...........................................................................81

3.7.3 Labview Software Architecture...........................................................86

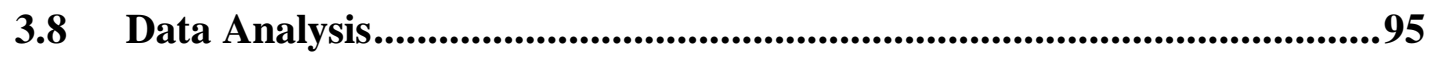

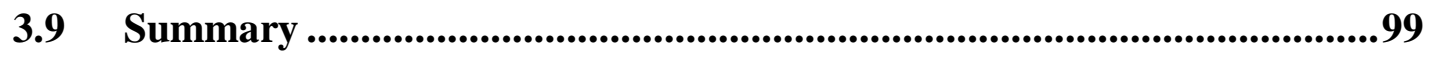

4 Practical Aspects of the DEMS Instrument ............................................... 101

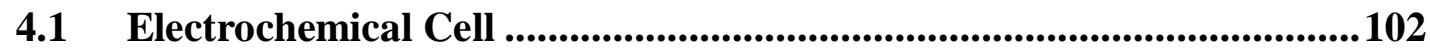

4.1.1 Potential Control..............................................................................103

4.1.2 Effect of Electrolyte Flow Rate.......................................................109

4.2 Performance of the Membrane Interface Material ................................119

4.3 Optimisation of the QMS ...................................................................124

4.3.1 Ion Source Parameters...............................................................................124

4.3.2 Quadrupole and SEM Parameters ......................................................127

4.4 Calibration of the DEMS Instrument ..................................................130

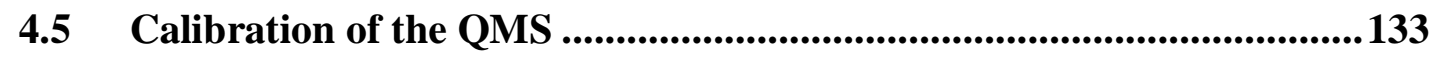

4.6 Further Considerations....................................................................137

4.6.1 Measurement Error ...................................................................137

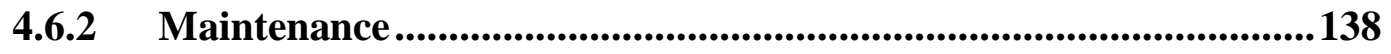

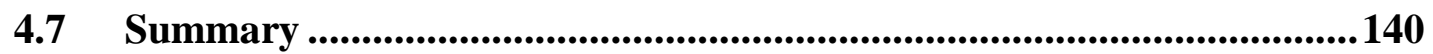




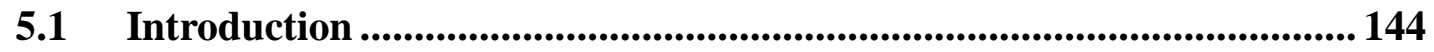

5.1.1 Background ........................................................................................... 145

5.1.2 Motivation................................................................................. 148

5.2 Experimental....................................................................................................... 150

5.2.1 Measurement Procedure ................................................................ 152

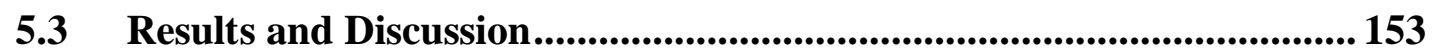

5.3.1 Electrochemical Surface Area Determination ................................... 153

5.3.2 Cyclic Voltammetry ............................................................................... 159

5.3.3 Chronoamperometry ................................................................. 167

5.3.4 Three-Dimensional Voltammetry ...................................................... 173

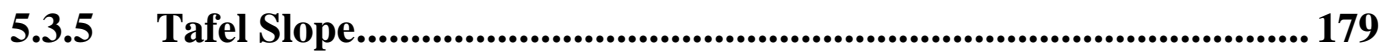

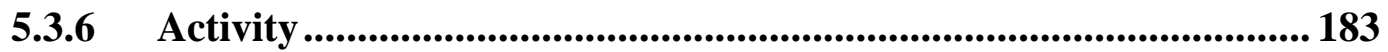

5.3.7 Potential Dependent Conversion ...................................................... 185

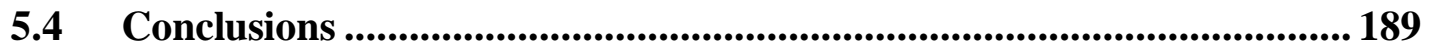

6 The Electrochemical Oxidation of HSAC Catalyst Supports .....................192

6.1 Introduction ........................................................................................... 193

6.1.1 Motivation............................................................................................. 196

6.1.2 Background ..................................................................................................... 197

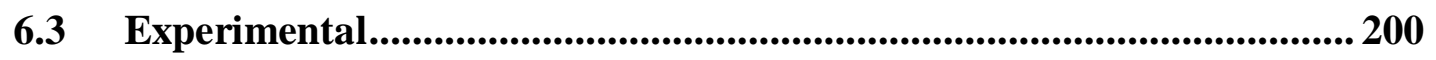

6.3.1 Electrochemical Oxidation Procedure ................................................ 202

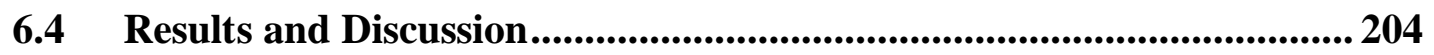

6.4.1 Determination of the Apparent Double-Layer Capacitance........... 204

6.4.2 Substrate Background Contributions............................................. 210 
6.4.3 Electrochemical Oxidation of HSAC Supports.............................212

6.4.5 Electrochemical Oxidation of HSAC Supported Pt Catalysts........242

6.4.6 Future Applications of DEMS in the Study of the COR ................249

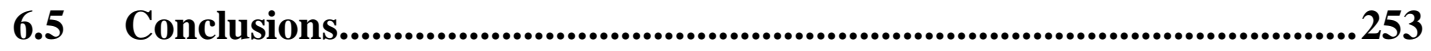

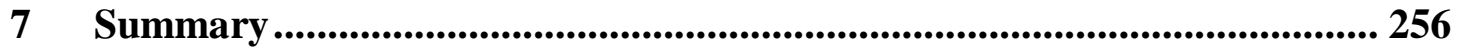

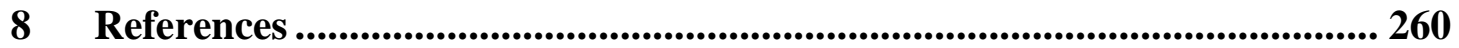

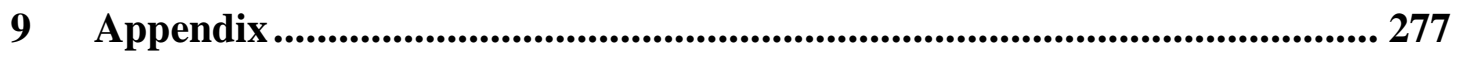

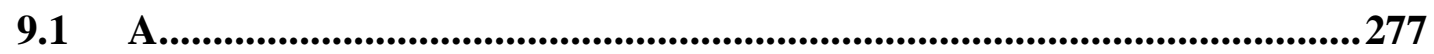

$9.2 \quad$ B 


\section{List of Figures}

Figure 1-1: A diagram illustrating the principles behind operation of the hydrogen fed PEMFC. .............................................................................. 2

Figure 1-2: A graph highlighting the origins of PEMFC performance losses........ 4

Figure 1-3: An outline of the experimental setup and numerous parameters that must be controlled during an in-situ MEA experiment for the characterisation of electrocatalyst materials....................................... 5

Figure 1-4: An illustration of an ex-situ electrochemical half-cell RDE setup that may be employed to characterise the performance of a PEMFC electrocatalyst material ex-situ. 7

Figure 2-1: An illustration of the typical components of a DEMS instrument..... 11

Figure 2-2: An illustration of the 'classic' DEMS cell construction. 13

Figure 2-3: A sketch of the thin-layer flow cell (Reproduced by permission of the American Chemical Society) [40]. 16

Figure 2-4: A drawing of the dual thin-layer flow cell (Reproduced by permission of The Electrochemical Society) [28]. 18

Figure 2-5: An illustration of the capillary inlet DEMS electrochemical cell....... 20

Figure 2-6: An illustration of the vacuum membrane distillation process through a microporous PTFE membrane (Reproduced by permission of Elsevier) [54].

Figure 3-1: A diagram presenting an overview of the various components contributing to the whole DEMS instrument setup. 35

Figure 3-2: Photograph of the DEMS instrument constructed as part of this thesis.

Figure 3-3: A technical drawing of the DEMS instrument construction highlighting the electrochemical cell, membrane interface and vacuum system including QMS. 37

Figure 3-4: A sketch of the three-electrode DEMS electrochemical flow cell setup. 40

Figure 3-5: Photographs of the DEMS electrochemical flow cell setup constructed as part of this thesis.

Figure 3-6: Exploded drawing of the modular DEMS electrochemical flow cell design and membrane interface constructed as part of this thesis. 42 
Figure 3-7: Drawing of the electrolyte flow regime through the dual-cyclone thinlayer electrochemical flow cell.

Figure 3-8: Illustration of the electrolyte flow regime through the DEMS linear dual thin-layer electrochemical cell flow through block.

Figure 3-9: An illustration of the membrane interface. .50

Figure 3-10: An illustration highlighting the chambers of the 3-stage differentially pumped vacuum system.

Figure 3-11: Exploded diagram of the DEMS instrument vacuum system \& QMS.

Figure 3-12: Illustration of the original 3-way flange QMS vacuum chamber housing (A) and modified 5-way QMS vacuum chamber housing (B). .56

Figure 3-13: A sketch of the DEMS vacuum chamber giving the respective pumping speeds $(S)$, conductances $(C)$ and operating pressure $(P)$ of the 3-stage differentially pumped vacuum system when the DEMS instrument is in operation.

Figure 3-14: Illustration of the DEMS adjustable aperture (left) and exploded image (right).

Figure 3-15: Illustration of the QMS cross-beam ion source highlighting possible molecular beam inlet orientations. .58

Figure 3-16: Technical drawing indicating the position and orientation DEMS vacuum aperture with respect to the cross-beam ion source.

Figure 3-17: Overview of instrumentation, control and data acquisition hardware and software employed in this DEMS instrument. .63

Figure 3-18: Image of DEMS electrochemical potentiostat hardware setup. .65

Figure 3-19: Image of custom built negative impedance device (NID) highlighting the potentiostat and cell connections. .66

Figure 3-20: A schematic of the original NID circuit design and electrochemical cell setup.

Figure 3-21: A schematic of an enhanced NID circuit design allowing adjustable $i R$-compensation developed for the DEMS instrument as part of this thesis.

Figure 3-22: Images depicting the QC 422 oscilloscope connections (left) and NI DAQ card used for QMS data acquisition (right). 
Figure 3-23: Example QMS analogue measurement signal outputs (intended for an oscilloscope) of the QC 422 for the measurement of two QMS channels.

Figure 3-24: Screenshot of the CV3 software front panel developed for the DEMS instrument. 76

Figure 3-25: Screenshot of the QMS settings control panel for DDE and DAQ... 77

Figure 3-26: Screenshot of the Macro software front panel further developed to incorporate the required DEMS instrument functionality............. 78

Figure 3-27: Image of the DC3 software front panel developed for the DEMS instrument.

Figure 3-28: Image of the KDS 200 syringe pump settings (left) and control panel (right) developed for the DEMS instrument. 80

Figure 3-29: Overview of the QMS calibration measurement hardware and software system.

Figure 3-30: Schematic of the analogue pressure gauge measurement system used for QMS calibration.

Figure 3-31: Image of the DEMS (QMS) calibration software front panel developed for the DEMS instrument. 85

Figure 3-32: A diagram of the electrochemistry Labview software conceptual architecture in standard CV, DC and MWE operational modes. .. 87

Figure 3-33: Diagram of the electrochemistry Labview software conceptual architecture in DEMS operation mode.

Figure 3-34: A diagram illustrating how the QMS data points are correlated to the electrochemistry data.

Figure 3-35: Screenshot of the DEMS save data file indicating the file format.... 94

Figure 3-36: Screenshot of the DEMS analysis software front panel developed as part of this thesis.

Figure 3-37: Screenshot of the queued state machine producer-consumer architecture of the DEMS Analysis software.

Figure 3-38: Diagram representing the structure of the data treatment command sequence (producer) and sequence processor (consumer) of the DEMS analysis software. 98

Figure 4-1: Equivalent circuits for the DEMS electrochemical cells that employ: (a) the stabilising resistor, or (b) the dual counter electrode setups to stabilise the potential control of the WE. 106 
Figure 4-2: An example CV series for ORR on polycrystalline $P t$ at $20 \mathrm{mV} \mathrm{s}$ for various electrolyte flow rates in the dual-cyclone thin-layer flow cell.

Figure 4-3: The dependence of the diffusion limited current, $I_{d l}$, on the electrolyte flow rate for the ORR, HOR and CO bulk oxidation reaction in the dual-cyclone thin-layer flow cell.

Figure 4-4: A graph highlighting qualitatively, the difference between the faradaic electrode current and the corresponding $I_{m / z=44}$ response during a CO-bulk oxidation measurement on polycrystalline Pt for the dual-cyclone thin-layer flow cell performed at various electrolyte flow rates. 112

Figure 4-5: The dependence of the QMS response time for bulk CO-oxidation on the electrolyte flow rate through the dual-cyclone thin-layer flow cell. 113

Figure 4-6: $\mathrm{CV}$ and $\mathrm{MSCV}$ of $\mathrm{CO}_{2}\left({ }^{\mathrm{m}} / \mathrm{z}=44\right)$ during $\mathrm{CO}$-bulk oxidation at various different electrolyte flow rates for the dual-cyclone thin-layer flow cell. 115

Figure 4-7: A plot demonstrating the dependence of DEMS cell correlation coefficient for the ORR on the electrolyte flow rate at various potential scan rates for the dual-cyclone thin-layer flow cell........ 116

Figure 4-8: A plot illustrating the dependence of DEMS cell $\mathrm{CO}_{2}$ collection efficiency, $N$, on electrolyte flow rate at various potential scan rates for the dual-cyclone thin-layer flow cell.

Figure 4-9: Membrane interface material vacuum test setup. 120

Figure 4-10: Total gas flux through porous membrane into an evacuated volume plotted as a function of the evacuated volume pressure.

Figure 4-11: Mass ion current converted to flux normalised to concentration of species in aqueous solution.

Figure 4-12: The effect of extraction plate voltage on QMS ion current at various focus plate voltages. 126

Figure 4-13:The mass scan analogue spectrum of vacuum chamber background at various $Q M S$ resolution values.

Figure 4-14: The effect of ion source current on QMS calibration constant. ..... 128

Figure 4-15: The effect of SEM Voltage on QMS Calibration Constant. 129

Figure 4-16: An example of three repeated CO-stripping DEMS measurements on HSAC Supported Tanaka $5 \mathrm{~nm} P t$ catalyst at $10 \mathrm{mV} \mathrm{s}^{-1}$,

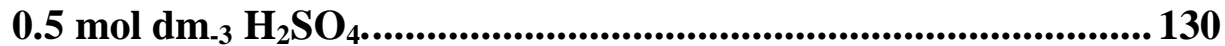


Figure 4-17: An example background subtracted and integrated CO-Stripping measurement for a $50 \mu \mathrm{g}_{\mathrm{Pt}} \mathrm{cm}^{-2}$ sample of $\mathrm{HSAC}$ supported $\mathrm{Pt}$ Tanaka $5 \mathrm{~nm}$ catalyst. 132

Figure 4-18: A schematic and photograph of QMS calibration volume setup. .. 134

Figure 4-19: An example QMS calibration measurement showing the calibration volume pressure decay and mass ion current response (a) and the corresponding relationship between gas flux and mass ion current (b). 135

Figure 5-1: A diagram of the electrochemical methanol oxidation reaction pathway. 147

Figure 5-2: A DEMS CO-stripping measurement for $50 \mu \mathrm{g} \mathrm{cm}^{-2}$ of the Pt $5 \mathrm{~nm}$ HSAC supported catalyst

Figure 5-3: A DEMS CO-Stripping measurement of Tanaka PtRu HSAC supported catalyst. 155

Figure 5-4: A DEMS measurement of the MOR on HSAC supported Pt 5 nm catalyst.

Figure 5-5: DEMS measurement of the methanol oxidation reaction on Tanaka HSAC supported PtRu catalyst. 161

Figure 5-6: A comparison of the MOR and CO-Stripping LSV of Pt $5 \mathrm{~nm}$ HSAC supported catalyst. 164

Figure 5-7: Comparison of the methanol oxidation and the $\mathrm{CO}$-stripping of the Tanaka HSAC support PtRu catalyst. 165

Figure 5-8: A plot of multiple chronoamperometric (current vs. time) measurements for the MOR at a number of electrode potentials (a) and corresponding $I_{\mathrm{CO} 2}$ response (b).

Figure 5-9: The comparison of sampled current chronoamperometry with CV for the methanol oxidation reaction on Tanaka HSAC supported $5 \mathbf{~ n m}$ Pt catalyst. 169

Figure 5-10: Comparison of the sampled current chronoamperometric measurement with $\mathrm{CV}$ for the methanol oxidation reaction on Tanaka HSAC supported PtRu catalyst. 171

Figure 5-11: Three dimensional voltammogram of $1.0 \mathrm{~mol} \mathrm{dm} \mathbf{~ m e t h a n o l ~}^{-3}$ mat oxidation on HSAC supported Tanaka PtRu catalyst. 173

Figure 5-12: Contour plot of $1.0 \mathrm{~mol} \mathrm{dm}^{-3}$ methanol oxidation on HSAC supported Pt catalyst. 174 
Figure 5-13: Contour plot of the conversion (coloured) of $1.0 \mathrm{~mol} \mathrm{dm} \mathbf{~ d m}^{-3}$ methanol oxidation to $\mathrm{CO}_{2}$ on HSAC supported Pt $5 \mathrm{~nm}$ catalyst.

Figure 5-14: Contour plot of the faradaic electrode current (a) and conversion (b) of $1.0 \mathrm{~mol} \mathrm{dm}{ }^{-3}$ methanol oxidation to $\mathrm{CO}_{2}$ on the HSAC supported Tanaka PtRu catalyst. 176

Figure 5-15: Tafel-slope of LSV for $1.0 \mathrm{~mol} \mathrm{dm}^{-3}$ methanol oxidation on HSAC supported Tanaka Pt $5 \mathrm{~nm}$ catalyst and MSLSV for $I_{\mathrm{CO} 2}$ at a potential scan rate of $10 \mathrm{mV} \mathrm{s}^{-1}$ (a) and corresponding chronoamperometric current samples at $420 \mathrm{~s}$ (b) electrolyte flow rate of $5 \mu \mathrm{l} \mathrm{s}$ 179

Figure 5-16: Tafel-slope of the LSV for $1.0 \mathrm{~mol} \mathrm{dm}$ methanol oxidation on HSAC supported Tanaka PtRu catalyst and MSLSV for $I_{\mathrm{CO}_{2}}$ at a potential scan rate of $10 \mathrm{mV} \mathrm{s}^{-1}$ (a) and corresponding chronoamperometric current samples at $420 \mathrm{~s}$ (b) electrolyte flow rate of $5 \mu \mathrm{l} \mathrm{s}{ }^{-1}$. 180

Figure 6-1: A graph illustrating the experimental procedure used to study the electrochemical oxidation processes of the carbon black samples. 202

Figure 6-2: CV of EC300 HSAC support (a) and corresponding potential dependence on the double layer electrode capacitance $(b)$ at various potential scan rates. 205

Figure 6-3: Measured capacitance of the HSAC support samples plotted as a function of carbon black sample loading. 206

Figure 6-4: CV and MSCVs of polycrystalline -Pt, -Au and GC electrodes. 210

Figure 6-5: A graph illustrating the origins of the observed current during an initial high potential excursion above $1.05 \mathrm{~V}_{\text {RHE }}$ 212

Figure 6-6: First high potential LSV $\left(5 \mathrm{mV} \mathrm{s} \mathrm{s}^{-1}\right)$ of the pristine $\operatorname{EC300}\left(50 \mu \mathrm{gc}_{\mathrm{c}} \mathrm{cm}^{-2}\right)$ and heat-treated samples 1-5 $\left.\left(85 \mu \mathrm{g} \mathrm{cm}^{-2}\right)\right]$.

Figure 6-7: Effect of heat treatment temperature on the complete and partial electrochemical oxidation charge evolved during the first high potential excursion to $2.0 \mathrm{~V}_{\mathrm{RHE}}$ (a) (EC300 normalised to $85 \mu \mathrm{g} \mathrm{\textrm {cm } ^ { - }}$ $\left.{ }^{2}\right)$

Figure 6-8: First four LSVs of the electrochemical COR of a pristine EC300 HSAC support in $0.5 \mathrm{~mol} \mathrm{dm}^{-3} \mathrm{H}_{2} \mathrm{SO}_{4}$.

Figure 6-9: First four LSVs of the electrochemical COR of graphitised Sample 5 $\left(3000{ }^{\circ} \mathrm{C}\right)$ HSAC support in $0.5 \mathrm{~mol} \mathrm{dm}^{-3} \mathrm{H}_{2} \mathrm{SO}_{4}$ 223

Figure 6-10: First four LSVs of the electrochemical COR of graphitised Sample 4 $\left(2100{ }^{\circ} \mathrm{C}\right) \mathrm{HSAC}$ support in $0.5 \mathrm{~mol} \mathrm{dm}^{-3} \mathrm{H}_{2} \mathrm{SO}_{4}$ 224 
Figure 6-11: Integrated charge of the partial (hollow markers) and complete (solid markers) electrochemical COR processes for each of the carbon black samples during the first four CVs between 1.05 and 2.05 $\mathrm{V}_{\mathrm{RHE}}$ in $0.5 \mathrm{~mol} \mathrm{dm}^{-3}$ 225

Figure 6-12: Effect of the lower potential limit on the electrochemical COR of electrochemically oxidised EC300 carbon black in $0.5 \mathrm{~mol} \mathrm{dm}^{-3}$ $\mathrm{H}_{2} \mathrm{SO}_{4}$ 227

Figure 6-13: Effect of the lower potential limit on the electrochemical COR of electrochemically oxidised and graphitised Sample $1\left(3200{ }^{\circ} \mathrm{C}\right)$

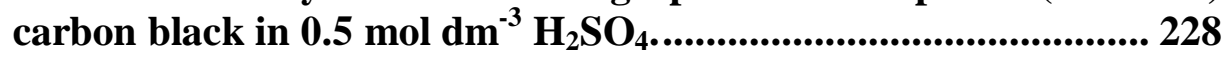

Figure 6-14: Effect of the lower potential limit on the electrochemical COR of electrochemically oxidised and graphitised Sample $3\left(2450{ }^{\circ} \mathrm{C}\right)$ carbon black in $0.5 \mathrm{~mol} \mathrm{dm}^{-3} \mathrm{H}_{2} \mathrm{SO}_{4}$. 229

Figure 6-15: Integrated charge of the partial (hollow markers) and complete (solid markers) electrochemical COR of the carbon black samples during the phase (iii) of the electrochemical treatment. 230

Figure 6-16: The change in the integrated charge of the complete electrochemical COR of the HSAC carbon samples for each successive $\mathrm{CV}$ as a function of the lower electrode potential limit in $0.5 \mathrm{~mol} \mathrm{dm}^{-3} \ldots . .231$

Figure 6-17: The total complete and partial oxidation charge evolved during the electrochemical treatment versus heat treatment temperature (a) and corresponding data normalised to BET surface area (b)...... 237

Figure 6-18: The apparent double layer capacitance for EC300 carbon measured before and after electrochemical oxidation (a) and increase in apparent double-layer capacitance for each carbon black following electrochemical oxidation (b). 238

Figure 6-19: The quinone/hydroquinone redox peak charge plotted as a function of the total partial and complete COR charge evolved during the electrochemical treatment (a). 239

Figure 6-20: The origins of the observed current during the initial potential excursion above $1.1 \mathrm{~V}_{\mathrm{RHE}}$ 242

Figure 6-21: First ten LSVs of the electrochemical COR of a (EC300) HSAC supported Pt $1 \mathrm{~nm}$ catalyst $\left(50 \mu \mathrm{g}_{\mathrm{Pt}} \mathrm{cm}^{-2}\right)$ on polycrystalline Au substrate in $0.5 \mathrm{~mol} \mathrm{dm}^{-3} \mathrm{H}_{2} \mathrm{SO}_{4}$ between 1.05 and $1.8 \mathrm{~V}_{\mathrm{RHE}}$ (a) .. 243

Figure 6-22: A plot illustrating the re-activation of the complete COR of a Pt $1 \mathrm{~nm}$ HSAC supported catalyst (following phase (ii) of the electrochemical treatment) by reducing the $\mathrm{WE}$ potential to $0.05 V_{\text {RHE}}$, analogous to the effect observed on pure EC300 carbon (see figure 6-12). 244 
Figure 6-23: CO Stripping Charge vs. Cumulative CO2 evolved of various HSAC supported Pt nanoparticle catalysts...........................................248

Figure 9-1: CV of the MOR on Pt (a) and PtRu (b) catalyst indicating the methylformate produced, and the ratio with the electrode current.

Figure 9-2: Tafel plot of the MOR on the Pt (a) and PtRu (b) catalysts. The Tafelslope for methylformate does not correspond for the Pt catalysts, but does for the PtRu catalysts in contrast to $I_{\mathrm{CO} 2}$....................... 277

Figure 9-3: First four LSVs of the electrochemical COR of a heat treated carbon black Samples 3, 2 and 1 in $0.5 \mathrm{~mol} \mathrm{dm}^{-3} \mathrm{H}_{2} \mathrm{SO}_{4}$......................... 281

Figure 9-4: Effect of the lower potential limit on the electrochemical COR of the graphitised (and electrochemically oxidised in phase (ii) of the electrochemical treatment) Samples 2,4 and 5 in $0.5 \mathrm{~mol} \mathrm{dm}^{-3}$ $\mathrm{H}_{2} \mathrm{SO}_{4}$ 282

Figure 9-5: LSVs of repeated high potential excursion to $1.85 \mathrm{~V}_{\mathrm{RHE}}$ on the electrochemical oxidation of the HSAC supported Pt $5 \mathrm{~nm}$ catalyst (a) and influence of the lower electrode potential limit following electrochemical oxidation (b) 283

Figure 9-6: LSVs of repeated high potential excursion to $1.85 \mathrm{~V}_{\mathrm{RHE}}$ on the electrochemical oxidation of the HSAC supported Pt $3 \mathrm{~nm}$ catalyst (a) and influence of the lower electrode potential limit following electrochemical oxidation (b) 283 


\section{List of Tables}

Table 2-1: Details of the DEMS microporous PTFE membrane specifications contained in the scientific literature.

Table 3-1: Details of the specifications of the two DEMS cell flow regimes. 46

Table 3-2: Details of the microporous PTFE membrane interface material candidates obtained for this DEMS instrument construction.

Table 3-3: Details of the QMS component operating pressure limits [85] 54

Table 3-4: EC 422 Electrometer Amplifier Range (AO = Analogue Output)....... 74

Table 4-1: DEMS electrochemical flow cell resistances between WE, CE, RE electrode compartments and membrane interface sampling compartment (SC). 104

Table 4-2: The total gas flux values extrapolated to 0.01 mbar vacuum pressure at $5 \mu \mathrm{s} \mathrm{s}{ }^{-1}$ electrolyte flow rate.

Table 4-3: List of numerous QMS calibration constants, $K^{0}$, for a number of gaseous species obtained for this DEMS instrument.

Table 5-1: Summary of CO-stripping measurement details and ECSA values of the Tanaka Pt $5 \mathrm{~nm}$ and PtRu HSAC supported catalysts.

Table 5-2: Summary of the Tafel-slopes and activities of the MOR on Pt $5 \mathrm{~nm}$ and PtRu electrocatalysts investigated in this study.

Table 5-3: A table summarising the MOR activities obtained from electrocatalytic studies on respective materials with relevant experimental details. . 184

Table 6-1: Details of the heat-treatment temperature, BET surface area and corresponding gravimetric and specific double layer capacitances for each carbon black samples investigated in this study. 207

Table 6-2: COR Tafel slopes $\left(\mathrm{I}_{\mathrm{CO} 2}\right)$ and corresponding symmetry constants for each HSAC during the first high potential excursion. 216

Table 9-1: List of experimental variables that must be considered when performing MOR activity measurements in an electrochemical half-cell using an RDE in acid electrolyte. 280 


\section{Acknowledgements}

I would first and foremost like to thank Prof. Matthias Arenz, whom will likely recall that in the interview and presentation for the Ph.D. position, I had to rather awkwardly respond to almost all questions regarding my master thesis with "I'm really sorry but I can't say. It's confidential”. Despite that, he decided to patiently guide me through my work and moulded me into an 'electrochemist' for which I am truly grateful.

This work, however, would not have even begun on the right track if it was not for the kind advice of Dr. Zenonas Jusys, whose knowledge and experience in DEMS is unrivalled. I would also like to thank Dr. Karl J.J. Mayrhofer whose enthusiasm provided me with a firm foundation in electrochemistry, and also Dr. Gustav Wiberg because when he wasn't occupied finishing his own work, or singing in a choir (or the lab...), he was most likely teaching me Labview programming; without his influence I would almost certainly still be treating and analysing data today. There are also the remaining members of the electrochemistry group whom supported my work, namely Katrin and Markus to thank, particularly for the good-times we had setting up the laboratory in Copenhagen with Neuhavn and The Moose being special highlights. I would also like to express my appreciation to Prof. Ulrich Heiz and his research group for the pleasant working environment, the TU-München Mechanische Werkstatt for their meticulous fabrication of the DEMS components, and the Elektronik Werkstatt for regularly letting me 'borrow' things.

Of course, the completion of this work was unachievable without the support of my friends and family. I owe an awful lot to Gustav, Martin and Aras for providing mutual distraction from the highs and lows of our Ph.Ds. It was always great to get away from the relentless grinding of vacuum pumps, whether it was off to a Biergarten, the calmness of a snooker table, or even a trip to the ski slopes. Finally, I would like to especially thank my Mum and Dad, Jason and Jasmine for their unconditional love and support, and Bärbel, Steffi, Marga and Horst for their unreserved kindness. Thank you all. 


\section{Introduction}

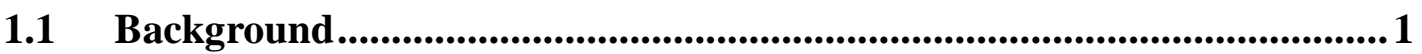

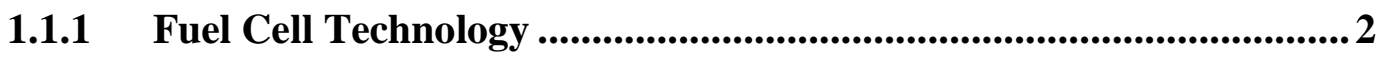

1.1.2 Electrocatalyst Development ...........................................................5

1.2 Outline and Objectives ........................................................................

At the beginning of a thesis dedicated to the construction of a differential electrochemical mass spectrometer (DEMS), it is essential to first present the broader significance of the instruments intended application in the fundamental study of fuel cell relevant electrochemical reaction processes.

\subsection{Background}

Rising concerns over climate change and future oil and gas supply security are stimulating a political and economic shift towards a policy of sustainable energy [1]. In order to achieve sustainable energy consumption, aspects of both renewable energy and energy efficiency are required to be developed and encouraged. Sources of renewable energy are, however, not necessarily new concepts with wind-turbines having been present across the landscape for some time. Nevertheless, changing the way in which we produce and supply energy is only one aspect; the energy must also be stored and used efficiently. It is in energy efficiency, therefore, that more efficient chemical-electrical energy conversion (CEEC) devices such as the fuel cell, will play an essential role in replacing today's inefficient energy conversion devices, such as the combustion engine [2]. In contrast to the combustion engine, CEEC devices are not restricted by the Carnot efficiency limitations and can therefore offer higher energy conversion efficiency for the endeavour of sustainable energy [3]. 


\subsubsection{Fuel Cell Technology}

The fuel cell CEEC device essentially converts chemical energy (contained in a fuel and oxidant) directly into electrical energy, and crucially does not involve any combustion. In principle, the device operates in a similar manner to an everyday CEEC, the battery; however, in contrast to the battery in which a limited quantity of the chemical energy source is stored internally, a fuel cell uses an external source of fuel and oxidant. An overview of the principle operation of a proton exchange membrane fuel cell (PEMFC) is depicted in figure 1-1.

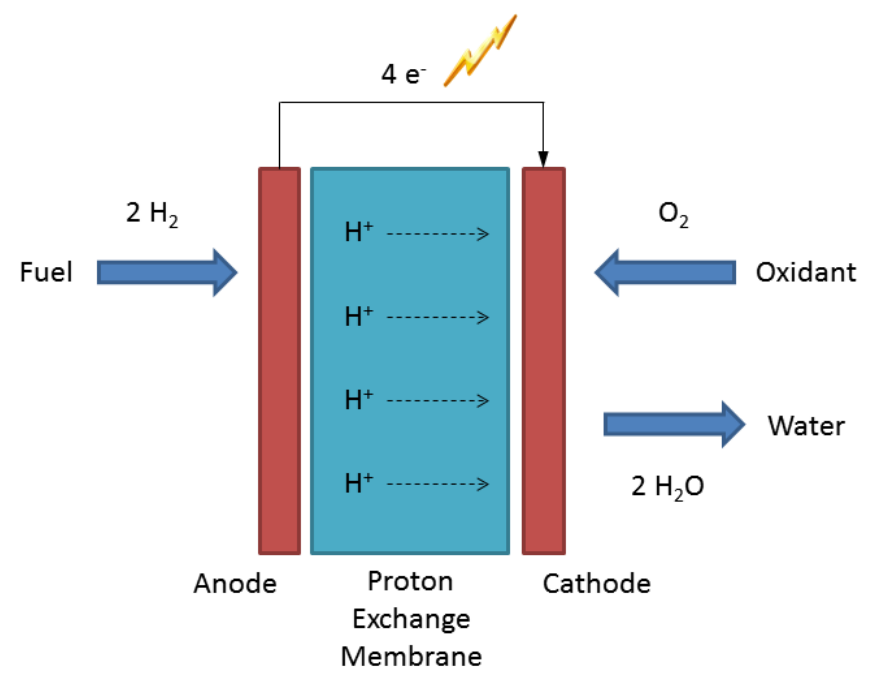

Figure 1-1: A diagram illustrating the principles behind operation of the hydrogen fed PEMFC. The CEEC device possesses two electrodes that are separated via a proton exchange membrane, and converts an external supply of fuel $\left(\mathrm{H}_{2}\right)$ at the anode, and oxidant $\left(\mathrm{O}_{2}\right)$ at the cathode into electricity and water $\left(\mathrm{H}_{2} \mathrm{O}\right)$.

The PEMFC consists of two platinum (Pt) electrodes: an anode and cathode which are separated by a proton exchange membrane (PEM) barrier. The device takes an external source of fuel and oxidant, in this case hydrogen $\left(\mathrm{H}_{2}\right)$ and oxygen $\left(\mathrm{O}_{2}\right)$, and generates electricity by electrocatalytically converting them into water. This process is a result of two electrochemical reactions which occur at each of the electrodes. At the anode electrode, the hydrogen is oxidised to produce protons and electrons:
Anode:
$H_{2} \rightarrow 2 H^{+}+2 e^{-}$
$\mathrm{E}^{+}=0.0 \mathrm{~V}_{\mathrm{RHE}}$ 
Once generated, both protons and electrons travel from the anode to the cathode electrode, however, they must do so via two different paths imposed by the properties of the PEM. On one hand, the PEM is ion conducting and therefore the protons created at the anode simply pass through the membrane barrier to the cathode. On the other, the PEM is an electrical insulating barrier which means that the electrons must travel to the cathode via an alternate electron conducting pathway through which the current is harnessed. The protons and electrons are then consumed at the cathode electrode in the reduction of oxygen in the following reaction:

$$
\text { Cathode: } \quad \frac{1}{2} \mathrm{O}_{2}+2 \mathrm{H}^{+}+2 e^{-} \rightarrow \mathrm{H}_{2} \mathrm{O} \quad \mathrm{E}^{-}=1.23 \mathrm{~V}_{\mathrm{RHE}}
$$

The only emission from the hydrogen fuelled PEMFC, therefore, is water vapour. The efficiency of the conversion of chemical into electrical energy in a fuel cell is determined by the potential difference between the anode and cathode electrode potentials. Theoretically, the maximum is $1.23 \mathrm{~V}$ determined by the difference between the thermodynamic half-cell potentials (i.e. $\mathbf{E}^{-}-\mathbf{E}^{+}$). Unfortunately, kinetic constraints for both anode and in particular, the cathode electrochemical reactions make it impossible for the thermodynamic reversible potential to be realised and large electrode overpotentials result, decreasing the energy conversion efficiency of the fuel cell CEEC device, as depicted in figure 1-2. 


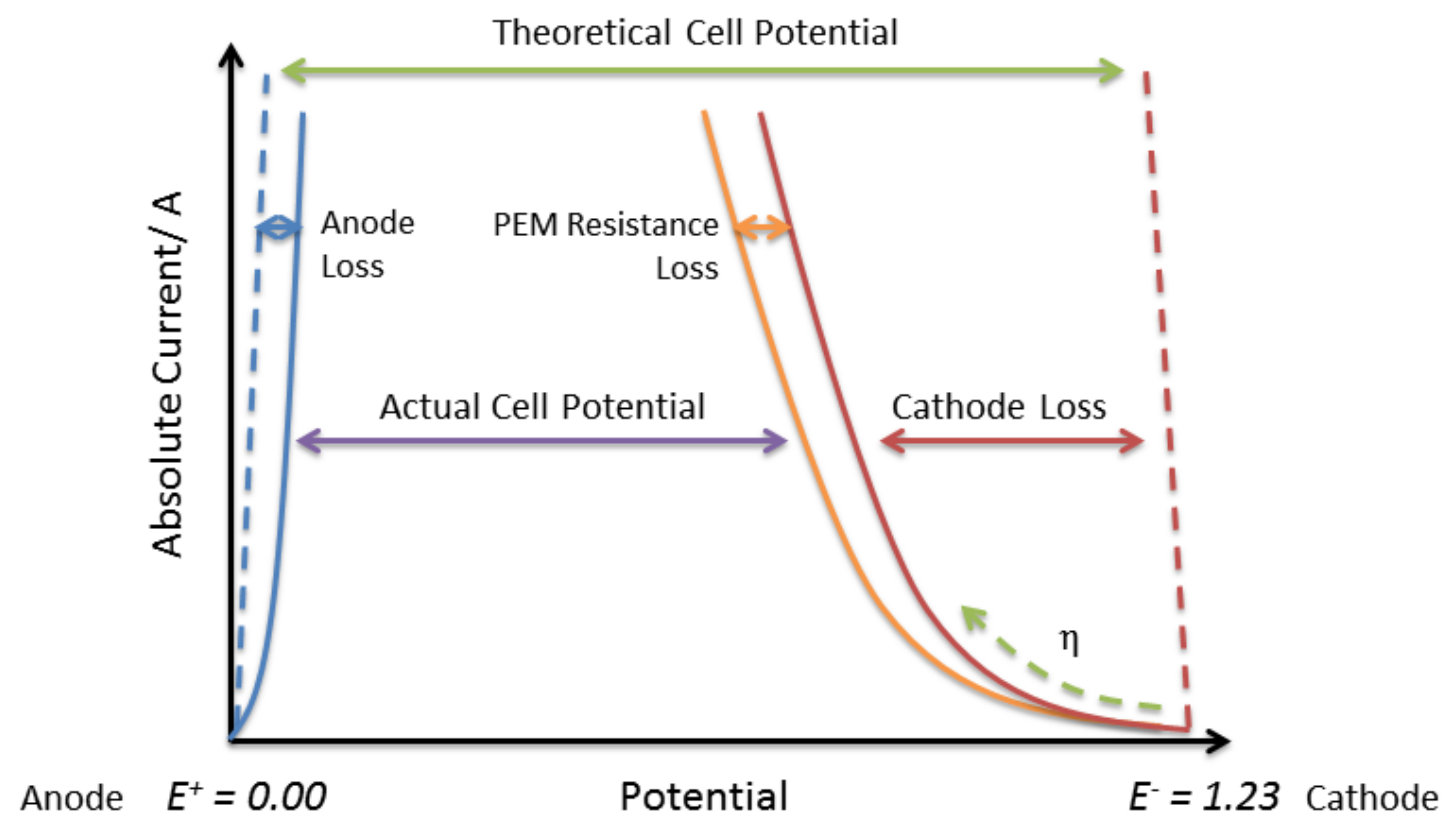

Figure 1-2: A graph highlighting the origins of PEMFC performance losses. The practical cell potential (ca. $0.6 \mathrm{~V})$ is significantly lower than the theoretical cell potential $(\sim 1.23 \mathrm{~V})$ resulting in a loss of energy conversion efficiency. A significant proportion of these losses arise from cathode due to the significant overpotential $(\eta)$ required for the oxygen reduction reaction on $\mathrm{Pt}$.

In order to achieve acceptable energy conversion efficiency (i.e. improve the cell potential), a significant quantity of expensive Pt electrocatalyst must be incorporated into the fuel cell membrane electrode assembly (MEA) to reduce the electrode overpotentials, particularly for the oxygen reduction reaction (ORR) at the cathode. In addition to that, is an extra quantity of Pt electrocatalyst required to ensure that the fuel cell also performs acceptably over its intended lifetime because the $\mathrm{Pt}$ electrocatalyst performance is not static but unfortunately deteriorates through use [4, 5]. Although adding Pt electrocatalyst can indeed provide an efficient and reliable PEMFC device, the net result of the significant Pt content required is a rather expensive and non-commercially viable CEEC device. Consequently, there is more than a strong incentive to develop electrocatalyst materials that are more active, more durable and less expensive than today's state-of-the-art Pt based fuel cell electrocatalysts [6, 7]. 


\subsubsection{Electrocatalyst Development}

There are two feasible approaches to the screening and characterisation of prospective PEMFC electrode materials, whereby the performance of an electrocatalyst is either evaluated in-situ, as part of the MEA of a fuel cell; or exsitu, in a three-electrode electrochemical half-cell setup, typically employing a rotating disc electrode (RDE) [7].

In the in-situ approach, the electrocatalyst is fabricated into the MEA, and its performance is assessed by measuring the current-voltage characteristics (or polarisation curves) of the cell. The exact potentials of the anode and cathode in such measurements in are in general not known precisely, only their potential difference. A simplified diagram illustrating the experimental parameters involved in such an experimental setup is depicted in figure 1-3.

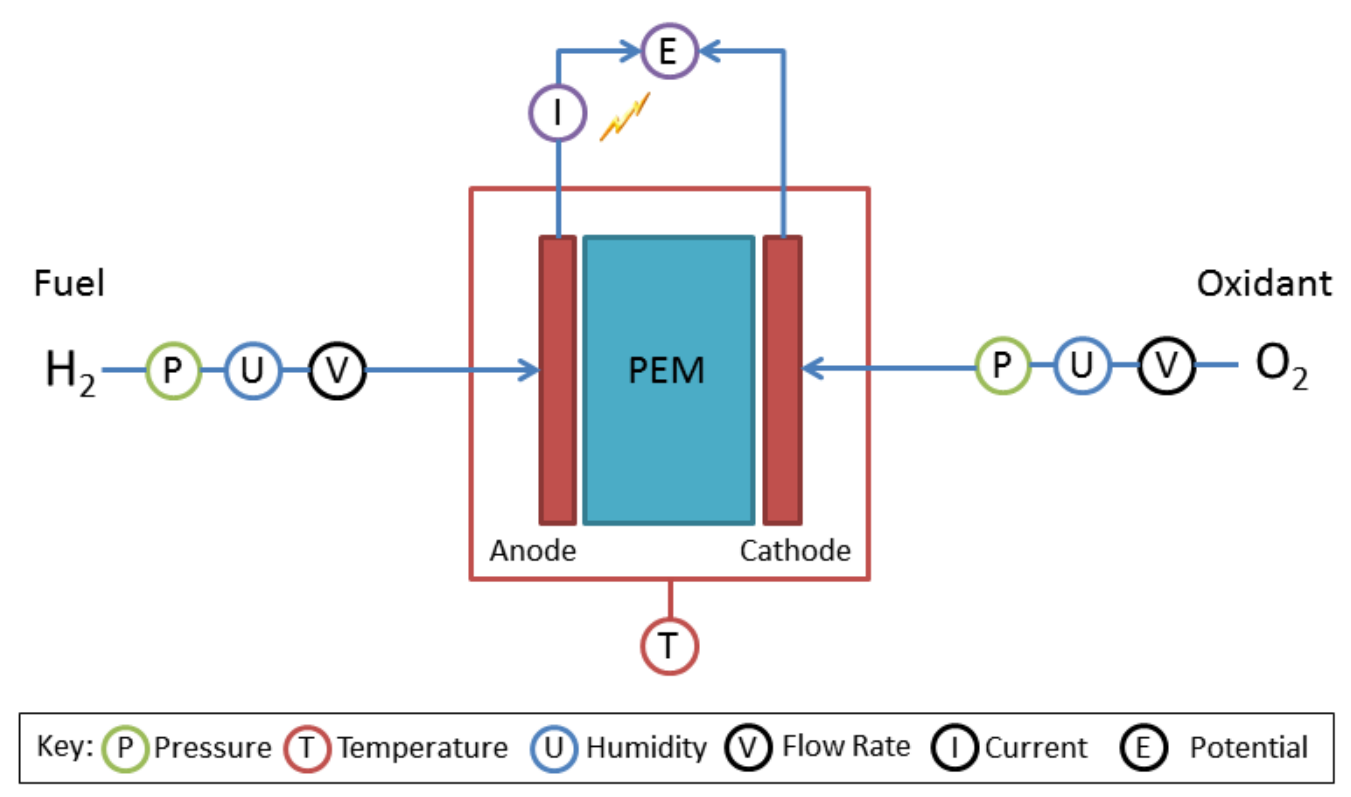

Figure 1-3: An outline of the experimental setup and numerous parameters that must be controlled during an in-situ MEA experiment for the characterisation of electrocatalyst materials. The electrocatalyst must be fabricated into an MEA and the current is measured as a function of cell potential. These types of experiments are rather complicated and assessing the performance of the electrocatalyst alone is far from trivial.

In order to assess the performance of an electrocatalysts in an MEA, meticulous control over the MEA fabrication and experimental variables such as fuel and oxidant pressure, flow rate, humidity, and fuel cell operating temperature is 
required. The cell performance of a particular MEA is typically often published, together with details of the operating temperature, reactant pressure, stoichiometry, MEA preparation, and cell construction etc. Whilst there is no doubt that the assessment of the performance of an electrocatalyst in the fuel cell environment is the ultimate test an electrocatalyst materials performance; controlling every aspect of the MEA fabrication and experimental parameters in order to deduce the performance of the electrocatalyst alone is far from trivial. Not only does the preparation of an MEA require great effort in the research environment, but there are also numerous MEA preparation methods which are often performed by-hand, with questionable reproducibility [8]. It is therefore even more difficult to compare in-situ electrocatalyst performance measurement observations between different research groups, and it has been suggested that the incorporation of a prospective electrocatalyst in an MEA should perhaps rather be left to experts in MEA fabrication [9]. The substantial effort, cost and difficulties associated with fabricating MEAs and reproducing experimental conditions with relatively slow accumulation of data, suggests that the in-situ assessment of electrocatalyst performance alone is ambiguous, and neither an intuitive nor cost effective approach to the screening and characterisation of prospective fuel cell electrocatalyst materials.

The second approach concerning fuel cell electrocatalyst development are ex-situ methods that typically involve fundamental research using an electrochemical half-cell in aqueous electrolyte, such as the three-electrode RDE setup depicted in figure 1-4. The three-electrode electrochemical half-cell setup consists of a working electrode (WE), counter electrode (CE) and reference electrode (RE), each of which are submerged in a conducting aqueous electrolyte. In this setup, the potential of the WE is controlled relative to the $\mathrm{RE}$ using a potentiostat. The $\mathrm{RE}$ is itself an electrochemical half-cell, whose potential remains at a constant, fixed value. The potentiostat therefore can be used to precisely manipulate the potential of the WE electrode relative to the $\mathrm{RE}$ using electroanalytical techniques such as cyclic voltammetry (CV) to study electrochemical processes. The CE meanwhile is required to balance the current flowing through the WE (no current flows through the RE). 


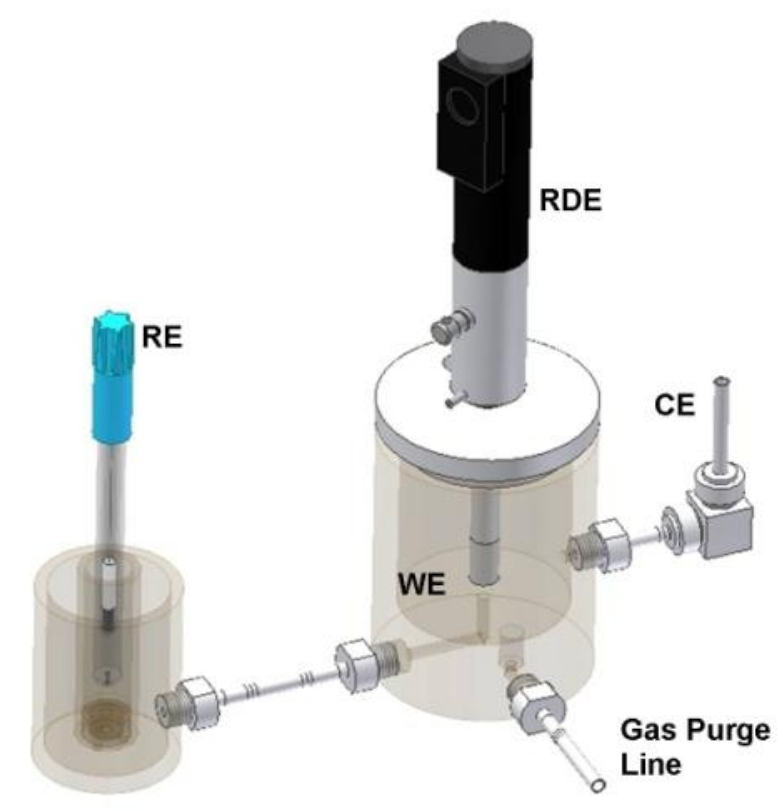

Figure 1-4: An illustration of an ex-situ electrochemical half-cell RDE setup that may be employed to characterise the performance of a PEMFC electrocatalyst material ex-situ. The electrocatalyst material is simply deposited on a WE substrate (i.e. no MEA fabrication is required). The setup is rather straightforward compared to in-situ methodologies with fewer experimental variables, and the current and WE potential relationship may be measured precisely through the use of a RE.

The performance and characteristics of electrocatalyst materials can then be accurately evaluated by measuring the current and WE potential relationship of the electrocatalytic reaction. The principle advantage of the ex-situ threeelectrode electrochemical half-cell over the in-situ MEA approach, in terms of electrocatalyst development, is essentially its capability to investigate model electrocatalyst materials. These experimental setups have, for example, been utilised in a number of fundamental studies with particular focus on the electrocatalysis of fuel cell reactions, typically the ORR [7, 10], hydrogen oxidation reaction (HOR) and methanol oxidation reaction (MOR) [11], which have been extensively studied on model electrodes such as polycrystalline [10], single crystal [12] and well-characterised alloy [11, 13-16] Pt surfaces, in order to gain a fundamental understanding of the reaction mechanisms and the relationship between catalyst structure and activity.

Fundamental studies on model catalysts, however, are not always entirely applicable to the more immediate concerns of fuel cell development, although in 
combination with theory [17] they do provide insights into how the ideal fuel cell electrocatalyst might be designed [11, 18, 19]. A more applied method, therefore, is to perform fundamental research studies in an electrochemical half-cell which directly approach problems associated with fuel cell development needs, such as the characterisation of the activity $[7,20-23]$, and durability $[24,25]$ of industrial fuel cell catalysts. In comparison to in-situ methods, there are fewer experimental variables to control in the electrochemical half-cell, no MEA fabrication is required and the potential of the WE can be controlled and measured precisely. Consequently, the experiments are comparatively straightforward and cost effective, allowing the more rapid accumulation of experimental data. Electrochemical half-cell setups may therefore be considered as a rather intuitive methodology for effective fuel cell electrocatalyst material screening and characterisation, as well as for fundamental research studies on fuel cell relevant electrochemical processes. This is of course achieved at the expense of the electrocatalyst material being characterised in an aqueous rather than humid gas phase environment of the fuel cell, and consequently the extrapolation of ex-situ observations to the MEA is not necessarily straightforward. A rather complementary approach is therefore crucial toward developing characterisation methodologies to accelerate fuel cell electrocatalyst development.

It is the more applied ex-situ approach to the fundamental study of fuel cell relevant processes, and development of electrocatalyst characterisation methodologies in an electrochemical half-cell that is taken in our laboratory. Nevertheless, such studies using standard RDE setups are not without their own limitations and ambiguities. For example, the study of complex and frequently overlapping electrochemical reactions is in principle limited to the clouded interpretation of a single electrode current. Consequently, more sophisticated techniques that allow the in-situ elucidation of electrochemical reactants, intermediates and products, in an electrochemical half-cell such as DEMS are an invaluable research tool. 


\subsection{Outline and Objectives}

The objective of this dissertation was to essentially design, construct and characterise a DEMS instrument, intended for fundamental research studies on fuel cell relevant electrochemical reaction processes. The thesis is organised in the almost chronological order of the design, construction and development processes, followed by two demonstrations of the DEMS application in research.

In order to achieve this goal it was important to first acquire an overview of the DEMS literature, and evaluate the various DEMS design solutions that have appeared previously. A review of the DEMS literature, with particular focus on previous electrochemical cell design solutions is presented in Chapter 2.

In Chapter 3, an overview of the experimental setup along with the design of the critical components of the DEMS instrument built as part of this work is then presented. This section is extended to included necessary details of the hardware and software that were required, or indeed developed to improve the operation and usability of the DEMS instrument.

Once a working DEMS instrument was established, it was crucial to then understand the practical aspects of the instrument and its operation, which is covered in Chapter 4. This includes the evaluation of the effect of experimental variables and a guide for optimising and calibrating the DEMS instrument.

In a first study, the research capabilities of the DEMS instrument is demonstrated in Chapter 5 that revisits the MOR on HSAC supported Pt and PtRu catalysts. Using DEMS, we are able to observe MOR products and evaluate the conversion of methanol to $\mathrm{CO}_{2}$ as a function of the WE potential using a variety of electroanalytical techniques.

Finally, in Chapter 6 the electrochemical oxidation of various HSAC supports is examined and is a topic which has received relatively little attention using DEMS. In this study, we are able to elucidate electrode currents that arise from partial and complete oxidation of the HSAC supports, as well as the oxygen evolution reaction that occurs on the polycrystalline Au substrate or supported Pt particles. 


\section{Differential Electrochemical Mass Spectrometry}

$2.1 \quad$ Principle of Operation ......................................................................................10

$2.2 \quad$ Instrument Design Solutions.............................................................................13

2.2.1 Electrochemical Cells ....................................................................................13

2.2.2 Membrane Interfaces...............................................................................22

2.2.3 Vacuum Systems \& Mass Spectrometer ….............................................25

2.3 Research Applications..........................................................................................27

2.3.1 Radio-Isotope Labelled Experimentation ................................................27

2.3.2 Characterisation of Organic Adsorbates ..............................................28

2.3.3 Study of the Electro-oxidation of Small Organic Compounds ..........28

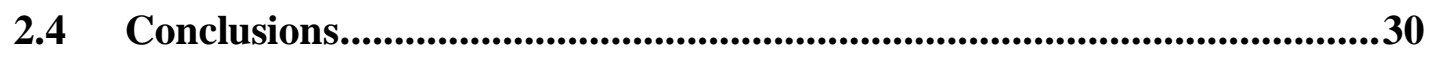

The intention of this chapter is to provide an overview of the DEMS technique, the instrument designs and example research applications. This involves the presentation and discussion of previous design solutions, which are separated into three parts: the electrochemical cell, membrane interface and the vacuum system of the mass spectrometer. Example experiments from the DEMS literature are then briefly described which highlight where a particular DEMS electrochemical cell design may be favoured. Ultimately, the appropriate design solution is determined by the intended research application of the DEMS instrument.

\subsection{Principle of Operation}

Differential Electrochemical Mass Spectrometry (DEMS) is essentially an analytical technique that combines electrochemical half-cell experimentation with mass spectrometry. This allows the in-situ, mass resolved observation of gaseous or volatile electrochemical reactants, reaction intermediates and/or products. By correlating the faradaic electrode current with relevant mass ion currents, ambiguous electrochemical reaction processes can be elucidated, whereas 
standard electrochemical techniques such as the RDE, are otherwise limited to the interpretation of a single electrode current. An overview of the DEMS instrument is depicted in figure 2-1.

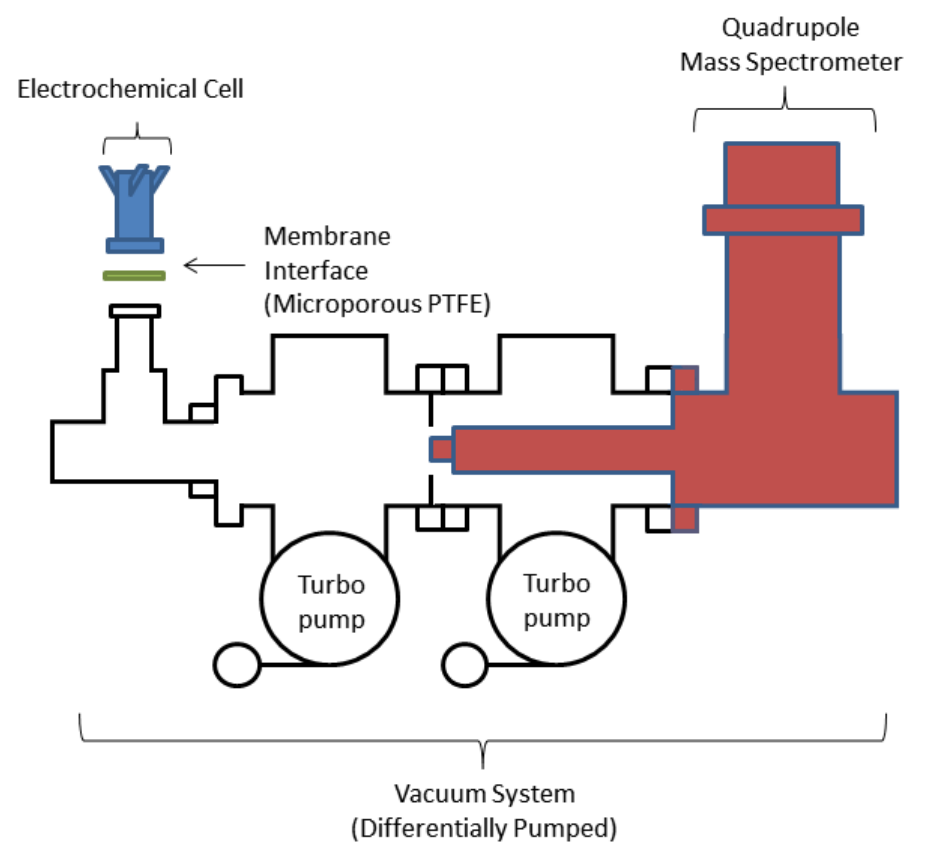

Figure 2-1: An illustration of the typical components of a DEMS instrument. The DEMS instrument consists of an electrochemical cell that is interfaced to a (2-stage) differentially pumped vacuum system containing a QMS via a microporous PTFE membrane interface.

The instrument essential consists of three crucial components: an electrochemical half-cell, a PTFE membrane interface, and a vacuum system including the quadrupole mass spectrometer (QMS). The role of the electrochemical half-cell is to enable controlled electrochemical experimentation at a WE of interest, and to allow reaction products to be transported to the membrane interface. This interface consists of a microporous PTFE membrane that partitions the aqueous electrolyte of the electrochemical cell from the high vacuum conditions required by the QMS. The hydrophobic nature of the membrane prevents the passage of aqueous electrolyte, whilst allowing dissolved gaseous, volatile and relatively nonpolar species to permeate, evaporating into the vacuum system. The permeating species may be subsequently observed online by monitoring the relevant mass ion current with QMS. The operation of the QMS, however, requires high vacuum conditions and consequently a specially designed, differentially pumped vacuum system is typically necessary. 
The DEMS technique was first established by Wolter and Heitbaum in 1984 [26], however, the origins of utilising mass spectrometry to study electrochemical processes via a PTFE interface may be traced back further to Gadde and Bruckenstein in 1971 [27]. The 'differential' term was chosen in order to distinguish between the time- and potential- resolved correlation of the mass ion and electrode current technique [26] from un-resolved integrating methods [27, 28]. The study of dissolved gases in aqueous solution using mass spectrometry via a membrane interface, however, may be traced back even further to Hoch and Kok in 1962 [29]. Because DEMS incorporates a membrane interface, the technique is closely related to the analytical technique referred to as membrane introduction mass spectrometry (MIMS). Essentially, the only features that distinguish DEMS from MIMS are the incorporation of an electrochemical halfcell, and the exclusive use of a PTFE microporous membrane interface in DEMS. Despite the almost 30 year history of DEMS, however, no commercial instrument is available and as a result DEMS has been largely limited to the academic research environment where it has been used in a range of fundamental research studies on a variety of different electrodes, albeit by a relatively small number of research groups. Consequently, a number of DEMS design solutions have appeared in the literature over the years, with particular variations in the electrochemical cell design. 


\subsection{Instrument Design Solutions}

\subsubsection{Electrochemical Cells}

The most flexible aspect of the DEMS instrument is the electrochemical cell design. A number of different DEMS cell designs have gradually evolved over the past 25 or so years, each tailored towards the study of different electrochemical reactions on various types and size of electrodes, ranging from porous [30] or smooth model electrodes [31] to HSAC precious metal catalysts [32] and even fuel cell MEAs [33]. Some of the more noteworthy cell designs will be presented in this section, along with a discussion of their respective advantages and limitations.

\subsubsection{The Classic Cell}

The DEMS technique was first demonstrated using the 'classic' DEMS cell [26]. The most defining feature of this cell is the porous membrane electrode whereby powdered electrode material is deposited directly onto the PTFE membrane interface to form the WE. An illustration of the classic DEMS cell construction is given in figure 2-2.

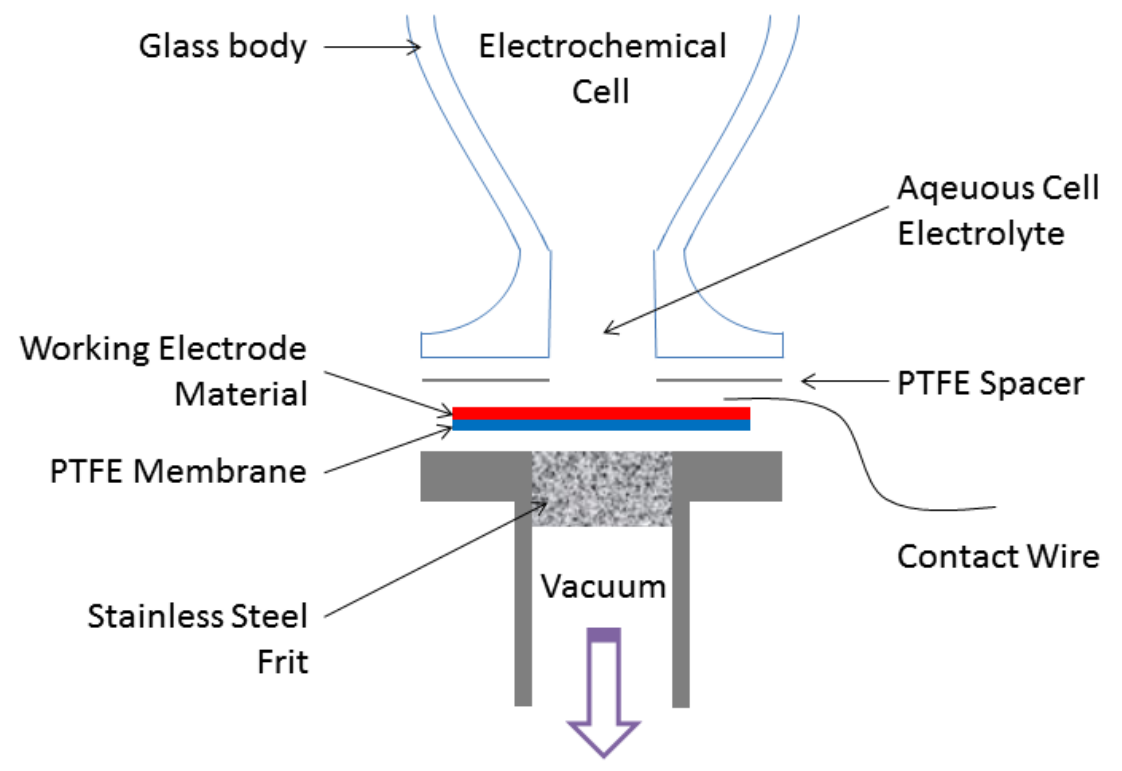

Figure 2-2: An illustration of the 'classic' DEMS cell construction. The WE material is deposited directly onto the microporous PTFE membrane interface. The CE and RE (not shown) are then positioned in the electrolyte above the membrane WE. 
The classic DEMS cell construction consists of a modified vacuum flange containing a stainless steel frit that mechanically supports the porous membrane WE. The membrane electrodes were originally prepared by painting a lacquer containing small metallic particles (e.g. Pt) directly onto the PTFE membrane [34]; however, this was soon adapted so that an electrocatalyst layer could be sputter deposited directly onto the PTFE membrane interface [30], presumably to provide a more homogeneous layer. The electrochemical cell and membrane electrode are sealed between the vacuum flange and a PTFE ring which is compressed by the body of the electrochemical cell compartment. The electrochemical cell body has been typically constructed of glass although a PTFE cell has also been demonstrated [35]. Electrical contact to the WE meanwhile is achieved using a platinum wire, and the $\mathrm{CE}$ and $\mathrm{RE}$ are typically located within the single electrochemical cell compartment, positioned above the porous membrane WE via the multiple connections situated in the cell hood. These connections also serve to allow electrolyte to be deaerated, whilst also allowing electrolyte to be pumped through the cell if desired.

The principle advantage of this classic DEMS cell design is a fast mass spectrometry response time, typically $<0.1 \mathrm{~s}$ and high collection efficiency of ca. 0.5 for lacquer and 0.9 for sputtered electrode [34]. Both are a consequence of the microscopic transport distances between the electrode surface and the vacuum side of the membrane. The cell may also be optimised to possess a small volume (ca. 1 to $2 \mathrm{ml}$ ) with static electrolyte, allowing very economical use of costly isotope labelled reactants for studies of electrochemical mechanisms [34]. The extremely close proximity of electrode material to the membrane interface does, however, introduce certain experimental difficulties. For instance, when using volatile reactants that readily evaporate through the membrane, their concentration at the electrode surface can be significantly reduced compared to the bulk, even with electrolyte convection. The competing process of evaporation through the membrane and adsorption of reactants onto the electrode surface could also lead to a shift in reaction equilibrium [34]. Moreover, whilst convection of electrolyte over the WE may be achieved by positioning the cell electrolyte inlet 
hosing close to the membrane electrode (perhaps to some extent analogous to a wall-jet configuration), the true mass transport of reactants is very likely to be difficult to define and control. This is problematic when studying either steady state or diffusion limited processes, although an adaptation of the classic cell design incorporating a rotating rod to induce convection did appear to resolve this [36]. The design is further limited to experimentation with materials that can be deposited onto the PTFE membrane interface i.e. it is therefore impossible to for example study technical or smooth bulk electrodes such as single crystal or even polycrystalline electrode surfaces. Furthermore, the characterisation of WE material sample loading or utilisation and homogeneity (which is crucial in electrocatalysis) on the non-conducting membrane is difficult to quantify. Finally, the RE is also positioned within the electrochemical cell which can introduce impurities which can have dramatic consequences on the electrochemistry observed [37].

The trends in electrocatalyst research since the early inception of DEMS led focus away from powdered electrode materials that the classic DEMS cell was originally designed for, to more model systems such as polycrystalline and single crystal electrodes. As a consequence of these limitations, few new research studies utilising this DEMS cell alone have appeared for the past 15 years (exception are ref. $[38,39])$, and hence its designation in this review as the classic DEMS cell. 


\subsubsection{Thin-Layer Cell}

The thin-layer design first appeared in 1990 and was initially developed to allow the DEMS study of smooth bulk electrodes i.e. polycrystalline or single crystal electrodes [40]. The DEMS thin-layer flow cell construction illustrated in figure 2-3.

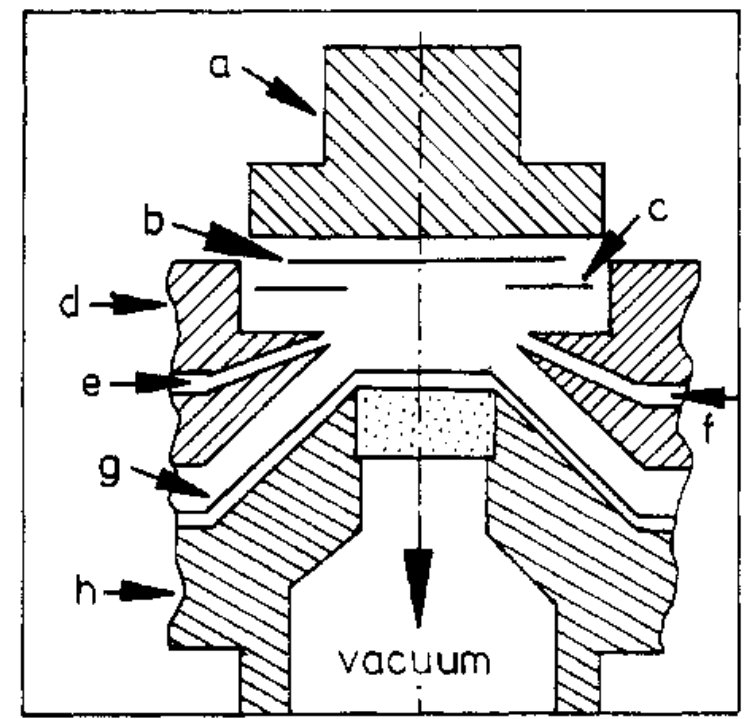

Figure 2-3: A sketch of the thin-layer flow cell (Reproduced by permission of the American Chemical Society) [40]. (a) electrode support, (b) platinum sheet (WE), (c) circular PTFE spacer, (d) cell body, (e) electrolyte outlet and connection to counter electrode, (f) electrolyte inlet, (g) porous PTFE membrane separating electrolyte and vacuum (support by a porous steel frit), (h) connection to vacuum chamber. Not shown, reference electrode connected by a third capillary. The WE in this cell design is separated from the membrane interface via a thin-layer of electrolyte allowing technical WE(s) to be used in DEMS.

In contrast to the 'classic' DEMS cell, the WE is now separated from the membrane interface via a thin-layer of electrolyte allowing considerably more flexibility in terms of the type of electrode that may be studied. Due to the miniature nature of the this cell design ( $\sim \mu \mathrm{L}$ volume [40]), the $\mathrm{CE}$ and $\mathrm{RE}$ are now placed in separate compartments, whose capillaries (which can be connected to either the electrolyte inlet or outlet) lead to the thin-layer WE compartment. However, the electrochemical reaction products must now diffuse from the electrode surface and across the thin layer before it may evaporate through the membrane to be detected. 
The obvious advantage of this design is improved versatility because a variety of well characterised, bulk model WEs may be studied, and the preparation of a porous membrane electrode is no longer necessary. Furthermore, the flow of electrolyte and reactants over the electrode may be defined more exactly and easily controlled compared to the classic DEMS cell. The separation of the WE from the membrane interface, however, results in inherently longer delay times, ca. 2 to $3 \mathrm{~s}[34,40]$, although the collection efficiency is rather high $\sim 0.9$ [34] under static electrolyte conditions. Under continuous electrolyte flow conditions efficiencies of only 0.2 or below are observed at practical flow rates of at least $1 \mu \mathrm{s}^{-1}$ [40]. Whilst the thin layer cell can still be utilised to determine product formation rates under electrolyte flow conditions, there are a number of serious drawbacks. Firstly, the diffusion of reaction products across the thin layer is competing with the flow of electrolyte through the cell and therefore, any reaction products formed at the electrode close to the cell outlet are unable to diffuse to the membrane before exiting the cell. The result of which is reduced collection efficiency. A second issue, regarding volatile and gaseous reactants is that these will in addition to reacting at the WE, also evaporate through the membrane leading to a significant decrease in the concentration gradient along the cell flow field. This DEMS thin-layer cell design is therefore more appropriate for the study of desorption products under a static electrolyte flow conditions [41-43]. As a consequence of the relatively poor cell collection efficiency during electrolyte flow conditions, in cases where convection of the WE is desired, the dual thinlayer flow cell is usually preferred.

Overall, the thin-layer cell is an ideal approach to the study of a variety of technical WE under static electrolyte conditions such as stripping or desorption measurements. However, in much electrochemical experimentation it is desirable to have a controlled and continuous flow of electrolyte and reactants over the WE surface for which the thin-layer cell is less appropriate. 


\subsubsection{Dual Thin-Layer Flow Cell}

A large quantity of the research performed in the past 10 years using DEMS has employed the dual thin-layer flow cell, depicted in figure 2-4.

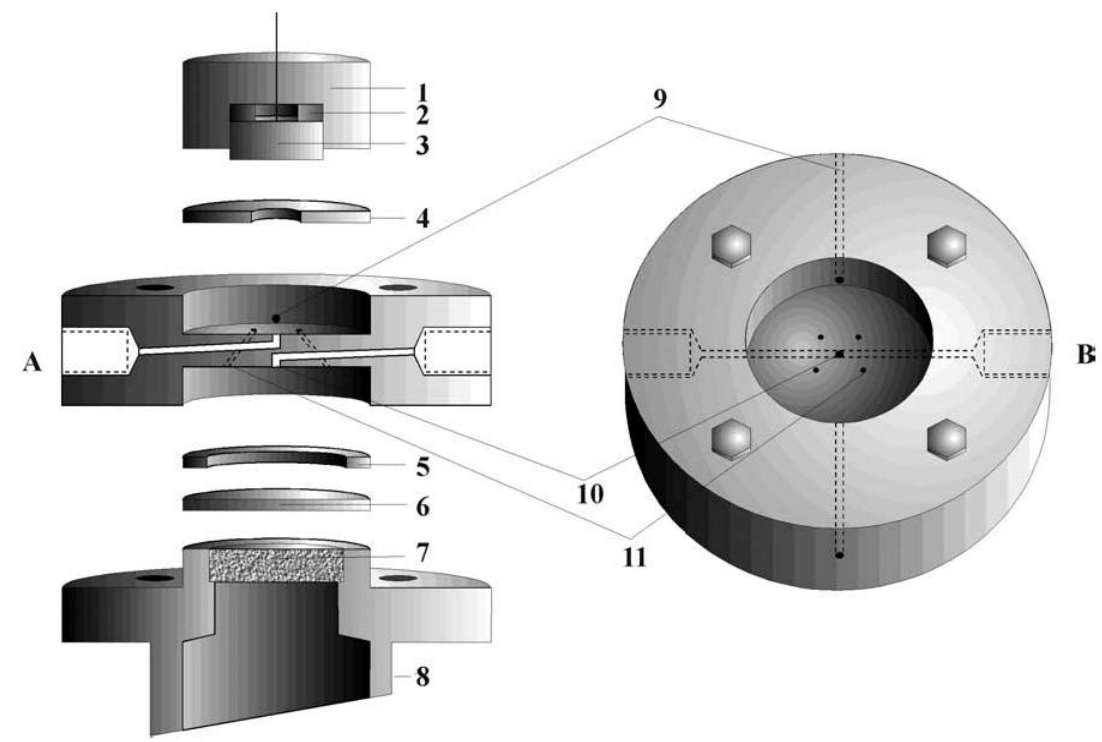

Figure 2-4: A drawing of the dual thin-layer flow cell (Reproduced by permission of The Electrochemical Society) [28]. (1) Kel-F support, (2) Kalrez, (3) electrode, (4) (5) Teflon spacer, (6) porous Teflon membrane, (7) stainless steel frit, (8) stainless steel connection to MS, (9) capillaries for flushing with Ar, (10) inlet-outlet capillaries, (11) connecting capillaries. (a) Cross-section and top-view (b) of Kel-F cell body. The separation of the WE and membrane interface into two separate compartments avoid the problems associated with the original thin-layer design during the study of electrochemical reactions under electrolyte flow conditions.

The cell was initially developed to incorporate an electrochemical quartz crystal microbalance (EQCM), for combined DEMS and EQCM studies [28] but has since then, also been used to combine DEMS with attenuated total reflectance fourier transform infrared spectroscopy (ATR-FTIR) $[44,45]$. In contrast to the thin-layer flow cell described previously, the WE and membrane are now divided into two separate thin-layer compartments: a WE compartment and a membrane interface sampling compartment. As a result, two key problems associated with the thin-layer DEMS cell are avoided: firstly, the bulk concentration of gaseous and volatile reactants at the WE are no longer depleted by evaporation through the membrane, and secondly the cell collection efficiency is enhanced to $\sim 0.4$ under electrolyte flow conditions [34]. This is a consequence of the increased retention time of the electrolyte over the membrane interface. The dual thin layer 
cell has been typically constructed with either passivated titanium or PCTFE (Kel-f).

The dual-thin layer cell design is better suited to the monitoring of continuous faradic reactions, with controlled hydrodynamics in the determination of reaction product formation rates and turn over frequencies. A drawback of the dual thinlayer flow cell, however, is a comparably slow response time, often ca. 2 s [46] or more because of the finite time required for the reaction products to be transported to the sampling compartment. Furthermore, the flow of electrolyte over the WE surface is not uniform and static regions exist making this particular cell difficult to compare to common hydrodynamic cells [47]. Finally, the thinlayers and capillaries linking the $\mathrm{CE}$ and $\mathrm{WE}$ to the $\mathrm{RE}$ are also of high resistance, which can introduce WE potential control issues, and the DEMS setup has required a modified 3-elecrode electrochemical half- cell setup which incorporates two $\mathrm{CE}$ coupled via an external resistor [46, 48, 49].

The advantage of the dual thin-layer flow cell design is the ability to now study continuous faradaic reactions under electrolyte flow conditions, avoiding the limitations of the thin-layer cell, namely the depletion of electrochemical reactants through the membrane interface and poor collection efficiency under flow conditions. 


\subsubsection{Capillary Inlet}

An alternative approach to positioning the WE close to the membrane interface, is to position a PTFE membrane covered capillary inlet vacuum interface close to WE [50-53]. The capillary inlet (also known as the pinhole inlet) DEMS technique is illustrated in figure 2-5.

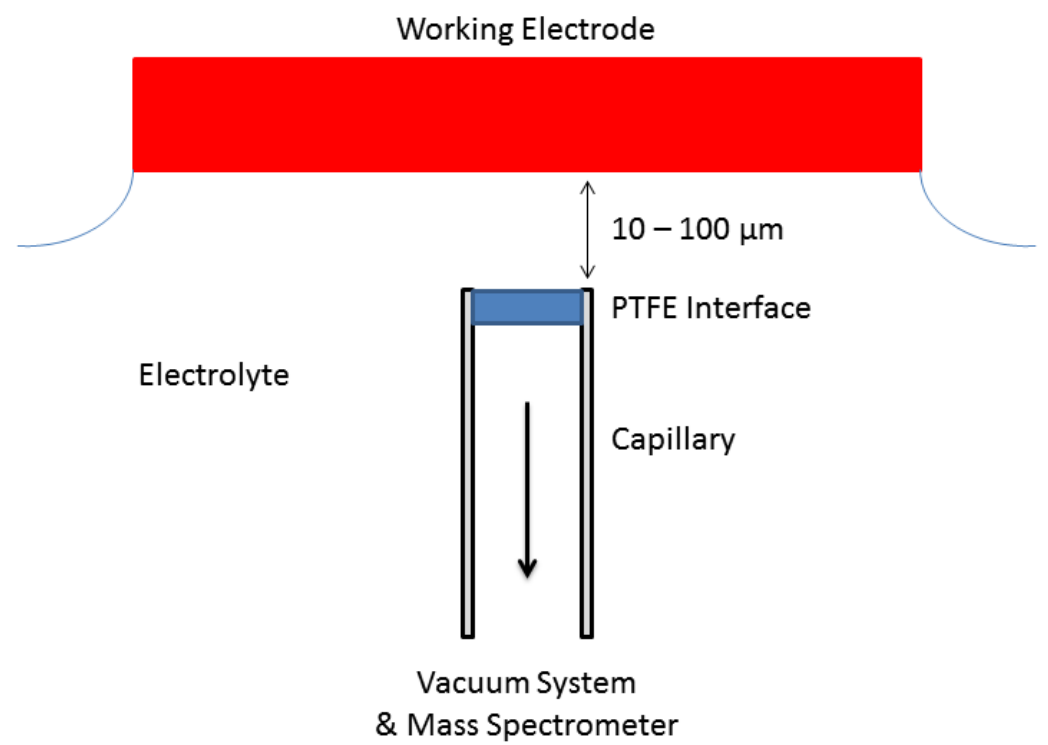

Figure 2-5: An illustration of the capillary inlet DEMS electrochemical cell. The microporous PTFE membrane is incorporated into the end of a capillary whose interface is then position closely to the WE surface. This allows the study of either (i) single-crystal electrodes in a hanging meniscus configuration, or (ii) multiple electrode arrays.

The inlet consists of capillary whose tip is covered by [50], or imbedded with [51] a microporous PTFE membrane material. The capillary, for example, consists of a $0.6 \mathrm{~mm}$ diameter glass tubing with an $0.3 \mathrm{~mm}$ inlet diameter which is positioned between 10 and $20 \mu \mathrm{m}$ away from the electrode surface [51]. Electrochemical reaction products which are formed close to the capillary inlet may then be either sampled by the membrane interface or simply diffuse away into the electrolyte. The quantity of electrochemical reaction species entering the vacuum system is therefore inherently significantly smaller compared to the regular membrane interface. The use of a capillary inlet was also extended in the multi-electrode scanning DEMS (SDEMS) cell allowing localised monitoring of reaction products over an electrode array $[52,53]$. Here, the capillary inlet is attached to a three- 
dimensional piezoelectric-driven positioning system so that it may be moved along the length of a band of electrodes of different type or composition for catalyst screening. Here the capillary inlet consisted of a PTFE capillary tube, $0.15 \mathrm{~mm}$ in diameter with $20 \mathrm{~nm}$ pore Gore-Tex membrane, and was typically positioned $100 \mu \mathrm{m}$ away from electrode [52].

The advantages of the capillary inlet system is essentially that DEMS can be used in combination with single crystal electrodes in the hanging meniscus configuration $[50,51]$ whereas the SDEMS system allows either a large number of electrode samples to be screened within a single experiment, or a particularly large electrode surface to be spatially mapped $[52,53]$. The use of a rather small membrane surface area and capillary inlet also offers some advantages in terms of the DEMS vacuum system design, which can be of a simpler because of a lower inherent gas flux through the capillary, although this will be discussed later in section 2.2.3. There are, however, a number of serious limitations. For instance, only a small fraction of the electrochemical reaction product is sampled resulting in an inherently lower sensitivity. Furthermore, the capillary inlet interface samples from a larger volume than the cylindrical volume between the capillary inlet and electrode surface, and the amount of electrochemical reactants that enter the membrane capillary is sensitive to the capillary inlet position relative to the electrode surface. This perhaps makes calibration and quantitative experimentation somewhat tricky. The superposition of the products planar diffusion away from the surface and the spherical diffusion to the capillary may also lead to complicated time dependence and longer response times [34]. Finally, the capillary inlet would be inappropriate for experimentation requiring convection over the electrode surface. 


\subsubsection{Membrane Interfaces}

In DEMS a microporous PTFE membrane is used to interface the aqueous electrolyte of the electrochemical cell with the high vacuum required by the mass spectrometer. The membrane acts as a selective barrier, preventing the passage of aqueous electrolyte into the vacuum system whilst allowing electrochemical reactions species to evaporate through into the vacuum to be detected by the mass spectrometer. The process is analogous to the vacuum membrane distillation which is depicted in figure 2-6.

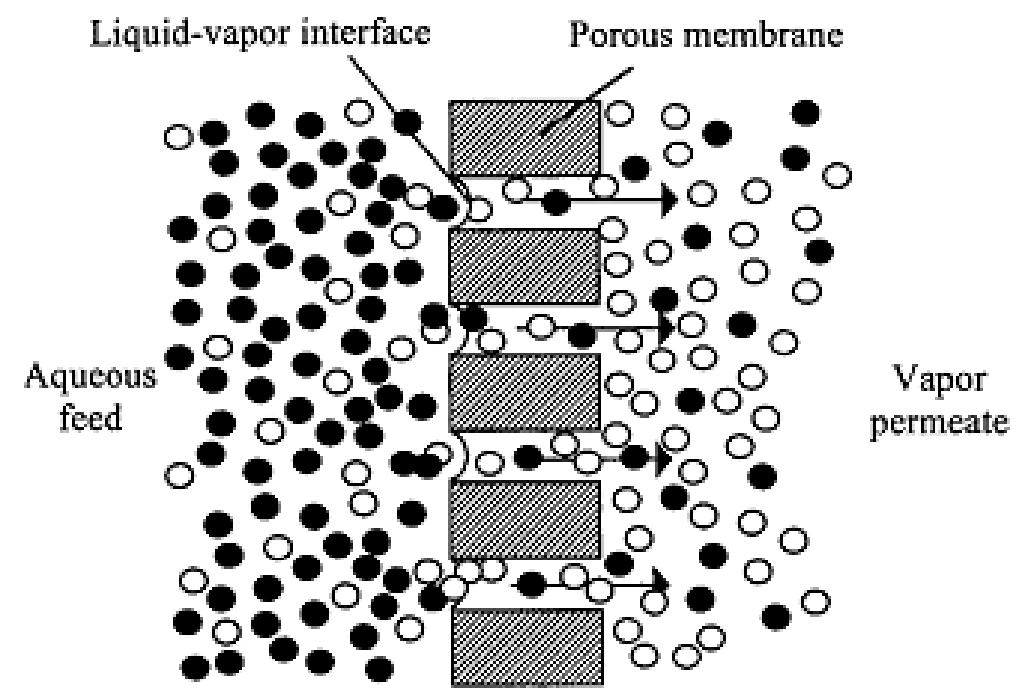

Figure 2-6: An illustration of the vacuum membrane distillation process through a microporous PTFE membrane (Reproduced by permission of Elsevier) [54]. The hydrophobic and microporous nature of the membrane interface prevents the passage of liquid electrolyte whilst allowing dissolved gaseous or volatile species to readily evaporate through the membrane pores.

The hydrophobic properties of the membrane prevent the penetration of the aqueous solution into the pores, resulting in a vapour-liquid interface at each pore entrance. The passive transport of species through the membrane pores is via the vapour phase and is instigated by the pressure drop across the membrane between the hydrostatic pressure of the aqueous electrolyte and the vacuum system of the mass spectrometer. The prevention of liquid transport through the hydrophobic PTFE membrane is rather dependent on a critical parameter, specifically the membrane pore size. For aqueous liquid the hydrophobic membrane must possess a critical pore radius of $<0.8 \mu \mathrm{m}$ in order to prevent 
liquid phase transport through the membrane [26]. This is a value determined by the kelvin law and is dependent on the surface tension of the liquid, the contact angle between the liquid and membrane and the applied pressure. For a given pore size, therefore, a critical hydrostatic penetration pressure exists above which the liquid phase will be transported through the membrane. Hydrophobic membranes with a narrow pore size distribution and high level of porosity are therefore preferred for this application [55]. The membrane specifications found in the DEMS literature, when provided, are summarised in table 2-1.

Table 2-1: Details of the DEMS microporous PTFE membrane specifications contained in the scientific literature. In general the pore dimensions are $\leq 0.2 \mu \mathrm{m}$ with thicknesses $\leq 110 \mu \mathrm{m}$.

\begin{tabular}{|c|c|c|c|c|c|}
\hline $\begin{array}{c}\text { Pore } \\
\text { Size/ } \\
\mu \mathrm{m}\end{array}$ & $\begin{array}{c}\text { Thickness/ } \\
\boldsymbol{\mu m}\end{array}$ & $\begin{array}{c}\text { Porosity/ } \\
\%\end{array}$ & Supplier & $\begin{array}{c}\text { Geometric } \\
\text { Area/ } \\
\mathbf{c m}^{2}\end{array}$ & $\begin{array}{c}\text { Literature } \\
\text { Source }\end{array}$ \\
\hline $\mathbf{0 . 2}$ & $\mathbf{6 0}$ & $\mathbf{5 0}$ & Scimat & - & {$[32, \mathbf{4 4 , 4 6 , 5 6 , 5 7 ]}$} \\
\cline { 1 - 1 } $\mathbf{0 . 1 7}$ & $\mathbf{6 0}$ & $\mathbf{5 0}$ & - & - & {$[58-60]$} \\
\hline $\mathbf{0 . 0 2}$ & 110 & - & $\begin{array}{c}\text { Schleicher } \\
\text { \& Schuell }\end{array}$ & - & {$[33,61]$} \\
\hline $\mathbf{0 . 0 2}$ & $\mathbf{5 0}$ & - & - & 1.13 & {$[28]$} \\
\hline $\mathbf{0 . 0 2}$ & $\mathbf{7 5}$ & $\mathbf{5 0}$ & Gore & $\mathbf{0 . 3 9}$ & {$[\mathbf{5 9}, \mathbf{6 2}]$} \\
\hline
\end{tabular}

In practice, the PTFE microporous membranes generally used in DEMS are typically $\sim 0.02 \mu \mathrm{m}$ in size with a thickness between 50 and $110 \mu \mathrm{m}$. By using even smaller membrane pore sizes molecular flow in the pores is ensured, and the influence of isotropic effects during the gas transport are avoided [26, 63]. Almost all studies using DEMS have been performed in aqueous electrolyte with the exception being studies in organic electrolytes such as propylcarbonate (PC), dimethylsulfoxide (DMSO) [64] and dimethylcarbonate [38] which prove to be non-wetting to the DEMS microporous PTFE membrane. There are of course also other membrane materials which may be suited to DEMS such as polypropylene and PVDF, although these are unlikely to possess any advantages in consideration of the superior durability, and chemical resistance of PTFE. Microporous PTFE membranes meanwhile, are generally widely available in flat and capillary configurations which can be unsupported; however, the PTFE membrane is more commonly laminated onto a nonwoven polypropylene support web to improve strength and handling. 
The use of a PTFE microporous membrane does impose certain limitations on the type of species that may be detected. In order for a species to readily evaporate through a PTFE membrane, it must be either gaseous or volatile, and relatively non-polar [34]. Although the closely related MIMS technique may employ a wide variety of membrane material, DEMS has so far exclusively used a microporous PTFE membrane interface because of a number it its properties, namely its robust and reliable hydrophobic character, mechanical stability, durability and chemical resistance in the electrochemical environment.

Despite the non-wetting properties of the PTFE microporous membrane, a significant quantity of aqueous electrolyte is still able to evaporate through the membrane pores and into the vacuum system owing to the significant pressure gradient across the membrane. It is therefore crucial that a reasonably sized vacuum system can sufficiently pump the continuous flux of gas penetrating the membrane and achieve the operating pressures required by the QMS. This parameter, however, is not a typical specification of PTFE membranes which are for instance, more commonly employed for filtration in HPLC or woven into high performance clothing. There are some literature values for the total gas flux value of a PTFE membrane, which was calculated to be $0.09 \mathrm{mbar}^{\mathrm{l}} \mathrm{s}^{-1}$ at $20{ }^{\circ} \mathrm{C}$ assuming a water vapour pressure of 23 mbar, and $0.4 \mathrm{mbar}^{-1}$ at elevated temperature of $40{ }^{\circ} \mathrm{C}$ [34]. Values other than these are rather scarce, and presumable depend on the exact specifications of the membrane i.e. pore size and thickness.

Finally, a membrane is not the only method of interfacing an electrochemical cell with a mass spectrometer. For example, a capillary had been used to sample the gas above a closed electrochemical cell [65] or thin-layer flow cells have been combined with electrospray mass spectrometry [66-68] and inductively coupled plasma atomic emission spectroscopy (ICP-AES) for trace metal analysis [69]. The techniques, however, do not offer the time and potential resolved capabilities of DEMS. 


\subsubsection{Vacuum Systems \& Mass Spectrometer}

Despite that the hydrophobicity and pore size of the microporous PTFE membrane prevents the transport of liquid water; a considerable quantity is still able to evaporate through the membrane pores into the vacuum system. Consequently, a steady flux of gas $\sim 0.09 \mathrm{mbar} 1 \mathrm{~s}^{-1} \mathrm{~cm}^{-2}$ that largely consists of water vapour (in addition to gaseous, volatile and non-polar species) enters the vacuum system [34]. The requirement of the vacuum system is to therefore pump at such a rate, that the necessary operating pressure of the mass spectrometer is obtained, typically $<1.0 \mathrm{E}^{-5}$ mbar. The exact design of the vacuum system can have important implications on the performance of the DEMS instrument, both in terms of pumping efficiency and mass spectrometer sensitivity [46]. However, the specifics of the DEMS vacuum system such as exact the operating pressures, membrane gas flux are seldom presented or discussed in the literature and the vacuum system is often simply described as being differentially pumped.

Differentially pumped vacuum systems are not specific to DEMS but are generally useful when requiring to measure trace constituents of gas at relatively high pressure, for example 1 mbar, using a mass spectrometer that must operate at $<10^{-5}$ mbar vacuum pressure. The differentially pumped vacuum system essentially consists of two or more, individually pumped vacuum chambers that are connected by a small aperture or skimmer. To illustrate why a single vacuum system is not sufficient, we can consider a reasonably sized vacuum system with a pumping speed of $300 \mathrm{l} \mathrm{s}^{-1}$ which must operate at $10^{-5}$ mbar pressure. The total gas flux that can be pumped by this system is $3 * 10^{-3}$ mbar $1 \mathrm{~s}^{-1}$. In order to interface this vacuum system to an electrochemical cell via a microporous PTFE membrane with a total gas flux of $0.09 \mathrm{mbar}^{-1} \mathrm{sm}^{-2}$ then the acceptable membrane interface geometrical area is $0.033 \mathrm{~cm}^{-2}$. Although a single pumping stage may be sufficient for the capillary inlet DEMS vacuum system [51], such as small membrane surface area is rather impractical for the classic and thin-layer DEMS cells, which employ somewhat higher geometrical areas $\sim 1 \mathrm{~cm}^{2}[28,40]$. In order to allow a larger membrane interface geometric area and therefore gas flux, a differentially pumped vacuum system must be employed. By using a vacuum system with two chambers, and allowing a relatively high vacuum 
pressure of $\sim 10^{-3}$ mbar in the first pumping chamber, with a pumping speed of ca. $901 \mathrm{~s}^{-1}$ a total gas flux of $0.09 \mathrm{mbar} 1 \mathrm{~s}^{-1} \mathrm{~cm}^{-2}$ can be pumped, allowing a more practical membrane geometric area of $\sim 1 \mathrm{~cm}^{2}$. The pressure in the $2^{\text {nd }}$ chamber, containing the QMS therefore, may be determined by controlling the conductance of gas between the $1^{\text {st }}$ and $2^{\text {nd }}$ pumping chamber using an aperture, in order to obtain the necessary operating pressure of the mass spectrometer $<10^{-5}$ mbar. For example, a $200 \mathrm{l} \mathrm{s}^{-1}$ pumping speed in the second chamber would allow an operating pressure of the mass spectrometer of $10^{-5} \mathrm{mbar}$ to be achieved using an aperture with a conductance of $\sim 2 \mathrm{mbar}^{1} \mathrm{~s}^{-1}$. A circular aperture with a radius of $0.85 \mathrm{~cm}$ would therefore be acceptable, and can be easily machined.

Previous DEMS instruments vacuum systems have employed a number of different types of pumps for the $1^{\text {st }}$ chamber, such as a rotary vane pump $[33,70]$, diffusion pump [36], or turbo pump [34] for the first pumping stage, however, all instruments used a turbo pump for the second pumping stage containing the mass spectrometer. It has been claimed that a higher sensitivity of the DEMS arose from positioning the ion source between the first and second DEMS chambers [46] although exact details were not given but presumably a higher pressure at the ion sources increases the number of ions produced relative to the vacuum system background. Once a differentially pumped vacuum system has been constructed and can accept a certain gas flux, the vacuum system is not necessarily limited to DEMS, as highlighted in a previous instrument [70], whereby the DEMS vacuum system and QMS was used in combination with thermo-gravimetric analysis. For the related MIMS technique, however, differentially pumped vacuum systems are generally not required because total gas fluxes through the membranes are much smaller owing to the different non-porous membrane types used [59]. 


\subsection{Research Applications}

The purpose of this section is to briefly analyse the types of experiments that may be performed using DEMS, and highlight when certain DEMS electrochemical cells are favoured.

\subsubsection{Radio-Isotope Labelled Experimentation}

The very first DEMS study using radio-isotope labelled reactants was entitled simply 'Does the oxide layer take part in the oxygen evolution reaction on Platinum' [71] and aimed to elucidate whether or not the PtO layer formed on Pt electrode $>0.9 \mathrm{~V}_{\mathrm{RHE}}$ was involved in the oxygen evolution at potentials $>1.5 \mathrm{~V}_{\mathrm{RHE}}$ according to the following reaction:

$$
2 \mathrm{H}_{2} \mathrm{O}+\mathrm{PtO}_{(\text {bulk })} \rightarrow \mathrm{O}_{2}+4 \mathrm{H}^{+}+4 \mathrm{e}^{-}+\mathrm{PtO} \mathrm{O}_{(\text {bulk })}
$$

This study nicely demonstrates the power of DEMS using radio-isotope labelled reactants, employing the classic DEMS cell. Although Pt electrodes possess relatively poor activity toward the oxidation of water, it is considered to be a model electrode useful for fundamental studies on the oxygen evolution reaction (OER) mechanism, and there was some debate concerning the role played by the Pt oxide layer. By using $\mathrm{H}_{2}{ }^{18} \mathrm{O}$ to form an oxide layer of $\mathrm{Pt}^{18} \mathrm{O}$ species, the DEMS instrument could be used to observe whether the $\mathrm{O}_{2}$ evolved during the oxidation of $\mathrm{H}_{2}{ }^{16} \mathrm{O}$ contained any ${ }^{16} \mathrm{O}^{18} \mathrm{O}$ species in circumstances where the oxide layer does take part, or if only ${ }^{16} \mathrm{O}^{16} \mathrm{O}$ species are formed. In this study, only ${ }^{16} \mathrm{O}^{16} \mathrm{O}$ species were observed, indicating that the oxide is not consumed during the OER. In this case the classic DEMS cell was quite suitable because the experiment purely concerned the fundamental study of a surface reaction and does not require the use of more technical bulk electrodes, and that the cell could be optimised to possess a small volume using little of the expensive radioisotope labelled water. 


\subsubsection{Characterisation of Organic Adsorbates}

The first application of the thin-layer DEMS cell, which allowed the use of smooth electrodes by separating the WE from the membrane interface, was used to study the cathodic desorption and hydrogenation of benzene to cyclohexane on an annealed $\mathrm{Pt}$ electrode, in $0.5 \mathrm{~mol} \mathrm{dm}^{-3} \mathrm{H}_{2} \mathrm{SO}_{4}$ [40]. The general procedure for this type of study is described by the following process:

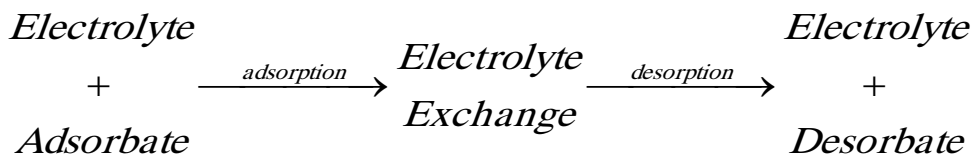

These types of experiments involve adsorbing a certain reaction species, such as benzene at $0.5 \mathrm{~V}_{\mathrm{RHE}}$, which is saturated in the starting cell electrolyte. The electrolyte is then exchanged with the pure electrolyte removing the saturated, whilst maintaining the WE potential at a fixed value. The WE potential is then swept to a potential where the pre-adsorbed species desorbs from the WE surface. In this study, it was observed that benzene partially desorbs without reaction in the $H_{\text {upd/ads }}$ potential region, however, at more negative potentials where the hydrogen evolution reaction takes place on $\mathrm{Pt}$, the benzene is hydrogenated and desorbs in the form of cyclohexane [40]. In adsorption and then desorption measurement such as these, where no convection of the electrolyte is require during the electrochemical experimentation (except to exchange electrolytes), the thin-layer cell is very suitable and can incorporate almost all types of technical electrode. Finally, in addition to benzene there are numerous other desorption studies such as acetone [72], bicyclic aromatics [73, 74], ethene [31, 41] systems, each employing the thin-layer cell.

\subsubsection{Study of the Electro-oxidation of Small Organic Compounds}

One of the first ever DEMS studies focused its attention towards the study of the MOR which is of fundamental research interest for DMFCs [62]. Since then the MOR [32, 46, 56, 75, 76] and other potential organic fuels that are oxidised to carbon dioxide, such as ethanol [57, 77-79] have been quite extensively studied 
using DEMS on a number of electrode systems. The overall MOR may be described by the following reaction formula:

$$
\mathrm{CH}_{3} \mathrm{OH}+\mathrm{H}_{2} \mathrm{O} \stackrel{\mathrm{E}}{\longrightarrow} \mathrm{CO}_{2}+6 \mathrm{H}^{+}+6 e^{-}
$$

However, it was long suspected that the MOR does not reach completion based on strange dependences of the MOR current on the convection of electrolyte using conventional RDE techniques [80]. This assumption could, however, not be confirmed using standard RDE techniques alone because only a single faradaic electrode current is observed. In contrast, DEMS is able to monitor in-situ both the consumption of methanol [81], and the generation of partially oxidised intermediates $[82]$ and carbon dioxide $[32,46,56,75,76]$ allowing the calculation of the MOR current efficiencies and study of the reaction mechanism. The dual-thin layer flow cell is particularly suitable for the study of the MOR under flow conditions owing to its advantages over the thin-layer flow cell during experiments using electrolyte convection i.e. there is less depletion of reactant in the electrolyte along the flow vector, and higher electrode collection efficiencies. 


\subsection{Conclusions}

The intention of this chapter was to provide an overview of the literature concerning the DEMS instrument, with particular focus on previous design solutions and their respective advantages and limitations, prior to the design of the DEMS instrument to be constructed in this thesis. The DEMS instrument requires the custom design and fabrication of three parts: the electrochemical half-cell, membrane interface and vacuum system.

Various DEMS electrochemical cell designs have appeared over the years with varying degrees of success. In recent years, few publications have appeared using the classic DEMS electrochemical cell that deposited the electrode material in the membrane interface, primarily because electrochemists today are rather more interested in the study of smooth electrode surfaces which cannot be incorporate into the microporous PTFE membrane and must therefore be separated. The most implemented DEMS cell design of the past 10 years or more, are the thinlayer, and in particular, the dual-thin layer electrochemical flow cells. In these designs the electrode and membrane are separated via either a thin-layer layer of electrolyte, or by placing them in two separate thin-layer compartments. These cells allow the use of bulk electrodes with sufficient sensitivity, whereby the thinlayer cell is favoured under static electrolyte conditions, whilst the dual-thin layer cell is more suited to continuous flow experiments. In general, the requirements of the electrochemical cell is to incorporate the electrode and vacuum interface of choice, allow controlled electrochemical experimentation at the WE, transport electrochemical products to the vacuum interface and obtain sufficient time and potential resolution.

All DEMS design solutions employ a microporous PTFE membrane in order to interface the aqueous electrolyte of the electrochemical cell and the vacuum system of the mass spectrometer. The membrane permeation process is analogous to vacuum membrane distillation. In order to prevent the passage of liquid water, the radius of the membrane pores must be $<0.08 \mu \mathrm{m}$. The use of a PTFE membrane does, however, impose certain restrictions on the electrolytes that may be used, along with the electrochemical reaction species that can be detected, 
because only dissolved gaseous, volatile and relatively non-polar species readily pass through the microporous membrane. Despite the hydrophobic properties of the PTFE membrane, a considerable amount of water still evaporates into the vacuum system[34], requiring a specifically design vacuum system.

The requirement of the DEMS vacuum system is to pump away the gas flux that passes through the PTFE membrane at such a rate that the necessary vacuum conditions required for the mass spectrometer are maintained. In order for this to be achieved using both a sensibly sized vacuum system, and a reasonable membrane interface surface area (or gas flux), a two-stage differentially pumped vacuum system is often employed. Such vacuum systems are not specific to DEMS but are often used when a gas at relatively high pressure $\sim 1$ bar must be measured online using mass spectrometry. Details of the total gas flux through the PTFE membrane, vacuum system and corresponding operating pressures of a DEMS instrument are, however, rather scarce in the literature.

DEMS has been utilised to study a variety of electrochemical reactions on various electrode systems. For instance, the classic DEMS has been used in combination with radio-isotope electrochemical reactants to elucidate the origins of electrochemical surface species. The development of the thin-layer cell for DEMS allowed the study of smooth electrodes and is particularly suitable to the study of electrode adsorption potential induced desorption products which do not require convection. However, convection can be desirable for experiments involving continuous faradaic reactions, such as the electrochemical oxidation of small organic compounds. In such cases the dual thin-layer flow cell is preferred.

Overall, there is no commercially available DEMS instrument and the design of the custom parts must be tailored toward the intended research application of the instrument. 


\section{Design and Construction of the DEMS Instrument}

3.1 Design and Development Process …………………..........................................33

3.2 DEMS Instrument Overview ......................................................................35

3.2.1 Principle Components....................................................................................37

3.2.2 Operating Hardware and Software ...........................................................38

3.4 Electrochemical Half-Cell Setup .....................................................................40

3.4.1 Dual Thin-layer Flow Cell Design .........................................................42

3.5 Membrane Interface ......................................................................................48

3.6 Vacuum System Design .......................................................................................52

3.6.1 Three-Stage Differential Pumping ..........................................................54

3.6.2 Tubular Aperture...................................................................................57

3.7 Instrumentation, Control and Data Acquisition ..........................................61

3.7.1 DEMS Measurement Setup ..........................................................................63

3.7.2 QMS Calibration Setup ................................................................................81

3.7.3 Labview Software Architecture..................................................................86

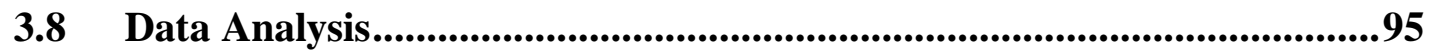

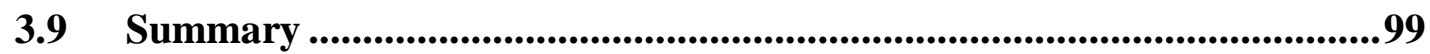

In this chapter, details of the DEMS instrument design and construction are presented. The DEMS instrument construction employs a dual thin-layer electrochemical flow cell and microporous PTFE membrane interfaced to a high vacuum system containing a QMS. The vacuum system design possesses a tubular aperture to control and direct the flux of gas through the cross-beam ion source, whilst a three-stage differentially pumped vacuum construction provided optimum operating pressures of the QMS in order to maximise instrument sensitivity. The actual functionality and usability of the DEMS instrument, however, also relies on additional instrumentation, control and data acquisition hardware and software. This includes the development of custom hardware such 
as the negative impedance device (NID), and custom Labview programmed electrochemical DEMS CV, Macro and QMS calibration software, along with a DEMS data analysis program. Overall, this provided a very capable, reliable and usable DEMS instrument.

\subsection{Design and Development Process}

When starting the design and development of any construction, a number of basic technical requirements must first be considered in order to provide a specific direction in which the project should proceed. In the construction of an instrument such as the DEMS, the intended application of the scientific apparatus was first defined: the key objective of this DEMS instrument was essentially to allow the in-situ observation of electrochemical reaction species, enabling the study and elucidation of electrochemical processes relevant to fuel cell technologies on both model and applied electrocatalysts. There were of course more general requirements considered such as reliability, functionality, performance, life-time and even price. Once these requirements were outlined (where possible) initial design concepts are thought-out, although in the case of the DEMS instrument the concept of interfacing an electrochemical cell with a mass spectrometer to study electrochemical reactions via a membrane interface has existed for some time. There were therefore, details of DEMS design solutions contained within the scientific literature, an overview of which was presented and discussed previously in Chapter 2. These of course aided considerably in the design and development of this instrument, and I am also extremely thankful to Dr. Z. Jusys for our friendly discussions concerning DEMS which offered an early insight into issues that must be considered during the design and development process. Once a design was finalised, a development phase followed in which the cycle of fabrication, evaluation and when necessary re-design or modification was followed until a satisfactory instrument construction was achieved.

This chapter which concerns the design and construction of the DEMS instrument begins with an overview of the whole DEMS instrument. The 
attention is then focused on the more detailed discussion of the designs of the parts more specific to DEMS, namely the electrochemical cell, membrane interface and vacuum system of the QMS. However, rather than beginning each section with a long laborious list of design requirement that are neither pleasant to read or indeed write, the designs themselves are simply presented with a discussion of the important features and critical aspects that were considered. The focus then shifts onto the necessary hardware and software required to operate the DEMS instrument. This largely includes commercial hardware for the electrochemistry, vacuum system and QMS, however, a custom NID was also developed to perform $i R$-compensation of the WE in the DEMS electrochemical half-cell. The majority of the software for the DEMS instrument consists of a number of custom software programs developed in the Labview graphical programming environment, with the exception of the commercial Quadstar 32-bit software required to operate the QMS. These software programs combine with the DEMS hardware in two different measurement setups: the first is for common DEMS experimentation, whereas the second is intended for the calibration of the QMS. These measurement setups are described, along with the conceptual architecture of the custom Labview software programs. The chapter finally ends with a presentation of the DEMS data analysis software architecture. 


\subsection{DEMS Instrument Overview}

The constituents of the overall DEMS instrument can be divided into two main categories: firstly, there are intrinsic DEMS components i.e. the electrochemical flow cell, membrane interface and vacuum system including QMS; and secondly, there are the instrumentation, control and data acquisition hardware and software that are essential to the operation of the DEMS instrument. An overview of the affiliation between the DEMS components, and various hardware and software required for the instrument to function is illustrated in figure 3-1.

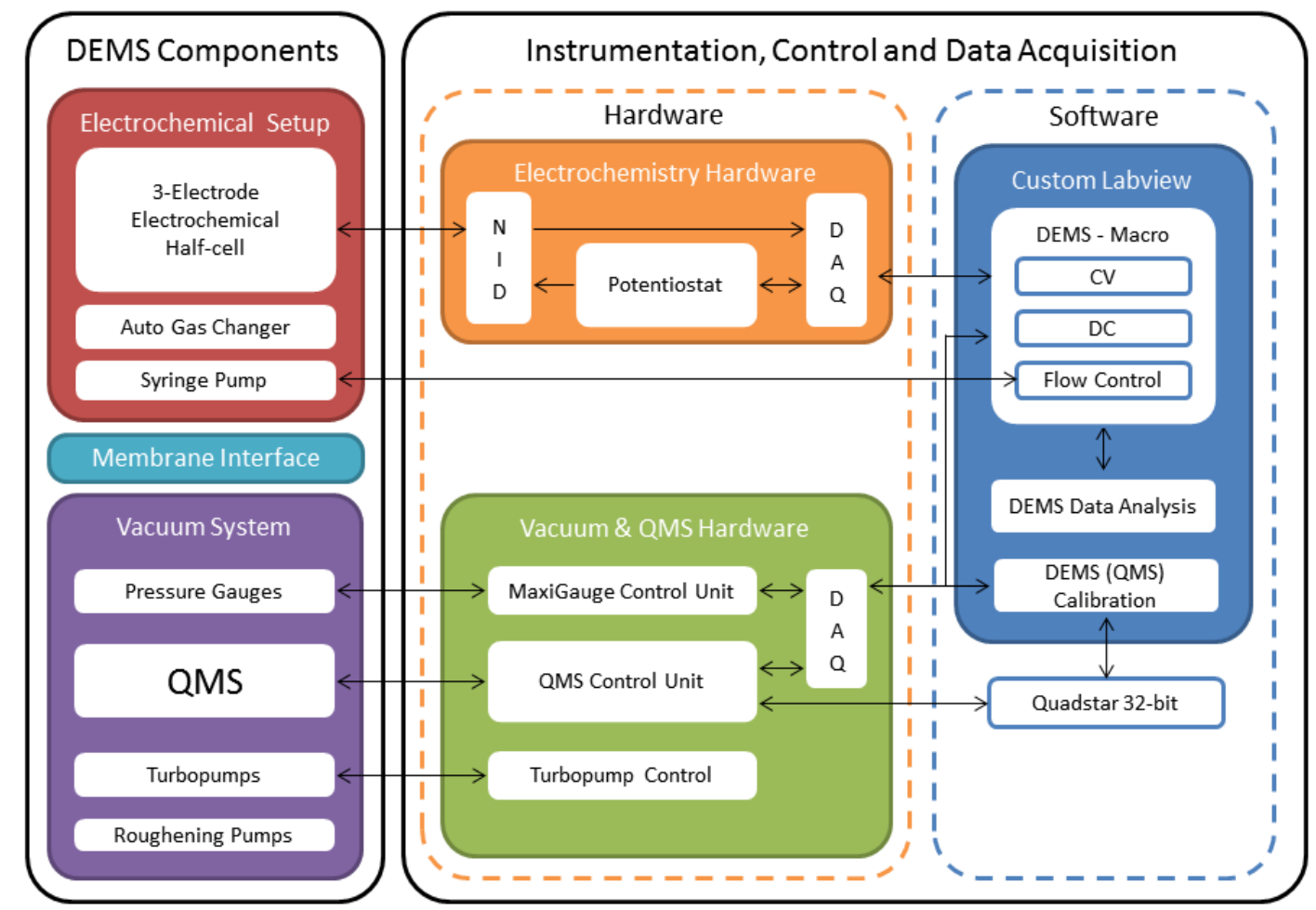

Figure 3-1: A diagram presenting an overview of the various components contributing to the whole DEMS instrument setup. The instrument consists of the key DEMS components, namely the electrochemical cell, membrane interface and vacuum system including QMS. The operation of the DEMS components of course relies on additional instrumental control and data acquisition hardware and software.

Although in DEMS we are essentially combining an electrochemical half-cell with mass spectrometry, they are nonetheless operated and controlled as discrete entities that happen to be (albeit intentionally) interfaced by a membrane. The instrumentation, control and data acquisition hardware is therefore divided up into those that are required to perform and measure the electrochemistry from 
those required to operate and measure the QMS mass ion currents and vacuum system pressures. The control of this instrumentation along with the acquisition and synchronisation of experimental data from the electrochemical, vacuum and QMS hardware is, however, combined using a number of custom programmed Labview software to significantly enhance the functionality and usability of the DEMS instrument facilitating its use as a research tool.

An overview of the actual DEMS instrument is depicted in the photograph in figure 3-2. The location of the electrochemical flow cell, vacuum system, electrochemistry hardware, and QMS and vacuum system hardware are each highlighted.

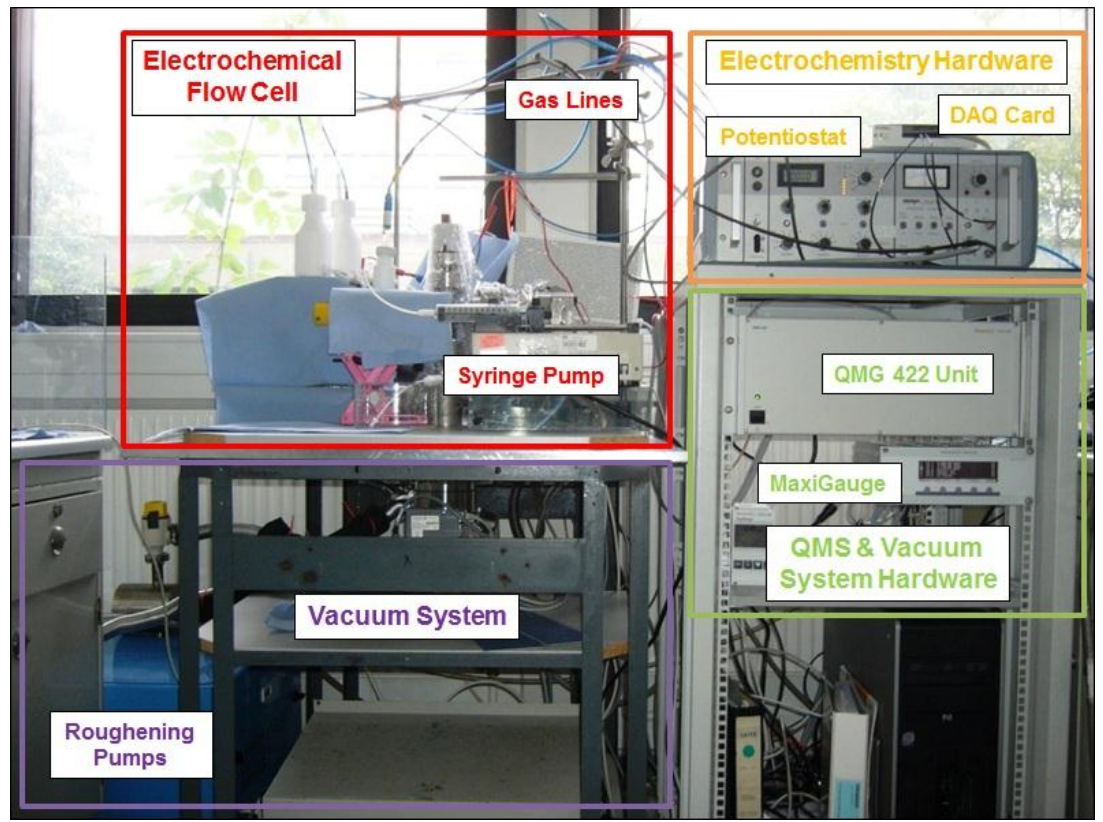

Figure 3-2: Photograph of the DEMS instrument constructed as part of this thesis. The electrochemical cell setup is located on a sturdy bench top (trolley) which serves to house and support the vacuum system and related hardware. Supplementary hardware for the electrochemistry, QMS and vacuum pressure measurement are meanwhile located on a separate equipment rack.

The electrochemical flow cell setup is situated on a sturdy aluminium bench top which also (along with the movable trolley) serves to house and support the vacuum system and turbo pump controllers. The rotary vane pumps required to back the turbo pumps are meanwhile located in a separate sound isolation box located to the left of the vacuum system. The electrochemistry, QMS and vacuum 
pressure control and measurement instrumentation are positioned on a separate hardware rack located to right of the vacuum chamber. These are then controlled by the DEMS user via a combination of the custom Labview DEMS software, and Quadstar 32-bit software on a Microsoft Windows based desktop computer (not shown in figure 3-2).

\subsubsection{Principle Components}

An overview of the principal instrument components which are inherent to DEMS, namely the electrochemical cell, membrane interface and vacuum system including QMS, of the instrument designed and constructed in this thesis is illustrated in figure 3-3.

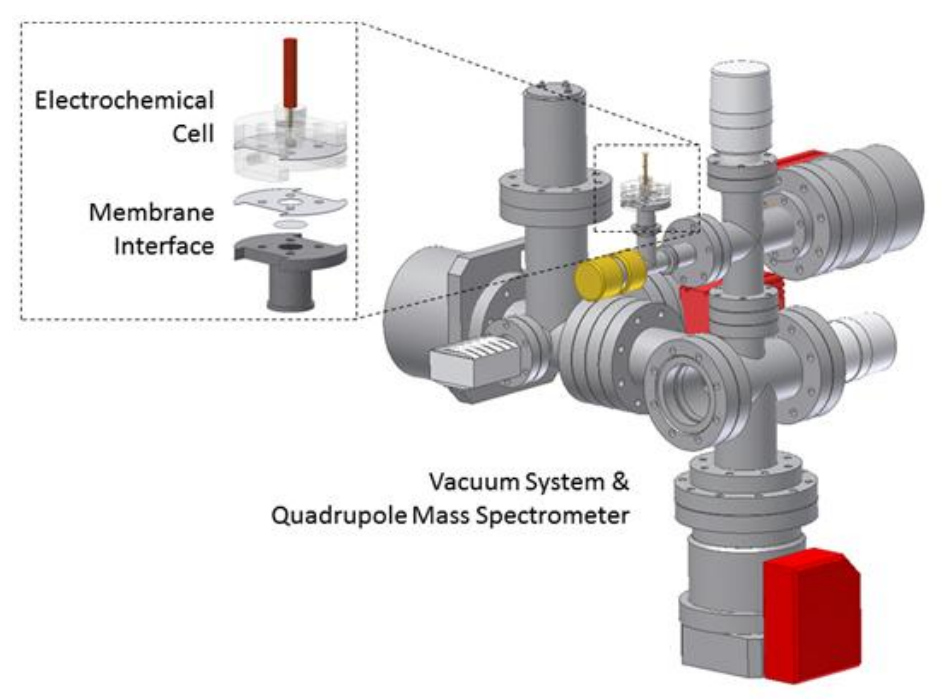

Figure 3-3: A technical drawing of the DEMS instrument construction highlighting the electrochemical cell, membrane interface and vacuum system including QMS. The electrochemical cell incorporating the WE and membrane interface is fixed to the vacuum flange of a leak valve connecting the vacuum system.

The electrochemical cell is of a dual thin-layer flow cell design, incorporating a WE compartment and membrane interface compartment which is attached to the DEMS vacuum system. By employing the cell in a three-electrode electrochemical half-cell setup (not shown in figure 3-3) the potential of the WE can be controlled to study electrochemical reactions. A controlled flow of electrolyte meanwhile 
transports the electrochemical reaction products away from the WE to the membrane interface where they may be sampled into the vacuum system.

The membrane is used to interface the aqueous electrolyte of the electrochemical cell with the high vacuum conditions of the QMS. In DEMS this is achieved using microporous PTFE membrane which is able to sample electrochemical reaction products in a process that is analogous to vacuum membrane distillation. The membrane prevents the passage of aqueous electrolyte whilst allowing dissolved gaseous, volatile and relatively non-polar species to evaporate into the vacuum system of the QMS.

The DEMS vacuum system is used to achieve the high vacuum conditions required for mass spectrometry. In this DEMS instrument, this was achieved using a three-stage differentially pumped vacuum system allowing the total flux of gas that enters the vacuum system from the membrane interface to be pumped at such a rate that the operating conditions of the QMS are maintained. The vacuum system incorporates a commercial Pfeiffer Vacuum QMA 422 quadrupole mass analyser with a $90^{\circ}$ off-axis SEM and cross-beam electron impact ion source (CB-IS). The QMS allows the relevant mass ion currents of electrochemical reaction species which evaporate through the membrane interface to be monitored in-situ with the electrochemistry performed at the WE.

Of course, in order for the electrochemical cell, vacuum system and QMS to function and combine as DEMS, they rely on a number of additional operating hardware and software.

\subsubsection{Operating Hardware and Software}

The DEMS operational hardware required for the electrochemistry includes a Wenking POS2 potentiostat to control and manipulate the potential of the WE during electrochemical experimentation. Precise control of the WE potential also necessitates the implementation of a custom designed and built NID to achieve $i R$-compensation of the solution (or stabilising) resistance: a rather uncommon device amongst electrochemical half-cell setups. Finally, a commercial precision 
syringe pump (KDS 220) was employed in order to control the electrolyte flow rate through the DEMS dual thin-layer electrochemical flow cell.

The vacuum system hardware includes the necessary roughening- and turbopumps, along with the corresponding turbo pump control units, vacuum pressure gauges including MaxiGauge pressure gauge control and measurement unit. The hardware required the QMS operation meanwhile, includes the QMG 422 control unit, Rf-generator and EP 422 electrometer amplifier.

The QMS is operated using the commercial Quadstar 32-bit software provided with the mass spectrometer. The DEMS instrument experimentation, however, is performed using custom Labview software including CV, DC and Macro software. A custom Labview program was further more designed and developed to allow the straightforward calibration of the QMS. Labview is a graphical programming environment that allows engineers and scientists to develop sophisticated measurement, test and control systems. Finally, in order to aid the analysis of DEMS data, custom Labview data analysis software was developed to perform common data treatment tasks that are specific to DEMS. 


\subsection{Electrochemical Half-Cell Setup}

An illustrated overview of the entire electrochemical flow cell setup is given in figure 3-4, that (described from left to right) consists of an electrolyte reservoir(s), CE compartment, the DEMS dual thin-layer flow cell containing WE and membrane interface mounted to the QMS vacuum system, a RE compartment and finally, the syringe pump flow control.

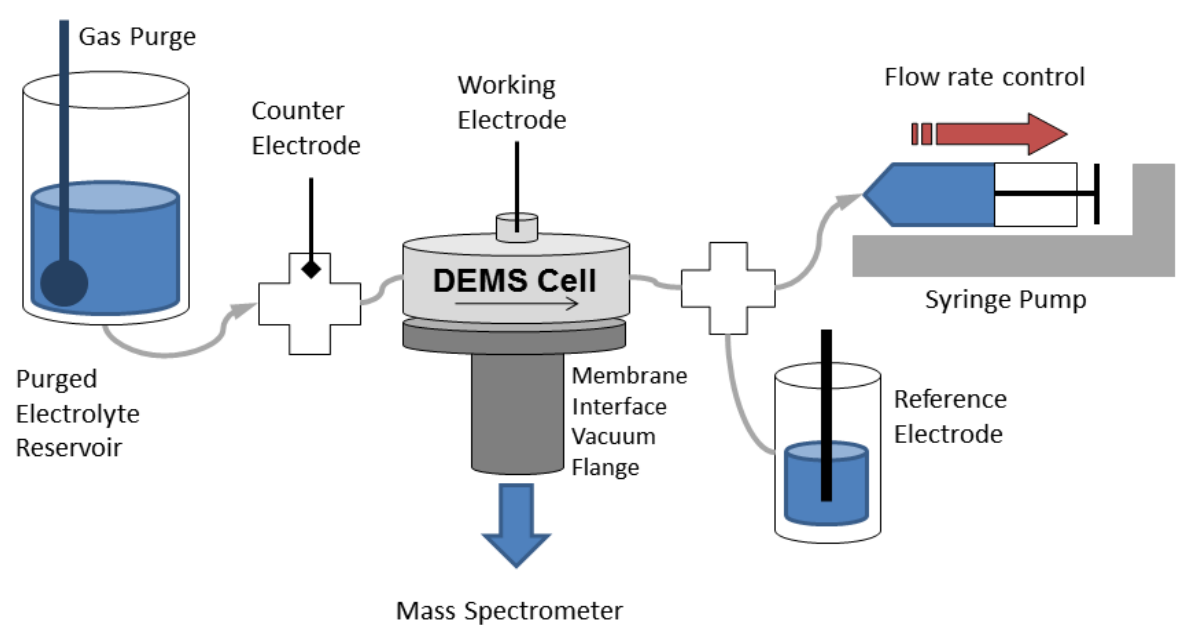

Figure 3-4: A sketch of the three-electrode DEMS electrochemical flow cell setup. The CE and RE separated from the WE and are positioned in separate sealed compartments on the cell inlet and outlet respectively. The electrolyte from a purged reservoir is allowed to flow through the cell at a volumetric rate determined by the syringe pump (operating in withdrawal mode) positioned on the cell outlet.

Each of the electrochemical flow cell components are interconnected using flanged $1.6 \mathrm{~mm}$ internal diameter commercial PTFE tubing with UNF $1 / 4$ inch 28 G fittings, typically used in high performance liquid chromatography (HPLC). The electrolyte reservoir(s) is attached via a PTFE 4-way connection which is also attached to the sealed CE compartment and the DEMS flow cell inlet. Note that the remaining connections of the 4-way flange may either be stoppered, connected to a second fluid reservoir or an electrolyte controlled drain line. The DEMS flow cell outlet is then attached to another 4-way connection, linking the sealed RE compartment and the syringe pump whereby the final connection is stoppered. The purged electrolyte is thus able to flow from the supply reservoir(s), through the DEMS flow cell (over the WE and then membrane interface) and into the 
syringe at a rate determined by the syringe pumping speed. The syringe pump is operated in withdrawal mode, at flow rates lower than the maximum flow of electrolyte through the cell, as determined by the hydrostatic pressure of the supply reservoir(s) and the size of the flow cell capillaries. The syringe pump thus effectively limits the flow of electrolyte, rather than sucking it through the cell. Images of the actual electrochemical flow cell setup are presented in figure 3-5.
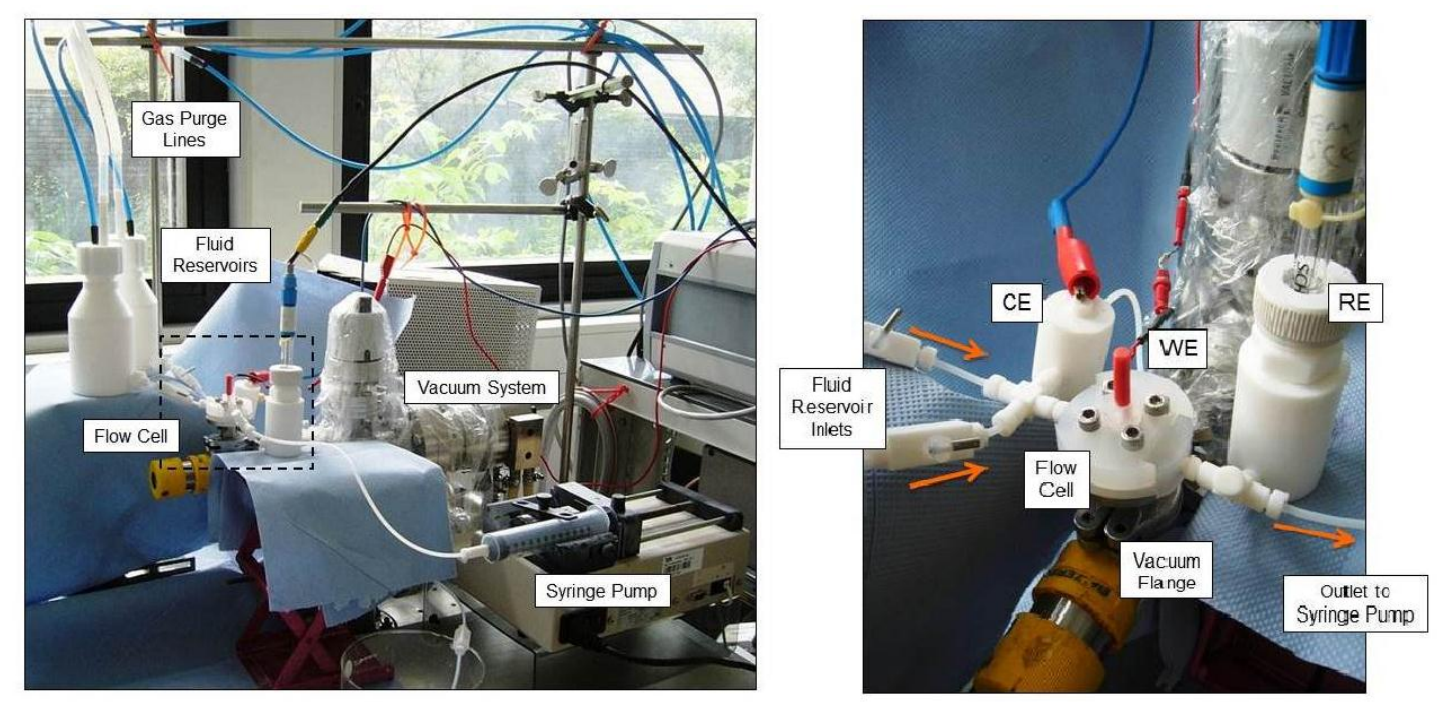

Figure 3-5: Photographs of the DEMS electrochemical flow cell setup constructed as part of this thesis. All cell components are fabricated from either PTFE or Kel-f, and are connected using $1.6 \mathrm{~mm}$ internal diameter PTFE tubing. The electrolyte flow rate is determined by the withdrawal of electrolyte from the gas purged electrolyte reservoirs.

It should be noted that the CE compartment does not lie in the path of the electrolyte between the supply reservoir(s) and DEMS flow cell, which is important in preventing electrochemical reaction products (such as $\mathbf{H}_{2}$ or $\mathbf{O}_{2}$ ) arising from the CE, from contaminating the inlet flow of electrolyte and interfering with the electrochemistry at the WE. For similar reasoning, the saturated calomel electrode (SCE) RE is also located on the exit of the electrochemical flow cell in order to avoid the contaminating effects of chlorides arising from the SCE before the electrolyte contacts the WE [37]. In general, the lengths of all interconnecting PTFE tubing are kept to a minimum with the intention of maintaining the purged state of the electrolyte from reservoir as it flows through the cell. All electrochemical flow cell reservoir(s), CR and RE compartments and connecting tubing, meanwhile, consist of PTFE whereas the 
dual thin-layer flow cell components are Kel-f (PCTFE), both of which are chemically inert to acidic and basic electrolytes that are commonly used in electrochemistry, such as $\mathrm{H}_{2} \mathrm{SO}_{4}$ and $\mathrm{KOH}$.

\subsubsection{Dual Thin-layer Flow Cell Design}

The DEMS electrochemical cell produced in this work is of a modular dual thin-layer flow cell design solution possessing two small (thin-layer) compartments connected via four narrow capillaries: an electrochemical cell volume containing the WE and membrane interface sampling compartment. An exploded overview of the DEMS electrochemical cell design and membrane interface is presented in figure 3-6.

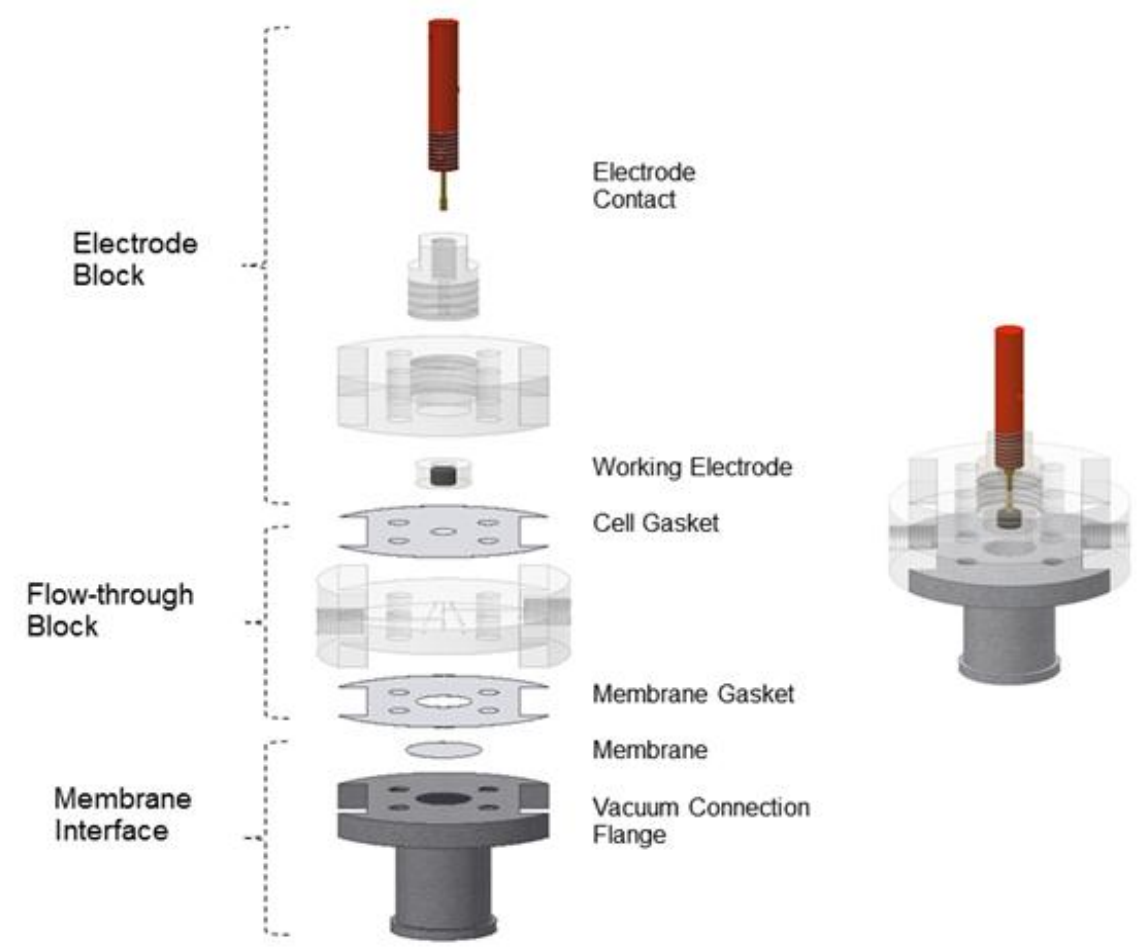

Figure 3-6: Exploded drawing of the modular DEMS electrochemical flow cell design and membrane interface constructed as part of this thesis. The cell components lock together and are tightened and sealed using four M4 bolts which screw into the vacuum flange. The WE and membrane interface compartment volumes are determined by the thickness of the PTFE gaskets. 
This cell construction can be divided into three principle interlocking components: (i) an electrode block, (ii) flow-through block (each fabricated from Kel-f) and (iii) a stainless steel KF vacuum connection flange. When these components are combined with the WE (including Kel-f screw piece), two PTFE gaskets and a microporous PTFE membrane; the electrochemical cell and membrane sampling compartments are formed which are partitioned by the flow-through block (5 mm thick). The cell and membrane gaskets are cut from commercial PTFE sheets, and are typically $0.25 \mathrm{~mm}$ thick. The gasket thickness and size of the holes cut through the centre of each PTFE gasket form and define the dimensions of the two compartments: (i) the electrochemical cell volume that is enclosed by the WE above, and flow-through block below, and (ii) the membrane interface sampling compartment, which is similarly enclosed by the flow-through block above, and microporous PTFE membrane below. The membrane is meanwhile supported by a porous metal stainless steel frit (40 $\mu \mathrm{m}$ pore size, Applied Porous Technologies $^{(}$) embedded in the centre of the vacuum flange connection, providing mechanical support to the membrane when it is exposed to the vacuum. Finally, each of these components are inserted together, fixed and sealed tight using four M4 bolts that screw directly into the vacuum flange.

The modular design presented here is intended to allow a considerable degree of flexibility and/or modification to the DEMS electrochemical cell parameters. Using this modular system, individual cell components can be modified, exchanged or replaced when necessary, without requiring a completely new cell construction. This is particularly beneficial in the cycle of construction, evaluation, modification and when necessary re-design or modification for a new research application, because it reduces the number of parts that are required to be fabricated minimising, time, labour and material costs. More importantly, this modular construction allows for the optimisation of cell parameters, for instance, the PTFE cell gaskets can be exchanged with gaskets of different dimensions to optimise cell volumes, or an alternative flow-through block could be employed to use a different electrolyte flow regime over the WE surface or membrane interface. In the long-term, parts which may deteriorate more quickly in time may also be more easily replaced. 
In regards to the electrolyte flow though the cell, the exact flow regimes over the WE and membrane interface is determined by both the arrangement of the inlet and exit capillaries of the flow-through block, and the shape of the punctured holes of the WE compartment gasket. In this thesis, two dual-thin layer designs were constructed: the dual-cyclone, and dual-linear thin-layer flow cell.

\subsubsection{Dual-Cyclone Flow Block}

The flow of electrolyte through the dual-cyclone thin-layer flow cell, including the flow regime over the WE and membrane interface is depicted in figure 3-7.

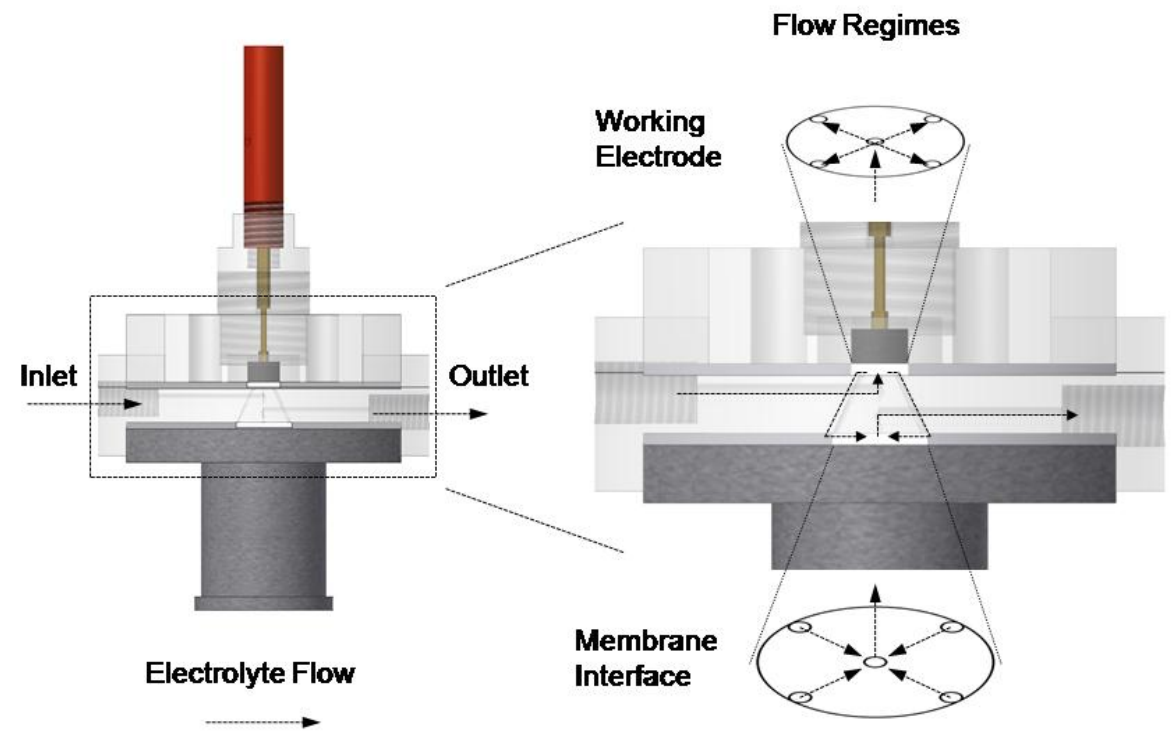

Figure 3-7: Drawing of the electrolyte flow regime through the dual-cyclone thin-layer electrochemical flow cell. The electrolyte flows from the centre of the WE outwards to four capillaries that interconnect the WE compartment with the membrane sampling compartment. The electrolyte flows through the membrane interface sampling compartment via four small capillaries, flowing from the outer edge of the compartment to the centre when it then exits the cell.

In this design, the electrolyte first enters a capillary leading to the WE compartment, where the electrolyte rises from an inlet located in the centre of the compartment. Electrolyte then flows upwards into the centre of the WE and outwards, in a radial fashion before exiting the WE compartment via four exit capillaries. These capillaries then transport the electrolyte towards the membrane 
interface sampling compartment. In contrast to the flow over the WE, the electrolyte enters the sampling compartment from four inlet capillaries located at the perimeter of the volume, and flows towards a central exit capillary that leads to the cell outlet. This flow regime is in principle identical to that described previously [28] and furthermore modelled in [34, 83].

\subsubsection{Dual-Linear Flow Block}

The flow regime through a second flow through module, the dual-linear thin-layer cell is depicted in figure 3-8.

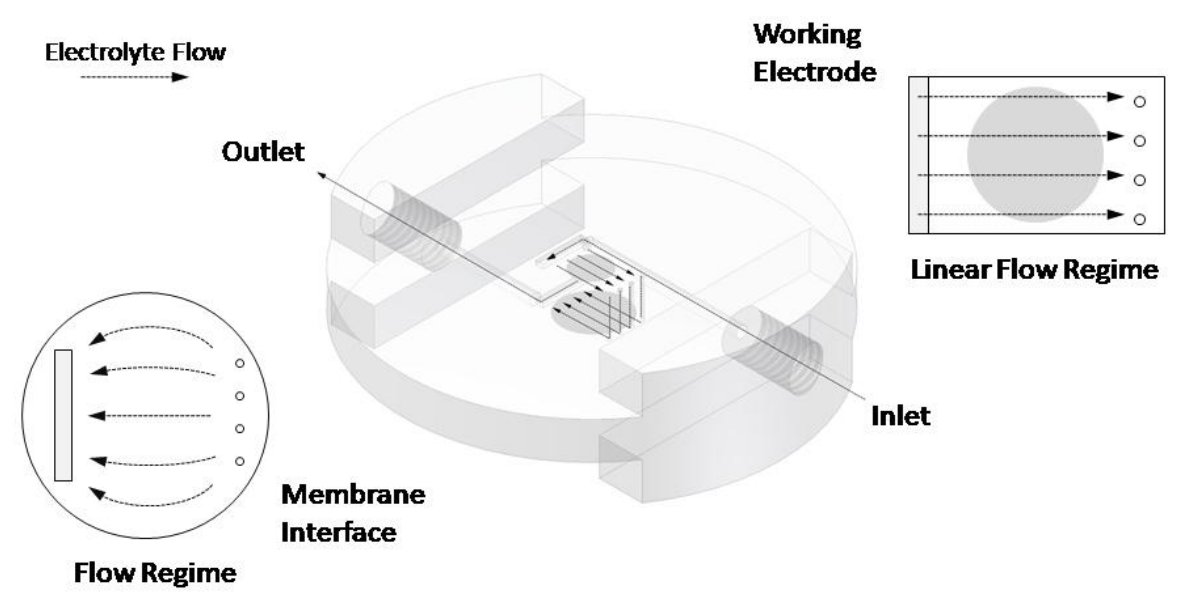

Figure 3-8: Illustration of the electrolyte flow regime through the DEMS linear dual thin-layer electrochemical cell flow through block. The electrolyte first enters a small volume which distributes the flow over the WE surface in a linear fashion. The electrolyte then passes through four capillaries into the membrane interface where it then flows to the cell outlet.

In this flow regime, the electrolyte flows through the inlet into an initial (dead) volume before then flowing into the thin-layer WE compartment in a linear fashion over the electrode surface. The electrolyte then passes through four capillaries, transporting it into the membrane interface thin-layer compartment and passes over the membrane interface before exiting the flow cell. This linear flow cell regime over the WE is not unlike that described previously [84]. The advantage of the linear flow regime is that it is comparatively common in contrast to the dual-cyclone flow regime. Essentially, the flow regime over the linear cell is 
more defined and better understood. The dimensions and volumes of the DEMS cells are summarised in table 3-1.

Table 3-1: Details of the specifications of the two DEMS cell flow regimes. The compartmental volumes are determined by the size and shape of the punctured holes in the cell PTFE gaskets.

\begin{tabular}{|c|c|c|c|}
\hline & & Cyclone & Linear \\
\hline \multirow{3}{*}{ 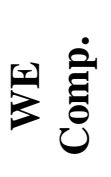 } & Length $x$ Width/ mm & $6(\varnothing)$ & $7 \times 5$ \\
\hline & Thickness/ mm & $\sim 0.2$ & $\sim 0.2$ \\
\hline & Volume/ $\mu \mathrm{l}$ & $\sim 5.6$ & $\sim 7$ \\
\hline \multirow{3}{*}{ 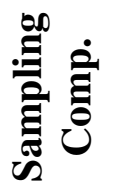 } & Diameter/ mm & $11(\varnothing)$ & $11(\varnothing)$ \\
\hline & Thickness/ mm & 0.2 (-membrane) & 0.2 (-membrane) \\
\hline & Volume/ $\mu \mathrm{l}$ & $\sim 9$ & $\sim 9$ \\
\hline
\end{tabular}

These small volumes and narrow connecting capillaries of the cells are a consequence of a number of design considerations. Firstly, by minimising the compartment and capillary volumes, the quantity of solution available for reaction species that arise from the WE electrode to diffuse away and dilute into is minimised. This therefore maximises the concentration of the analyte species entering the membrane sampling compartment which in turn, helps to enhance the flux of diffusing species to the membrane interface surface whilst minimises the delay between the creation of species at the WE and subsequent detection in the QMS, and the residence time of the species in the electrochemical cell after the electrochemical reaction has ceased. All of these considerations are crucial to the performance of the DEMS instrument which is later characterised in section 4.1. These narrow connecting capillaries and small cell volumes, however, do have a detrimental effect on the electrochemistry (discussed later in section 4.1.1) in terms of potential control and stability. This necessitates the modification of the standard three-electrode electrochemical setup, employing a stabilising resistor and negative impedance device (NID) described in detail later in section 3.7.1.1.3.

To summarise, the modular DEMS electrochemical flow cell design presented here allows controlled electrochemistry at the WE of interest and incorporates a PTFE membrane that interfaces the cell electrolyte with the vacuum of a QMS. 
This is achieved by employing a dual thin-layer flow cell (using either dual -cyclone or -linear flow regimes) of modular design that allows easy modification of cell parameters, the exchange of WE substrate or sample, and replacement or alteration of the cell components, whether that is to modify the electrolyte flow regime or membrane type. The performance and characteristics of the dual-cyclone thin-layer electrochemical cell setup are meanwhile presented and evaluated in section 4.1. 


\subsection{Membrane Interface}

All previous DEMS instruments have utilised a microporous PTFE membrane to interface the electrochemical cell with the vacuum system of the QMS (see Chapter 2 section 2.2.2) and this DEMS instrument is indeed no different simply because microporous PTFE membranes are most suitable for this application. Such materials have a wide range of applications in electrons, pharmaceuticals, laboratories and manufacturing and are consequently commercially available. The key to designing and constructing the membrane interface is to obtain a suitable commercially available membrane material, define an appropriate geometric area and decide how to form and seal the membrane between the electrochemical cell and vacuum flange.

The first and foremost requirement of the microporous PTFE membrane is that it must not be permeable to aqueous electrolyte, which can in principle be ensured by choosing a membrane pore radius $<0.8 \mu \mathrm{m}[26]$. Nevertheless, an appreciable amount of aqueous electrolyte still evaporates through the microporous membrane owing to a significant pressure gradient across the membrane interface, as was highlighted previously in Chapter 2. The pore size itself, however, does not tell us too much about the total flux of gas that will pass through the DEMS membrane interface when exposed to the vacuum, which is actually the crucial parameter required to design the DEMS membrane interface and differentially pumped vacuum system. Unfortunately, the total gas flux through a microporous membrane separating aqueous electrolyte and a vacuum is not a specification of the commercial membranes although it will be determined by the membrane properties i.e. pore size, pore density, thickness, and geometrical area of the membrane, not to mention the temperature of the aqueous electrolyte and pressure gradient across the membrane. The only conceivable approach toward obtaining an appropriate membrane material, therefore, is to source a number of commercial microporous PTFE membrane samples and test their suitability.

A number of potentially suitable commercial microporous PTFE membrane samples were obtained from three manufacturers, whose specifications (namely 
pore size) were chosen based on the details provided in previous DEMS literature which were summarised previously in Chapter 2 section 2.2.2 table 2-1. The manufacturer specifications of the membrane samples obtained for this DEMS instrument are presented in table 3-2.

Table 3-2: Details of the microporous PTFE membrane interface material candidates obtained for this DEMS instrument construction. (Note * refers to Mullen-Hydrostatic values)

\begin{tabular}{|c|c|c|c|c|}
\hline Manufacturer & Ref No. & Pore Size/ $\boldsymbol{\mu m}$ & Thickness/ $\mu \mathrm{m}$ & $\begin{array}{c}\text { Water Entry } \\
\text { Pressure/ kPa }\end{array}$ \\
\hline \multirow{2}{*}{ Gore } & MMT-316 & $\mathbf{0 . 1}$ & $127 \pm 9$ & 4.8 \\
\cline { 2 - 5 } & MMT-335 & $\mathbf{0 . 0 3}$ & $124 \pm 11$ & 16.1 \\
\hline \multirow{2}{*}{ Donaldson } & $\# 1302$ & 0.2 & 89 & $620^{*}$ \\
\cline { 2 - 5 } & $\# 6860$ & 0.4 & 152 & 517 \\
\hline & $\# 6865$ & 1.5 & 152 & 117 \\
\hline Whatman & & 0.2 & - & - \\
\hline Donaldson & $\# 1301$ & $\mathbf{0 . 1}$ & 89 & $8^{*}$ \\
\hline Donaldson & $\# 1314$ & 1.0 & 89 & $140^{*}$ \\
\hline
\end{tabular}

The material obtained from both Gore and Donaldson Membrane Solutions were conveniently available in A4 sheets allowing the desired shape and size of the membrane to be cut out (using a hole-punch, for example). There are then two possible approaches to testing the suitability of the membrane candidates: the first approach, which was taken in this project, is essentially trial and error, whereby an electrochemical cell and vacuum system is designed and constructed, and a range of membrane materials are obtained and tested. Either the electrochemical cell or vacuum system (or even both) is then modified to achieve a balanced system with appropriate vacuum pressures and sensitivity. Another approach would have involved constructing a membrane test setup using a small vacuum chamber, pressure gauge and roughening pump, using which the gas flux through the membrane is determined in an experimental setup similar to that described later in section 3.7.2. Once the gas fluxes have been established, it is then possible to choose upon a suitable membrane geometric area and then design the vacuum system (or vice versa) based on the expected total gas flux. In hindsight this latter approach would have perhaps been somewhat wiser, but came rather as an afterthought. 
The incorporation of the membrane material in the DEMS electrochemical flow cell is illustrated in the electrochemical cell cross-section of figure 3-9. The membrane is located between the PTFE membrane compartment gasket and the vacuum flange, and is positioned so that it covers the porous stainless steel frit entrance to the vacuum chamber. When the electrochemical cell components are combined, fixed and PTFE gaskets compressed using the four M4 bolts which fix into the vacuum flange the membrane sampling compartment volume is formed, as depicted in figure 3-9.

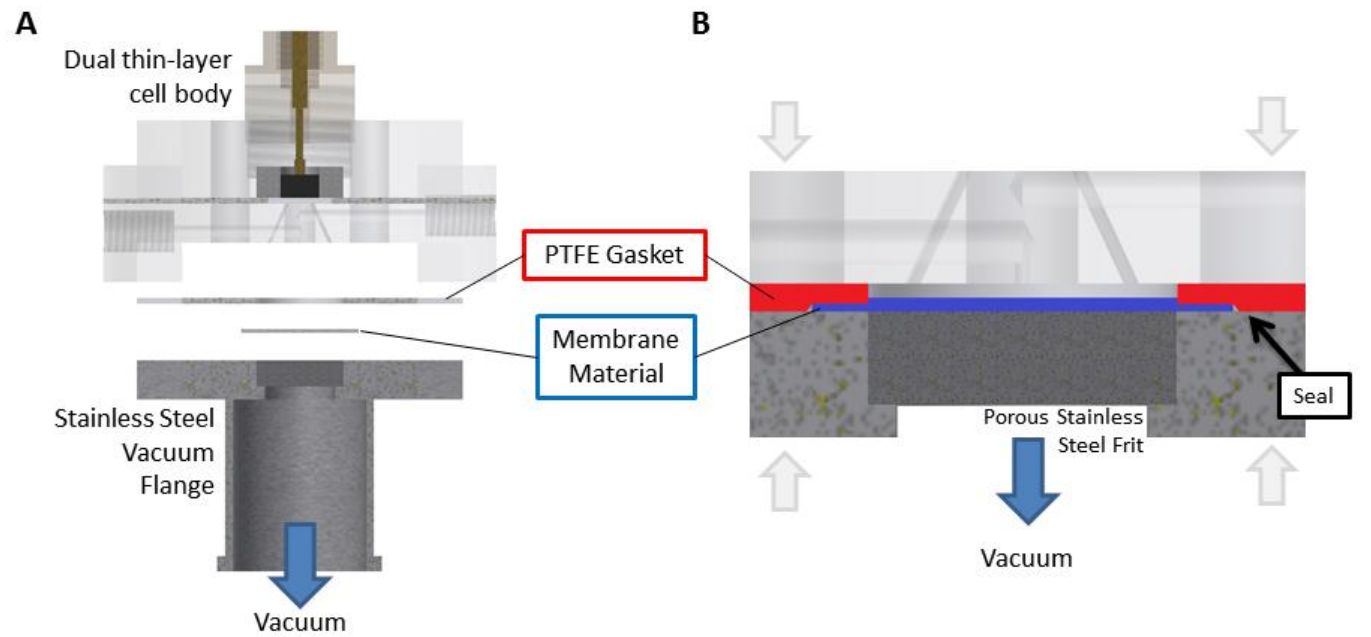

Figure 3-9: An illustration of the membrane interface. The microporous membrane material is mechanically supported by a stainless steel frit, and sealed between the cell flow through block and vacuum flange via a PTFE gasket. When compressed the PTFE sheet forms a gas tight seal around the edges of the membrane material between the PTFE gasket and vacuum flange connection.

In practice there are few crucial points to consider when dealing with the membrane interface: firstly, it is absolutely necessary that the membrane completely covers the porous metal frit to prevent aqueous electrolyte from entering the vacuum chamber. Secondly, it is equally important that the PTFE gasket is compressed against the flange so that it forms a seal around the membranes circumference. The compression of the membrane material against the flange alone is not sufficient because the membrane substrate is still highly gas porous. Any additional gas leaking through the membrane will add to the total gas flux entering the vacuum system resulting in higher pressure and larger QMS mass ion current background signals. The third necessity is that the 
aqueous cell electrolyte completely covers the membrane surface before the DEMS vacuum system is exposed to the membrane so that the vacuum is formed i.e. without the aqueous electrolyte (forming a seal) air will simply pass through the membrane with little or no resistance which could potentially destroy the vacuum equipment.

Ideally, it is desirable to maximise the geometric area of the membrane to volume of electrolyte ration in order to attain as high sensitivity as possible by collecting as much of the electrochemical reaction species. However, the larger the geometrical area of the membrane is, the greater the gas flux will be, and therefore the more vacuum system pumps will be required. 


\subsection{Vacuum System Design}

The purpose of the DEMS vacuum system is to achieve the high vacuum conditions required for the reliable operation of the QMS. In terms of the DEMS instrument, the vacuum system must be capable of sufficiently pumping away the total flux of gas that passes through the membrane interface at such a rate that the operating pressure of the QMS is maintained. The key to constructing a vacuum system of appropriate design, therefore, was to balance the constant flux of gas entering the vacuum chamber via the membrane interface, against the overall pumping speed of the vacuum system. This was achieved by designing and developing the three-stage differentially pumped vacuum system illustrated in figure 3-10, with each of the three chambers highlighted in green, blue and yellow.

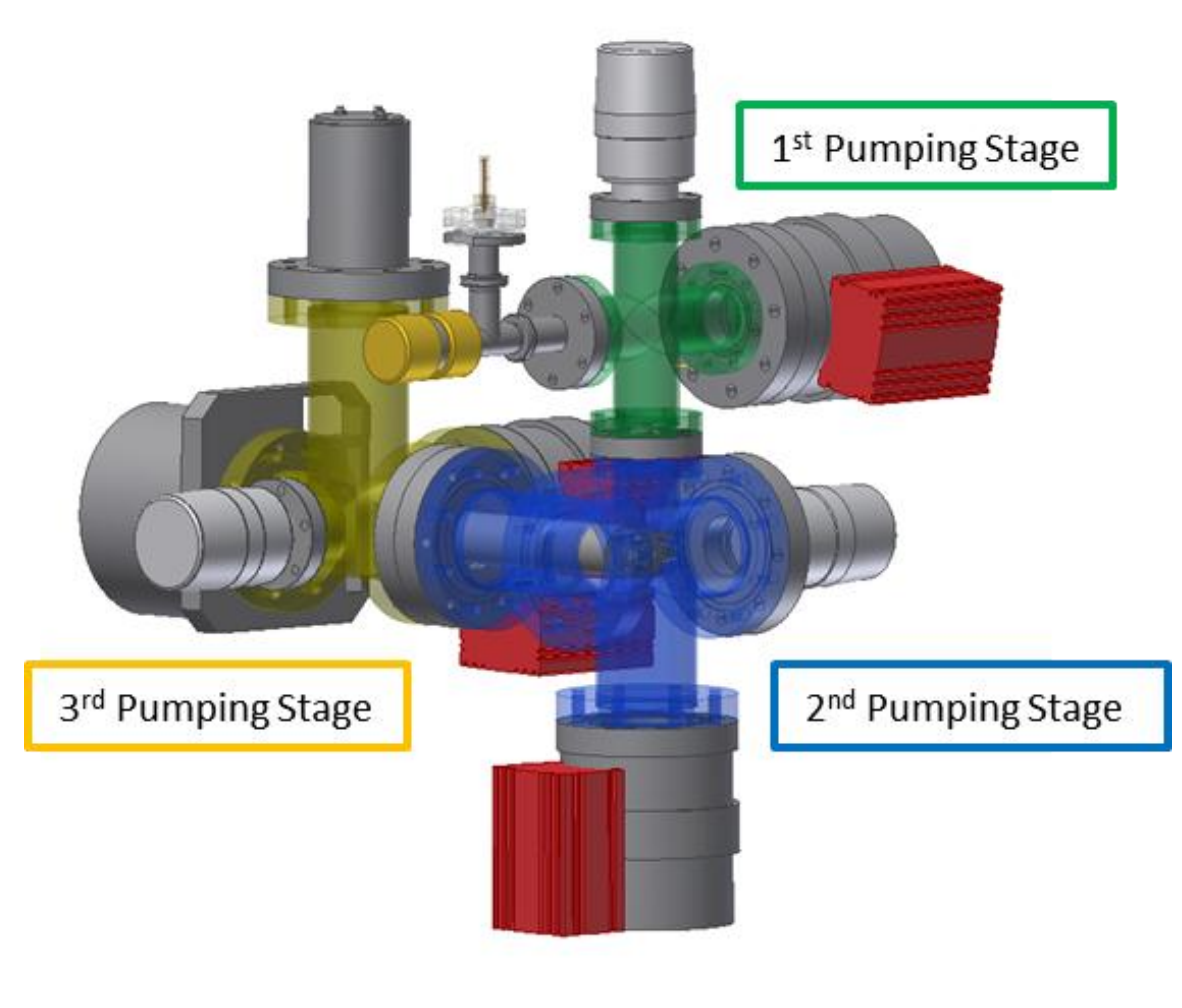

Figure 3-10: An illustration highlighting the chambers of the 3-stage differentially pumped vacuum system. The $1^{\text {st }}$ pumping stage (green) operates at a rather high vacuum pressure and is connected via a tubular aperture to the $2^{\text {nd }}$ pumping stage containing the QMS ion source. The $3^{\text {rd }}$ pumping stage meanwhile is located in the chamber connecting the quadrupole and SEM of the QMS.

The three-stage differentially pumped DEMS instrument vacuum system possesses three connected vacuum chambers that each operate at different 
vacuum pressures. These are evacuated by individual turbo molecular pumps (two $70 \mathrm{l} \mathrm{s}^{-1}$ and a single $300 \mathrm{ls}^{-1}$ ) that are backed by two rotary vane pumps. The electrochemical cell including membrane interface, is attached via a KF flange to a leak valve connection to the vacuum system. An exploded diagram of the 3-stage differentially pumped vacuum system created in this work is depicted in figure 3-11.

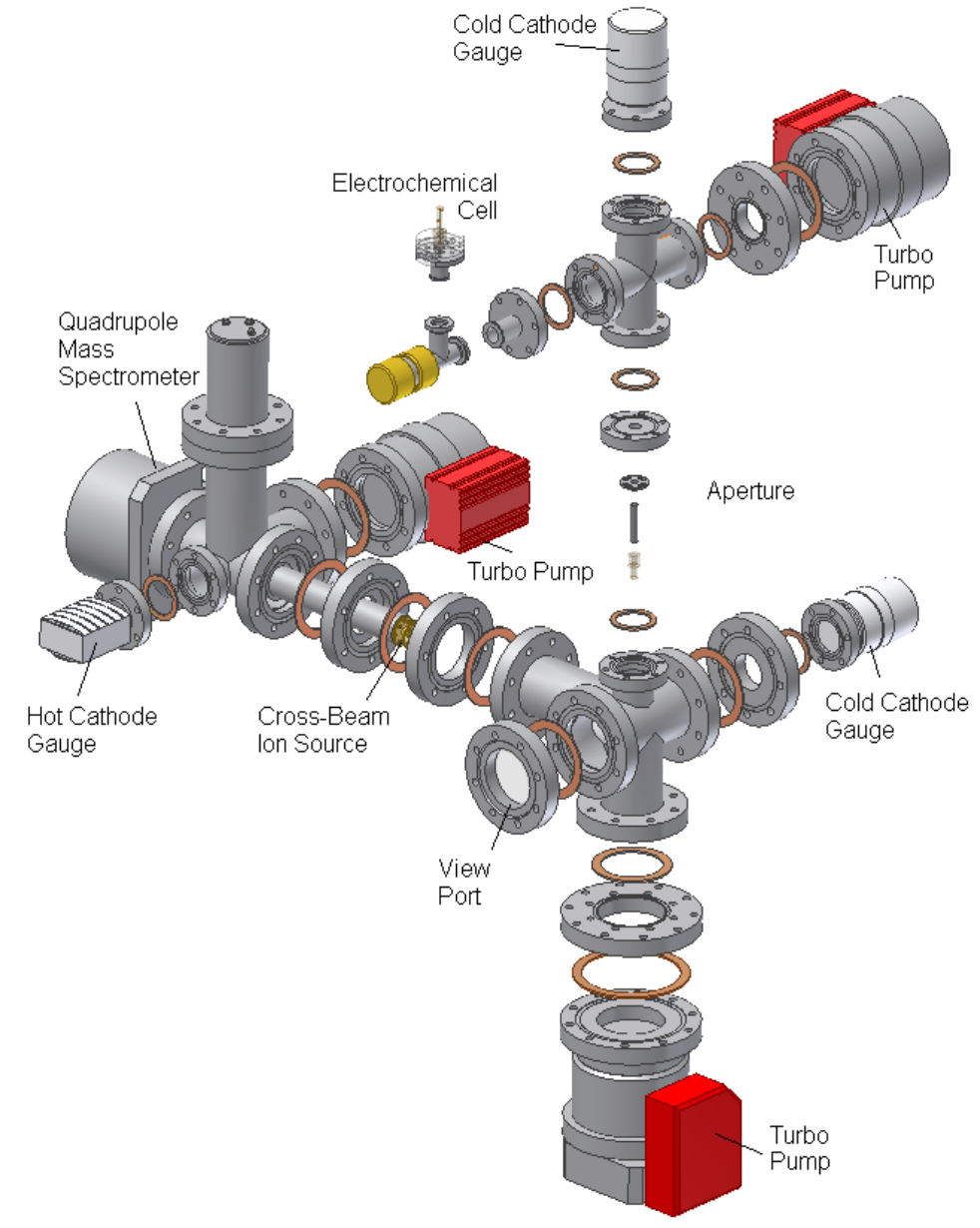

Figure 3-11: Exploded diagram of the DEMS instrument vacuum system \& QMS. The three pumping stages are connected via two apertures which control the flux of gas between each chamber to achieve the necessary differential pumping. Each chamber is evacuated by a turbo molecular pump whereby each pressure is measured by a vacuum gauge.

The leak valve connecting the electrochemical cells membrane interface is attached to a $1^{\text {st }}$ pumping stage, evacuated by a relatively small $70 \mathrm{ls}^{-1}$ turbo molecular pump, backed by a single rotary vane pump. The $1^{\text {st }}$ pumping stage is then coupled to a $2^{\text {nd }}$ pumping stage whose cavity contains the ion source and 
front section of the quadrupole rods of the QMS, which is evacuated using a larger $300 \mathrm{I} \mathrm{s}^{-1}$ turbo molecular pump. The relative pressure difference between these two chambers is determined by the conductance of gas passing from the $1^{\text {st }}$ into the $2^{\text {nd }}$ pumping stage, which can be controlled using an aperture of certain dimension. An additional $3^{\text {rd }}$ pumping stage (highlighted in yellow) is located at the $90^{\circ}$ axis between the quadrupole and SEM of the QMS, and is separated from the $2^{\text {nd }}$ chamber using a flange that partially encloses the cross-section of the outer quadrupole casing. This $3^{\text {rd }}$ pumping stage lowers the pressure at the SEM relative to the $2^{\text {nd }}$ pumping stage using another $701 \mathrm{~s}^{-1}$ turbo molecular pump, and allows a slightly higher gas pressure at the ion source relative to the SEM.

The key features that differentiate this vacuum system from DEMS instruments, is the use of three differentially pumped vacuum chambers, and the tubular aperture which is orientated at a $90^{\circ}$ angle with respect to the quadrupole axis. Further description and details of these features, namely the three-pumping stages and aperture will be presented in the following two sections, respectively.

\subsubsection{Three-Stage Differential Pumping}

The pressure requirements of the vacuum system design are essentially defined by the upper operating pressure limits of the QMS ionisation, mass ion selection and detection processes. The reliable upper operating vacuum pressure limit provided by the manufacturer varies slightly for each component of the QMS, and is summarised in table 3-3.

Table 3-3: Details of the QMS component operating pressure limits [85]. When operating the QMS with the SEM the vacuum pressure must be nearly two orders of magnitude lower than with the faraday cup.

\begin{tabular}{|c|c|}
\hline QMS Component & Operating Pressure/ mbar \\
\hline Cross Beam Ion-source & $<\mathbf{1 . 0} * \mathbf{1 0}^{-4}$ \\
\hline Quadrupole & $<\mathbf{1 . 0} * \mathbf{1 0}^{-4}$ \\
\hline Faraday Cup & $<\mathbf{1 . 0} * \mathbf{1 0}^{-4}$ \\
\hline Secondary Electron Multiplier (SEM) & $<\mathbf{5 . 0} * \mathbf{1 0}^{-6}$ \\
\hline
\end{tabular}


The absolute upper operating pressure limit is dependent upon the QMS detection mode, either using the Faraday Cup, or Secondary Electron Multiplier (SEM). If the whole QMS is positioned in a single vacuum chamber, then the upper operational pressure limit is determined by the QMS mass ion detection mode. For measurements where the detected ions are measured using the Faraday Cup (during which time the SEM is turned off), the upper operating pressure is in the $1.0 * 10^{-4}$ mbar, whereas for SEM operation, the upper operating pressure limit is much lower at $5.0 * 10^{-6}$ mbar. These pressures are easily achievable in a closed vacuum chamber, however, in DEMS we require a constant gas flux through the membrane to continuously enter the chamber, but be pumped away at such a rate that the operating pressures of the QMS are maintained.

In order to achieve these pressure using a single vacuum chamber there are two possibilities. Either, the gas flux through the membrane is controlled to an acceptable value by adjusting the size of the membrane inlet (possible with capillary inlets), or we employ a differentially pumped vacuum system. Two-stage differentially pumped vacuum systems have been successfully employed in DEMS previously [34, 36, 46, 59], which poses the question why is it that a three-stage system has been constructed here. Well, there are two reasons related to the performance and reliability of the QMS. In terms of performance or rather, sensitivity of the QMS, it is desirable to employ the SEM which considerably amplifies the mass ion current, whilst at the same time maintaining a relatively high pressure at the ion source in order to maximise the number of ions produced. In terms of reliability, however, the SEM should only operate at vacuum pressures $<5.0 * 10^{-6}$ mbar, whereas the CB-ion source can operate at pressures up to $1.0 * 10^{-4}$ mbar. If the SEM is operated at pressure above $5.0 * 10^{-6}$ mbar then the performance and lifetime of the SEM is severely compromised [85]. A pressure difference between the CB-ion source and SEM of the QMS is therefore desirable.

A differential pressure difference between the CB-ion source and SEM was achieved therefore, by modifying the original vacuum housing of the QMS. This modification from the original chamber to the 5-way cross chamber is depicted in figure 3-12. 


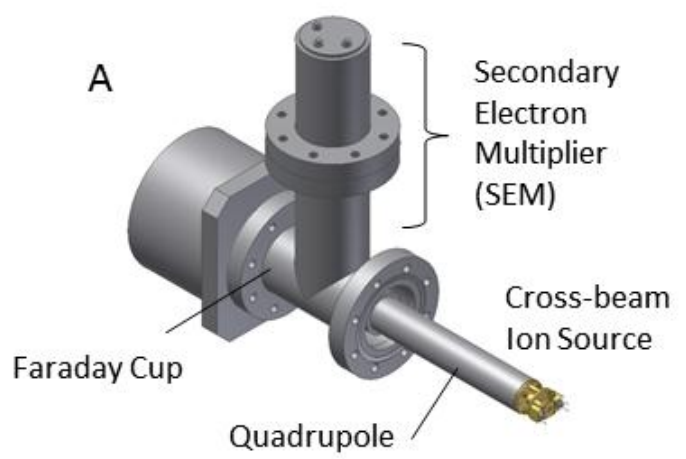

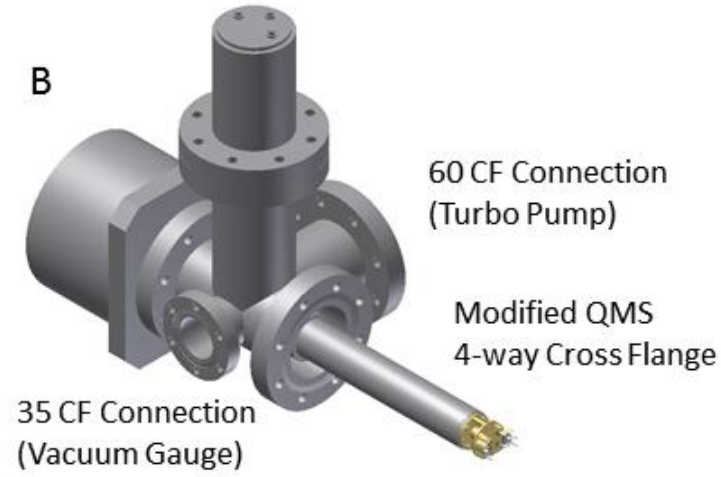

(Vacuum Gauge)

Figure 3-12: Illustration of the original 3-way flange QMS vacuum chamber housing (A) and modified 5-way QMS vacuum chamber housing (B). The original QMA 422 vacuum chamber was adapted to incorporate a flange connection for the turbo pump and pressure measurement gauge allowing the 3-stage differentially pumped vacuum system design.

The addition of two vacuum flanges to the QMS housing allows an additional turbo-pump and pressure gauge to be placed between the SEM and ion source. An additional flange is then positioned over the quadrupole to limit the conductivity of the vacuum tube in order to achieve a larger pressure difference between the CB-ion source $\left(2^{\text {nd }}\right.$ chamber) and SEM $\left(3^{\text {rd }}\right.$ chamber $)$. The resulting vacuum pressures, calculated tube conductances and effective pumping speeds are provided in the schematic in figure 3-13.

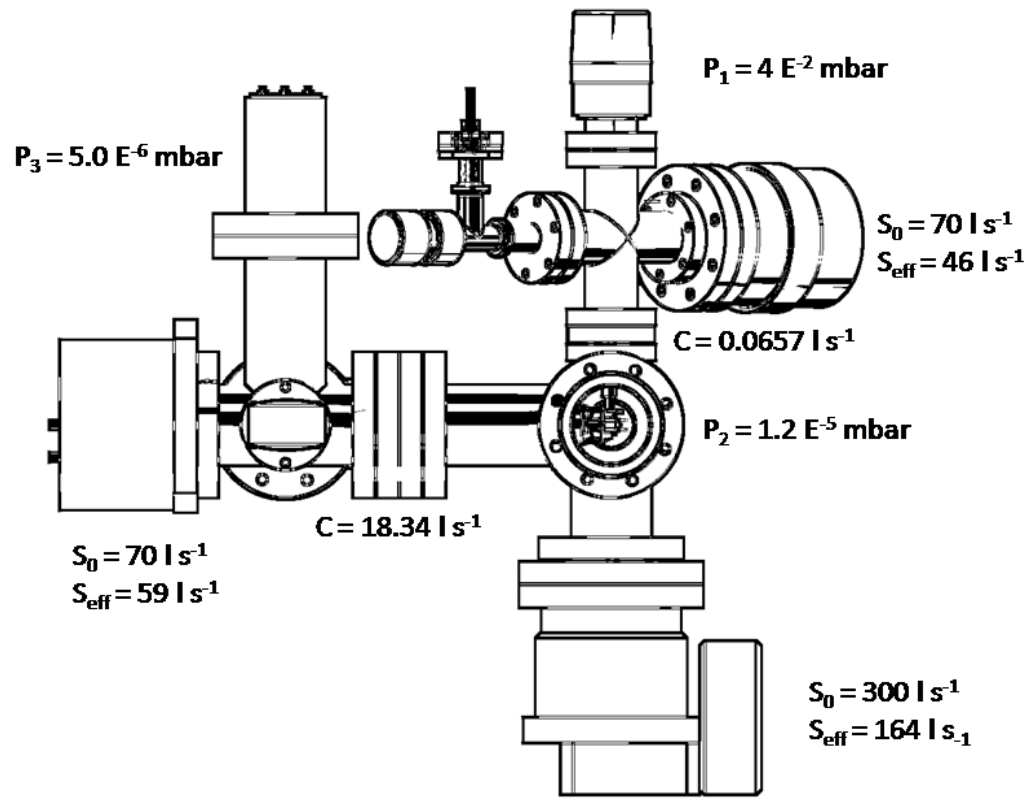

Figure 3-13: A sketch of the DEMS vacuum chamber giving the respective pumping speeds (S), conductances $(C)$ and operating pressure $(P)$ of the 3-stage differentially pumped vacuum system when the DEMS instrument is in operation. The effective vacuum pumping speeds and gas conductance of the apertures are determined by the internal dimensions of the components. 
The turbo molecular pumps located in the $2^{\text {nd }}$ and $3^{\text {rd }}$ pumping stages are backed using a single rotary vane pump. The vacuum pressures are monitored by two cold cathode gauges in the $1^{\text {st }}$ and $2^{\text {nd }}$ chamber, and a hot cathode gauge located in the $3^{\text {rd }}$ chamber. Once the DEMS electrochemical cell is attached to the vacuum system, and the valve exposing the membrane interface is fully opened, a constant gas flux into the vacuum chamber results. This gives typical steady operating vacuum pressures of $\sim 10^{-2}, \sim 2 * 10^{-5}$ and $\sim 5^{*} 10^{-6}$ mbar in the $1^{\text {st }}, 2^{\text {nd }}$ and $3^{\text {rd }}$ chambers, respectively.

\subsubsection{Tubular Aperture}

The tubular aperture of the DEMS instrument in this design serves two essential functions. Its primary function is to limit the conductance of gas between the $1^{\text {st }}$ and $2^{\text {nd }}$ differentially pumped vacuum chambers so that a considerable lower pressure in the $2^{\text {nd }}$ chamber is achieved. Its second function is to guide the flux of gas originating in the first chamber into the $\mathrm{CB}$-ion source of the QMS. This was achieved using the adjustable tubular aperture design depicted in figure 3-14.
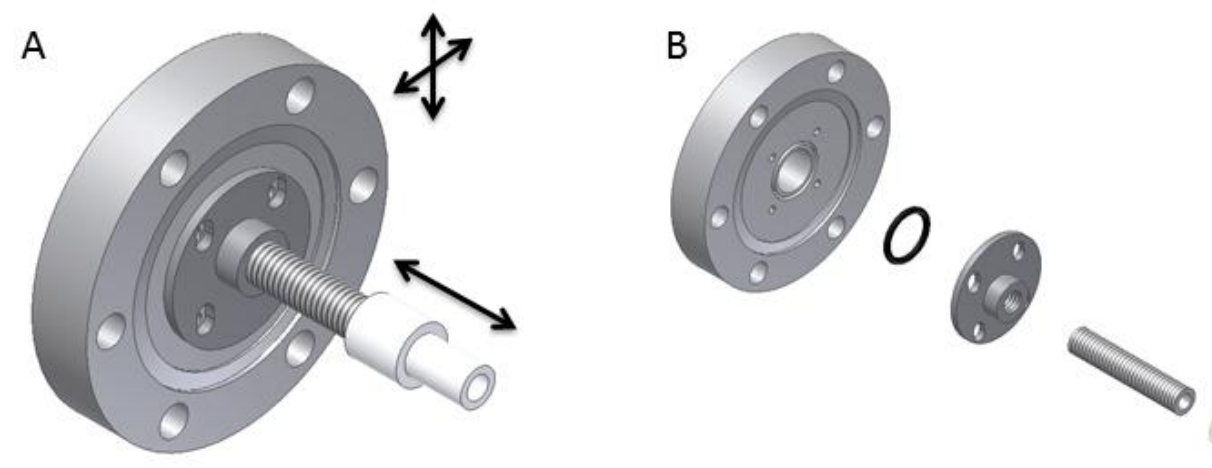

Figure 3-14: Illustration of the DEMS adjustable aperture (left) and exploded image (right). The design allows the conductance to be varied by changing the internal diameter and length of the tube. The adjustable nature of the design allows the aperture outlet to be position closely to the QMS ion source entrance.

The adjustable aperture is mounted onto NW35 CF vacuum flange, and sealed using a Viton O-ring. The tubular section of the aperture is screwed into the 
mounting plate and is therefore adjustable in the vertical direction by screwing or unscrewing the tubing. The mounting plate itself also possesses oval holes allowing some adjustment in the horizontal direction. The exact dimensions of the aperture, most significantly the internal diameter and length of the tube determines its gas conductance. The dimensions of the tubular aperture can therefore be tuned to give the necessary differential pressure between the $1^{\text {st }}$ and $2^{\text {nd }}$ pumping chambers. The aperture used here is $5 \mathrm{~mm}$ internal $\emptyset$ and $8 \mathrm{~mm}$ in length, allowing a gas flux of $\sim 0.07 \mathrm{l} \mathrm{s}^{-1}$. Finally, the tubular aperture is capped with the glass ceramic material, Macor ${ }^{\circledR}$ to prevent discharging from the CB ion source to the conducting aluminium part of the aperture, so that the aperture may be positioned in close proximity.

By positioning the aperture close to the $\mathrm{CB}$ ion source, and therefore directing the molecular beam directly into the CB-ion source, the partial pressure of gas leaking into the $2^{\text {nd }}$ chamber through the ion source is increased with respect to the surrounding vacuum chamber. The adjustability of the aperture is important therefore, because the positioning of the tubular aperture over the cross-beam ion source can have a significant effect on the performance of the QMS. There are two possible entrances into the CB ion source as shown in figure 3-15.

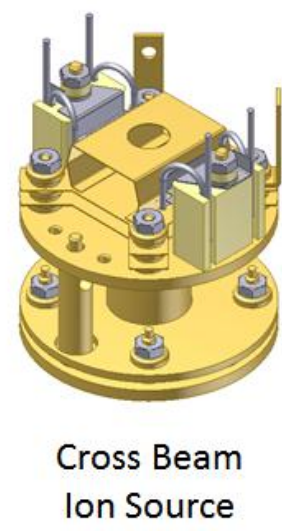

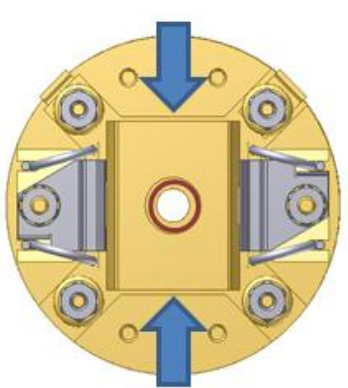

Front View

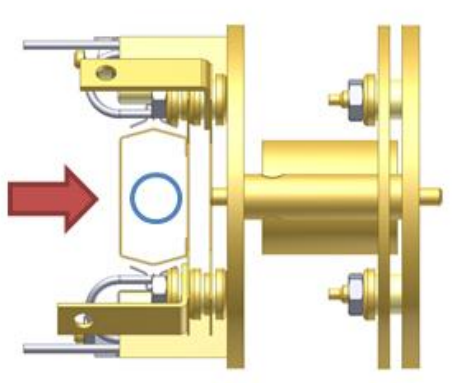

Top View

Figure 3-15: Illustration of the QMS cross-beam ion source highlighting possible molecular beam inlet orientations. The Wehnelt chamber of the ion source possesses two possible entrances which may be either in-line or perpendicular to the axis of the quadrupole. The perpendicular entrance is rather more open and was the chosen orientation of the aperture used in this DEMS instrument. 
The molecular beam exiting the aperture may be directed either along (indicated with the red) or perpendicular (indicated in blue) to the quadrupole axis in order to enter the CB-ion source ionisation area (Wehnelt Cage). In contrast to the smaller front entrance (indicated in red) the perpendicular entrance (either above or below the Wehnelt cage) is much larger, and the aperture can more easily be positioned correctly. The protruding molecular beam will therefore more easily pass through the $\mathrm{CB}$ ion source rather than hitting the outer face of the Wehnelt cage and bouncing off into the surrounding vacuum chamber. In this DEMS instrument therefore, the aperture and protruding molecular beam was orientated perpendicularly to the quadrupole axis. This is as depicted in figure 3-16 where the exit of the tubular aperture $\left(\right.$ Macor $^{\circledR}$ tip) into the $2^{\text {nd }}$ pumping stage is positioned directly over the entrance to the $\mathrm{CB}$ ion source.
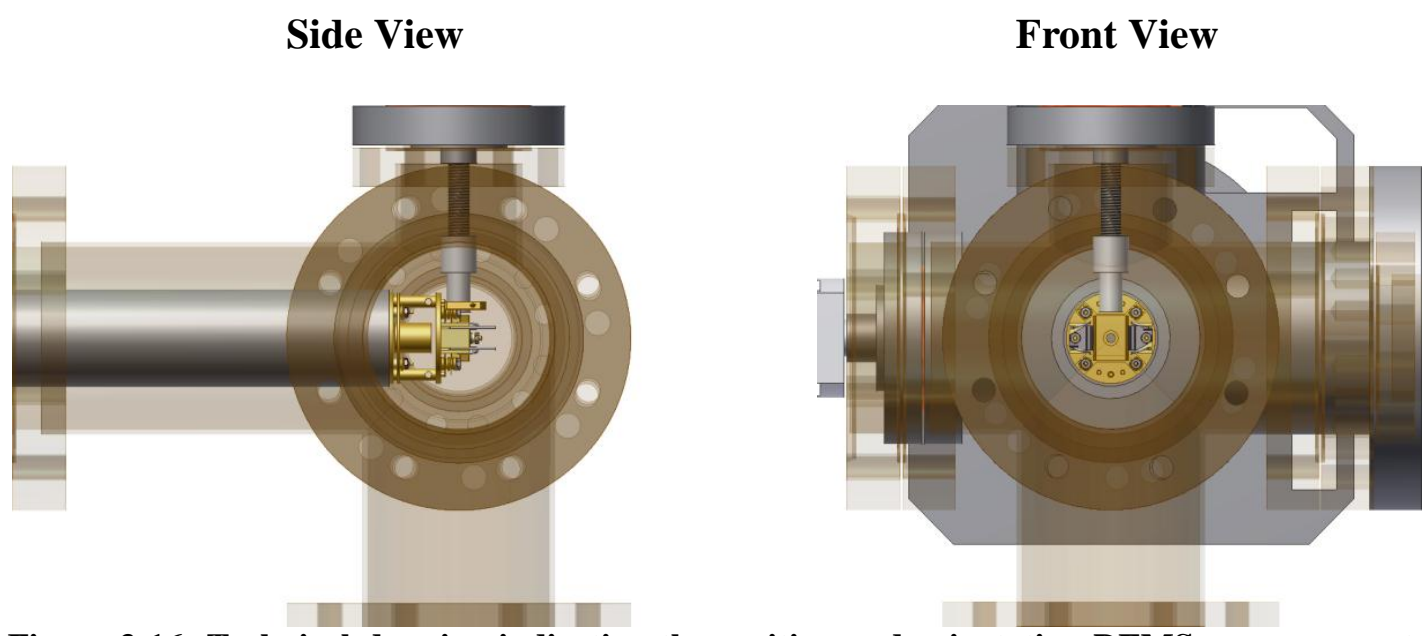

Figure 3-16: Technical drawing indicating the position and orientation DEMS vacuum aperture with respect to the cross-beam ion source. The aperture outlet is positioned directly over the ion source directing a large proportion of the gas entering the $2^{\text {nd }}$ vacuum chamber through the ion source. This effectively raises the pressure of the ion source enhancing the number of ionised species, increasing the sensitivity of the QMS.

A tubular aperture is not necessarily the only possible solution because a simple hole would suffice in order to control the conductance of gas between chambers. The tubular aperture, however, not only possess the advantage that the protruding molecular beam can be position directly over the $\mathrm{CB}$ ion source but also that is more easily fabricated compared to the simple hole i.e. the exact conductance is sensitive to the dimensions of a hole aperture with little room for error, whereas a tube of a certain diameter may be adjusted in length. In cases 
where it would be perhaps desirable to tune the conductance of the aperture, alternative design solutions include an inexpensive variably control aperture designed in ref. [86] and realised in ref. [87], or alternatively a simple leak valve could be placed between vacuum chambers.

Finally, in cases where a tubular aperture separates vacuum chambers with differential operating pressures of ca. 1 mbar and possess a molecular beam protruding from the exit of the tube as the gas expands into the lower pressure vacuum chamber, it is possible that the sensitivity of the QMS could be enhanced further by the addition of a chopper and lock-in amplifier to reduce measurement 1/f noise [88]. This more complicated system, however, was not employed in this experimental setup. Another alternative would have been to employ a closed ion source which are commercially available [89]. 


\subsection{Instrumentation, Control and Data Acquisition}

The purpose of this section is to present the details of hardware instrumentation, control and data acquisition (DAQ) devices, as well as custom Labview software setup for the DEMS instrument. Its aim is to provide an overview of the most important aspects of the hardware and software setup, and when necessary guide the reader to where further, relevant information may be found i.e. in technical notes and manuals etc. The hardware instrumentation can be separated into those that are required for the electrochemistry, such as the potentiostat, NID and syringe pump, from those required by the vacuum system and QMS, such as turbo pumps, pressure control units and signal amplifiers. In common to many pieces of the DEMS instrument hardware, however, is the use of DAQ cards and software to both control instrumentation and record measurement data.

Whilst historically, analogue function generators and xy-plotters were essential in electrochemical experimentation, such devices ought to have no place in a stateof-the-art electrochemistry laboratory. Data acquisition hardware and graphical software programming tools such as Labview offer electrochemists the possibility to meticulously control potentiostats amongst other experimental parameters programmatically, with precise data acquisition, real time data plotting and recording. Although commercial electrochemical instrumentation software is available, their functionality or customisability cannot be compared to a welldesigned Labview based electrochemistry software. Furthermore, custom data treatment software that performs common treatment tasks can also allow rather efficient post-measurement data treatment and analysis. Hence, a customised Labview software was the approach taken in our electrochemistry laboratory, further details of which may also be found in ref. [90]. In short, the role of the traditional analogue function generator is replaced by a software generated digital signal that is output via a digital to analogue converter (DAC) device. Computerised rather than manual control allows experimental measurement parameters and techniques to be performed meticulously, with defined measurement sequences and protocols. Whereas previously, analogue signals were recorded and displayed using an X-Y recorder, plotter or oscilloscope, data is more often today transferred to computer using an analogue-to-digital 
converter (ADC) device. The data can then be easily displayed and recorded in an appropriate format that is tuned to the measurement type. This is particularly useful in electrochemistry were a single CV can measured relatively quickly, and consequently a large quantity of data can be accumulated in a relatively short period of time, making it equally important to be able to analyse measurement data efficiently. The advantages of a custom Labview programmed instrument control and data acquisition led to the extension of the approach taken with the RDE and MWE methodologies, to the DEMS instrument. Overall, the instrument requires two measurement setups, one for DEMS experimentation and another for QMS calibration. 


\subsubsection{DEMS Measurement Setup}

An overview of the communication between the various instrumentation, control and data acquisition hardware and software required to operate the DEMS instrument acquire measurement data during experimentation is presented in figure 3-17.

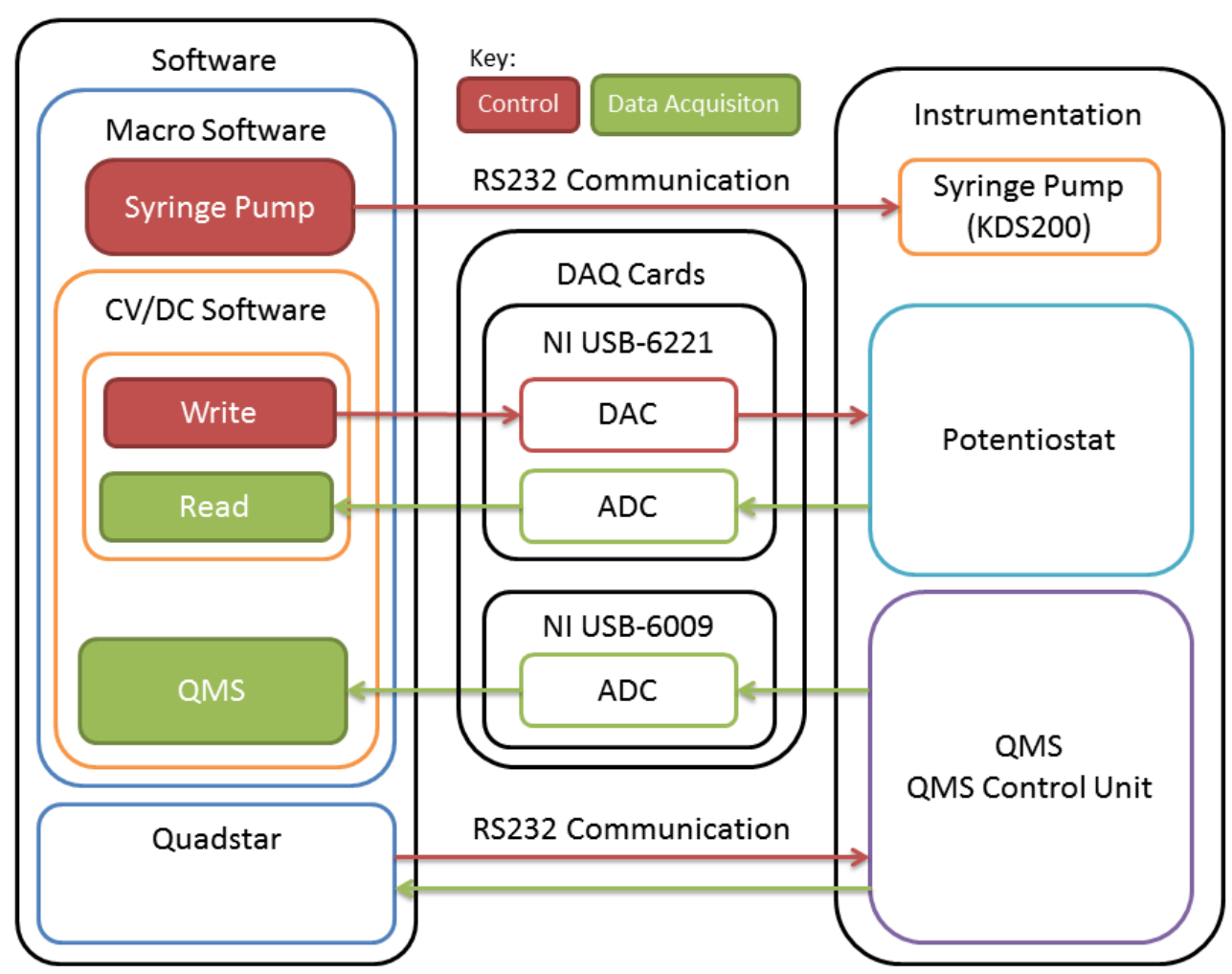

Figure 3-17: Overview of instrumentation, control and data acquisition hardware and software employed in this DEMS instrument. The custom electrochemistry software is used to control the potentiostat and syringe pump for the electrochemical cell, and acquire data from the potentiostat and QMS. The commercial 32-bit software meanwhile is used to exclusively to operate the QMS.

The instrumental setup relies on two sets of software which control or record data from three instruments (the syringe pump, potentiostat and QMS control unit) using either two DAQ cards, or RS232 data communication. The first piece is the commercial Quadstar 32-bit program which is used simply for the operation of the QMS. The second piece of software includes there are the custom developed Labview software programs: Macro and $\mathrm{CV}$ which control, measure, display and save the DEMS electrochemical data. These applications were primarily developed for RDE and MWE methodologies; however, as part of this thesis the 
software was developed further and extended to incorporate the additional functionality required to acquire data from the QMS. Both the electrochemical and QMS experimental data is consequently recorded, treated, synchronised, displayed and saved in real-time. Finally, the Macro software also incorporates programmatic control over the electrochemical flow cell syringe pump for the control of the electrolyte flow rate. Overall, this custom Labview program approach considerably increases the functionality and usability of the DEMS instrument.

\subsubsection{Electrochemistry Hardware}

The DEMS electrochemical flow cell setup relies on the control of three pieces of hardware: the syringe pump to control the electrolyte flow rate through the electrochemical cell, the potentiostat to control the WE potential in electrochemical experimentation, and finally, the NID to achieve $i R$-compensation.

\subsection{Syringe Pump}

The electrochemical flow cell setup employed in this DEMS instrument is of a flow cell nature and therefore requires the continuous passage of electrolyte through the electrochemical cell at a precisely controlled rate. In this setup, this is achieved by placing a syringe pump on the flow cell outlet and operating it in withdrawal mode which limits the flow of electrolyte through the cell to a certain rate. The syringe pump employed is a KDS210 infusion withdrawal pump using either a 25 or $50 \mathrm{ml}$ syringe with typical withdrawal rates of between 60 and $600 \mu \mathrm{l} \mathrm{min}{ }^{-1}$. Refer to syringe pump manual for further details on its operation [91]. Finally, although manual control of the syringe pump during DEMS experimentation is of course possible, in certain experiments it can be desirable to have computer control over the syringe pump which can be achieved using RS232 communication, and Labview VIs which were incorporated into the custom Macro program. The flow control software is later described in 3.7.1.3.5. 


\subsection{Potentiostat}

The potentiostat employed in this DEMS instrument is a Banks Wenking POS 2 potentiostat which in may be controlled using either manually with the internal scan generator, an external function generator, or via computer control using the SCSI interface. In this setup depicted in figure 3-18, the potentiostat is controlled via its potential control analogue input by the DAQ which effectively functions as an external function generator but is controlled by the $\mathrm{CV}$ and Macro software.

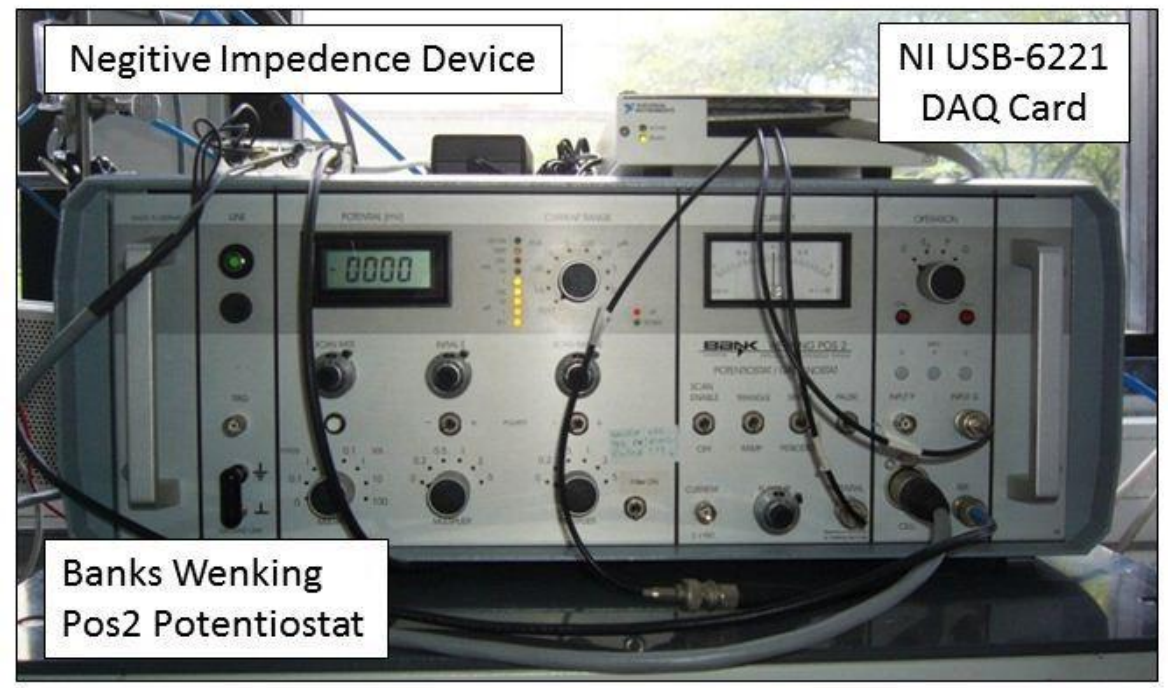

Figure 3-18: Image of DEMS electrochemical potentiostat hardware setup. The custom NID device is used to apply $i R$-compensation, whilst the DAQ card is used to both control the potentiostat via the custom Labview electrochemistry software and measure the WE potential from the potentiostat and electrode current via the NID.

The DAQ simultaneously applies the desired WE potential and measures the corresponding measurement potential and current. The current range meanwhile is controlled using the DAQ card digital outputs via the SCSI interface on the rear of the potentiostat. The instrument operation mode i.e. whether it set to off, open circuit potential, potentiostatic or galvanostatic, however, must be performed manually. In principle, the potentiostat and control system is identical to the RDE setups used in the rest of our electrochemistry laboratory, with the exception that a NID device is incorporated into the electrochemical cell setup. The NID device is required for two reasons: firstly, the positive feedback $i R$ - 
compensation scheme of this potentiostat does not function properly, and secondly, the NID circuit has some advantages over positive feedback schemes to compensate highly resistive electrochemical cells.

\subsection{Negative Impedance Device}

The negative impedance device (NID) is an operational amplifier based circuit that is positioned between the WE of the electrochemical cell and potentiostat WE input connection. An overview of the in-house built NID and its connections is depicted in figure 3-19.

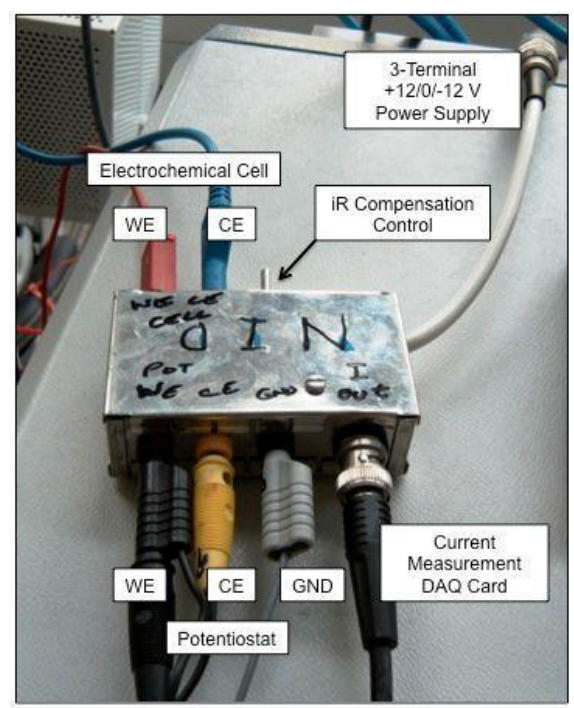

Figure 3-19: Image of custom built negative impedance device (NID) highlighting the potentiostat and cell connections. The NID is located between the potentiostat and the WE electrode, allowing adjustable $i R$-compensation of the solution (or in this case stabilising) resistance. In this setup the DAQ measures the current response via the NID rather than the potentiostat.

The circuit functions as a 'negative resistor', and adjusts the potential applied to the actual WE relative to the potentiostat output, according to the current that flows through the WE (and therefore NID) and the choice of circuit resistances. A general schematic of the NID circuitry is given in figure 3-20. The potential difference across the NID, $V_{c}$, is described by the following equation:

$$
V_{c}=-i R_{m} \frac{R_{1}}{R_{2}}
$$


In a circuit where $R_{1}$ and $R_{2}$ are of equal resistances, $V_{c}$ is purely determined by the resistance of $\boldsymbol{R}_{m}$ and the current flowing through the WE. By measuring the ohmic resistance between the Luggin-capillary and WE, and then applying an equivalent $\boldsymbol{R}_{m}$ resistor, complete compensation of the solution resistance $\boldsymbol{R}_{s}$ may be achieved.

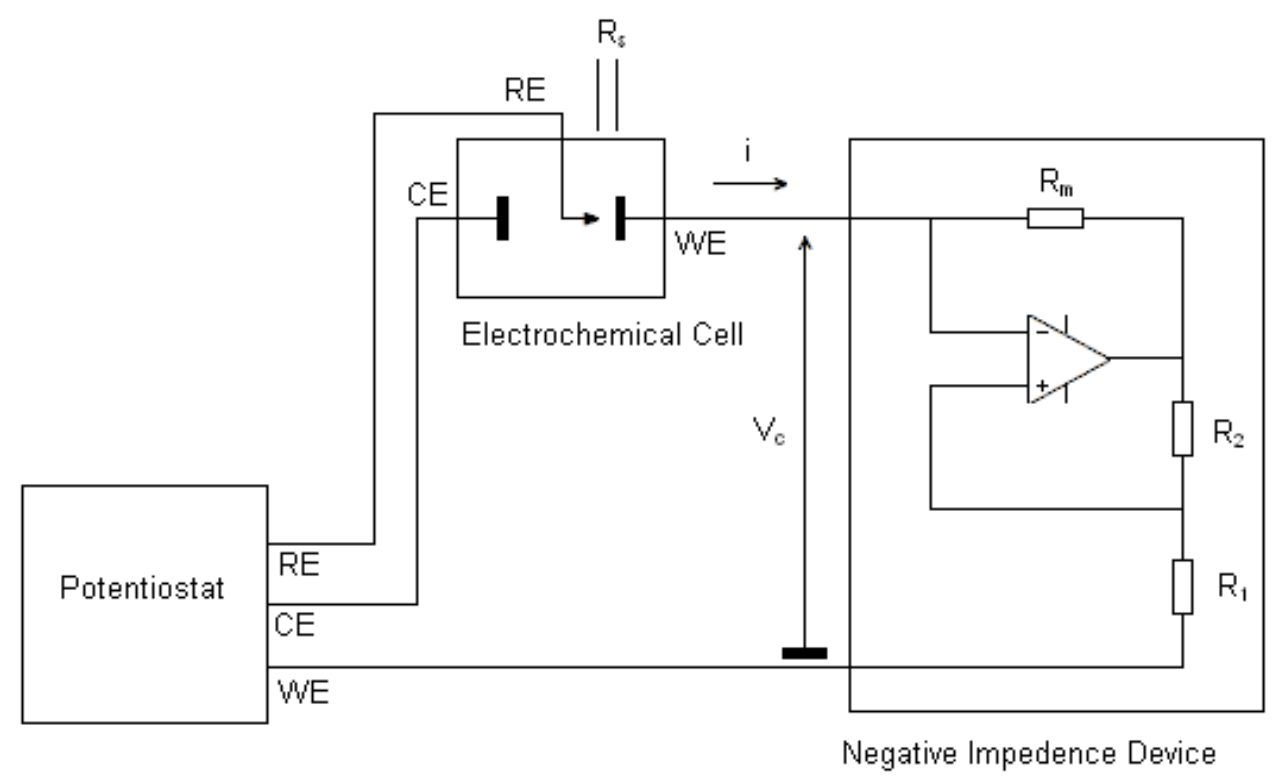

Figure 3-20: A schematic of the original NID circuit design and electrochemical cell setup. The circuit functions as a negative resistor and can be used to compensate the solution resistance in an electrochemical cell without interference on the potentiostat circuit. The amount of $i R$-compensation is typically determined by the size of the resistor, $R_{\mathrm{m}}$.

This particular NID circuit design, however, is only suitable in circumstances where $\boldsymbol{R}_{s}$ is both known exactly, and is constant during the course of an entire experiment or set of experiments which is not often the case. Furthermore, when using the NID over compensation of the $R_{s}$ may be possible, in contrast to the positive feedback circuitry employed in analogue potentiostats, where complete and over compensation results in potentiostat instability and oscillation [92, 93]. The usefulness of the NID device circuit design shown figure 3-20 is therefore limited but could be significantly enhanced if the amount of $\boldsymbol{R}_{s}$ it compensates could be more easily adjusted.

A modified NID circuit given in figure 3-21 was therefore developed (which is contained in the housing shown in figure 3-19) for the DEMS electrochemical cell 
setup and is a modification of the NID designs previously reported [94]. Note that in this case the solution resistance is replaced with an artificial stabilising resistance, $\boldsymbol{R}_{s t}$, which is necessary to stabilise the DEMS electrochemical flow cell and is discussed in more detail in Chapter 4 section 4.1.1.

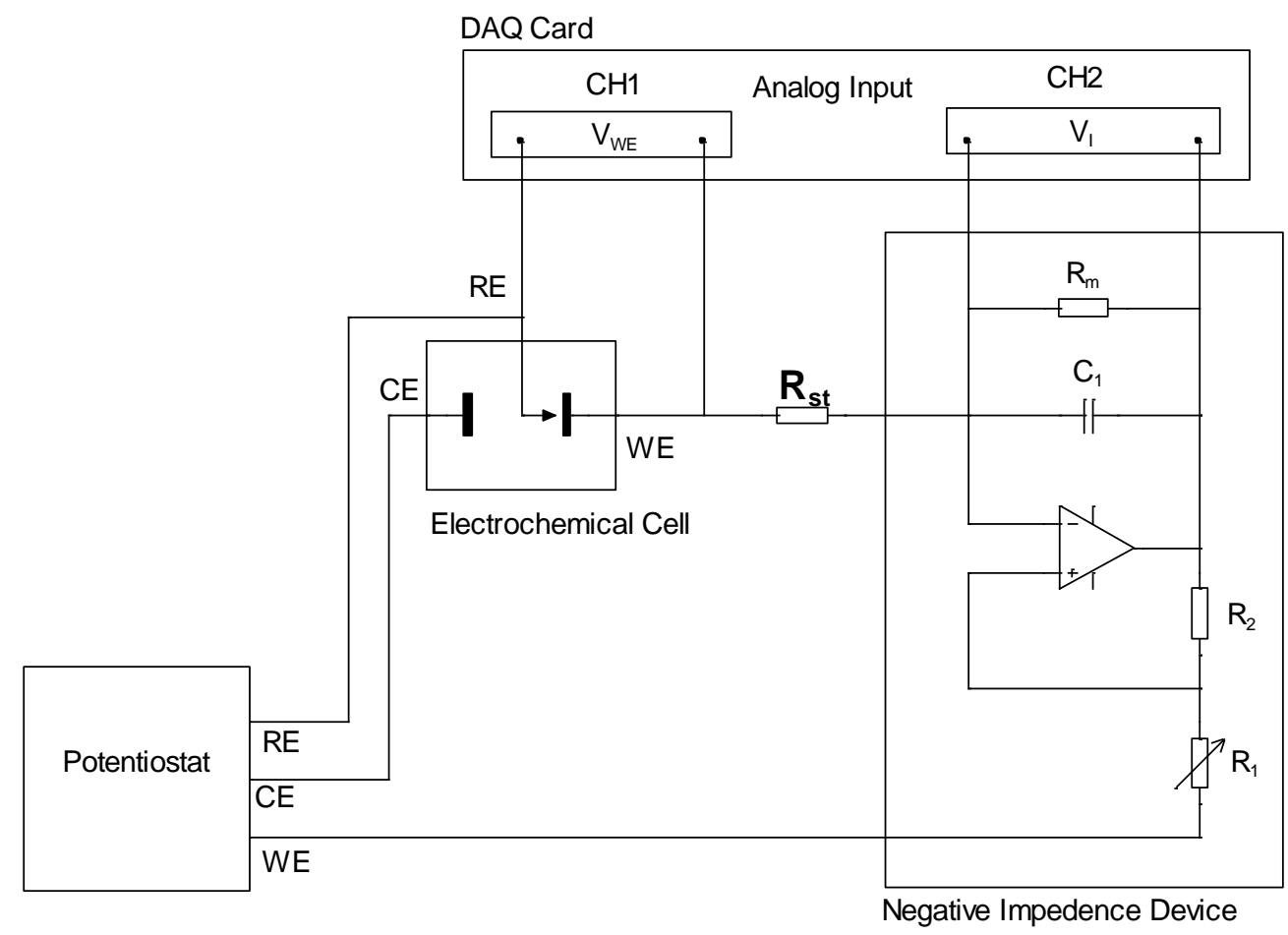

Figure 3-21: A schematic of an enhanced NID circuit design allowing adjustable $i R$-compensation developed for the DEMS instrument as part of this thesis. The circuit allows the amount of $i R$-compensation of the solution (or stabilising) resistance to be adjusted by the variable resistor (potentiometer) $R_{I}$ whilst enabling the WE current to be measured across a fixed $\boldsymbol{R}_{m}$ resistor using a DAQ card.

In this circuit, the extent of $\boldsymbol{R}_{s}$ compensation can be adjusted using a variable resistor, $R_{1}$, in combination with a fixed, $R_{2}$ and $R_{m}$ resistors. An $R_{m}$ value of $1.0 \mathrm{k} \Omega$ was chosen in order to give a $1.0 \mathrm{~V}$ across the resistor when a current of 1.0 mA flows through the WE which can easily be measured using standard DAQ cards. A combination of $R_{1}$ and $R_{2}$ values of 10 and $50 \mathrm{k} \Omega$ resistances, respectively, offers a flexible range of $R_{s}$ compensation from 0 to $200 \Omega$ using this NID circuit. Another feature is the $0.01 \mu \mathrm{F}$ filter capacitor, $C_{1}$, which allows AC frequencies $>10 \mathrm{kHz}$ to bypass $R_{m}$ so that measurement noise is also reduced.

The introduction of the stabilising resistor, $R_{s}$, also has the additional advantage that the actual WE potential can be measured using a DAQ channel that directly 
measures the potential between the WE and RE, as indicated as $\mathrm{CH} 1$ in figure 3-21. The actual potential, current relationship is therefore measured directly, rather than applying an $i R$ correction in post measurement data treatment. Post data treatment $i R$ correction of the potential for large uncompensated resistances in a CV for instance, will introduce errors into the measured current, particularly for electrode processes dependent upon scan rate. The WE potential measured directly using the DAQ card, used in combination with an NID circuit could be a useful compromise in experiments where it is necessary to work in with poorly conducting electrolytes. The true current and potential relationship is therefore measured rather than performing post measurement data correction, which for large resistance will be incorrect. The device also allows adjustable $i R$ compensation and measurement of the WE current, without directly interfering with the potentiostat control circuit. It should be noted, however, that the NID can only be used with the potentiostat functioning in potentiostatic mode i.e. $i R$ compensation of the potential is not possible in galvanostatic operating modes, however, for galvanostatic measurements the current is generally not varied but static and thus the post measurement $i R$ correction is acceptable.

\subsection{Electrochemical Setup DAQ Card}

The potentiostat is controlled from the custom Labview software via a National Instruments USB-6221 DAQ device. This is identical to the DAQ devices used in the RDE setups in the electrochemistry lab, although in this setup the current is measured via the NID rather than the potentiostat current output. The DAQ card possesses two analogue outputs, one of which is used to control the WE potential via the potentiostat (or rotation in the RDE setups), and 16 analogue inputs, two of which are used to measure the applied WE potential and current. The digital outputs meanwhile are used to programmatically control the potentiostat current measurement range. Although this card could also have been used to acquire analogue data from the QMS, a separate DAQ card was instead used to simplify the software programming and maintain the electrochemistry software 
architecture described later in section 3.7.3. For more details of the electrochemistry DAQ setup refer to ref. [90].

\subsubsection{Quadrupole Mass Spectrometer Hardware}

The operation of the QMA 400 requires a number hardware including the QMH 400 high frequency generator, EP 422 electrometer preamplifier and the QMA 422 control unit that make up the whole QMS. The average user would perhaps not need to be aware of most aspects of this hardware which is simply controlled using the Quadstar 32-bit software. However, the DEMS user should be aware of the method in which QMS data is acquired from the instrument and the importance of choosing the correct amplifier range for the mass ion current. 


\subsection{QMG 422}

Data from the QMS may be obtained in real-time using either DDE communication between Labview and Quadstar, or via the oscilloscope connections located on the back of the QC 422 module, as indicated in figure 3-22 (A) (Note that DDE communication is software rather than analogue hardware based, DDE is discussed in more detail in section 3.7.3.1.2.). The DAQ hardware employed to acquire the QMS analogue signal is National Instruments USB-6008 card with 8 analogue inputs, as depicted in figure 3-22(B).
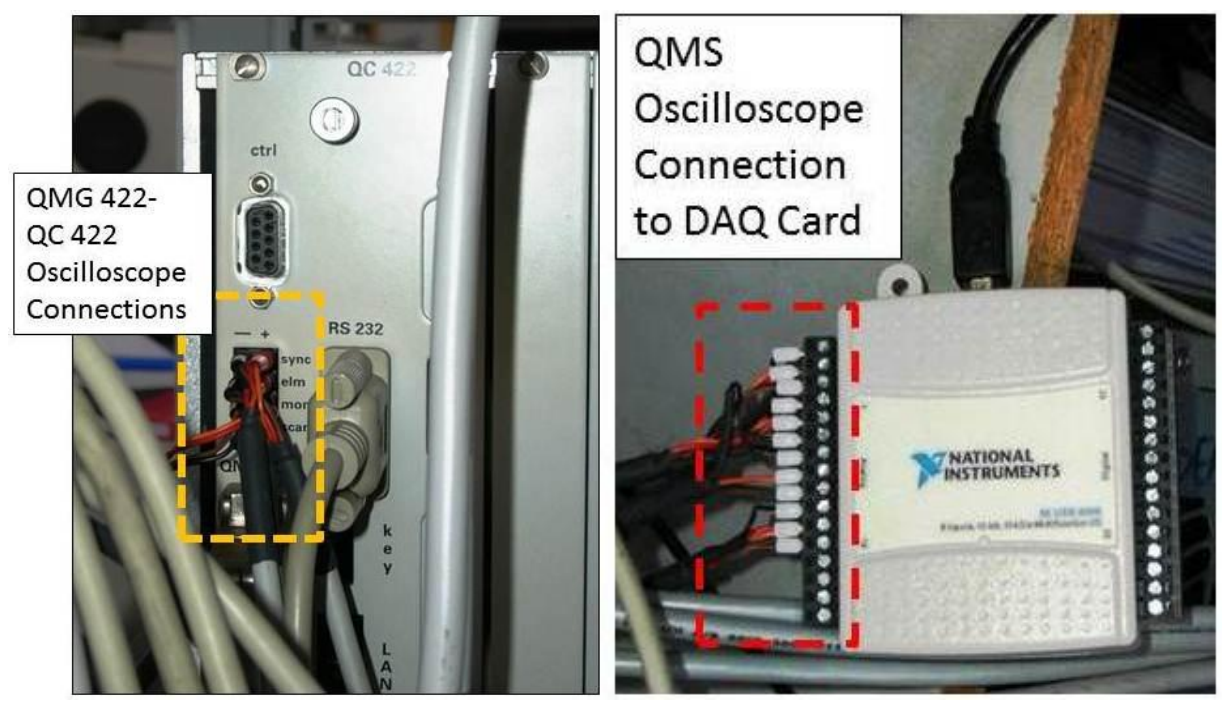

Figure 3-22: Images depicting the QC 422 oscilloscope connections (left) and NI DAQ card used for QMS data acquisition (right). The DAQ card is used to acquire the QC 422 oscilloscope voltage output which is then treated by the custom Labview software to acquire the measurement data.

The analogue output signal of the QC 422 unit labelled trig (trigger), elm (elementary), scan (scan) and mon (monitor). The exact nature and composition of these analogue output signals are dependent on the measurement mode of the QMS i.e. whether in mass scan or mass ion detection modes. Because the mass ion detection mode of the QMS is employed in DEMS, only the analogue outputs in this mode will be explained here, however, a more comprehensive overview is given in reference [85]. An example analogue signal output of the QC 422 unit during the MID or two different mass ion ratios is depicted in figure 3-23. 


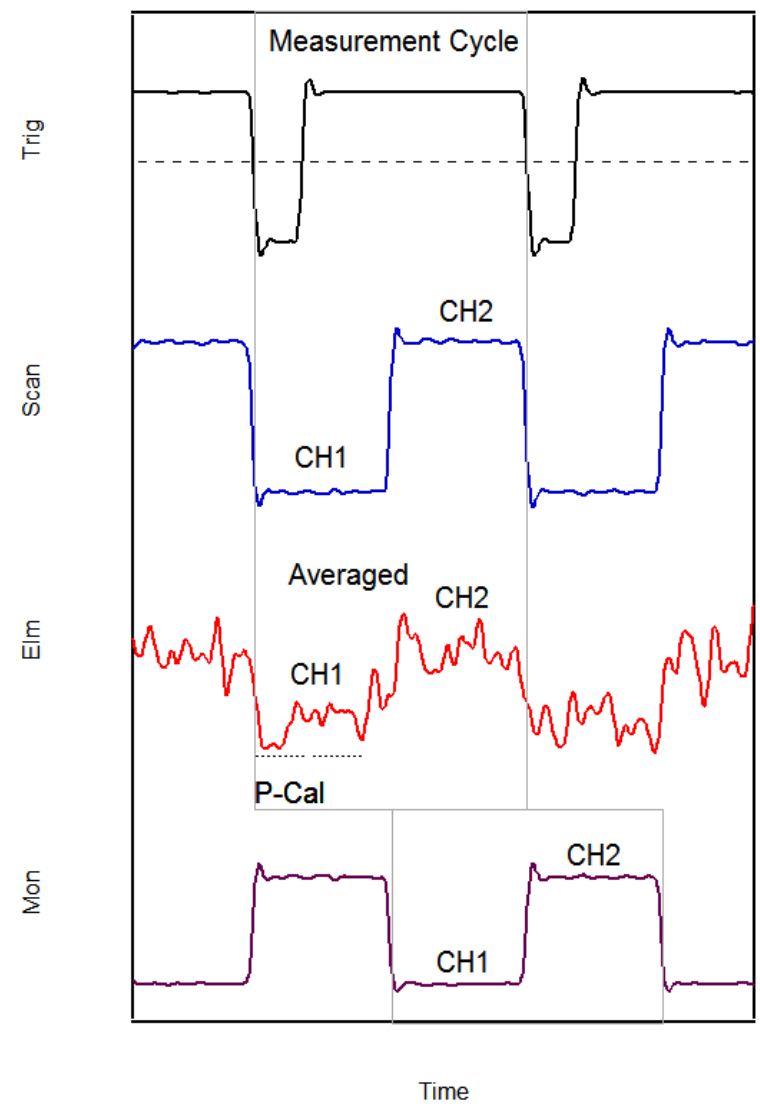

Figure 3-23: Example QMS analogue measurement signal outputs (intended for an oscilloscope) of the QC 422 for the measurement of two QMS channels. The Trig output defines the start of a measurement cycle, whilst scan corresponds to the selected mass. The raw electrometer output (Elm - from the EP 422 amplifier) is then treated and averaged by the QC 422 unit which outputs a corresponding fixed value (Mon). Note that the Mon output is delayed by one measurement channel.

Each fall in the trigger analogue output voltage (from 5 to $0 \mathrm{~V}$ ) indicates the onset of a new QMS measurement cycle. The selected mass ion current channels (defined in the Quadstar 32-bit software) are then cycled through, as indicated by a corresponding change in the scan output voltage which is proportional to the selected mass ion charge ratio of the QMS. The change in measurement channel mass corresponds to a change in the raw amplifier output signal $(\mathrm{elm})$. In the QC 422 this signal is averaged and output in the monitor analogue output delayed by a single channel. The monitor output voltage signal is determined by both the channel mass ion current, and the analogue output setting defined in the Quadstar 32-bit software measurement parameters. This -may be either linear or various logarithmic scales, depending on measurement mode. Details of the analogue output to current conversion equations may be found in the QMG 422 
manual. The length of time spent on the measurement channel is determined by two adjustable parameters in the Quadstar 32-bit software: $p$-cal and dwell. The p-cal value, typically 1.0, determines how long the channel is set before the mass ion current signal is averaged. This time is necessary in order to allow the amplifier to rise, overshoot and stabilise before the mass ion current is averaged. The dwell time then corresponds to the time over which the mass ion current signal is averaged before switching to another measurement channel. Although not clear from the QMG 422 manual, the total time for each channel is the p-cal factor multiplied by the dwell time, plus the dwell time. In the DEMS Labview software the VI is capable of measuring either the elm or mon channel. In most cases where the analogue output from the EP 422 amplifier is linear, then elm is the preferred where the QMS signal is averaged in the Labview software. The part of the signal being averaged can therefore be assessed and the QMS settings easily optimised. 


\subsection{EP 422 Amplifier}

The EP 422 electrometer amplifies the mass ion current signal output from the SEM (or faraday cup depending on QMS mode) and converts the signal into a proportional voltage between 0 and $10 \mathrm{~V}$. This signal is then filtered, treated and averaged by the QC 422 bus (according to the Quadstar 32-bit filter parameter) in the QMG 422 unit which communicates the measurement value to the Quadstar 32-bit software. Despite that the Quadstar 32-bit software possesses options for eight amplification ranges, the EC 422 circuitry in fact only possess four (physical) amplification ranges, which should be considered in the optimisation of the DEMS instrument. These are noted in table 3-4.

Table 3-4: EC 422 Electrometer Amplifier Range (AO = Analogue Output). The EP 422 device only possesses four physical amplification ranges, despite that the Quadstar 32-bit software allows one of eight ranges to be selected.

\begin{tabular}{|c|c|c|c|}
\hline AO Range & $\begin{array}{c}\text { Measurement } \\
\text { Range/ } \\
\text { A }\end{array}$ & $\begin{array}{c}\text { Sensitivity/ } \\
\mathbf{A ~ V}^{-1}\end{array}$ & $\begin{array}{c}\text { Rise Time/ } \\
\text { ms }\end{array}$ \\
\hline $\mathbf{1 0}^{-5}, \mathbf{1 0}^{-6}$ & $\mathbf{1 0}^{-5}$ & $\mathbf{1 0}^{-6}$ & $\mathbf{5 0}$ \\
\hline $\mathbf{1 0}^{-7}, \mathbf{1 0}^{-8}$ & $\mathbf{1 0}^{-7}$ & $\mathbf{1 0}^{-8}$ & 90 \\
\hline $\mathbf{1 0}^{-9}, \mathbf{1 0}^{-10}$ & $\mathbf{1 0}^{-9}$ & $\mathbf{1 0}^{-10}$ & 1.9 \\
\hline $\mathbf{1 0}^{-11}, \mathbf{1 0}^{-12}$ & $\mathbf{1 0}^{-11}$ & $\mathbf{1 0}^{-12}$ & $\mathbf{2 . 6}$ \\
\hline
\end{tabular}

Much of the QMS signal noise appears to arise from the EP 422 amplifier rather than the ion source or SEM. Ideally, the signal arising from the SEM is as large as possible so that the amplifier range can be set to a lower amplification range to minimise the measurement signal noise. This, however, is not always possible at reasonable SEM voltages, however, in general it is preferred that the SEM signal is in the upper decade of the amplifier output range i.e. in the $10^{-7}$ amplifier range, the mass ion current signal should ideally be in the $10^{-7}$ region $(1-10 \mathrm{~V})$ rather than the lower $10^{-8}$ range $(0-1 \mathrm{~V})$. 


\subsubsection{DEMS Measurement Software}

A description of the software related to the DEMS experimental setup, namely the potentiostat, syringe pump, vacuum gauges and QMS will be presented in the following sub-sections. The majority of this software, with the exception of the commercial Quadstar 32-bit program, is Labview based software programs designed or developed as part of this thesis for the DEMS instrument. Through thoughtfully designed Labview based software, the functionality, usability and productivity of any experimental setup can be considerably enhanced. Consequently, in order to aid experimentation using the DEMS instrument, additional functionality was incorporated into two previously written in-house software applications, the $\mathrm{CV}$ and Macro programs that were originally developed for RDE and MWE methodologies [90]. The descriptions of the custom Labview based software architectures and virtual instruments (VIs) presented here are intended to aid the understanding and interpretation of the graphical code. The commercial Quadstar software which controls the QMS, however, will be addressed first.

\subsection{Quadstar 32-bit}

The Quadstar 32-bit software is a modular application for QMS models 422/421 and QMS 200 Prisma $^{\text {TM }}$. The standard software is capable of performing qualitative and quantitative analyses and is user programmable. There are a number of programs that make up the Quadstar 32-bit software, however most commonly used are the Parset (Parameter Setup), TuneUp, Measure, and Dispsav (Display Save) programs. During DEMS operation the Quadstar 32-bit measure program is launched either manually by the user or by the $\mathrm{CV}$ or Macro program during initialisation depending on the measurement mode. In both cases the QMS is operated in MID Versus Time mode and runs using pre-defined Measure MID parameter (*.mip) file created in the Parset program. For more information on the Quadstar 32-bit software refer to the software documentation [95]. 


\subsection{CV3}

The CV3 program is a custom developed Labview program designed to perform CV electroanalytical experiments with DEMS. The software is an extension of the previous version (CV2) which was originally designed for SWE and MWE electrochemical setups [90]. In CV3 additional functionality which is required for the QMS component of the DEMS instrument was incorporated to aid DEMS experimentation, however, the control of the electrochemical experimentation essentially remains unchanged. The CV QMS software graphical user interface (GUI) is given in figure 3-24.

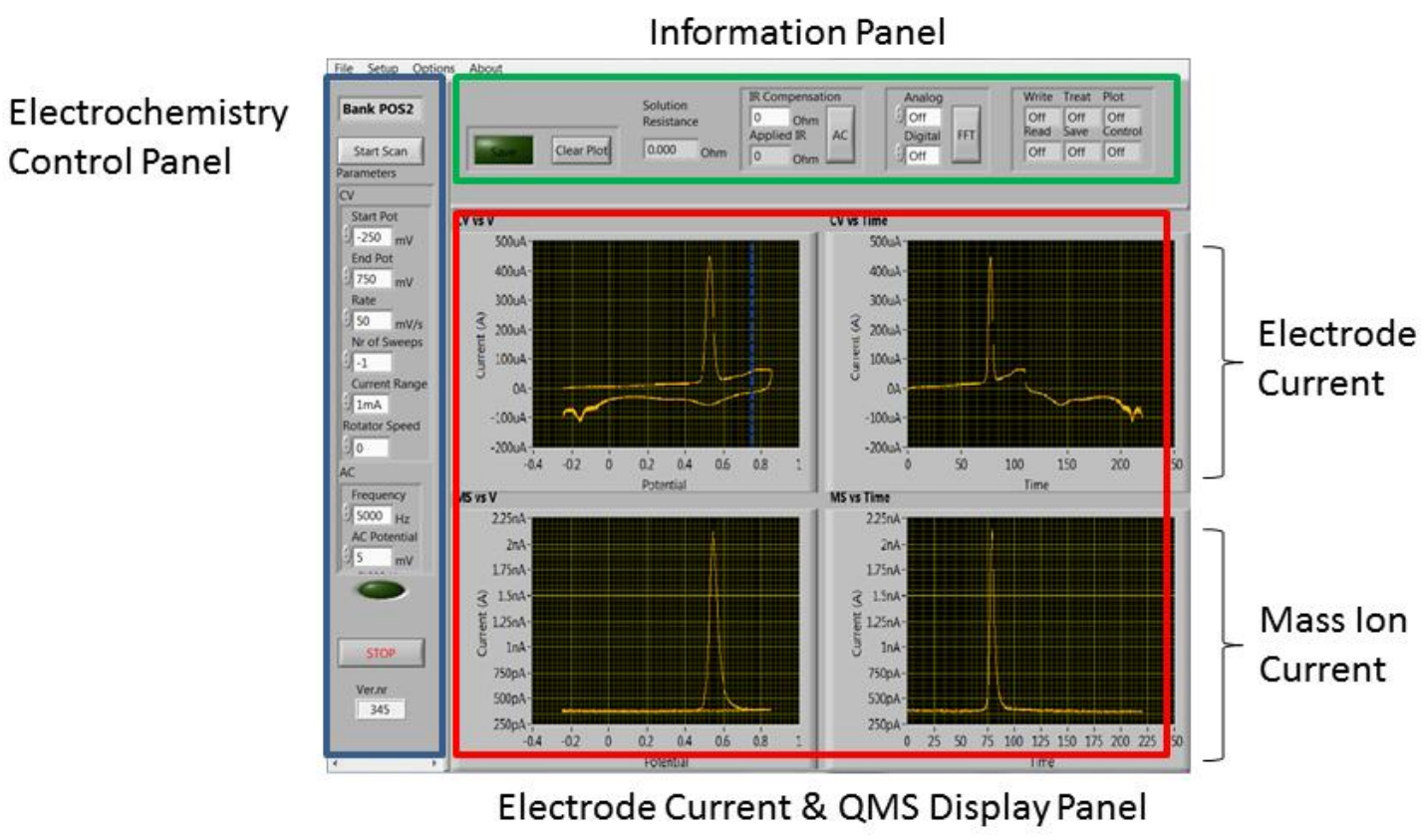

Figure 3-24: Screenshot of the CV3 software front panel developed for the DEMS instrument. The CV3 software incorporates DEMS functionality combining CV electroanalytical experimentation with the acquisition, synchronisation, display and save of QMS mass ion current data in real-time.

In the DEMS operation mode the faradaic electrode current and QMS data are measured, synchronised, displayed and saved in real time. The CV3 software control panel allows users to manually control the CV electroanalytical parameters such as potential range, scan rate etc. in real-time during an electrochemical experiment. In DEMS operation, QMS data is also measured, synchronised with the electrochemical and displayed along with the electrochemical data. In contrast to the standard SWE and MWE modes, the 
experimental data is display as both a function of the WE potential, and time allowing easier interpretation of the QMS mass ion currents.

The QMS data acquisition (referred to as DEMS mode) can be setup in the CV3 (and Macro or DC software described later) using a sub-panel whose user interface is given in figure 3-25. Here QMS data acquisition method must be defined (whether DDE or analogue DAQ) and the preferred QMS channel to display (if more than one).

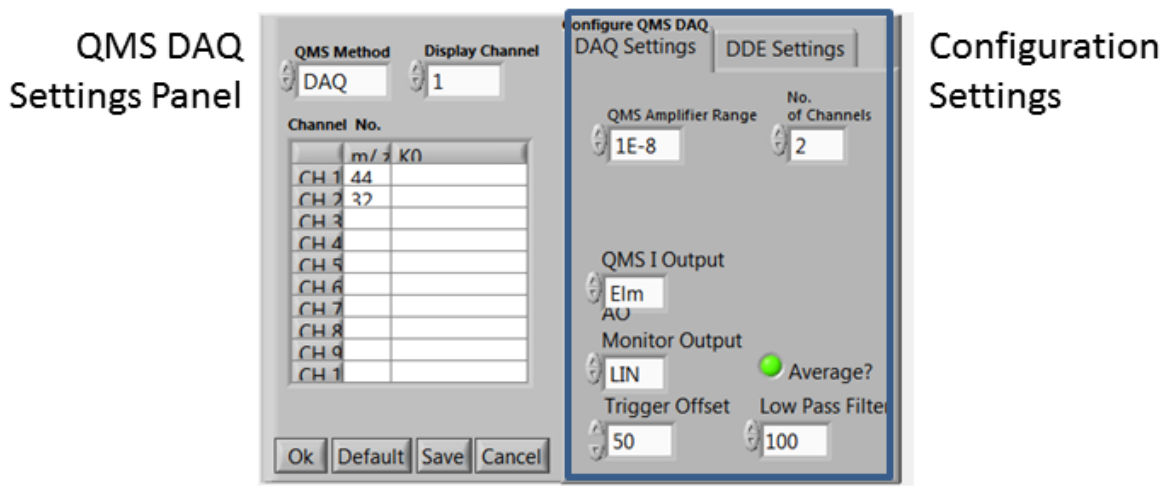

Figure 3-25: Screenshot of the QMS settings control panel for DDE and DAQ. The pop-up panel allows the QMS data acquisition settings to be defined prior to the measurement. Of particular importance is that the number of channels and channel mass labels $(\mathrm{m} / \mathrm{z})$ are defined, and that all DAQ settings correlate to the QMS parameters defined in the Quadstar 32-bit software when using the QMS DAQ method.

Of particular importance here are the analogue DAQ settings, which define how the analogue signal from the QMG 44 unit is acquired, filtered, averaged and converted into a mass ion current. The settings here should correspond to the analogue output, amplifier range defined in QMS measurement parameters if the Quadstar 32-bit software otherwise the calculated mass ion current values will be incorrect. The settings for the DDE communication on the other hand will usually not require changing. The method in which data is obtained by the Labview QMS read VIs is described in sections 3.7.1.2.2 and 3.7.3.1.2.

The CV3 allows comprehensive user control over CV experimentation in real-time for SWE, MWE and DEMS apparatus; however, for more meticulous control of the electrochemical parameters and the use of other electroanalytical techniques such as chronoamperometry the Macro software is preferred. 


\subsection{Macro}

The custom Macro electrochemistry software program allows users to employ a wide range of electroanalytical techniques to study the electrochemistry of a reaction system. The software allows users to pre-define and execute a measurement sequence of electroanalytical techniques programmatically allowing meticulous control of electrochemical experimentation. The GUI of the Macro software is shown in figure 3-26.

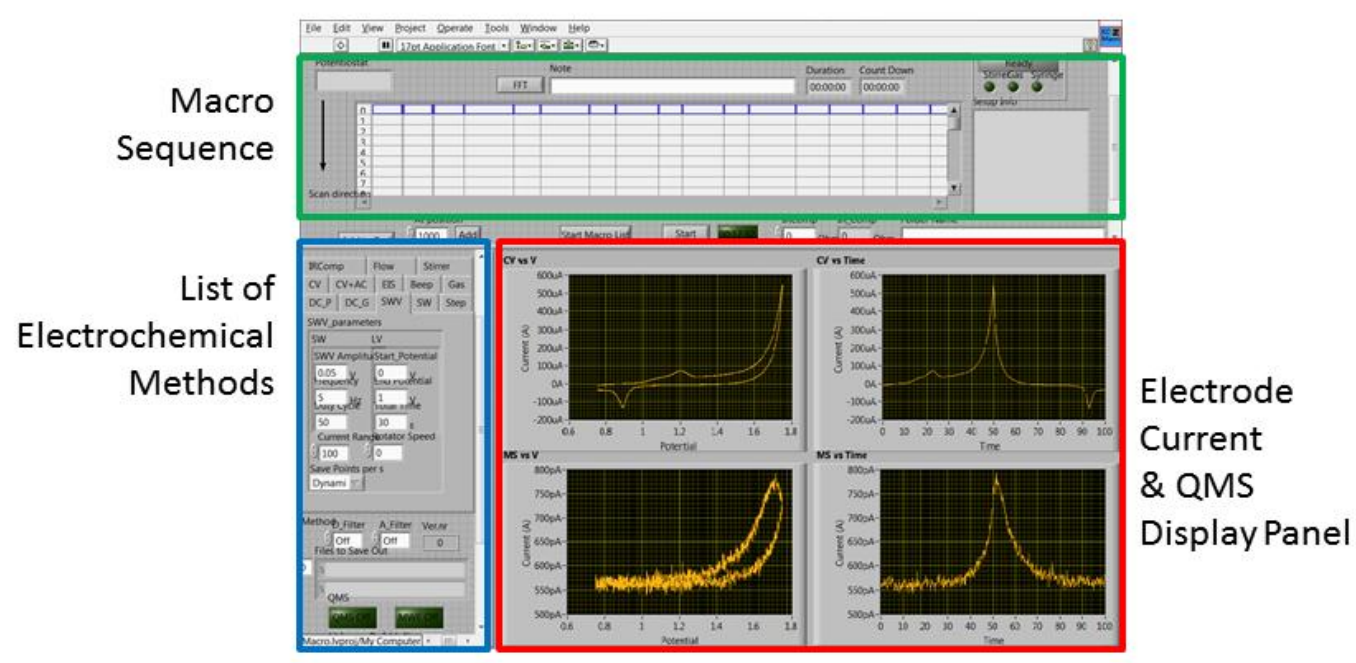

Figure 3-26: Screenshot of the Macro software front panel further developed to incorporate the required DEMS instrument functionality. The Macro software allows experimenters to define a sequence of electroanalytical measurements (such as DC and CV), or parameter changes (e.g. flow rate) allowing meticulous control of experimentation.

The user first chooses from a list of electroanalytical techniques located to the lower left of the panel, defines the measurement parameters and adds this to the Macro sequence list located at the top of the front panel. Once a list of electroanalytical methods has been defined, the user can then execute the sequence and the measurements are performed. This powerful software was adapted to incorporate the CV3 described previously, the DC3 and Syringe Pump Flow control VI's discussed in the following subsections to provide the DEMS functionality. The DEMS functionality was not incorporated into all electroanalytical methods such as fast square wave voltammetry, because they are 
of limited practical use in DEMS. However, the architecture of the QMS measurement incorporation in the CV3 and DC3 VI's should provide a template to incorporate the QMS in other electroanalytical methods.

\subsection{DC3}

The DC3 software is designed to perform either chronoamperometric or chronopotentiometric electrochemical experimentation. The user interface of the DC software is depicted in figure 3-27.

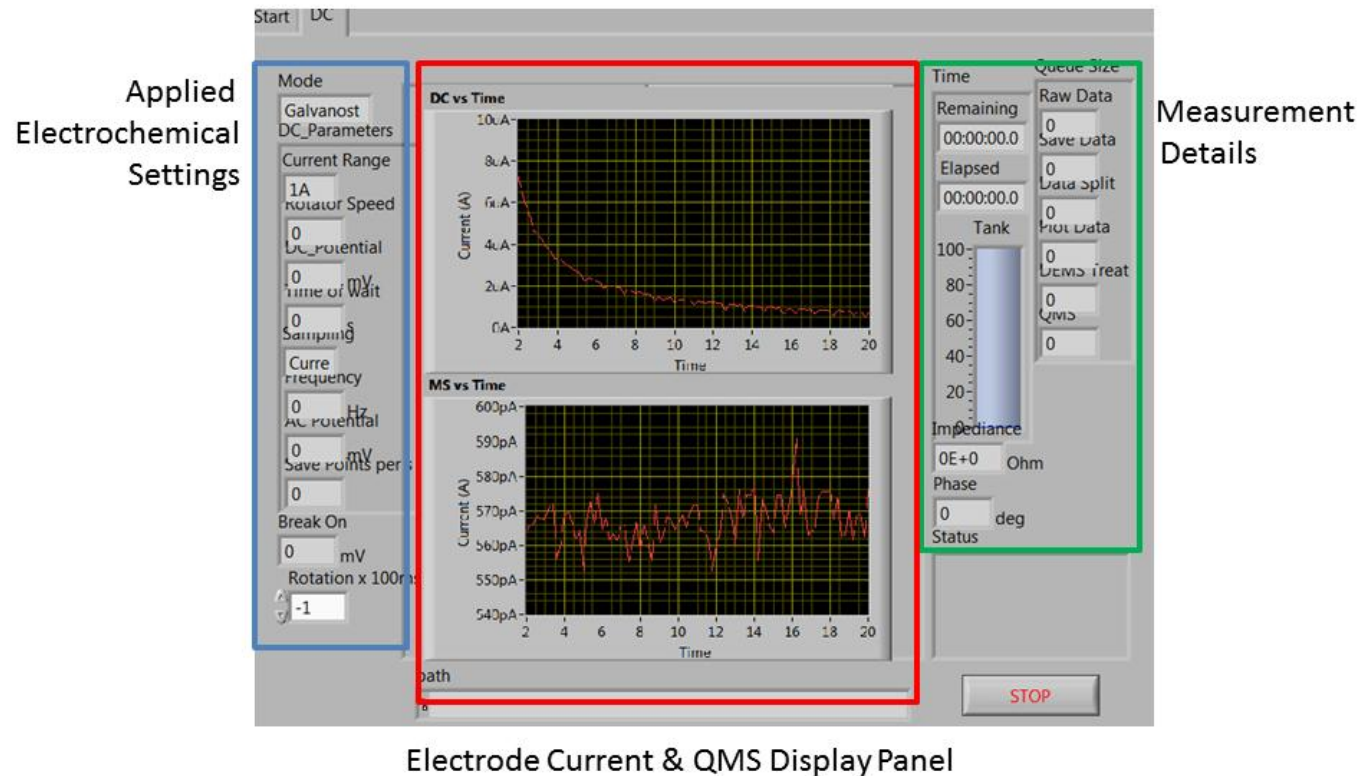

Figure 3-27: Image of the DC3 software front panel developed for the DEMS instrument. The necessary QMS functionality required for the DEMS instrument was incorporated into the DC3 software, allowing chronoamperometric and chronopotentiometric DEMS measurements.

Users can define the electrochemical parameters i.e. WE potential and time. The software is, however, more commonly used in the Macro software where a sequence of DC3 program executions can be defined, for instance in potential step measurements. 


\subsection{Syringe Pump Control}

The syringe pump control software is used to control the KDS200 syringe pump via the computer rather than manual interface. The GUI for the stand alone flow control application is given in figure 3-28.

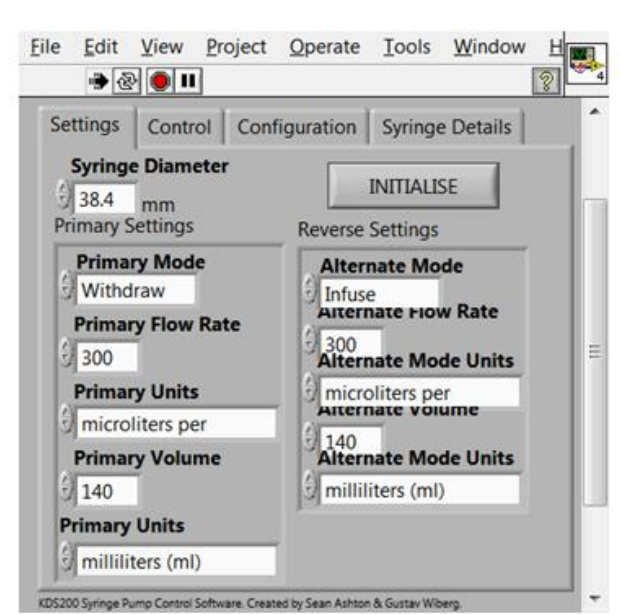

Settings Tab

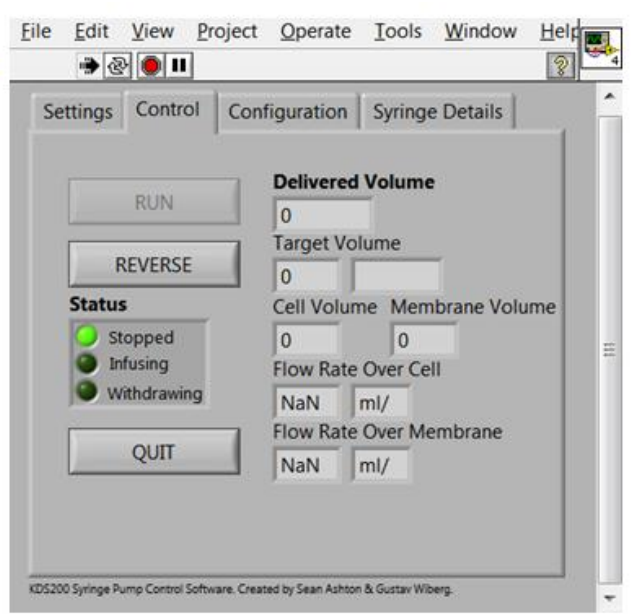

Control Tab

Figure 3-28: Image of the KDS 200 syringe pump settings (left) and control panel (right) developed for the DEMS instrument. The functionality of this software was incorporated into the Macro software allowing the programmatic control of the syringe pump which is often desirable in DEMS experimentation.

The communication between the syringe pump and program occurs via and R232 interface and must first be initiated. Details of the operational mode, syringe pump dimensions, volume and the desired flow rate must then be communicated to the syringe pump. Once this is achieved, the program can then control the operation and direction of the syringe pumping. In this form, however, the program is of limited use simply because it is quite easy to make the same changes manually via the syringe pump interface. However, programmatic control of the flow rate is far more useful when incorporated in to the Macro software in order to start or stop the pump, and manipulate the electrolyte flow during the course of an electrochemical experiment. It also eases the documentation of the experimental parameters which can be written into the DEMS measurement data file. 
These electrochemistry software tools combined allow comprehensive control over the DEMS instrument for common measurement tasks. However, another measurement setup is required in order to perform calibration procedures required for the QMS.

\subsubsection{QMS Calibration Setup}

An overview of the measurement setup for the simultaneous acquisition of QMS and pressure data used for the calibration procedure described later in Chapter 4 section 4.5 is depicted in figure 3-29.

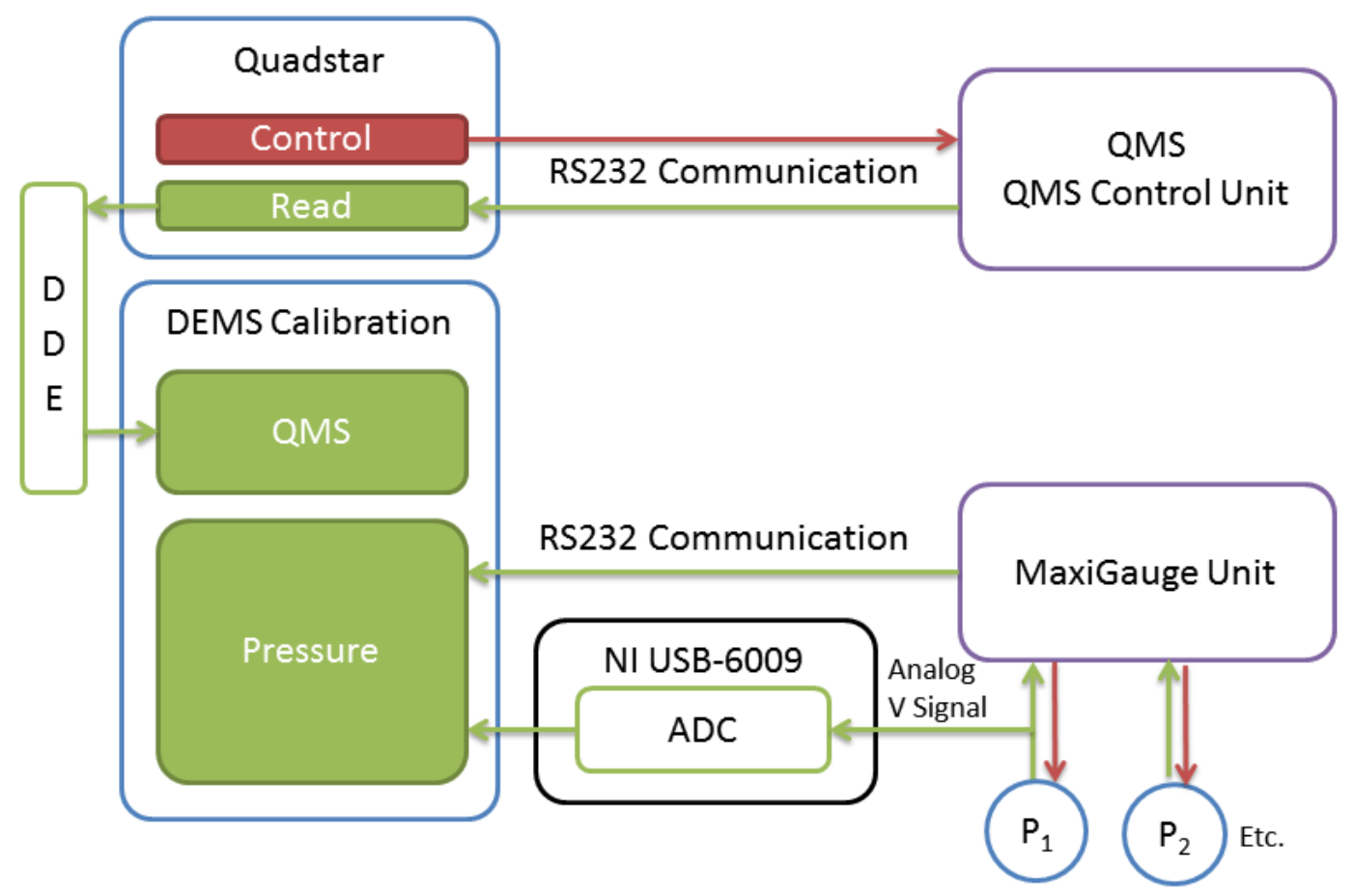

Figure 3-29: Overview of the QMS calibration measurement hardware and software system. The calibration procedure requires the precise measurement and correlation between the QMS mass ion current and pressure of a calibration volume.

In order for the QMS to be calibrated, the pressure of a calibration volume and the mass ion current of the relevant species must be measured accurately and perfectly synchronised. In the QMS calibration measurement setup, pressure data may be acquired from the vacuum gauge control units using either or both a DAQ card and RS232 data communication. The DAQ card is used to acquire the raw analogue signal voltage output of the pressure gauge, whilst RS232 
communication are averaged values provided by the Maxi Gauge unit. In general, the DAQ of the analogue signal is preferred for the pressure measurement of the calibration volume. In the DEMS calibration software, however, the QMS data should be acquired using DDE communication between Labview and Quadstar software. In this case DDE communication is necessary simply because the DAQ card that is usually used to acquire QMS data during DEMS operation is now used to measure the vacuum gauge pressures.

\subsubsection{Vacuum System Hardware}

The DEMS vacuum pressure is monitored using a variety of vacuum gauges, namely two pirani gauges, two cold cathode gauges and a hot cathode gauge. An additional capacitance gauge is also used for the calibration of the QMS. Each of these gauges were purchased from Pfeiffer Vacuum, and are all powered and controlled using the Maxi Gauge control unit. There is of course additional vacuum hardware such as the turbo and rotary vane pumps (including their control units), however, these are neither especially adapted nor specific to DEMS. For a comprehensive overview of vacuum technology the reader is instead directed to ref. [96-100].

\subsection{Vacuum Gauges}

The DEMS vacuum system employs five permanent vacuum gauges to monitor the vacuum pressure. The $1^{\text {st }}$ and $2^{\text {nd }}$ pumping stages employ a IKR 261 compact cold cathode gauge which is able to monitor high vacuum pressures between $10^{-2}$ and $2 * 10^{-9}$ mbar. The $3^{\text {rd }}$ pumping stage employs a hot cathode gauge which is able to monitor vacuum pressures between 1100 and $5^{* 10^{-10}}$ mbar. The cold cathode gauge could not be used on the $3^{\text {rd }}$ pumping stage because of the disruptive influence of the magnet on QMS. Two pirani gauges are meanwhile used to measure the vacuum pressure between the back of the turbo pumps and the rotary vane (roughening) pumps. 
For the calibration of the QMS an addition gauge is used to measure the pressure of the calibration volume which is attached to the DEMS vacuum system in place of the electrochemical cell. In this setup, a capacitance manometer gauge with ceramic diagram was employed to monitor the vacuum pressure of the calibration volume. These types of gauge are able to measure the vacuum pressure very precisely, although they are limited to a comparably narrow pressure range of $\sim 2$ decades. The chosen gauge for calibration was a capacitance gauge capable of monitoring vacuum pressure between 1 and 110 mbar. Each of the vacuum gauges require the MaxiGauge vacuum measurement and control unit to operate.

\subsection{MaxiGauge}

The MaxiGauge control unit is required to operate and monitor the six Pfeiffer Vacuum pressure gauges used by the DEMS instrument. When operated the MaxiGauge first identifies the attached vacuum gauge, provides the necessary power for operation, and then monitors the pressure gauge measurement voltage output which it averages and converts into a pressure value that is then displayed on the MaxiGauge display panel. This allows users to observe the vacuum system pressure which is often all that is desired.

For the QMS calibration procedure described later in Chapter 3 section 4.5, it is necessary to accurately measure the pressure decay of a calibration volume and precisely correlate this with a corresponding QMS mass ion current. In order to do this, however, we once again require a data acquisition setup to record the vacuum pressure. Pressure measurements can be acquired from the MaxiGauge unit via a R232 interface and programmatic communication between the device and Labview software. This is an acceptable solution when monitoring gradual changes in vacuum pressure where time resolution is not critical because the DAQ rate is relatively slow, and irregular due to the nature of RS232 communication. This is problematic for the QMS calibration procedure outlined in Chapter 3 where the pressure changes relatively quickly and the measurement is very time critical. A faster alternate method of acquiring the vacuum pressures 
with precise time resolution is to measure the original pressure voltage output arising from the pressure gauge using a DAQ card. The vacuum gauge manufacturer, however, does not make this straightforward because the Maxi Gauge unit does not possess any analogue signal outputs, and the nature of modern vacuum gauges does not make it easy to probe the signal directly. However, by construction a special adapter cable which lies between the pressure gauge and control unit, the vacuum gauge measurement output may be probed without impinging on the vacuum gauge operation. The adapter cable therefore lies between the connection between the pressure gauge and Maxi Gauge unit, as depicted in figure 3-30.

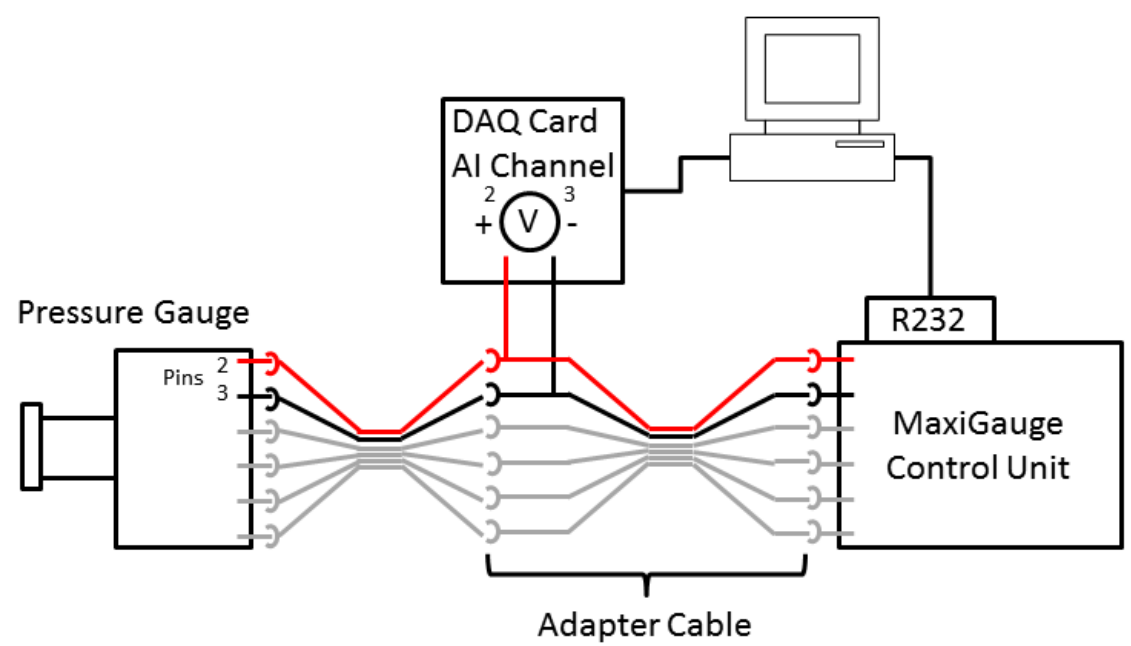

Figure 3-30: Schematic of the analogue pressure gauge measurement system used for QMS calibration. An adapter cable was created to directly probe the pressure dependant potential output of the pressure gauge to precisely acquire the analogue signal using the DAQ card rather than via the averaged measurement obtained through the MaxiGauge (which is not time resolved).

The vacuum measurement voltage output can therefore be measured directly and converted into the corresponding pressure via a certain equation provided in the gauge operating manuals (see ref. [96-98]). For the capacitance gauge in the QMS calibration setup the output between 0.1 and $10 \mathrm{~V}$ simply corresponding to a pressure range of 1 and 100 mbar. For more details of the MaxiGauge operation refer to the operating manual [99]. The acquisition and synchronisation of the QMS and vacuum pressure meanwhile is achieved using the QMS calibration software. 


\subsubsection{QMS Calibration Software}

The DEMS (QMS) calibration software was programmed in order to facilitate the calibration of the QMS and avoid the tedious and time consuming nature of the calibration procedure which in some fields is sometimes avoided unless absolutely necessary for a certain analysis. The calibration software is used to measure, synchronise, display and record the QMS mass ion current and calibration vacuum gauge pressure simultaneously. Once a QMS calibration measurement has been performed, a data treatment panel is also included in the software which is capable of importing and treating the calibration measurement data to extract the calibration constant. The DEMS (QMS) calibration software GUI is shown in figure 3-31.

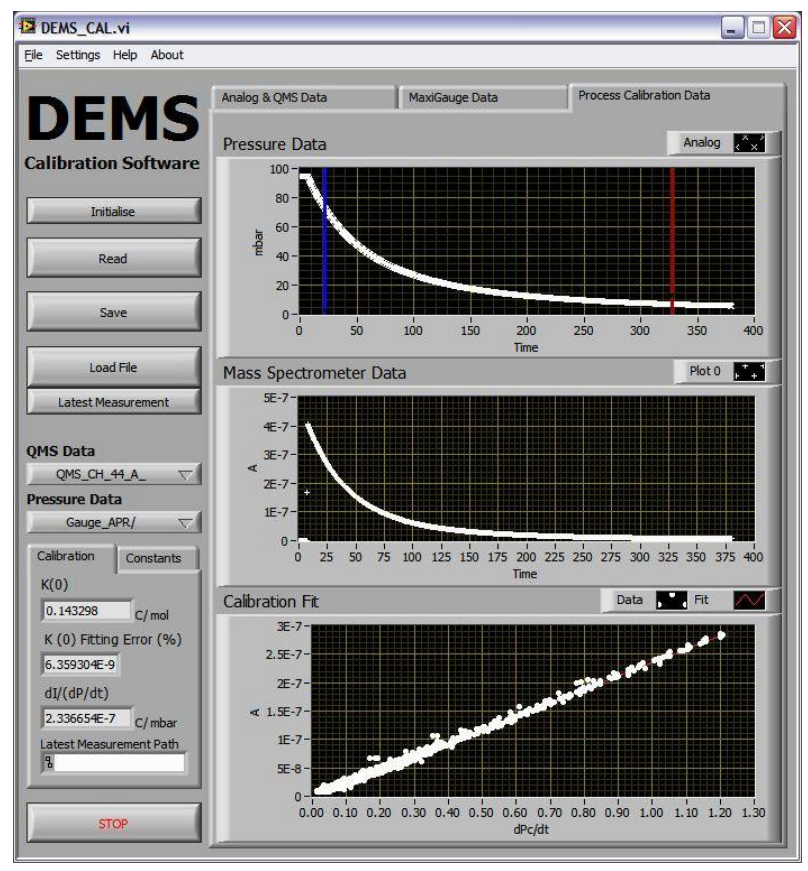

Figure 3-31: Image of the DEMS (QMS) calibration software front panel developed for the DEMS instrument. The DEMS (QMS) calibration software is capable of both measuring, displaying and recording pressure and QMS mass ion current data in real-time. Following a calibration measurement procedure, the software is also imports and treats the calibration data to provide the calibration constant, $K^{0}$.

In order to calibrate the QMS instrument, the pressure of a calibration volume and DEMS vacuum chamber must be recorded in parallel to the mass ion currents relevant to the species of interest. Once initialised and started the software begins to read, synchronise and display the QMS and pressure gauge 
data. When the calibration measurement is ready to be performed, the user can then chooses to begin to save the data points which are written to a text (.txt) file in a tab delimited format. The calibration procedure can then performed and once finished, the user can then stop the saving of measurement data points. The calibration procedure can then be repeated to ensure reproducibility of the measurement, and then stopped. The DEMS (calibration) files are then saved to a user defined folder name organised in a folder structure according to the measurement date Further details of the calibration setup and measurement methodology are present in section 4.5 .

\subsubsection{Labview Software Architecture}

The purpose of this section is to present an overview of the architecture of the custom Labview programmed electrochemistry software (CV3), and how the required DEMS functionality has been incorporated. Rather than attempting to document and explain the graphical code which would take a thesis in itself (and would not be appreciated) the conceptual architecture of the code will be described from a top level perspective. This should at least aid in the understanding of the principle structure of the Labview code to those with little or no programming experience, whilst assisting an experienced Labview programmer to interpret the structure of graphical code. The architecture of the CV3 (and DC3) software will first be presented in the standard single working electrode (SWE) and multiple working electrode (MWE) modes. This is then followed by an explanation of how required DEMS functionality was incorporated into the software. The architecture presented here can in principle be used for many programming solutions.

In Labview based graphical programming it is good practice to encapsulate segments of code with a specific function or task within a Labview virtual instrument (VI) in order to ease the readability and management of the code from a higher programming level. The top level architecture of the CV3 and DC3 Labview VIs, when operating in either SWE or MWE mode (i.e. no DEMS functionality) is conceptualised in figure 3-32. 


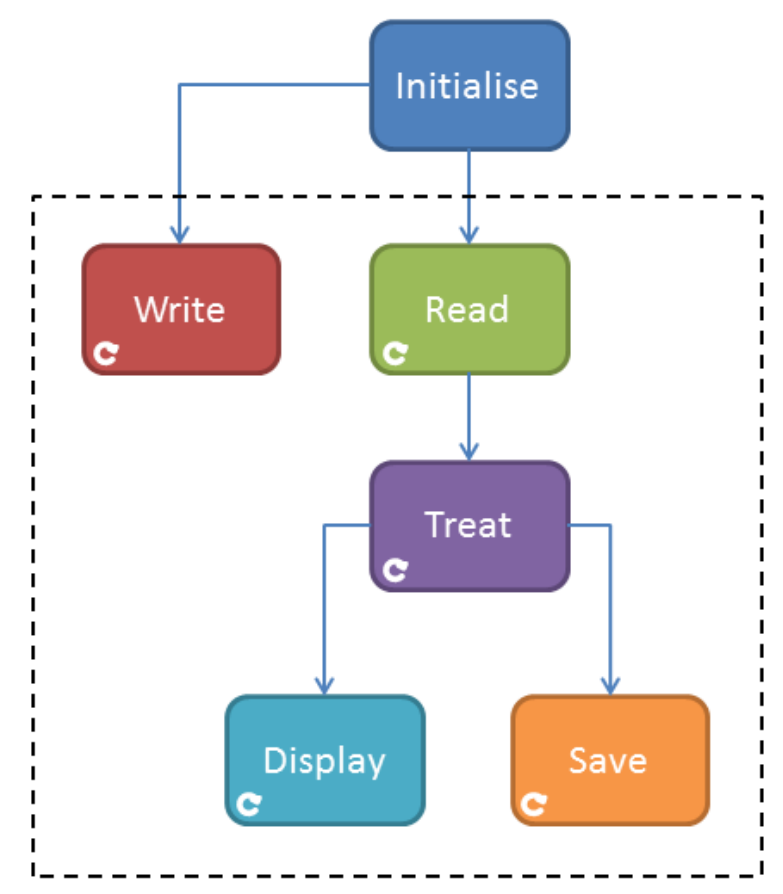

Figure 3-32: A diagram of the electrochemistry Labview software conceptual architecture in standard CV, DC and MWE operational modes. The software possesses a write VI to control the potentiostat whilst the read measures the potentiostat response. The electrochemical data is then passed to the treat VI where it is treated before it is displayed (in the GUI) and saved. By passing the data between the VIs using Labview Queues, the VIs are able to operate at different execution speeds allowing easy synchronisation of the processes.

In the SWE or MWE modes the software relies on the execution of six VIs which are each responsible for a different task. When the program is started an initialisation VI(s) executes once (during start or restart) before five VIs execute, each with their own specific purpose. In the CV3 software the initialisation VI(s) prepare the DAQ card and obtains the setup, measurement mode and experimental parameters defined by the user in the GUI. The relevant parameters are then passed onto the Write and Read VIs which execute simultaneously. The Write VI is responsible for writing the potential perturbation values (such as for a potential ramp or fixed potential value) to the DAQ card buffer which is transformed into an analogue voltage output that is applied to the analogue potentiostat WE potential control input. At the same instant, as defined by a trigger, the Read VI begins to read the DAQ analogue input signals which arise from the potentiostat measurement voltage outputs i.e. the measured WE current and potential. This data is then passed to the Treat VI which transforms 
the data, for example, by converting the current voltage measurements into the corresponding current, and averaging the raw measurement data. In the CV3 software, this Treat VI is designed to average the electrochemistry measurement data to a single data point per $\mathrm{mV}$ (or per second in DC mode) independent of the potential scan rate. The Treat VI then outputs the treated data to the Display, and a Save VIs. The Display VI plots the measured data in the appropriate format i.e. either current vs. potential (CV3), or current and potential versus time (DC3). The Save VI meanwhile writes the measured data to a tab delimited text file (.txt). The synchronisation and timing of these VIs is aided considerably using a set of built-in Labview Queue VIs. The Queue VIs allows data to be passed and buffered between each of the five VIs, allowing each VI to execute at different speeds. By furthermore placing the VIs in separate CPU threads, and assigning the VIs and execution priority, the execution of time critical VIs such as the Write and Read VIs can be prioritised over the Treat, Display or Save VIs which are not time critical and can execute at slower rates, or with a slight delay without losing measurement data. For more details of the aspects of the Labview program in SWE and MWE modes may be found in ref. [90].

In order to reduce management of the Labview programming and utilise the already powerful existing CV2 and Macro software for SWE and MWE methods, the required DEMS functionality was incorporated into the existing CV2 software architecture, rather than construct a new separate piece of software. In order to incorporate the required functionality of DEMS in the CV3, DC3 and Macro software, two additional VIs, the QMS Read and DEMS Treat VIs were introduced, whilst the Save and Display VIs were modified to offer more flexibility (i.e. the same core VI's can be used in SWE, MWE and DEMS modes). The conceptual architecture of the electrochemistry software in DEMS operation mode is presented in figure 3-33. 


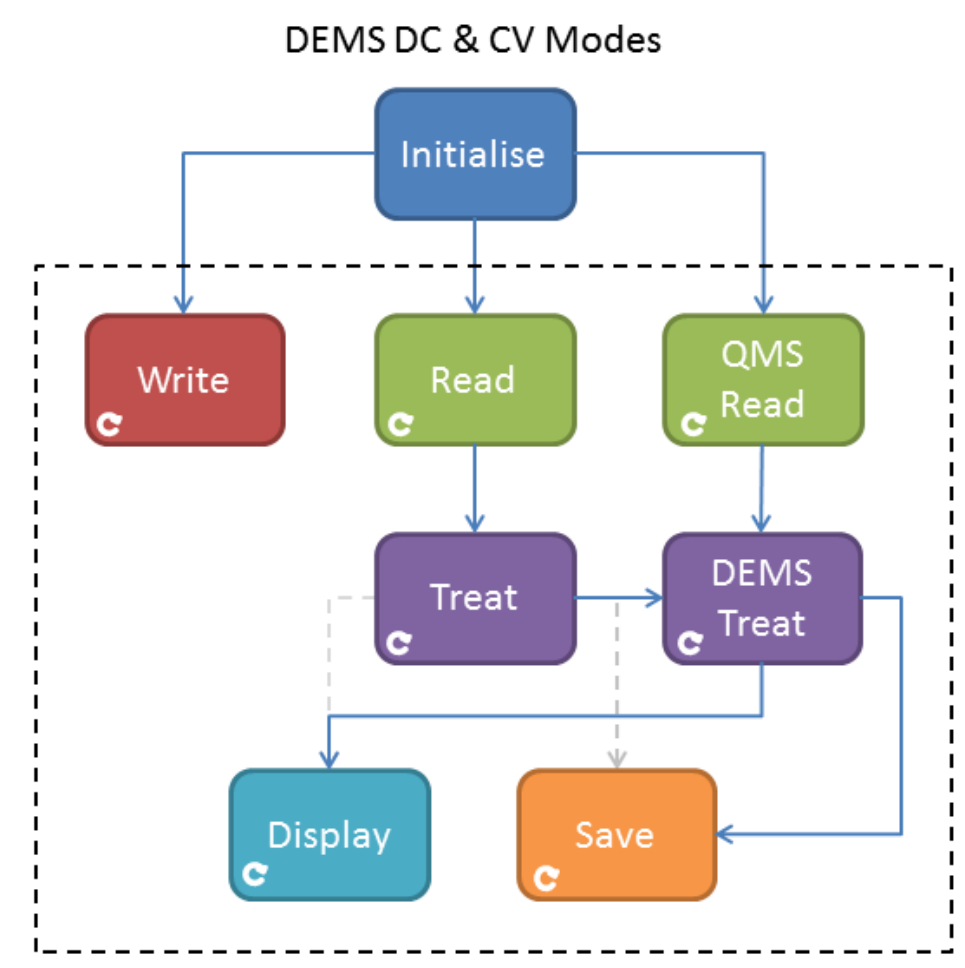

Figure 3-33: Diagram of the electrochemistry Labview software conceptual architecture in DEMS operation mode. In this mode the Queue from the Treat VI to the Display (normally used in CV/MWE modes) is cancelled whilst the Queue to Save is diverted to the DEMS Treat VI. This VI synchronises QMS measurement data (from QMS Read) with the electrochemical data. The DEMS Treat then passes the combined electrochemical and QMS data to the Display and Save VIs.

The QMS Read VI is responsible for acquiring data from the QMS, which may be obtained using two modes, either analogue DAQ or DDE software communication, which are described in more detail in paragraph 3.7.3.1.2. This VI then sends data via a Labview Queue VI to a DEMS Treat VI. In DEMS mode the functionality of the standard Treat VI is changed and instead of sending data directly to the Display and Save VIs, the data for the Display VI is cancelled whereas the data intended for the Save VI is re-directed via a queue to the DEMS Treat VI. The DEMS Treat VI then synchronises the electrochemical data with the QMS data in real-time before the new data is passed to the Display and Save VIs which plot and save the combined electrochemistry and QMS data in the desired format. By using this structure, the electrochemistry VIs are largely unchanged in the DEMS operation mode and we simply introduce two new VIs and re-direct the treated electrochemistry data. A more detailed description of 
the Labview VIs used in the DEMS software is described in the following subsections.

\subsection{Initialise VI}

The purpose of the QMS Initialise VI(s) is to perform the all the necessary functions that must be done prior to the start of the DEMS measurement. All the parameters that are relevant to the QMS initialisation and measurement are placed into Labview global parameter class called QMS_Config.lvclass. This contains all details relevant to the VIs required for QMS VIs such as the QMS status, DAQ measurement or DDE service details. These parameters may be manipulated using the QMS settings dialogue box presented previously in section 3.7.1.3.2. The QMS configuration settings are furthermore stored in the same 'ini.txt' file which retains details of the electrochemical setup parameters (such as potentiostat id, measurement mode etc.) when the software is closed. Once the measurement software is started the initialisation VI(s) execute and prepare the DEMS instrument for operation. In DDE QMS data acquisition mode this initialisation procedure also involves launching the Quadstar 32-bit software, which is used more regularly by the DEMS (QMS) calibration software rather than CV3 etc.

\subsection{QMS Read VI}

The QMS Read VI is responsible for acquiring the QMS data points, which may be achieved in either of two ways: (i) by using DDE communication with Quadstar 32-bit, or (ii) via an external DAQ card that reads the amplified mass ion current signal from the oscilloscope connections on the QMG 442 control unit. DDE is a technology that enables communication between software applications in the Microsoft ${ }^{\odot}$ Windows operating system environment. In this case, DDE is used to communicate the QMS data points obtained by Quadstar 32-bit measure program with the Labview software VI. Alternatively, raw QMS data may be measured using a DAQ card that records the signal output from the analogue 
outputs (AOs) in the back of the QMG 422 unit. The DAQ card records the signal from sync, scan and either elm, the raw amplifier AO signal, or mon, the measured value averaged by the QMG 422 unit. Details of how the QMS data is extracted from the oscilloscope connection of the QMG 422 unit is described in section 3.7.1.2.1.

In general, data acquisition via the oscilloscope connection is favoured over DDE for a number of reasons. Firstly, DDE communication Quadstar must be initialised controlled and closed using the Labview VIs which locks any manual control of Quadstar 32-bit software program by the user when the CV3, DC3 or Macro software is operating. This is unsatisfactory because the Labview software program must be restarted each time the QMS settings are change, and DDE can use considerable computer resources. In contrast, using the DAQ card the Quadstar 32-bit measure software runs independently of the Labview VIs and QMS settings are easily adjusted when desired. An additional advantage of using the DAQ card, is that the raw electrometer signal may be assessed using the elm analogue output. The raw signal can then be treated using appropriate analogue signal filters and averaged using Labview rather than (blindly) relying on the QMG 422 units and Quadstar 32-bit DDE or mon oscilloscope output which is less flexible and more of a black box. In cases where unusual QMS settings are used the QMG 422 unit data output can deviate significantly from the actual values, particularly when inappropriate filters, $\mathbf{p - c a l}$ values or QMS parameters are applied. Finally, DDE generally requires more computer resources than the DAQ method, which not only limits the ability of the computer to perform other tasks but there is also a risk of time critical data points to arrive late or be lost. Regardless of the method used to acquire QMS data, once obtained the data points are sent to the DEMS Treat VI.

\subsection{DEMS Treat VI}

The DEMS Treat VI is responsible for synchronising the QMS data points with the electrochemistry data points, a process which is crucial to the precision of the instrument. However, combining data from two separate sources that measure at 
different sampling rates (which can both change depending on the measurement parameters) can be quite tricky, particularly when DAQ rates are fairly slow (such as the QMS) in the desire that no measurement points are discarded. In the electrochemistry Treat VI, the measured WE current is treated to provide a single data point per $\mathrm{mV}$, regardless of the potential scan rate of a $\mathrm{CV}$, or a single data point per second for chronoamperometry (DC3). The data sampling frequency of the QMS is, however, comparably slow compared to the electrochemical measurement, and is furthermore sensitive to the QMS parameter settings, particularly in terms of the number of different mass ion current channels measured, and their corresponding p-cal and dwell times. Owing to the relatively slow sampling rate of the QMS, the QMS data cannot simply be averaged down to provide a single data point per $\mathrm{mV}$ (unless a single mass ion current is measured in the QMS DAQ mode, however this is generally not the case).

The time, current and potential data from the electrochemistry Treat VI which is sent to the DEMS Treat VI has been averaged to provide a single data point per $\mathrm{mV}$ (or $\mathrm{s}^{-1}$ in the DC3) independent of the potential scan rate. The QMS data point frequency is, however, comparably slow and cannot be simply averaged down to a single point per $\mathrm{mV}$, particularly for scan rates > ca. $5 \mathrm{mV} \mathrm{s}^{-1}$. In order to allow some flexibility in terms of the possible CV scan rates, along with QMS settings (e.g. dwell times) the DEMS Treat VI synchronises the QMS data with the electrochemistry data differently for cases where the QMS sampling frequency is faster than a single data point per $\mathrm{mV}$, from cases where it is slower. The manner used in the DEMS Treat VI to synchronise the QMS data with the electrochemical data in is illustrated in figure 3-34. For situations where the QMS sampling rate is faster than a single data point per $\mathrm{mV}$, the QMS data points are simply averaged. This may be the case when few QMS channels are measured and/or and the potential scan rate in the $\mathrm{CV}$ is slow. More commonly, however, the QMS data point frequency is slower than one point per $\mathrm{mV}$ and the QMS data is retained rather than averaging the faradic current data down to the QMS sampling frequency (this way we do not lose any of the electrochemical data). Another approach could have been to extrapolate QMS points where a data point 
was missing, however, this was considered highly inadvisable (one would lose track of the true measurement data).

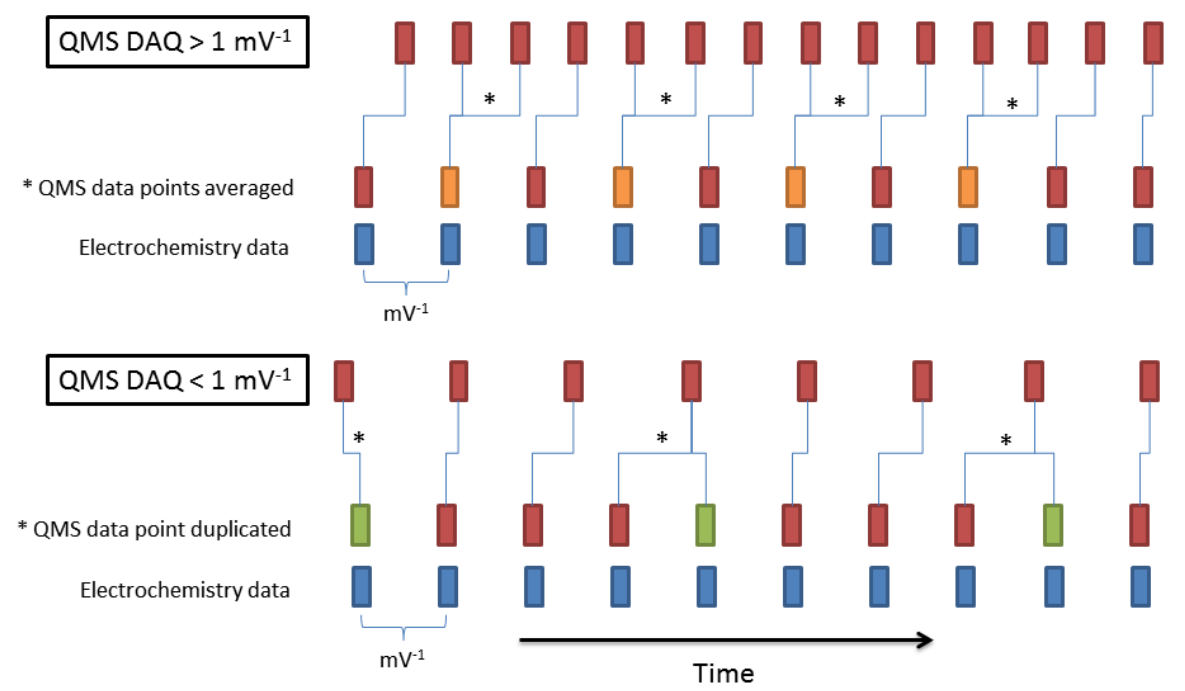

Figure 3-34: A diagram illustrating how the QMS data points are correlated to the electrochemistry data. When the QMS DAQ speed is faster than $1 \mathrm{mV} \mathrm{s}^{-1}$, the QMS data points are averaged, whereas when it is slower, the QMS data points are duplicated when a new data point is missing. This allows a flexible range of scan rates to be used without needing to endlessly adjust QMS parameters or lose electrochemistry measurement data.

Overall, the DEMS Treat VI allows a flexible degree of variation in electrochemistry scan rates, and QMS sampling frequency without loss of electrochemistry data. The duplication of the QMS data points, however, necessitates the removal of the duplicate data points during post measurement data treatment, particularly where smoothing of the QMS data is desired (this removal is performed using the DEMS data analysis program)

\subsection{Plot VI}

The QMS plot VI, displays the electrochemical and QMS data that was synchronised in the QMS treat VI. In contrast to the CV plot, the electrochemical and QMS data is plotted as both a function of both potential and time. This way the QMS mass ion currents are more easily interpreted as a function of time, compared to the electrochemistry which is best analysed as a function of the 
electrode potential. The Display VI also retains data from the previous three CVs in the display of the latest $\mathrm{CV}$ so that the experimenter can observe changes in the electrochemistry of the WE, for instance when changing electrochemical parameters or assessing stability and reproducibility of a system. In the CV3 and DC3 programs the display VI incorporates the appropriate plots for SWE, MWE and DEMS techniques rather than using separate VIs making the Labview code more manageable.

\subsection{Save VI}

The Save VI is responsible for saving the DEMS measurement data in '.txt' text file format. The Save VI was adapted so that the VI would work in more general cases, i.e. it functions for each of the SWE, MWE and DEMS modes of the software. The DEMS data file produced is a tab delimited text file, as shown in figure 3-35 containing details of the experimental parameters (either auto assigned or defined in the CV/Macro by the user), electrochemistry data (time, WE potential and WE current) and in DEMS mode the QMS data.

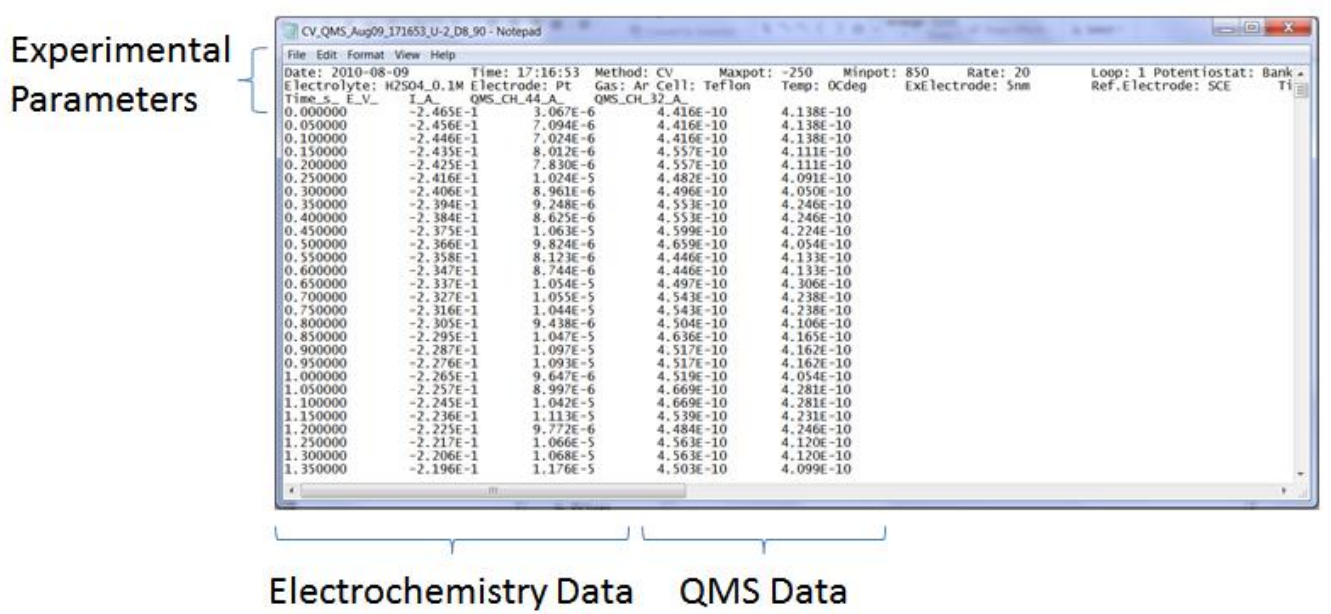

Figure 3-35: Screenshot of the DEMS save data file indicating the file format. The experimental parameters relevant to the electrochemical measurement are stored in the file header. The electrochemistry and QMS mass ion current channel data are then written in tab delimited columns. This allows experimental data to be easily imported into common data analysis software such as excel or Igor Pro, or the custom Labview programmed DEMS Analysis software. 
A new DEMS data file is created for each $\mathrm{CV}$, or chronoamperometric measurement, and is stored in a user defined folder name that is organised in a folder hierarchy according to date, month and year. The tab delimited text file format was chosen because it is easily read and can be accessed or imported into the majority of data analysis software.

\subsection{Data Analysis}

Data treatment can be very time consuming and in situations where tasks are repetitive, specifically designed data treatment software can greatly enhance productivity. Consequently, a custom Labview software program was also developed in order to perform common measurement data treatment tasks that are specific to DEMS. The DEMS Analysis software GUI is depicted in figure 3-36.

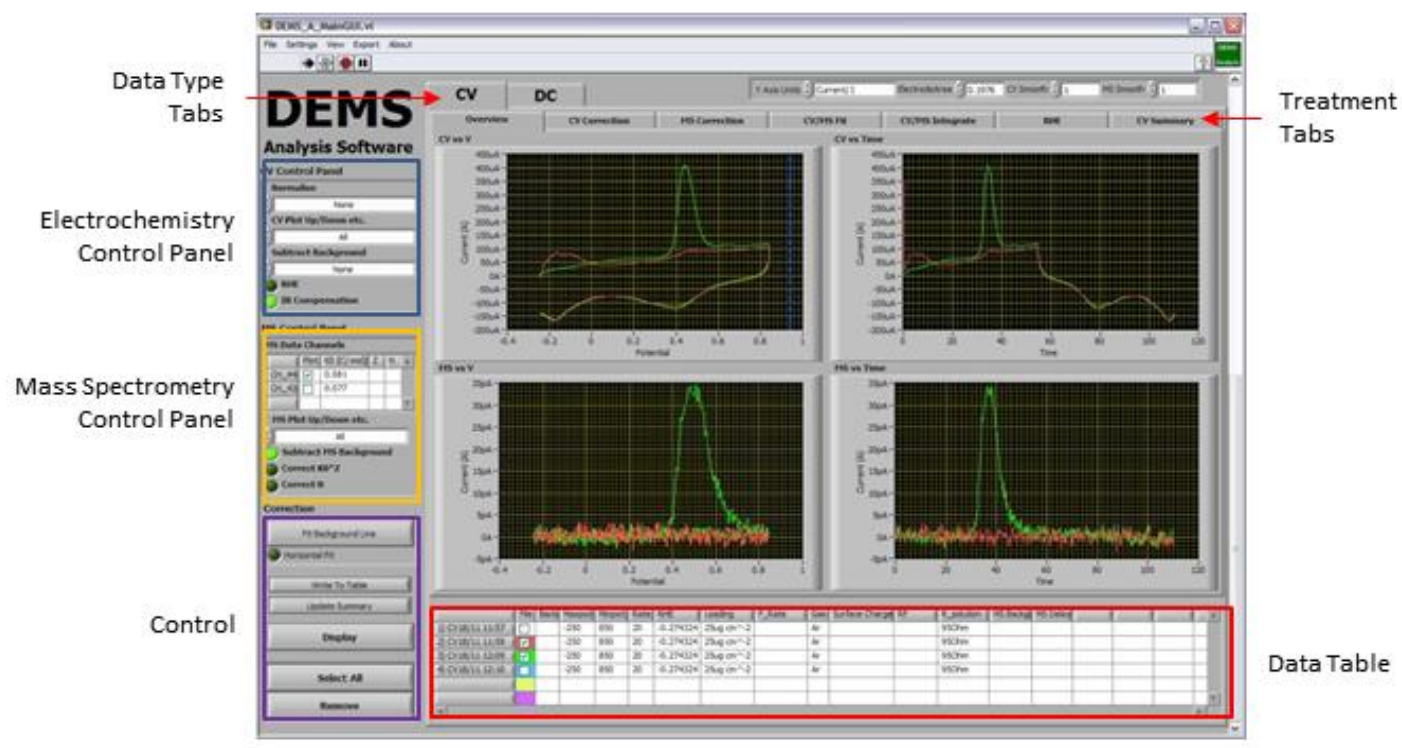

Figure 3-36: Screenshot of the DEMS analysis software front panel developed as part of this thesis. The DEMS analysis software allows common, necessary data analysis and treatment tasks that are specific to DEMS to be performed quickly and easily. The software also allows treated data to be exported to other analysis programs such as excel or Igor Pro.

The GUI is arranged so that common display or data treatment options are always available and visible to the user on the left hand side of the panel. A 
number of tabs are meanwhile located in the graphical view, which are each organised by specific task, for instance, there are tabs for background subtraction and integration. The DEMS measurement data .txt files may be imported into the program via the runtime header which are then added to the interactive table at the bottom of the GUI displaying the file names along with important measurement parameters such as the potential range, scan rate, solution resistance etc. Using this table, users are able to easily choose the data files to be displayed and treat in the necessary manner. The data treatment tasks for the faradaic current and mass ion current such as, background subtraction, normalisation or post measurement $i R$-compensation etc. are then applied on the selected data files and plotted in the display panel. This allows data to be treated in a matter of a few minutes rather than hours if the data were to be treated manually in either Excel or Igor Pro.

In comparison to the custom programmed electrochemistry measurement software, the graphical code of the DEMS data analysis software is comparatively more straightforward, with a (hopefully) clearer structure. The graphical code of the software will therefore be discussed more specifically, in order to direct future users or programmers of the software to adding additional functionality to the DEMS analysis software if and when desired for special data treatment tasks. An annotated image of the top level graphical code of the DEMS data analysis program is shown in figure 3-37. The program essentially possesses three loops: an initialise loop (which executes when the program starts), event (or producer) and processer (or consumer) loops which execute depending on the user input from the GUI. 


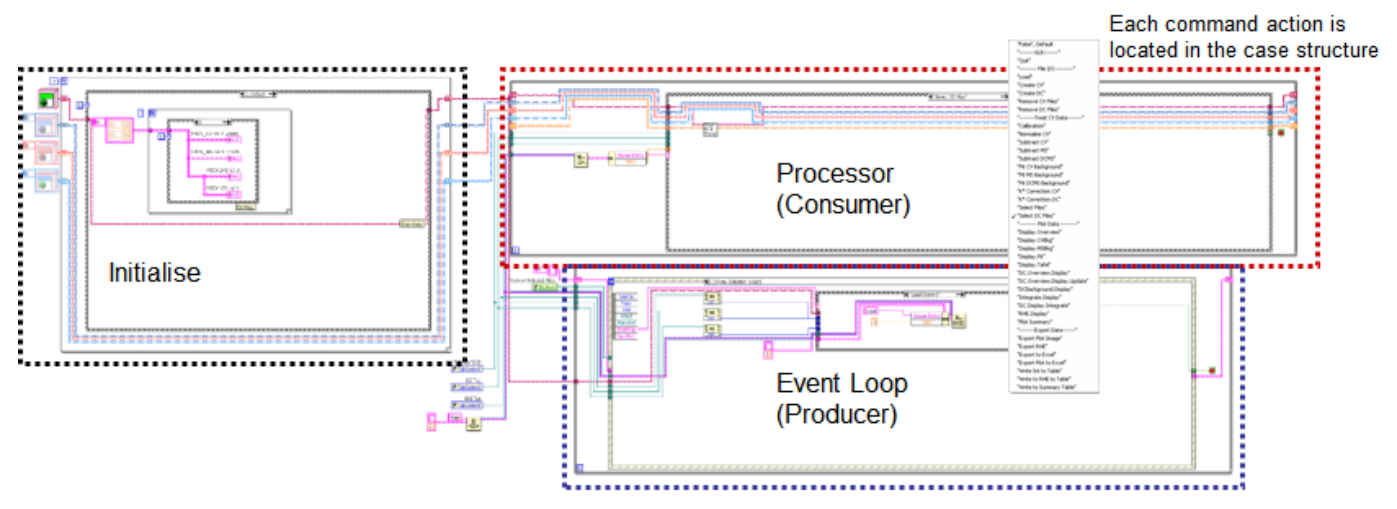

Figure 3-37: Screenshot of the queued state machine producer-consumer architecture of the DEMS Analysis software. The event loops is responsible for the programmatic response to user events (producer), whereas the processor (consumer) executes the corresponding code. This architecture is a very useful method of solving many programming tasks.

The initialise loop only executes once when the Labview program is started, and is responsible for resetting the GUI and writing the necessary front panel references to a class VI. The event and processor loop then execute continuously. The event loop handles all user inputs by recognising an event, such as a user mouse click on a GUI button which corresponds to a certain task such as import data, or display. The event loop then executes and produces a command (or set of commands) which is passed to the processor loop. The processor loop has two main functions: (i) it imports and stores the raw DEMS data in the shift register of the processors while loop in a file data class and (ii) it performs all the necessary data treatment tasks. Although these tasks will be specific to the data treatment being performed, it generally involves creating an interpolated duplicate of the raw DEMS data, which is then treated in the desired manner before being plotted in the DEMS analysis display. The data treatment tasks are, however, determined by the user and in turn the event loop. The communication between the event loop and the processor loop is handled using the Labview Queue VIs, and is depicted in figure 3-37. 


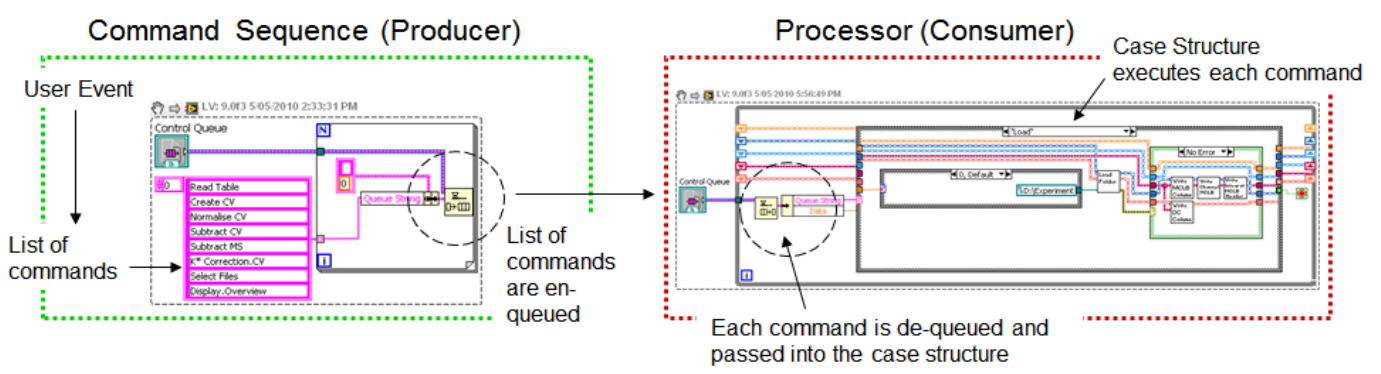

Figure 3-38: Diagram representing the structure of the data treatment command sequence (producer) and sequence processor (consumer) of the DEMS analysis software. The event loop produces an array of strings which contain the tasks necessary to execute the user event. This list of commands is then executed on the imported electrochemical data, treating the data in the desired way.

When the user executes a certain event such as display, the event loop produces a list of commands (or arrays of strings) which must be performed to display that data. This array of strings is then enqueued into the Labview queue in the event loop (producing commands), which are then de-queued in the corresponding order in the processor (which consumes the commands). Each of these commands (or strings) correspond to a case in the processor while loop case structure. The processor then de-queues each string and executes the corresponding case in the processor case structure for each loop iteration. These array of strings typically involve a read table (to read the data table values), create $\mathrm{CV}$ (which duplicates and interpolates the raw data into a treat data class), a series of data treatment steps (to treat the treat data class) and then a select file command (which obtains the chosen files to be displayed) and finally, a display file command.

This structure should all the easy addition of new data treatment tasks by simply creating a new case in the processor case structure, and adding the corresponding string to the array of strings, which may be desired in the future depending upon data treatment tasks. Furthermore, the software also possesses an export to Excel and text file function that allows treated DEMS experimental data to be further analysed in either Excel or Igor Pro for special analysis or the production of publication quality figures. The structure of the DEMS analysis program used here is in principle a queued state machine producer consumer architecture which is very powerful and can be used to solve a wide range of programming tasks, further details of which may be found in ref. [101]. 


\subsection{Summary}

The aim of this chapter was to present details of the DEMS instrument design, along with a description of the required hardware and software that were developed in order for the instrument to not only be functional, but also usable for experimentation.

An electrochemical half-cell was designed that is in principle, of very close geometry to the dual thin-layer DEMS flow cell design presented in Chapter 2. This allows electrochemical experimentation on bulk and thin-film electrodes but requires a flow of electrolyte. This was achieved by withdrawing electrolyte from an electrolyte supply reservoir using a precision syringe pump. In this particular cell construction a certain amount of flexibility was incorporated into this design to, for instance, allow the exchange of individual cell parts such as the flow block to give a different flow regime over the WE, and is considered rather intuitive to the cell development processes. Furthermore, the electrochemical half-cell construction possesses an all-Teflon or Kel-f flow pathway for the aqueous electrolyte, allowing alkaline solutions to potentially be used without contamination from glass constituents.

The membrane interface consists of a microporous PTFE membrane which is mechanically supported by a stainless steel frit. The total flux of gas through the membrane when separating the aqueous electrolyte from the vacuum system is crucial to obtaining the necessary vacuum pressure required to operate the QMS. It is also crucial to ensure that the membrane is sealed between the vacuum and the cell, preventing an additional leak of air into the vacuum system increasing the vacuum system pressure and background QMS mass ion currents. In general, the membrane interface compartment should possess a large geometric area to volume ratio in order to ensure high collection of the electrochemical reaction species.

The vacuum system was of a differentially pumped design, consisting of three vacuum chambers. The membrane interface is attached to a $1^{\text {st }}$ pumping stage which operates at a rather high pressure of $10^{-2}$ mbar. This is attached to a $2^{\text {nd }}$ pumping stage operating at $10^{-5}$ mbar. The $1^{\text {st }}$ and $2^{\text {nd }}$ vacuum chambers are 
connected via tubular aperture which directs the flux of gas entering from $1^{\text {st }}$ chamber through the CB-ion source of the QMS. This was to allow a higher pressure at the ion source in order to maximise the sensitivity of the QMS. Finally, a $3^{\text {rd }}$ pumping stage also lowers the pressure of the chamber containing the SEM of the QMS to $<5.0 * 10^{-6} \mathrm{mbar}$ range required for long-term, reliable operation of the SEM.

The operation and overall usability of the DEMS instrument, however, relies on a number additional commercial and custom developed hardware and software produced as part of this thesis. The electrochemical setup employed a commercial BANKS Pos2 analogue potentiostat, a NI DAQ card and custom-built NID to control the electrochemistry and apply appropriate $i R$-compensation. The QMS and vacuum pressure gauges were meanwhile controlled via commercial hardware. Potentiostat control and measurement was achieved using NI DAQ card and custom Labview CV/DC and Macro software, originally developed for the RDE methodology. As part of this these, however, the software were further developed in order to incorporate the mass spectrometry aspects of the DEMS instrument. The resulting software with added DEMS functionality allows electrochemical data to be measured, synchronised, recorded and displayed alongside QMS measurement data in real-time. The acquisition of QMS data meanwhile was achieved using either DDE software communication, or via a NIDAQ card to record the oscilloscope output of the QMS control unit. A separate hardware and software setup was also constructed using NI DAQ cards and inhouse Labview programmed software to calibrate the QMS. Finally, a custom data analysis program was also developed in order to perform data treatment tasks that are common in DEMS. 


\section{Practical Aspects of the DEMS Instrument}

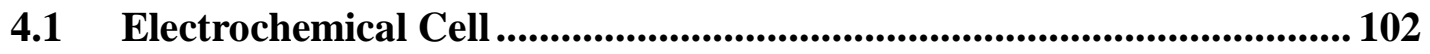

4.1.1 Potential Control ........................................................................ 103

4.1.2 Effect of Electrolyte Flow Rate ........................................................ 109

4.2 Performance of the Membrane Interface Material ............................... 119

4.3 Optimisation of the QMS.......................................................................... 124

4.3.1 Ion Source Parameters .................................................................. 124

4.3.2 Quadrupole and SEM Parameters............................................... 127

4.4 Calibration of the DEMS Instrument ............................................... 130

4.5 Calibration of the QMS ................................................................ 133

4.6 Further Considerations ................................................................... 137

4.6.1 Measurement Error ...................................................................... 137

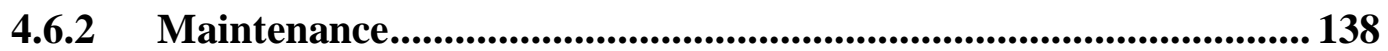

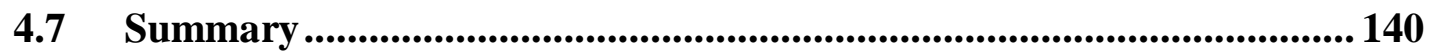

This chapter concerns the more practical aspects of the DEMS instrument, covering the instrument characterisation, influence of experimental variables and measurement parameters, calibration procedures and finally, error and maintenance considerations. The characterisation and evaluation of the DEMS instrument is crucial to finding optimum experimental parameters, discovering possible areas of improvement during the development process, and to understanding the capabilities and constraints of the instrument before any research is conducted. This process is separated into the evaluation of the three principle DEMS components, to re-iterate the electrochemical cell, the membrane interface, and the vacuum system including QMS whose capabilities combine towards the overall performance of the instrument. Following this, the methodologies and setups used to optimise and calibrate the QMS and the DEMS instrument as a whole are presented. The contents of this chapter should serve to provide a practical understanding of the DEMS instrumental variables and 
document calibration procedures so that the instrument can be used in confidence by future users.

\subsection{Electrochemical Cell}

A thorough understanding of the DEMS electrochemical cell characteristics is necessary in order to differentiate experimental observations that purely arise from instrumental parameters or measurement methodology, from those that actually result from the electrochemistry of the WE. An immediately apparent and undesirable characteristic of the DEMS electrochemical flow cell is its inherent ability to induce potentiostat instability causing oscillation of the WE using conventional three-electrode setups. Such instabilities can typically arise from high resistances in cell constructions which employ rather narrow or long connecting capillaries between working, counter and reference electrodes. The first obstacle therefore, was to diagnose and resolve this crucial issue in order to achieve reliable and precise potential control of the WE, without which we cannot perform experimentation. Once a stable control of the electrochemistry was achieved, the influence of electrochemical cell parameters, namely the electrolyte flow rate through the cell, is investigated. This was of prime interest because the electrolyte flow rate not only influences the electrochemistry at the WE, but also the QMS response impacting the DEMS instrument as a whole. More specifically, the electrolyte flow rate affects the observed diffusion limited current, $I_{d l}$, the response time and correlation between the faradaic and observed QMS mass ion current, and finally, the DEMS cell collection efficiency. In the following subsections, the influence of experimental parameters on the characteristics of the dual-cyclone thin-layer electrochemical cell measurement is presented and assessed. The methodologies employed here to characterise this cell should also be useful for the optimisation, assessment and comparison of different DEMS electrochemical cell designs fabricated in the future. 


\subsubsection{Potential Control}

The ability of the potentiostat to precisely and reliably control the potential of the WE is of vital importance to electrochemical experimentation. The potential control of the WE in the dual-cyclone thin-layer electrochemical flow cell setup fabricated for this DEMS instrument, however, is unstable and the WE potential oscillates using standard three-electrode electrochemical cell arrangements. This essentially means that electrochemical experimentation is impossible under the desired experimental conditions, and a solution to achieve reliable potential control was of vital importance to the operation of the DEMS instrument.

The instability and oscillation of a potentiostat can arise as a result of a number of issues regarding the characteristics of the electrochemical cell configuration, such as: (i) a high RE impedance, (ii) high CE resistance (iii) high cell capacitance and (iv) a too small a solution resistance ca. $<1 \Omega$ between WE and Luggin-capillary, typically arising from excessive $i R$-compensation of the solution resistance. The most common causes of potentiostat stability are high cell resistances between electrodes, and therefore often the easiest solution to stabilise a potentiostat is to reduce the resistances between the WE, RE and CE by simply positioning the electrodes closer together. Unfortunately, this is largely not possible in the DEMS electrochemical cell, although we can reduce resistances by widening the connecting capillaries of the electrode compartments and/or increasing the concentration of the electrolyte. These modifications certainly help to reduce the cell resistances but not to the extent that reliable potentiostat stability is achieved. In such cases where high resistances are a problem but the cell design cannot be changed, there are certain modifications to the threeelectrode electrochemical cell setup, such as those described in ref. [102], that can be used to stabilise the potentiostat despite the high cell resistances. For example, instability arising from high RE impedances can be reduced by employing a low impedance reference probe (i.e. Pt wire) positioned close to the WE that is coupled to the RE via a capacitor, or via a high frequency shunt in the form of a capacitor connecting $R E$ and $C E$ [102]. A full explanation of the origins of potentiostat instability (such as the electronic circuitry theory and physics) and its resolution is some way beyond the scope of this chapter, however, a 
comprehensive overview of the circumstances that cause this instability may be found in references [92, 103]. Unfortunately, these methods were found (by trial and error) not to satisfactorily resolve the issues of the DEMS electrochemical cell, suggesting that despite high cell resistances being less than ideal, they were not necessarily the main cause of the instability.

The first step toward diagnosing the origins of the potentiostat instability of the dual-cyclone thin-layer DEMS cell was to determine the solution resistances between the WE, CE and RE. The solution resistances determined between the electrode compartments of the dual-cyclone thin-layer flow cell in various electrolyte and concentrations are presented table 4-1. These were determined using a two-electrode setup using AC voltammetry.

Table 4-1: DEMS electrochemical flow cell resistances between WE, CE, RE electrode compartments and membrane interface sampling compartment (SC). Solution resistances were determined using AC voltammetry $\left(5 \mathrm{mV}, 5000 \mathrm{~Hz}\right.$ at $\left.20 \mathrm{mV} \mathrm{s}^{-1}\right)$ in a two-electrode electrochemical cell setup employing platinised $\mathrm{Pt}$ wires. The cell resistances are generally rather large but can be reduced by employing more concentrated electrolyte.

\begin{tabular}{|c|c|c|c|}
\cline { 2 - 4 } \multicolumn{1}{c|}{} & \multicolumn{3}{c|}{ Electrolyte Resistance/ $\mathrm{k} \Omega$} \\
\hline \multirow{2}{*}{$\begin{array}{c}\text { Electrode } \\
\text { Position }\end{array}$} & \multicolumn{2}{|c|}{$\mathrm{HClO}_{4}$} & $\mathrm{H}_{2} \mathrm{SO}_{4}$ \\
\cline { 2 - 4 } & $\mathbf{0 . 1 0 ~ \mathrm { mol } \mathrm { dm } ^ { - 3 }}$ & $1.00 \mathrm{~mol} \mathrm{dm}^{-3}$ & $\mathbf{0 . 5 0} \mathrm{mol} \mathrm{dm}^{-3}$ \\
\hline $\mathrm{CE} \leftrightarrow \mathrm{WE}$ & 12 & 1.4 & 2.5 \\
\hline $\mathrm{RE} \leftrightarrow \mathrm{WE}$ & 17 & 2.7 & 4.9 \\
\hline $\mathrm{CE} \leftrightarrow \mathrm{RE}$ & 29 & 5 & 10 \\
\hline $\mathrm{WE} \leftrightarrow \mathrm{SC}$ & 7 & - & - \\
\hline
\end{tabular}

It is immediately apparent that the electrochemical cell resistances in $0.10 \mathrm{~mol} \mathrm{dm}^{-3}$ are quite large, and can therefore be partly attributed to the potentiostat instability. The resistance between WE and RE, however, may not be quite that problematic in consideration that problems resolved in the literature examples were typically $>50 \mathrm{k} \Omega$ [102], although the $\mathrm{CE}$ to $\mathrm{WE}$ resistance in DEMS electrochemical cell is much higher than typical cells that do not isolate the CE from the WE compartment (such as RDE cells). By employing more concentrated and thus more conductive electrolyte, these cell solution resistances are roughly reduced by a factor of $\sim 10$, to much more reasonable values. Despite 
these reductions in cell resistances, potentiostat stability is still not achieved using a standard three-electrode setup and therefore an alternative modification to the electrochemical half-cell configuration was required.

The origins of the potentiostat instability was in fact found to arise because of too of a small solution resistance, $R_{s}, \leq$ ca. $1 \Omega$ between the WE and Luggin-capillary. Potentiostats typically require a certain amount $R_{\mathrm{s}}$ in order that conditions of potentiostat oscillation are not met, which is why $100 \% i R$-compensation is generally not possible (using analogue $i R$-compensation schemes) although it can be minimised to errors of $\sim 1 \mathrm{mV}$ (the typical error of a RE) by choosing certain cell parameters [92]. The DEMS electrochemical cell, however, does not possess a Luggin-capillary in the traditional sense of a narrow capillary leading to the WE. Instead, the electrochemical flow cell lines between the WE and RE are analogous to the Luggin-capillary, and the point at which these cell lines intersect the WE and $\mathrm{CE}$ current path can be considered to be the Luggin-capillary entrance. This would lie somewhere between the electrochemical flow cell inlet entrance to the WE compartment and the holes joining the WE compartment to membrane interface, although this cannot be defined precisely. Because this distance is relatively small $<0.2 \mathrm{~mm}$ the solution resistance is too small and the conditions at which the potentiostat oscillates is met, which poses somewhat of a dilemma in DEMS because the thin-layer is crucial to the performance of the instrument i.e. we cannot simply increase the layer thickness to the extent that the solution resistance becomes significant enough without impeding on the DEMS instruments performance. An alternative solution was therefore needed.

The solution in the case of this DEMS electrochemical cell setup was to simply stabilise the potentiostat by artificially increasing the solution resistance by adding a stabilising resistor between the $\mathrm{WE}$ and potentiostat, the equivalent circuit of which is shown in figure 4-1 (a). 
a) Stabilising Resistor

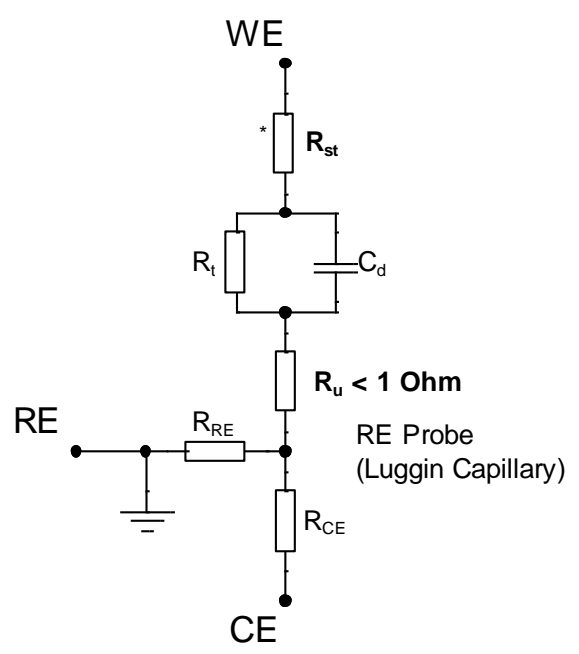

b) Dual Counter Electrode

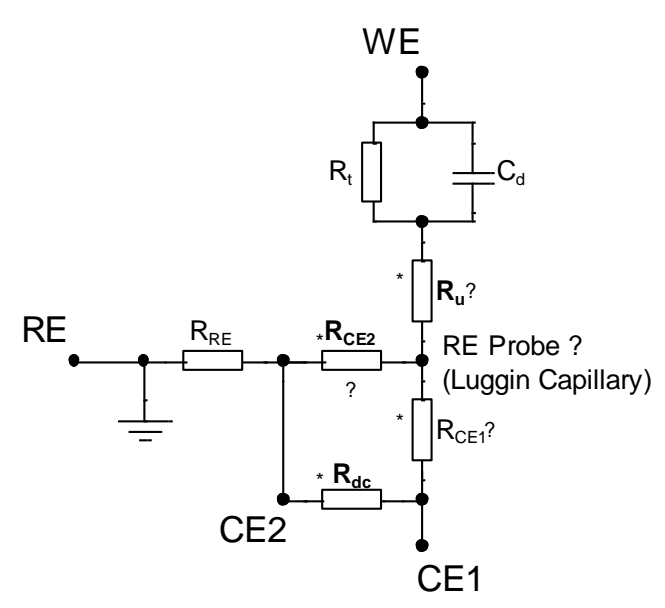

Figure 4-1: Equivalent circuits for the DEMS electrochemical cells that employ: (a) the stabilising resistor, or (b) the dual counter electrode setups to stabilise the potential control of the WE. Modifications to standard circuit are marked with a *. Key: stabilising resistor $\left(R_{s}\right)$, charge transfer resistance $\left(\boldsymbol{R}_{t}\right)$, dual counter resistor $\left(\boldsymbol{R}_{d c}\right)$ and uncompensated $\left(\boldsymbol{R}_{u}\right)$, reference electrode $\left(\boldsymbol{R}_{R E}\right)$ and counter electrode $\left(\boldsymbol{R}_{C E}\right)$ solution resistances, and double layer capacitance $\left(\boldsymbol{C}_{d l}\right)$.

The size of resistor required to stabilise the potentiostat is most easily found by trial and error, however, it is advantageous to use the lowest reliable resistance value possible in order to minimise the uncompensated WE resistance. For reference, the potentiostat was stabilised using a resistance of $\sim 110 \Omega, \sim 25 \Omega$ and $\sim 25 \Omega$ in $0.1,1.0 \mathrm{~mol} \mathrm{dm}^{-3} \mathrm{HClO}_{4}$ and $0.05 \mathrm{~mol} \mathrm{dm}^{-3} \mathrm{H}_{2} \mathrm{SO}_{4}$ respectively. In general, these errors are unacceptably large, introducing WE potential errors of 110 and $25 \mathrm{mV}$ at $1 \mathrm{~mA}$ of current (without employing $i R$-compensation) which is particularly undesirable in cyclic voltammetry when electrode currents are potential scan rate dependent. Of course $i R$-compensation could be applied using the potentiostat, however, compensation using conventional analogue potentiostats that employ positive feedback compensation schemes may not be beneficial at all due to the origin of the potentiostat instability of this cell i.e. by trying to compensate for the stabilising resistor we quickly reintroduce potentiostat oscillation and lose potential control of the WE. It is worth noting that these problems may be avoidable using a potentiostat that employs a digital $i R$-compensation scheme. 
An alternative $i R$-compensation technique that does not intrude upon the potentiostat control amplifier circuitry is the negative impedance convertor device (NID) [94], the technical details (i.e. circuitry) of which were presented previously in Chapter 3 section 3.7.1.1.3. The NID device was successful in reducing the uncompensated stabilising resistance, $R_{\text {st }}$, from $\sim 110 \Omega$ to $\sim 60 \Omega$ in $0.10 \mathrm{~mol} \mathrm{dm}^{-3} \mathrm{HClO}_{4}$ and from $\sim 25 \Omega$ to an acceptable $\sim 10 \Omega$ in $1.0 \mathrm{~mol} \mathrm{dm}^{-3}$ $\mathrm{HClO}_{4}$ and $0.5 \mathrm{~mol} \mathrm{dm}^{-3} \quad \mathrm{H}_{2} \mathrm{SO}_{4}$ electrolytes. This more reasonable uncompensated resistance can then be corrected post measurement to obtain close to the actual potential current relationship. The combination of the stabilising resistor and NID to apply $i R$-compensation allows potentiostat stability along with the measurement (using AC voltammetry) and minimisation of the uncompensated solution resistance. Potentiostat instability, however, is not specific to the dual-thin layer electrochemical cell fabricated for this DEMS instrument and other methods have been used to stabilise the potentiostat, such as the dual-counter electrode cell setup.

In the dual-CE cell arrangement, a second CE is placed on the DEMS cell outlet and is coupled to the primary $\mathrm{CE}$ (on the inlet) via a resistor, as shown in the equivalent circuit in figure 4-1 (b). Although it is not entirely clear how exactly this cell configuration functions, it presumably produces a similar effect to the stabilising resistor, of increasing the effective solution resistance. The second $\mathrm{CE}$ seemingly shifts the effective position of the Luggin-capillary entrance so that the solution resistance is increased to the WE by allowing a proportion of the cell current to pass through the cell outlet to the second CE. The location where the RE probe intercepts the current path between the WE and CE is shifted closer to the RE, increasing the solution resistance and stabilising the potentiostat. The dual-CE setup, however, cannot be considered to be a well-established or even documented solution for resolving potentiostat stability (i.e. the author could not find any literature outside DEMS on the dual-CE configuration) and it is not clear how exactly this cell configuration is working, or whether, for example, the solution resistance is current dependant, changing the effective position of the Luggin-capillary and therefore uncompensated solution resistance. Furthermore, there have been no indications in previous DEMS literature of the typical uncompensated solution resistance or whether it can be measured at all in such a 
configuration and if so, whether $i R$-compensation of the solution resistance is possible. It is therefore proposed that the stabilisation of a potentiostat used in the DEMS electrochemical dual thin-layer flow cell via a stabilising resistor on the WE connection to potentiostat in combination with $i R$-compensation using the NID (or possibly via a potentiostat that employs digital $i R$-compensation scheme), is a more transparent, reliable and understood solution to resolve potentiostat instability compared to the dual-CE arrangement employed in previous studies.

To summarise, the origins of the potentiostat stability in the three-electrode setup of the dual-cyclone thin-layer cell was found to largely arise from a small solution resistance between the $\mathrm{WE}$ and the effective Luggin-capillary rather than large cell resistances. By employing a stabilising resistor between the WE and potentiostat, the solution resistance of the electrochemical cell can be effectively increased, therefore stabilising the potentiostat allowing potential control of the WE. By using a NID for $i R$-compensation, the uncompensated resistance introduced by the stabilising resistor can be reduced to acceptable values of $\sim 10 \Omega$. Although in DEMS we are indeed very interested in the in-situ observation of electrochemical reaction species using mass spectrometry, this should not be at the expense of the quality and precision of the electrochemical measurement. 


\subsubsection{Effect of Electrolyte Flow Rate}

\subsubsection{Diffusion Limited Current}

The first and probably most straightforward characteristic of the dual-cyclone thin-layer electrochemical flow cell to determine, is the relationship between the diffusion limited current for a reaction process, $I_{d l}$, and the volumetric electrolyte flow rate, $V_{f}$. Diffusion limited currents arise when the rate of electrochemical reaction is limited by the diffusion of reactants from the bulk to the electrode surface, rather than kinetics of the electrode reaction. In hydrodynamic cells such as the DEMS flow cell, the $I_{d l}$ is related by a certain expression to the $V_{f}$ of the electrolyte containing the reaction species over the WE surface. In general, such hydrodynamic cells may be separated into two categories: (i) uniformly accessible electrode, such as the RDE and (ii) non-uniformly accessible electrodes such as the channel flow cell. The principle difference between these two categories is whether or not the $I_{d l}$ scales proportionally to the geometric area of the electrode: if the $I_{d l}$ scales then the electrode is considered uniformly accessible. The dual-cyclone (and -linear) thin-layer flow cell(s) fall into the latter non-uniformly accessible electrode category. In the case of the dual-cyclone thin-layer flow cell, which does not possess a typical flow regime over the WE, it is particularly important to both understand the relationship between the diffusion limited current and electrolyte flow rate in order to compare the dual-cyclone cell to more common flow regimes. This is especially true for experiments where the reactant of interest is in low concentration in the electrolyte.

The relationship between the $I_{d l}$ and the $V_{f}$. can be most easily determined by measuring the $\mathrm{CV}$ for the oxygen reduction reaction (ORR) of oxygen saturated electrolyte at a series of volumetric electrolyte flow rates. An example series of CVs for the oxygen reduction reaction (ORR) on polycrystalline Pt electrode in the dual-cyclone thin-layer flow cell is presented in figure 4-2. The CVs clearly exhibit the same characteristic features observed for the ORR using a RDE i.e. a kinetically limited region at potentials $>0.8 \mathrm{~V}_{\mathrm{RHE}}$, a diffusion limited region below $0.4 V_{\text {RHE }}$ and the so-called mixed region lying between. 


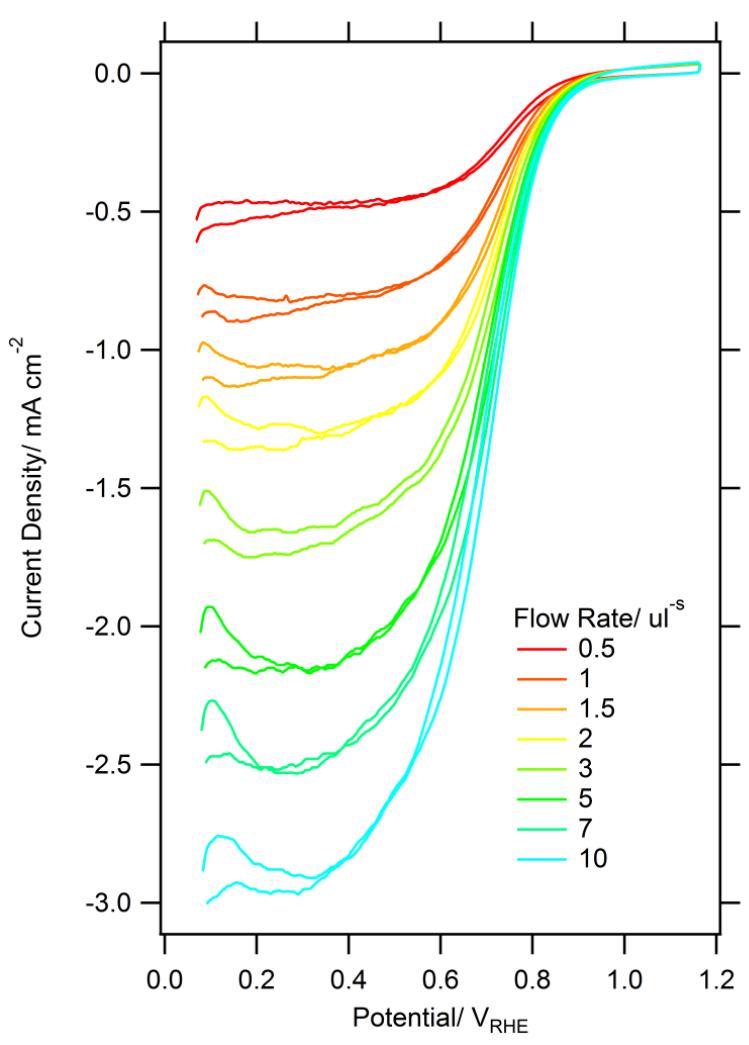

Figure 4-2: An example CV series for ORR on polycrystalline $\mathrm{Pt}$ at $20 \mathrm{mV} \mathrm{s} \mathrm{s}^{-1}$ for various electrolyte flow rates in the dual-cyclone thin-layer flow cell. The diffusion limited current is reached at $\sim 0.25 \mathrm{~V}_{\mathrm{RHE}}$ and increases with increasing volumetric flow rates.

The maximum diffusion limited current occurs at $\sim 0.3 \mathrm{~V}_{\mathrm{RHE}}$ for each flow rate similarly to that observed using RDE (when increasing rotation), and increases with increasing electrolyte flow rate. For non-uniformly accessible hydrodynamic electrodes the $I_{d l}$ scales according to a number of constants multiplied by the volumetric flow rate raised to a power. For example, the relationship between the diffusion limited current and the volumetric flow rate for a channel electrode is given by the following equation:

$$
I_{d l}=0.952 n F c_{\infty} D^{2 / 3}\left(h^{2} d\right)^{-1 / 3} W x^{2 / 3} V_{f}^{1 / 3} \quad \text { ref. [104] }
$$

Where, $n=$ no. of electrons, $F=$ faraday constant, $D=$ diffusion coefficient for the reacting molecule, $h=1 / 2$ height of flow channel, $d=$ flow channel diameter, $w=\mathrm{WE}$ width and $x=$ position relative to start of electrode and $V_{f}=$ volumetric flow rate (flow parameter). These terms will be constant for a certain reaction in the cell, and therefore the $I_{d l}$ will essentially scale with the $V_{f}$. multiplied by a 
certain power. Note that this is generally true for all non-uniformly accessible electrodes [104]. For the dual-cyclone thin-layer flow cell the $I_{d l}$ was found to scale with $V_{f}$. to the power of a $1 / 3$, as shown in figure 4-3.

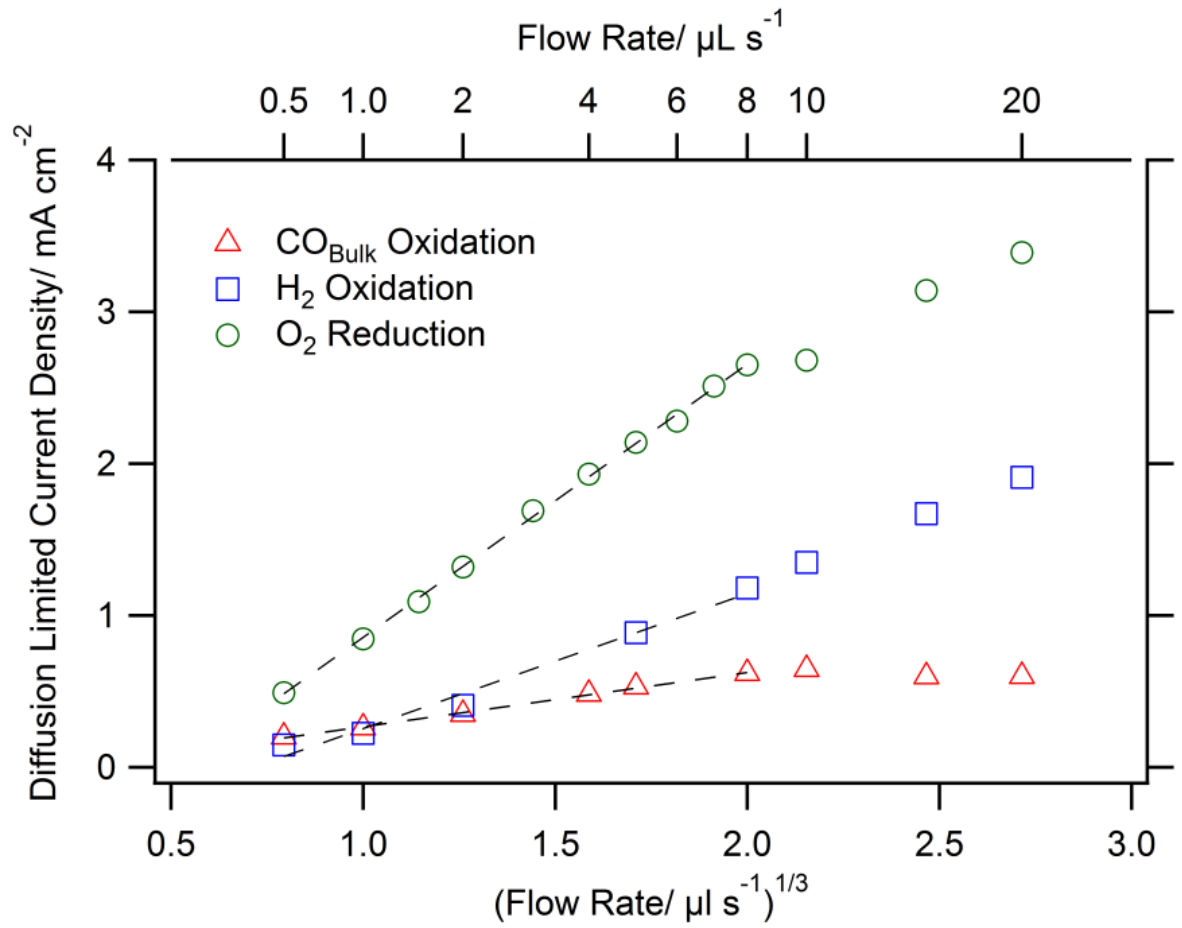

Figure 4-3: The dependence of the diffusion limited current, $I_{d l}$, on the electrolyte flow rate for the ORR, HOR and CO bulk oxidation reaction in the dual-cyclone thin-layer flow cell. The diffusion limited current scales with the volumetric flow rate according to a power of $1 / 3$ for flow rates below $10 \mu \mathrm{l} \mathrm{s}{ }^{-1}$.

The $I_{d l}$ scales linearly with the volumetric flow rate, within the range between 0.5 and $10 \mu \mathrm{s} \mathrm{s}^{-1}$ before deviating at higher flow rates. This value of $1 / 3$ is comparable to the value of given in ref. [34] for another dual-cyclone thin-layer cell (although with presumably slightly different dimensions) of 0.4 which was suggested to be analogous to the wall-jet flow cell. The wall-jet electrode, however, scales with the volumetric flow rate according to the power of $3 / 4$ [104]. The exact values, however, will be dependent on the cell dimensions. Finally, the loss of linearity observed in figure 4-3 at flow rates $\geq 10 \mu \mathrm{l} \mathrm{s}^{-1}$ suggests that the flow of electrolyte becomes less stable (perhaps indicating more turbulent flow) and that lower flow rates should therefore be favoured for DEMS experimentation. 


\subsubsection{Response Time}

The QMS response time, or rather the delay between the formation of a species at the WE and its subsequent detection by the QMS was measured as a function of the electrolyte flow rate. This delayed response is largely determined by the finite time required for the reaction species to be transported away from the WE to the membrane surface, and is therefore strongly dependent upon the electrolyte flow rate. The relationship between the volumetric flow rate and the QMS response time is first shown quantitatively in figure 4-4 via the LSV of CO-bulk oxidation and corresponding mass ion current, $I_{m / z=44}$ on polycrystalline $P t$ at various electrolyte flow rates.

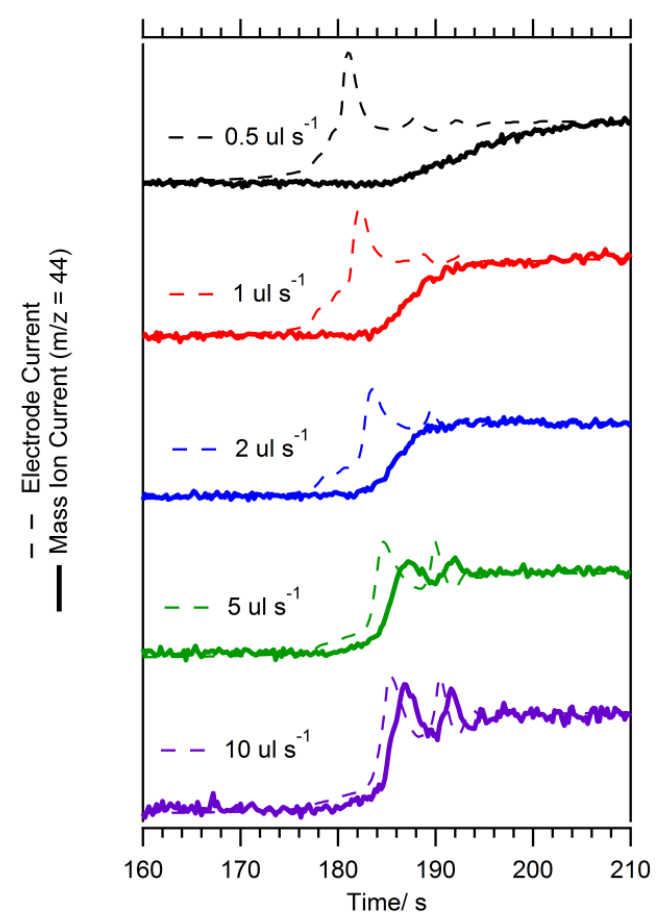

Figure 4-4: A graph highlighting qualitatively, the difference between the faradaic electrode current and the corresponding $I_{m / z=44}$ response during a CO-bulk oxidation measurement on polycrystalline $P t$ for the dual-cyclone thin-layer flow cell performed at various electrolyte flow rates. The QMS response time decreases whilst the reproduction of the electrochemical features improves with increasing electrolyte flow rate.

Here we see that the time difference between the generation of carbon dioxide and the mass ion current response predictably decreases with increasing flow rate, owing to the reduced transport time of reaction products in solution from the WE to the membrane. At the very low flow rate of $\sim 0.5 \mu \mathrm{l} \mathrm{s}{ }^{-1}$ the QMS response time 
is some $15 \mathrm{~s}$ long, whereas this is reduces to below $1 \mathrm{~s}$ at $10 \mu \mathrm{l} \mathrm{s}{ }^{-1}$. In order for the QMS measurement signal to correlate with the electrode potential, the time of the mass ion current must be corrected for in post measurement data treatment. It also evident from figure 4-4, that the reproduction of the features observed in the electrochemistry by the QMS current is also dependent on the flow rate (this will be addressed via the correlation in the next section). The precise dependence of the QMS response time on the electrolyte flow rate through the dual-cyclone thinlayer cell was determined using potential step chronoamperometry of bulk CO oxidation on polycrystalline $\mathrm{Pt}$, and is presented quantitatively in figure 4-5.

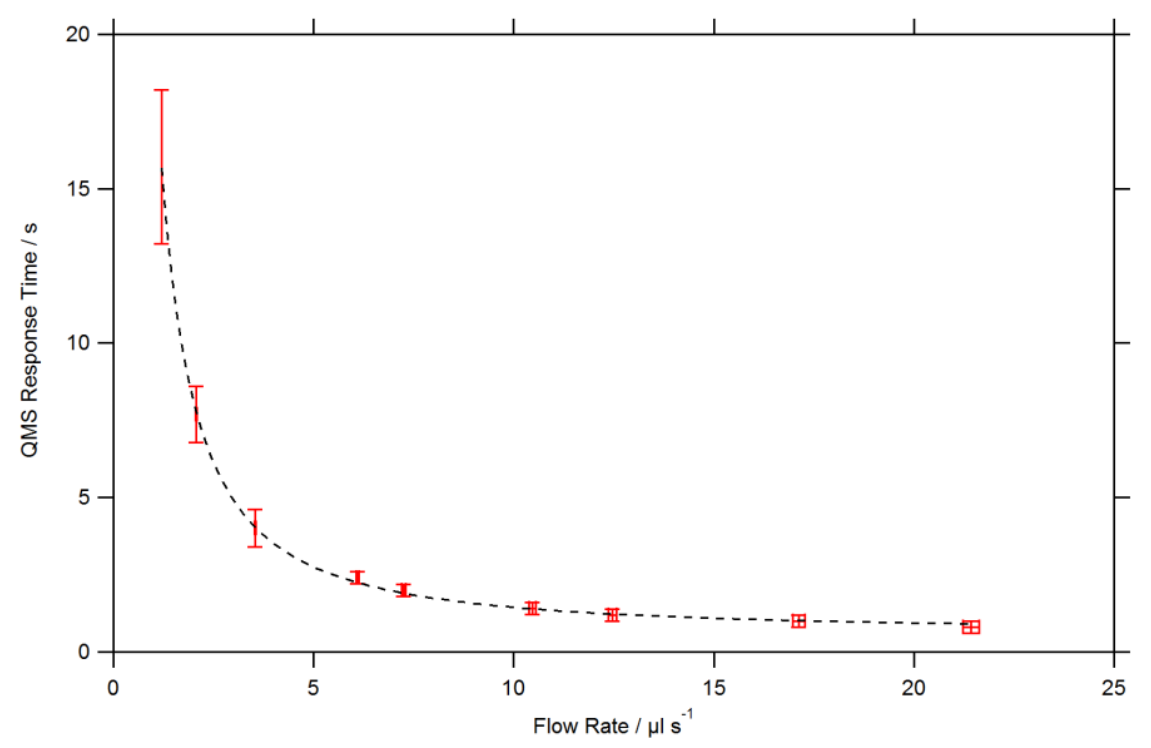

Figure 4-5: The dependence of the QMS response time for bulk CO-oxidation on the electrolyte flow rate through the dual-cyclone thin-layer flow cell. The large error bars indicating the variability in the response time suggests slight but significant fluctuation in the flow of electrolyte through the cell at low flow rates.

The QMS response time decays in a power law fashion from around $15 \mathrm{~s}$ to below $1 \mathrm{~s}$ the electrolyte flow rate increases $>10 \mu \mathrm{l} \mathrm{s}{ }^{-1}$. The large error bars present at flow rates below $4 \mu \mathrm{s}^{-1}$ demonstrates that the QMS response time is very sensitive to slight fluctuations in the precise flow rate in this range. These can, for instance, arise from minor disturbances of the DEMS flow cell PTFE tubing which induces turbulence in the electrolyte within the thin-layers. At flow rates above $4 \mu \mathrm{s} \mathrm{s}^{-1}$ the QMS response time gradually approaches a steady value below $1 \mathrm{~s}$ and the response time is significantly more reproducible. The correction of the QMS data in potential and time therefore, can be acceptably performed without 
error. If one was to only consider the QMS response time in choosing the optimum electrolyte flow rate, values greater than $4 \mu \mathrm{s}^{-1}$ would be acceptable. There are however, other characteristics of the DEMS instrument which must also be considered, such as correlation and collection efficiency.

\subsubsection{Correlation}

The degree of correlation between the electrode current arising from a certain reaction at the WE, and the subsequent QMS response to the electro-generated species is a strong function of both the electrolyte flow rate and potential scan rate. The difference between the $\mathrm{CV}$ for $\mathrm{CO}$ bulk oxidation on Pt and the mass spectrometric cyclic voltammogram (MSCV) for $I_{m / z=44}$ is first illustrated qualitatively at various electrolyte flow rates in figure 4-6. The correlation between the $\mathrm{CV}$ and MSCV improves as the electrolyte flow rate through the cell is increased. This is indicated for instance, by the reproduction of the CO-bulk oxidation peak observed in all the CVs at $\sim 1.0 \mathrm{~V}_{\mathrm{RHE}}$ but is only resolved in the MSCV at rather electrolyte flow rates exceeding $10 \mu \mathrm{l} \mathrm{s}$. Furthermore, the presence of carbon dioxide remaining in the MSCV (and therefore the electrochemical cell) during the reverse potential sweep after $\mathrm{CO}$ oxidation has ceased at $\sim 0.9 \mathrm{~V}_{\mathrm{RHE}}$ is longer at lower electrolyte flow rates. This effect is associated with the residence time of the reaction product in the electrochemical cell compartment, which at lower electrolyte flow rates is more prone to diffusion and mixing in the electrochemical cell thin-layers. Furthermore, the degree of correlation will also be dependent on the potential scan rate of the $\mathrm{CV}$ whereby fast scan rates or electrode processes are inherently more difficult to reproduce. Such qualitative observations, however, a somewhat unsatisfactory by themselves and it would be far more useful if the correlation between the $\mathrm{CV}$ and MSCV is expressed in numbers so that optimum experimental parameters can be identified. Fortunately, there are a number of statistical methods exist such as correlation coefficient or goodness of fit, which are designed to quantify the degree of fitting, or correlation between two data sets. Typically, these statistical methods are used to assess the fit of a mathematical model to true experimental data. By defining 
the measured WE current as our true data and the QMS response as our modelled data, the correlation of the QMS response can be assessed quantitatively.

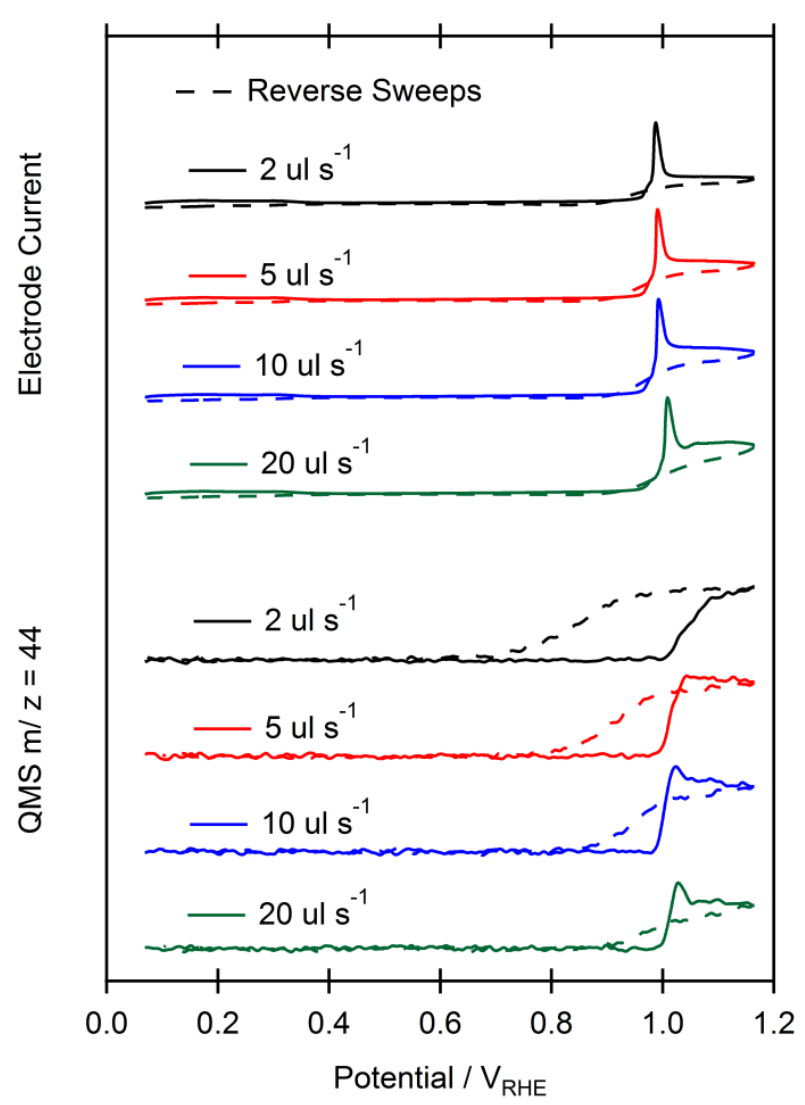

Figure 4-6: $\mathrm{CV}$ and $\mathrm{MSCV}$ of $\mathrm{CO}_{2}(\mathrm{~m} / \mathrm{z}=44)$ during $\mathrm{CO}$-bulk oxidation at various different electrolyte flow rates for the dual-cyclone thin-layer flow cell. The correlation of the QMS mass ion current with the electrode current improves with increasing electrolyte flow rate. The residence of the electrochemical reaction species in the electrochemical cell also decreases.

The dependence of the correlation coefficient for the $\operatorname{MSCV}\left({ }^{m} / z=32\right)$ on the electrolyte flow rate and various potential scan rates during the ORR on polycrystalline $\mathrm{Pt}$ is give in figure 4-7. These quantitative observations indicate that the correlation of the MSCV improves with increasing electrolyte flow rate, and decreasing potential scan rate. In general, the correlation of the MSCV was relatively poor for the high $\mathrm{CV}$ scan rates of 50 and $100 \mathrm{mV} \mathrm{s}$ at all electrolyte flow rates. 


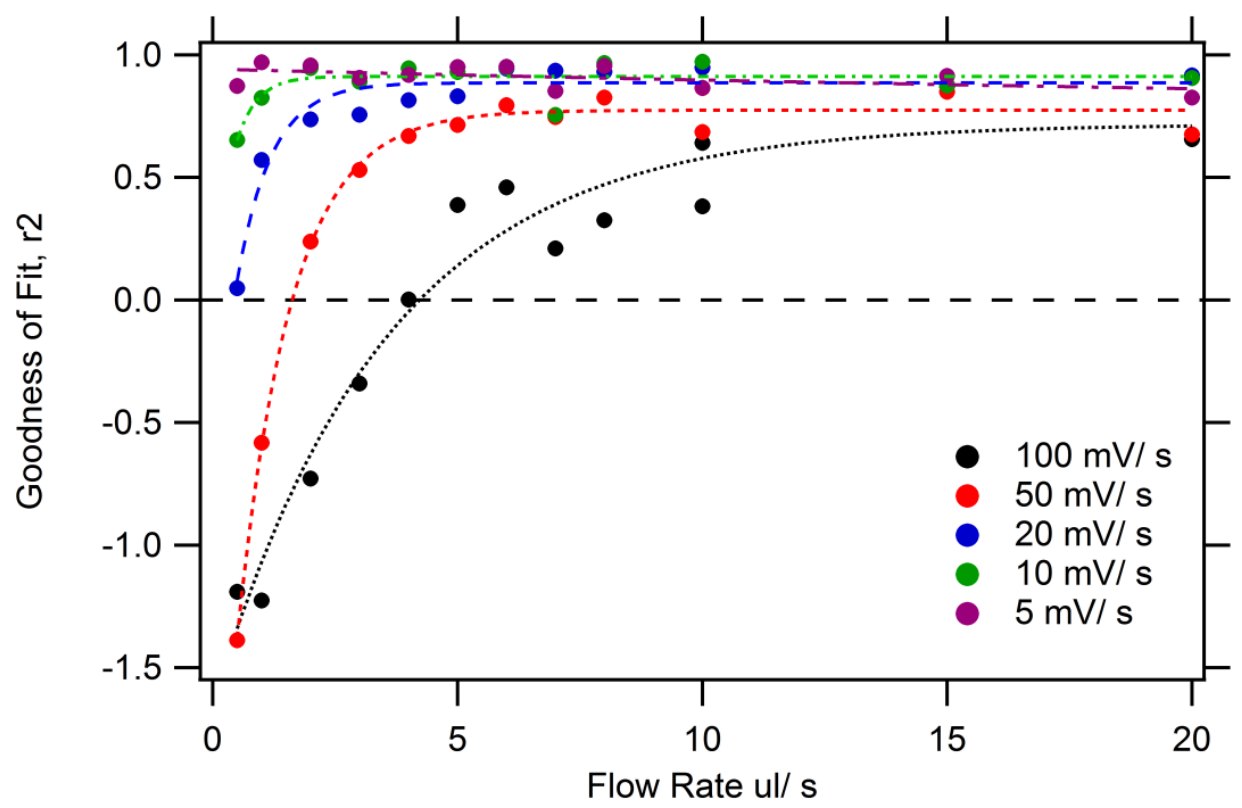

Figure 4-7: A plot demonstrating the dependence of DEMS cell correlation coefficient for the ORR on the electrolyte flow rate at various potential scan rates for the dual-cyclone thin-layer flow cell. Acceptable correlation (close to 1.0) between the electrode current and QMS mass ion current is achieved at potential scan rates $\leq 20 \mathrm{mV} \mathrm{s}^{-1}$ and at flow rates $\geq 4 \mu \mathrm{l} \mathrm{s} \mathrm{s}^{-1}$.

Acceptable correlation of the MSCV can be, however, achieved at scan rates of 5, 10 and $20 \mathrm{mV} \mathrm{s}^{-1}$ by employing flow rates of at least $3 \mu \mathrm{l} \mathrm{s}$. The exact values of the correlation will of course be dependent on the nature of the electrochemical reaction and the features of the $\mathrm{CV}$, of which the ORR is a rather simple example. In order to identify an optimum electrolyte flow rate, we must finally assess the DEMS cell collection efficiency. 


\subsubsection{Collection Efficiency}

The DEMS cell collection efficiency is simply the fraction of reaction products produced at the WE that then evaporate through the PTFE membrane interface into the vacuum system to be detected by the QMS. The collection efficiency, therefore, has a direct influence on the detection limit and sensitive of the QMS and is ideally close to 1.0 . Although high collection efficiencies $>0.9$ can be obtained with electrolyte convection, any flow of electrolyte through the electrochemical cell will reduce the retention time of the electrochemical species above the membrane interface. The time frame in which the electrochemical species can therefore diffuse to the membrane surface is diminished and thus the collection efficiency falls. The DEMS cell collection efficiency obtained from CO bulk oxidation on polycrystalline $\mathrm{Pt}$ is presented as a function of electrolyte flow and potential scan rate in figure 4-8. Note that the method of determining the DEMS cell collection efficiency, $N$, required for calibration of the instrument is outlined later in section 4.4.

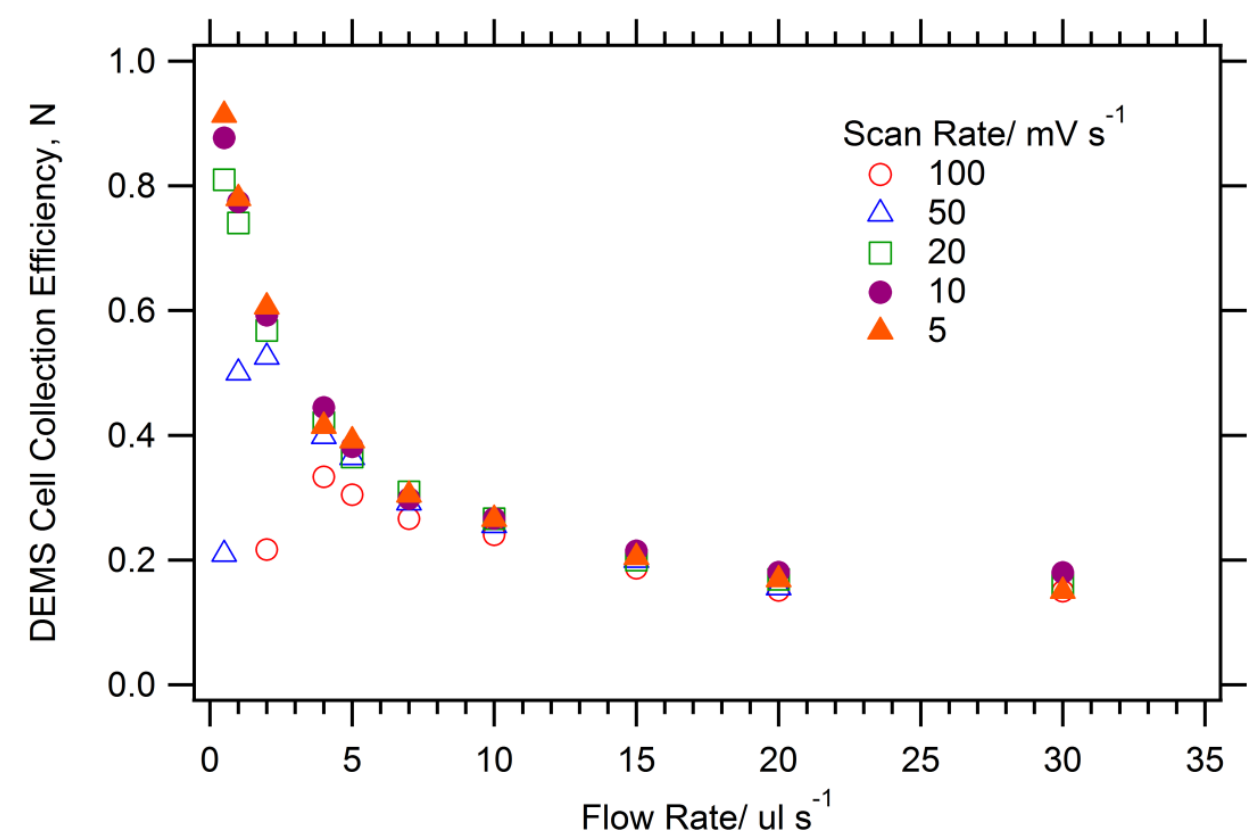

Figure 4-8: A plot illustrating the dependence of DEMS cell $\mathrm{CO}_{2}$ collection efficiency, $\mathrm{N}$, on electrolyte flow rate at various potential scan rates for the dual-cyclone thin-layer flow cell. The DEMS collection efficiency decreases with increasing electrolyte flow rate, beginning to stabilise at rates $\geq 4 \mu \mathrm{s} \mathrm{s}^{-1}$ and is independent of potential scan rate. 
The DEMS cell collection efficiency decreases as the electrolyte flow rate through the cell is increased. A collection efficiency of $\sim 0.9$ can be achieved using with very low electrolyte flow rates, however, this decreases rapidly until $4 \mu \mathrm{s}^{-1}$ before steadying and plateauing at flow rates $>15 \mu \mathrm{s}^{-1}$. Furthermore, the collection efficiency is independent of potential scan rates of $50 \mathrm{mV} \mathrm{s}$ or lower, and flow rates greater than $3 \mu \mathrm{l} \mathrm{s}{ }^{-1}$. Note that the discrepancies at high scan rates and low flow rates arise due to the failure for the total QMS peak response to be produced in the corresponding $\mathrm{CV}$, and the integration of the QMS data is therefore incomplete. The collection efficiency is therefore concluded to be independent of the potential scan rate. On one hand, a high collection efficiency is desired to maximise the instrument sensitivity, which would favour electrolyte flow rates $<3 \mu \mathrm{l} \mathrm{s}{ }^{-1}$, however, the sensitive dependence of the collection efficiency on the exact electrolyte flow rate $<3 \mu \mathrm{s}^{-1}$ may lead to difficulties in terms of reproducibility for quantitative analysis if the flow rate is not absolutely stable. Consequently, an electrolyte flow rate $<3 \mu \mathrm{s} \mathrm{s}{ }^{-1}$ is not considered to be optimum. Finally, the performance in terms of collection efficiency at a flow rate of $5 \mu \mathrm{l} \mathrm{s}$ is comparable to a value of 0.3 provided in ref. [34]. 


\subsection{Performance of the Membrane Interface Material}

There are two properties of the PTFE membrane that are critical to the performance of the DEMS instrument: (i) the total gas flux, and (ii) the selective permeability. The total flux of gas through the membrane from the aqueous phase to the vacuum determines the size of the vacuum system necessary and/or the geometric area of the membrane interface as discussed in Chapter 3 section 3.5. The selective permeability of the membrane meanwhile, determines if and how easily certain species are able to evaporate through into the vacuum system. Presented in this section are methods in which to evaluate both of these membrane characteristics.

There are a number of methods in which the total gas flux through a membrane may be determined to evaluate if the membrane is appropriate. For example, if the pumping speed of a simply vacuum system is well-known, then the gas flux can be easily determined by simply measuring the vacuum system pressure. There are, however, two problems associated with this method. The first is that the total gas flux through the membrane will be to some extent dependent on the vacuum system pressure, and the secondly there is the risk of destroying the turbo pumps if the membrane is inappropriate. A rather simple and robust test setup to assess the membrane performance is therefore somewhat desirable. The total gas flux through the membrane material may be evaluated using a straightforward setup consisting of a small vacuum chamber, pressure gauge, roughening pump and the DEMS electrochemical cell membrane interface, as illustrated in figure 4-9. The membrane sample is setup in the DEMS flow cell to form the membrane vacuum interface, and electrolyte is passed through the cell (almost as if a DEMS experiment were to be performed). The operating roughening pump may then be allowed to evacuate the volume by opening gate valve (V1) which will have one of two consequences. Either a vacuum is not achieved, in which case the membrane material is completely unsuitable, or a vacuum is achieved to give a vacuum pressure $<50 \mathrm{mbar}$, in which case the membrane may be suitable. 


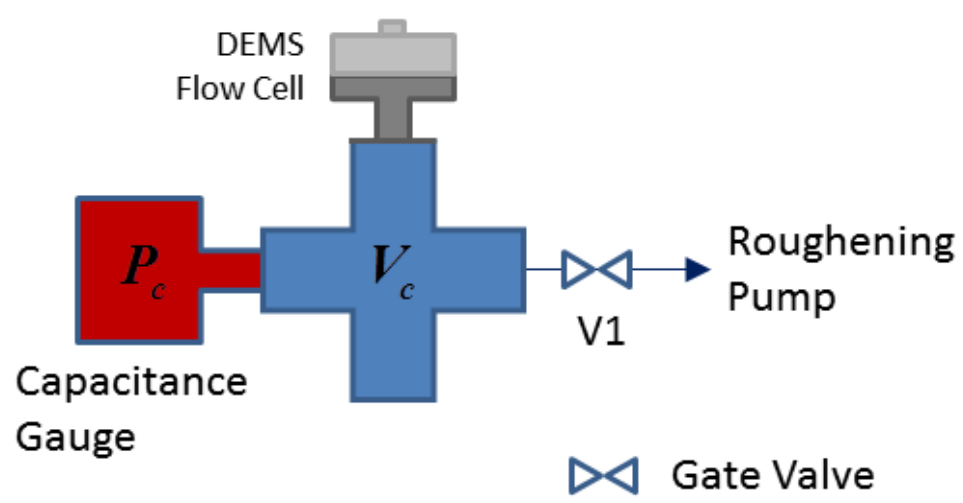

Figure 4-9: Membrane interface material vacuum test setup. The roughening pump is used to evacuate the vacuum chamber volume, whilst the pressure is monitored using a capacitance gauge. By shutting the gate valve V1 the total gas flux through the membrane is measured as a function of the vacuum pressure.

If the pumping is then rapidly closed off using the gate valve (V1), the corresponding increase in the pressure of the vacuum volume can be used to determine the flux of gas through the membrane according to the following equation:

$$
J=\boldsymbol{V}_{c} \frac{\delta \boldsymbol{P}_{c}}{\delta \boldsymbol{t}}
$$

In order to extract the total gas flux, the vacuum pressure must be recorded accurately as a function of time. This is achieved using the DEMS (QMS) calibration and measurement setup described in Chapter 3 section 3.7.2. The total gas flux, $J$, through a number of the microporous PTFE membrane candidates is plotted as a function of the vacuum pressure in figure 4-10. 


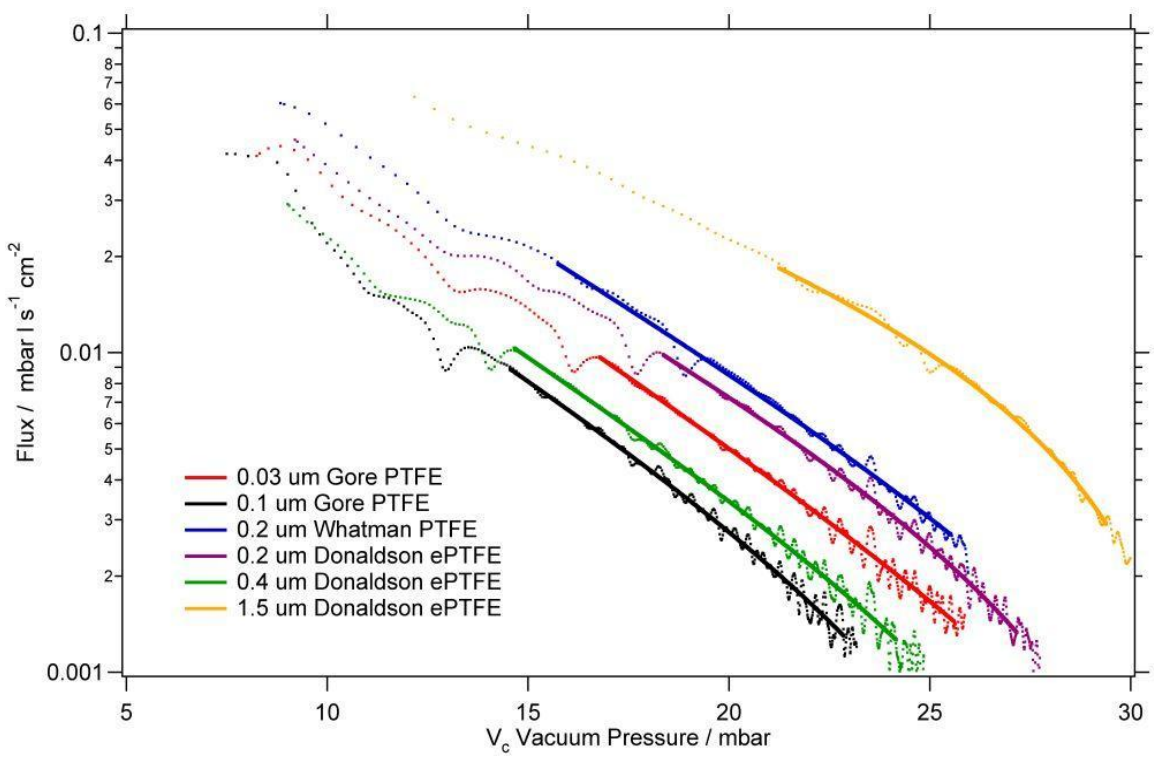

Figure 4-10: Total gas flux through porous membrane into an evacuated volume plotted as a function of the evacuated volume pressure. The flux of gas permeating the microporous membrane is dependent on the vacuum pressure applied.

This plot shows that the gas flux through the membrane decreases with increasing vacuum pressure. This trend approximately follows an exponential decay, and through fitting the total gas flux may be extrapolated to vacuum pressures expected for the vacuum system $\sim 0.01$ mbar. Through fitting a number of these measurements, and extrapolating to a vacuum pressure 0.01 mbar, gas fluxes approximating those of the membrane when attached to the vacuum system can be obtained. These values are given in table 4-2.

Table 4-2: The total gas flux values extrapolated to $0.01 \mathrm{mbar}$ vacuum pressure at $5 \mu \mathrm{s} \mathrm{s}^{-1}$ electrolyte flow rate. The total gas fluxes of the potentially suitable membrane interface materials lie between 0.1 and $1.0 \mathrm{mbar}^{1} \mathrm{~s}^{-1} \mathrm{~cm}^{-2}$.

\begin{tabular}{|c|c|c|c|}
\hline Manufacturer & $\begin{array}{c}\text { Pore Size/ } \\
\boldsymbol{\mu m}\end{array}$ & $\begin{array}{c}\text { Thickness/ } \\
\boldsymbol{\mu m}\end{array}$ & $\begin{array}{c}\text { Total Gas Flux at } \\
\mathbf{0 . 0 1} \text { mbar Vacuum } \\
\text { Pressure }\end{array}$ \\
\hline Gore & $\mathbf{0 . 0 3}$ & $127 \pm 9$ & $\mathbf{0 . 1 1 4 6}$ \\
\hline & $\mathbf{0 . 1}$ & $\mathbf{1 2 4} \pm 11$ & $\mathbf{0 . 1 4 6 3}$ \\
\hline Whatman & $\mathbf{0 . 2}$ & & $\mathbf{0 . 3 8 6 6}$ \\
\hline Donaldson & $\mathbf{0 . 2}$ & $\mathbf{8 9}$ & $\mathbf{0 . 2 3 5 1}$ \\
\hline & $\mathbf{0 . 4}$ & 152 & $\mathbf{0 . 1 2 1 1}$ \\
\hline & 1.5 & 152 & $\mathbf{0 . 9 3 2 8}$ \\
\hline
\end{tabular}

The total gas flux extrapolated from the measurement data to the DEMS vacuum system pressure for the Gore and Donaldson membrane pore sizes of $\leq \mathbf{0 . 4} \boldsymbol{\mu m}$ are 
comparable to the calculated values of $0.09 \mathrm{mbar}^{\mathrm{s}} \mathrm{s}^{-1}$ at $25{ }^{\circ} \mathrm{C}[26]$ and $0.4 \mathrm{mbar}^{-1}$ at $40{ }^{\circ} \mathrm{C}$ [34]. There is no particular trend concerning the relationship between the micropore size and total gas flux in this data, however, this is likely attributed to the precision of the measurement. In practical terms there is little difference between pore sizes for the microporous Gore membranes.

The second property of the membrane of relevance to DEMS is the selectivity. The selectivity of the membrane will not only determine which species evaporate into the vacuum system, but also how easily, which in turn directly influences the sensitivity and detectability of the DEMS instrument. A measure of the ability of a certain species to pass through the membrane is the DEMS collection efficiency. A method for determining the DEMS cell collection efficiency, $\mathrm{N}$, for $\mathrm{CO}_{2}$ using CO-Stripping is later described in section 4.4. There is, however an alternative method whereby the electrolyte containing a certain concentration of is simply flowed through the cell (no electrochemistry is involved), and the relevant mass ion current is monitored as a function of flow rate. The membrane sample compartment collection efficiency, $f$, is thus given by:

$$
\boldsymbol{f}=\frac{\boldsymbol{I}_{m / z=i}}{K_{i}^{0} c_{i} V_{f}}
$$

Where $c_{\mathrm{i}}=$ concentration of species in solution and $v_{f}=$ volumetric flow rate through the cell. This experiment was performed for a number dissolved gaseous species, and the collection efficiency of the membrane sampling compartment converted to flux is plotted as a function of flow rate in figure 4-11. 


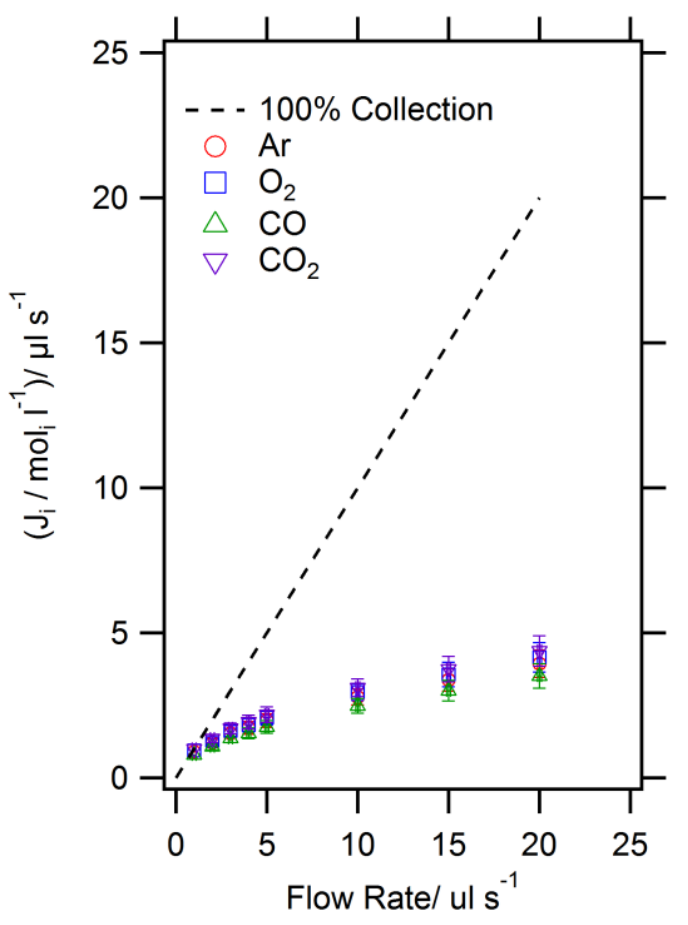

Figure 4-11: Mass ion current converted to flux normalised to concentration of species in aqueous solution. The permeability of the dissolved gaseous species are almost identical within experimental error, and are not limited by the membrane permeability but rather the diffusion of species from the bulk electrolyte to the membrane pore entrances.

The fluxes of gaseous and volatile species are almost the same within the experimental uncertainty. For very volatile species the flux through the membrane is limited by the diffusion of gaseous species to the membrane pores, and consequently it is unlikely that any significant difference between different membrane materials to the detection of gases be observed. The membrane interface collection efficiency therefore is as much, if not more of a measure of the performance of the membrane interface design rather than the membrane material. It would, however, be worthwhile to determine and compare the membrane interface collection efficiency for less volatile species which may be limited by their passage through the membrane should the experiment desire so. For less volatile species such organic molecules, the permeability through the membrane is significantly less [34]. 


\subsection{Optimisation of the QMS}

The optimisation of the QMS parameters is necessary in order to maximise the DEMS instrument sensitivity and stability, producing better quality QMS data with maximised signal to noise ratio and therefore higher detection and quantification limits. Whilst some brief guidelines for optimising the QMS are presented in the operating manuals $[85,95,105]$, a more detailed and qualitative description with figures that clearly demonstrate the effects of the QMS tuning parameters are presented here.

The optimisation of the ion source begins by admitting a controlled gas leak into the vacuum system. Ideally, this is a molecular of interest, such as carbon dioxide, so that vacuum conditions similar to those during the DEMS instrument operation are obtained. This can be achieved by attaching a gas line (or by simply admitting air) to the vacuum system inlet valve, and partially opening the inlet leak-valve until the appropriate vacuum system pressure is achieved. The QMS ion source settings are then optimised using the Quadstar 32-bit Tune Up application in the Tune Ion Source window. Here, the QMS settings can be manipulated and the effect on the observed mass ion current can be evaluated in real time. QMS parameters can then be systematically varied and the averaged mass ion current response value recorded. In order to tune the QMS sensitivity, it is crucial to first optimise the ion source parameters followed by the quadrupole and SEM.

\subsubsection{Ion Source Parameters}

The ion source settings can have a significant impact on the sensitivity of the QMS because the ion source settings will determine how many ions are produced, and are guided to the quadrupole for mass selection. This can involve modifying the ion acceleration voltage, however, the emission current, and the extraction and focus plate voltages are the most common parameters required to be optimised. 


\subsubsection{Emission Current}

The emission (or electron) current determines how many electrons are emitted from the ion source filament(s). Typical emission currents for the cross-beam ion source are either exclusively 0.1 or $1.0 \mathrm{~mA}$ [105]. The number of ions produced at the ion source is given by the following relationship:

$$
I_{k+}=i_{-}^{*} i_{e}^{*} S^{*} p_{k}
$$

Where $I_{k+}=$ ion current, $i_{-}=$emission current, $I_{e}=$ mean path length of the electrons, $s=$ differential ionization effect cross-section, and $p_{k}=$ partial pressure of the gas component. In general, an ion current of $1.0 \mathrm{~mA}$ are preferred because it will generate ten times more ion current, although a lower emission current may be preferred under certain circumstances, such as when the CB-ions source is operated at pressures approaching $10^{-4}$ mbar [105].

\subsubsection{Focus \& Extraction Plate Voltages}

The focus and extraction plate voltages determine the acceleration and focusing of the ions into the quadrupole mass filter. The focus and extraction plate voltages are not mutually exclusive and so their values can only be tuned by systematically varying the voltages and recording the currents until the optimum parameters are found. The dependence of the mass ion current on the extraction plate voltage at various focus plate voltages for each of the two $\mathrm{CB}$-ion source filaments are shown in figure 4-12. 

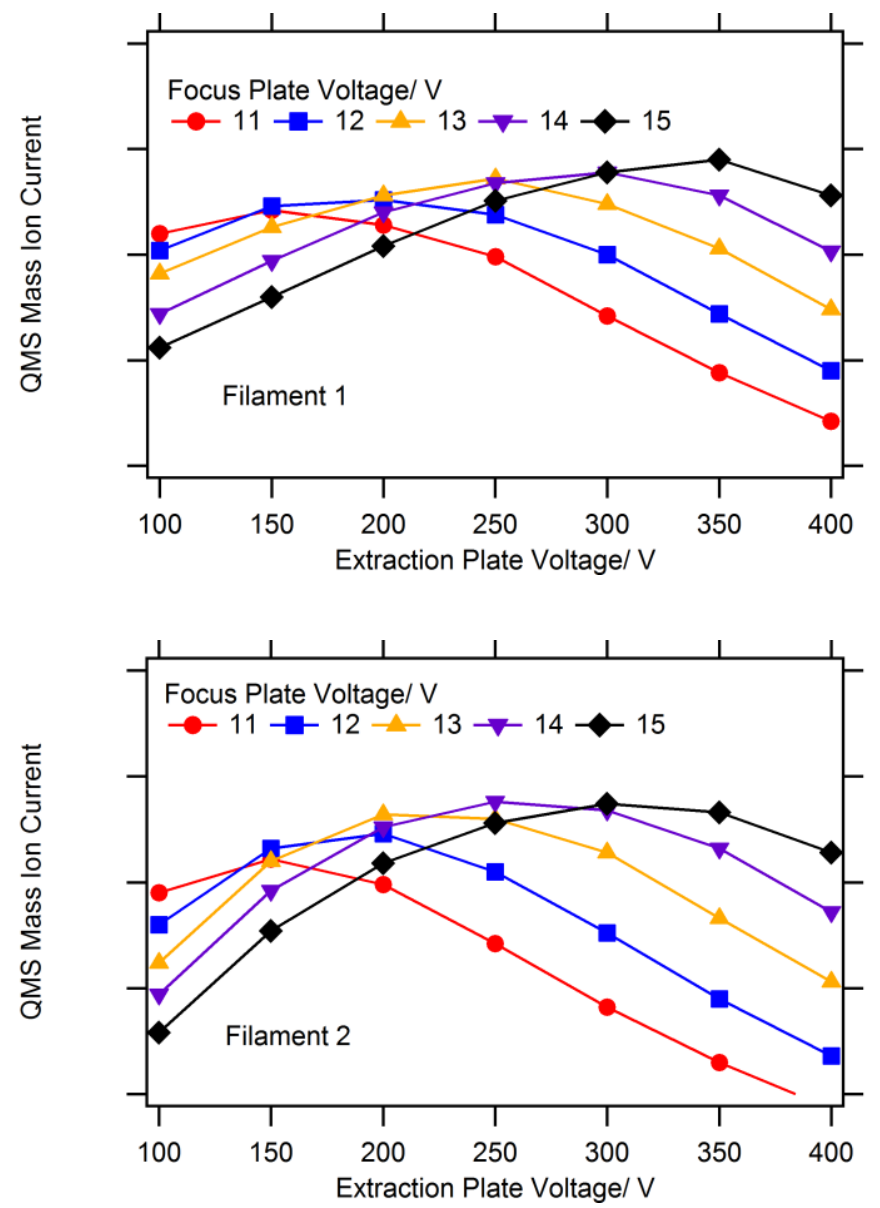

Figure 4-12: The effect of extraction plate voltage on QMS ion current at various focus plate voltages. A mutual voltage combination for the mass ion current peak maximum should be evaluated based on these curves. Optimum settings based on this data would suggest a focus plate voltage of $14 \mathrm{~V}$ and extraction plate voltage of $250 \mathrm{~V}$, although there is no definitively optimal setting for both filaments.

Typical focus plate voltages lie in a narrow voltage range between 11 and $15 \mathrm{~V}$, however, the extraction plate voltage range can be much wider, spanning from 100 to $400 \mathrm{~V}$. From these plots, a mass ion current peak maximum that is common for both filaments at a certain focus and extraction plate voltage should be evaluated for normal operation when both filaments are simultaneously running. If more than a single peak maximum exists then the lower focus plate voltage combinations are be preferred. If the values for each filament are significantly different then mechanical damage, or contamination of the ion optics or filaments may have occurred [85]. 


\subsubsection{Quadrupole and SEM Parameters}

Once the ion source settings are optimised, the next step is to optimise the resolution, SEM voltage and deflection plate voltage.

\subsubsection{Resolution}

In analytical chemistry the term resolution usually refers to the degree of peak separation whereby larger values correspond to better separation, however, in the Quadstar 32-bit software a larger resolution is in fact less separation of the mass ion current peaks (which can cause some confusion), as depicted in the background mass spectrum of the DEMS vacuum system in figure 4-13.

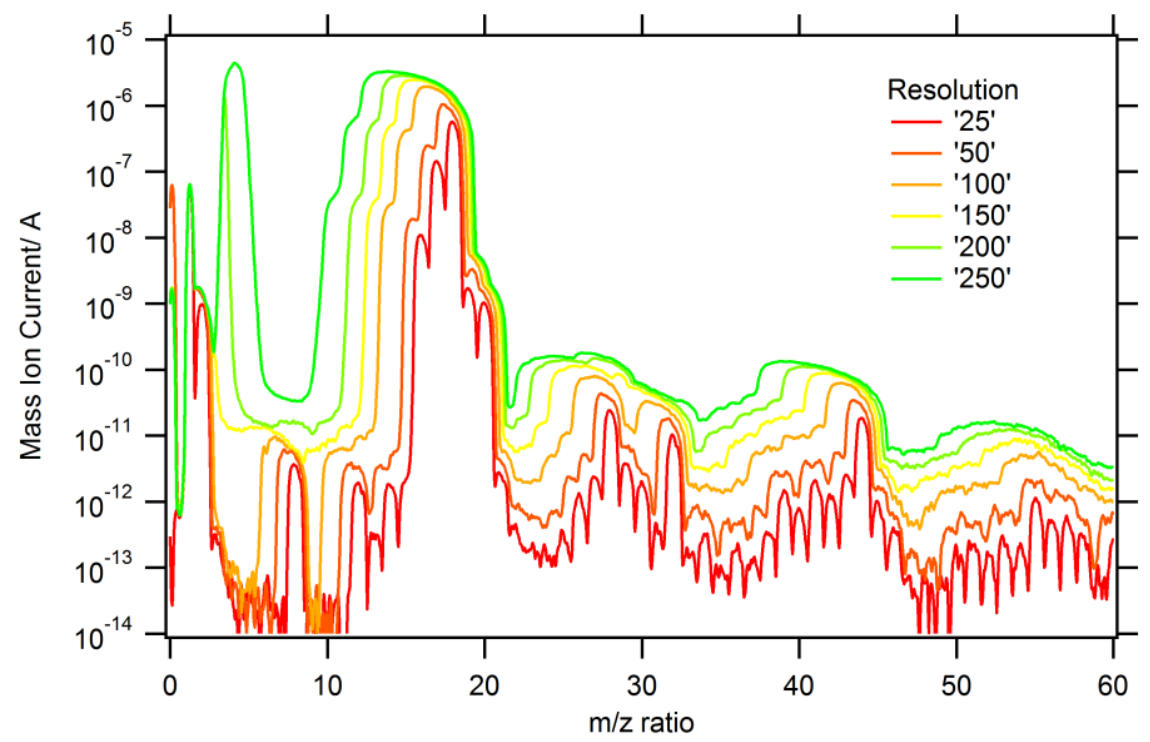

Figure 4-13:The mass scan analogue spectrum of vacuum chamber background at various QMS resolution values. The selectivity of the quadrupole to a certain mass decreases within increasing the resolution parameter. Higher resolution values are desirable to increase QMS sensitivity when the mass of interest is not surrounded by mass ions of the similar magnitude.

The resolution in this case reflects the precision of the mass selection and therefore the number of ions that are transmitted through the mass filter. When operating the QMS in mass ion current mode, the resolution can be raised in order to increase the quadrupole transmission and hence mass ion current and sensitivity of the QMS. In cases where the mass ion signal of surrounding masses 
are one or two orders of magnitude less than the mass of interest, then the resolution can be safely increased without interference. The resolution therefore should be optimised for each mass ion current of interest.

\subsubsection{Deflection Plate Voltage}

The deflection plate voltage is responsible for deflecting the mass selected ions exiting the quadruple into the SEM. According to the Pfeiffer vacuum manual, the deflection plate voltage should be optimised each time the SEM voltage is changed [85, 105]. A plot of deflection plate voltage versus mass ion current at various SEM voltages is therefore shown in figure 4-14.

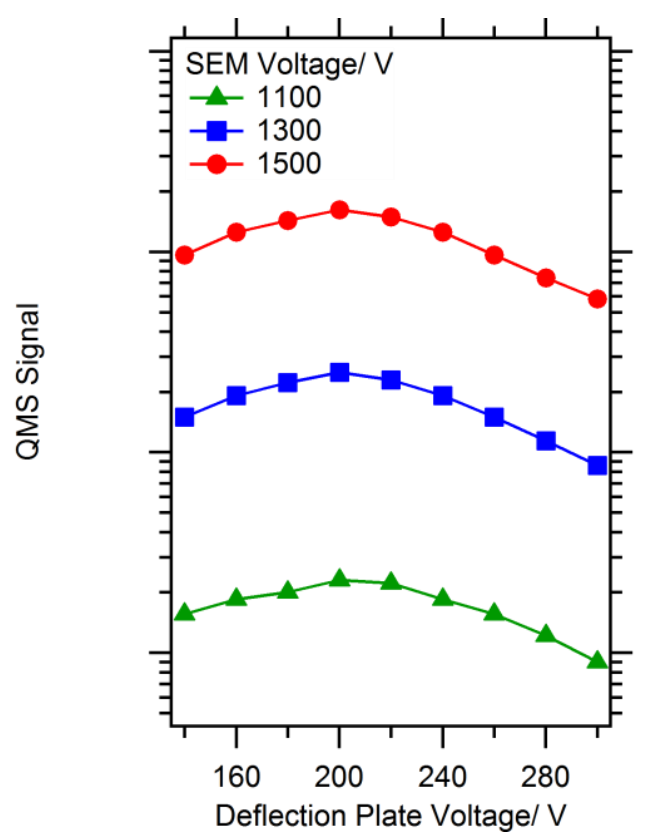

Figure 4-14: The effect of ion source current on QMS calibration constant. The deflection voltage is only slightly dependant on the SEM voltage. It is therefore not strictly necessary to adjust the deflection plate voltage each time the SEM voltage is changed.

In this plot, however, the peak deflection voltage was $\sim 200 \mathrm{~V}$ for a wide range of SEM voltages (1100 to $1500 \mathrm{~V})$. The influence of the SEM voltage on the optimum deflection plate voltage did not appear to have a hugely significant impact. 


\subsubsection{SEM Voltage}

The SEM is a device which multiplies the particle beam. The voltage applied to the SEM affects the number of secondary electrons that are produced and thus has a significant effect on the overall amplification of the mass ion current. The effect of the SEM voltage on the measured QMS calibration constant for carbon dioxide mass ion fragments is shown in figure 4-15.

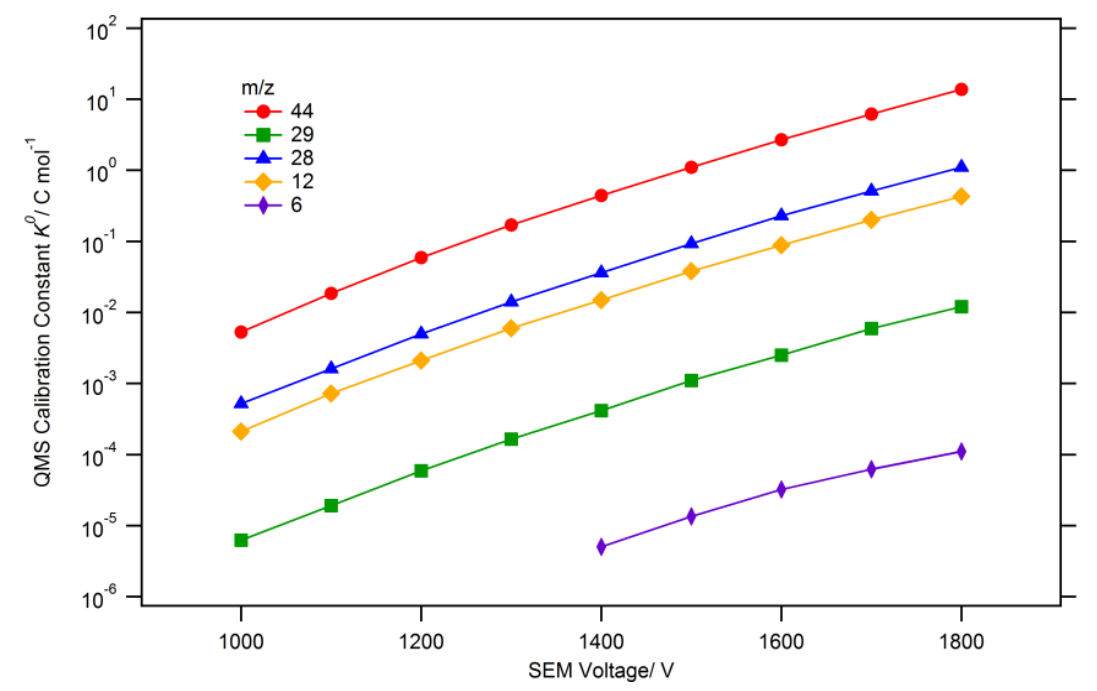

Figure 4-15: The effect of SEM Voltage on QMS Calibration Constant. A $200 \mathrm{~V}$ increase in the SEM voltage increases the amplification of the mass ion signal (and therefore the calibration constant) by a factor of $\sim 10$.

Here we see that an increase in the SEM voltage increases the mass ion fragment calibration constant significantly. As a general guide, an increase in SEM voltage of $200 \mathrm{~V}$ raises the calibration constant by a factor of $\sim 10$. It is, however, in advisable to operate the SEM at voltages below $1100 \mathrm{~V}$ or greater than $1600 \mathrm{~V}$ (above which the amplification begins to be non-linear). Furthermore, the mass ion current output should in general not exceed $10^{-6} \mathrm{~A}$ for any considerable length of time to maintain SEM lifetime. Of particular importance concerning the amplification of the mass ion current signal, is the amplification range of the EP 422 amplifier which amplifies the SEM output to an appropriate voltage to be recorded by the QC 422 bus. This is discussed in more detail in Chapter 3 section 3.7.1.2.2. 


\subsection{Calibration of the DEMS Instrument}

The calibration of the DEMS instrument can be simply achieved using a welldefined reaction at the $\mathrm{WE}$, and then measuring the mass ion current of the corresponding reaction product. The faradaic current, $I_{F}$, and $I_{Q M S}$ and mass ion current are then related by a constant, $K^{*}$, whereby:

$$
\boldsymbol{K}^{*}=\frac{\boldsymbol{z} \boldsymbol{I}_{Q M S}}{\boldsymbol{I}_{\boldsymbol{F}}}
$$

For carbon dioxide, $K^{*}$ may be most straightforwardly obtained using the $\mathrm{CO}$ Stripping measurement procedure. An example of three calibrating CO-Stripping measurements, including background $\mathrm{CV}$ in Argon saturated electrolyte on a HSAC-supported Pt catalyst are shown in figure 4-16.
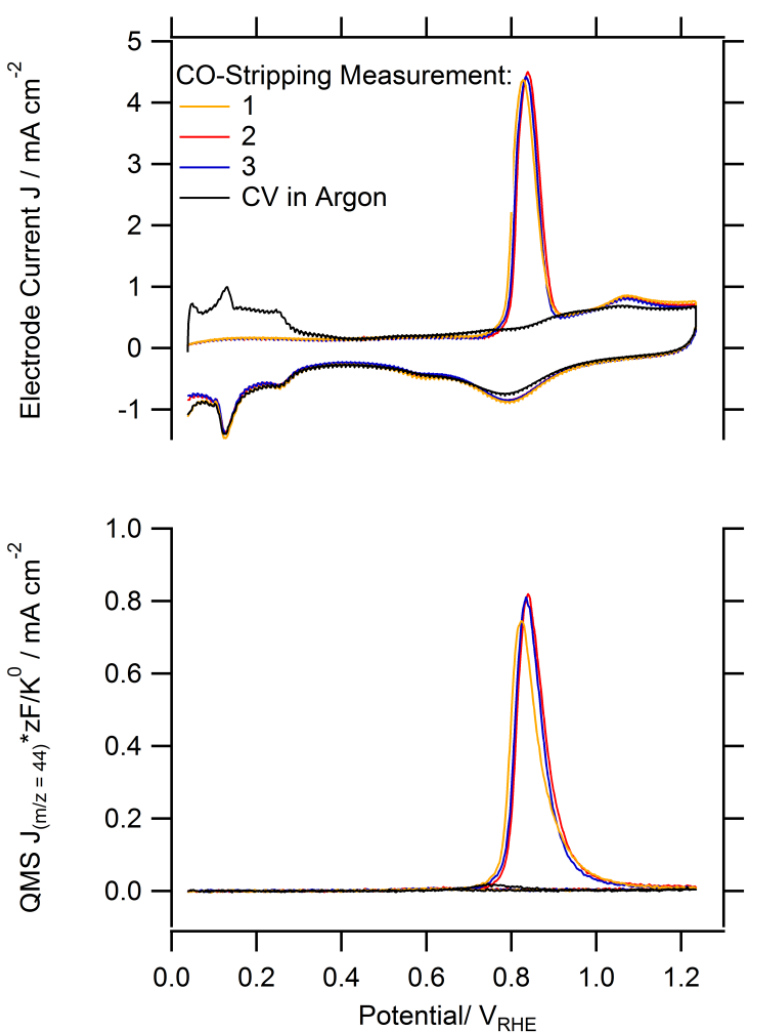

Figure 4-16: An example of three repeated CO-stripping DEMS measurements on HSAC Supported Tanaka $5 \mathrm{~nm}$ Pt catalyst at $10 \mathrm{mV} \mathrm{s}$, $0.5 \mathrm{~mol} \mathrm{dm}_{-3} \mathrm{H}_{2} \mathrm{SO}_{4}$. The figure demonstrates the precision and reproducibility of the DEMS instrument under stable electrolyte flow cell conditions. 
The CO-Stripping procedure is later discussed in more detail elsewhere in this thesis (see section 5.3.1) but briefly, the measurement involves adsorbing a saturated layer of $\mathrm{CO}_{\text {ads }}$ species on $\mathrm{Pt}$ and then oxidising this layer to carbon dioxide during a CV. This produces an oxidative electrode current peak corresponding to the $\mathrm{CO}_{\text {ads }}$ oxidation charge which corresponds to the electrochemical active surface area (ECSA) of the Pt surface. The faradaic electrode charge arising from the oxidation of the $\mathrm{CO}_{\text {ads }}$ layer on the WE, is therefore related to the mass ion charge $(\mathrm{m} / \mathrm{z}=44)$ by a certain constant, such that:

$$
K^{*}=\frac{z Q_{m / z=44}}{Q_{F}}
$$

Where $z=$ the number of electrons, $Q_{F}=$ faradaic current charge and $Q_{m / z=44}=$ integrated mass ion current response. In order to appropriately integrate these peaks the background $\mathrm{CV}$ for the Pt catalyst in Ar saturated solution must be subtracted from the CO-Stripping $C V$, and the $I_{\mathrm{m} / \mathrm{z}=44}$ background signal (where no electrode reaction occurs) must be subtracted from the measured response to the $\mathrm{CO}_{2}$, as was the case for $\mathrm{CV}$ and $\mathrm{MSCV}$ integration in figure 4-17. The evaluation of the $K^{*}$ from the CO-Stripping measurement, however, is an over simplification because $K^{*}$ contains two terms: (i) the DEMS cell collection efficiency, $N$, and (ii) the calibration constant for the relevant species of the QMS, $K^{0}$, so that in general:

$$
\boldsymbol{K}^{*}=\frac{\boldsymbol{K}^{\oplus} \boldsymbol{N}}{\boldsymbol{F}}
$$

While the parameter $K^{0}$ is independent of the electrochemical cell; $K^{*}$, must be measured each time in order to determine $N$. This collection efficiency therefore is dependent upon the DEMS electrochemical cell parameters, as we saw previously in section 4.1.2.4. If $K^{0}$ is known, then $K^{*}$ may be measured, from which a value for $N$ is obtained for each set of experiments. The exact value of $N$ is dependent upon the flow rate of electrolyte over the membrane, which in turn is determined by the cell geometry, thin-layer thickness and the permeability of the analyte species through the membrane. 


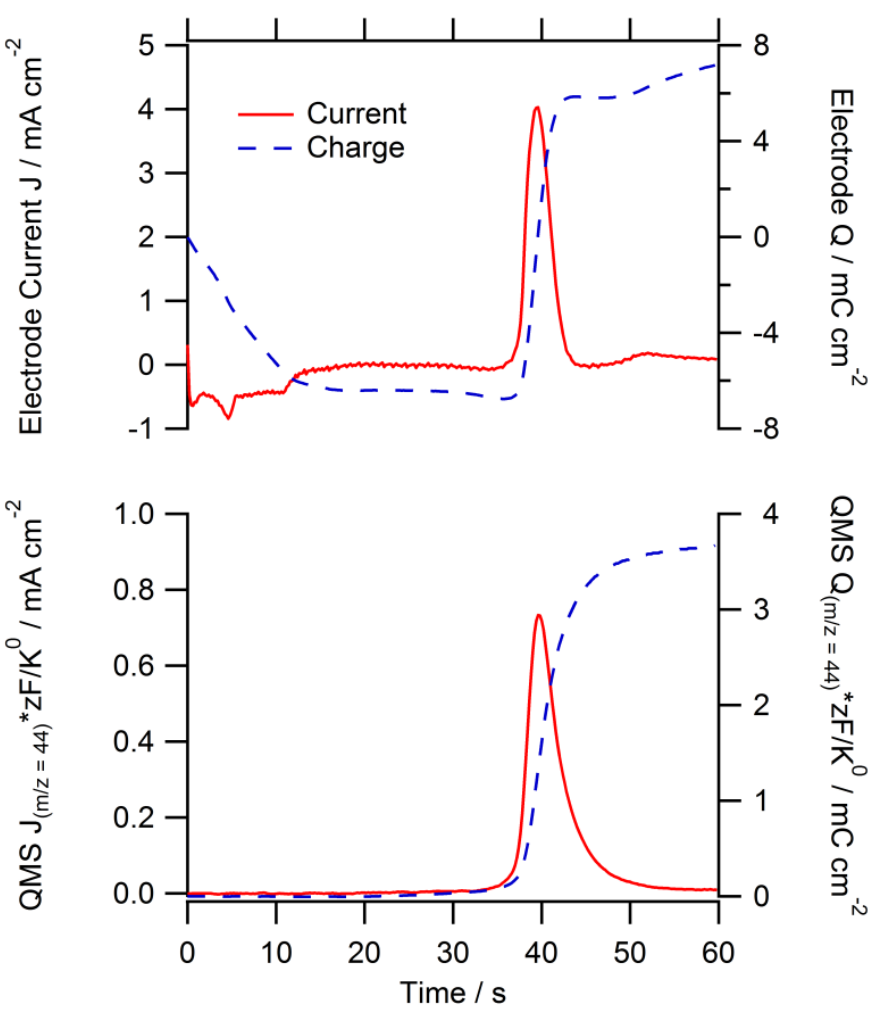

Figure 4-17: An example background subtracted and integrated CO-Stripping measurement for a $50 \mu \mathrm{g}_{\mathrm{Pt}} \mathrm{cm}^{-2}$ sample of HSAC supported Pt Tanaka $5 \mathrm{~nm}$ catalyst. By subtracting the background $\mathrm{CV}$ in Argon saturated electrolyte, capacitive and surface reaction contributions to the charge are removed, giving the charge associated with the oxidation of the $\mathrm{CO}_{\text {ads }}$ layer only.

Typical values for $N$ for the dual-cyclone thin-layer cell are 0.3 at a flow rate of $5 \mu \mathrm{s}^{-1}$. Although $K^{*}$ may be used, it is limited in use because each mass ion current channel (i.e. $\mathrm{m} / \mathrm{z}$ ratio) must be calibrated for each experiment. In contrast $K^{0}$ can be determined for a range of molecules independent of the DEMS cell parameters. Therefore, if $K^{0}$ is known, then $N$ can be measured using a single CO-Stripping experiment, thus calibrating the DEMS cell. This of course only remains true for similar types of molecules, whose collection efficiency are essentially equal, such as dissolved gaseous species. However, the $N$ for a volatile organic molecule can be expected to be much lower than for a gaseous species such as carbon dioxide. However, if the ratio between the two collection efficiencies is known then calibration could be achieved for the two different species using a single CO-Stripping measurement. This will need to be confirmed experimentally. 


\subsection{Calibration of the QMS}

Correct calibration of the QMS is very useful in order to perform quantitative DEMS studies that correlate measured mass ion currents with the faradaic electrode current. This may be achieved by leaking a known flux of pure gas into the DEMS vacuum system, and measuring the corresponding mass ion current response, as formerly described in ref. [34]. The mass ion current, $I_{i}$, recorded by the QMS is directly proportional to the flux of species, $J_{i}$, into the vacuum chamber, related by a calibration constant, $K^{0}$.

$$
I_{i}=K^{\oplus} J_{i}
$$

The gas flux is most easily measured using a calibration volume, $V_{c}$, whereby the flux of species, $J_{i}$ entering a vacuum from $V_{c}$ is described by:

$$
J_{i}=\frac{V_{c}}{R T}\left(\frac{d p_{c}}{d t}\right)
$$

Where, $R$ is the ideal gas constant $\left(0.08\right.$ mbar $\left.1 \mathrm{~s}^{-1}\right), T$ is the calibration volume temperature $(\sim 298 K)$ and $p_{c}$ is the pressure (mbar) of the calibration volume $\left(V_{c}\right)$. It therefore follows that:

$$
I_{i}=K^{\Theta}\left(\frac{V_{c}}{R T}\right)\left(\frac{d p_{c}}{d t}\right)
$$

By plotting $I_{i}$ against $\left(\frac{d p_{c}}{d t}\right)$ a slope of is obtained.

Because $V_{c}, R$ and $T$ are known or can be easily measured, the QMS calibration constant, $K^{0}$, can be acquired. The exact value of $K^{0}$, however, is dependent on a combination of the vacuum system, all QMS settings, and of course the particular molecule of interest.

An illustration of the QMS calibration setup is depicted in figure 4-18, and consists of a capacitance gauge, calibration gas line and roughening pump which are each attached to a calibration volume that is connected to the DEMS vacuum 
system via a control valve. The calibration volume was $40 \mathrm{~cm}^{3}$ determined using a method describe in ref. [106].
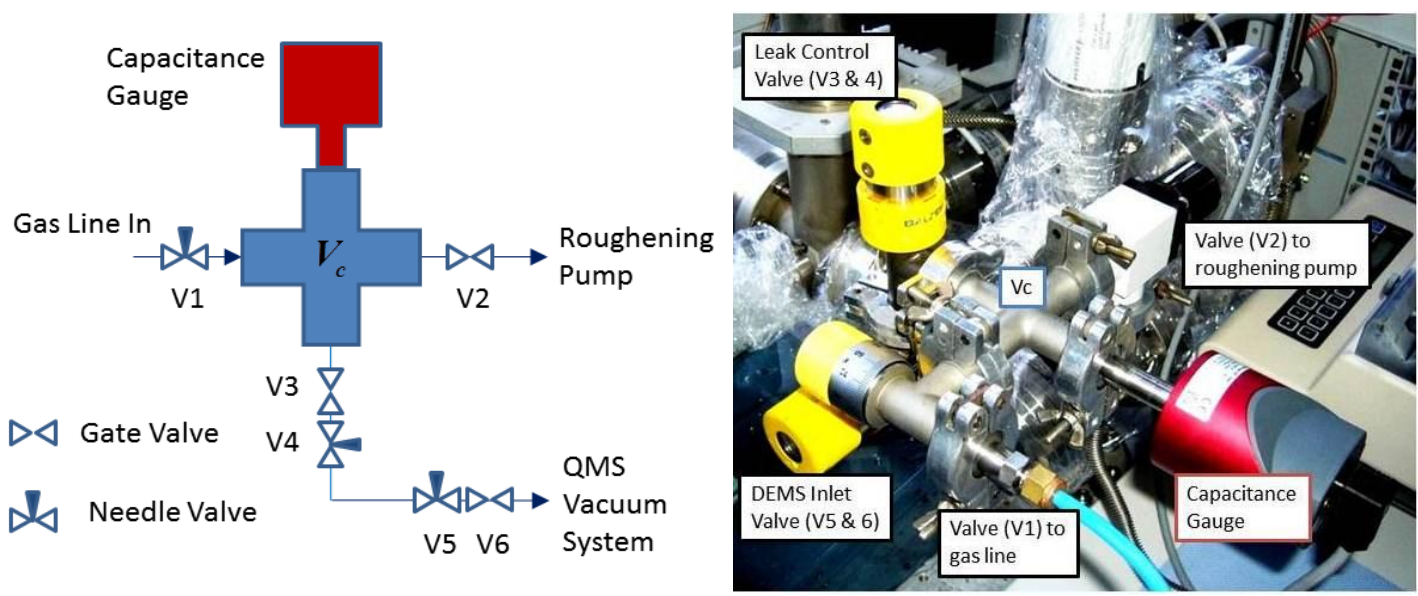

Figure 4-18: A schematic and photograph of QMS calibration volume setup. The QMS calibration setup consists of a small vacuum chamber, capacitance pressure gauge and numerous valve connections connecting the calibration gas line, roughening pump and QMS vacuum system. The calibration gas is leaked from the vacuum chamber in the QMS whilst monitoring both the calibration volume pressure decay and relevant mass ion current.

A typical QMS calibration procedure commences as follows:

i) The calibration volume is first evacuated using the roughening pump.

ii) Gate valve 2 is then closed, and the calibration gas is slowly leaked in via needle valve $\mathrm{V} 1$ into the evacuated volume until a pressure of 90 mbar is obtained.

iii) The needle valve V4 is then partially opened (ca. $51 / 5$ turns) the degree to which determines the leak rate of the calibration volume in preparation for the instrument calibration. At this point gate valve V3 remains closed.

iv) The gate valve V6 and pin valve V5 of the DEMS vacuum system inlet valve should then be opened slowly until full open.

v) Once the calibration measurement and software setup (described in section'3.7.2) is acquiring, displaying and recording the pressure and mass ion current data, the calibration measurement is ready to be performed. 
vi) In order to allow the flux of gas to enter the vacuum system, gate valve $V 3$ is opened rapidly, and the calibration gas contained within $V_{c}$ flows into the DEMS vacuum system.

The response of the corresponding mass ion currents is then measured in conjunction with the pressure decay inside the calibration volume using the QMS calibration measurement setup and custom Labview calibration software. An example measurement is shown in figure 4-19 (a) along with the respective calibration curve (b).
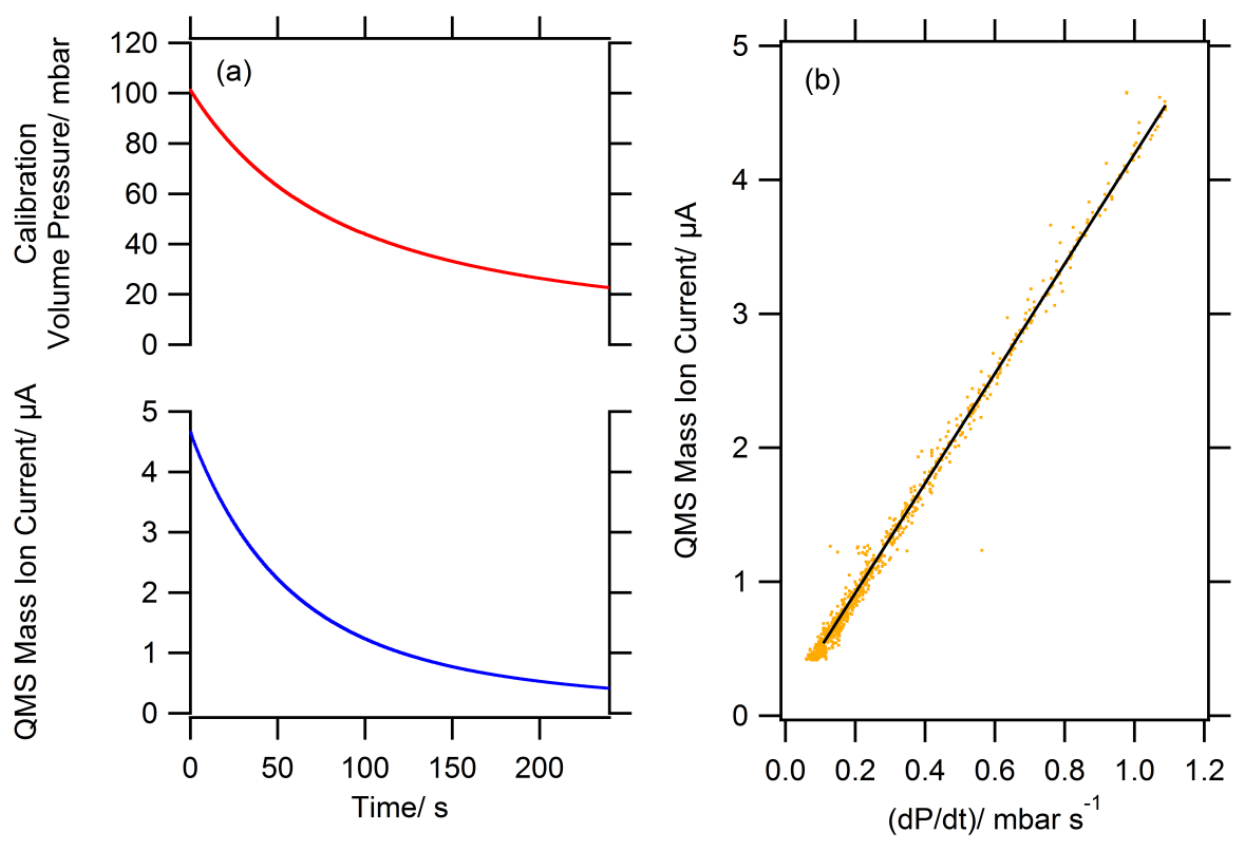

Figure 4-19: An example QMS calibration measurement showing the calibration volume pressure decay and mass ion current response (a) and the corresponding relationship between gas flux and mass ion current (b). The gas flux is determined by the conductance of the valves and the pressure difference between the calibration volume and DEMS vacuum system.

The mass ion current of the relevant channel $(\mathrm{m} / \mathrm{z}$ ratio) is linearly proportional to the flux into the DEMS vacuum system, and a very linear calibration curve can be obtained giving a value $K^{0}$. A table of typical QMS calibration values for a number of gaseous species are given in table 4-3. The calibration constants are of course dependent on the gaseous species along with the exact settings of the QMS and nature of the vacuum system. 
Table 4-3: List of numerous QMS calibration constants, $K^{0}$, for a number of gaseous species obtained for this DEMS instrument. QMS parameters: Emission current $1 \mathrm{~mA}$, Ion ref. $85 \mathrm{eV}$, Cathode 70 eV, Focus plate 14.0 V, Extraction $250 \mathrm{~V}$, Field Axis $10.50 \mathrm{~V}$, Deflection $200 \mathrm{~V}$ and Resolution 30. Measurements are reproducible to within $\sim 0.005 \mathrm{C} \mathrm{mol}^{-1}$.

\begin{tabular}{|c|c|c|c|c|c|c|}
\hline & \multirow[t]{2}{*}{ Molecule } & \multirow[t]{2}{*}{$\mathbf{m} / \mathbf{z}$} & \multicolumn{2}{|c|}{ SEM Voltage/ V } \\
\hline & & & & & 1300 & 1600 \\
\hline \multirow{5}{*}{ 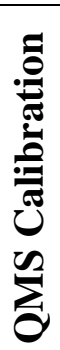 } & \multirow{5}{*}{ 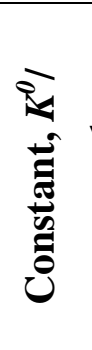 } & \multirow{5}{*}{${ }^{\bar{\Xi}}$} & Ar & 40 & 0.18 & 2.15 \\
\hline & & & $\mathbf{O}_{2}$ & 32 & 0.12 & 1.61 \\
\hline & & & $\mathbf{H}_{2}$ & 2 & 0.06 & 0.78 \\
\hline & & & $\mathrm{CO}_{2}$ & 44 & 0.19 & 2.65 \\
\hline & & & $\mathrm{CO}$ & 28 & 0.16 & 2.03 \\
\hline
\end{tabular}

This calibration technique may also be extended to volatile organic compounds such a methylformate, however, an injection port and heating of the calibration volume would be necessary in order to introduce the sample and prevent the condensation of the species onto the inner walls of the calibration volume, highlighted in ref. [34]. There are of course other methods of QMS calibration such as by, for example, gas quantities described previously in ref. [107]. 


\subsection{Further Considerations}

\subsubsection{Measurement Error}

All measurements are subject to errors that contribute to the uncertainty of a measured value. It is therefore important to be able quantify errors in the measurement, or the assumptions in data treatment in order to distinguish between true experimental observations and those that arise from measurement errors to avoid possible misinterpretation of data.

In experimentation where the DEMS instrument is used for purely qualitative observations, then clearly errors that are less of an issue and reproducibility of the qualitative observations is. However, when the DEMS instrument is used in a quantitative manner (which is far more interesting), then the sources of error for the DEMS instrument are dependent on a number of contributing factors which we need to be aware of. In order to quantify the measured QMS mass ion current, and calculate the equivalent faradaic current for an electrode, the following equation is used:

$$
I_{F_{Q M S}}=\frac{I_{Q M S} z F}{K^{0} N}
$$

The error in the equivalent faradaic current calculated from the QMS mass ion current contains an error that are propagated from other experimental uncertainties, namely $K^{0}, N$ and $I_{\mathrm{QMS}}$ as given in the following equation:

$$
\delta \boldsymbol{I}_{F_{Q U S}}=\left|\boldsymbol{I}_{F_{Q M S}}\right| \cdot \sqrt{\left(\frac{\delta \boldsymbol{I}_{Q M S}}{\boldsymbol{I}_{Q M S}}\right)^{2}+\left(\frac{\delta \boldsymbol{K}^{\Theta}}{\boldsymbol{K}^{\Theta}}\right)^{2}+\left(\frac{\delta \boldsymbol{N}}{\boldsymbol{N}}\right)^{2}}
$$

These experimental uncertainties, namely $\delta I_{Q M S}, \delta K^{0}$ and $\delta N$ are defined in the following manner. The uncertainty in the calibration constant, $K^{0}$, can be easily accessed by performing multiple calibration measurements and taking the standard deviation of the measurements. The error in DEMS cell collection efficiency, $N$, may be obtained from performing multiple CO stripping measurements prior to an experiment, and represents the most significant error, 
typically $\sim 10 \%$. The error in the measured $\mathrm{I}_{\mathrm{QMS}}$, however, is slightly more difficult to define because the absolute value relies on a background correction of the mass ion current signal which can be subject to gross errors if the background is ill-defined and subtracted improperly. In the calculation of the DEMS instrument error bars in this thesis, the mass ion current uncertainty, $\delta I_{Q M S}$, is defined as the QMS detection limit which is 2.5 multiplied by the standard deviation of the mass ion current signal noise.

All these errors are of course susceptible to gross errors. If the $I_{Q M S}$ background current is unstable or ill-defined and subtracted incorrectly, then the background corrected values are then subject to a potentially large systematic error, and obviously in such cases the interpretation of experimental data should obviously be treated with caution. Similarly, the sensitivity of the DEMS cell collection efficiency, $N$, on the electrolyte flow rate can also lead to a large systematic error if the electrolyte flow fluctuates or is momentarily disturbed or impeded effecting measurement results. These gross errors, however, should be easily spotted by an experienced user. Finally, the sensitivity of the QMS will to some extent deteriorate and drift through use (changing $K^{0}$ for the various species) as the ion source filaments and SEM wear out. The calibration constant therefore should be assessed periodically, or when it has not been used for some time.

\subsubsection{Maintenance}

In order to maintain the DEMS instrument and (hopefully) provide many years of reliable use, the following aspects of maintenance concerning the electrochemical flow cell, membrane interface and vacuum system should be considered.

Regarding the DEMS electrochemical flow cell, all PTFE and Kel-f parts should be regularly cleaned and boiled in line with the standard PTFE electrochemical RDE setup protocol. In certain circumstances it is not strictly necessary to reconstruct the DEMS cell for each daily measurement and the flow block can be left attached to the membrane interface and vacuum flange; however, the thin- 
layers of the cell should be extensively purge with Millipore water after, and prior to experimentation. It is also important to monitor for corrosion on the DEMS cell vacuum flange and steel frit. Another aspect of the electrochemical flow setup concerning maintenance, is the syringe pump and flow cell lines. The performance of the syringe will degrade through use, particularly as it is generally in contact with acid electrolyte which hardens the rubber seal over time and will likely be the cause of inconsistent flow, although deterioration in the sealing of the flow line connections can also be problematic. Furthermore, the thread of the syringe pump mechanism is also prone to being damaged if the mechanism of the pump is exposed to resistance in the flow.

The microporous PTFE membrane material is very durable, and can in principle by used for many days of experimentation without adversely affecting performance provided that the DEMS cell is not dismantled. The PTFE cell gaskets are re-usable, but the stainless steel metal frit and flange are prone to slight corrosion.

In general, the vacuum system requires the typical maintenance procedures vacuum hardware, such as monitoring the condition of the turbo pumps (by sound and the current drawn), particularly that pumping the $1^{\text {st }}$ pumping stage (which operates at rather high vacuum pressure), and replacing their oil reservoirs on a yearly basis. Of particular concern regarding the DEMS instrument system is the appreciable amount of aqueous acidic electrolyte that evaporates into the vacuum system. Whilst, the turbo-pumps appear to be largely unaffected by this (but only time will tell), the roughening pumps are prone to being damaged. This is because the aqueous acid electrolyte condenses in the roughening pump oil, which not only changes the composition of the oil but also begins to corrode the pumps mechanical parts. Consequently, it is recommended that after using the DEMS instrument the roughening pumps are operated with the gas ballast valve open for $\sim 1$ to 2 hours to assist in purging the aqueous contaminants from the vacuum pump oil. Although helping, the gas ballast will not completely resolve the problem. The roughening pump oil should therefore be periodically replaced, every 2 to 3 months depending on the use of the DEMS, 
particularly the Trivac D16B pump which backs the $1^{\text {st }}$ pumping stage, and thus pumps more acid vapours.

\subsection{Summary}

The purpose of this chapter was to present an understanding of the practical aspects of the DEMS instrument, concerning the influence and optimisation of experimental parameters, the settings of the QMS, the performance of the membrane whilst to also document the instrument calibration procedures.

As a result of the narrow capillaries and thin-layer of the electrochemical cell design, potential control of the WE cannot be achieved without modifying the electrochemical setup. The origin of the potentiostat oscillation in this cell was too small of a solution resistance $(<1 \Omega)$ between WE and the Luggin-capillary. The potentiostat was consequently stabilised by adding a resistance of $\sim 30 \Omega$ to the WE connection, which effectively acts as an artificial solution resistance. This rather high artificial solution resistance was then compensated for using a NID to provide a more acceptable $\sim 10 \Omega$ uncompensated resistance in $0.5 \mathrm{~mol} \mathrm{dm}^{-3}$ to minimise systematic errors, without impinging on the potentiostat circuitry.

The performance of the DEMS instrument was found to be strongly dependent on the electrochemical cell variables, particularly the electrolyte flow rate. The diffusion limited current for the ORR, HOR and CO-bulk oxidation was observed to scale with the volumetric flow rate by a power of $1 / 3$. Of more importance to DEMS, however, is the influence of the flow rate on the QMS response time, the degree of correlation between the faradaic electrode current and the mass ion current as well as the DEMS cell collection efficiency. By systematically assessing the effect of flow rate on these parameters, optimised flow rates were found to be between $\sim 4$ and $10 \mu \mathrm{s} \mathrm{s}^{-1}$ at potential scan rates $\leq 20 \mathrm{mV} \mathrm{s}^{-1}$ giving DEMS cell collection efficiencies in the range of 0.4 to 0.1 .

The performance of prospective microporous membrane materials was characterised in terms of its permeability and selectivity. The permeability of the membrane was assessed using a test setup which measured the total gas fluxes 
through the wetted membrane when exposed to a vacuum. Typical total gas fluxes for suitable DEMS membranes were in the range of 0.1 and $0.2 \mathrm{mbar} \mathrm{l} \mathrm{s}^{-1}$ at room temperature. In terms of selectivity, the instrument is very capable of detecting dissolved gaseous electrochemical products but is not suitable for the in-situ detection of polar or ionic electrochemical reaction species. If there is a desire in the future to use alternative membrane materials, or non-aqueous electrolytes then these combination should be performed on a test setup, rather than risk the flooding of the DEMS vacuum system

The optimisation of the QMS parameters is crucial toward maximising the singleto-noise ratio of the DEMS instrument and henceforth the detection limit of the instrument. An overview of the effect of QMS parameters on the measured mass ion current was presented in order to aid in the understanding and tuning of the QMS. The majority of the noise was found to arise from the amplification of the SEM signal by EP 422, and it is of particular importance that this is set manually in the appropriate amplification range.

The calibration of the DEMS instrument meanwhile involves the determination of the DEMS cell collection efficiency, and calibration of the QMS. In principle, the calibration of the DEMS collection efficiency should be performed prior to experiment using CO-Stripping. The QMS calibration values for a certain species should be measured each time a QMS parameter is changed, and occasionally thereafter to monitor changes in the QMS performance. The sensitivity of the instrument, however, is defined by the DEMS cell collection efficiency, and the single-to-noise ratio of the QMS.

The sources of DEMS error include those that are common to all electrochemical half-cells, such as the systematic error introduced by uncompensated solution resistance, impurities, incomplete gas purging of the electrolyte and shifts in the potential of the RE. Of more specific concern for DEMS are errors that can arise from unstable electrolyte flow rate which can have effect on the diffusion limited current, the uncertainty in DEMS calibration constant, QMS response time and correlation. Finally, the DEMS instrument also requires the standard maintenance routines for vacuum equipment, however, the condition of the oil in 
the roughening pumps should be particularly monitored carefully due to the detrimental effect that the traces of acidic electrolyte which pass through the membrane interface have on the oil composition, and corrosion of mechanical parts. 


\section{Methanol Oxidation on HSAC Supported Pt and PtRu Catalysts}

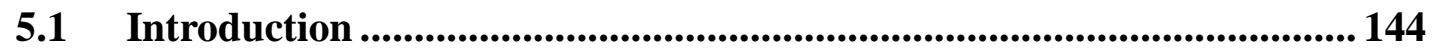

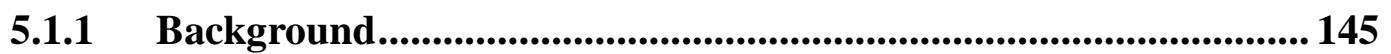

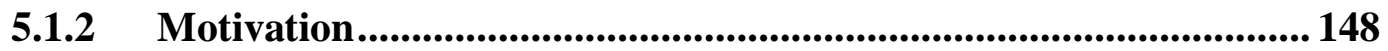

5.2 Experimental..................................................................................... 150

5.2.1 Measurement Procedure ................................................................ 152

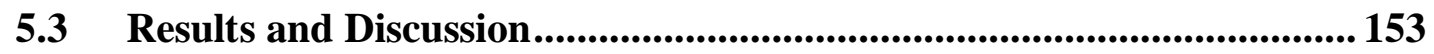

5.3.1 Electrochemical Surface Area Determination ................................ 153

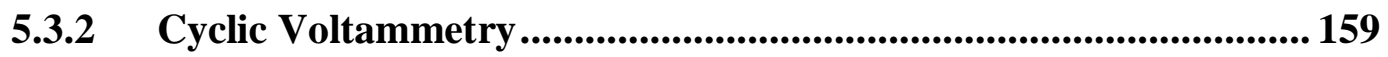

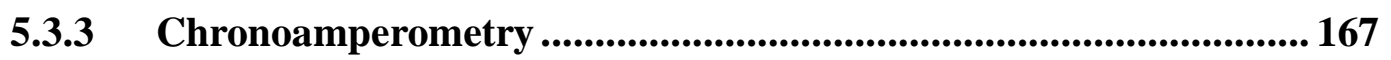

5.3.4 Three-Dimensional Voltammetry ................................................ 173

5.3.5 Tafel Slope..................................................................................................... 179

5.3.6 Activity ............................................................................................. 183

5.3.7 Potential Dependent Conversion ................................................ 185

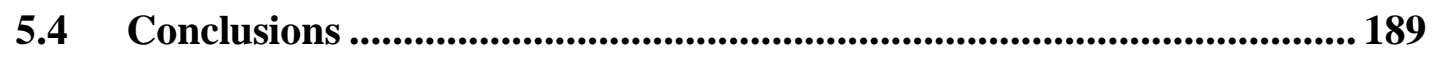

In this first demonstration of this DEMS instrument research application, the study of the methanol oxidation reaction (MOR) on HSAC supported -Pt and -PtRu catalysts is revisited. The objective of this study was to not only demonstrate the research capabilities of the DEMS instrument constructed in this thesis but to also examine the electroanalytical techniques that are commonly employed to study and assess the MOR reaction and electrocatalyst activities using RDE. The results presented here, however, do not simply repeat previous observations but elucidate the contrasting potential dependent conversion of the MOR to $\mathrm{CO}_{2}$ on $\mathrm{Pt}$ and PtRu catalysts. Three-dimensional voltammetry is furthermore applied for the first time combined with DEMS and utilised to describe the MOR, encapsulating the potential, current and time relationship of 
the reaction system within a single contour plot. The observations demonstrate that the MOR on the Pt catalyst correlates directly with the conversion of methanol to $\mathrm{CO}_{2}$; however, this relationship was not apparent in the PtRu catalyst signifying a key difference in the MOR mechanism and rate determining step.

\subsection{Introduction}

Direct methanol fuel cell (DMFC) CEEC devices possess the obvious advantage over compressed hydrogen fuelled PEMFCs that the liquid methanol fuel can be easily transported and stored using existing infrastructure. Unfortunately, even with rather high electrocatalyst loadings $\left(4 \mathrm{mg}_{\mathrm{Pt}} \mathrm{cm}^{-2}[108]\right)$ and elevated temperature $\left(>100{ }^{\circ} \mathrm{C}\right) \mathrm{DMFCs}$ only achieve relatively poor power densities (100 to $\left.250 \mathrm{~mW} \mathrm{~cm}^{-2}[108]\right)$ in contrast to hydrogen fed PEMFCs $\left(700 \mathrm{~mW} \mathrm{~cm}^{-2}\right.$ with catalyst loadings of $0.6-0.8 \mathrm{mg}_{\mathrm{Pt}} \mathrm{cm}^{-2}$ [7]). This comparatively poor power density is a result of the critical technical obstacle concerning DMFC development, namely the large operating electrode overpotentials required for both the methanol oxidation reaction (MOR) at the anode, and oxygen reduction reaction (ORR) at the cathode [109]. Despite that these limitations inhibit DMFC application in transportation, passive DMFCs with low power densities (6 to

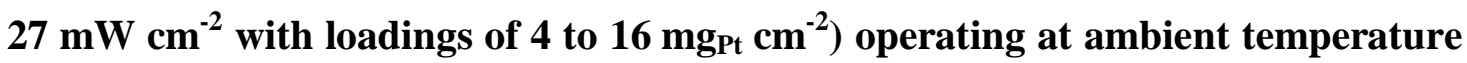
$\left(<40{ }^{\circ} \mathrm{C}\right)$ are nonetheless rather appealing to the portable electronic device market where they possesses a number of potential advantages over $\mathrm{Li}$ ion battery technology. For instance, the theoretical specific energy density of the liquid methanol fuel is $6000 \mathrm{Wh} \mathrm{kg}^{-1}$ which is some ten times higher compared to the $\mathrm{Li}$ ion battery at $600 \mathrm{Wh} \mathrm{kg}^{-1}$ [108]. Small portable DMFCs therefore, not only offer consumers the possibility that their portable electronic devices to be used for longer, but also allows instantaneous recharge (or rather refill), as opposed to the hours required to recharge a battery. Regardless of application, in order for the DMFC development targets to be met a reduction in the precious metal electrocatalyst loading is essential [108]. Although the large overpotential for the oxygen reduction reaction (common to all PEMFCs) and methanol 
crossover (diffusion of methanol from anode to cathode) contribute significantly to the performances losses of the DMFC, the overpotential required for the MOR at the anode is of even greater concern. Extensive research efforts have consequently focused on the study of the MOR, particularly in electrochemical half-cells in an endeavour to understand the fundamental aspects of the reaction mechanism and the relationship between electrocatalyst structure and activity $[14-16,110,111]$ to aid in the development of more active and cheaper electrocatalyst materials. Even so, rather expensive PtRu based electrocatalysts are still by far the most active anode materials for the MOR, although there is certainly an incentive to develop alternative non-Pt based electrocatalyst materials [112].

\subsubsection{Background}

The complete electrochemical oxidation of methanol to carbon dioxide is described by the following overall reaction formula:

$$
\mathrm{CH}_{3} \mathrm{OH}+\mathrm{H}_{2} \mathrm{O} \stackrel{\mathrm{E}}{\longrightarrow} \mathrm{CO}_{2}+6 \mathrm{H}^{+}+6 e^{-} \quad \mathrm{E}^{0}=0.046 \mathrm{~V}
$$

Although the low standard redox potential for the MOR is theoretically favourable for a CEEC device, reasonable MOR rates are unfortunately not achieved using even the most active Pt-based electrodes until a positive overpotential of several hundred millivolts is applied, reducing the practical performance of the DMFC device. This overpotential is necessary because the complete MOR proceeds via multiple reaction steps involving partially oxidised intermediates, the most significant of which is the strongly adsorbed $\mathrm{CO}_{\text {ads }}$ species that poisons the Pt electrocatalyst surface inhibiting further methanol adsorption, as described by:

$$
\mathrm{CH}_{3} \mathrm{OH} \underset{\mathrm{Pt}}{\stackrel{E_{1}}{\longrightarrow}} \mathrm{Pt}-\mathrm{CO}_{\mathrm{ads}}+4 \mathrm{H}^{+}+4 e^{-}
$$

The oxidation of the $\mathrm{CO}_{\text {ads }}$ species on $\mathrm{Pt}$ in turn requires the dissociative adsorption of water to form $\mathrm{OH}_{\text {ads }}$ species, as given by the following formula: 


$$
\mathrm{H}_{2} \mathrm{O} \underset{\mathrm{Pt}}{\stackrel{\mathrm{E}_{2}}{\longrightarrow}} \mathrm{Pt}-\mathrm{OH}_{\mathrm{ads}}+\mathrm{H}^{+}+\boldsymbol{e}^{-}
$$

The oxidation of the $\mathrm{CO}_{\text {ads }}$ species may then proceed via the bifunctional reaction of adsorbed $\mathrm{CO}_{\mathrm{ads}}$ and $\mathrm{OH}_{\mathrm{ads}}$ species:

$$
\mathrm{Pt}-\mathrm{CO}_{\mathrm{ads}}+\mathrm{Pt}-\mathrm{OH}_{\mathrm{ads}} \stackrel{\mathrm{E}_{3}}{\longrightarrow} 2 \mathrm{Pt}+\mathrm{CO}_{2}+\mathrm{H}^{+}+\mathrm{e}^{-}
$$

The adsorption of $\mathrm{OH}_{\mathrm{ad}}$ is therefore critical to the oxidation of $\mathrm{CO}_{\mathrm{ads}}$ and in turn the MOR; however, $\mathrm{OH}_{\mathrm{ads}}$ species are not formed on pure Pt surfaces until much higher electrode potentials i.e. $E_{2} \gg E_{1}$. As a consequence of the self-poisoning nature of the MOR and the limitation imposed by the slow adsorption of $\mathrm{OH}_{\text {ads }}$ on Pt necessary for the oxidation of poisoning $\mathrm{CO}_{\text {ads }}$ species, pure Pt materials are generally considered rather poor MOR catalysts. Certain alloys of Pt such as PtRu have, however, proved to exhibit superior MOR activity in comparison to pure Pt. On PtRu this improvement is attributed to the adsorption of $\mathbf{O H}_{\text {ads }}$ species on Ru surface atoms at lower electrode potentials, and is similarly described by the formula:

$$
\mathrm{H}_{2} \mathrm{O} \underset{\mathrm{Ru}}{\stackrel{E_{4}}{\longrightarrow}} \mathrm{Ru}-\mathrm{OH}_{\mathrm{ads}}+\mathrm{H}^{+}+\boldsymbol{e}^{-}
$$

The consequence of the adsorption of $\mathrm{OH}_{\text {ads }}$ on $\mathrm{Ru}$ at lower electrode potentials, i.e. $E_{4}<E_{2}$, is that the oxidation of $\mathrm{CO}_{\text {ads }}$ on $\mathrm{Pt}$ can then proceed by the bifunctional reaction given by:

$$
\mathrm{Ru}-\mathrm{OH}_{\mathrm{ads}}+\mathrm{Pt}-\mathrm{CO}_{\mathrm{ads}} \stackrel{E_{5}}{\longrightarrow} \mathrm{Pt}+\mathrm{Ru}+\mathrm{CO}_{2}+\mathrm{H}^{+}+2 e^{-}
$$

The poisoning $\mathrm{CO}_{\text {ads }}$ species are therefore oxidised at lower overpotentials, liberating the Pt surface and allowing methanol to adsorb and reaction proceed at lower potentials, where $E_{5} \sim E_{4}$. The combined action of the $\mathrm{Pt}$ and $\mathrm{Ru}$ atoms is often referred to as being co-catalytic, whilst the $\mathrm{CO}_{\text {ads }}$ oxidation mechanism can be considered bifunctional in both senses of the term i.e. the reaction proceeds via two different adsorbed functional groups $\left(\mathrm{CO}_{\mathrm{ads}}\right.$ and $\left.\mathrm{OH}_{\mathrm{ads}}\right)$ involving two different adsorption sites (Pt and Ru). 
The poisoning $\mathrm{CO}_{\text {ads }}$ species, however, is not the only partial MOR intermediate involved in the complete MOR to carbon dioxide. A number of detailed mechanisms have been proposed in the scientific literature, some of which are quite elaborate [113, 114] while others more straightforward [81]. The MOR mechanism presented in figure 5-1 is of the latter straightforward variety and is based on MOR products and predominantly adsorbed surface species observed using in-situ methods such as DEMS and FTIR. For example, DEMS has been previously used to measure the consumption of methanol [81], and/or the production of methylformate $\left(\mathrm{HCOOCH}_{3}\right)$ and carbon dioxide $[32,46,56,75,81$, 115], whereas surface spectroscopy techniques such as FTIR [116] or singlepotential alteration infrared spectroscopy (SPAIRS) [14] have primarily observed $\mathrm{CO}_{\text {ads }}$ species along with some additional absorption bands that have been assigned to various adsorbed species such as $\mathrm{COH}_{\text {ads }}$ and $\mathrm{CHOO}_{\text {ads. }}$ Formaldehyde yields of the MOR have meanwhile been observed ex-situ using fluorescence spectroscopy $[117,118]$.

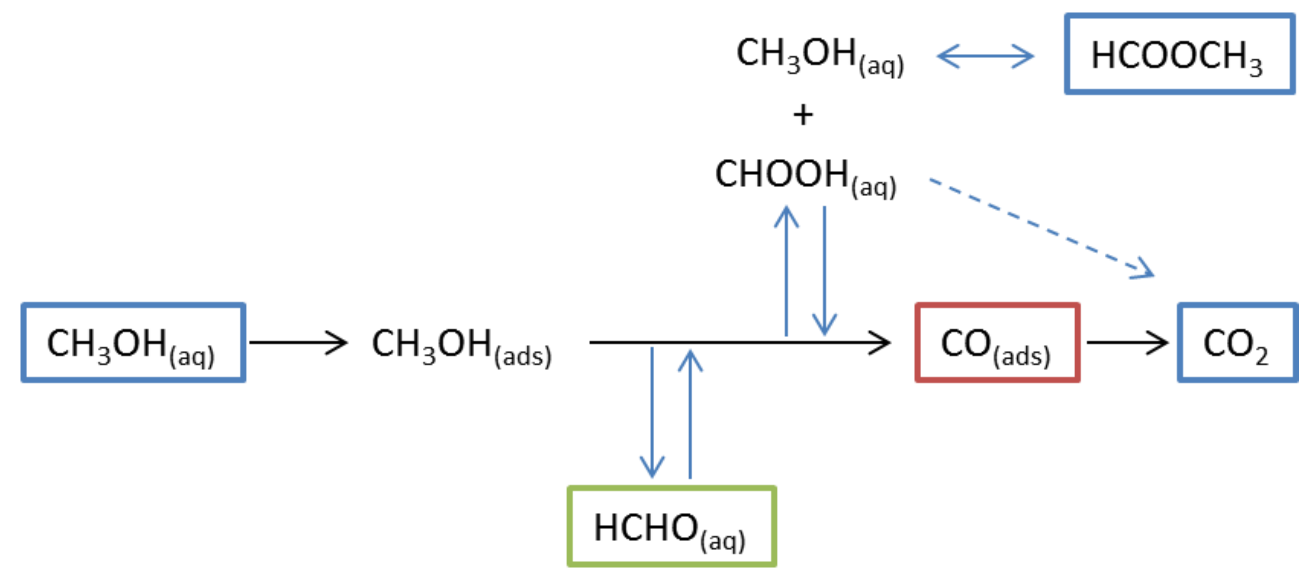

Figure 5-1: A diagram of the electrochemical methanol oxidation reaction pathway. Methanol $\left(\mathrm{CH}_{3} \mathrm{OH}\right)$, methylformate $\left(\mathrm{HCOOCH}_{3}\right)$ and $\mathrm{CO}_{2}$ (blue) have been observed using DEMS, $\mathrm{CO}_{\text {ads }}$ (red) using FTIR/SPAIRS whilst formaldehyde (HCHO - green) has been observed (ex-situ) using fluorescence spectroscopy. In order for methanol to be complete oxidise to $\mathrm{CO}_{2}$ all desorbed partially oxidised MOR intermediates must be further oxidised.

At ambient temperature $\left(<40{ }^{\circ} \mathrm{C}\right)$ the MOR is initiated via the dissociative adsorption of methanol on the Pt surface and may proceed via two pathways termed direct and in-direct. In the direct pathway, the reaction proceeds directly to $\mathrm{CO}_{\text {ads }}$ which must then be oxidised to evolve $\mathrm{CO}_{2}$ yielding the full six electrons. 
Alternatively, methanol may only be partially oxidised to weakly adsorbed intermediates that can desorb in the form of either formaldehyde [117] or formic acid $[32,46,56,75,81,115]$, yielding only two or three electrons respectively. The desorbed formaldehyde and formic acid partial MOR products must therefore re-adsorb onto the electrocatalyst surface in order to oxidise fully to $\mathrm{CO}_{2}$, a process that can be termed the in-direct pathway. To further complicate the MOR system, these desorbed partial MOR products may also undergo chemical reaction with species in the electrolyte. For example, it has been noted that formaldehyde largely exists in the form of methylene glycol $\left(\mathrm{H}_{2} \mathrm{C}(\mathrm{OH})_{2}\right)$ in acid electrolyte whose oxidation may also proceed via two pathways [119, 120]. Similarly, formic acid may again, oxidise via two pathways or alternatively undergo an acid catalysed esterification reaction with methanol in solution to form methylformate (as observed using DEMS [115]) which electrochemically oxidises to yield $\mathrm{CO}_{2}$ and methanol [121].

Overall, in order for the complex MOR processes to be considered complete, all desorbed partial MOR products must re-adsorb and oxidise completely to $\mathrm{CO}_{2}$ in order for full conversion to be achieved yielding the full six electrons per adsorbing methanol molecule.

\subsubsection{Motivation}

In light of the extensive research efforts that have already been exhausted on the study of the MOR on various Pt and PtRu based catalysts under numerous experimental conditions, what can yet another electrochemical half-cell study on the MOR hope to achieve. Well, there are three main aspects to the motivation for this study. The first actually concerns the performance of DEMS instrument rather than the study of MOR itself. It is more than reasonable to choose a topic which has already been relatively extensively studied using DEMS [32, 52, 62, 75, $81,122]$ in order to first demonstrate and assess the research capabilities of the instrument constructed as part of this thesis. This, however, does not mean to say that this investigation simply repeats the observations of previous DEMS studies. The second aspect of the motivation for this study was to evaluate using DEMS, 
the electroanalytical techniques often employed using standard RDE methods to characterise and compare the electrocatalytic activity of prospective MOR catalysts. In doing so, this study will highlight that there is no consistently employed method to evaluate and compare the activity of such materials toward the MOR, with many studies in particular focusing on rather high electrode potentials > $0.5 \mathrm{~V}_{\text {RHE}}$. The final aspect, concerns whether new insights can be offered into the MOR by, for example, applying more sophisticated electroanalytical methods such as three-dimensional voltammetry combined with DEMS, despite all that has been done previously. 


\subsection{Experimental}

Each electrocatalyst sample (Tanaka Pt 5 nm $50.6 \%$ wt. Pt on, and Tanaka $78 \%$ wt. PtRu (1:1) catalysts supported on EC300 HSAC) was prepared by ultrasonically dispersing the catalyst powder in $50 \mathrm{ml}$ of Millipore Milli-Q water. The measurement sample was prepared by pipetting $20 \mu \mathrm{L}$ of the aqueous catalyst suspension onto a mirror polished $5 \mathrm{~mm} \emptyset$ glassy carbon WE substrate to give an electrode loading of $50 \mu g_{\text {metal }} \mathrm{cm}^{-2}$. All measurements were performed in $0.5 \mathrm{~mol} \mathrm{dm}^{-3} \mathrm{H}_{2} \mathrm{SO}_{4}$ electrolyte prepared in Millipore Milli-Q water ( $<18.3 \mathrm{MOhm} \mathrm{cm}{ }^{-1}$, TOC $<5$ ppb) using concentrated $\mathrm{H}_{2} \mathrm{SO}_{4}$ (Normaton; Merck, Germany). The MOR reaction measurements were meanwhile performed in the $\mathrm{H}_{2} \mathrm{SO}_{4}$ acid electrolyte containing a methanol concentration of $1.0 \mathrm{~mol} \mathrm{dm}^{-3}$ (Pro analysis, Merck, Germany).

The DEMS instrument setup consists of a dual-cyclone thin-layer electrochemical flow cell, $0.03 \mu \mathrm{m}$ microporous PTFE membrane (Gore) interface, a 3-stage differentially pumped high vacuum system and QMS (Pfeiffer Vacuum QMA 422) as was described previously in Chapter 0 . The electrochemical and membrane interface thin-layer compartments are formed and sealed tight using $250 \mu \mathrm{m}$ thick PTFE gaskets resulting in volumes of $\sim 4 \mu \mathrm{L}$ and $\sim 7 \mu \mathrm{L}$ in the WE and membrane interface compartment, respectively. The $\mathrm{CE}$ was positioned in a sidecompartment located to the side of the flow cell inlet, whilst the RE is located in a sealed compartment positioned on the outlet of the flow cell. . An electrolyte flow rate of $5 \mu \mathrm{s} \mathrm{s}^{-1}$ through the thin-layer flow cell was maintained using a KDS 200 precision syringe pump, located on the flow outlet, withdrawing electrolyte from an Ar (purity 5.0) gas purged reservoir. In order to avoid potentiostat oscillations that arise because of high CE, RE solution resistances to the WE and low solution resistance to the Luggin-capillary in dual thin-layer flow cell, a stabilising resistor $(\sim 30 \Omega)$ was added in series to the WE connection, and compensated for using a custom built NID. The resulting uncompensated solution resistance, typically $\sim 10 \mathrm{Ohm}$, was then corrected for during post measurement data treatment

The faradaic current arising from the complete MOR reaction to $\mathrm{CO}_{2}, \mathrm{I}_{\mathrm{CO}}$, was calculated from the mass ion current, $I_{\mathrm{m} / \mathrm{z}=44}$, using the following equation: 


$$
I_{C O_{2}}=\frac{\boldsymbol{I}_{m / z=44} \cdot 6 \boldsymbol{F}}{\boldsymbol{K}_{C O_{2}}^{\ominus} N_{C O_{2}}}
$$

The QMS calibration constant, $K^{0}$, for $\mathrm{CO}_{2}$ at $\mathrm{m} / \mathrm{z}=44$ was $2.95 \mathrm{C} \mathrm{mol}^{-1}$, the DEMS cell collection efficiency, $N$, for $\mathrm{CO}_{2}$ was determined experimental using CO stripping prior to the experiment, typically 0.3 at an electrolyte flow rate of $5 \mu \mathrm{s} \mathrm{s}^{-1}$. The WE surface is continuously supplied with fresh $1.0 \mathrm{~mol} \mathrm{dm}^{-3}$ methanol in acid solution, and a total of 6 electrons are assumed to arise for each $\mathrm{CO}_{2}$ observed. The background current signal for $I_{m / z=44}$ was subtracted post measurement, whereby the background was defined as the stable $I_{m / z=44}$ signal observed when no electrode reaction took place. The $I_{\mathrm{CO} 2}$ was furthermore used to determine the conversion (expressed as a fraction, also commonly referred as current efficiency in previous DEMS papers) of the MOR to $\mathrm{CO}_{2}$ using the following equation:

$$
\text { Conversion }=\frac{I_{\mathrm{CO}_{2}}}{I_{F}}
$$

In addition to $I_{m / z=44}$, the $I_{m / z=60}$ corresponding to methylformate $\left(\mathrm{CHOOCH}_{3}\right)$ was also measured during the MOR measurements presented in this work. The $I_{m / z=60}$ signal, however, was (unfortunately) not calibrated in this work and all observation of $I_{m / z=60}$ are purely qualitative. Consequently, the results and discussion of the $I_{m / z=60}$ measurements has been largely omitted in this study; however, the data is presented in Appendix A for future reference.

All electrode potentials meanwhile are given versus the RHE determined using the HOR/HER in hydrogen saturated electrolyte determined using a polycrystalline Pt electrode prior to experimentation. 


\subsubsection{Measurement Procedure}

The catalyst samples were initially characterised using $\mathrm{CV}$ at various scan rates in Argon saturated solution, by cycling the potential between 0.05 and $1.15 \mathrm{~V}_{\mathrm{RHE}}$ for the Pt catalyst, and 0.05 and $0.85 \mathrm{~V}_{\mathrm{RHE}}$ for the PtRu catalyst. A CO-Stripping measurement was then performed in order to determine the ECSA of the catalyst samples prior to the study of the MOR. Following the CO-Stripping measurement, the electrolyte was then exchanged with electrolyte containing $1.0 \mathrm{~mol} \mathrm{dm}^{-3}$ concentration of methanol by switching the electrolyte reservoir and purging the electrochemical cell for ca. 5 minutes, during which the potential of the WE was maintained at $0.05 \mathrm{~V}_{\mathrm{RHE}}$.

The electrochemistry of the HSAC supported -Pt and -PtRu catalysts toward the MOR was first characterised under potential dynamic conditions using CV performed at $10 \mathrm{mV} \mathrm{s}^{-1}$ between 0.05 and $1.15 \mathrm{~V}_{\text {RHE }}$ for the Pt catalyst and 0.05 and $0.85 \mathrm{~V}_{\mathrm{RHE}}$ for the PtRu catalyst. The MOR on the respective catalyst samples was then studied under potentiostatic conditions using a sequence of chronoamperometric measurements. This sequence involved stepping the WE potential from $0.05 \mathrm{~V}_{\mathrm{RHE}}$ to the desired potential of study, progressively increasing the potential in $50 \mathrm{mV}$ steps from 0.435 to $0.835 \mathrm{~V}_{\mathrm{RHE}}$ in the case of the Pt catalyst, and $0.435 \mathrm{~V}$ to $0.735 \mathrm{~V}_{\mathrm{RHE}}$ for the PtRu catalyst in $50 \mathrm{mV}$ steps holding for $420 \mathrm{~s}$. Between each chronoamperometric measurement three CV were recorded to 'reset' the catalyst and monitor any apparent changes in the $\mathrm{CV}$. By performing this series of multiple chronoamperometric measurements between the potential region of interest, three-dimensional plots can be constructed to encapsulate the time, potential and current relationship using Wavemetrics $^{\circledR}$ Igor Pro 6.21 contour plot graph function. This is most easily achieved by arranging the series chronoamperometric data in a single $X, Y$ and $Z$ wave and then building the contours using ca. x16 interpolation. The 3dimensional plot matrix was then constructed from the contours using the Contour $Z$ function. 


\subsection{Results and Discussion}

\subsubsection{Electrochemical Surface Area Determination}

In this study on the MOR, the ECSA of the HSAC Pt and PtRu catalysts were first determined using CO-Stripping, whereby a saturated layer of $\mathrm{CO}_{\text {ads }}$ species is adsorbed at a potential of $0.05 \mathrm{~V}_{\mathrm{RHE}}$ which is subsequently oxidised during a linear sweep voltammogram (LSV). In DEMS this serves two purposes: firstly, it can be used to calibrate the DEMS cell collection efficiency $\left(\mathrm{N}_{\mathrm{CO} 2}\right)$, and secondly, it is a measure of the ECSA of the catalyst sample. The results from a typical DEMS CO-Stripping measurement of the HSAC Tanaka Pt $5 \mathbf{~ n m}$ catalyst is given in figure 5-2.
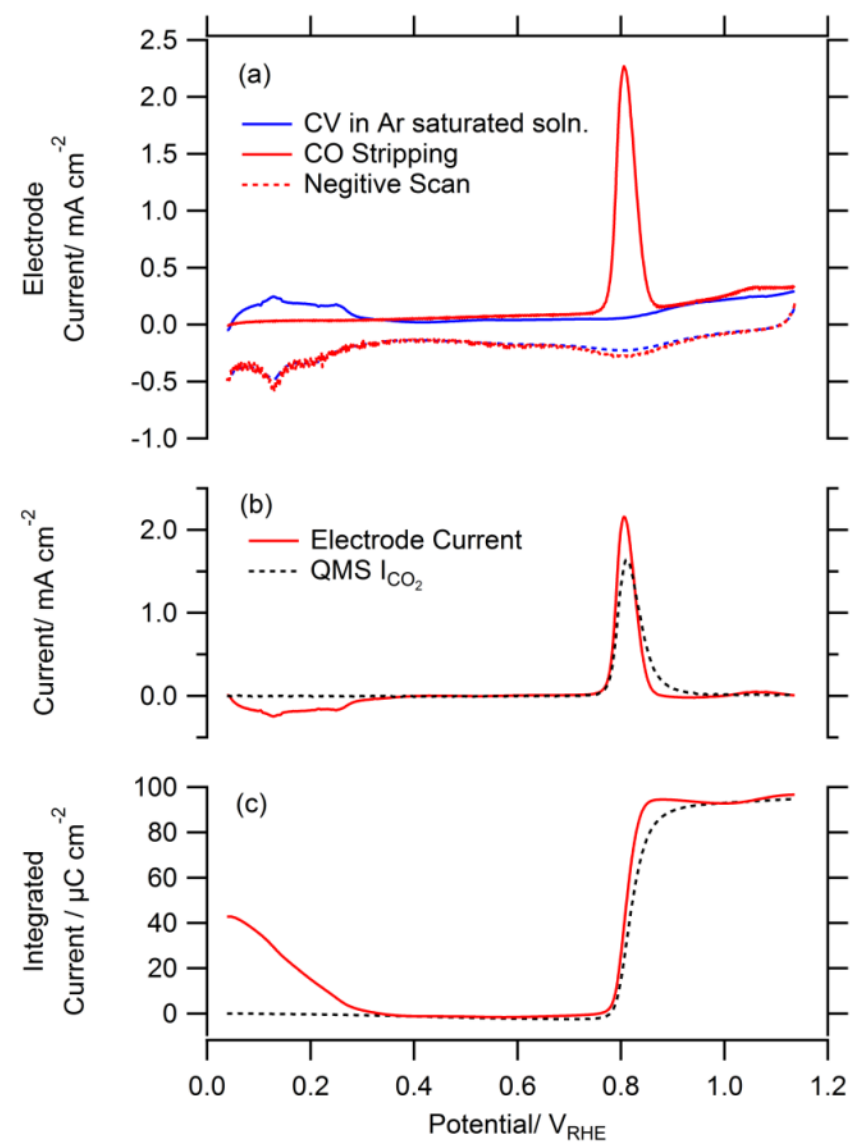

Figure 5-2: A DEMS CO-stripping measurement for $50 \mu \mathrm{g} \mathrm{cm}^{-2}$ of the Pt $5 \mathrm{~nm}$ HSAC supported catalyst.(a) CO-Stripping and background $\mathrm{CV}$ in Argon saturated electrolyte, (b) corresponding background subtracted CO-Stripping LSV and $I_{\mathrm{CO} 2}$ and (c) charge, performed at $10 \mathrm{mV} \mathrm{s}$ in $0.5 \mathrm{~mol} \mathrm{dm}^{3} \mathrm{H}_{2} \mathrm{SO}_{4}$. The background subtracted CO-Stripping charge can be almost exclusively attributed to oxidation of the saturated $\mathrm{CO}_{\mathrm{ads}}$ layer. 
Focusing firstly on the $\mathrm{CV}$ in Argon saturated electrolyte in figure 5-2(a), we observe the standard features expected from a pure Pt catalyst. In the positive potential scan the hydrogen under potential desorption $\left(\mathrm{H}_{\mathrm{upd}}\right)$ between 0.05 and $0.35 \mathrm{~V}_{\mathrm{RHE}}$ is clearly present, followed by the double layer region between 0.35 and $\sim 0.74 V_{\text {RHE }}$ before we begin to oxidise the Pt surface with $O H_{\text {ads }}$ species and finally forming PtO as the potential exceeds $0.9 \mathrm{~V}_{\mathrm{RHE}}$. In the reverse potential scan, these Pt oxide species are then reduced, with a visible hysteresis that exhibits a reduction peak at $\sim 0.8 \mathrm{~V}_{\mathrm{RHE}}$, which is again followed by a narrowed double layer region before we observe the reduction current of hydrogen adsorption on Pt between 0.35 and $0.05 \mathrm{~V}_{\mathrm{RHE}}$. The currents in the $\mathrm{CV}$ of the HSAC-supported Pt catalyst in Argon saturated electrolyte are dominated by the faradic currents arising from the surface reactions that occur between the Pt and aqueous electrolyte; however, these also contain ill-defined capacitive currents that arise from the whole electrode surface area, including that of the HSAC support and WE substrate.

Prior to the CO-Stripping CV the WE potential is held at $0.05 \mathrm{~V}_{\mathrm{RHE}}$ and the electrode surface to exposed to dissolved $\mathrm{CO}$ gas that adsorbs onto the Pt surface, displacing the $\mathrm{Pt}-\mathrm{H}_{\mathrm{ads}}$ species and forming a saturated $\mathrm{Pt}-\mathrm{CO}_{\mathrm{ads}}$ layer. This is indicated by the disappearance of the $\mathrm{H}_{\text {upd }}$ feature in the upward potential scan prior to $\mathrm{CO}_{\text {ads }}$ oxidation. In the positive potential sweep of the saturated $\mathrm{CO}_{\mathrm{ads}}$ covered $\mathrm{Pt}$ electrode, the current is dominated by double-layer capacitive currents until $0.74 \mathrm{~V}_{\mathrm{RHE}}$ where we observe a sharp increase in current arising from the oxidation of the saturated $\mathrm{CO}_{\text {ads }}$ layer. Once the $\mathrm{CO}_{\mathrm{ads}}$ is completely oxidised to $\mathrm{CO}_{2}$ freeing the $\mathrm{Pt}$ surface, the $\mathrm{CV}$ returns to the form observed prior to the CO-Stripping measurement. The CO-Stripping CV, however, still contains capacitive currents arising from the HSAC support, WE substrate and doublelayer, along with the faradaic $P t$ surface oxidation currents that are present in the $\mathrm{CV}$ in Argon saturated solution. These additional current contributions in the CO-stripping measurement may be removed by subtracting the $\mathrm{CV}$ in Argon, yielding both the CO-stripping and inverse $\mathrm{H}_{\text {upd }}$ charge, as shown in the LSV of figure 5-2(b). This is compared with the corresponding faradaic current MSLSV for $I_{\mathrm{CO}_{2}}$ calculated (assuming $2 \mathrm{e}^{-}$) from the simultaneously measured $I_{m / z=44}$ using DEMS, where it can be seen that the potential dependent curves of the $I_{F}$ and $I_{C O 2}$ 
do not perfectly overlap. This broadening in the $I_{\mathrm{CO} 2}$ is due to the diffusion and mixing of the $\mathrm{CO}_{2}$ generated at the electrode surface in the DEMS, and is a typical limitation of the technique, nonetheless, the overall calibrated charge in figure 5-2(c) is the same. At this point, it is immediately clear from a simple CO-Stripping measurement why $\mathrm{CO}_{\text {ads }}$ species that do not begin to oxidise (in a saturated layer) until $0.74 \mathrm{~V}_{\mathrm{RHE}}$ might inhibit the MOR on a pure Pt surface at DMFC anode relevant potentials $<0.5 \mathrm{~V}_{\mathrm{RHE}}$.

The significantly earlier onset potential of the oxidation of a saturated $\mathrm{CO}_{\text {ads }}$ layer on the HSAC supported PtRu catalyst is seen in figure 5-3.
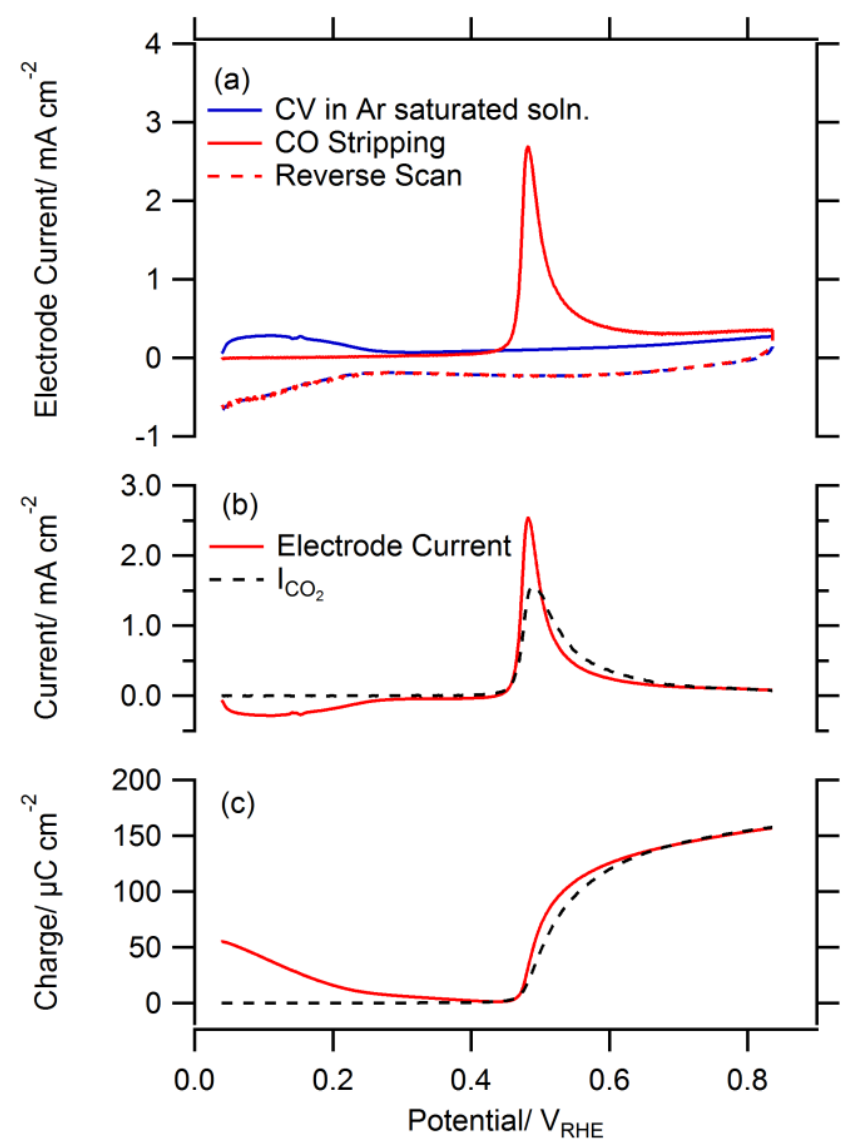

Figure 5-3: A DEMS CO-Stripping measurement of Tanaka PtRu HSAC supported catalyst. (a) CO-Stripping and background $\mathrm{CV}$ in Argon saturated solution, (b) corresponding background subtracted CO-Stripping LSV and $I_{\mathrm{CO} 2}$ and (c) charge, performed at $10 \mathrm{mV} \mathrm{s}^{-1}$ in $0.5 \mathrm{~mol} \mathrm{dm}^{3}$ $\mathrm{H}_{2} \mathrm{SO}_{4}$. The charge arising from the background subtracted $\mathrm{CO}$-Stripping measurement can be almost exclusively attributed to the oxidation of the saturated $\mathrm{CO}_{\text {ads }}$ layer. 
The CV of the PtRu catalysts recorded in Argon saturated solution exhibits similar features to those observed on pure $\mathrm{Pt}$ catalyst, although the terms $\mathbf{H}_{\text {upd, }}$, double layer and oxidation/reduction regions are (strictly speaking) only relevant to the CV of pure Pt catalysts, these terms will be used to identify the same potential regions for the $P$ tRu catalyst. $A H_{\text {upd }} / \mathrm{H}_{\text {ads }}$ feature is present in the $\mathrm{CV}$ of PtRu between 0.05 and $0.28 \mathrm{~V}_{\mathrm{RHE}}$, although in this instance the process is assumed to occur equally on both the Ru and Pt surface atoms based on the CV of pure Ru electrodes [111, 123]. In contrast to the pure Pt catalyst, however, there is no longer a clear distinction between the $\mathbf{H}_{\text {upd, double layer and }}$ oxidation/reduction regions of the $\mathrm{CV}$. This is a consequence of additional faradaic current arising from the earlier oxidation of the $\mathrm{Ru}$ surface atoms forming $\mathrm{Ru}-\mathrm{OH}_{\mathrm{ads}}$ species as early as $0.2 \mathrm{~V}_{\mathrm{RHE}}$ following $\mathrm{H}_{\mathrm{upd}}$ and continuing across the double layer potential region where the $\mathrm{Pt}$ surface also begins to oxidise. The surface oxide species are subsequently reduced in the downward potential scan, analogous to the pure $\mathrm{Pt}$ electrode which is assumed to be reversible. Following the adsorption of dissolved CO gas at $0.05 \mathrm{~V}_{\mathrm{RHE}}$ on the PtRu catalyst, the $\mathrm{H}_{\text {upd }}$ feature in the upward potential scan of CO-Stripping sweep is again clearly supressed suggesting that a saturated layer of $\mathrm{CO}_{\text {ads }}$ has formed on both the $\mathrm{Pt}$ and $\mathrm{Ru}$ surface atoms (the adsorption of $\mathrm{CO}_{\text {ads }}$ on $\mathrm{Ru}$ is known from CO-Stripping measurements performed on pure Ru electrodes [111]). The saturated $\mathrm{CO}_{\mathrm{ads}}$ layer also appears to have suppressed some small oxidative currents between 0.35 and $0.4 \mathrm{~V}_{\mathrm{RHE}}$ that are present in the $\mathrm{CV}$ in Argon saturated solution but not in the subsequent CO-stripping sweep which may suggest that the $\mathrm{Ru}-\mathrm{CO}_{\mathrm{ads}}$ species inhibit the formation of $\mathrm{Ru}-\mathrm{OH}_{\mathrm{ads}}$ in this potential region. The onset of the saturated $\mathrm{CO}_{\text {ads }}$ layer oxidation on the PtRu catalyst occurs at 0.4 $\mathrm{V}_{\text {RHE}}, \sim 350 \mathrm{mV}$ earlier than that observed on the pure Pt catalyst. Following the rapid onset of $\mathrm{CO}_{\text {ads }}$ oxidation peaking at $0.48 \mathrm{~V}_{\mathrm{RHE}}$ we observe an initially rapid fall in current which then begins to slow at $0.52 \mathrm{~V}_{\mathrm{RHE}}$ broadening the $\mathrm{CO}_{\text {ads }}$ oxidation peak across a full $400 \mathrm{mV}$ range. This is significantly broader than the symmetric CO-Stripping peak observed on the pure Pt catalyst which possessed a peak width of only $120 \mathrm{mV}$. Once again, the CV in Argon saturated electrolyte can be subtracted to yield the $\mathrm{CO}$-stripping and $\mathrm{H}_{\text {upd }}$ charge and the $\boldsymbol{I}_{\mathrm{CO} 2}$ observed does not perfectly overlap with the faradaic electrode current. The 
oxidation of a saturated $\mathrm{CO}_{\text {ads }}$ layer on the PtRu catalyst at $0.5 \mathrm{~V}_{\mathrm{RHE}}$ is significantly earlier than on the $P t 5 \mathrm{~nm}$ catalyst, and is promisingly within DMFC anode relevant potentials $<0.5 \mathrm{~V}_{\mathrm{RHE}}$.

The ECSA measurement values and DEMS cell collection efficiency obtained from CO-stripping experiments averaged over ten HSAC -Pt and -PtRu catalyst samples are summarised in table 5-1.

Table 5-1: Summary of CO-stripping measurement details and ECSA values of the Tanaka Pt $5 \mathrm{~nm}$ and PtRu HSAC supported catalysts. The ECSA of the PtRu catalysts is comparable to the Pt 5nm suggesting similar particle sizes, while the DEMS cell collection efficiencies are identical.

\begin{tabular}{|c|c|c|}
\hline & Pt $5 \mathrm{~nm}$ & PtRu \\
\hline $\mathrm{H}_{\text {upd }}$ Charge/ $\mathrm{mC} \mathrm{cm}^{-2}$ & $6.2 \pm 6$ & $4.7 \pm 10$ \\
\hline CO Stripping Charge/ $\mathrm{mC} \mathrm{cm}^{-2}$ & $12.6 \pm 10$ & $14.5 \pm 14$ \\
\hline $\mathrm{H}_{\text {upd }}$ : CO-Stripping Charge Ratio & $1: 2$ & 1: $\sim 3$ \\
\hline Roughness Factor/ $50 \mu g_{\text {metal }}$ & 31.5 & 37.18 \\
\hline ECSA/ $\mathrm{m}^{2} \mathrm{~g}_{\text {metal }}{ }^{-1}$ & 63 & 74 \\
\hline DEMS Cell Collection Efficiency/ $\mathrm{N}_{\mathrm{CO} 2}$ & $0.3 \pm 0.03$ & $0.3 \pm 0.03$ \\
\hline
\end{tabular}

Here we see that the mass normalised CO-Stripping charges and ECSA are reasonably comparable for both the HSAC -Pt 5 nm and -PtRu catalyst samples. An important observation is that the DEMS cell collection efficiencies for both the Pt and PtRu catalysts were observed to be identical. This in itself might not seem overly significant, however, it has been noted in previous DEMS studies on PtRu based catalysts that the observed faradaic CO-Stripping charge contains significant current arising from a 'large and undefined capacitive contribution due to the large double-layer current' $[32,75]$ and therefore the ECSA can be overestimate by up to $50 \%$, but is be avoided using DEMS. The connotations of this claim is that investigations of PtRu using standard RDE methodologies overestimate, by an unknown factor, the ECSA of the PtRu samples, rendering any subsequent comparison of ECSA normalised activities systematically erroneous. Whilst the investigation into this claim was not the focus of this study, in principle the calculated DEMS collection efficiency, $N$, obtained during the CO-Stripping measurement should be statistically lower for the PtRu catalysts, if indeed the CO-stripping charge is systematically overestimated because of 
undefined capacitive current contributions. For this PtRu catalyst, however, this was clearly not the case, although there is a discrepancy in the ratio of the $\mathbf{H}_{\text {upd }}$ and CO-Stripping charges should be 1:2 (as is the case on Pt electrodes) but is 1: 3 for the PtRu catalyst.

A crucial difference between the observations in the CO-Stripping measurements performed in this study in comparison to the observations in previous studies [32, 75] could be attributed to data treatment rather than the measurement methodology itself. In this study the background CV in Argon saturated solution was subtracted prior to the integration of the CO-Stripping peak for both HSAC supported -Pt and -PtRu catalysts. The background capacitive contributions from the Pt/PtRu surface, the WE substrate and HSAC support are consequently removed and there is no longer an issue regarding the possible over-estimation of the CO-Stripping charge. For PtRu catalysts this background subtraction is perhaps even more important given the greater width of the characteristic CO-Stripping peak $(\sim 350 \mathrm{mV})$, typically $\sim 3$ to 4 times broader than that on $\mathrm{Pt}$ $(150 \mathrm{mV})$ with inherently more capacitive contributions. In the previous studies, however, it was not clear if the background CV was subtracted prior to integration, and because it was not explicitly stated then it can only be assumed that this was not the case $[32,75]$. The limitation of the findings in the study here, is that it only compares a single PtRu catalyst with $\mathrm{Pt}$, and therefore the observation warrants additional investigation on a number of PtRu catalyst samples in order to clarify this further. A consistent methodology, however, is crucial for electrocatalyst development if we consider the importance of the ECSA on electrocatalytic reaction rates and the comparison of specific (surface area normalised) activities between different electrode materials and research groups. 


\subsubsection{Cyclic Voltammetry}

In this study, the MOR on the HSAC Pt catalyst was first probed using CV. The steady state $\mathrm{CV}$ and $\mathrm{I}_{\mathrm{CO} 2} \mathrm{MSCV}$ for the MOR, along with the corresponding calculated conversion of methanol to $\mathrm{CO}_{2}$ on a $\mathrm{HSAC}$ supported $\mathrm{Pt} 5 \mathrm{~nm}$ catalyst is shown in figure 5-4.
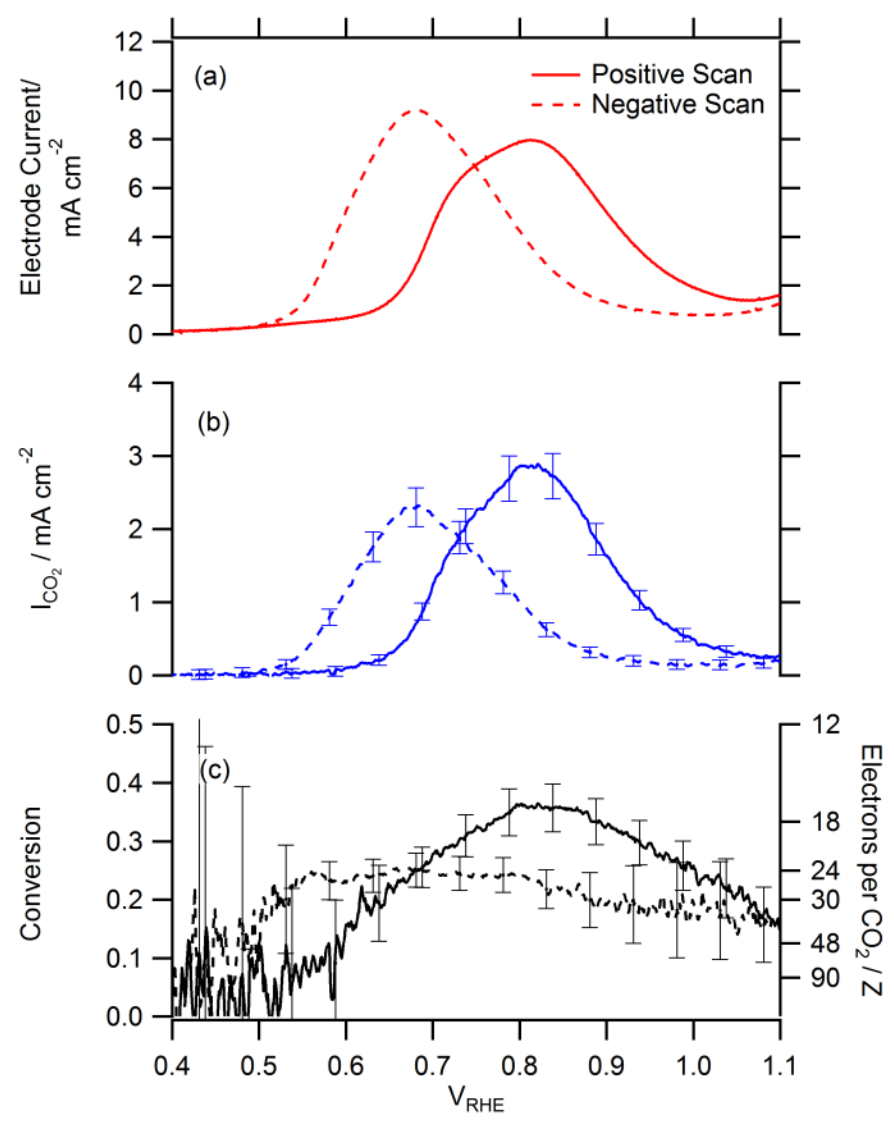

Figure 5-4: A DEMS measurement of the MOR on HSAC supported Pt $5 \mathrm{~nm}$ catalyst.CV of $1.0 \mathrm{~mol} \mathrm{dm}^{-3}$ methanol oxidation on HSAC supported Tanaka Pt $5 \mathrm{~nm}$ catalyst (a), corresponding MSCV for $I_{\mathrm{CO} 2}(\mathrm{~b})$, and resulting calculated conversion of methanol to $\mathrm{CO}_{2}(\mathrm{c})$, at $10 \mathrm{mV} \mathrm{s}$ in $0.5 \mathrm{~mol} \mathrm{dm}^{3} \mathrm{H}_{2} \mathrm{SO}_{4}$ and an electrolyte flow rate of $5 \mu \mathrm{s} \mathrm{s}^{-1}$. The conversion of the MOR to $\mathrm{CO}_{2}$ is far from complete, and is furthermore potential dependent.

The CV closely resembles the characteristic form of the potential dependence of the MOR reaction reported previously [46, 124, 125], with the onset of the MOR in the positive potential sweep occurring at $\sim 0.55 \mathrm{~V}_{\mathrm{RHE}}$ rising rapidly until $0.70 \mathrm{~V}_{\mathrm{RHE}}$ before slowing and peaking at $0.82 \mathrm{~V}_{\mathrm{RHE}}$. At higher potentials the MOR current then decays significantly reaching a minimum at $1.05 \mathrm{~V}_{\mathrm{RHE}}$. In the reverse potential sweep, the $\mathrm{CV}$ resembles the upward sweep but with a 
prominent hysteresis, whose peak shifts downwards to $0.65 \mathrm{~V}_{\mathrm{RHE}}$. The peak current on the negative scan is furthermore $1.2 \mathrm{~mA} \mathrm{~cm}$ higher compared than the peak current in the positive going scan. The relative peak heights of the $\mathrm{CO}_{2}$ observed in the MSCV during the forward and reverse potential sweep is, however, inverse to the $\mathrm{CV}$ and is best expressed in terms of conversion. During the forward potential scan, the conversion of the MOR to $\mathrm{CO}_{2}$ increases with increasing potential, beginning at $\sim 0.1$ and rising to $\sim 0.4$ at $0.82 \mathrm{~V}_{\mathrm{RHE}}$ before decaying to $\sim 0.15$ as the potential is increased further. In the reverse potential scan, the conversion of methanol to $\mathrm{CO}_{2}$ rises only slightly, remaining steady at around 0.25 until falling sharply as the MOR halts. At all electrode potentials, it is clear that we do not come close to the complete conversion of methanol to $\mathrm{CO}_{2}$ and that the majority of the oxidation of methanol on Pt is only the partial MOR. The highest conversion, however, corresponds to the peak MOR rate indicating a close link between the MOR rate and the conversion of methanol to $\mathrm{CO}_{2}$.

Although in this DEMS study we were able to present the potential dependence of the conversion of methanol to $\mathrm{CO}_{2}$, this has not to the authors knowledge been previously presented using $\mathrm{CV}$ in the DEMS scientific literature. The conversion of $\sim 0.25$ averaged over the whole MOR CV in $1.0 \mathrm{~mol} \mathrm{dm}^{-3}$ methanol is nonetheless comparable to literature values of 0.28 for polycrystalline Pt [81], but is somewhat lower than the $\mathbf{0 . 6 5}$ reported previously on a HSAC support pure Pt [46] in $0.1 \mathrm{~mol} \mathrm{dm}^{-3}$ methanol. Although still far less than 1.0, the averaged conversion values observed using DEMS have been shown to strongly depend on the experimental parameters $[56,81,122]$.

The steady $\mathrm{CV}$ and MSCV of $I_{\mathrm{CO} 2}$ for the MOR, along with the corresponding calculated conversion of the MOR to $\mathrm{CO}_{2}$ over a HSAC supported PtRu catalyst is shown in figure 5-5. 

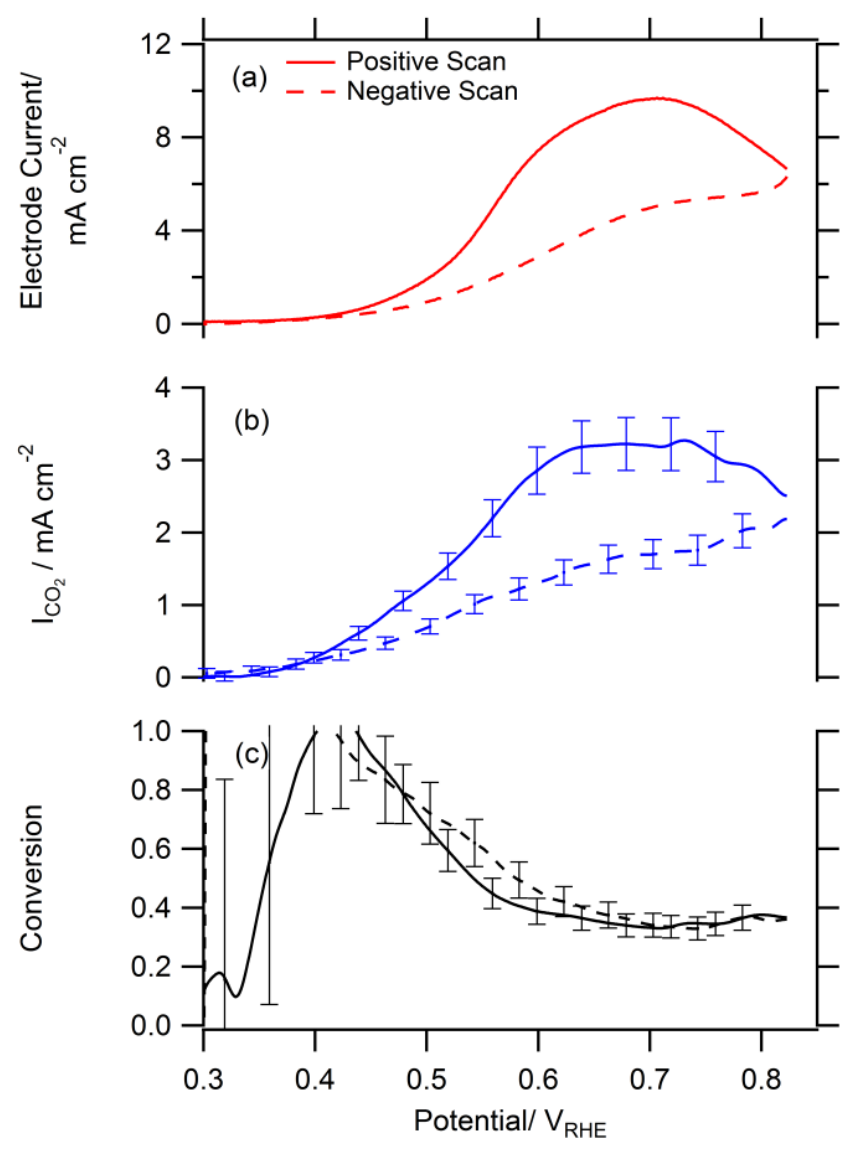

Figure 5-5: DEMS measurement of the methanol oxidation reaction on Tanaka HSAC supported PtRu catalyst. CV of $1.0 \mathrm{~mol} \mathrm{dm}^{-3}$ methanol oxidation on HSAC supported Tanaka PtRu catalyst (a), corresponding MSCV for $\mathrm{I}_{\mathrm{CO} 2}$ (b), and resulting calculated conversion of methanol to $\mathrm{CO}_{2}$ (c) at $10 \mathrm{mV} \mathrm{s}^{-1}$ in $0.5 \mathrm{~mol} \mathrm{dm} \mathrm{H}_{2} \mathrm{SO}_{4}$ and an electrolyte flow rate of $5 \mu \mathrm{s} \mathrm{s}^{-1}$. The conversion of the MOR to $\mathrm{CO}_{2}$ on PtRu is potential dependent, decreasing with increasing MOR rate.

The CV closely resembles the characteristic form of the potential dependence of the MOR reaction observed in previous studies [32, 124], with the onset of the MOR in the positive potential sweep occurring at $0.33 \mathrm{~V}_{\mathrm{RHE}}$ rising rapidly until $\sim 0.60 \mathrm{~V}_{\mathrm{RHE}}$ before slowing and peaking at $\sim 0.70 \mathrm{~V}_{\mathrm{RHE}}$. At higher potentials the MOR current begins to decay, however, the $\mathrm{CV}$ in this case is limited to an upper potential of $0.85 \mathrm{~V}_{\mathrm{RHE}}$ to prevent the preferential dissolution of Ru at more positive potentials [111]. In the reverse potential sweep, the MOR current is $\sim 50 \%$ lower with little or no hysteresis, and no clear peak in the observed current or MSCV. Significantly, the corresponding potential dependent conversion of the MOR to $\mathrm{CO}_{2}$ on the PtRu catalyst is strikingly different to the potential dependent conversion on the pure Pt catalyst. In the case of the PtRu catalyst, using DEMS we observe that the conversion actually decreases from $>0.7$ at 
$0.45 \mathrm{~V}_{\mathrm{RHE}}$ to $\sim 0.35$ at $0.7 \mathrm{~V}_{\mathrm{RHE}}$ despite the increase in electrode potential and MOR rate. This trend is furthermore very similar during both the positive and negative potential sweep, suggesting little change in the MOR mechanism, despite the significant smaller electrode current.

Similarly to the Pt catalyst, the potential dependence of the conversion of the MOR to $\mathrm{CO}_{2}$ during a $\mathrm{CV}$ on the MOR on a HSAC supported PtRu surface has not been reported previously in the DEMS scientific literature. The average conversion of the MOR to $\mathrm{CO}_{2}$ observed in this experiment of $\sim 0.5$ in $1.0 \mathrm{~mol} \mathrm{dm}^{-3}$ methanol is lower than the average value of $\sim 0.9$ (expressed as slightly above 6 electrons per $\mathrm{CO}_{2}$ ) in $0.1 \mathrm{~mol} \mathrm{dm}^{-3}$ given previously [32]. The higher averaged conversion on the PtRu $\sim 0.5$ catalyst in comparison to $\sim 0.25$ on the Pt $5 \mathrm{~nm}$ catalyst is meanwhile in agreement with previous comparisons [122]; however, again the exact values have been shown to be dependent on the experimental parameters $[56,81,122]$. The trends for higher methanol conversion to $\mathrm{CO}_{2}$ with slower electrolyte flow rates [81], lower methanol concentrations [81, 122] and higher catalyst loadings [56] observed in these previous studies, are strongly indicative of a MOR mechanism which involves more than a single adsorption step i.e. the MOR largely proceeds via the in-direct pathway involving desorbed intermediates.

In the interpretation of the contrasting potential dependant conversion trends observed during the $\mathrm{CV}$, we must be aware that the $I_{\mathrm{CO} 2}$ and therefore conversion is to some extent overestimated during the onset of the MOR in the positive potential sweep. At potentials below the onset of the MOR $<0.35 \mathrm{~V}_{\mathrm{RHE}}$ the electrode surface is already covered in partial MOR products arising from the dissociative adsorption of methanol at potentials as low as $0.1 \mathrm{~V}_{\mathrm{RHE}}$ (not shown in these measurements but examples may be found in ref. [11, 14]) which have been deduced to largely consist of $\mathrm{CO}_{\text {ads }}$ species $[32,46]$. The oxidation of these species would therefore yield fewer than the six electrons per $\mathrm{CO}_{2}$ assumed for the oxidation of methanol in the calculation of the $I_{\mathrm{CO} 2}$ leading to the overestimation of the conversion until all the previously adsorbed partial MOR products have been oxidised. The coverage of these $\mathrm{CO}_{\text {ads }}$ species has been observed to be $\sim 0.75$ for pure Pt catalysts [46] and $<0.4$ for PtRu [32] which may suggest Pt is most 
overestimated. Assuming that the partially oxidised MOR products oxidise at the onset of the MOR LSV, we can deduce that the charge arising from the oxidation of the previously adsorbed partial MOR products has dissipated prior to $\sim \mathbf{0 . 6 0}$ and $\sim 0.43 \mathrm{~V}_{\mathrm{RHE}}$ for the Pt and PtRu catalyst respectively. The observed potential dependent trends are therefore still valid; however, we should be aware that the conversion is to some extent overestimated during the course of the $\mathrm{CV}$.

Before rationalising the observed potential dependence, it is crucial to first examine the relationship between the MOR and oxidation of $\mathrm{CO}_{\text {ads }}$ (which has been well documented in the scientific literature) by comparing the LSV of the MOR and the CO-Stripping measurement, and to later confirm the potential dependent conversion trend of the MOR using sampled current chronoamperometry which is extended to three-dimensional voltammetry. 


\subsubsection{Relationship between Methanol and $\mathrm{CO}_{a d s}$ Oxidation}

The relationship between the $\mathrm{CO}_{\text {ads }}$ oxidation and the MOR on Pt HSAC supported catalyst was examined by comparing the positive MOR LSV and $I_{\mathrm{CO}_{2}}$ mass spectrometric LSV (MSLSV) with the electrode current for oxidation of a $\mathrm{CO}_{\text {ads }}$ saturated layer, as shown in figure 5-6(a).
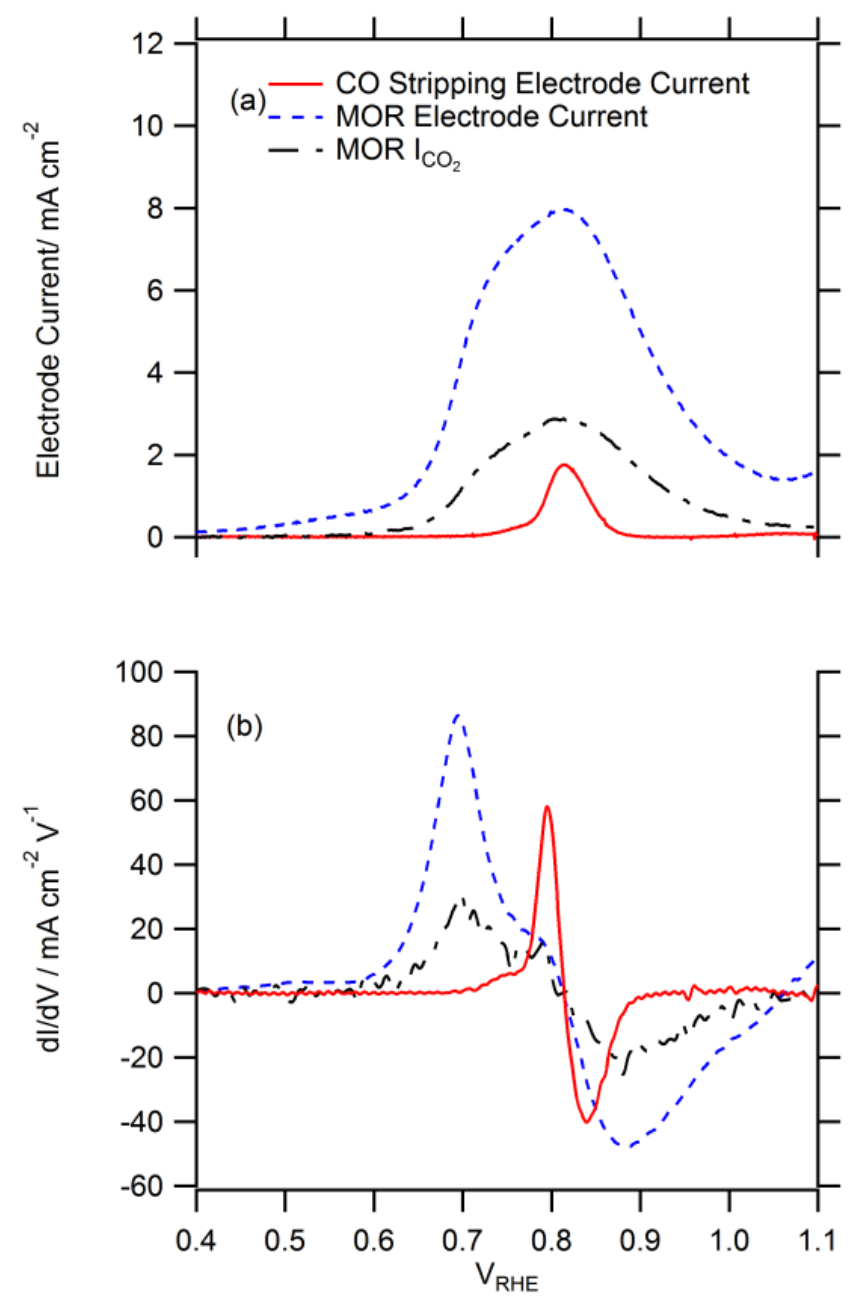

Figure 5-6: A comparison of the MOR and CO-Stripping LSV of Pt $5 \mathrm{~nm}$ HSAC supported catalyst. LSVs for electrode current and $I_{\mathrm{CO}_{2}}$ (a) and corresponding differential voltammogram (b) of HSAC supported Tanaka Pt $5 \mathrm{~nm}$ catalyst in $0.5 \mathrm{~mol} \mathrm{dm}^{-3} \mathrm{H}_{2} \mathrm{SO}_{4}$ supporting electrolyte during $1.0 \mathrm{~mol} \mathrm{dm}^{-3}$ methanol oxidation and CO stripping, with a potential scan rate of $10 \mathrm{mV} \mathrm{s}$ and electrolyte flow rate of $5 \mu \mathrm{s}^{-1}$. The key features of the LSV for the MOR on the Pt catalyst are closely related to the $\mathrm{LSV}$ of the oxidation of a saturated $\mathrm{CO}_{\text {ads }}$ layer.

In the CV and MSLSV ( $\left.I_{C O 2}\right)$ we observe that the MOR peak current potential and CO-stripping peak current closely coincide. This correlation is more precisely observed in the differential voltammogram in figure 5-6(b), whereby the 
MOR and CO-Stripping LSV peak maximums (where $\mathrm{dI} / \mathrm{dV}=0$ ) coincide at precisely $0.815 \mathrm{~V}_{\mathrm{RHE}}$. An additional feature of the differential voltammogram, are the peaks that indicate the potential at which the current rises fastest during the LSV. This peak MOR potential in the positive going potential scan is the same for both the electrode current, and $I_{\mathrm{CO} 2}$ which is found at $0.695 \mathrm{~V}_{\mathrm{RHE}}$, which interestingly coincides closely with the onset potential for the oxidation of a saturated monolayer of $\mathrm{CO}_{\text {ads. }}$. Nonetheless, there is clearly a close relationship between the ability of the Pt catalyst to oxidise a saturated $\mathrm{CO}_{\text {ads }}$ layer and the MOR rate on the HSAC Pt catalysts.

If we compare the MOR LSV and $I_{\mathrm{CO} 2}$ MSLSV to the oxidation of a saturated $\mathrm{CO}_{\text {ads }}$ layer on the PtRu HSAC supported catalyst in figure 5-7, we observe fewer similarities between the features of the MOR CV and CO-Stripping LSV.
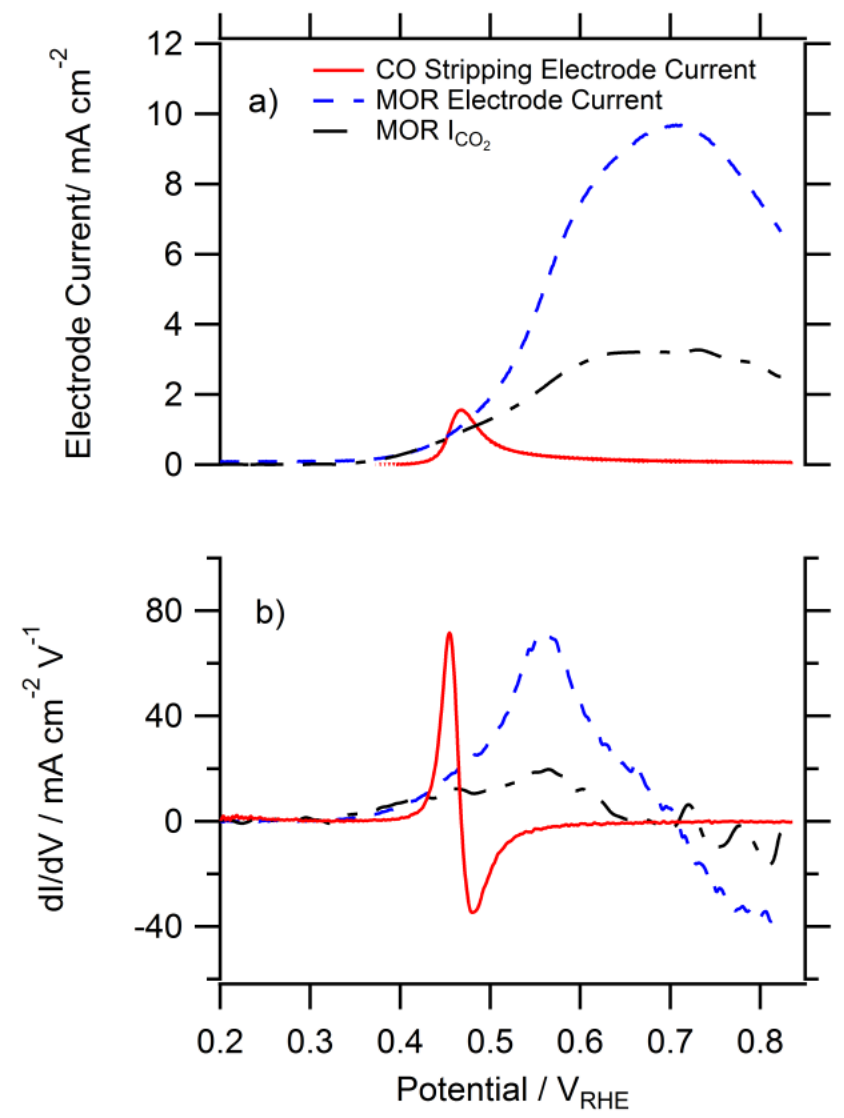

Figure 5-7: Comparison of the methanol oxidation and the CO-stripping of the Tanaka HSAC support PtRu catalyst. LSV and MSLSV (a) and corresponding differential voltammogram (b) of HSAC supported Tanaka PtRu catalyst in $0.5 \mathrm{~mol} \mathrm{dm}^{-3} \mathrm{H}_{2} \mathrm{SO}_{4}$ supporting electrolyte during $0.1 \mathrm{~mol} \mathrm{dm}^{-3}$ methanol oxidation and CO stripping. The features of the $\mathrm{CV}$ for the MOR on the PtRu catalyst do not correlate closely with the oxidation of a saturated $\mathrm{CO}_{\text {ads }}$ layer. 
The oxidation of a saturated layer of $\mathrm{CO}_{\text {ads }}$ on the PtRu catalyst occurs at $\sim 0.4 \mathrm{~V}_{\mathrm{RHE}}$, peaking at $0.46 \mathrm{~V}_{\mathrm{RHE}}, 100 \mathrm{mV}$ lower in potential than the peak MOR current at $0.56 \mathrm{~V}_{\mathrm{RHE}}$ observed in the LSV and MSLSV. The MSLSV for $I_{\mathrm{CO} 2}$ however, possesses a much broader peak that remains fairly constant between 0.64 and $0.74 \mathrm{~V}_{\mathrm{RHE}}$. The highest potential dependence on the current of the MOR also follows the CO-stripping onset by $200 \mathrm{mV}$. Although the earlier onset of the MOR at 0.33 $V_{\text {RHE }}$ on the PtRu catalysts compared with $0.55 V_{R H E}$ on the Pt catalyst of the MOR can be explained by the co-catalytic mechanism of the PtRu surface and the ability of $\mathrm{Ru}-\mathrm{OH}_{\text {ads }}$ species to oxidise poisoning $\mathrm{Pt}-\mathrm{CO}_{\text {ads }}$ which prevent the initial dissociative adsorption of methanol; the MOR electrode current (and therefore reaction rate) does not appear to be so closely related to the ability for the HSAC supported PtRu catalyst to oxidise $\mathrm{CO}_{\text {ads }}$ species.

It is clear from these comparisons that the relationship between the MOR and the oxidation of a saturated $\mathrm{CO}_{\text {ads }}$ layer is quite different for the $\mathrm{Pt}$ in comparison to the PtRu catalyst. Whereas the features of the LSV of the MOR on the Pt catalysts correspond meticulously with the features of the CO-Stripping measurement indicating a close relationship between the two, the features of the MOR and CO-Stripping LSVs of PtRu catalyst are somewhat different. All measurement observations so far have, however, been performed using potentiodynamic electroanalytical techniques, and it is therefore not clear at this point if these trends are reflected in potentiostatic steady-state conditions. Consequently, the observations made of the MOR using $\mathrm{CV}$ were then compared with sampled current chronoamperometry in the following section. 


\subsubsection{Chronoamperometry}

Chronoamperometry is a powerful electrochemical technique for studying electrochemical systems by monitoring the current as a function of time at a certain fixed potential, and has been used previously in kinetic studies on the MOR on Pt and PtRu based catalysts [126-132]. Example chronoamperometric curves for the MOR, and corresponding $I_{\mathrm{CO} 2}$ on the PtRu catalyst performed at numerous electrode potentials are given in figure 5-8.
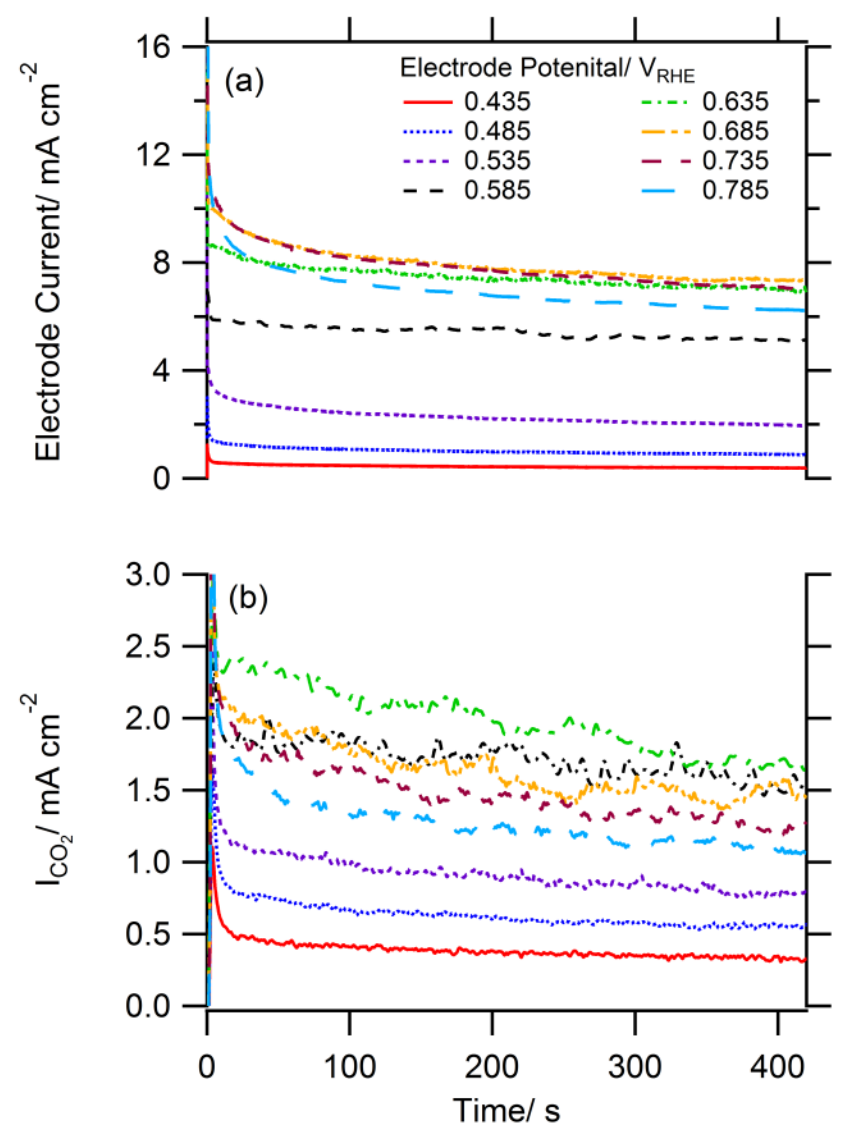

Figure 5-8: A plot of multiple chronoamperometric (current vs. time) measurements for the MOR at a number of electrode potentials (a) and corresponding $I_{\mathrm{CO} 2}$ response (b). Each chronoamperometric measurement was performed by stepping the electrode potential from $0.05 \mathrm{~V}_{\mathrm{RHE}}$ to the desired electrode potential which was progressively increased in $50 \mathrm{~V}$ steps from 0.435 to $0.785 V_{\text {RHE}}$.

In the current response to the potential step there is the typical initial capacitive current spike, along with MOR current which quickly decays to a steadier faradaic MOR current after $\sim 1 \mathrm{~s}$, the exact value depending on the size of the potential step. The MOR current then decays gradually over time at all potentials 
during the course of the chronoamperometric measurement, a trend that is also observed in the corresponding $I_{\mathrm{CO} 2}$. The presentation and discussion of chronoamperometric data at multiple potentials is, however, somewhat difficult to visualise using such current vs. time plots and consequently the current from a multiple of chronoamperometric measurements at various potentials are often sampled at a certain time and plotted as function of electrode potential - a technique that is often referred to as sampled current chronoamperometry. This aids in expressing chronoamperometric data as a function of potential which in turn allows the comparison of chronoamperometric measurements with CVs. 
The sampled current chronoamperometric measurements sampled at $420 \mathrm{~s}$ for the MOR on the Pt $5 \mathrm{~nm}$ catalyst, corresponding $\mathrm{I}_{\mathrm{CO} 2}$ and calculated conversion of methanol to $\mathrm{CO}_{2}$ is given in figure 5-9. Note that the traces of the $\mathrm{CV}$ are also indicated on the plot to aid in the comparison of techniques.

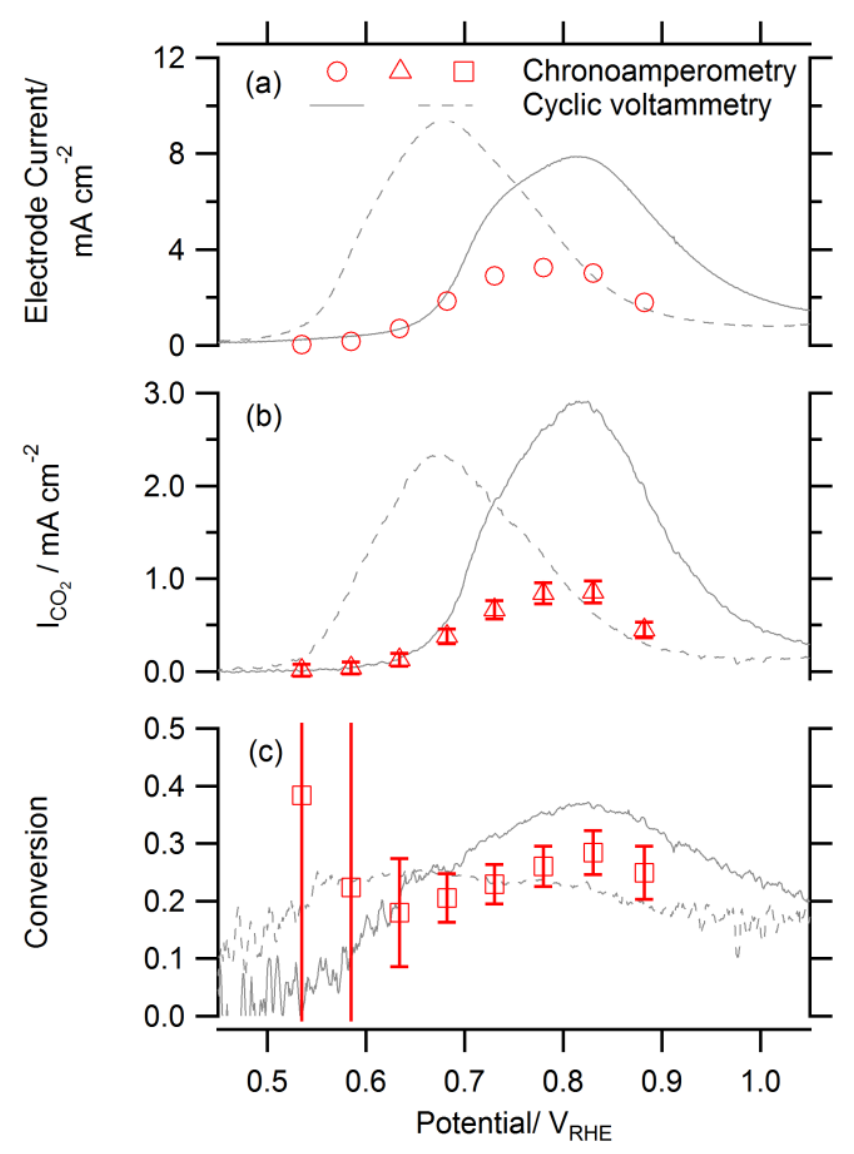

Figure 5-9: The comparison of sampled current chronoamperometry with $\mathrm{CV}$ for the methanol oxidation reaction on Tanaka HSAC supported $5 \mathrm{~nm} P t$ catalyst. Sampled current chronoamperometry of $1.0 \mathrm{~mol} \mathrm{dm}^{-3}$ methanol oxidation (a), corresponding $I_{\mathrm{CO} 2}$ (b), and resulting calculated conversion of methanol to $\mathrm{CO}_{2}(\mathrm{c})$, sampled at $420 \mathrm{~s}$ at an electrolyte flow rate of $5 \mu \mathrm{s} \mathrm{s}^{-1}$. The CV from figure 5-4 is also shown (in grey) for direct comparison. The sampled current chronoamperometric measurements confirm the trends observed in the CV.

In general, the trends observed in the chronoamperometric data are in agreement with those observed in the $\mathrm{CV}$. The steady-state electrode and $I_{\mathrm{CO} 2}$ correspond reasonably well with the $\mathrm{CV}$ at potentials $<0.75 \mathrm{~V}_{\mathrm{RHE}}$, where there is relatively little MOR, however, the steady-state faradaic current deviates to significantly lower values at higher electrode potentials indicating that the MOR rate has decayed significantly over time on the $\operatorname{Pt} 5 \mathrm{~nm}$ catalyst. The peak electrode 
current potential also appears to have shifted to lower potentials compared to the $\mathrm{CV}$, however, the peak mass ion current still corresponds well with $I_{\mathrm{CO} 2}$. Furthermore, the observed conversion during the potentiostatic measurements on the MOR to $\mathrm{CO}_{2}$, is lower ( 0.1) than suggested by $\mathrm{CV}$, although the trend for greater conversion at the peak MOR current potential remains the same.

The trend of increasing conversion with MOR rate is in agreement with a previous DEMS study which observed a slight increase in conversion of the MOR to $\mathrm{CO}_{2}$ based on two chronoamperometric measurements at 0.65 and $0.75 \mathrm{~V}_{\mathrm{RHE}}$ [76]. This trend also corresponds to the (ex-situ) measurement of formaldehyde yields using fluorescence spectroscopy following chronoamperometric measurements of the MOR on polycrystalline Pt [117]. Here, formaldehyde yields decreased with increasing MOR rates and potentials ranging from 40 to $5 \%$ yields between 0.25 to $0.5 \mathrm{~V}_{\mathrm{Ag} / \mathrm{AgCl}}$ (ca. 0.5 to $0.75 \mathrm{~V}_{\mathrm{RHE}}$ ) respectively. The conversion of the MOR to $\mathrm{CO}_{2}$ on a pure Pt electrode using chronoamperometry has also been previously reported to be rather high $\sim 0.85$ at $0.55 \mathrm{~V}_{\mathrm{RHE}}$ using SDEMS during a potentiostatic measurement sampled at $300 \mathrm{~s}$ [52]. Unfortunately, it is not possible to extract a comparable value from the data in figure 5-9 because the very small electrode and $I_{\mathrm{CO} 2}$ currents observed at these potentials induce significant errors in the resulting calculated conversion. Furthermore, there is no trend in these measurement data to suggest that the conversion is increasing towards lower electrode potentials and the high conversion at this potential claimed previously can perhaps be treated with some caution. Overall, it is clear that the study of the MOR activity using CV is not representative of the time resolved steady state MOR electrode current of the HSAC supported Pt catalyst obtained using chronoamperometry, with significantly lower MOR electrode current and conversion to $\mathrm{CO}_{2}$ observed.

The sampled-current chronoamperometric measurements taken at $420 \mathrm{~s}$ for the MOR on the PtRu catalyst, corresponding $\mathrm{I}_{\mathrm{CO} 2}$ and calculated conversion to $\mathrm{CO}_{2}$ is given in figure 5-10. 

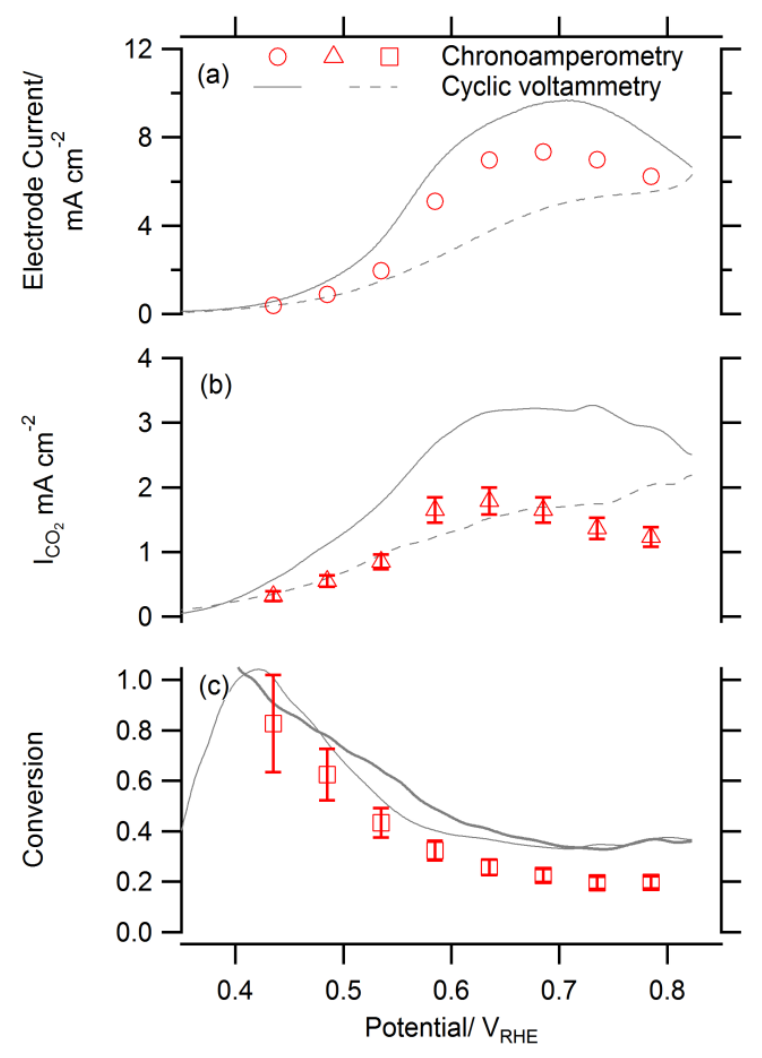

Figure 5-10: Comparison of the sampled current chronoamperometric measurement with $\mathrm{CV}$ for the methanol oxidation reaction on Tanaka HSAC supported PtRu catalyst. Sample-current chronoamperometry of $1.0 \mathrm{~mol} \mathrm{dm}^{-3}$ methanol oxidation on HSAC supported Tanaka PtRu catalyst (a), corresponding $I_{\mathrm{CO}_{2}}$ (b), and resulting calculated conversion of methanol to $\mathrm{CO}_{2}$ (c), sampled at $420 \mathrm{~s}$, with an electrolyte flow rate of $5 \mu \mathrm{s}^{-1}$. The $\mathrm{CV}$ from figure 5-5 is shown for comparison. The sampled current chronoamperometric measurements confirm the trends observed in the CV.

It is clear in this plot that the potentiostatic measurements follow the same trend as the CV; however, the steady state faradaic currents are all $\sim 20 \%$ lower across the whole potential range in contrast to the $P t 5 \mathrm{~nm}$ catalyst which was $\sim 60 \%$ lower. The chronoamperometric data also confirms the trend for decreasing conversion with higher electrode potentials on the PtRu catalyst, however, the steady-state conversions are $\sim 15 \%$ lower in conversion across the whole potential range compared to the $\mathrm{CV}$. The conversion of the MOR to $\mathrm{CO}_{2}$ at $0.55 \mathrm{~V}_{\mathrm{RHE}}$ of 0.3 is comparable to the conversions of 0.2 to 0.3 observed using DEMS on a PtRu catalysts with a composition of 20-40 \% wt. Ru [52]. The potential dependence of the MOR on the PtRu catalyst meanwhile is in agreement with the observations hinted at in ref. [76]; although the data presented here is without question more conclusive. The generally higher conversion observed for 
the MOR on the PtRu catalyst compared to $P t$ is also in agreement with previous observations [81]. Measurements of formaldehyde yields using fluorescence spectroscopy following chronoamperometry, however, did not follow this trend, although very low yields $(<3 \%)$ for formaldehyde were observed on HSAC supported PtRu (1:1) catalysts [118].

Overall, the sampled current chronoamperometric measurements confirm the potential dependence of the MOR, however, both the electrode current and conversion of methanol to $\mathrm{CO}_{2}$ are lower under steady-state conditions. Part of the loss of MOR electrode current may be attributed to the adsorption of impurities as assumed in previous studies [16, 126, 133], although in the case of industrial electrocatalysts with large ECSA, the influence of adsorbing impurities is not comparable to model electrocatalysts which possess considerably ECSA (and are thus more sensitive to electrolyte impurities).

Although the chronoamperometric measurements are very useful in assessing the steady-state MOR currents, which is more crucial to DMFC development, it is unfortunate that in performing these measurements and creating plots of this type, much of the experimental data is actually discarded. For example, it is not easy to convey how the current reached a certain current value over time, as a function of potential in such a two dimensional format. Another possibility is to plot the three measurement variables (potential, current and time) in the form of a three-dimensional voltammogram, which to the author's knowledge has not been previously applied to the study of the MOR. Such plots can aid in visualising the experimental data and by providing a new perspective could allow further interpretation of the time dependent behaviour of the MOR system to be made. 


\subsubsection{Three-Dimensional Voltammetry}

The three-dimensional voltammogram that is depicted in figure 5-11(a) was constructed to encapsulate all the data contained within a series of chronoamperometry measurements, rather than discarding much of the data as if the case in sampled current chronoamperometry. This allows the visualisation of the current, time and potential relationship within a single plot, which could allow further interpretation of the electrochemical data and reaction mechanisms.

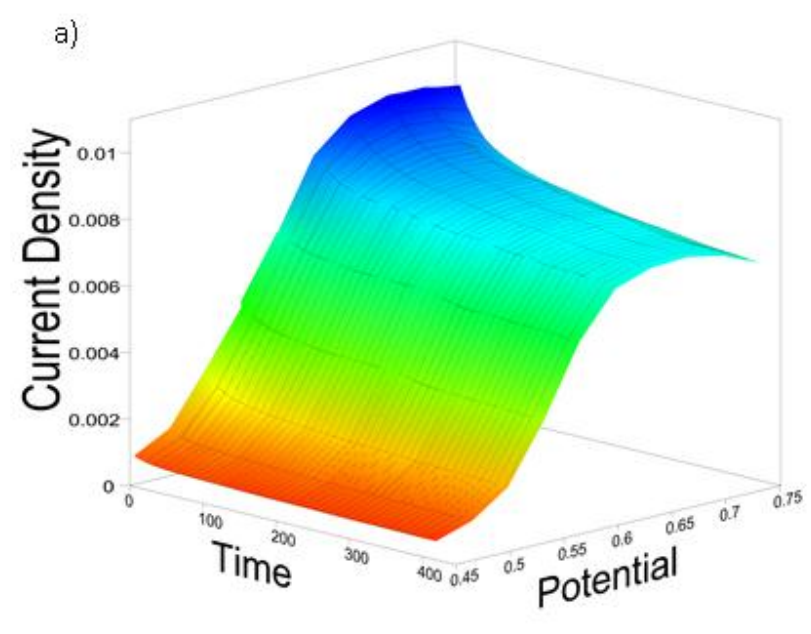

b)

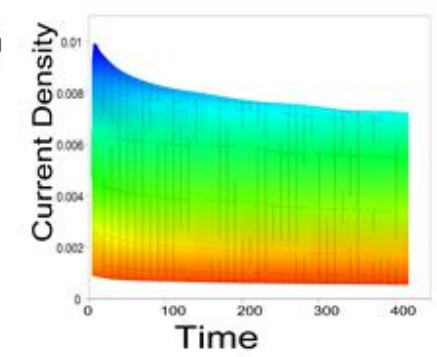

c)

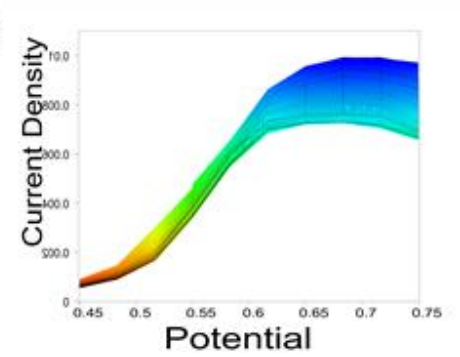

Figure 5-11: Three dimensional voltammogram of $1.0 \mathrm{~mol} \mathrm{dm}^{-3}$ methanol oxidation on HSAC supported Tanaka PtRu catalyst. The 3-dimensional plot encapsulates the current $\left(\mathrm{A} \mathrm{cm}^{-2}\right)$, potential $\left(V_{R H E}\right)$ and time (s) relationship of the MOR within a single plot.

By rotating the orientation of the three dimensional plot we can also observe the current versus time figure 5-11(b), and the current versus potential relationships figure 5-11(c). Although this three-dimensional voltammogram aids in visualising the reaction system, it is impossible to deduce specific information from the plot in its current form. In a previous study on an unrelated reaction system that also produced these three-dimensional plots, much of the data was after all presented and analysed in the form used for sampled current chronoamperometry [134] although the author compared different points in time. A potentially valuable alternative representation of the three-dimensional data which could offer a new perspective and interpretation of a reaction system (which to author's knowledge 
has not been used previously to describe an electrochemical half-cell reaction) is the contour surface plot.

\subsubsection{Contour Surface Plot}

The contour surface plot represents three dimensional data by plotting slices of the current data in a two dimensional format, allowing the mapping of the current, potential and time relationship of the reaction system in a single interpretable format. The current contours describing the MOR on the HSAC supported Pt catalyst is presented in figure 5-12.

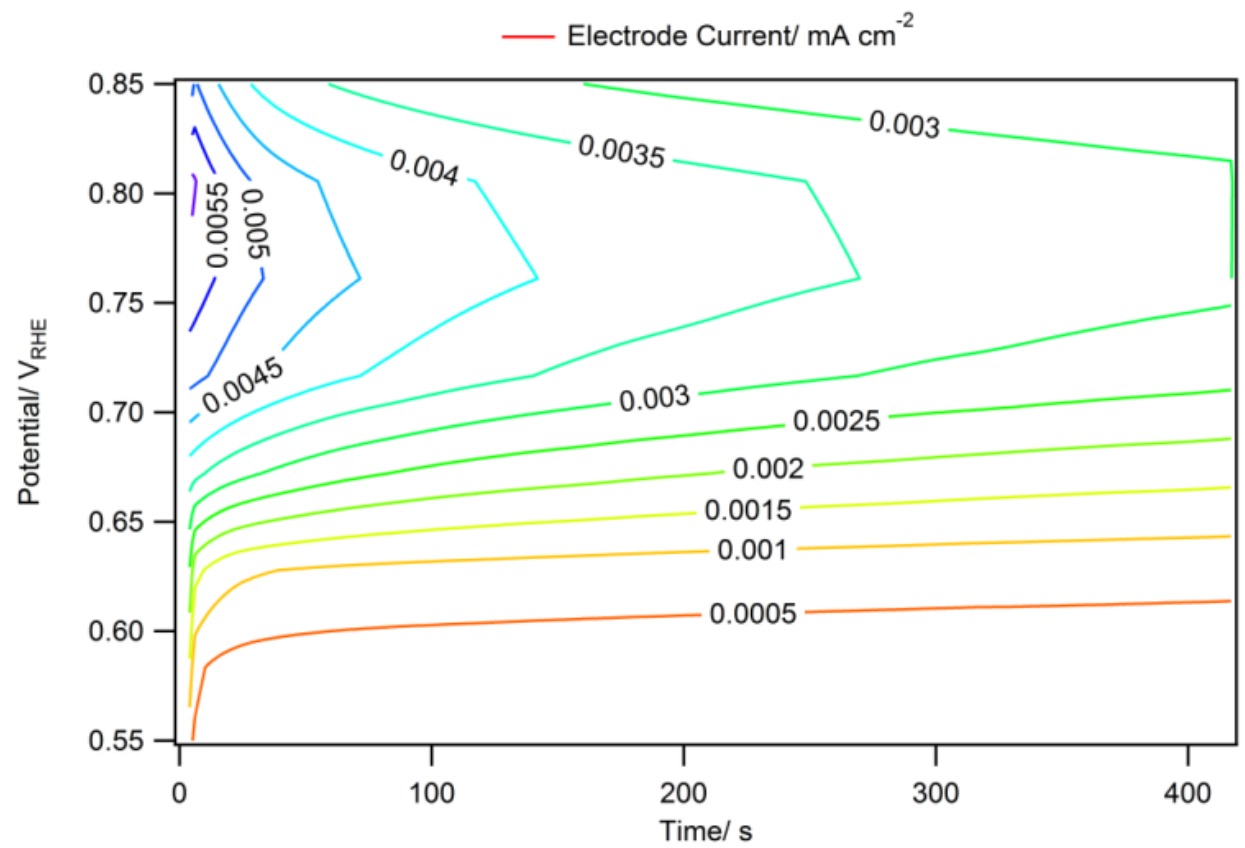

Figure 5-12: Contour plot of $1.0 \mathrm{~mol} \mathrm{dm}$ methanol oxidation on HSAC supported Pt catalyst. Electrolyte flow rate of $5 \mu \mathrm{l} \mathrm{s}$ in $0.5 \mathrm{~mol} \mathrm{dm}^{-3} \mathrm{H}_{2} \mathrm{SO}_{4}$, contour units in $\mathrm{A} \mathrm{cm}^{-2}$. The contour plots of the three-dimensional plot provide a new perspective on the MOR.

The fan shaped form of the current contours identifies the potential region of the MOR system on the Pt catalyst, and its change in potential over time as the current decreases. This brings greater focus on the potential region between $\sim 0.58$ and $0.68 \mathrm{~V}_{\text {RHE }}$ where the MOR current is relatively stable over the course of the experiment, in comparison to potentials $>0.7 \mathrm{~V}_{\mathrm{RHE}}$ the MOR electrode current decays more rapidly with time, whilst furthermore highlighting the lack 
MOR current $<0.55 \mathrm{~V}_{\mathrm{RHE}}$. The form of the curves furthermore resemble those of chronopotentiometric measurements (a.k.a. galvanostatic polarisation curves) such as those given in ref. [135].

By extending this technique to incorporate DEMS data for quantitative measurement of the $\mathrm{CO}_{2}$, the conversion of the MOR over the whole current, potential and time relationship may also be expressed in the form of the contour plot. In order to compare the relationship between the conversion of the MOR and the faradaic electrode current, the conversion contour plot is overlaid onto the faradaic electrode current in figure 5-13.

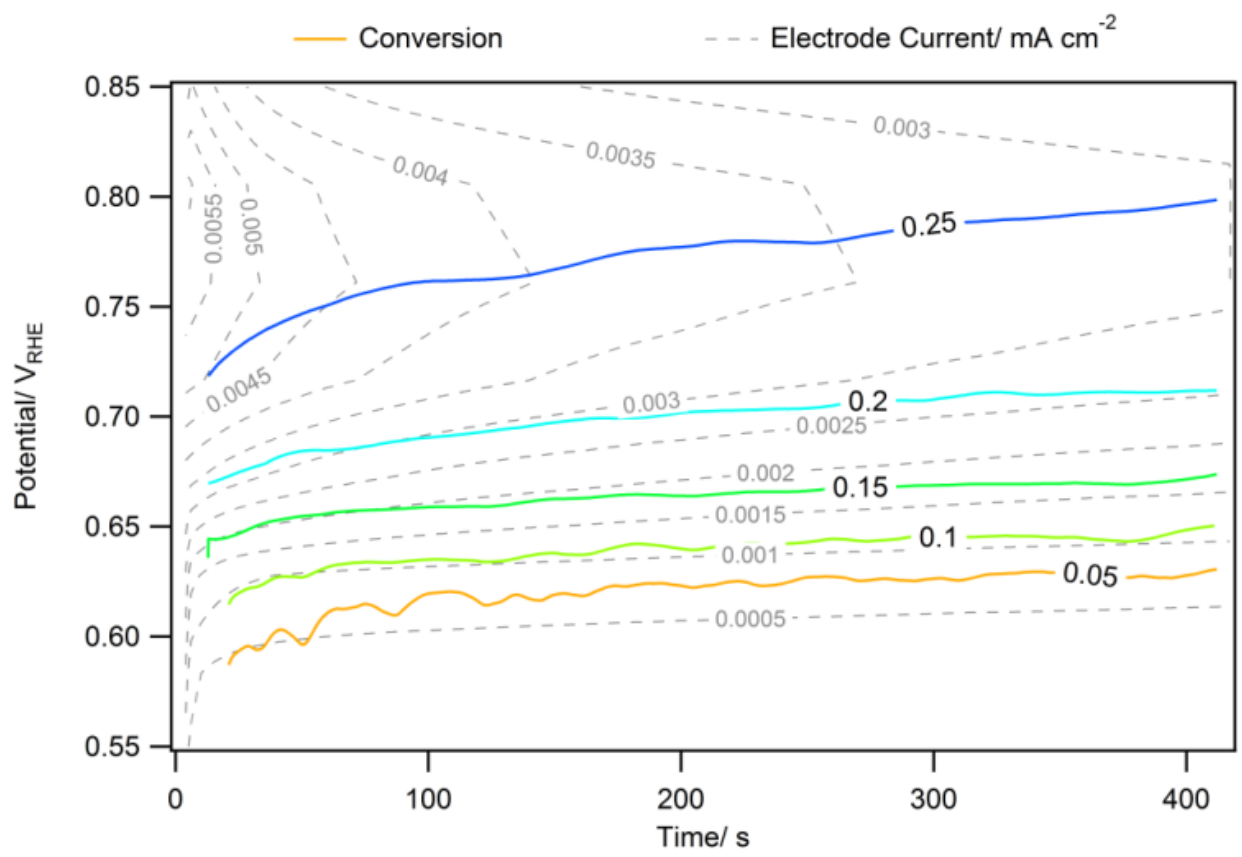

Figure 5-13: Contour plot of the conversion (coloured) of $1.0 \mathrm{~mol} \mathrm{dm}^{-3}$ methanol oxidation to $\mathrm{CO}_{2}$ on HSAC supported Pt $5 \mathrm{~nm}$ catalyst. Electrolyte flow rate of $5 \mu \mathrm{l} \mathrm{s}{ }^{-1}$ in $0.5 \mathrm{~mol} \mathrm{dm}^{-3} \mathrm{H}_{2} \mathrm{SO}_{4}$, and contours (greyed) of the faradaic electrode current given in figure 5-12. The contours of the conversion of the MOR to $\mathrm{CO}_{2}$ closely correlate with the contours of the faradaic electrode current.

Here we can observe that the form of the contours of the conversion correlate closely with the contours of the electrode current, particularly in the lower potential regions $<0.7 \mathrm{~V}_{\mathrm{RHE}}$. This is able to illustrate the region in which there is the crucial link between the observed MOR current and the conversion of the MOR to $\mathrm{CO}_{2}$ (and hence $\mathrm{CO}_{\text {ads }}$ oxidation) over the whole MOR system within the time frame of the experiment, an observation suggested previously by the $\mathrm{CV}$ and 
chronoamperometric measurements in this study but represented fully in the contour plot.

The corresponding contour plots representing the electrode current and conversion of the MOR to $\mathrm{CO}_{2}$ on the PtRu catalyst is presented in figure 5-14.
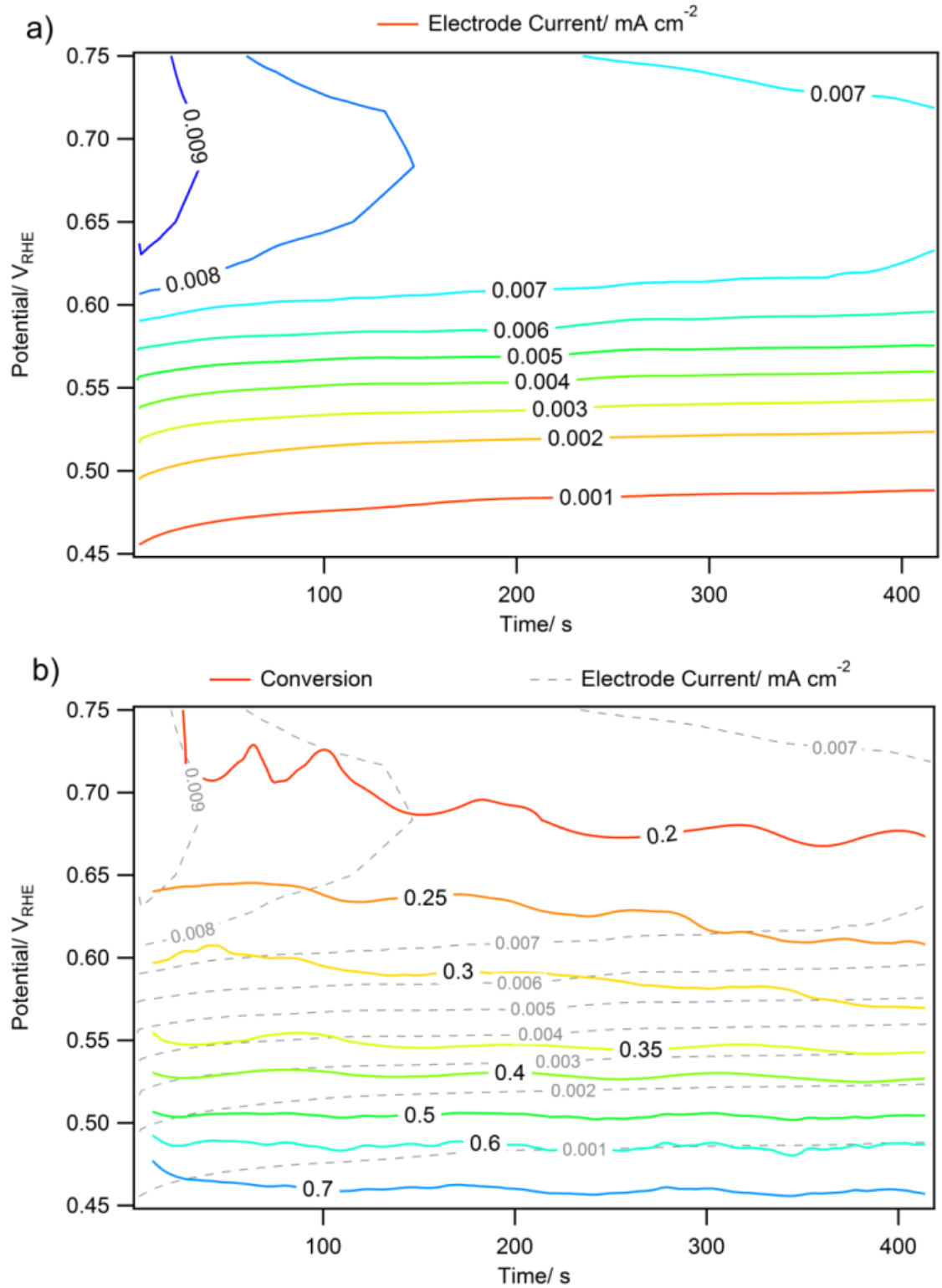

Figure 5-14: Contour plot of the faradaic electrode current (a) and conversion (b) of $1.0 \mathrm{~mol} \mathrm{dm}^{-3}$ methanol oxidation to $\mathrm{CO}_{2}$ on the HSAC supported Tanaka PtRu catalyst. Electrolyte flow rate of $5 \mu \mathrm{l} \mathrm{s}^{-1}$ in $0.5 \mathrm{~mol} \mathrm{dm}^{-3} \mathrm{H}_{2} \mathrm{SO}_{4}$. The contours of the faradaic electrode current are also displayed in grey in (b) for comparison. The contours of the conversion of the MOR to $\mathrm{CO}_{2}$ do not correlate with the contours of the faradaic electrode current. 
While the fan shaped contours of the MOR electrode current observed on the PtRu catalyst in figure 5-14(a) are analogous to those observed on $\mathrm{Pt}$, the contours of the conversion of the MOR to $\mathrm{CO}_{2}$ on the PtRu depicted in figure 5-14(b) do not resemble the shape and form of the electrode current, as was the case on the pure $\mathrm{Pt}$ catalyst. The conversion clearly decreases with time, particularly as the potential is increased $>0.55 \mathrm{~V}_{\mathrm{RHE}}$; however, despite this decrease the electrode current of the MOR is maintained. This would suggest that the oxidation of $\mathrm{CO}_{\text {ads }}$ species is less crucial of the MOR rate on the PtRu catalyst, and that the MOR mechanism is not only changing as a function of potential, but also as a function of time during the course of the chronoamperometric measurement. Although the rationalisation of this observation is not entirely clear, this change may represent a gradual alteration in the surface morphology of the PtRu catalyst surface which begins to occur at potentials as low as $0.55 \mathrm{~V}_{\mathrm{RHE}}$, an observation that cannot be deduced from the electrode current alone. For example, the Pt character of PtRu electrodes has been shown to gradually increase with potential cycling up to $0.7 \mathrm{~V}_{\text {RHE }}$ [136] whilst the preferential dissolution of Ru has also been observed in DMFC anodes [137]. Essentially, by measuring the MOR at potentials using chronoamperometry above $0.55 \mathrm{~V}_{\text {RHE }}$ we perhaps irreversibly change the surface morphology of the PtRu catalyst.

\subsubsection{Potential Uses of Contour Surface Plots in Electrochemistry}

The contour plots presented in this section offer a new perspective on the MOR, allowing the current, potential and time relationship of the reaction system to be expressed in a single interpretable format. Besides being a tool to aid in the visualisation of the MOR system (or indeed any electrochemical reaction system), it can also aid in new interpretation, identify regions of interest or to confirm findings over the whole reaction system during the course of a half-cell measurement. Combined with techniques such as DEMS, the plots can furthermore be used to map the conversion or product distributions of the reaction system to at least contribute towards our understanding of the MOR system in electrochemical half-cell. Moreover, the three-dimensional plots are 
conceivably very useful in aiding in the planning of chronopotentiometric measurements which are often notoriously difficult to perform in fundamental studies. In such measurement, meticulous knowledge of the current, potential and time relationship of a reaction system is required to ensure that the potential of the electrode (whilst not under direct control) is coerced within an acceptable or desired potential region. This is crucial in electrocatalysis because the structure of the electrode is sensitively dependent on the potential (often irreversibly so) and avoiding high potentials that can modify or destroy pristine electrode samples is essential. This is of course assuming that the electrochemistry of an electrode reaction is not influenced by the electroanalytical technique itself. For instance, the oscillation of the electrode potential that can be observed in some reaction systems (such as formic acid oxidation) using chronopotentiometric measurements cannot be resolved using chronoamperometry.

Although three-dimensional plots have been suggested a number of times, and demonstrated for a limited number of reaction systems [134], there are rather few or no other examples of their use in electrochemistry to convey specific information. This is certainly, partly because of the limitations described in ref. [134] such as the lack of automated setups along with instrumentation restrictions, but there is also the difficulty in expressing the data contained in the threedimensional plot which although look nice and are useful for educational purposes, are not particularly interpretable for more quantitative information. Hopefully current contour plots help to convey this information and could be potentially useful in encapsulating and elucidating many electrochemical processes in more than just a purely qualitative way.

Having established an understanding of the MOR system using CV, chronoamperometry and three-dimensional voltammetry, it is now useful to evaluate the kinetics and activity of the Pt and PtRu catalysts towards the MOR. 


\subsubsection{Tafel Slope}

The logarithmic current vs. potential plot (or Tafel plot) and corresponding $I_{\mathrm{CO} 2}$ for the MOR on the HSAC supported Pt catalyst measured using both $\mathrm{CV}$ and sampled current chronoamperometry is presented in figure 5-15 (a) and (b) respectively.

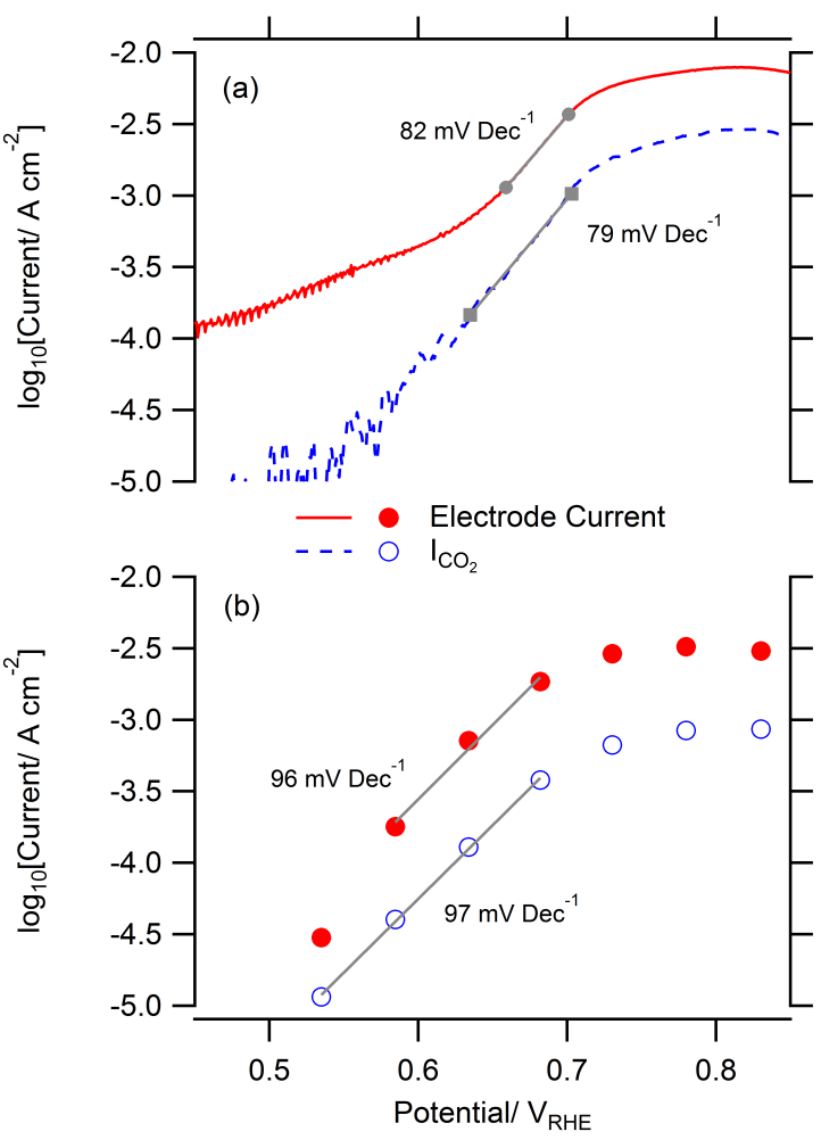

Figure 5-15: Tafel-slope of LSV for $1.0 \mathrm{~mol} \mathrm{dm}^{-3}$ methanol oxidation on HSAC supported Tanaka Pt $5 \mathrm{~nm}$ catalyst and MSLSV for $I_{\mathrm{CO} 2}$ at a potential scan rate of $10 \mathrm{mV} \mathrm{s}^{-1}$ (a) and corresponding chronoamperometric current samples at $420 \mathrm{~s}(\mathrm{~b})$ electrolyte flow rate of $5 \mu \mathrm{l} \mathrm{s}{ }^{-1}$. The Tafel slope of the faradaic electrode current closely corresponds to that of $I_{\mathrm{CO} 2}$.

The linear region (or rather Tafel-slope region) observed between 0.65 and $0.7 \mathrm{~V}_{\mathrm{RHE}}$ for the MOR of $82 \mathrm{mV} \mathrm{Dec}^{-1}$ of current on the Pt catalyst during the LSV is mirrored precisely with the Tafel-slope of $79 \mathrm{mV} \mathrm{Dec}^{-1}$ for the $I_{\mathrm{CO} 2}$ between 0.55 and $0.7 \mathrm{~V}_{\text {RHE }}$ observed using DEMS, and corresponds closely with literature values of $80 \mathrm{mV} \mathrm{Dec}{ }^{-1}[15,126]$. Although the Tafel behaviour is only observed over less than one decade of current during the LSV, the potential range is extended when using sampled current chronoamperometry (there are for instance no influences from capacitive currents), although a slightly shallower 
slope of $96 \mathrm{mV} \mathrm{Dec}^{-1}$ is observed. The linearity in the LSV between 0.45 and 0.60 $\mathrm{V}_{\mathrm{RHE}}$ for the electrode current meanwhile has less significance owing to the rather small electrode currents involved, a large proportion of which is likely to be capacitive in nature and are therefore short lived. Nonetheless, the Tafel-slope for $I_{\mathrm{CO} 2}$ corresponds to the MOR electrode current on the Pt catalyst and identifies the potential region where the current scales exponentially with the electrode potential and is should be the region of interest in the studies of electrode kinetics.

The Tafel-slopes observed for the electrode current, and $I_{\mathrm{CO} 2}$ for the MOR on the PtRu catalyst during LSV and sampled current chronoamperometry are given in figure 5-16.

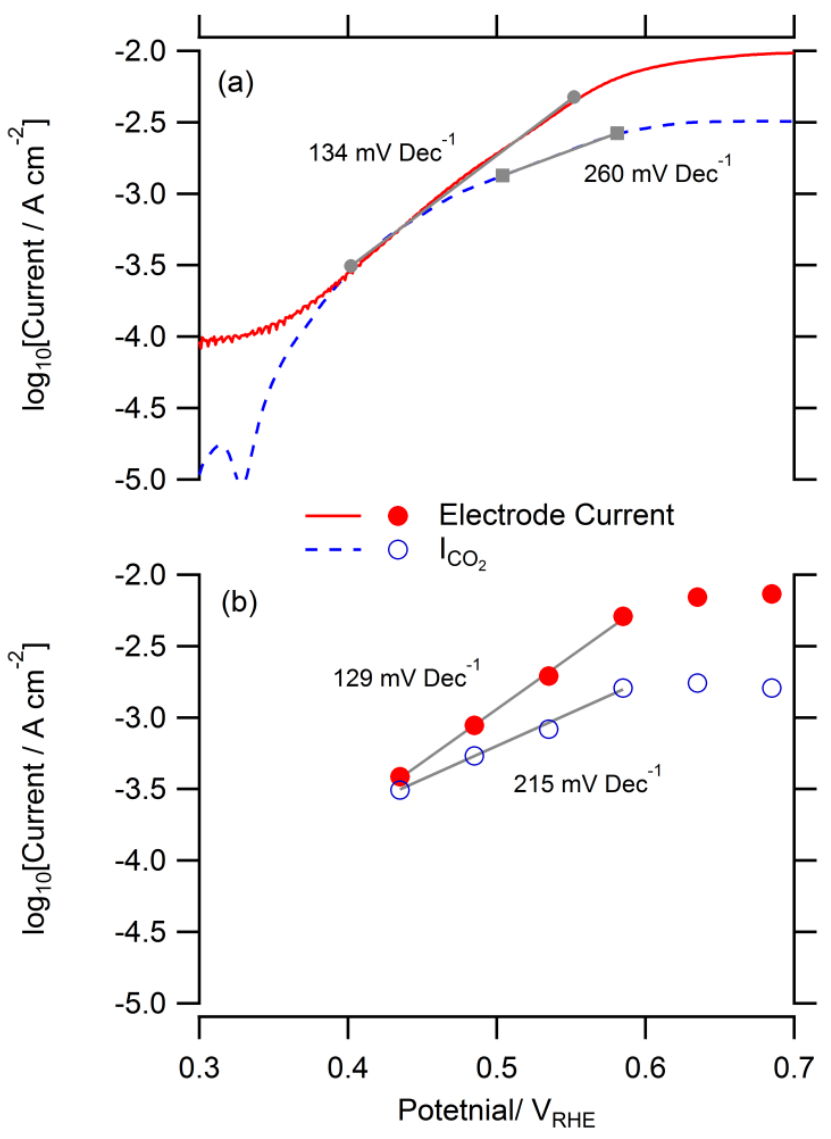

Figure 5-16: Tafel-slope of the LSV for $1.0 \mathrm{~mol} \mathrm{dm} \mathrm{dm}^{-3}$ methanol oxidation on HSAC supported Tanaka PtRu catalyst and MSLSV for $I_{\mathrm{CO} 2}$ at a potential scan rate of $10 \mathrm{mV} \mathrm{s}$ (a) and corresponding chronoamperometric current samples at $420 \mathrm{~s}$ (b) electrolyte flow rate of $5 \mu \mathrm{s}^{-1}$. The Tafel slope of the faradaic electrode current does not correspond to that of $I_{\mathrm{CO} 2}$.

In the both the LSV and the sampled chronoamperometric measurement of the MOR on the PtRu catalyst, a clear Tafel-slope of 134 and $129 \mathrm{mV} \mathrm{Dec}^{-1}$ is 
observed over more than one decade of current between 0.40 and $0.55 \mathrm{~V}_{\mathrm{RHE}}$. This value is within the range of literature values obtained from previous electrochemical half-cell studies (typically RDE) such as $115 \mathrm{mV} \mathrm{Dec}{ }^{-1}$ for PtRu/C [133], $100 \mathrm{mV} \mathrm{Dec}^{-1}$ on PtRu/Nanotubes [128], $195 \mathrm{mV} \mathrm{Dec}^{-1}$ on PtRu/Vulcan [126], 180-195 mV Dec ${ }^{-1}$ on PtRu surface alloys [15] and $115 \mathrm{mV} \mathrm{Dec}^{-1}$ in $\mathrm{HClO}_{4}$ [138] and finally $\sim 110 \mathrm{mV} \mathrm{Dec}{ }^{-1}$ observed for PtRu/C in a DMFC [139]. The corresponding $I_{\mathrm{CO} 2}$ signal, however, clearly does not follow the same trend with only slight linearity observed between 0.50 and $0.55 \mathrm{~V}_{\mathrm{RHE}}$ in the $\mathrm{CV}$ with a rather shallow linear slope of 260 and $215 \mathrm{mV} \mathrm{Dec}{ }^{-1}$. The curve of the $I_{C O 2}$ signal in the LSV below $0.37 \mathrm{~V}_{\mathrm{RHE}}$ is also comparatively steep, however, in this potential region we cannot distinguish between the MOR and the oxidation of previously adsorbed partial MOR products. Overall, the Tafel slope of the $I_{\mathrm{CO} 2}$ does not correlate to the MOR electrode current on the PtRu catalyst in contrast to Pt where they were identical, which perhaps reflects the differences in the MOR rate determining step (rds).

A detailed discussion of the circumstances where mechanistic information can and cannot be extracted from the Tafel-slope is beyond the scope of this DEMS study on the MOR, and the reader is instead directed to ref. [140] (Chapters 4 and 12). Briefly, the key points are that the transfer coefficient extracted from the Tafel-slope for a multi-step reaction can allow interpretation of the rds of the reaction based on the number of electrons involved in the rds, and the total number of electrons in the reaction assuming all other reaction steps are comparatively fast. However, the extraction of such mechanistic information is not possible for multi-step reactions that are either bifunctional and/or involve a significant coverage of adsorbed intermediates [140], such as the MOR where rather unusual Tafel-slopes are generally observed. Consequently, the significance of the Tafel slopes observed for the MOR can and has often only been discussed in a very qualitative manner, whereby it is acknowledged that the Tafel-slope does not carry its usual meaning [15]. This statement is sometimes referenced [128] but rarely elaborated on with the exception of ref. [126]. Here it was suggested that it still reflects the nature of the rds (common to all multi-step reactions) whereby, the differences in the Tafel-slope of the MOR on Pt and PtRu were rationalised by proposing that Tafel-slopes of $<130 \mathrm{mV} \mathrm{Dec}{ }^{-1}$ are typical for 
surface reaction $\mathrm{rds}$ such as $\mathrm{CO}_{\text {ads }}$ oxidation on $\mathrm{Pt}$, whereas Tafel-slopes $>130 \mathrm{mV} \mathrm{Dec}^{-1}$ can be expected from a chemical rds i.e. the dissociative adsorption of methanol on PtRu. The difference in the MOR rds on the Pt and PtRu catalyst is also supported by the observation of different activation energies (typically $\sim 60 \mathrm{~kJ} \mathrm{~mol}^{-1}$ on PtRu vs. 17 to $29 \mathrm{~kJ} \mathrm{~mol}^{-1}$ for Pt), and that the MOR on $P t$ is essentially zero order at high methanol concentrations $>0.5 \mathrm{~mol} \mathrm{dm}^{-3}$ [15].

The correlation between the Tafel-slope for the MOR electrode current and $I_{\mathrm{CO} 2}$ on the Pt catalyst (not to mention the close correlation between the MOR and $\mathrm{CO}_{\text {ads }}$ oxidation) is clearly in accordance with the well accepted conclusion that the $\mathrm{CO}_{\text {ads }}$ is the rds. In contrast, the lack of correlation between the Tafel-slope of the MOR electrode current and $I_{\mathrm{CO}_{2}}$ on the PtRu catalyst would on the other hand suggest that $\mathrm{CO}_{\text {ads }}$ oxidation is not the rds for the MOR on PtRu, assuming all $\mathrm{CO}_{2}$ is produced via $\mathrm{CO}_{\text {ads }}$ oxidation. Nonetheless, there is still a clear correlation between the ability of the PtRu catalyst surface to oxidise $\mathrm{CO}_{\text {ads }}$ and the onset of the MOR. The poisoning $\mathrm{CO}_{\text {ads }}$ species therefore, can be concluded to still be inherently responsible for the necessary overpotential for the MOR, even if the oxidation of the $\mathrm{CO}_{\text {ads }}$ is not the rds and reasonable reaction rates.

Although the Tafel-slope may provide some qualitative information of the rds, which DEMS can help elucidate, there is no doubt from a more practical aspect that the reaction kinetics i.e. activity and Tafel-slope are especially useful in the modelling and engineering of a fuel cell. The linearity of the Tafel-slope identifies the potential region in which the MOR is determined by the electrode kinetics, anything above or below can be considered blockage of the electrocatalyst surface (either via $\mathrm{CO}_{\text {ads }}$ or $\mathrm{OH}_{\text {ads }}$ species). For the Pt catalyst, the Tafel-slope region was observed to lie between 0.55 and $0.68 \mathrm{~V}_{\mathrm{RHE}}$ whereas for the PtRu catalyst this region lie between 0.4 and $0.58 V_{R H E}$, giving a narrow potential region where the activity of the two catalysts can be compared (i.e. between 0.55 to $0.58 \mathrm{~V}_{\mathrm{RHE}}$ ) in the following section. 


\subsubsection{Activity}

The assessment and comparison of electrocatalytic activities is crucial toward the understanding of the fundamental relationship between activity and catalyst structure and toward the development or selection of suitable electrocatalyst materials. The activities of the mass and specific activities of the Pt and PtRu catalysts investigated in this study are summarised in table 5-2.

Table 5-2: Summary of the Tafel-slopes and activities of the MOR on Pt $5 \mathrm{~nm}$ and PtRu electrocatalysts investigated in this study. Values were taken from within the potential window that exhibited Tafel-slope behaviour.

\begin{tabular}{|c|c|c|c|c|c|c|c|c|}
\hline & \multirow{2}{*}{ Method } & \multirow{2}{*}{$\begin{array}{c}\text { Tafel } \\
\text { Slope/ } \\
\text { mV Dec }\end{array}$} & \multicolumn{5}{|c|}{ Potential/ $\mathbf{V}_{\mathbf{R H E}}$} & \\
\hline & & & 0.45 & 0.50 & 0.55 & 0.60 & 0.65 & \\
\hline \multirow{4}{*}{ 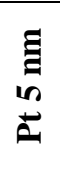 } & \multirow{2}{*}{ CV } & \multirow{2}{*}{82} & - & - & - & - & 19.4 & mA mg metal $^{-1}$ \\
\hline & & & - & - & - & - & 0.030 & $\mathbf{m A ~ c m}$ real $^{-1}$ \\
\hline & \multirow{2}{*}{ Chrono-A } & \multirow{2}{*}{72} & - & - & 1.6 & 7.1 & 22.0 & $\mathrm{~mA} \mathrm{mg}_{\text {metal }}{ }^{-1}$ \\
\hline & & & - & - & 0.001 & 0.006 & 0.023 & 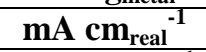 \\
\hline \multirow{4}{*}{$\vec{z}$} & \multirow{2}{*}{ CV } & \multirow{2}{*}{134} & 19.4 & 47.5 & 107.5 & - & - & $\mathbf{m A ~ m g}_{\text {metal }}{ }^{-1}$ \\
\hline & & & 0.021 & 0.052 & 0.116 & - & - & $\mathbf{m A ~ c m}$ real $^{-1}$ \\
\hline & \multirow{2}{*}{ Chrono-A } & \multirow{2}{*}{129} & 13.3 & 30.1 & 72.6 & - & - & $\mathrm{mA} \mathrm{mg}_{\text {metal }}{ }^{-1}$ \\
\hline & & & 0.010 & 0.024 & 0.053 & - & - & $\mathbf{m A ~} \mathbf{c m}_{\text {real }}{ }^{-1}$ \\
\hline
\end{tabular}

These values were taken from the $\mathrm{CV}$ and chronoamperometric measurements at a number of potentials within the Tafel-slope potential region. The only overlap between the Tafel-slope regions of the MOR on the Pt and PtRu catalyst in these measurements is at $0.55 \mathrm{~V}_{\mathrm{RHE}}$ using chronoamperometry, whereby the mass activity of the PtRu catalyst is almost two orders of magnitude greater than Pt. If we then compare the potentials at $\sim 19.4 \mathrm{~mA} \mathrm{mg}_{\text {metal }}{ }^{-1}$ of current we observe a $\sim 200 \mathrm{mV}$ improvement in the performance of the catalyst. Either way, it is clear that the PtRu catalyst is by far the most active and suitable MOR catalyst with measurements at electrode potentials $\geq 0.6 \mathrm{~V}_{\mathrm{RHE}}$ bearing little relevance to the development of DMFC anodes. An issue arises, however, if we try to compare the MOR activities in this study with those of other catalyst materials based on the measurements of different research groups. It has in the past been noted that the comparison of MOR activities between different research groups were not unambiguous, citing differences in the 'history' of the electrodes, duration of the measurements, and/or different concentrations of the supporting electrolyte [126]. 
This can be considered an understatement of a situation that has not improved in the past 10 years, as is illustrated in table 5-3 which gives details of the literature values and methodologies employed in the determination of MOR activities.

Table 5-3: A table summarising the MOR activities obtained from electrocatalytic studies on respective materials with relevant experimental details. References with * indicate data which was taken from figures because no specific values are given in either the text or table. Note that the $\mathrm{Ag} / \mathrm{AgCl}$ reference electrode potential is typically $\mathbf{- 0 . 2 5} \mathrm{V}_{\mathrm{RHE}}$.

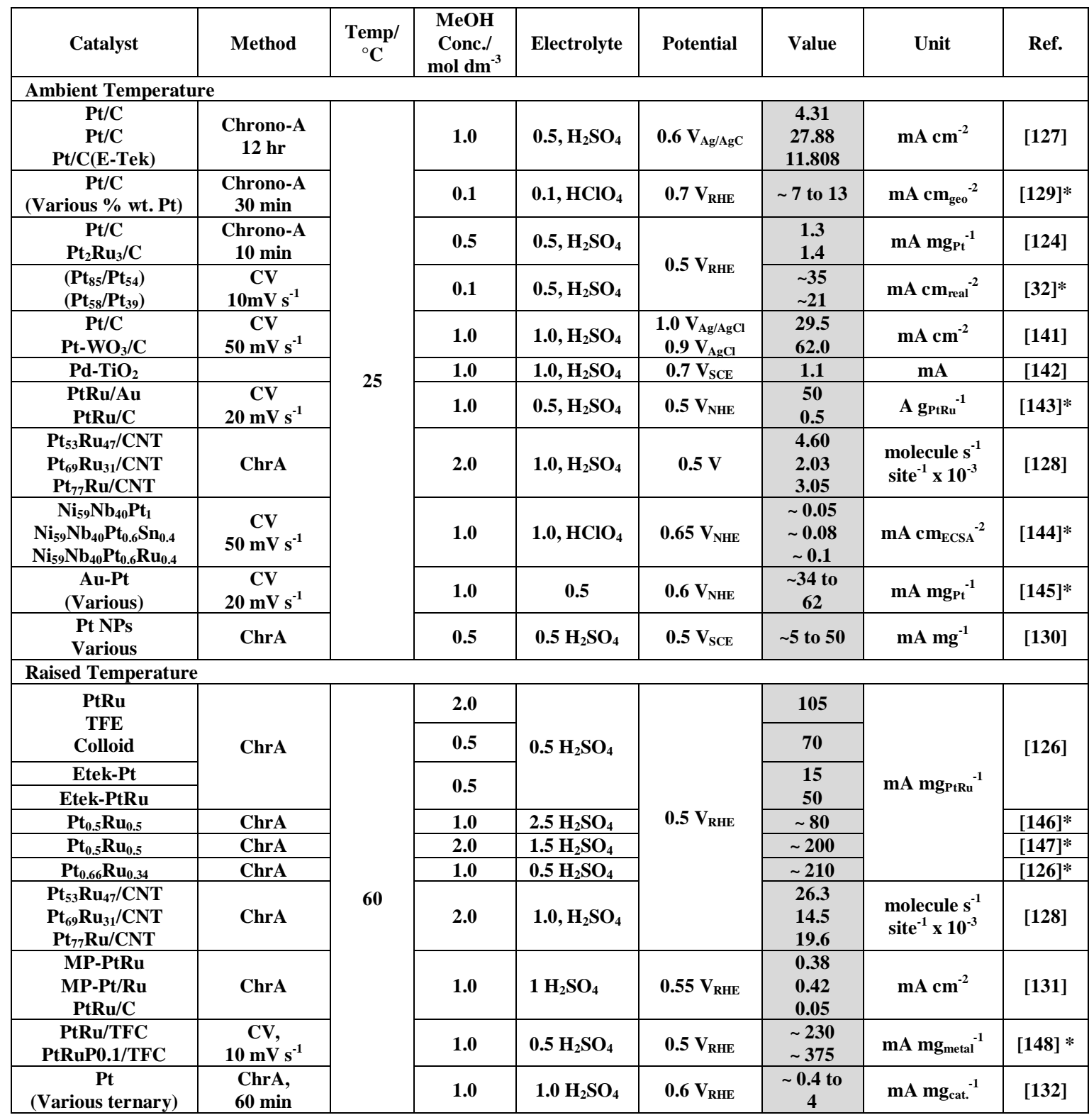

It is immediately clear that there are a number of variations in the electroanalytical techniques employed, which are performed in various different concentrations of electrolyte who probe the MOR at inconsistent WE potentials 
that are referenced to various different $R E$ with a wide range of MOR activity values normalised in a number of different ways. The problem is not only identifying activities that are comparable to those in this study, but also identifying values from different studies that are comparable to each other because there is clearly no commonly accepted approach to determining and comparing the MOR activities of prospective catalyst materials.

This issue has been raised previously, although quite briefly in 1999, calling for the need of a standardised method for comparing prospective DMFC anode catalysts for the MOR [109]. In fuel cell related electrocatalyst research and development, these problems are unfortunately not isolated to the study of the MOR, but is also prevalent in, and also previously highlighted for the study of ORR activities [7]. This has since led to an effort to present and scrutinise the ORR methodology and offer suggested measurement guidelines [20], which were later improved upon in ref. $[90,149]$ and demonstrated in ref. [10], to facilitate the meaningful comparison between ORR activities of different electrocatalyst materials. In light of the experimental details presented in table 5-3, it would appear that the same approach is urgently needed to facilitate meaningful comparison between MOR electrocatalysts, particularly where claims of novel materials with 'high' activity are made. The attention here was to highlight this issue rather than discuss ways in which it should be resolved (this is after all a DEMS study); however, some discussion of the issues in defining a methodology are given in Appendix A for future reference.

\subsubsection{Potential Dependent Conversion}

By investigating the MOR on HSAC supported -Pt and -PtRu catalyst using CV, sampled current chronopotentiometry and three-dimensional voltammetry combined with DEMS, a contrast in the potential dependence of the conversion of the MOR to $\mathrm{CO}_{2}$ between the two catalysts became apparent, suggesting a key difference in the MOR mechanism and its potential dependence on each respective catalyst. Whereas on the $P t$ catalyst a close positive correlation was observed between the MOR electrode current and the conversion of methanol to 
$\mathrm{CO}_{2}$, the conversion of methanol to $\mathrm{CO}_{2}$ on the PtRu catalyst was found to be rather inversely correlated to the MOR electrode current. In order to discuss perhaps why we observed this inverse relationship on the PtRu electrocatalyst, it is necessary to first deliberate the possible explanations for the potential dependence of the MOR on the $\mathrm{Pt}$ catalyst in relation to the current understanding of the MOR mechanism.

In order for methanol to adsorb on the Pt surface and the MOR to proceed, the poisoning $\mathrm{CO}_{\text {ads }}$ species must be oxidise in order to free active sites for the dissociative adsorption of methanol. The oxidation of the $\mathrm{CO}_{\text {ads }}$ species is in turn reliant on the bifunctional adsorption of and reaction with $\mathbf{O H}_{\text {ads }}$ species, whose adsorption on Pt has two opposing consequences on the MOR depending on $\mathbf{O H}_{\text {ads }}$ surface coverage. At low coverage, the $\mathbf{O H}_{\text {ads }}$ species can be considered as a promoter of the MOR by oxidising the primarily surface poisoning $\mathrm{CO}_{\text {ads }}$ species generated from the MOR, whereas at high surface coverage, the $\mathrm{OH}_{\text {ads }}$ species themselves begin to block the electrode surface inhibiting the adsorption of methanol. The coverage of $\mathrm{OH}_{\text {ads }}$ species, which is strongly dependent on potential, is therefore crucial to the MOR which is understood to proceed via two generalised pathways: the direct pathway, whereby the MOR proceeds via a single dissociative adsorption step oxidising to $\mathrm{CO}_{\text {ads }}$ and then $\mathrm{CO}_{2}$; and the in-direct pathway, whereby partial MOR products (such as formaldehyde and methylformate) desorb from the surface and may or may not re-adsorb and further oxidise to $\mathrm{CO}_{2}$. In general, the calculated conversion of the MOR on the Pt catalyst is far from complete, averaging at $\sim 0.25$ or less in these measurements, indicating that the majority of MOR is only partially oxidised under the experimental conditions suggesting that the MOR largely proceeds via the indirect pathway. The partial MOR products, however, generally do not re-adsorb and react further to $\mathrm{CO}_{2}$. Even so, the experimental results for the MOR reaction showed a strong correlation between the conversion to $\mathrm{CO}_{2}$ and henceforth the oxidation of $\mathrm{CO}_{\text {ads }}$ and the coverage of $\mathrm{OH}_{\text {ads }}$ species. These results indicated that at rather high coverage of either the $\mathrm{CO}_{\mathrm{ads}}$ or $\mathrm{OH}_{\mathrm{ads}}$ species, the conversion was poorer than at the peak MOR potential. 
If we consider that in the MOR we are not observing the charge transfer kinetics of the MOR because of the influence of adsorbed intermediates, but rather the rds and therefore the degree of poisoning of the $\mathrm{Pt}$ surface by either or both $\mathrm{CO}_{\text {ads }}$ or $\mathrm{OH}_{\text {ads, }}$ we can deduce that at the peak MOR current potential, the surface blockage is at a minimum and the most ensembles of $\mathrm{Pt}$ atoms required for either methanol or desorbed partial MOR products to adsorb (or re-adsorb) and oxidise are available. The minimised coverage of blocking species on the electrode could therefore conceivably promote both the direct and in-direct MOR pathways. For instance, a higher population of $\mathrm{Pt}$ ensembles required for the direct MOR pathway would be conceivably greater, whereas desorbed partial MOR products will also have more active sites available to re-adsorb and oxidise further. Each possibility may be equally valid and neither one can be discounted, although the majority of the MOR is still only partial oxidation, even at the peak current potential.

On PtRu the MOR mechanism was observed to be somewhat different. At ambient temperature, only the Pt component of the PtRu surface is understood to be active for the initial dissociative adsorption of methanol [16]. In order for the MOR to commence, $\mathrm{Pt}-\mathrm{CO}_{\text {ads }}$ species must be oxidised, a process that is facilitated in the bifunctional reaction with adsorbed $\mathrm{Ru}-\mathrm{OH}_{\mathrm{ads}}$ species at low potentials. Again, the methanol may dissociatively adsorb reacting via either the direct or in-direct pathway to $\mathrm{CO}_{2}$. The measurements in this study, however, indicate that the conversion at low potentials is rather high despite that the MOR rate is fairly low which would suggest that the coverage of $\mathrm{Pt}-\mathrm{CO}_{\text {ads }}$ species, at least on the active site for the dissociative adsorption of methanol is considerable. This is in contrast to the observations on the pure Pt catalyst and it would seem unlikely that either of MOR pathways would be favoured at low potentials, until we consider the possible secondary role of the Ru component of the PtRu surface. Although $\mathrm{Ru}$ is not active for the initial adsorption of methanol at room temperature [11, 14], pure Ru electrodes have been observed to possess some activity for the oxidation of both formaldehyde [120] and formic acid [14] partial MOR products. It is therefore proposed that the role of $\mathrm{Ru}$ in the MOR under these experimental conditions is more than purely providing the $\mathrm{OH}_{\mathrm{ads}}$ species to oxidise the $\mathrm{CO}_{\text {ads }}$ on $\mathrm{Pt}$ at low potentials, but is to also oxidise partial MOR 
products that desorb from the Pt surface in the in-direct MOR pathway. The decrease in the conversion with increasing MOR rate and potential on the PtRu catalyst may then be rationalised in two ways. Firstly, the increasing MOR rate on the Pt surface will generate a larger number of desorbed partial MOR products which will eventually exceed the oxidising capacity of the Ru surface. Secondly, the ability of the Ru to oxidise these intermediates will also change (similarly to $\mathbf{P t}$ ), initially increasing as $\mathbf{O H}_{\text {ads }}$ are adsorbed until at a certain point where it peaks and diminishes as the coverage of $\mathrm{OH}_{\mathrm{ads}}$ species increases. Although the ability of the Ru component to oxidise partial MOR products has in general been neglected in the discussion of the MOR mechanism, there is evidence to support this in the scientific literature. For example, formic acid oxidation on pure Ru electrode commences at $\sim 0.35 V_{\text {RHE }}$ peaking at $0.5 V_{\text {RHE }}$ before decreasing with increasing electrode potential [14]. This is closely related to the adsorption of $\mathrm{OH}_{\text {ads, }}$ which has be probed by studying the blocking effect of $\mathrm{OH}_{\text {ads }}$ on the HOR on pure Ru electrodes, whereby the HOR reaction decreases between $0.2 V_{R H E}$ and $0.5 V_{R H E}$ as $O_{\text {ads }}$ species form on the Ru surface [150] analogous to the effect of $\mathrm{OH}_{\text {ads }}$ on the HOR on pure Pt electrodes at $0.9 \mathrm{~V}_{\mathrm{RHE}}$ [90]. If we furthermore consider that $\mathrm{Ru}$ is not active for the initial dissociative adsorption of methanol $<60^{\circ} \mathrm{C}$, the re-adsorption of desorbed partial MOR products do not compete with methanol for the adsorption on active sites on $\mathrm{Ru}$ in contrast to competitive re-adsorption on Pt (assuming the active sites for both methanol and partial MOR products on Pt are the same). The overall conversion is therefore determined by the relative rates of the predominantly partial MOR on the Pt surface to produce desorbed intermediates which may subsequently re-adsorb and oxidise on Ru.

These finding will have less significance to electrochemical half-cell measurements or DMFCs which operate at temperatures $>60{ }^{\circ} \mathrm{C}$ where methanol is known to adsorb on pure $\mathrm{Ru}$ electrode and therefore generally participate more in the MOR. The measurements do, however, highlight that rather high conversion of the MOR can be observed on the PtRu catalyst under these experimental conditions, albeit to low MOR overpotentials and rates. The findings may have more relevance to portable DMFC devices that operate close to room temperature, however, the conversion of the $\mathrm{MOR}$ to $\mathrm{CO}_{2}$ may not have 
major significance because partial MOR products will still eventually be oxidised to $\mathrm{CO}_{2}$, unless the altered composition of the DMFC fuel is a major practical problem. The findings at least serve to clarify the difference in the MOR mechanisms for Pt and PtRu that occur during the measurement in the fundamental studies in electrochemical half-cells, indicating that the MOR mechanism is dependent on the electrode potential and highlighting that it should not be overlooked that the role of $\mathrm{Ru}$ is more than just the promotion of the MOR via the adsorption of $\mathrm{OH}_{\text {ads }}$ species.

\subsection{Conclusions}

This study set out to not only demonstrate the research capabilities of the DEMS instrument, but to also re-visit the MOR on HSAC supported Pt and PtRu catalysts. Although the MOR has been extensively studied for many years using a variety of techniques, DEMS was still able to provide new insights into the MOR, demonstrating the contrast between MOR mechanisms on Pt and PtRu catalysts. Furthermore, contrary to previous DEMS studies the CO-Stripping charge of the PtRu catalyst this was found not to be overestimated if the background CV in Argon saturated electrolyte is subtracted prior to the integration of the CO-Stripping charge.

An extremely close relationship was observed between the LSV of a saturated $\mathrm{CO}_{\text {ads }}$ layer, and the MOR electrode current and corresponding $\mathrm{I}_{\mathrm{CO} 2}$ on the $\mathrm{Pt}$ catalyst. The peak conversion of the $\mathrm{MOR}$ to $\mathrm{CO}_{2}$ of $\sim 0.35$ corresponded precisely to the CO-Stripping and MOR electrode current peak potential at $0.815 \mathrm{~V}_{\text {RHE}}$. Furthermore, the Tafel-slopes for the MOR electrode current and corresponding $I_{\mathrm{CO} 2}$ were seen to be almost identical at $\sim 80 \mathrm{mV} \mathrm{Dec}{ }^{-1}$ during CV and $\sim 96 \mathrm{mV} \mathrm{Dec}{ }^{-1}$ using sampled current chronoamperometry. This observation is in accordance to the widely accepted understanding that the oxidation of the poisoning $\mathrm{CO}_{\text {ads }}$ species is the rds for the MOR on pure Pt catalysts. The close relationship between the MOR rate and $\mathrm{CO}_{\text {ads }}$ oxidation on the Pt catalysts, however, was not mirrored by the observations of the MOR on the PtRu catalyst. On the PtRu catalyst, the highest conversion of the MOR to $\mathrm{CO}_{2}$ was observed at 
rather low reaction rates decreasing from $>0.7$ at $0.4 \mathrm{~V}_{\mathrm{RHE}}$ to $\sim 0.2$ at $0.6 \mathrm{~V}_{\mathrm{RHE}}$. Furthermore, the Tafel slope of $\sim 130 \mathrm{mV} \mathrm{Dec}^{-1}$ for the electrode current of the MOR was not reflected in the $I_{C O 2}$ whose slope was $\sim 200 \mathrm{mV} \operatorname{Dec}^{-1}$ with significantly less linearity. This would support the interpretation that the oxidation of $\mathrm{CO}_{\text {ads }}$ is not the rds of the MOR on PtRu catalysts but rather the initial dissociative adsorption of methanol on $\mathrm{Pt}$, although the poisoning $\mathrm{CO}_{\text {ads }}$ species can still attributed to the significant electrode overpotential required for the MOR to proceed. The trends in the MOR mechanism were furthermore confirmed over the entire current, time and potential relationship of the MOR measurement using 3-dimensional voltammetry, which was for the first time used to study the MOR, as well as in combination with DEMS. The resulting current contour plots provided a visual aid of the MOR system by encapsulating the current, potential and time relationship contained in multiple chronoamperometric within a single interpretable plot. These plots revealed changes in the MOR mechanism on the PtRu catalyst over time, during chronoamperometric measurements at $\geq 0.55 \mathrm{~V}_{\mathrm{RHE}}$ and was attributed to potential induced changes in the surface morphology of the PtRu catalyst.

The mass activity of the MOR on the PtRu catalyst (72.7 $\mathrm{mA} \mathrm{mg}_{\text {metal }}{ }^{-1}$ ) meanwhile was observed to be almost two orders of magnitude greater than the Pt catalysts (1.6 mA mgetal ${ }^{-1}$ ) at the comparable potential of $0.55 \mathrm{~V}_{\mathrm{RHE}}$ using chronoamperometry. The comparison of these values to other studies was, however, found to be extremely ambiguous because there is no commonly accepted and applied methodology to assess the activity of prospective MOR catalysts materials. These inconsistencies are cannot be intuitive to MOR electrocatalyst development, particularly where claims of 'high activity' on novel materials are made, and guidelines analogous to those suggested for the ORR are proposed to be beneficial, although for the MOR there are certainly greater difficulties in defining guidelines in comparison to the ORR methodology using RDE.

Perhaps the most significant insight into the MOR of this study was the contrast in the potential dependent conversion of the MOR to $\mathrm{CO}_{2}$ on Pt and PtRu catalysts. On Pt the conversion of the MOR to $\mathrm{CO}_{2}$ was directly related to its 
ability to oxidise $\mathrm{CO}_{\text {ads }}$ species. The contrasting trend on the PtRu catalyst, where relatively high conversion was observed at low MOR rates $\left(<0.6 \mathrm{~V}_{\mathrm{RHE}}\right)$ and ambient temperature $(\sim 298 \mathrm{~K})$ was attributed to the ability of $\mathrm{Ru}$ to oxidise desorbed partial MOR reaction products such as formaldehyde and formic acid that arise from the partial MOR on the Pt component of the PtRu surface. The decrease in conversion with increasing potential on PtRu was rationalised by the difference in the relative rates of the MOR on the $\mathrm{Pt}$, and the re-adsorption and oxidation of partial MOR products on Ru.

Overall, these findings serve to provide greater insight into the electrochemical half-cell study of the MOR, highlighting that the ability of Ru to oxidise partial MOR products may be significant at low overpotentials allowing rather high MOR conversion to $\mathrm{CO}_{2}$ at ambient temperature. 


\section{The Electrochemical Oxidation of HSAC Catalyst Supports}

6.1 Introduction 193

6.1.1 Motivation 196

6.1.2 Background 197

6.3 Experimental 200

6.3.1 Electrochemical Oxidation Procedure 202

6.4 Results and Discussion 204

6.4.1 Determination of the Apparent Double-Layer Capacitance 204

6.4.2 Substrate Background Contributions 210

6.4.3 Electrochemical Oxidation of HSAC Supports 212

6.4.5 Electrochemical Oxidation of HSAC Supported Pt Catalysts.........242

6.4.6 Future Applications of DEMS in the Study of the COR 249

6.5 Conclusions. 253

A fundamental understanding of the electrochemical oxidation behaviour of carbon blacks used to support finely dispersed Pt particles is crucial to the design of mitigating strategies that prevent the deterioration of PEMFC performance, particularly in automotive applications. In this study, DEMS is utilised to investigate and compare the electrochemical oxidation tendencies of a pristine EC300 carbon black and five graphitised carbon blacks (heat-treated between 2100 to $3200{ }^{\circ} \mathrm{C}$ ). The study is then briefly extended to EC300 HSAC supported Pt nanoparticle catalysts. By monitoring the $\mathrm{CO}_{2}$ and $\mathrm{O}_{2}$ produced in-situ, it is possible to elucidate the contributions of the different electrochemical reactions to the overall faradaic electrode current observed during high potential excursions $>1.35 \mathrm{~V}_{\text {RHE}}$. The initial partial and complete electrochemical oxidation of EC300 was found to be considerably greater than the graphitised samples. However, during repeated potential excursions the electrochemical oxidation tendencies 
were observed to be dynamic, exhibiting three trends that are not only influenced by the amount of electrochemical oxidation but also the lower electrode potential limit. Finally, the behaviour of the EC300 HSAC supported Pt catalysts was found to be largely the same as the pure EC300 carbon black, however, an additional peak is observed after electrochemical oxidation attributed to the oxidation of CO species on Pt.

\subsection{Introduction}

The key aspects of PEMFC research and development are focused towards resolving issues concerning the cost, efficiency and durability of the CEEC device. Although every component of the PEMFC contributes to at least one of these aspects (to variable extents) the most fundamentally crucial is the electrocatalyst material of the MEA. In terms of durability, several processes such as PEM thinning, gas diffusion layer degradation, electrocatalyst (Pt) particle sintering and HSAC support corrosion have been found to contribute to the PEMFC performance deterioration [151], of which the latter two factors are directly related to the characteristics of the electrocatalyst material. In particularly demanding PEMFC applications such as in an automobile, the degradation in the performance is considerable [4] and consequently, a thorough understanding of the failure mode(s) for each FC component in the development mitigating strategies to improve PEMFC endurance are urgently required [151]. Of particular concern to the PEMFC lifetime is the number of start-stop events [4, 152-156], during which the potential of the cathode may reach potentials up to $1.75 V_{\text {RHE }}$ [155] evolving considerable quantities $\mathrm{CO}_{2}$ [157] corroding the electrocatalyst HSAC support and rapidly deteriorating the PEMFC performance.

There are essentially two types of approach to the study of PEMFC degradation processes: (i) lifetime testing (LT) and (ii) accelerated stress testing (AST) [151, 158]. LT involves operating the PEMFC as it is intended to be used, which although certainly provides the most reliable indication of durability, is both time consuming and expensive with slow accumulation of data. The interpretation of 
LT data is also complicated by the wide range of parameters that can influence the long term durability PEMFCs [5]. Consequently, AST is a considerably more popular approach to assessing PEMFC durability, simply because data can be accumulated relatively quickly, under controlled conditions leading to more rapid developments. These ASTs can (similarly to the approach in assessing PEMFC electrocatalyst activities as described in Chapter 1) take two forms, and involve either in-situ or ex-situ methodologies.

In-situ AST methods typically involve fabricating an MEA and subjecting it to specific treatments under controlled conditions that push the boundaries of the intended operating region of the PEMFC in order to stress and accelerate the degradation of the MEA components. In such experiments the performance of the MEA is often monitored as a function of the AST parameter(s), such as current load, current load cycles or operating temperature $[4,156,159-161]$. The effect of the AST treatment on the MEA components may then be assessed by analysing the condition of the MEA after a degradation treatment [159, 161-163], identifying process such as cathode thinning, Pt particle loss and Pt precipitation $[162,163]$. There are also experiments which monitor corrosion processes online, for example, by measuring and correlating the $\mathrm{CO}_{2}$ concentration in cathode PEMFC exhaust gas using mass spectrometry [164, 165] or sampled gas chromatography $[156,160]$ with the deteriorating in the PEMFC performance. Although undoubtedly essential, in-situ methods are still relatively expensive and require the control of a large number of experimental variables (temperature, pressure, humidity etc.) and fabrication of the MEA. The screening of electrocatalysts or HSAC support materials, as well as the precise elucidation of degradation processes to specific PEMFC components (whose deterioration may not be uniform but localised [161]) is by no means trivial. A complementary approach using ex-situ AST techniques are therefore useful in order to enhance our fundamental understanding of the degradation processes of specific components, and to screen prospective HSAC support materials in order to facilitate the development of mitigating strategies that enhance the PEMFC lifetime. 
Ex-situ AST methods involve assessing the individual component of an MEA, such as the study of the electrocatalyst material in a three-electrode electrochemical half-cells, which have been used to measure the electrochemical oxidation of model carbons [166, 167], carbon blacks [168-170] and HSAC supported Pt catalysts [24, 25, 168]. These studies typically involve applying a certain electrochemical treatment, using either CV $[24,25,171]$, chronoamperometry [168] or potential pulse $[168,172]$ perturbation to a potential of at least 1.2 $\mathrm{V}_{\mathrm{RHE}}$, with the intention of electrochemically oxidising the carbon or electrocatalyst material. The anodic electrode current observed is then often referred to as corrosion current [169] or it is assumed to correspond with the loss of carbon material [168]. The electrochemical AST treatment is then compared to a measurable property such as the apparent electrode double-layer capacitance [168] or the nature of the surface oxygen containing functional groups [172]. In electrochemical half-cell studies of HSAC supported Pt catalyst corrosion, however, the analysis of the electrode current is further complicated by the electrochemical reactions of the supported Pt particles, in particular the oxidation of the Pt surface $>0.74 \mathrm{~V}_{\mathrm{RHE}}$ and the oxygen evolution reaction (OER) $>1.5 \mathrm{~V}_{\mathrm{RHE}}$. Using standard electrochemical half-cell methods therefore, it is impossible to decipher the carbon oxidation current based on the electrode current alone. Consequently, a measurable property of the electrocatalyst such as the ECSA of the supported Pt particles is often related to a parameter of the corrosion treatment, such as number of CVs [24, 25, 171], potential pulses [166] or potential hold time [168, 173], analogous to in-situ AST approaches. These methodologies have also been enhanced by utilising analytical techniques such as transmission electron microscopy (TEM) [24, 25], secondary electron microscopy (SEM) [167], atomic force microscopy (AFM) [171, 173], X-ray photoelectron microscopy (XPS) $[166,171,173]$ and EQCM [170]. For example, non-destructive TEM has been used to visually analyse the changes that occur to a specific electrocatalyst particle following a certain electrochemical treatment, identifying particle loss as the major cause of ECSA decrease during potential cycles up to $1.4 \mathrm{~V}_{\mathrm{RHE}}$ at $25{ }^{\circ} \mathrm{C}$. Alternatively, corrosion half-cell studies may also focus on the dissolution pure Pt electrodes [174]. 
Standard electrochemical half-cell techniques are nevertheless limited to the clouded interpretation of a single electrode current, even though a number of different electrochemical reaction processes are known to occur in the high potential regions $>1.2 \mathrm{~V}_{\text {RHE}}$, particularly in the study of HSAC supported Pt catalysts. The DEMS technique is therefore potentially very useful in the fundamental study of electrochemical oxidation processes by quantitatively measuring $\mathrm{CO}_{2}$ and $\mathrm{O}_{2}$, and has already been demonstrated in a handful of qualitative corrosion studies $[30,48,49]$.

\subsubsection{Motivation}

The intention of this study was to investigate the electrochemical oxidation tendencies of a number of HSAC supports (a.k.a. carbon blacks), and to develop a methodology in which DEMS can be used to study and compare their carbon oxidation reaction (COR) processes effectively. In order to approach this problem, a comparison using $\mathrm{CV}$ is made between the electrochemical oxidation tendencies of a pristine EC300 HSAC and five graphitised samples (of the same raw carbon black material) prepared at various different temperatures between 2100 and $3200{ }^{\circ} \mathrm{C}$. Rather than focusing this study on electrode potentials within a PEMFC relevant region to observe effects of a quite subtle nature and attempt to interpret them claiming direct PEMFCs relevance, this study will instead probe relatively high electrode potentials $\leq 2.05 \mathrm{~V}_{\mathrm{RHE}}$ with the intention of revealing clear trends in the electrochemical oxidation using DEMS. The electrochemical treatment will demonstrate the contrasting electrochemical oxidation tendencies of the various HSAC support samples to repeated high potential excursions, along with the possibly significant influence of the lower electrode potential limit. The study is then briefly extended to the electrochemical oxidation of EC300 HSAC supported Pt nanoparticle catalysts. Using DEMS, we are able to elucidate the contributions of the partial and complete COR processes, and the OER to the observed faradaic electrode current, relating the HSAC weight loss to the Pt ECSA loss. The methodology and experimental findings presented in this study should offer a 
valuable foundation for future DEMS electrochemical half-cell studies on the electrochemical oxidation processes of HSAC supports.

\subsubsection{Background}

The HSACs that are commonly used to support finely dispersed Pt particles are grouped in a classification of material termed 'carbon blacks' which are manufactured via the incomplete combustion (or thermal decomposition) of hydrocarbons under controlled conditions. Carbon blacks are rather high purity carbonaceous materials (typically $\geq 97 \%$ wt. carbon compared to the $\sim 60 \%$ carbon content of ash) that possess distinct, although wide ranging physiochemical properties that are useful in a number of industrial applications. In electrochemistry, carbon blacks possess the useful combination of acceptable electron conductivity, along with chemical and electrochemical compatibility with the electrochemical environment. The amorphous carbon black materials are considered to be formed from sections of graphene layers with little order parallel to the layer planes [175]. A carbon black particle possesses an aciniform structure (like a 'bunch of grapes'), which retains greater order of the graphene planes in the outer layers of the microstructure compared to the more disordered core. The high surface area and compatibility of carbon blacks with the electrochemical environment make them almost ideal supports for finely dispersed Pt electrocatalyst particles; however, they are inherently prone to being electrochemically oxidised and consumed in the PEMFC environment, deteriorating the electrocatalyst performance.

The complete oxidation of HSAC supports to $\mathrm{CO}_{2}$ may be described by the following overall reaction mechanism:

$$
\mathrm{C}+2 \mathrm{H}_{2} \mathrm{O} \rightarrow \mathrm{CO}_{2}+4 \mathrm{H}^{+}+4 e^{-} \quad \mathrm{E}^{0}=0.207 \mathrm{~V}_{\mathrm{SHE}}
$$

The low standard electrode potential of $0.207 \mathrm{~V}_{\text {SHE }}$ would suggest that carbon would be rather unstable in the fuel cell environment, however, because of the high irreversibility of the reaction, the complete oxidation of carbon remains 
relatively slow within typical PEMFC operating potentials i.e. $\leq 1.2 V_{\text {RHE }}$ [176]. Although the study of HSAC support corrosion in the PEMFC environment has only relatively recently received greater attention [177], the corrosion of HSAC supports in the phosphoric acid fuel cell (and half-cells aqueous electrolyte) is comparably well-studied owing to the more corrosive environment (due to higher operating temperatures). These earlier studies observed that the electrochemical oxidation of carbon involves both the complete oxidation to $\mathrm{CO}_{2}$ or partial oxidation of the carbon to either oxygen containing surface functional groups or CO [172, 178]. Although a number of mechanisms have been proposed to explain the COR processes, none are considered complete and can explain all the experimental observations [178], which often produce apparently contradictory results, as discussed in ref. [169]. The carbon corrosion mechanism(s) therefore remain rather speculative, although the inconsistent findings could be attributed to differences in experimental conditions, use of inconsistent terminology and both of the complexity of the COR reaction processes and the difficulties in characterising the inhomogeneous carbon black materials as discussed in ref. [169]. The COR processes that occur on carbon black supports are determined by a number of concurrent phenomena that are influenced by the bulk and surface characteristics of the carbon material [169]. Crucial bulk properties include the d-spacing (a measure of the graphitic character) and BET area that are each determined by the microstructure of the carbon black, whereas the surface characteristics are defined by the nature and concentration of surface functional groups. Although there are a number of classifications of functional groups present on the surface of the carbon blacks, those containing oxygen are undoubtedly the most significant. These oxygen containing functional groups not only determine many of the physiochemical properties of the carbon blacks, but also strongly influence the COR processes and may be classified into three groups: acidic, basic and neutral, each possessing different reactivities. Acidic oxygen functional groups include carboxylic or weaker acids such as alcohols and hydroquinones, whereas neutral functional groups include quinones and lactones. Basic surface oxygen functional groups meanwhile are thought to resemble heterocyclic pyran rings [179]. 
A crucial advantage of carbon blacks is that by controlling the synthesis and/or subsequent treatment, it is to some extent possible to tune desirable properties of the material toward its intended application. For example, heat-treatment in an inert atmosphere between ca. 1000 and $2700{ }^{\circ} \mathrm{C}$ is often used to graphitise raw carbon blacks, which essentially induces the stacking and order of the graphene layers decreasing the amorphous characteristics of the carbon microstructure. This treatment is an effective method of tuning the characteristics of the carbon black because between the two extremes forms of carbon: graphite and amorphous, there are a wide range of properties. Graphitisation, however, not only affects the structure of the carbon blacks but also changes the nature and concentration of surface functional groups. For instance, even mild heattreatment $<450{ }^{\circ} \mathrm{C}$ is able to reduce the surface concentration of carboxylic acid, carbonyl and lactone surface functional groups, which are directly related to the volatile content of the carbon black [180]. The influence of heat treatment, however, will of course be dependent on both the bulk and surface characteristics of the raw carbon black material, and the heat-treatment temperature, atmosphere and duration. 


\subsection{Experimental}

Each carbon black stock suspension was prepared by ultrasonically dispersing the powdered HSAC (EC300 and 5 heat-treated) in Millipore Milli-Q water to give solution concentration of $2.5 \mu g_{c} \mu l^{-1}$ (EC300) and $4.3 \mu g_{c} \mu l^{-1}$ (Samples 1 to 5). Details of the heat-treated (graphitised) samples, heat treatment temperatures and BET areas are presented later in section 6.4.1 table 6-1. Each measurement sample was then prepared by pipetting $20 \mu \mathrm{L}$ of an aqueous catalyst suspension onto a mirror polished $5 \mathrm{~mm} \emptyset$ polycrystalline Au WE substrate and dried in air to provide sample loadings of $50 \mu \mathrm{g}_{\mathrm{c}} \mathrm{cm}^{-2}$ (EC300) and $85 \mu \mathrm{g}_{\mathrm{c}} \mathrm{cm}^{-2}$ (Samples 1 to 5). The EC300 HSAC supported Pt nanoparticle catalyst samples were meanwhile prepared in an analogous manner to provide carbon loadings of $50 \mu \mathrm{g}_{\mathrm{c}} \mathrm{cm}^{-2}$. The particle sizes and \% wt. Pt of the HSAC supported nanoparticles catalysts are as follows: $1 \mathrm{~nm}-\mathbf{1 9 . 4} \%, 3 \mathrm{~nm}-\mathbf{4 6 . 0} \%$ and $5 \mathrm{~nm}-\mathbf{5 0 . 6} \%$.

The DEMS electrochemical flow cell setup used in this study is identical to that described previously in section 5.2. All experiments were performed at room temperature $\left(295 \mathrm{~K}\right.$ ) in aqueous $0.5 \mathrm{~mol} \mathrm{dm}^{-3} \mathrm{H}_{2} \mathrm{SO}_{4}$ (Normaton; Merck, Germany) electrolyte prepared Millipore Milli-Q water $\left(<18.3 \mathrm{MOhm} \mathrm{cm}^{-1}\right.$, TOC $<5$ ppb). The DEMS cell collection efficiency determined in each experiment using CO-Stripping on a polycrystalline Pt electrode, and the QMS was calibrated for $\mathrm{CO}_{2}$ and $\mathrm{O}_{2}$ at $\mathrm{m} / \mathrm{z}=44$ and $\mathrm{m} / \mathrm{z}=32$, respectively. The evolution of $I_{\mathrm{m} / \mathrm{z}=44}$ and $I_{\mathrm{m} / \mathrm{z}=32}$ was followed online by the QMS, and the faradaic current corresponding to the production of $\mathrm{CO}_{2}$ and $\mathrm{O}_{2}$ was calculated using the respective calibration constants $K^{0}$ and measured cell collection efficiency $N$.

The $I_{\mathrm{CO} 2}$ currents are calculated from the $I_{m / z}=44$ using the following equation:

$$
I_{\mathrm{CO}_{2}}=\frac{I_{m / Z=44} 4 F}{K_{\mathrm{CO}_{2}}^{\mathrm{N}} N_{\mathrm{CO}_{2}}}
$$

The values for $K_{C O 2}^{0}$, was $2.95 \mathrm{C} \mathrm{mol}^{-1}$, and $\mathrm{N}$ was typically $\sim 0.30$ at a flow rate of $5 \mu \mathrm{s} \mathrm{s}^{-1}$. A total of four electrons are assumed to be produced for each $\mathrm{CO}_{2}$ evolved. The detection limit of the DEMS instrument for $\mathrm{CO}_{2}$ is $2.754 \mathrm{ug}_{\mathrm{c}} \mathrm{hr}^{-1}$ and 


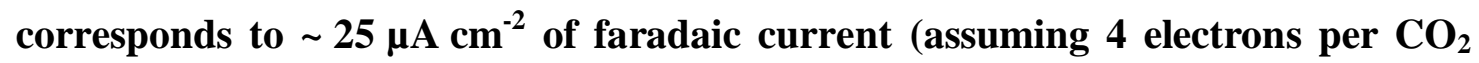
molecule).

The equivalent faradaic current arising from the oxygen evolution reaction was calculated using the following equation:

$$
I_{O_{2}}=\frac{I_{m / z=32} 4 F}{K_{O_{2}}^{\dagger} N_{C O_{2}}}
$$

The values for $K_{O 2}^{0}$, was $1.61 \mathrm{C} \mathrm{mol}^{-1}$ and we assume that the $N_{O 2}$ is identical to that of $\mathrm{N}_{\mathrm{CO} 2}$ which are found to be experimentally the same, within the error of the DEMS calibration measurement.

The remaining anodic electrode charge that does not arise from either the complete COR of the HSAC support or the OER on the polycrystalline Au background was assumed to arise from the partial COR, according to the following equation:

$$
Q_{P a r t i a l ~ C O R}=Q_{F}-Q_{C O_{2}}-Q_{Q_{2}}
$$

All electrode potentials meanwhile are given versus the RHE determined using the HOR/HER in hydrogen saturated electrolyte determined using a polycrystalline Pt electrode prior to experimentation. 


\subsubsection{Electrochemical Oxidation Procedure}

In accordance with the objective of this study, the electrochemical measurement procedure was designed with the intention of obtaining an overview of the electrochemical oxidation tendencies of the carbon black samples. Prior to the electrochemical oxidation treatment, the samples were first characterised using $\mathrm{CV}$ between 0.05 and 1.0 $\mathrm{V}_{\mathrm{RHE}}$ at various scan rates between 0.02 and $1.0 \mathrm{~V} \mathrm{~s}^{-1}$. The potential of the WE was then stepped to $1.05 \mathrm{~V}_{\mathrm{RHE}}$ in preparation for the electrochemical oxidation study on the HSAC samples, which involved the comparison of three experimental phases. For clarity, the three phases of these potential perturbations are depicted in figure 6-1.

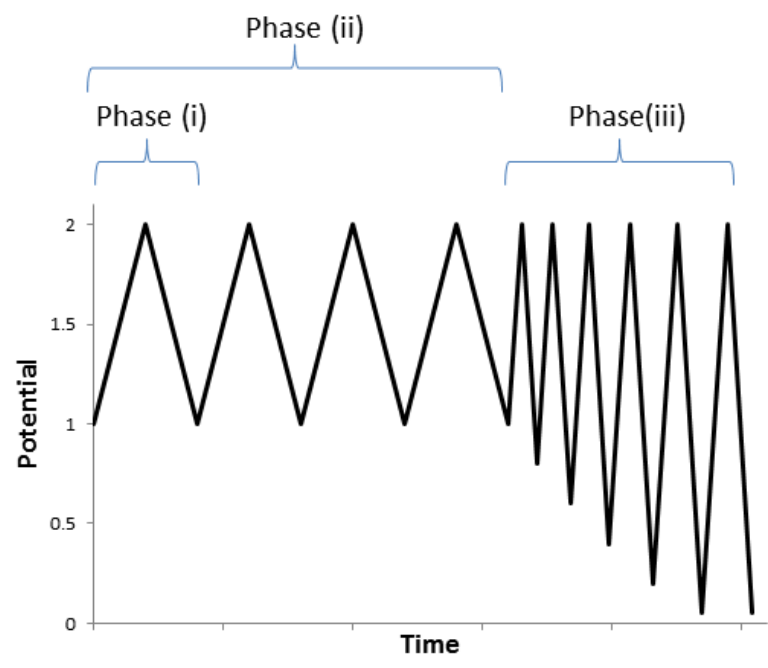

Figure 6-1: A graph illustrating the experimental procedure used to study the electrochemical oxidation processes of the carbon black samples. The procedure aims to compare the electrochemical oxidation tendencies of the HSAC supports in three phases: (i) the initial oxidation (ii) effect of repeated high potential oxidation and (iii) effect of low potential limit.

The three phases of the electrochemical treatment commenced as follows:

(i) The very first high potential excursion between 1.05 and $2.05 V_{\text {RHE }}$ to represent the initial electrochemical oxidation of the HSAC supports at $5 \mathrm{mV} \mathrm{s}^{-1}$. 
(ii) A further three CVs between 1.05 and 2.05 $\mathrm{V}_{\mathrm{RHE}}$ to study and compare the changes in the electrochemical oxidation tendencies of the HSAC samples at $5 \mathrm{mV} \mathrm{s}^{-1}$.

(iii) The effect of the lower potential limit of the CV on the electrochemical oxidation tendencies of the now oxidised HSAC support samples by progressively lowering the lower CV potential limit between 1.05 and $0.05 \mathrm{~V}_{\mathrm{RHE}}$ in steps of $200 \mathrm{mV}$ maintaining an upper electrode potential limit of 2.05 $\mathrm{V}_{\mathrm{RHE}}$ in six subsequent CVs at $20 \mathrm{mV} \mathrm{s} \mathrm{s}^{-1}$.

After the electrochemical oxidation treatment the oxidised HSAC samples were then characterised once again using $C V$ between 0.05 and $1.05 V_{R H E}$ at various scan rates. 


\subsection{Results and Discussion}

\subsubsection{Determination of the Apparent Double-Layer Capacitance}

In electrochemistry it is often desirable to be able to quantify the mass and/or ECSA of the electrode material of interest in-situ of the electrochemical half-cell experiment, which in the study of precious metal catalysts the ECSA may be relatively straightforwardly determined using a probe molecule. For example, the

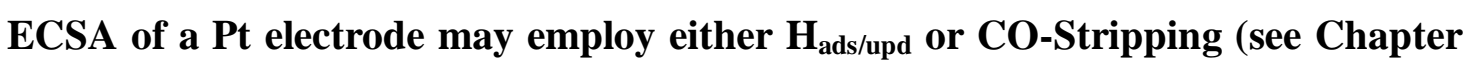
4 and 5 sections 4.5 and 5.3.1) techniques, whereas non-Pt catalysts such as Ag the ECSA can be measured via a metal-stripping (such as $\mathrm{Pb}$ ) procedure [10, 149, 181]. However, for surfaces where a probe molecule does not adsorb (such as carbon surfaces) the in-situ determination of the mass and/or ECSA is comparatively problematic with no common methodology. Consequently, a method that utilises the property of double-layer capacitance was developed as part of this study in order to quantify the mass of the carbon sample on the WE substrate using CV in-situ, prior to electrochemical experimentation.

An example series of CVs for the EC300 sample black performed at a number of scan rates between 0.05 and $1.05 V_{\text {RHE }}$ is shown in figure 6-2 (a). An almost rectangular shaped voltammogram is observed which appears to scale proportionally with the potential scan rate, both of which are characteristic of capacitive processes. The capacitance of the electrode surface can be extracted from the current in the $\mathrm{CV}$ using the following equation:

$$
Q=\frac{I_{+}-I_{-}}{2 V}
$$

Where $Q=$ capacitive charge, $I_{+}$and $I$. are the currents of the positive and negative going potential sweeps, and $v=$ potential scan rate. The resulting apparent double-layer capacitance of the electrode may then be plotted as a function of the electrode potential as given in figure 6-2 (b). Here we observe that the capacitance does not remain constant for all potentials between 0.05 and 1.05 $\mathrm{V}_{\text {RHE }}$ but instead possesses a parabolic relationship with the electrode 
potentials during the $\mathrm{CV}$ (ranging from 3.6 to $3.8 \mathrm{mF} \mathrm{cm} \mathrm{cm}^{-2}$ ), which is furthermore slightly dependent upon the potential scan rate, converging at higher rates.
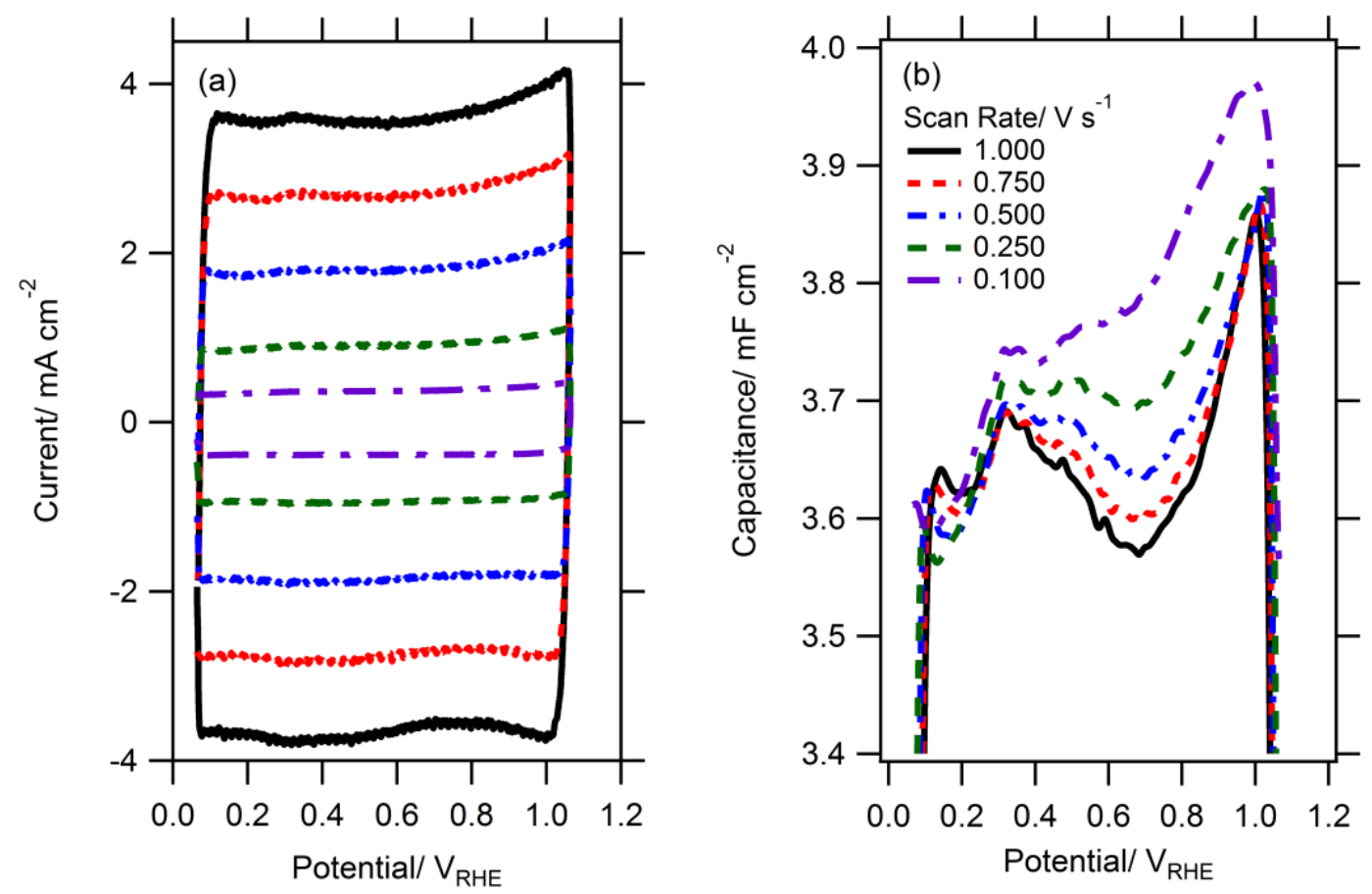

Figure 6-2: CV of EC300 HSAC support (a) and corresponding potential dependence on the double layer electrode capacitance (b) at various potential scan rates. Measurement performed in Ar saturated $0.5 \mathrm{~mol} \mathrm{dm}^{-3} \mathrm{H}_{2} \mathrm{SO}_{4}$ electrolyte.

The largely featureless $\mathrm{CV}$ and scaling of electrode current with scan rate strongly indicate that the electrode current is dominated by capacitive currents determined by the interface between the carbon electrode and electrolyte. It is generally assumed that the whole internal structure of the carbon is wetted by the electrolyte, although this may not be strictly the case for microporous carbons [182]. The parabolic relationship between capacitance and electrode potential is in agreement with the general trend observed using $\mathrm{CV}$ but would ideally be constant for all potentials where no faradaic electrode reactions occur [182]. This has been quite extensively studied, and it has been noted that there may complications with porous electrodes because of distributed capacitance effects where it is speculated that the exterior of the porous carbon charges faster than the interior and that less of the surface is therefore accessible at fast scan rates $[181,182]$. Nonetheless, a slight dependence of the double-layer capacitance on the CV scan rate does not impact on aims of the methodology. 
Although, the minimum in the double-layer capacitance vs. potential curves (see figure 6-2(b)) are most often quoted in the literature [183] for the purpose of scaling the apparent double-layer capacitance with sample loading a value at any potential where no faradaic current is observed can be used. The apparent double-layer capacitance of the carbon samples taken at $0.25 \mathrm{~V}_{\mathrm{RHE}}$ is plotted as a function of the sample loading on the WE substrate in figure 6-3.

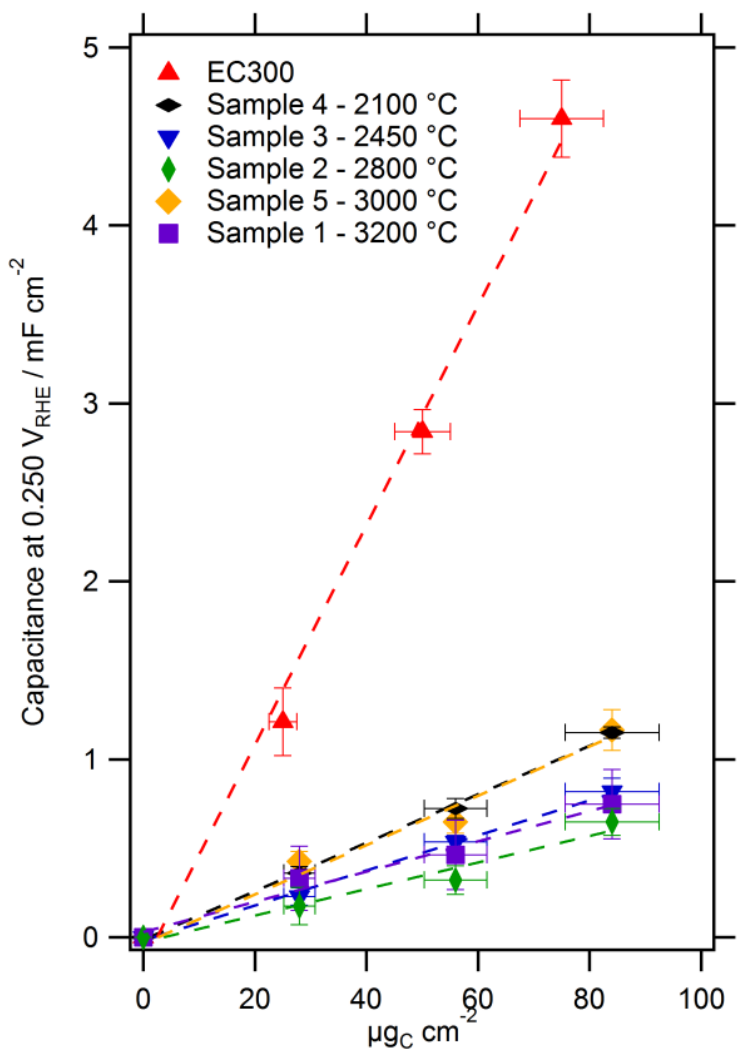

Figure 6-3: Measured capacitance of the HSAC support samples plotted as a function of carbon black sample loading. The WE substrate background corrected capacitance scales linearly with the sample loading of the carbon blacks.

In this plot we observe that the electrode capacitance scales linearly within the applied range of sample loading of each carbon black, and we can clearly see that the dependence of the capacitance of the EC300 carbon on the sample loading is significantly greater than all the heat-treated samples. Details of gravimetric and specific capacitance (BET normalised) of the carbon samples extracted from figure 6-3, along with details of the BET area of the carbon samples are summarized in table 6-1. 
Table 6-1: Details of the heat-treatment temperature, BET surface area and corresponding gravimetric and specific double layer capacitances for each carbon black samples investigated in this study.

\begin{tabular}{|c|c|c|c|c|}
\hline Sample & $\begin{array}{c}\text { Treatment } \\
\text { Temp. / } \\
{ }^{\circ} \mathrm{C} \\
\end{array}$ & $\begin{array}{l}\text { BET } \\
\text { Area/ } \\
\mathbf{m}^{2} \mathbf{g}^{-1} \\
\end{array}$ & $\begin{array}{c}\text { Gravimetric } \\
\text { Capacitance/ } \\
\text { F g }^{-1} \\
\end{array}$ & 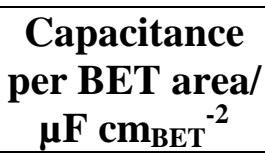 \\
\hline EC300 & - & 720 & $61.2 \pm 4.0$ & 8.5 \\
\hline (4) & 2100 & 247 & $14.1 \pm 0.8$ & 5.7 \\
\hline (3) & 2450 & 230 & $10.6 \pm 1.5$ & 9.4 \\
\hline (2) & 2800 & 218 & $7.5 \pm 1.0$ & 3.4 \\
\hline (5) & 3000 & 195 & $13.9 \pm 1.4$ & 7.1 \\
\hline (1) & 3200 & 135 & $8.5 \pm 0.9$ & 6.2 \\
\hline
\end{tabular}

The BET area of the EC300 carbon black $\left(720 \mathrm{~m}^{2}{ }_{\text {BET }} \mathrm{g}^{-1}\right)$ is significantly greater than that of the heat-treated $\left(\leq 247 \mathrm{~m}_{\text {BET }}^{2} \mathrm{~g}^{-1}\right)$ samples, which decreases further with increasing graphitisation temperature, particularly at $3200{ }^{\circ} \mathrm{C}$ $\left(135 \mathrm{~m}_{\text {BET }}^{2} \mathrm{~g}^{-1}\right)$. This observation is roughly mirrored by the gravimetric capacitance owing to the dependence of the double-layer capacitance on the surface area of the electrode, which in principle should (but not necessarily in practice) be rather independent of the electrode material and only on the surface area of the interface. For very porous carbons, for example, it is has been observed that the gravimetric capacitance of the porous carbon does not increase above $\sim 90 \mathrm{~F} \mathrm{~g}_{\mathrm{c}}^{-1}$ for carbons with specific surface areas $>1200 \mathrm{~m}^{2} \mathrm{~g}^{-1}$ although this is far higher than the carbon samples in this study [184]. The double-layer capacitance normalised per BET area are, however, not constant ranging from between 3.4 and $8.5 \mu \mathrm{F} \mathrm{cm}^{-2}$ exhibiting no particular trend with heat treatment temperature. The range of capacitance values are comparable to literature values,

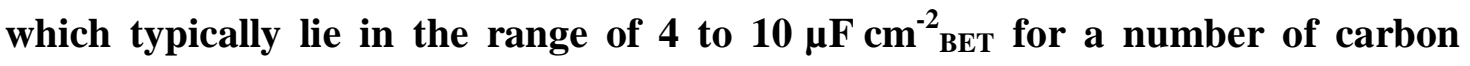
blacks in various electrolyte [183, 184]. More specifically, the $8.5 \mu \mathrm{F}$ cm $_{\text {BET }^{-2}}$ observed for the EC300 carbon is closely comparable to the $8 \mu \mathrm{F} \mathrm{cm}_{\mathrm{BET}^{-2}}$ of Vulcan XC-72 [183]. The sample loading can therefore be calculated using the following simple equation:

$$
W_{\text {sample }}=\frac{C_{\text {total }}-C_{\text {substrate }}}{C_{g}}
$$


Where $W_{s}=$ sample loading, $C_{\text {total }}=$ total electrode capacitance, $C_{\text {substrate }}=\mathrm{WE}$ substrate capacitance and $C_{g}=$ gravimetric capacitance (pre-determined) of the carbon sample.

The significance of this methodology is that the carbon black sample loading on the WE substrate may be quantified in-situ rather than relying on the deposited carbon sample remaining on the WE substrate during the course of the measurements, which in practice is prone to detaching from the WE substrate when first submerged in electrolyte reducing the actual sample loading. By measuring the sample loading in-situ the experiment may be validated or adjusted to the actual loading. Furthermore, given the importance of quantifying the electrode sample size in electrocatalysis, it may also be possible to adopt a similar methodology to quantify the sample loading of non-precious metal porphyrin ring ORR catalysts. The methodology could also be extended to the study of HSAC supported Pt catalysts by pre-adsorbing a saturated $\mathrm{CO}_{\text {ads }}$ layer on Pt to supress faradaic electrode processes and limiting the upper potential limit to below ca. $0.5 \mathrm{~V}_{\mathrm{RHE}}$ (at avoid oxidising the $\mathrm{CO}_{\text {ads }}$ layer) during the $\mathrm{CV}$. The electrode capacitance of the whole HASC supported Pt catalyst may be determined, similarly to a method used to assess the double-layer capacitance of an MEA in ref. [159].

It is, however, important to note that the scaling of the gravimetric capacitance with the carbon loading, particularly for the lower surface area graphitised carbons (namely, samples with BET areas $\leq 250 \mathrm{~m}^{2} \mathrm{~g}^{-1}$ ) is by no means one hundred per cent reliable and is prone to considerable error, predominantly arising from the potentially significant influence of the WE substrate background capacitance contributions. The capacitance of the WE substrate is ideally substantially smaller than the sample material and must remain constant for each experiment. The capacitance of a typical GC substrate (specific capacitance of $25 \mu \mathrm{F} \mathrm{cm}^{-2}$ [185]) however, was observed to be $\sim 1$ to $2 \mathrm{mF} \mathrm{cm}$ which is a quite considerable background equivalent to a $\sim 20$ to $30 \mu \mathrm{g}_{\mathrm{C}} \mathrm{cm}^{-2}$ loading of EC300 and much more for the graphitised samples. A polycrystalline Au WE substrate is in comparison better suited because it only possesses a capacitance of $\sim 0.35 \mathrm{mF} \mathrm{cm}^{-2}$. Some common sense is therefore required in the interpretation of 
data because changes in the WE substrate capacitance, which may arise from the leakage of electrolyte into the sides of the WE substrate or from changes in the substrate surface, can and will invalidate the double-layer capacitance measurement. A final important note is that we can only assume that the current observed is double-layer capacitance (hence the use of 'apparent') because it can also contain current contributions from reversible surface redox processes which are undefined. Although this will not influence the usefulness of this methodology for assessing the loading of a pristine carbon black sample on a WE substrate at the beginning of an electrochemical experiment, it will influence the interpretation of the specific capacitance and the changes in the apparent doublelayer capacitance that can occur as a result of an electrochemical treatment, as we will be noted later in this study. 


\subsubsection{Substrate Background Contributions}

At the beginning of the study on the electrochemical oxidation of carbon blacks, it was crucial to choose an appropriate WE substrate that (ideally) does not contribute to the faradaic electrode corrosion current, or evolve any $\mathrm{CO}_{2}$ in the potential region of interest, here $<2.05 \mathrm{~V}_{\mathrm{RHE}}$. The electrochemical oxidation of the commonly employed GC substrate, and a polycrystalline Au substrate were evaluated with comparison of the polycrystalline Pt (for reference) in figure 6-4.

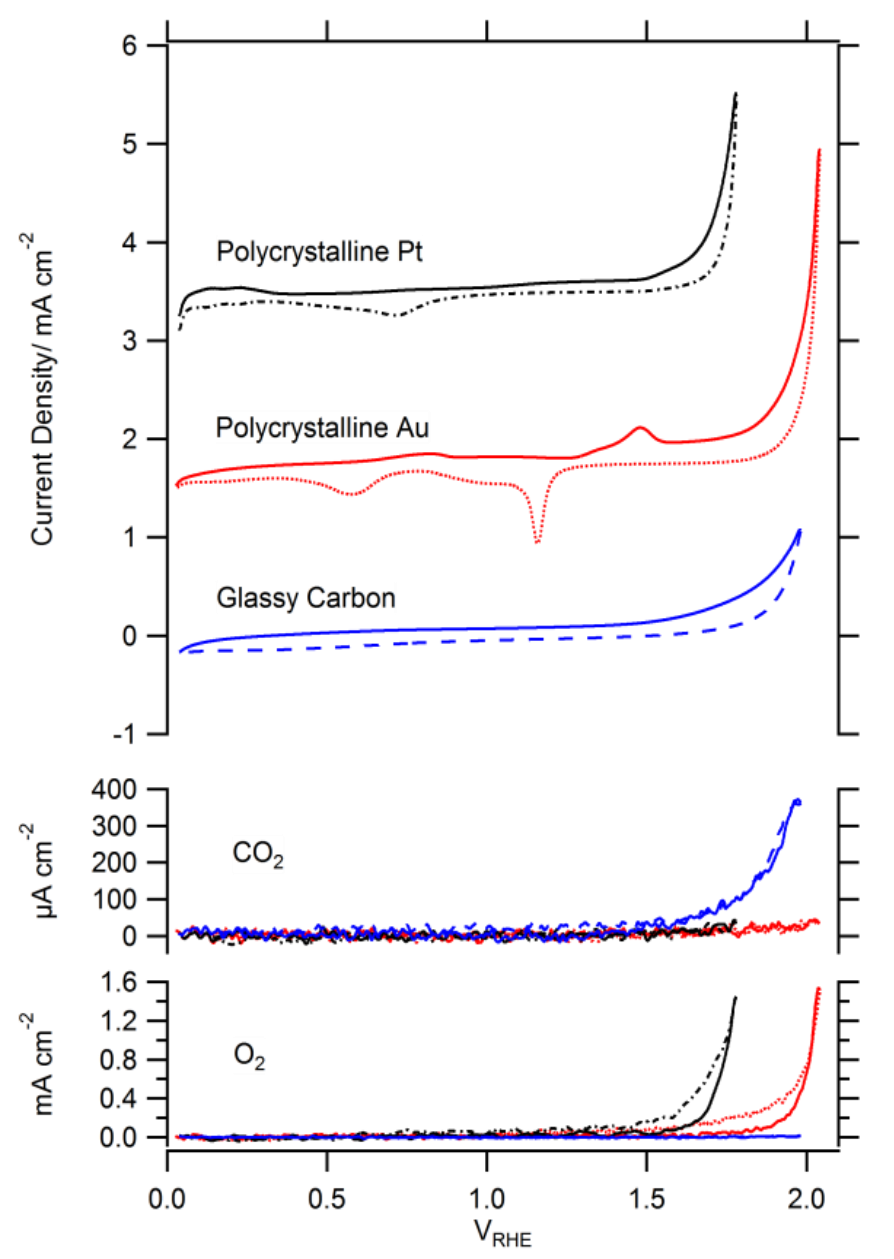

Figure 6-4: CV and MSCVs of polycrystalline-Pt, -Au and GC electrodes. Measurments performed at $20 \mathrm{mV} \mathrm{s}^{-1}$ in $0.5 \mathrm{~mol} \mathrm{dm}{ }^{-3} \mathrm{H}_{2} \mathrm{SO}_{4}$ and $300 \mu \mathrm{min}^{-1}$, with corresponding $\mathrm{CO}_{2}\left({ }^{\mathrm{m}} /{ }_{\mathrm{z}}=44\right)$ and $\mathrm{O}_{2} \quad(\mathrm{~m} / \mathrm{z}=32)$ mass ion currents. The commonly used GC substrate is observed to electrochemically oxidise to $\mathrm{CO}_{2}$ at potentials above $1.5 \mathrm{~V}_{\mathrm{RHE}}$ giving rise to undesirable WE substrate background contributions in the study of the electrochemical oxidation of carbon blacks.

The GC substrate exhibits rather small electrode current dominated by capacitive currents in the potential region $<1.4 \mathrm{~V}_{\mathrm{RHE}}$. At higher potentials, 
however, the GC substrate electrochemically oxidises yielding $\mathrm{CO}_{2}$. In comparison, the polycrystalline Au substrate exhibits pronounced features in the $\mathrm{CV}<1.5 \mathrm{~V}_{\mathrm{RHE}}$ which are attributed to the oxidation and reduction of the Au surface. At potentials above $1.7 \mathrm{~V}_{\mathrm{RHE}}$ the electrode current on polycrystalline Au is dominated by the OER yielding $\mathrm{O}_{2}$. For reference, the OER on the polycrystalline Pt surface occurs at the lower potential of $1.5 \mathrm{~V}_{\mathrm{RHE}}$.

Despite that GC is widely considered as one of the most stable, chemically inert form of carbon, the appearance of electrode current corresponding to the electrochemical oxidation of the $\mathrm{GC}$ to $\mathrm{CO}_{2}$ at potentials $>1.4 \mathrm{~V}_{\mathrm{RHE}}$ is far from an ideal characteristic of the WE substrate background in the study of the electrochemical oxidation of HSAC supports. This effectively limits the study of the COR of HSAC supports to the region $<1.4 \mathrm{~V}_{\mathrm{RHE}}$, although GC substrates have been used in previous DEMS studies on the COR [48, 49]. In comparison, a polycrystalline Au substrate does not introduce any background $\mathrm{CO}_{2}$, evolving only $\mathrm{O}_{2}$ at potentials $>1.7 \mathrm{~V}_{\mathrm{RHE}}$ allowing rather higher electrode currents to be studied. In consideration that we are primarily interested in $\mathrm{CO}_{2}$ the potential limit can conceivably be raised above $1.7 \mathrm{~V}_{\mathrm{RHE}}$, however, in practice the maximum potential is limited by the quantity of $\mathrm{O}_{2}$ evolved in the OER on the polycrystalline Au to $<2.05 \mathrm{~V}_{\mathrm{RHE}}$. At higher potentials the rate of OER produces an excessive amount of $\mathrm{O}_{2}$ which forms bubbles in the thin-layer and flow cell lines causing a loss of contact between the working, counter and reference electrodes. Finally, the observation of $\mathrm{CO}_{2}$ is not in agreement with the claim that the oxygen evolution reaction on $\mathrm{GC}$ electrodes in $0.1 \mathrm{~mol} \mathrm{dm}^{-3}$ in $\mathrm{HClO}_{4}$ occurs at 1.0 $\mathrm{V}_{\mathrm{SCE}}\left(\sim 1.35 \mathrm{~V}_{\mathrm{RHE}}\right)$; however, these measurements were based on RDE measurements alone [186]. 


\subsubsection{Electrochemical Oxidation of HSAC Supports}

\subsubsection{Phase (i) - First High Potential Excursion}

The contributions of the various electrochemical process towards the overall faradaic electrode current observed during the first potential sweep (phase(i)) from 1.05 to $2.05 \mathrm{~V}_{\mathrm{RHE}}$ of the pristine carbon black samples deposited on a polycrystalline Au substrate were elucidated using DEMS. An example measurement, highlighting the relative contributions of the different electrochemical reaction processes that occur during the initial high potential excursion for the EC300 carbon $>1.05 \mathrm{~V}_{\mathrm{RHE}}$ is first illustrated in figure 6-5.

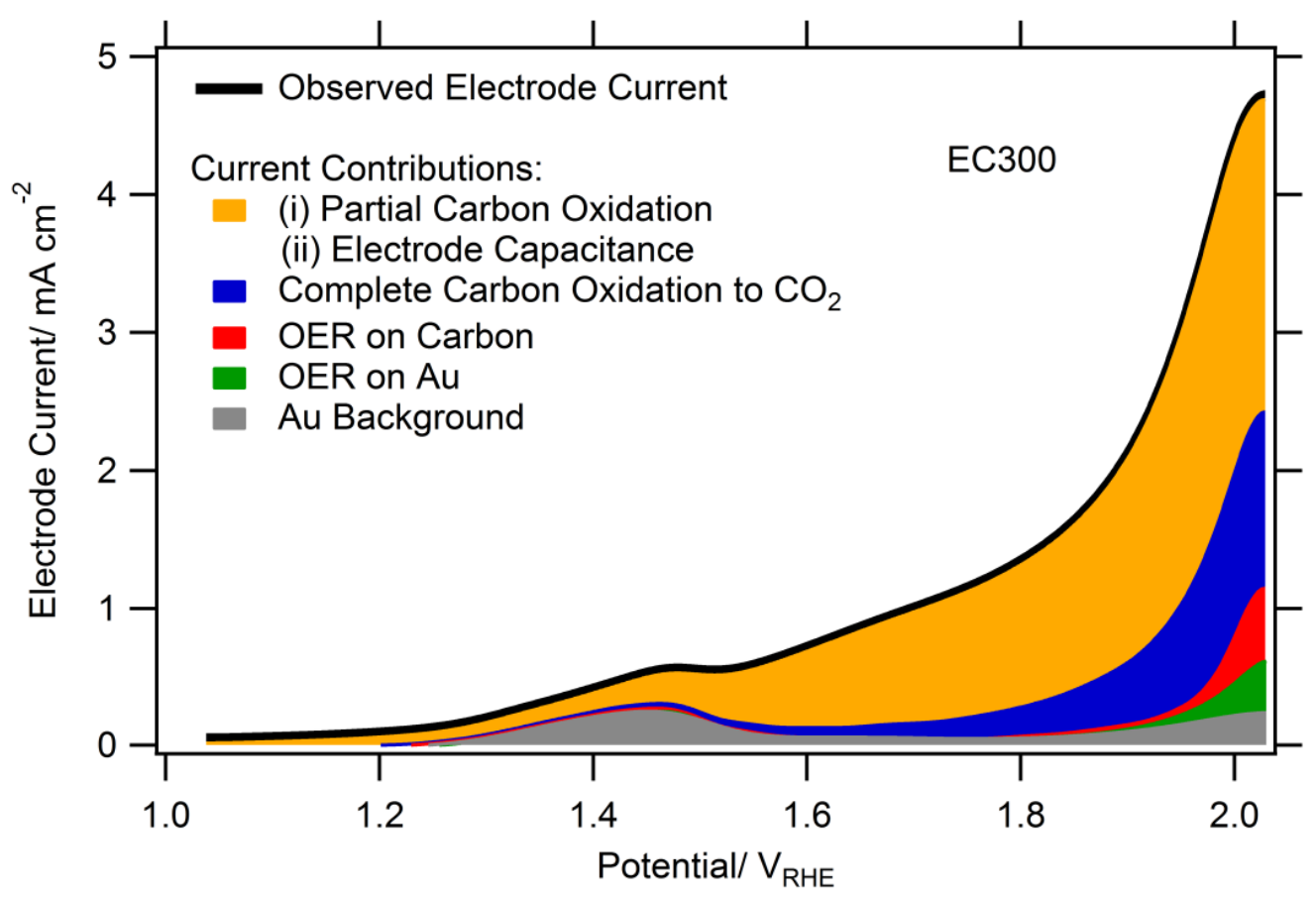

Figure 6-5: A graph illustrating the origins of the observed current during an initial high potential excursion above $1.05 \mathrm{~V}_{\mathrm{RHE}}$. Pure EC300 HSAC support $\left(50 \mu \mathrm{g}_{\mathrm{Pt}} \mathrm{cm}^{-2}, 0.5 \mathrm{~mol} \mathrm{dm}^{-3} \mathrm{H}_{2} \mathrm{SO}_{4}\right.$ and $5 \mathrm{mV} \mathrm{s}^{-1}$ ). The electrode current at these high potentials is dominated by the partial and complete COR of the HSAC support.

At potentials $<1.2 \mathrm{~V}_{\mathrm{RHE}}$ the electrode currents are dominated by double-layer capacitance currents until the potential exceeds $\sim 1.2 \mathrm{~V}_{\mathrm{RHE}}$ where we begin to observe the polycrystalline Au substrate surface oxidation peak (refer to figure 6-4 previously). As the potential sweeps above $>1.2 \mathrm{~V}_{\mathrm{RHE}}$ there is a steady increase in electrode current which is accompanied by the complete $\mathrm{COR}$ to $\mathrm{CO}_{2}$. 
As the electrode potential is raised even further to $>1.7 \mathrm{~V}_{\mathrm{RHE}}$ the complete COR begins to surge in an exponential manner. Above $1.9 \mathrm{~V}_{\mathrm{RHE}}$ we then observe the OER on the polycrystalline Au substrate with some evidence to indicate that a small amount of $\mathrm{O}_{2}$ is evolved from the EC300 HSAC support (although as we will see this is specific to EC300 and is short-lived). The quantity of $\mathrm{CO}_{2}$ and $\mathrm{O}_{2}$ observed using the calibrated DEMS instrument was found to be clearly insufficient to account for all electrode current observed during the high potential excursion for each of the carbon samples. In this study, the remaining current is assumed to arise from the partial COR of the HSAC support which may be to surface oxide functional groups or $\mathrm{CO}$, both of which are understood to occur (to varying degrees) simultaneously with the complete $\mathrm{COR}$ to $\mathrm{CO}_{2}$ (see ref. [178]).

In order to begin to compare the electrochemical oxidation tendencies of various HSACs, an initial comparison between the first high potential excursion between 1.05 and 2.05 $V_{\text {RHE }}$ for each of the carbon black samples is given in figure 6-6. It is immediately clear that the electrode current observed on the EC300 above 1.0 $\mathrm{V}_{\mathrm{RHE}}$ is significantly greater than for graphitised samples (despite that the EC300 mass loading is almost half in this figure i.e. $50 \mathrm{vs.} 85 \mu \mathrm{gc}_{\mathrm{c}} \mathrm{cm}^{-2}$ ). For all the carbon samples, the observed electrode current coincides with the $\mathrm{COR}$ to $\mathrm{CO}_{2}$, exhibiting a clear trend for decreasing COR rates with increasing graphitisation temperature. The peak observed at $1.45 \mathrm{~V}_{\mathrm{RHE}}$ and the $\mathrm{O}_{2}$ observed $>1.7 \mathrm{~V}_{\mathrm{RHE}}$ in each of the measurements can be solely attributed to the OER on the polycrystalline Au substrate, with the exception of the EC300 sample where some additional $\mathrm{O}_{2}$ was observed in the initial excursion. Finally, the overlap of the $\mathrm{O}_{2}$ evolved in the OER on the polycrystalline Au substrate for heat-treated sample demonstrate the precision and reproducibility of the DEMS measurement. 

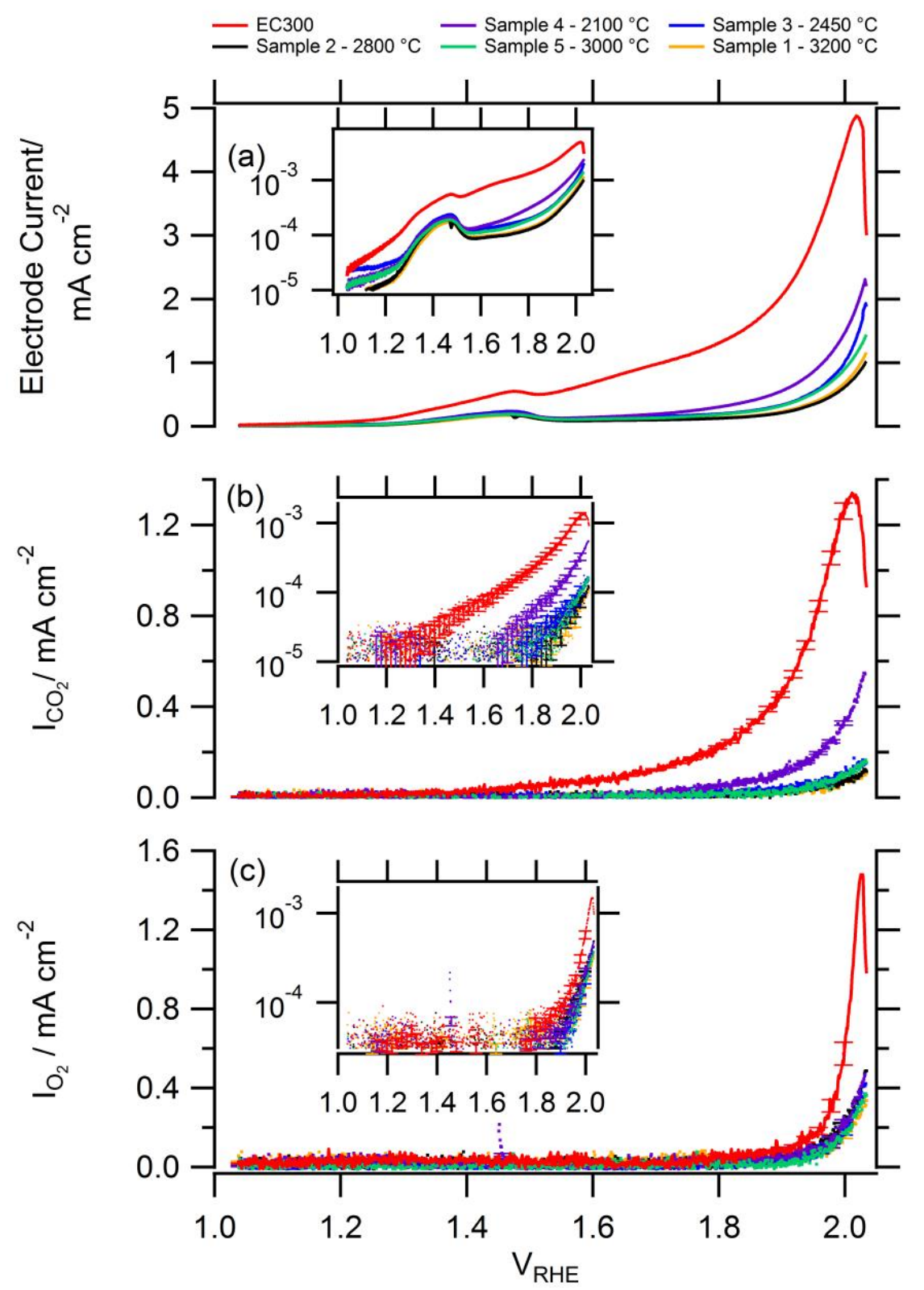

Figure 6-6: First high potential LSV $\left(5 \mathrm{mV} \mathrm{s}^{-1}\right)$ of the pristine $\operatorname{EC300}\left(50 \mu \mathrm{gg}_{\mathrm{c}} \mathrm{cm}^{-2}\right)$ and heattreated samples 1-5 $(85 \mu \mathrm{g} \mathrm{cm})$. In Argon saturated $0.5 \mathrm{~mol} \mathrm{dm}^{-3} \mathrm{H}_{2} \mathrm{SO}_{4}$ electrolyte on a polycrystalline Au substrate (a) $\mathrm{I}_{\mathrm{CO} 2}$ (b) and $\mathrm{I}_{\mathrm{O} 2}$ (c). The corresponding $\log \mathrm{I}$ vs. $\mathrm{V}_{\mathrm{RHE}}$ plots are inset. The rate of electrochemical oxidation of the HSAC support is comparably high for the pristine EC300 sample with an observed trend for decreasing COR rate with increasing graphitisation temperature.

In the example figure 6-5, it was highlighted that the COR of the EC300 HSAC support to $\mathrm{CO}_{2}$ coincided with additional electrode current that was attributed to partial COR processes. By subtracting the $I_{C O 2}, I_{O 2}$ and Au WE substrate background current contributions measured using DEMS from the overall 
observed electrode current, the currents attributed to the partial oxidation of the carbon black can be deduced. The integrated currents corresponding to the charge arising from the complete and partial COR on the carbon black samples during the first potential excursion to $2.05 \mathrm{~V}_{\mathrm{RHE}}$ are summarised as a function of the heat-treatment temperature in figure 6-7.
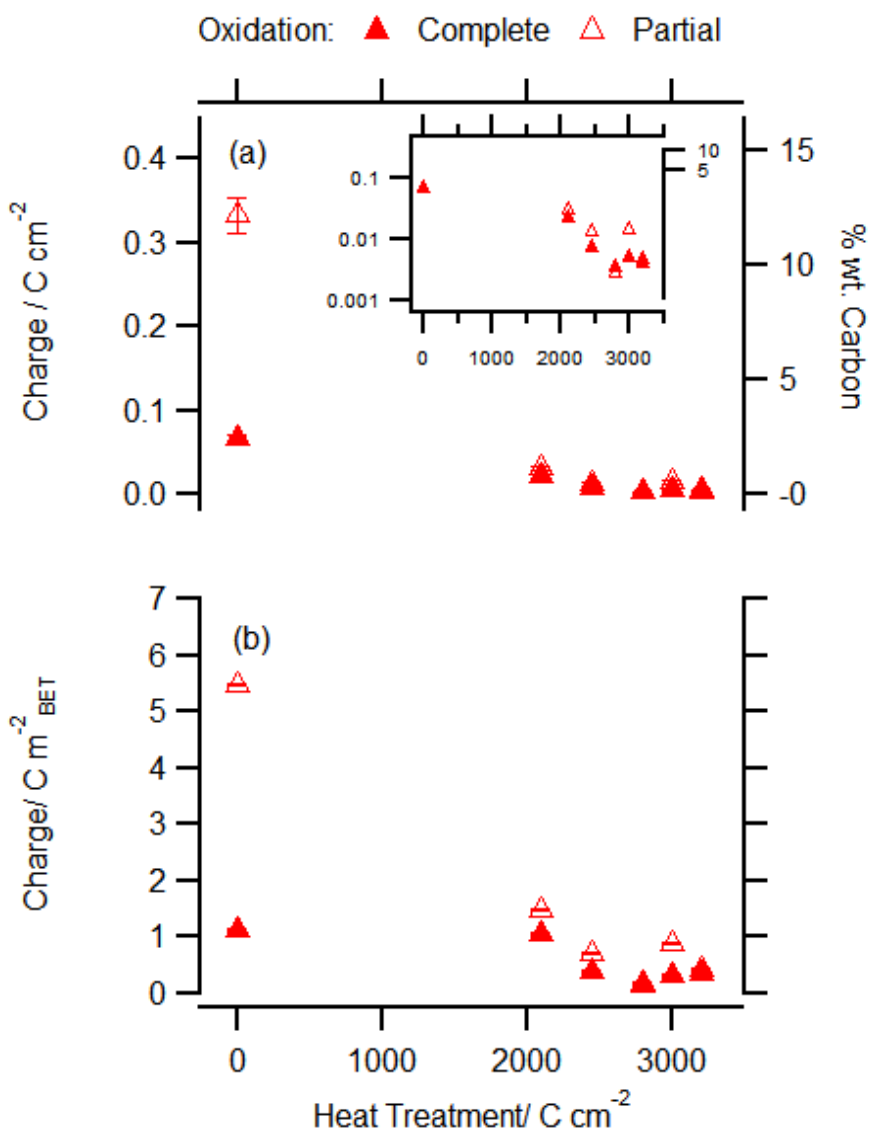

Figure 6-7: Effect of heat treatment temperature on the complete and partial electrochemical oxidation charge evolved during the first high potential excursion to $2.0 \mathrm{~V}_{\text {RHE }}$ (a) (EC300 normalised to $85 \mu \mathrm{g} \mathrm{cm}^{-2}$ ). Corresponding oxidation current normalised to BET surface area (b) in $0.5 \mathrm{~mol} \mathrm{dm}^{-3} \mathrm{H}_{2} \mathrm{SO}_{4}$ for the various HSAC samples. The quantity of partial COR is significantly higher than complete COR on the EC300 sample. The amount of partial and complete COR is highest on the EC300 HSAC support. The mass normalised quantity of complete electrochemical COR of the EC300 is, however, normalised to BET are the values are much more comparable.

In this plot we observe that the mass normalised charge for the complete and partial COR of the carbon blacks in general decreases with increasing graphitisation temperature. The complete COR charge evolved by the EC300 is almost one order of magnitude greater than the graphitised samples treated $\geq 2450{ }^{\circ} \mathrm{C}$. However, the charge corresponding to the partial oxidation of the 
EC300 HSAC support is significantly larger than complete COR in contrast to the heat-treated samples where the charges are of similar magnitude. Finally, by normalising the COR charges to the BET surface area of the HSAC samples it is observed that the specific complete COR charge evolved from the EC300 and graphitised sample $4\left(2100{ }^{\circ} \mathrm{C}\right)$ are quite similar.

In the logarithmic current vs. potential plots shown in the inset of figure 6-6, a linear Tafel-slope region for the $I_{\mathrm{CO}_{2}}$ and $I_{\mathrm{O}_{2}}$ can be observed in the oxidation of each of the HSAC samples, although in general these regions cover less than one decade of current. The $\mathrm{CO}_{2}$ detection limit potential, Tafel-slope and corresponding transfer coefficient $(\alpha * n)$ extracted from the observed $I_{\mathrm{CO} 2}$ are summarised in table 6-2.

Table 6-2: COR Tafel slopes $\left(\mathrm{I}_{\mathrm{CO} 2}\right)$ and corresponding symmetry constants for each HSAC during the first high potential excursion. DEMS $I_{\mathrm{CO} 2}$ detection limit equivalent to $25 \mu \mathrm{A} \mathrm{cm}{ }^{-2}$. In cases where two linear regions were observed, two Tafel slopes are given, whereby the *values corresponds to the slope in the lower potential region $<1.8 \mathrm{~V}_{\mathrm{RHE}}$. The $\alpha^{*} \mathrm{n}$ obtained from these slopes are comparable to values used in kinetic models of the HSAC support degradation in PEMFCs.

\begin{tabular}{|c|c|c|c|c|}
\hline Sample & $\begin{array}{c}\text { Graphitisation } \\
\text { Temp. } /{ }^{\circ} \mathbf{C}\end{array}$ & $\begin{array}{c}\mathbf{C O}_{2} \text { Detection } \\
\text { Limit Potential/ } \\
\text { V }_{\text {RHE }}\end{array}$ & $\begin{array}{c}\text { Tafel slope/ } \\
\mathbf{m V ~ D e c ~}^{-1}\end{array}$ & $\alpha^{* n}$ \\
\hline EC300 & - & 1.35 & $\begin{array}{c}* 413 \pm 6 \\
229 \pm 2\end{array}$ & $\begin{array}{c}\mathbf{0 . 1 4 3} \\
\mathbf{0 . 2 5 8}\end{array}$ \\
\hline$(4)$ & 2100 & 1.68 & $\begin{array}{c}* 307 \pm 11 \\
170 \pm 2\end{array}$ & $\mathbf{0 . 1 9 2}$ \\
& & $\mathbf{0 . 3 4 8}$ \\
\hline$(3)$ & 2450 & 1.84 & $200 \pm 9$ & $\mathbf{0 . 2 9 5}$ \\
\hline$(2)$ & 2800 & 1.88 & $206 \pm 11$ & $\mathbf{0 . 2 8 7}$ \\
\hline$(5)$ & 3000 & 1.88 & $167 \pm 6$ & $\mathbf{0 . 3 5 4}$ \\
\hline$(1)$ & 3200 & 1.93 & $170 \pm 9$ & $\mathbf{0 . 3 4 8}$ \\
\hline
\end{tabular}

In this table we precisely see that the $\mathrm{CO}_{2}$ detection limit potential for the complete COR of the carbon blacks increases with increasing heat-treatment temperature, with clear potential steps between the EC300, sample $4\left(2100{ }^{\circ} \mathrm{C}\right)$, and the remaining graphitised samples $\left(>2450{ }^{\circ} \mathrm{C}\right)$. For the EC300 and sample 4 $\left(2100{ }^{\circ} \mathrm{C}\right)$ carbons, two Tafel-slope linearities are observed in the logarithmic plot above and below $1.8 \mathrm{~V}_{\mathrm{RHE}}$. Below $1.8 \mathrm{~V}_{\mathrm{RHE}}$ the slopes are comparatively shallow $\left(\geq 307 \mathrm{mV} \mathrm{Dec}{ }^{-1}\right.$ ); however, above $1.8 \mathrm{~V}_{\mathrm{RHE}}$ the Tafel-slopes for all the carbon 
black samples are comparable $\left(\sim 200 \pm 30 \mathrm{mV} \mathrm{Dec}^{-1}\right)$ with no clear relationship with the heat-treatment temperature.

\subsection{Discussion of Initial Electrochemical Oxidation Processes}

It is uncommon to begin the discussion of experimental data with the limitations of the study, however, it is necessary in this case in order to focus the discussion on how the DEMS instrument can be used to study the COR, rather than overly speculate on the possible COR mechanism(s) that occur during each phase of the electrochemical treatment. The obvious limitation here, is that we simply do not know enough about the bulk and surface characteristics of the pristine carbon black materials i.e. BET surface areas alone are not enough. For the discussion in this study we instead have to make the following (reasonable) assumptions concerning the relative bulk and surface characteristics of the pristine EC300 and graphitised samples: (i) the EC300 carbon black possesses the least graphitic character and the highest oxygen content, whereas (ii) the heat-treated samples possess more graphitic character, which increases with rising heat-treatment temperature that in turn, decreases the BET area and oxygen content. These assumptions are based on previous observations that the oxygen content is in general proportional to the BET surface area [169] (although heat-treated samples tend to have lower oxygen content) and that heat-treatment organises the graphene layers reducing the amorphous nature of the carbon decreasing the $d$ spacing (and decreasing BET area) [187]. For more in-depth discussion of the differences in the initial COR tendencies and their mechanism(s), a greater effort in the characterisation of the pristine carbon samples using a range of methods (along with BET) is required. For example, techniques such as X-ray diffraction (XRD) can be used to determine the interlayer d-spacing and deduce the graphitic character of the carbon [169, 187], whereas $i R$-spectroscopy [188] or XPS [172] may be used to identify the nature and (possibly) concentration of the surface oxygen containing functional groups that are initially present on the pristine carbon samples. 
In phase (i) of the electrochemical treatment, DEMS was able to elucidate the proportion of electrode current arising from the complete $\mathrm{COR}$ to $\mathrm{CO}_{2}$, and the OER on polycrystalline Au evolving $\mathrm{O}_{2}$ during the high potential excursion to 2.05 $\mathrm{V}_{\mathrm{RHE}}$. By subtracting the calibrated $I_{\mathrm{CO} 2}$ and $I_{O_{2}}$ observed using DEMS, the remaining electrode current attributed to the partial COR can be deduced, an assumption that the complete COR occurs in parallel with the partial COR of carbon blacks, based on the findings of previous studies [176]. The partial COR current was observed to be quite significant, contributing toward $\sim 70 \%$ of the COR charge for the pristine EC300 and in general $\sim 50 \%$ for the graphitised samples during the first potential excursion to $2.05 \mathrm{~V}_{\mathrm{RHE}}$ and may proceed to both oxygen containing surface functional groups and CO [166, 176], the latter of which of course could also conceivably elucidated using DEMS. In light of the extensive partial COR current observed, the in-situ observation of $\mathrm{CO}$ would certainly be worthwhile measurement using DEMS to evaluate the quantity of $\mathrm{CO}$ in the future, although not necessarily straightforward and is discussed in more detail later in section 6.4.6. Nonetheless, the significant partial COR current observed would suggest that it should not be assumed that the whole electrochemical oxidation current arises from the complete COR of carbon black to $\mathrm{CO}_{2}$ and necessarily leads to carbon weight loss.

The lower complete and partial COR rates observed for the graphitised carbon black samples (with a tendency to decrease with increasing heat-treatment temperature) in comparison to the pristine $\mathrm{EC300}$ is in agreement with the wellestablished understanding that the heat-treatment and consequent graphitisation of carbon blacks tend to increase their resistance to chemical and electrochemical oxidation [178]. These finding are, for instance, in agreement with a previous DEMS study on an unspecified carbon black, whereby the $\mathrm{CO}_{2}$ evolved from a heat-treated material was found to be lower than that of the pristine sample 1.5 $\mathrm{V}_{\mathrm{RHE}}$ [48]. In an electrochemical half-cell study performed at $1.2 \mathrm{~V}_{\mathrm{RHE}}$ and $80{ }^{\circ} \mathrm{C}$, the corrosion current of non-graphitised carbons were observed to be in general, proportional to their BET area; however, graphitised carbons performed better by factor of $\sim 5$, in general agreement with the findings for the BET normalised partial and complete COR charges for the samples heat-treated $\geq 2450{ }^{\circ} \mathrm{C}$ [168]. In the PEMFC environment meanwhile, graphitised carbon 
MEAs have been found to perform up to 35 times better than non-graphitised carbon blacks at $1.2 \mathrm{~V}_{\mathrm{RHE}}$, and $\sim 5$ times better during start-stop corrosion cycles in the fuel cell environment [156].

Although the complete and partial COR rates of the graphitised samples were all found to be slower than the pristine EC300 carbon black, there appears to be a more significant decrease in the COR rates when increasing the heat-treatment temperature from 2100 to $\geq 2450{ }^{\circ} \mathrm{C}$. This would suggest that the changes in the microstructure and type and/or concentration of oxygen containing surface functional groups during the graphitisation of this particular carbon black at temperatures of $2450{ }^{\circ} \mathrm{C}$ and above, is more desirable in terms of achieving greater electrochemical oxidation corrosion resistance (at least initially), with a trend for increasing resistance with higher heat-treatment temperatures. This is in agreement with the trend observed in ref. [189] whereby heat-treatment at $2500{ }^{\circ} \mathrm{C}$ appeared to significantly decrease the specific COR rate (normalised to BET area) of the carbon black compared to lower temperature heat-treatment at $2000{ }^{\circ} \mathrm{C}$. The significantly greater COR rates on the EC300 is likely be attributed to the higher concentration the electrochemical oxidisable oxygen-containing surface functional groups that are initially present on the pristine HSAC support, which can be considered as reactive centres for the COR processes [169]. Ex-situ characterisation of the bulk and surface characteristics of the pristine carbon samples, however, is needed in order to shed more light on the differences between the initial oxidation rates of the pristine EC300 and heat-treated carbon samples, without which the rationalisation is purely speculative.

The pristine EC300 carbon exhibited two features that are not observed in the graphitised samples: (i) significant partial COR current representing $\sim 70 \%$ of the total charge (compared to $\sim 50 \%$ for the graphitised samples), and (ii) some evidence of $\mathrm{O}_{2}$ evolution that is not attributed to the polycrystalline Au WE substrate. The significant proportion of partial COR contributions to the overall current is in agreement with previous findings that the proportion of partial COR dominates the complete COR for pristine carbon blacks (in contrast to their graphitised counterparts) during the initial electrochemical oxidation, but decreases with time so that the complete COR eventually becomes dominant [190]. 
The origin of the observed $\mathrm{O}_{2}$ in the initial potential excursion is meanwhile unclear, and rather unexpected although the OER is thought to occur on certain carbon systems [186] (although the measurement on GC in figure 6-4 questions this). In phases (ii) and (iii) of the treatment, however, we will later observe that $\mathrm{O}_{2}$ is specific to the initial potential sweep and is not observed in any of the subsequent potential excursions. If we consider that the $\mathrm{O}_{2}$ feature coincides with a rapid increase in the $\mathrm{COR}$ rate, the $\mathrm{O}_{2}$ evolved may have been non-oxidatively released from sub-surface voids which opened as a result of the complete COR (rather than the oxidation of water), although this speculation.

Using DEMS we were also able to extract kinetic information on the complete COR by extracting the $I_{C O 2}$ Tafel-slope and the potential at which $\mathrm{CO}_{2}$ was first observed. In table 6-2 this potential was carefully termed the $\mathrm{CO}_{2}$ 'detection limit potential' rather than 'onset potential' because the complete COR can occur at lower potentials but at rates that are simply below the detection limit of the DEMS instrument. No $\mathrm{CO}_{2}$ below $1.35 \mathrm{~V}_{\mathrm{RHE}}$ was observed on the EC300 carbon, $1.68 \mathrm{~V}_{\mathrm{RHE}}$ on the mildly graphitised carbon $\left(2100{ }^{\circ} \mathrm{C}\right)$, or $1.84 \mathrm{~V}_{\mathrm{RHE}}$ on the more heavily graphitised carbons $\left(\geq 2450{ }^{\circ} \mathrm{C}\right)$ at a detection limit that is equivalent to a carbon weight loss of $2.754 \mu \mathrm{gchr}^{-1}$ evolved via the complete electrochemical COR, or $25 \mu \mathrm{A} \mathrm{cm}^{-2}$ of faradaic current (assuming $4 \mathrm{e}^{-}$per $\mathrm{CO}_{2}$ ). Previous DEMS studies have however, observed $\mathrm{CO}_{2}$ at potentials as low as $0.9 \mathrm{~V}_{\mathrm{RHE}}$ on NORIT BRX (BET of $\sim 1800 \mathrm{~m}^{2} \mathrm{~g}^{-1}$ [191]) [30], Vulcan XC-72 (BET of $\sim 238 \mathrm{~m}^{2} \mathrm{~g}^{-1}$ [192]) [49, 193] and an unspecified porous carbon with high oxygen content [48]. This may suggest that the EC300 (BET of $\sim 720 \mathrm{~m}^{2} \mathrm{~g}^{-1}$ ) carbon is more resistant to the complete COR than Vulcan XC-72 (a commonly employed PEMFC catalyst support) despite its higher BET area; however, the comparison between DEMS studies is not necessarily straightforward because previous studies were not quantitative, and the $\mathrm{CO}_{2}$ detection limit of the DEMS instruments are not given. The sample loadings of carbon material in the two more recent DEMS studies of $61 \mu \mathrm{g}_{\mathrm{c}} \mathrm{cm}^{-2}$ [193], $142 \mu \mathrm{g}_{\mathrm{c}} \mathrm{cm}^{-2}$ [48] are nevertheless comparable to this study (50 and $85 \mu g_{c} \mathrm{~cm}^{-2}$ ) although a much higher loading of $5 \mathrm{mg}_{\mathrm{c}} \mathrm{cm}^{-2}$ was used in the earliest DEMS study [30] which could therefore prove to be more sensitive. Possible approaches to improving the DEMS instrument sensitivity are later discussed in section 6.4.6. 
The Tafel-slopes extracted from the observed $I_{C O 2}$ meanwhile, were in the range of $200 \pm 30 \mathrm{mV} \mathrm{Dec}{ }^{-1}$ above $1.8 \mathrm{~V}_{\mathrm{RHE}}$, although shallower slopes of 413 and $307 \mathrm{mV} \mathrm{Dec}^{-1}$ were observed on the EC300 and mildly graphitised HSAC in the potential region below $1.8 \mathrm{~V}_{\mathrm{RHE}}$. These slopes are slightly steeper than the $270 \mathrm{mV} \mathrm{Dec}{ }^{-1}$ obtained from the measured faradaic electrode current using chronoamperometry in phosphoric acid at the lower potential 0.8 and $1.2 \mathrm{~V}_{\mathrm{SHE}}$ between 135 and $148{ }^{\circ} \mathrm{C}$ for Vulcan XC-72 and its graphitised counterpart heat treated at $2700{ }^{\circ} \mathrm{C}$ [194] (and similarly ref. [195]). The corresponding transfer coefficients $\left(\alpha^{*} n\right)$ obtained from the Tafel-slopes meanwhile can be compared to values used in various PEMFC corrosion studies such 0.275 [152], 0.66 [177] 1.0 [153] which measured and/or modelled the carbon corrosion over a range of temperature, potential and time. The values obtained in the electrochemical cell environment of this DEMS study, ranged between 0.143 and 0.354 , which are more comparable to the value of 0.275 used in ref. [152]. The usefulness of the Tafel-slopes and their kinetic constants is, however, not entirely clear at this stage. It may be tempting to extrapolate the Tafel-slopes down in potential to obtain COR rates at more practical PEMFC potentials ca. $<1.2 \mathrm{~V}_{\mathrm{RHE}}$ (with considerable errors) however, this would assume that the potential dependence of the COR processes are rather constant over time, and that the COR rate is independent of the degree of previous oxidation, neither of which will be shown to be the case.

Overall, phase (i) of the electrochemical treatment has established that the complete $\mathrm{COR}$ to $\mathrm{CO}_{2}$ occurs in combination with a significant amount of partial COR, particularly on the pristine EC300 carbon black. The electrochemical COR rates are found to be significantly lower on the graphitised samples, particularly when heat treated $\geq 2450{ }^{\circ} \mathrm{C}$ with a trend of decreasing COR rates with increasing heat-treatment temperature observed. In order to speculate further on the COR mechanism(s) the HSAC supports require greater characterisation using techniques such as XRD and $i R$-spectroscopy or XPS. The observations so far, however, are only relevant to the COR processes in the very first high potential excursion. 


\subsubsection{Phase (ii) - Repeated High Potential Excursions}

A further three high potential CVs were performed between 1.05 and $2.05 V_{\text {RHE }}$ in phase (ii) of the electrochemical treatment in order to assess the changes that occur in the COR tendencies of each of the carbon black samples. The observed behaviours can be separated into three trends represented by the EC300, and heat treated samples $5\left(300{ }^{\circ} \mathrm{C}\right)$ and $4\left(2100{ }^{\circ} \mathrm{C}\right)$, given in figure 6-8, figure 6-9 and figure 6-10 respectively. The data for the remaining samples are given in Appendix B.

In figure 6-8 we observe the first trend, which was that following the very first positive high potential excursion the electrochemical COR of the EC300 support slowed significantly in the reverse potential sweep, coming to rather abrupt end.

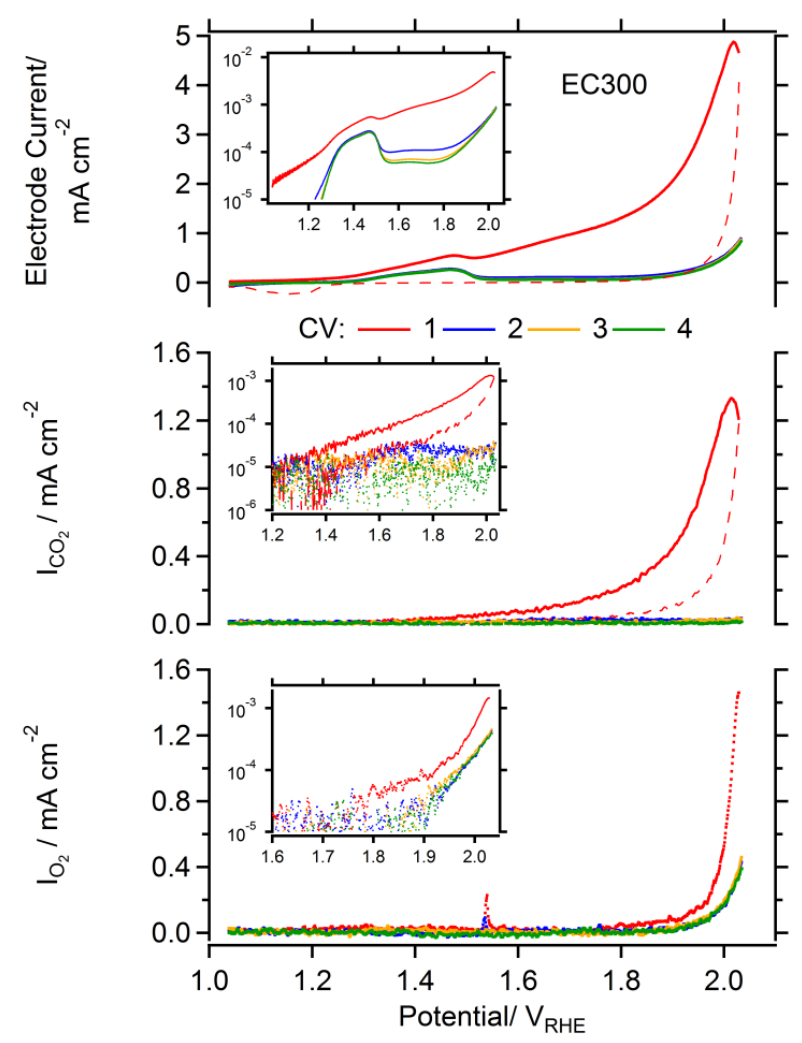

Figure 6-8: First four LSVs of the electrochemical COR of a pristine EC300 HSAC support in $0.5 \mathrm{~mol} \mathrm{dm}^{-3} \mathrm{H}_{2} \mathrm{SO}_{4}$. EC300 sample loading of $50 \mathrm{ug}_{\mathrm{c}} \mathrm{cm}^{-2}$ on polycrystalline Au WE substrate. Following a rather high amount of $\mathrm{COR}$ in the first $\mathrm{CV}$, the COR ceases almost completely in subsequent CVs between 1.05 and 2.05 $\mathrm{V}_{\mathrm{RHE}}$. 
In the three subsequent LSVs between 1.05 and $2.05 \mathrm{~V}_{\mathrm{RHE}}$, very little or no $\mathrm{CO}_{2}$ was observed and only small amounts of electrode current are present above the Au substrate background (seen logarithmic plot inset). Furthermore, there is no evidence of the $\mathrm{O}_{2}$ evolution from the $\mathrm{EC300}$ following the first $\mathrm{CV}$, whereby the $\mathrm{O}_{2}$ measured in the three subsequent CVs can be solely attributed to the OER on the polycrystalline Au WE substrate.

The second trend, exhibited by the more heavily graphitised samples $3\left(2450{ }^{\circ} \mathrm{C}\right)$, $2\left(2800{ }^{\circ} \mathrm{C}\right), 5\left(3000{ }^{\circ} \mathrm{C}\right)$ and $1\left(3200{ }^{\circ} \mathrm{C}\right)$, was that the electrochemical oxidation rate gradually increased for each successive $\mathrm{CV}$, which is represented by the CVs of sample $5\left(3000{ }^{\circ} \mathrm{C}\right)$ in figure 6-9.

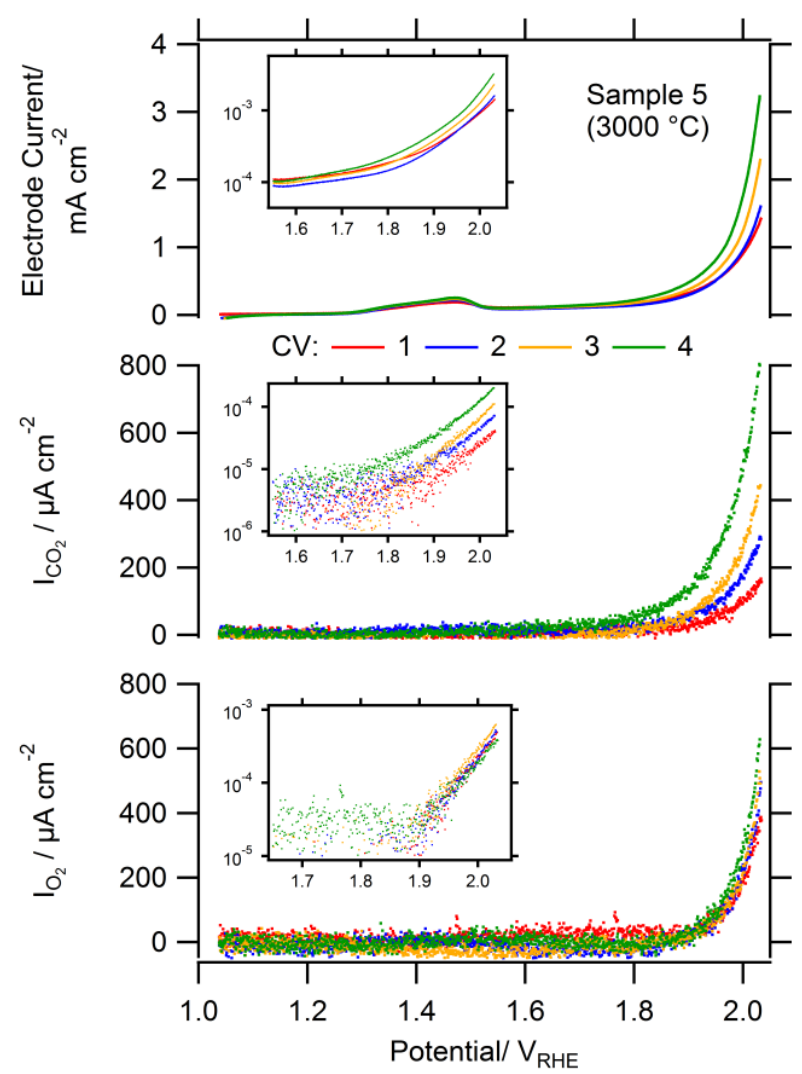

Figure 6-9: First four LSVs of the electrochemical COR of graphitised Sample $5\left(3000{ }^{\circ} \mathrm{C}\right) \mathrm{HSAC}$ support in $0.5 \mathrm{~mol} \mathrm{dm}{ }^{-3} \mathrm{H}_{2} \mathrm{SO}_{4}$. Sample 1 loading of $85 \mathrm{ug}_{\mathrm{c}} \mathrm{cm}^{-2}$ on polycrystalline Au WE substrate. The rate of electrochemical oxidation of the highly graphitised support increases with each subsequent $\mathrm{CV}$ between 1.05 and 2.05 $\mathrm{V}_{\mathrm{RHE}}$.

The electrode current and corresponding $I_{C O 2}$ clearly increase with each successive LSV whilst the Tafel-slopes of the $I_{C O 2}$ (see insert) appears to remain 
fairly constant ( $\left.167 \mathrm{mV} \mathrm{Dec}^{-1}\right)$. All $\mathrm{O}_{2}$ observed can meanwhile be attributed to the OER polycrystalline Au WE substrate background only.

The final trend demonstrated by sample $4\left(2100{ }^{\circ} \mathrm{C}\right)$, was that the rate of electrochemical oxidation increased during the first few LSVs (1 to 3), but then decreased in the final fourth LSV of the phase (ii) electrochemical treatment, as shown in figure 6-10.
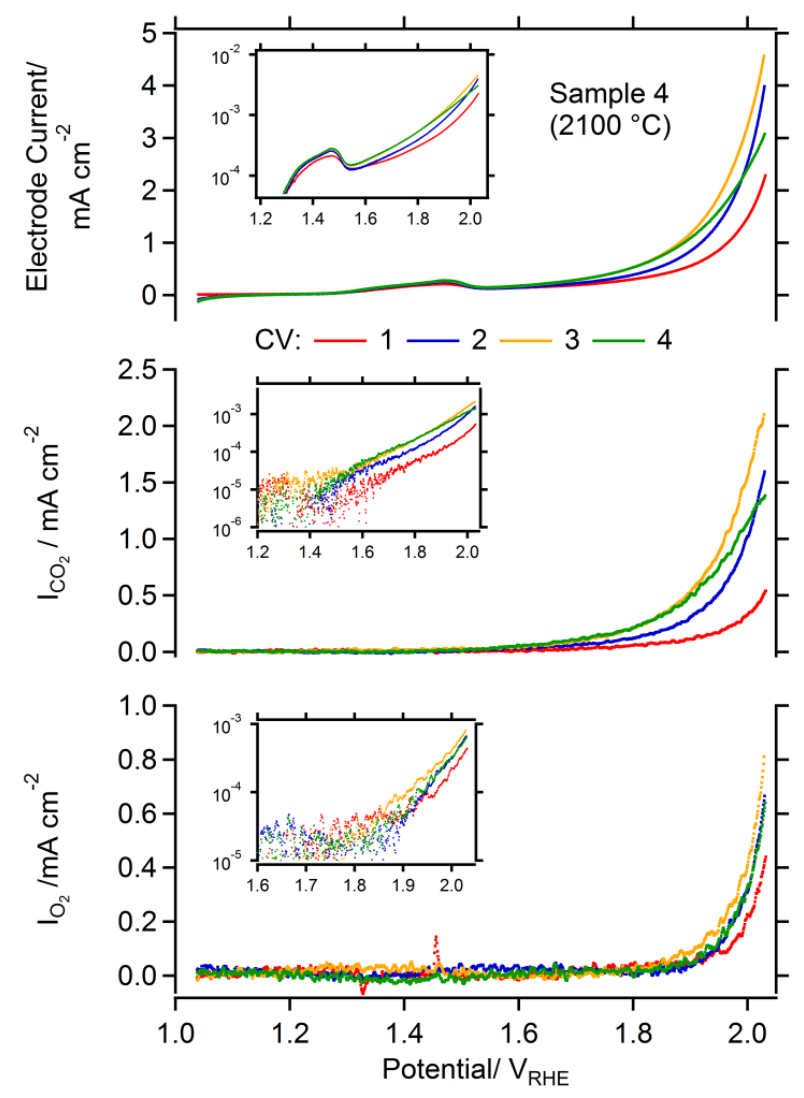

Figure 6-10: First four LSVs of the electrochemical COR of graphitised Sample $4\left(2100{ }^{\circ} \mathrm{C}\right)$ HSAC support in $0.5 \mathrm{~mol} \mathrm{dm}^{-3} \mathrm{H}_{2} \mathrm{SO}_{4}$. Sample 4 loading of $85 \mathrm{ug}_{\mathrm{c}} \mathrm{cm}^{-2}$ on polycrystalline Au WE substrate. The rate of electrochemical oxidation of the mildly graphitised support initially increases in the first three successive CVs, before being observed to decrease in the final $\mathrm{CV}$ between 1.05 and $2.05 \mathrm{~V}_{\text {RHE}}$.

The small changes observed in the measured $\mathrm{O}_{2}$, however, reflect the quality of this particular measurement rather than any $\mathrm{O}_{2}$ evolution on the carbon. Again, the Tafel-slopes remain reasonably constant (see inset).

In order to reveal these qualitative trends more quantitatively, the electrode charge evolved which can attributed to the complete and partial COR processes 
during each $\mathrm{CV}$ are plotted as a function of the cumulative charge corresponding to complete $\mathrm{COR}$ (obtained from $I_{\mathrm{CO}_{2}}$ ). By viewing the data in this way we are able to bring greater focus onto the dependence of the partial and complete COR processes on the prior quantity of complete COR of the carbon black sample, and therefore the significance of each CV. The data obtained during the first four CVs (whereby each point corresponds to the charge in each CV) for the carbon samples is summarised in figure 6-11.
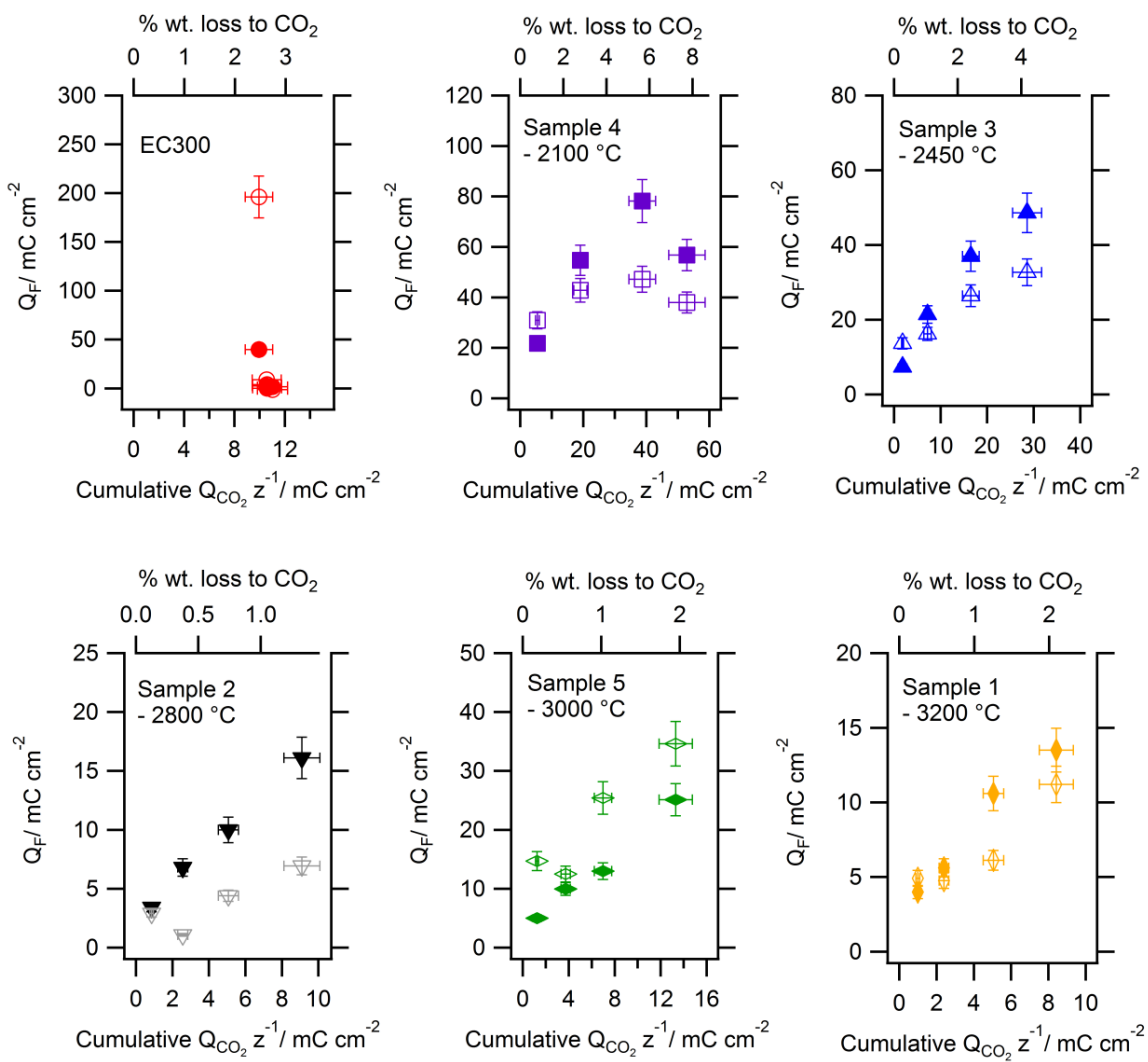

Figure 6-11: Integrated charge of the partial (hollow markers) and complete (solid markers) electrochemical COR processes for each of the carbon black samples during the first four CVs between 1.05 and $2.05 \mathrm{~V}_{\mathrm{RHE}}$ in $0.5 \mathrm{~mol} \mathrm{dm}{ }^{-3}$ Three trends are observed, either the quantity of electrochemical COR is: (i) initially high and ends abruptly (EC300), (ii) initially increases and then falls (Sample 4) or (iii) increases (Samples 1-3 and 5), for each successive CV.

In these plots, we can more clearly observe the three trends exhibited by the carbon blacks interpreted from the LSVs (in previous figures) in a more quantitative manner. In all cases, we see that the complete COR occurs simultaneously with the additional electrode charge attributed to the partial COR process. For the EC300, the faradaic charge arising from the partial COR 
significantly dominates that of complete $\mathrm{COR}$ to $\mathrm{CO}_{2}$ in the first $\mathrm{CV}$ whereas for heat-treated samples the relative charges are far more comparable. In the case of the mildly graphitised carbon black sample $4\left(2100{ }^{\circ} \mathrm{C}\right)$ the charge arising from the COR reached a maximum during the third $C V$. The complete and partial COR of the remaining more graphitised HSAC samples $\left(\geq 2450{ }^{\circ} \mathrm{C}\right)$ meanwhile increase with progressive oxidation. At this point in the electrochemical oxidation treatment, the EC300 and graphitised samples $4\left(2100{ }^{\circ} \mathrm{C}\right)$ and $3\left(2450{ }^{\circ} \mathrm{C}\right)$ have undergone the most complete $\mathrm{COR}$ to $\mathrm{CO}_{2}$ and consequently lost the most significant weight of carbon $(\sim 3,8$ and $4.5 \%$ respectively), although the COR have either ceased (EC300) or show signs of slowing (Sample 4). The more heavily graphitised samples (heat-treated $\geq 2800{ }^{\circ} \mathrm{C}$ ) have in comparison proven to be far more resistant to the complete COR and lost significant less weight in carbon $(<2.5 \%)$, although the complete COR rates are in those cases clearly increasing.

In the first four CVs (phase (i) and (ii) the electrode potential was scanned between 1.05 and $2.05 \mathrm{~V}_{\mathrm{RHE}}$, and the potential of the carbon black is therefore always in thermodynamically favoured carbon oxidation potential region i.e. > $0.207 \mathrm{~V}_{\mathrm{RHE}}$. Consequently, the next step of the electrochemical treatment was to investigate the effect of lowering electrode potential limit, and therefore the possible influence of the reduction on the now oxidised (in phase (i) and (ii)) carbon blacks in phase (iii) of the treatment.

\subsubsection{Phase (iii) - Reduction of the Lower Potential Limit}

In phase (iii) of the electrochemical treatment, the effect of the lower electrode potential limit on the COR of the oxidised (in phase (ii)) carbon blacks was investigated by progressively decreasing the lower potential limit from the 1.05 $\mathrm{V}_{\mathrm{RHE}}$ (lower limit in phase (ii)), to $0.05 \mathrm{~V}_{\mathrm{RHE}}$ in steps of $200 \mathrm{mV}$ in each of the six subsequent CVs. The observations made can be again be categorised into three trends, influenced by the consequence of the lower potential limit, each of which is represented by the EC300 and heat-treated Samples $1\left(3200{ }^{\circ} \mathrm{C}\right)$ and 3 $\left(2450{ }^{\circ} \mathrm{C}\right)$ in figure 6-12, figure 6-13 and figure 6-14 respectively. The 
corresponding data for the remaining samples are given in Appendix B. The first trend, exhibited by the EC300 sample is shown in figure 6-12.
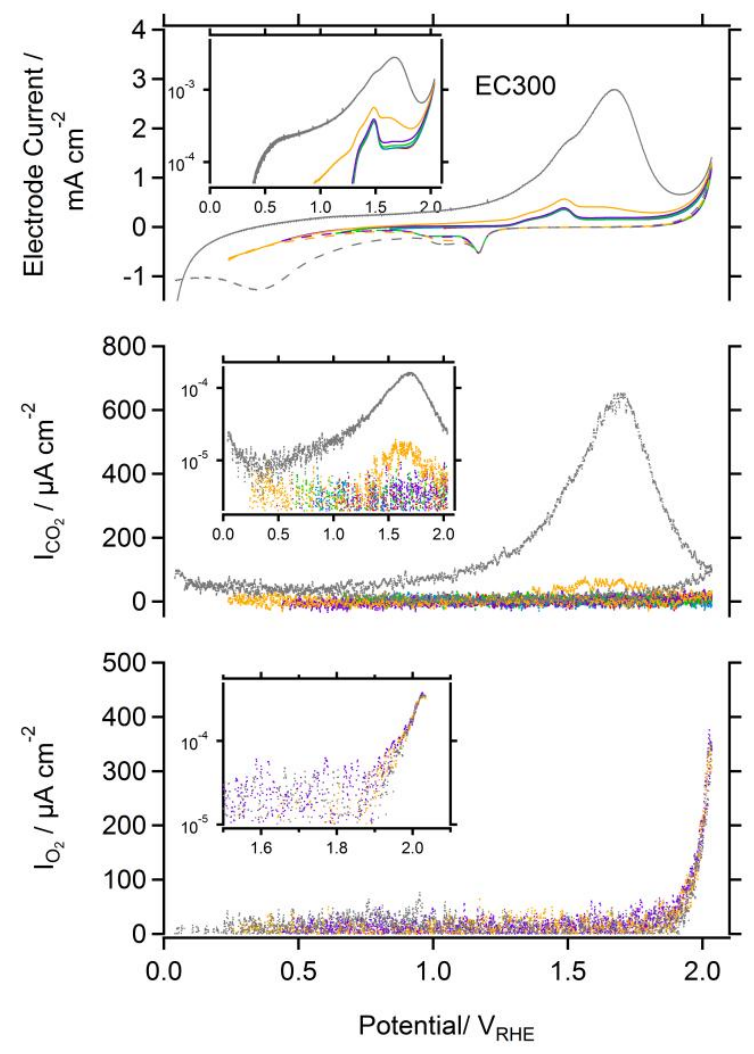

\begin{tabular}{|l}
\hline Lower Potential Limit $\left(\mathrm{V}_{\mathrm{RHE}}\right)$ : \\
$-1.04(1 \mathrm{st})-0.64(3 \mathrm{rd})-0.24(5 \mathrm{th})$ \\
$-0.84(2 \mathrm{nd})-0.44(4 \mathrm{th})-0.04(6 \mathrm{th})$ \\
\hline
\end{tabular}

Figure 6-12: Effect of the lower potential limit on the electrochemical COR of electrochemically oxidised EC300 carbon black in $0.5 \mathrm{~mol} \mathrm{dm}{ }^{-3} \mathrm{H}_{2} \mathrm{SO}_{4}$. Sample loading of $50 \mathrm{ug}_{\mathrm{c}} \mathrm{cm}^{-2}$ on a polycrystalline Au WE substrate. The electrochemical COR is significantly re-activated by reducing the lower electrode potential limit to $<0.25 \mathrm{~V}_{\mathrm{RHE}}$ of the electrochemically oxidised EC300 sample.

By lowering the WE potential to $0.05 \mathrm{~V}_{\mathrm{RHE}}$ and reducing the EC300 HSAC, we observe that the quantity of electrochemical oxidation current that occurs during the subsequent positive high potential sweep is not only significant, but also begins at the considerably lower electrode potential of $\sim 0.5 \mathrm{~V}_{\mathrm{RHE}}$ peaking at $\sim 1.7 \mathrm{~V}_{\text {RHE}}$. Furthermore, the reduction of the HSAC appears to coincide with some non-oxidative evolution of $\mathrm{CO}_{2}$ at potentials $<0.207 \mathrm{~V}_{\mathrm{RHE}}$. 
The second electrochemical oxidation trend was exhibited by the HSAC carbon samples heat treated at the higher temperatures $\geq 2800{ }^{\circ} \mathrm{C}$, and is demonstrated by sample $1\left(3000{ }^{\circ} \mathrm{C}\right)$ in figure 6-13.
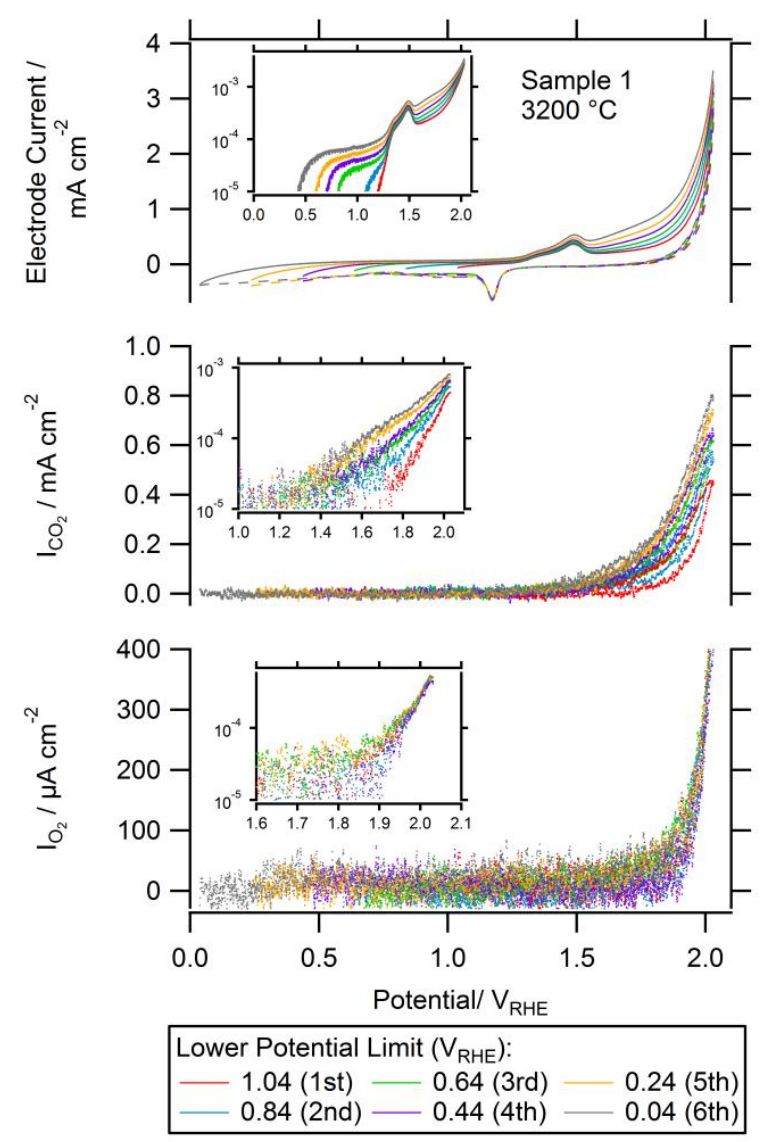

Figure 6-13: Effect of the lower potential limit on the electrochemical COR of electrochemically oxidised and graphitised Sample $1\left(3200{ }^{\circ} \mathrm{C}\right)$ carbon black in $0.5 \mathrm{~mol} \mathrm{dm}^{-3} \mathrm{H}_{2} \mathrm{SO}_{4}$. Sample loading of $85 \mathrm{ug}_{\mathrm{c}} \mathrm{cm}^{-2}$ on a polycrystalline Au WE substrate. The lower electrode potential limit does not appear to influence the electrochemical COR of the partially electrochemically oxidised highly graphitised HSAC sample.

In contrast to the profound effect that the reduction of the EC300 carbon black had on the complete $\mathrm{COR}$ to $\mathrm{CO}_{2}$, these heat-treated samples are seemingly unaffected with no oxidation peak at $1.7 \mathrm{~V}_{\mathrm{RHE}}$. Instead the samples follow the same increasing trend in the COR rates observed in phase (ii) of the experiment.

The final trend is observed for both the mildly heat-treated samples $4\left(2100{ }^{\circ} \mathrm{C}\right)$ and $3\left(2450{ }^{\circ} \mathrm{C}\right)$, the latter of which is given in figure 6-14. 

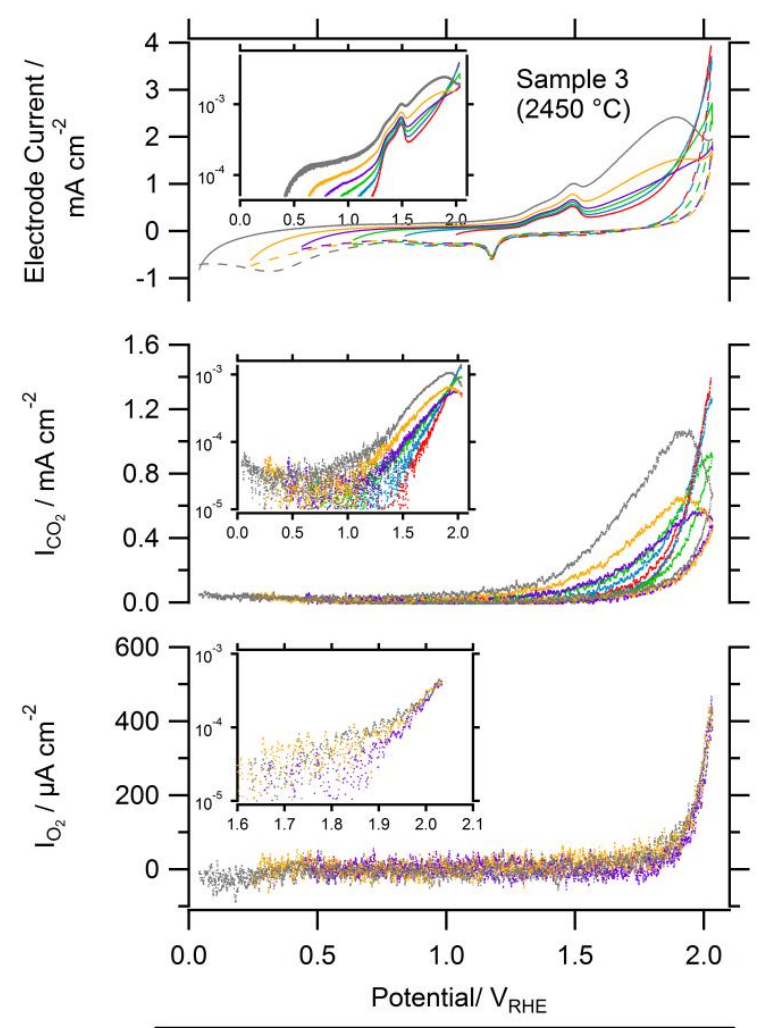

Lower Potential Limit $\left(\mathrm{V}_{\mathrm{RHE}}\right)$ :
$-1.04(1 \mathrm{st})-0.64(3 \mathrm{rd})-0.24$ (5th)
$0.84(2 \mathrm{nd})-0.44(4 \mathrm{th})-0.04(6 \mathrm{th})$

Figure 6-14: Effect of the lower potential limit on the electrochemical COR of electrochemically oxidised and graphitised Sample $3\left(2450{ }^{\circ} \mathrm{C}\right)$ carbon black in $0.5 \mathrm{~mol} \mathrm{dm}^{-3} \mathrm{H}_{2} \mathrm{SO}_{4}$. Sample loading of $85 \mathrm{ug}_{\mathrm{c}} \mathrm{cm}^{-2}$ on a polycrystalline Au WE substrate. The electrochemical COR is re-activated by reducing the lower electrode potential limit to $<0.25 \mathrm{~V}_{\mathrm{RHE}}$ of the partially electrochemically oxidised mildly graphitised HSAC sample.

By lowering the WE potential to $0.05 \mathrm{~V}_{\mathrm{RHE}}$ and reducing the carbon surface, a COR peak at $\sim 1.7 \mathrm{~V}_{\mathrm{RHE}}$ along with a hint of the non-oxidative release of $\mathrm{CO}_{2}<$ $0.207 V_{\text {RHE }}$ which coincides with reduction processes of the carbon black, as was observed on the EC300 sample. In this case, however, the electrochemical oxidation of the mildly graphitised carbon blacks had not ceased during the first four CVs (although sample 4 was decreasing) in phase (ii) of the treatment, and consequently the COR is enhanced rather than re-initiated. In all of the measurements during phase (ii and iii) the $\mathrm{O}_{2}$ evolved can again be attributed to the OER on the polycrystalline Au substrate.

In order to once again, summarise the trends observed in the phase (iii) of the electrochemical treatment, a plot of the partial and complete electrochemical 
$\mathrm{COR}$ charges is given as a functional of the cumulative $\mathrm{CO}_{2}$ evolved for each $\mathrm{CV}$ in figure 6-15. In the interpretation of this summary plot, it should be kept in mind that for each point, corresponding to each $\mathrm{CV}$, the lower limit was progressively decreased by $200 \mathrm{mV}$, from 1.05 to $0.05 \mathrm{~V}_{\mathrm{RHE}}$.
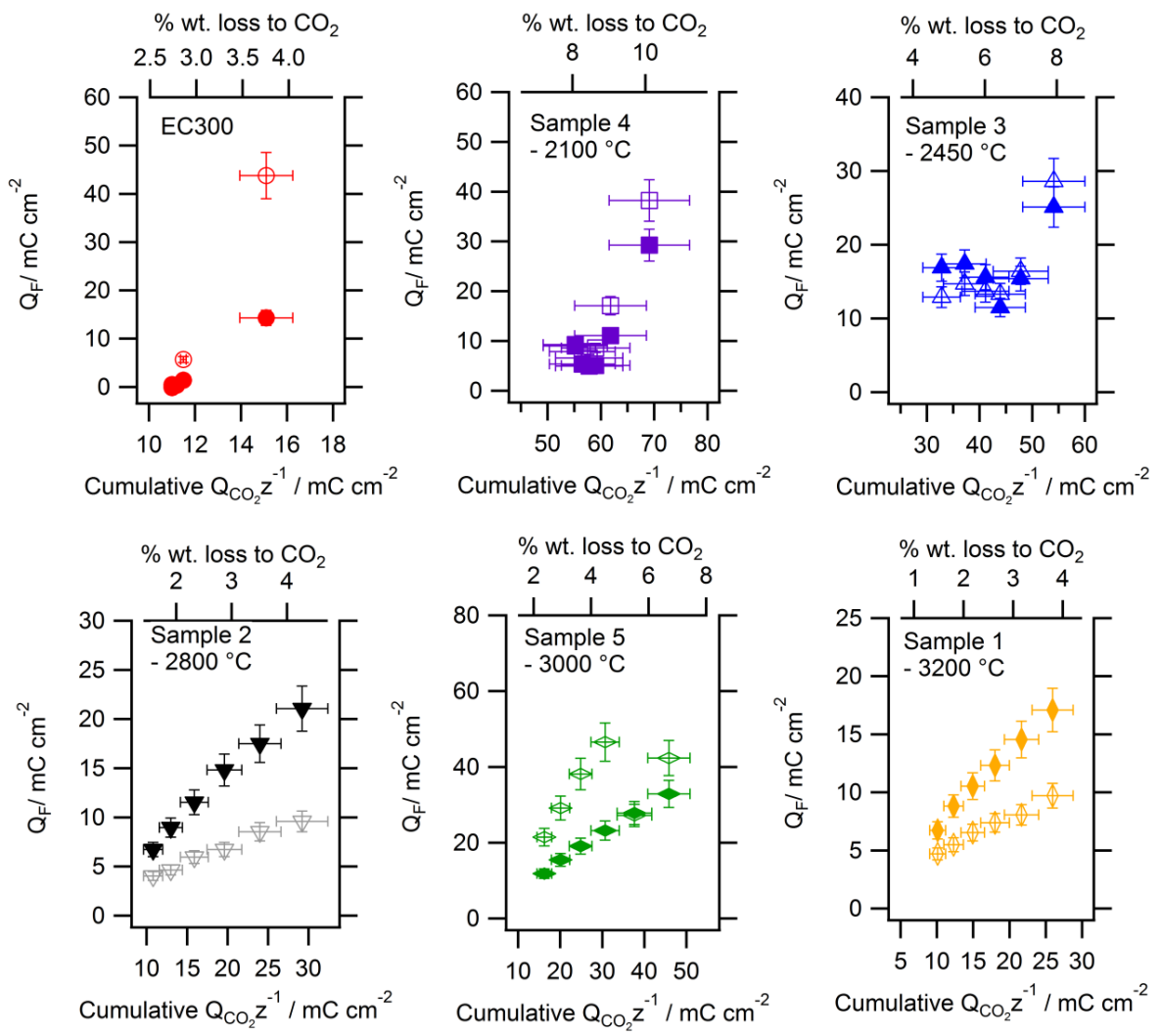

Figure 6-15: Integrated charge of the partial (hollow markers) and complete (solid markers) electrochemical COR of the carbon black samples during the phase (iii) of the electrochemical treatment. The lower potential limit was progressively decreased from 1.05 to, $0.05 V_{R H E}$ in $200 \mathrm{mV}$ steps maintaining an upper limit of $2.05 \mathrm{~V}_{\mathrm{RHE}}$ in $0.5 \mathrm{~mol} \mathrm{dm}^{-3}$ Three trends are observed, either: (i) re-activated (EC300), (ii) enhanced (Samples 3-4) or (iii) unaffected (Samples 1-2 and 5), by lowering potential limit to $<0.25 \mathrm{~V}_{\mathrm{RHE}}$.

In this figure we observe that both the partial and complete COR of the carbon surface occur simultaneously on all the carbon blacks (analogous to phase (ii)). For the EC300 and mildly graphitised samples (samples $3\left(2450{ }^{\circ} \mathrm{C}\right)$ and 4 $\left(2100{ }^{\circ} \mathrm{C}\right)$ ), the charge corresponding to the partial and complete oxidation is increased significantly upon lowering the electrode potential to $0.05 \mathrm{~V}_{\mathrm{RHE}}$. In contrast, there appears to be little or no effect on the electrochemical oxidation of the carbon samples treated at the higher temperatures $\left(\geq 2800{ }^{\circ} \mathrm{C}\right)$, although it is difficult in this plot to interpret with any certainty. In order to bring greater 
focus to the influence of the lower electrode potential limit therefore, the differential complete COR charge for each subsequent $\mathrm{CV}$ is plotted as a function of the lower electrode potential limit in figure 6-16.
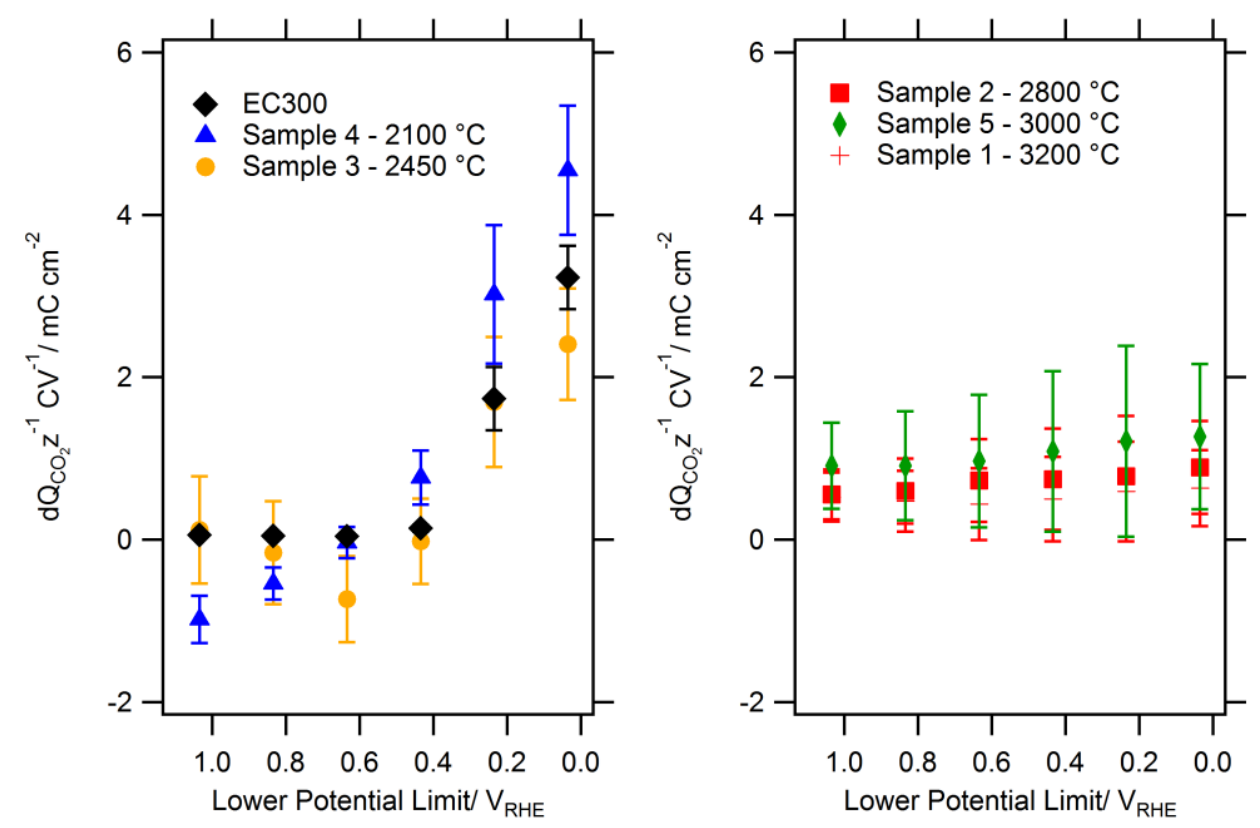

Figure 6-16: The change in the integrated charge of the complete electrochemical COR of the HSAC carbon samples for each successive $\mathrm{CV}$ as a function of the lower electrode potential limit in $0.5 \mathrm{~mol} \mathrm{dm}^{-3}$. The electrochemical COR of EC300 and mildly graphitised carbon black samples are enhanced in the subsequent high potential excursion by reducing the electrode potential < $0.45 V_{\text {RHE. }}$

This plot clearly shows that by decreasing the lower electrode potential limit to $<0.44 \mathrm{~V}_{\mathrm{RHE}}$, the complete COR charge increases in the subsequent $\mathrm{CV}$ on the EC300 and mildly heat-treated $\left(2100\right.$ to $\left.2450{ }^{\circ} \mathrm{C}\right)$ samples which exhibited the peak at 1.7 $\mathrm{V}_{\mathrm{RHE}}$. In contrast, the electrochemical oxidation COR processes of the samples treated at higher temperatures $\left(>2800{ }^{\circ} \mathrm{C}\right)$ remain uninfluenced by the lowering of the electrode potential limit.

\subsection{Discussion of the Long-term COR Tendencies}

Phases (ii) and (iii) of the electrochemical oxidation treatment demonstrated that the COR processes are dynamic, revealing three trends in the behaviour of the carbon black samples to repeated electrochemical oxidation, which may 
furthermore be influenced by the reduction of the carbon. Despite the initially high rates of the complete and (in particular) partial COR on the EC300, the oxidation of the carbon surface was observed to be comparatively short-lived. In contrast, the COR rates on the more heavily graphitised samples (heat-treated $\geq 2800{ }^{\circ} \mathrm{C}$ ) progressively increased during phase (ii) of the treatment whereas in the final trend exhibited by the mildly graphitised samples $\left(\leq 2450{ }^{\circ} \mathrm{C}\right)$, the $\mathrm{COR}$ rate initially increased before showing signs of decreasing in phase (ii) or early in phase (iii) of the electrochemical treatment. In phase (iii) of the experiment, it was then found that by decreasing the lower electrode potential limit to $<0.240 \mathrm{~V}_{\mathrm{RHE}}$ and reducing the carbon, the complete and partial COR rates of the EC300 and mildly graphitised carbon blacks could be re-initiated or enhanced, giving rise to a new COR feature peaking at $1.7 \mathrm{~V}_{\mathrm{RHE}}$. In stark contrast, the COR processes of the heavily graphitised carbons were seemingly unaffected by the lower electrode potential limit, simply continuing the trend observed in phase (ii) of the electrochemical treatment. Essentially, the COR rates can progressively decrease or increase, and by reducing the carbon, become re-initiated or enhanced in subsequent oxidation.

Although there is no commonly accepted mechanism describing the COR processes, previous work has stressed the importance of the oxygen containing surface functional groups on both the initial and long-term reactivity of carbon blacks [169, 176]. Often, the initial COR rates have been found to correlate positively with the oxygen content of the pristine carbon material (i.e. concentration of oxygen-containing surface functional groups), whereas various terms such as phase-, protective-, stable- or surface-oxide, oxide intermediates and complexes, have been used to try and describe the apparent influence of the more stable oxygen-containing surface functional groups on COR during the course of an experiment (see discussion and references in ref. [169]). It has previously been proposed previously that many of the 'apparently contradictory results and interpretations (either electrochemical or gas phase oxidation [studies - prior to 1989]) could be reconciled by invoking the relative stability of these oxides as the main discriminating factor' going further to state that 'the more stable oxides might play the role of decreasing the active surface area (ASA) of the carbon' whereas 'the formation of each product molecule, $\mathrm{CO}$ or $\mathrm{CO}_{2}$, is accompanied by 
regenerations of a free site and subsequent formation of another complex' [169] citing ref. [196, 197]. It is this approach of considering the relative stability of the oxygen containing surface functional groups formed, and their influence on the electrochemically oxidisable surface area (EOSA) of the carbon that will be used to rationalise the trends observed in this study.

The initial rate of the COR processes on the carbon black will ultimately be determined by the nature and concentration of the electrochemically oxidisable surface functional groups that can be considered to act as nucleation sites for either the complete oxidation to $\mathrm{CO}_{2}$, or partial oxidation to either $\mathrm{CO}$ or surface oxygen containing functional group(s) i.e. they define the EOSA of the carbon. If we consider that the $\mathrm{COR}$ to either $\mathrm{CO}_{2}$ or $\mathrm{CO}$ must involve the breakage of one or more $\mathrm{C}-\mathrm{C}$ bonds which will in-turn deteriorate the microstructure of the carbon generating at least one new active site, then these COR processes can be considered to either maintain or increase the EOSA (unless of course the carbon microstructure is completely consumed). Alternatively, if the partial COR proceeds to form oxygen containing surface functional group(s) that are stable (i.e. non-electrochemically oxidisable), then any further oxidation to $\mathrm{CO}_{2}$ or $\mathrm{CO}$ will cease at this site, effectively decreasing the EOSA and consequently lowering the COR rate. The long-term electrochemical oxidation processes of the carbon black, therefore, will be determined by the nature of the oxygen containing surface functional groups that are formed after the initial oxidation of the pristine carbon sample, which in turn will be influenced by the carbon black microstructure, and experimental conditions.

In the first trend observed for the EC300 carbon, the complete and partial COR processes came to an abrupt end following relatively fast initial COR rates. A sceptical interpretation may have been (given the nature of the abrupt end to the COR processes) that the carbon sample is simply lost from the WE substrate; however, in phase (iii) of the electrochemical treatment we observed that this was simply not the case and in any case the quantity of $\mathrm{CO}_{2}$ evolved is anyway far from insufficient $(\sim 4 \%$ wt.) to account for the consumption of the sample. The EOSA of the EC300 carbon is simply diminishing to zero during the initial potential sweep. One partial explanation for this observation may be that only a 
certain proportion of the EC300 carbon (given the in-homogeneous nature of carbon blacks) is readily electrochemically oxidised, ceasing the COR once consumed. In previous gas phase [198] and fuel cell studies [176], for example, it has been reported that following the oxidation of the carbon black, the more amorphous core of the carbon structure appears to have preferentially oxidised over the graphene outer layers. The outer carbon layer of the aciniform structure consequently remains largely intact and a hollow structure is formed. This phenomena has been used to explain the apparent decrease in the COR of a nongraphitised carbon-black (Vulcan XC-72) in the PEMFC environment [156] and can also be considered a contributing factor to the decrease in the EOSA (and COR rate) in this study. The significant partial COR charge, however, suggest another contributing factor toward the abrupt end in the COR of the EC300 carbon. If the COR of the carbon surface favours the formation of stable nonelectrochemically oxidisable oxygen-containing surface functional groups (as suggested by the partial COR charge and also phase (iii) of the measurement), then the EOSA of the EC300 carbon would decrease slowing the COR. The abrupt end in the COR on the EC300 carbon would suggest that the effect is so strong that it completely diminishes the EOSA. This behaviour would be analogous to the description of a protective oxide described in a previous carbon corrosion study [199].

The COR rates on the more heavily graphitised carbons $\left(\geq 2800{ }^{\circ} \mathrm{C}\right)$ on the other hand was initially rather low, but progressively increased with cumulative electrochemical oxidation, suggesting that the EOSA of the carbon is increasing during the course of the experiment. An increase in the EOSA could conceivably arise from a number of consequences of the COR processes. For instance, the breakage of $\mathrm{C}-\mathrm{C}$ bonds would deteriorate the ordered structure of the graphene planes, conceivably opening sub-surface voids (as suggested in ref. [200]) forming micropores [201] and increasing the total surface area, along with the EOSA. Alternatively, it has also been suggested that the electrochemical oxidation of carbon black only develops after a critical concentration of oxygen-containing functional groups on the surface [169], although it is not entirely clear why this should be unless it influences the nature of the oxygen-containing functional groups that are formed. Either way, the apparent increase in the COR rate of the 
progressively electrochemically oxidised-heavily graphitised carbons $\left(\geq 2800{ }^{\circ} \mathrm{C}\right)$ is analogous observations in a previous DEMS study which found that the $\mathrm{CO}_{2}$ generated at $1.5 \mathrm{~V}_{\mathrm{RHE}}$ from oxidised-graphitised carbon sample is greater than that of the pristine graphitised (as well as raw) carbon black counterpart [48]. In the PEMFC environment, however, the complete COR rates of graphitised carbon containing MEAs have been observed to be rather constant [156]. The mildly graphitised carbons $\left(\leq 2450{ }^{\circ} \mathrm{C}\right)$ meanwhile appear to exhibit a combination of both the COR tendencies shown for the pristine EC300 and heavily graphitised carbons, which became more evident in phase (iii) of the electrochemical treatment.

The COR trends observe in phase (ii) of the experiment, are complicated by the potentially significant influence of the lower potential limit, or rather the reduction of the apparently stable oxygen-containing surface functional group(s) proposed to have formed during phase (i) and (ii) of the experiment. The partial and complete COR of the EC300 and mildly graphitised HSAC supports may be re-activated or enhanced by lowering the electrode potential to $<0.240 \mathrm{~V}_{\mathrm{RHE}}$. Although that the stable oxygen-containing surface functional group(s) cannot be electrochemically oxidised, it appears that by reducing these functional groups the carbon surface can then be completely oxidised via an alternate pathway, avoiding the formation of the stable oxygen-containing functional group. The partial and complete CORs on the heavily graphitised HSAC supports meanwhile, were in contrast unaffected by the lower potential limit, suggesting that the partial COR of the heavily graphitised carbon (with more graphitic character) does not form these non-electrochemical oxidisable oxygen-containing surface functional group(s) which upon being reduced may be oxidised in the subsequent high potential excursion. The formation of the passivating non-electrochemically oxidisable functional group(s) has a significant impact on the total COR charge evolved over the course of the electrochemical treatment and will be discussed later.

It is premature to start discussing the identity of the oxidising and more stable oxygen-containing functional groups, however, if we consider that the carbon black microstructure are essentially organic molecular structures (albeit rather 
large ones) then an understanding of carbon corrosion mechanism(s) can conceivably utilise much from the field of organic electrochemistry in order to aid in the understanding of the relative reactivities of the surface oxygen-containing functional groups. For example, the oxidation of the carboxylic acid functional group is well-studied in organo-electrosynthesis because of its importance in the Kolbe reaction. Carboxylic acids may electrochemically oxidise to yield a $\mathrm{CO}_{2}$ molecule and either a radical or carbocation intermediate, the latter of which may then react further via nucleophile substitution, deprotonation or $\mathrm{C}-\mathrm{C}$ bond cleavage/rearrangement forming a new functional group [202]. Alcohols meanwhile can be oxidised to carboxylic acids or ketones [203] whilst hydrocarbons may undergo direct anodic oxidation under certain conditions [204]. Lactones on the other hand are not electrochemically oxidisable (or there are no examples) but can be reduced to carboxylic acids, diols or aldehydes [205]. These are just a handful of the reactions that surface functional groups present on the carbon surface can undergo, and a glance inside ref. [206, 207] will quickly demonstrate just how complex the COR processes may be.

After establishing some understanding of the dynamic behaviours of the COR processes, along with the influence of the reduction of the carbon, it is now appropriate to evaluate the overall effect of the electrochemical treatment on the carbon samples in the following section. 


\subsubsection{Overall Effect of Electrochemical Oxidation Treatment}

The total quantity of the complete and partial COR charges evolved during the electrochemical oxidation treatment are summarised as a function of the heattreatment temperature in figure 6-17.

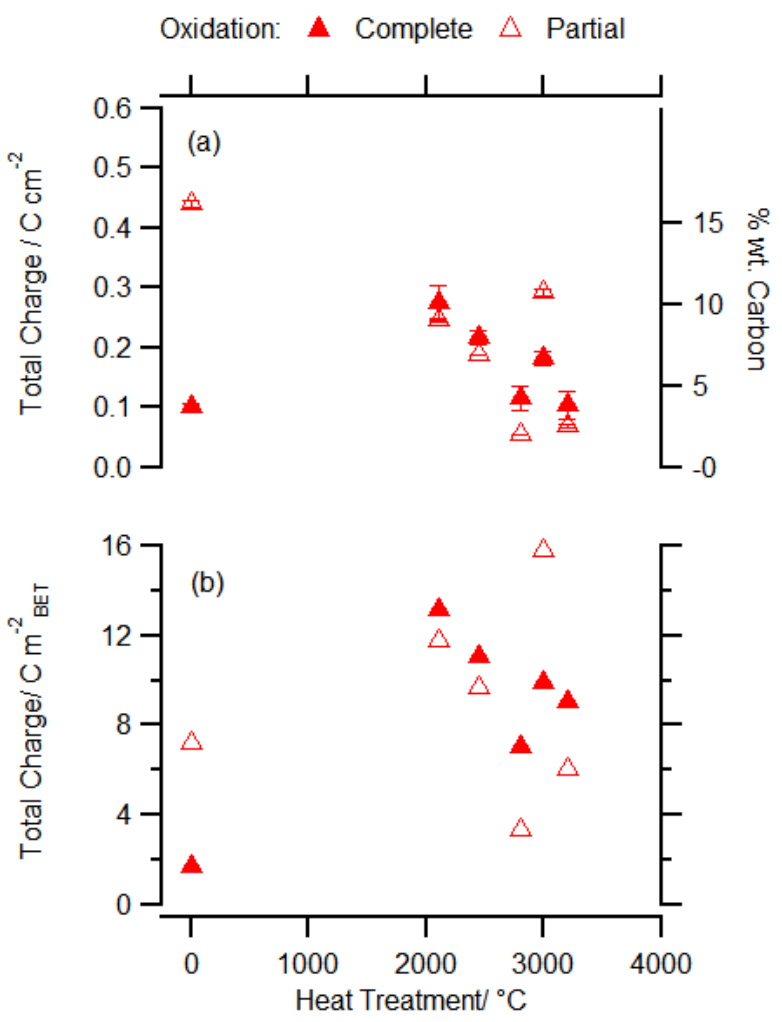

Figure 6-17: The total complete and partial oxidation charge evolved during the electrochemical treatment versus heat treatment temperature (a) and corresponding data normalised to BET surface area (b). The complete and partial COR charges decrease with increasing graphitisation temperature. The partial COR dominates on pristine EC300 exhibiting less COR charge normalised to BET are than the graphitised materials (EC300 sample normalised to $85 \mu \mathrm{g}_{\mathrm{c}} \mathrm{cm}^{-2}$ ).

The complete COR of the graphitised HSAC supports resulted in a carbon weight loss of between 10 and $4 \%$ wt. with generally higher resistance observed for the more heavily graphitised samples. The ratios of the complete to the partial COR charges on the graphitised samples are $\sim 1: 1$ with the exception of sample 5 $\left(3000{ }^{\circ} \mathrm{C}\right)$ where the partial $\mathrm{COR}$ current appeared to be greater. The carbon weight loss of the non-graphitised EC300 carbon black to $\mathrm{CO}_{2}$ was overall comparable to the more heavily graphitised samples. When normalised to the BET area of the samples, the specific complete COR charge evolved from the EC300 is in fact less than half that of the most graphitised materials $\left(\geq 2800{ }^{\circ} \mathrm{C}\right)$. 
At the end of the electrochemical treatment the $\mathrm{CV}$ of the HSAC support samples were evaluated, analogous to the initial measurement used to determine the apparent double layer capacitance (as described previously in section 6.4.1). The capacitance determined using $\mathrm{CV}$ of the pristine EC300 is compared to the capacitance after the electrochemical oxidation treatment in figure 6-18 (a).
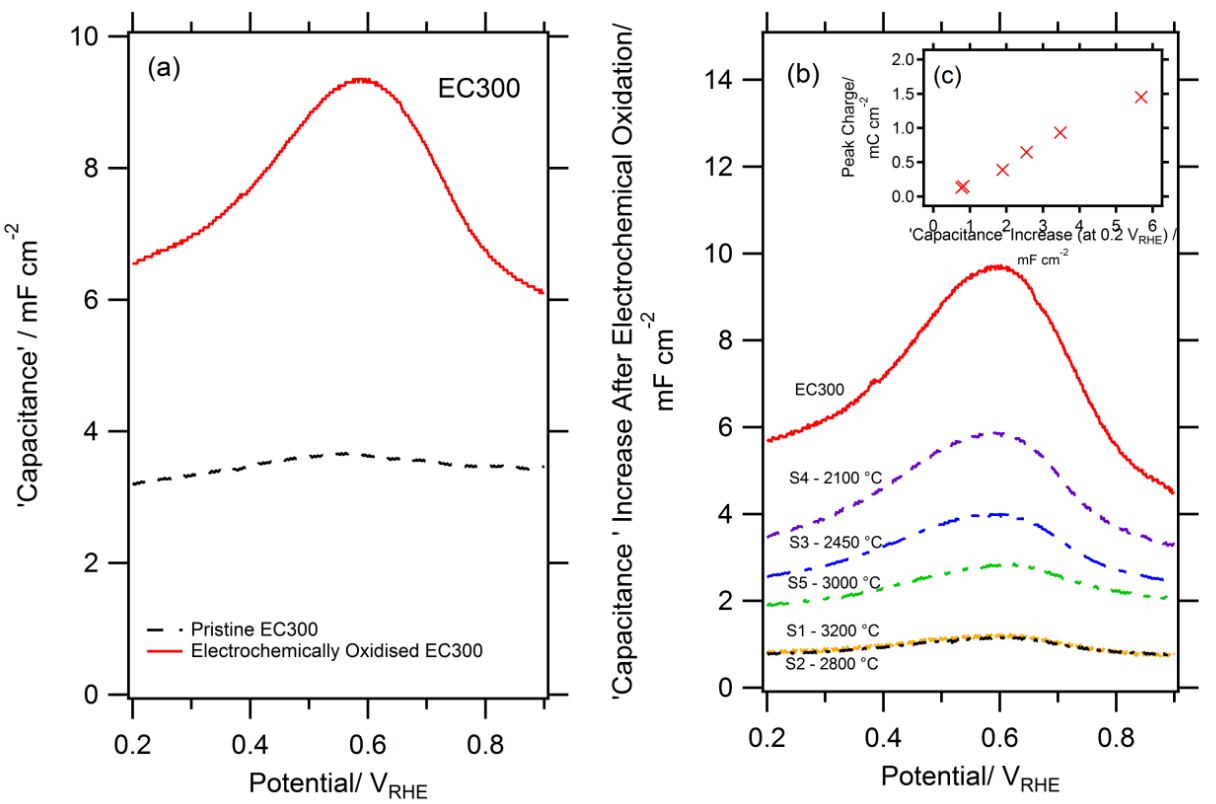

Figure 6-18: The apparent double layer capacitance for EC300 carbon measured before and after electrochemical oxidation (a) and increase in apparent double-layer capacitance for each carbon black following electrochemical oxidation (b). The double-layer capacitance of the HSAC sample substrate is observed to have increased following the electrochemical oxidation treatment. The capacitance at $0.2 \mathrm{~V}_{\mathrm{RHE}}$ scales linearly with the integrated charge of the peak observed at $0.6 \mathrm{~V}_{\mathrm{RHE}}$ (c) attributed to the reversible oxidation of surface oxide functional groups

The apparent double-layer capacitance of the EC300 sample has not only clearly increased significantly but a clear peak corresponding to reversible oxidation of the carbon surface has appeared at $\sim 0.6 \mathrm{~V}_{\mathrm{RHE}}$ following the electrochemical treatment is formed. The difference between the capacitance before and after the electrochemical oxidation procedure for each of the carbon samples is given in figure 6-18 (b). Here we observe that the apparent double-layer capacitance increases for all samples, coinciding with the formation of a peak at $0.6 \mathrm{~V}_{\mathrm{RHE}}$ which is a characteristic of the $\mathrm{CV}$ of a number of carbons and is attributed to the redox active quinone/hydroquinone surface functional groups [208]. In general, the size of the apparent double-layer capacitance and peak at $0.6 \mathrm{~V}_{\mathrm{RHE}}$ decreases 
with increasing graphitisation temperature. Finally, the capacitance at $0.2 \mathrm{~V}_{\mathrm{RHE}}$ and the integration of the peak at $0.6 \mathrm{~V}_{\mathrm{RHE}}$ (background capacitance at $0.2 \mathrm{~V}_{\mathrm{RHE}}$ subtracted) is shown to be directly correlated in figure 6-18 (c).

The relationship between the total complete and partial COR charges evolved during the course of the electrochemical treatment and the quinine/hydroquinone peak charge for each of the HSAC samples are given in figure 6-19 (a) (and normalised to their BET areas in (b)).
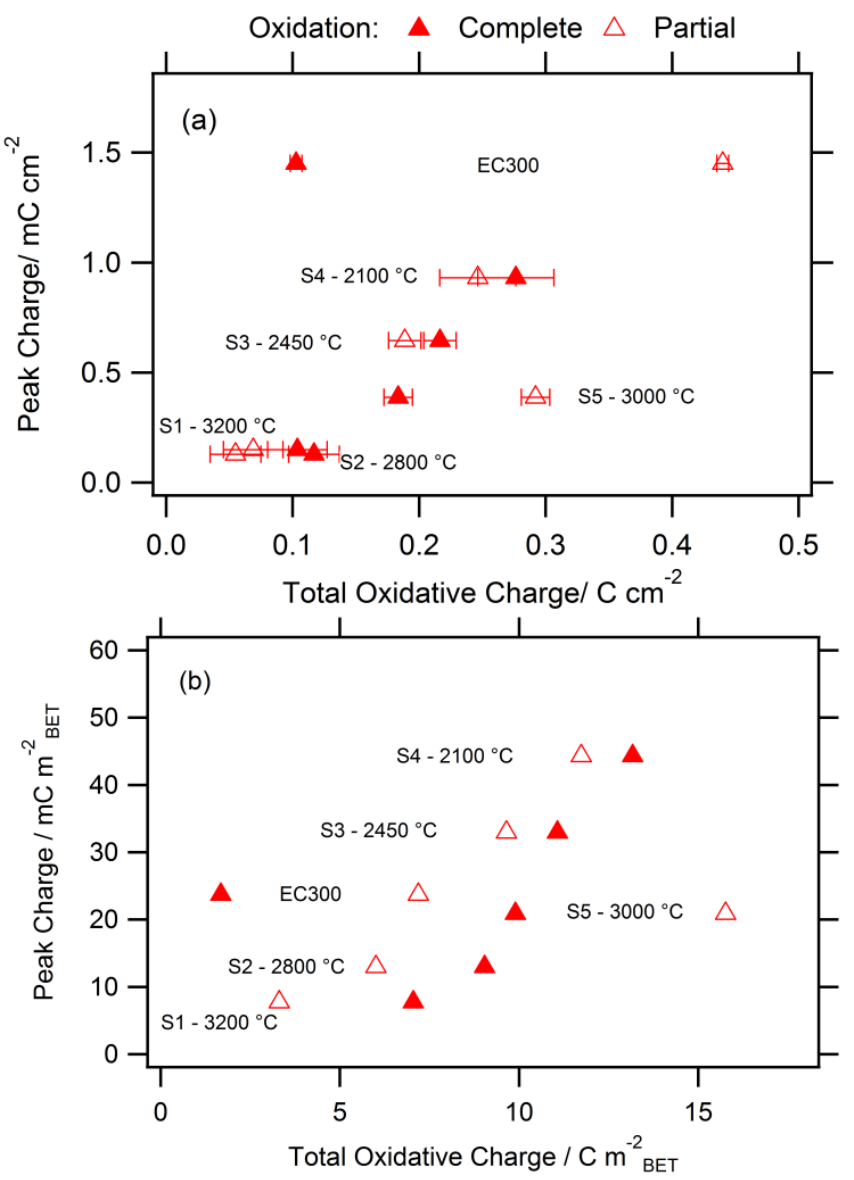

Figure 6-19: The quinone/hydroquinone redox peak charge plotted as a function of the total partial and complete COR charge evolved during the electrochemical treatment (a). Corresponding data normalised to BET surface area (b). The peak integration scales with the total partial COR current.

It appears that the peak charge (and in turn the increase in capacitance) is directly correlated to the partial and complete COR charge evolved during the electrochemical oxidation treatment with the exception of two points, the complete COR charge of the EC300 sample, and the partial COR of sample 5 
$\left(3000{ }^{\circ} \mathrm{C}\right)$. The partial $\mathrm{COR}$ charge measured for carbon sample 5 is believed to be an anomaly arising from changes in the WE substrate background, which will be discussed in the next section.

\subsection{Discussion of the Overall Effect of the Electrochemical Oxidation}

The overall mass normalised complete COR charge evolved during the course of the electrochemical treatment followed in the order of mildly graphitised $\left(\leq 2450{ }^{\circ} \mathrm{C}\right)>$ heavily graphitised $\left(\geq 2800{ }^{\circ} \mathrm{C}\right) \sim$ pristine EC300 $(>\mathrm{BET}$ normalised), which is in stark contrast to the complete COR charge evolved following the initial potential sweep: pristine EC300 > mildly graphitised > heavily graphitised. Clearly, an initially fast complete COR rate (although very significant when supporting catalyst particles) does not necessarily dictate the long-term electrochemical COR behaviour of the carbon black. The lower overall total COR charge arising from the EC300 compared to the mildly graphitised $\left(\leq 2450{ }^{\circ} \mathrm{C}\right)$ can be attributed to the rapid formation of the passivating nonelectrochemically oxidisable functional group which quickly reduces the EOSA, as suggested by the significant partial $\mathrm{COR}$ charge that does not lead to $\mathrm{CO}_{2}$ during the initial potential excursion. The lower complete COR charge evolved on the heavily graphitised carbons $\left(\geq 2800{ }^{\circ} \mathrm{C}\right)$ meanwhile can be simply attributed to its superior resistance to electrochemical oxidation; no evidence to suggest that the passivating non-electrochemically oxidisable functional group(s) is formed (which when reduced $<0.24 \mathrm{~V}_{\mathrm{RHE}}$ can be oxidised to $\mathrm{CO}_{2}$ via an alternative pathway). Although on the other hand the mildly graphitised carbon exhibited evidence to suggest that the non-electrochemically oxidisable species had formed in phase (i) and (ii), it was not to the extent that the EOSA was completely diminished and consequently (combined with inferior resistance to oxidation) a significant amount of complete and partial COR occurred. Depending on the carbon therefore, the non-electrochemically oxidisable oxygen-containing functional groups may have a significant influence on the long term oxidation processes, however, its stability is dependent on the lower electrode potential limit. 
Finally, a significant increase in the apparent double-layer electrode capacitance, along with the appearance of a peak at $0.6 \mathrm{~V}_{\mathrm{RHE}}$ (attributed to the hydroquinone/quinone redox processes) appeared after the electrochemical treatment was observed for all the carbon samples. This increase, along with the appearance of a peak at $0.6 \mathrm{~V}_{\mathrm{RHE}}$ is in agreement with the observations of many studies which observed a an increase in the apparent double-layer capacitance and the formation of a peak at $\sim 0.6 \mathrm{~V}_{\mathrm{RHE}}$ following electrochemical oxidation [166, 168, 170-172, 209]. In this study, a close relationship between the quinone/hydroquinone peak at $0.6 \mathrm{~V}_{\mathrm{RHE}}$ (as well as the increase in the apparent double-layer capacitance at $0.25 \mathrm{~V}_{\mathrm{RHE}}$ ) and the total COR charges evolved during the electrochemical measurement was revealed. There were, however, two discrepancies: (i) the complete COR charge of the EC300 was too low, and (ii) the total partial COR charge for Sample 5 heat treated at $3000{ }^{\circ} \mathrm{C}$ was somewhat large, and inconsistent with similarly heat-treated Samples 2 and1 (2800 and $3200{ }^{\circ} \mathrm{C}$ ). If, however, we closely evaluate the CV and MSCV of Sample 5 $\left(3000{ }^{\circ} \mathrm{C}\right.$ ) during phase (iii) of the electrochemical treatment (see Appendix B figure 9-4) it appears that the OER current is much more significant than all other measurements indicating a change in the WE substrate background contributions. This highlights the limitation of the DEMS measurement, in the sense that the partial COR can only be deduced by subtracting the polycrystalline Au WE substrate current and $I_{\mathrm{CO} 2}$ from the overall faradaic electrode current. Any unexpected change in the WE substrate background will therefore invalidate the partial COR charge deduced, which appears to be the case for this particular measurement. For example, the leakage of electrolyte around the sides of the WE substrate during the course of the measurement increases the polycrystalline Au WE substrate ECSA and henceforth the OER current, leading to the overestimation of the partial COR charge. It can therefore be concluded that the quinone/hydroquinone redox peak (and/or apparent double layer capacitance) scales proportionally with the partial COR and not the complete COR, as suggested by the EC300 sample. The final section of this study will briefly compare the observations of the pure EC300 sample, with that of EC300 HSAC supported Pt catalysts. 


\subsubsection{Electrochemical Oxidation of HSAC Supported Pt Catalysts}

After establishing the electrochemical oxidation tendencies of the pure EC300 HSAC support in section 6.4.3, the study was briefly extended to the investigation of EC300 HSAC supported Pt nanoparticle catalysts for comparison of the COR processes. An example measurement that shows the various electrochemical reactions that occur during a high potential excursion for the EC300 HSAC supported Pt $5 \mathrm{~nm}$ nanoparticle catalyst (analogous to phase (i) of the experiment in section 6.4.3.1) elucidated using DEMS is illustrated in figure 6-20.

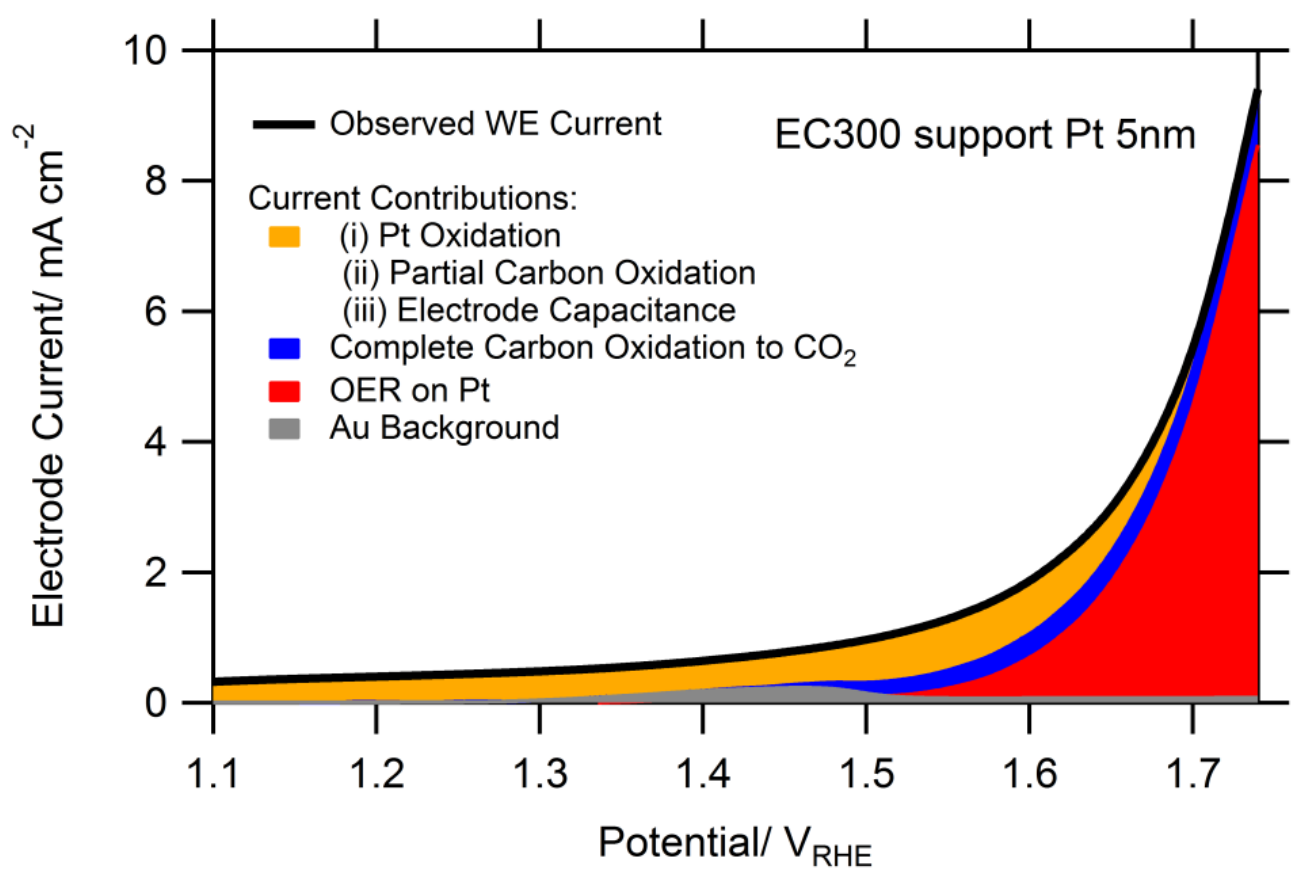

Figure 6-20: The origins of the observed current during the initial potential excursion above $1.1 V_{\text {RHE }}$. Tanaka $5 \mathrm{~nm}$ EC300 HSAC supported catalyst on Au substrate $\left(50 \mu \mathrm{g} \mathrm{cm}^{-2}, 0.5 \mathrm{~mol} \mathrm{dm}^{-3}\right.$ $\mathrm{H}_{2} \mathrm{SO}_{4}, 5 \mathrm{mV} \mathrm{s}^{-1}$ ). The electrode currents above $1.4 \mathrm{~V}_{\mathrm{RHE}}$ are dominated by the OER on the Pt nanoparticles with only a relatively small proportion of current arising from the complete COR to $\mathrm{CO}_{2}$.

During the potential excursion to $1.8 \mathrm{~V}_{\mathrm{RHE}}$ the electrode current observed $<1.35 \mathrm{~V}_{\mathrm{RHE}}$ is dominated by currents arising from the multilayer oxidation of the Pt surface as well as contributions from capacitive currents. As the WE potential rises above 1.35 $\mathrm{V}_{\mathrm{RHE}}$ the oxidation of the HSAC support to $\mathrm{CO}_{2}$ commences, closely followed by the evolution of $\mathrm{O}_{2}$ arising from OER on the Pt surface commencing at $1.5 \mathrm{~V}_{\mathrm{RHE}}$. At potentials exceeding $1.5 \mathrm{~V}_{\mathrm{RHE}}$ the faradaic electrode current is clearly dominated by the OER, arising from the rather fast OER rate 
on Pt. The significant quantity of $\mathrm{O}_{2}$ produced during the OER on Pt imposes a constraint on the upper potential limit that can be studied in comparison to the pure carbon sample as discussed previously in 6.4.2. Consequently, the study of the HSAC supported Pt catalysts was limited to and upper potential of $1.8 \mathrm{~V}_{\text {RHE}}$.

In figure 6-21 (a) the behaviour of the HSAC supported Pt $1 \mathrm{~m}$ catalysts to repeated high potential excursions is shown in an experiment analogous to that of phase (ii) in section 6.4.3.2 of the electrochemical treatment. Note that in this measurement the number of potential excursions is extended to ten rather than four in the previous electrochemical treatment (see Appendix B Figure 9-5 and Figure 9-6 for analogous measurements on HSAC supported Pt 5 and $3 \mathrm{~nm}$ catalysts).
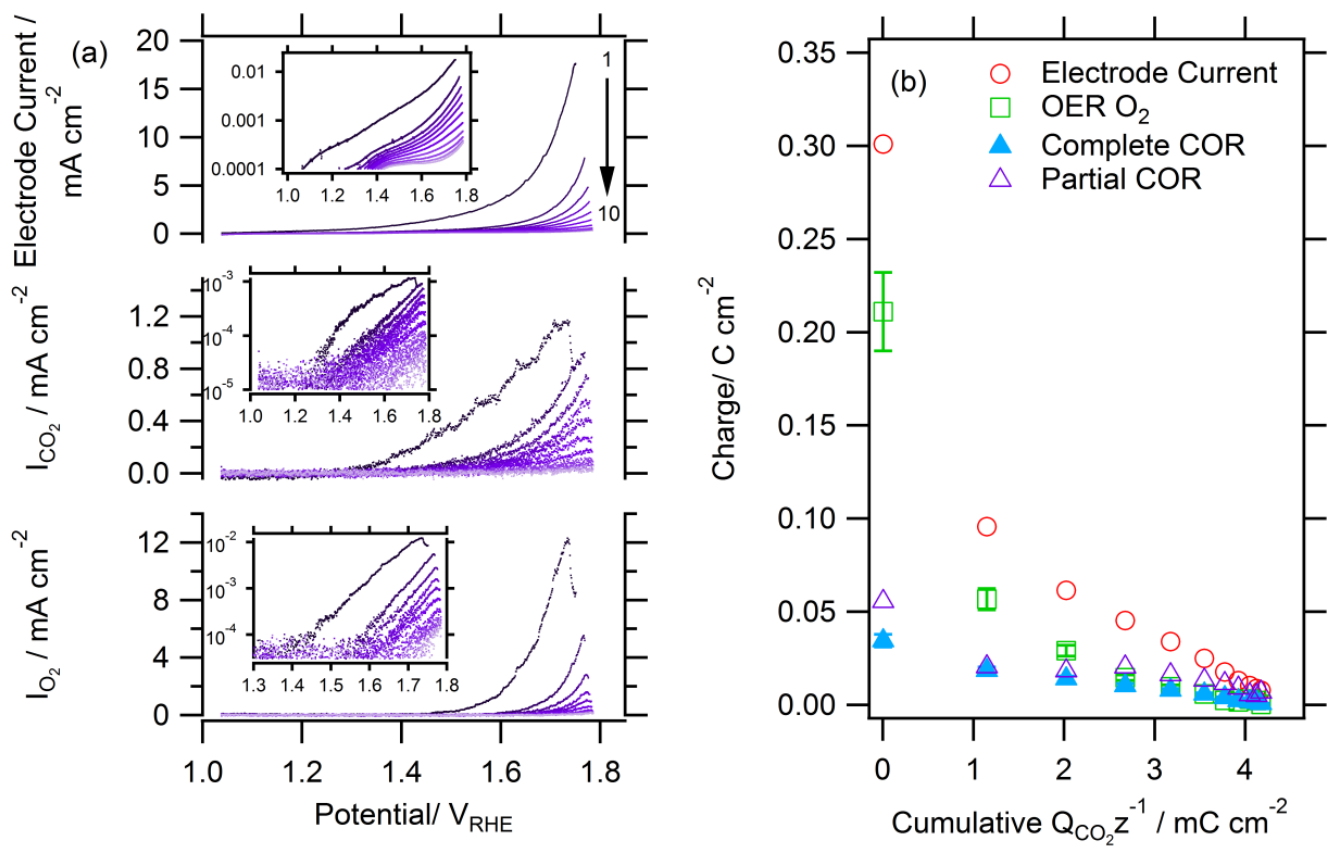

Figure 6-21: First ten LSVs of the electrochemical COR of a (EC300) HSAC supported Pt 1nm catalyst $\left(50 \mu \mathrm{g}_{\mathrm{Pt}} \mathrm{cm}^{-2}\right)$ on polycrystalline Au substrate in $0.5 \mathrm{~mol} \mathrm{dm}^{-3} \mathrm{H}_{2} \mathrm{SO}_{4}$ between 1.05 and 1.8 $\mathrm{V}_{\mathrm{RHE}}$ (a). Corresponding charges of the electrochemical processes during each sweep plotted as a function of the cumulative $\mathrm{CO}_{2}$ charge evolved (b). Following a rather high amount of COR in the first $\mathrm{CV}$, the COR gradually ceases in subsequent $\mathrm{CVs}$ between 1.05 and $2.05 \mathrm{~V}_{\mathrm{RHE}}$.

During the repeated LSVs of the HSAC support Pt catalyst, the faradaic electrode current is observed to decay rapidly with each subsequent potential sweep, corresponding to a significant decrease in complete $\mathrm{COR}\left(\mathrm{I}_{\mathrm{CO} 2}\right)$ of the EC300 supports, along with the OER $\left(I_{O 2}\right)$ on the Pt nanoparticles. The relative 
decay in the charges of the reactions (including the partial COR) for each subsequent sweep are plotted as a function of the $\mathrm{CO}_{2}$ evolved in figure 6-21(b). In this figure we observe that the OER initially dominates the total charge evolved during the first two potential excursions but is less substantial in subsequent sweeps where the partial and complete COR charges become more significant as the total electrode charge diminishes to almost zero.

In the final phase of the electrochemical treatment of the EC300 HSAC supported Pt $1 \mathrm{~nm}$ catalyst, the lower potential limit was reduced to $0.05 \mathrm{~V}_{\mathrm{RHE}}$ (analogous to phase (iii) in section 6.4.3.4) to observe the effect of reducing the HSAC support on the subsequent potential excursion to $1.8 \mathrm{~V}_{\mathrm{RHE}}$, as given in figure 6-22.
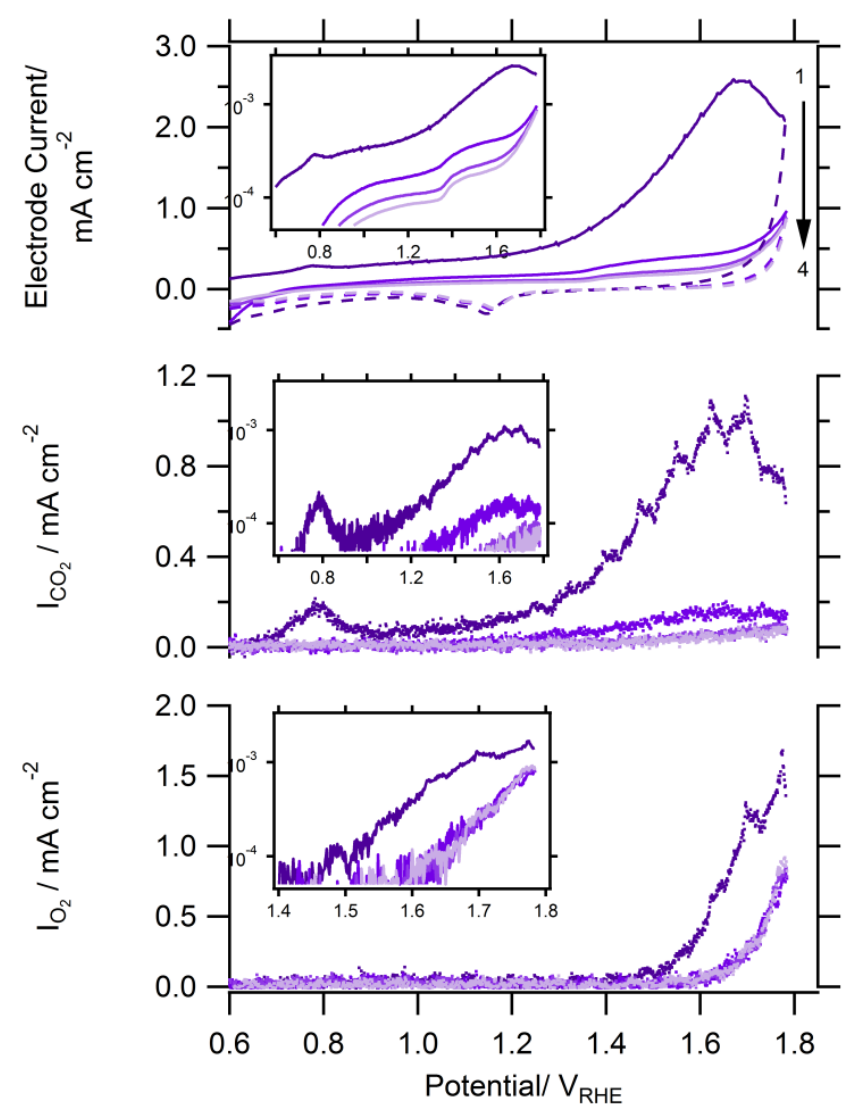

Figure 6-22: A plot illustrating the re-activation of the complete COR of a Pt $1 \mathrm{~nm}$ HSAC supported catalyst (following phase (ii) of the electrochemical treatment) by reducing the WE potential to $0.05 \mathrm{~V}_{\mathrm{RHE}}$, analogous to the effect observed on pure EC300 carbon (see figure 6-12).

By lowering the potential to $0.05 \mathrm{~V}_{\mathrm{RHE}}$ the complete COR of the EC300 HSAC support is re-activated as indicated in the electrode current and $I_{\mathrm{CO} 2}$ in the 
subsequent high potential sweep, forming a peak at $\sim 1.7 \mathrm{~V}_{\mathrm{RHE}}$ analogous to that observed on the pure EC300 carbon in figure 6-12 section 6.4.3.4. Furthermore, a small additional $\mathrm{CO}_{2}$ peak is observed at $0.75 \mathrm{~V}_{\mathrm{RHE}}$ whilst the OER seems to also be enhanced in the first subsequent potential sweep at $1.5 \mathrm{~V}_{\mathrm{RHE}}$, shifting to the high potential of $1.6 \mathrm{~V}_{\mathrm{RHE}}$ in the following three sweeps. It should be noted, however, that the peak at $0.75 \mathrm{~V}_{\mathrm{RHE}}$ is not necessarily reproducible (see Appendix B, figure 9-5 and figure 9-6) and that it cannot be guaranteed that no residual CO was present in the electrolyte because CO-Stripping measurements were also performed.

\subsubsection{Discussion of HSAC Supported Pt Catalyst Oxidation Processes}

By investigating the electrochemical oxidation tendencies of the HSAC supported Pt $1 \mathrm{~nm}$ catalyst three important observations were made. Firstly, during the initial high potential excursion to $1.8 \mathrm{~V}_{\mathrm{RHE}}$ the faradaic current contains contributions from the complete $\mathrm{COR}$ to $\mathrm{CO}_{2}$ of the HSAC support, but is dominated by the OER on the Pt nanoparticles. Secondly, the electrode, complete COR and OER charges all decreased significantly during repeated excursions, decaying to almost zero after ten potential sweeps and finally, by lowering the electrode potential to $0.05 \mathrm{~V}_{\mathrm{RHE}}$ the complete COR of the HSAC support was significantly reinitiated in the subsequent high potential sweep analogous to the pure EC300 with an additional peak feature at $0.8 \mathrm{~V}_{\mathrm{RHE}}$ that may be observed.

In this study of EC300 HSAC supported catalysts no $\mathrm{CO}_{2}$ was observed during the initial excursion until a potentials $>1.35 \mathrm{~V}_{\mathrm{RHE}}$, analogous to the observations on the pure EC300 carbon black in section figure 6-5. In previous DEMS studies, however, two additional peaks have been seen in the mass ion current for $\mathrm{CO}_{2}$ at $\sim 0.8[30,49]$ and $0.95 \mathrm{~V}_{\mathrm{RHE}}[30]$ during the CVs of the HSAC supporting Pt catalysts that were not present for pure carbon blacks NORIT BRX [30] and Vulcan XC-72 [49]). Again, it is difficult to compare between the findings of these DEMS studies because previous they are largely qualitative, the carbon blacks studied are different (which can have a wide range of bulk and surface properties) and the detection limit of the DEMS instrument used is not given. Furthermore, 
the initial potential excursions were not shown in either of the previous DEMS studies.

Repeated potential excursions from between 1.05 and $1.8 \mathrm{~V}_{\mathrm{RHE}}$ resulted in the rapid decrease of both the faradaic, OER and complete COR current during subsequent sweeps. The decrease in the OER current can be attributed to two processes: (i) the decrease in the OER kinetics of the supported Pt nanoparticles decreases and (ii) more importantly, the overall ECSA of the supported Pt decreases i.e. Pt nanoparticles detach from the HSAC support during these high potential excursions. These conclusions are based on kinetic studies of the OER on Pt which demonstrate the overpotential decreases with the extended oxidation of the Pt surface (i.e. multilayer Pt-Oxide formation) and observations using nondestructive TEM which show that Pt particles have detached from the HSAC support following extended potential cycling to a potential of $1.4 \mathrm{~V}_{\mathrm{RHE}}[24,25]$. Another important observation in these repeated potential excursion measurements was that the complete $\mathrm{COR}$ to $\mathrm{CO}_{2}$ was also observed to decay completely, analogous to the measurement on the pure EC300 which is attributed to the formation of a non-electrochemically oxidisable oxygen-containing surface functional group (see section 6.4.3.4.1), although the processes required additional potential sweeps (ten vs. one) owing to the lower, upper electrode potential limit (1.85 $\left.\mathrm{V}_{\mathrm{RHE}} \mathrm{vs.} 2.05 \mathrm{~V}_{\mathrm{RHE}}\right)$.

The EC300 HSAC supported Pt catalyst not only exhibited the same behaviour as the pure EC300 sample during repeated high potential excursions, but also responded in the same way to a decrease in the potential to $0.05 V_{\text {RHE }}$ i.e. following the reduction of the passivated carbon surface, the electrochemical oxidation is re-initialised during the subsequent sweeps. On the HSAC supported Pt catalysts the significantly large peak at $1.7 \mathrm{~V}_{\mathrm{RHE}}$ (observed on the pure EC300) was accompanied by a second peak at $\sim 0.75 \mathrm{~V}_{\mathrm{RHE}}$, analogous to that observed in previous DEMS studies on NORIT BRX and Vulcan XC-72 [30, 49] carbons supporting $\mathrm{Pt}$, that were not present during the initial potential excursion of the pristine EC300 support Pt catalysts in this study. The correlation of the small peak at $0.75 \mathrm{~V}_{\mathrm{RHE}}$ and the peak of CO-Stripping measurement led to the interpretation that the $\mathrm{Pt}$ is oxidising $\mathrm{CO}_{\text {ads. }}$ Based on the additional $\mathrm{CO}_{2}$ peak 
observed on the Pt supporting carbons, it was concluded in both previous studies that Pt catalyses the corrosion of the carbon support [30, 49]. The problem with this conclusion is that it is not supported by other experiments in electrochemical half-cells which in general require $>1.2 \mathrm{~V}_{\mathrm{RHE}}$ to corrode HSAC supported Pt catalysts.

At least part of this discrepancy arises from the definition of carbon corrosion. Carbon corrosion (whether induced by temperature or electrode potential) arises from the oxidation of the carbon surface, which may be via the complete COR to $\mathrm{CO}_{2}$, or the partial COR to either surface oxygen functional groups or CO. Regardless of the corrosion product (whether $\mathrm{CO}_{2}$, surface oxygen functional group(s), CO or even volatile organic compounds (VOCs)) the result of the carbon oxidation is essentially the breakage of $\mathrm{C}$ - $\mathrm{C}$ bonds, which deteriorates the microstructure of the carbon black reducing its ability to act as a support for finely dispersed Pt catalyst particles. Using this definition, if the role of the Pt supported is to purely catalyse the oxidation of $\mathrm{CO}$ species (generated by the partial $\mathrm{COR}$ ) to $\mathrm{CO}_{2}$, then $\mathrm{Pt}$ cannot be considered to catalyse the corrosion of the carbon support. The end gaseous product (whether $\mathrm{CO}_{2}$ or $\mathrm{CO}$ that is later oxidised to $\mathrm{CO}_{2}$ via $\mathrm{Pt}$ ) is of little significance to the corrosion of the carbon black because the corrosion occurred in the partial COR step. In contrast, the subsequent oxidation of $\mathrm{CO}$ to $\mathrm{CO}_{2}$ is very significant to the observations of corrosion experiments which monitor the $\mathrm{CO}_{2}$ produced.

\subsubsection{Relationship between HSAC Support Oxidation and ECSA of Pt}

Regardless of the COR rates on the HSAC support, the most crucial relationship in terms of PEMFC durability is the relationship between the quantity of COR and the ECSA of the finely dispersed $\mathrm{Pt}$ particles. In the preceding electrochemical treatment, the ECSA of the HSAC supported Pt $1 \mathrm{~nm}$ catalyst was determined using CO-Stripping prior to phase (i), following phase (ii) and at the end of the electrochemical treatment (after phase (iii)). The relationship between the ECSA of $\mathrm{Pt}$ and the $\mathrm{CO}_{2}$ evolved in the complete $\mathrm{COR}$ is given in figure 6-23. The ECSA values for the 3 and $5 \mathrm{~nm}$ for an analogous measurement 
are also given; however, phase (ii) of the electrochemical treatment was limited to only four CVs and the CO-Stripping measurement was only performed after phase (iii) of the electrochemical treatment. The two treatments (A and B) are therefore not directly comparable.

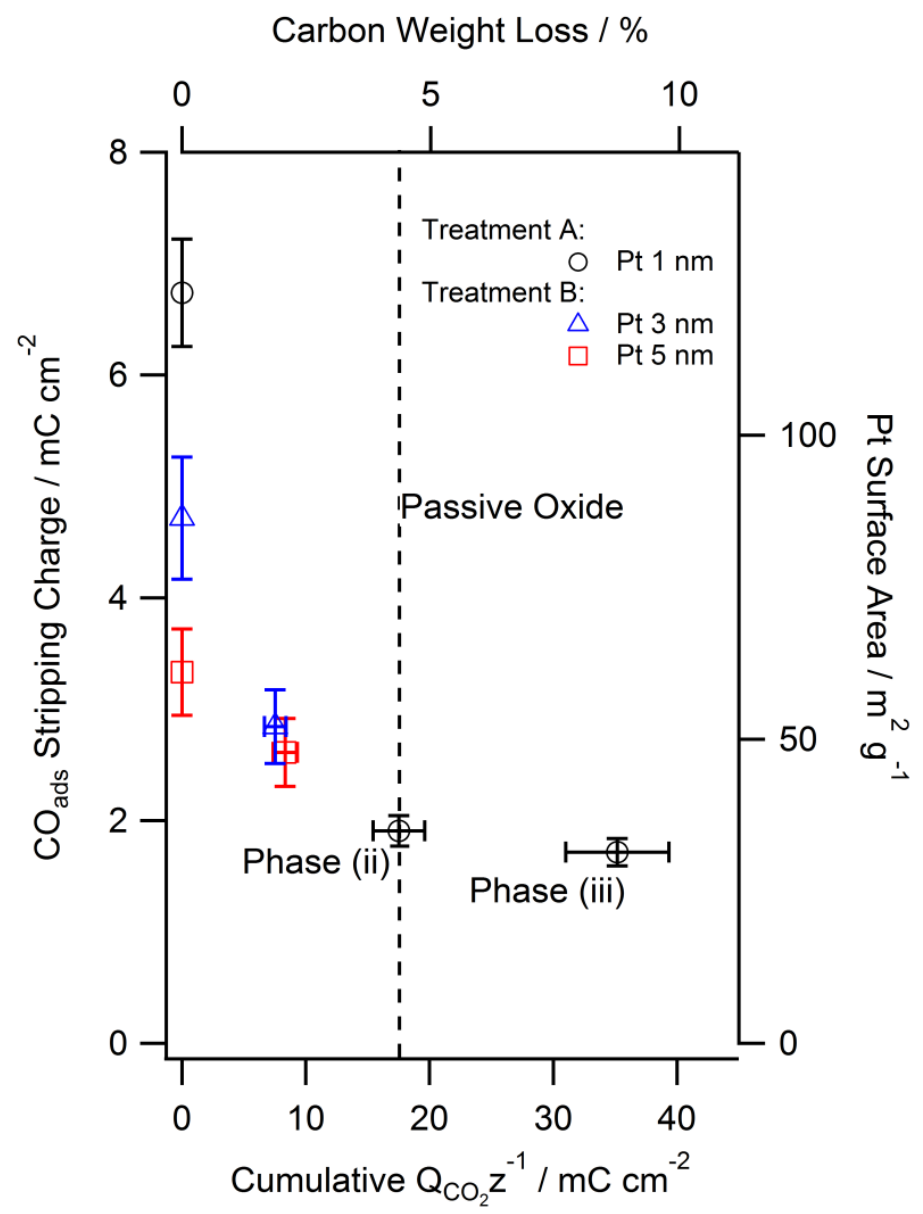

Figure 6-23: CO Stripping Charge vs. Cumulative CO2 evolved of various HSAC supported Pt nanoparticle catalysts. The CO-Stripping charge for the 3 and $5 \mathrm{~nm}$ catalysts are normalised to $14 \mu \mathrm{gPt} \mathrm{cm} \mathrm{cm}^{-2}$ (whilst the $\mathrm{CO}_{2}$ evolved is for $50 \mu \mathrm{g}_{\mathrm{c}} \mathrm{cm}^{-2}$ support). There are two key observations: (i) a carbon weight loss of only 3-4 \% results in a significant ECSA loss of the supported Pt particles and (ii) the passive oxide is formed at $4 \% \mathrm{wt}$. resulting in a $72 \%$ loss in the ECSA. The reduction and oxidation of the passive oxide does not appear to further influence the loss in ECSA.

After phase (ii) of the electrochemical treatment for the HSAC supported Pt 1 m catalysts (in Treatment $\mathrm{A}$ with ten sweeps to form the passivating oxide) the total ECSA of the Pt nanoparticles decreased by a significant $72 \%$ corresponding to a carbon \% wt. loss of only $4 \%$. After then reducing the electrode potential to $0.05 V_{\text {RHE }}$ to re-initialise the COR processes in the subsequent sweep (performed twice) evolving another $4 \%$ wt. in carbon, the total ECSA of the Pt $1 \mathrm{~nm}$ 
particles is largely unaffected decreasing by only a further $13 \%$. The losses of ECSA for the 3 and $5 \mathrm{~m}$ catalysts are meanwhile $\sim 40$ and $\sim 20 \%$ after $\sim 2 \%$ wt. loss of carbon via the complete $\mathrm{COR}$ to $\mathrm{CO}_{2}$.

The key objective of figure 6-23 is to demonstrate the ability of DEMS to correlate the quantity of $\mathrm{CO}_{2}$ evolved during the complete $\mathrm{COR}$ (via the integration of the $I_{\mathrm{CO} 2}$ during the $\mathrm{CV}$ ) with the decrease in the total ECSA of the support Pt nanoparticles (determined using CO-Stripping). In doing so, it is observed that the ECSA loss of the supported Pt catalysts is diminished significantly, even with rather low quantities of carbon weight loss (via the complete COR) highlighting that for all the discussion of the long-term COR processes, the initial electrochemical oxidation processes of the carbon is indeed most significant; the large quantities of $\mathrm{CO}_{2}$ evolved in phase (iii) of the experiment had little impact on the ECSA.

\subsubsection{Future Applications of DEMS in the Study of the COR}

In order discuss the potential future of DEMS in the study of the COR, it is crucial to first establish how the limitations of the instrument impose certain restrictions on the experimental design, primarily the electrode potential region of investigation. In this study, the electrochemical oxidation of the HSAC support was explored at rather high potentials up to $2.05 \mathrm{~V}_{\mathrm{RHE}}$ which is $\sim 0.3$ to $0.6 \mathrm{~V}$ higher than the potential induced at the cathode during the PEMFC start-stop events $\left(\leq 1.75 \mathrm{~V}_{\mathrm{RHE}}\right)$ and a further $\sim 0.8 \mathrm{~V}$ greater than those experienced when the PEMFC is idle $\left(\leq 1.2 \mathrm{~V}_{\mathrm{RHE}}\right)$. Extrapolating the observations in this study down to more PEMFC relevant potentials therefore, is not necessarily straightforward. The possible region of study using DEMS is determined by two aspects of the experiment: (i) the rate of the complete COR of the carbon black sample, and (ii) the $I_{\mathrm{CO} 2}$ detection limit, equivalent to a carbon weight loss (to $\mathrm{CO}_{2}$ ) of $2.8 \mathrm{ug}_{\mathrm{c}} \mathrm{hr}^{-1}$ or a complete COR current of $\sim 25 \mu \mathrm{A} \mathrm{cm}$ (assuming 4 electrons) for the DEMS instrument constructed in this thesis. Although DEMS was able to observe $\mathrm{CO}_{2}$ at potentials as low as $1.35 \mathrm{~V}_{\mathrm{RHE}}$ (at the detection limit) on the pristine EC300 carbon black, for the reliable quantification of $\mathrm{CO}_{2}$ (determined 
by the quantification limit $\sim 250 \mu \mathrm{A} \mathrm{cm}^{-2}$ ) requires potentials approaching 1.6 V RHE.

Although the sensitivity of the DEMS instrument imposes certain constraints on the experimental parameters that may be used, the ability to differentiate between the origin of the electrode currents that arise from the complete COR to $\mathrm{CO}_{2}$, the OER on either the polycrystalline Au or Pt nanoparticles and by deduction the partial COR is no doubt invaluable. By exploring potential up to 2.05 $\mathrm{V}_{\mathrm{RHE}}$, this study revealed clear COR processes and trends in the behaviours of the EC300 and graphitised carbon blacks samples which exhibited interesting and unpredictable behaviour which most likely generates more questions than answers. Hopefully, this work will provide the foundation for future DEMS and combined electrochemical half-cell studies on the COR because significantly more need be done to improve our fundamental understanding of the COR processes.

This DEMS study exclusively employed CV as the technique to analyse the electrochemical behaviour, however, having established some understanding of the COR tendencies of the carbon blacks it will be interesting in future DEMS studies to employ chronoamperometry in the study of the COR. Without prior knowledge of the electrochemical oxidation tendencies of the carbons these are difficult to plan and interpret, particularly when by measuring the COR the surface of the carbon black is changed. Quantitative studies using DEMS would likely, however, require rather high complete COR rates and henceforth high electrode potentials to precisely measure the quantity of $\mathrm{CO}_{2}$ i.e. it will likely be difficult to reliably subtract the mass ion current background.

An important area of improvement to the study of the COR processes, particularly in light of the significant partial COR current, would be to extend the QMS measurement to the detection of $\mathrm{CO}(\mathrm{m} / \mathrm{z}=28)$ which has been observed in an earlier qualitative DEMS study [30]. Although incorporating an additional measurement channel to the QMS may seem like a rather straightforward enhancement to the methodology, it is in fact not necessarily easy to implement. If we consider the contributions to $\mathrm{m} / \mathrm{z}=28$ mass ion current channel, such as the large $\mathrm{N}_{2}$ background, the contributions of $\mathrm{CO}_{2}$ fragment ions along with the 
sacrifices that would be made in the signal to noise ratios for $\mathrm{m} / \mathrm{z}=44$ and 32 by introducing an additional channel, some effort in optimising the QMS parameters will be required.

A possible area of future interest which was touched upon in this study, is for DEMS to quantitatively relate the amount of carbon black weight loss (from the observed $\mathrm{CO}_{2}$ and perhaps $\mathrm{CO}$ in the future) with the ECSA decrease of the supported Pt particles (preferably using $\mathrm{H}_{\text {upd }}$ ) during repeated high potential excursions which could be of significant fundamental interest to PEMFC development. This could also be combined with complementary non-destructive TEM measurements to observe the changes in the catalysts structure.

A quite demanding enhancement to the DEMS experiment would be to combine a EQCM with DEMS, the useful combination of which has been previously demonstrated in the study of the electro-oxidation of $\mathrm{CO}$ on Pt and PtRu [28]. The usefulness of EQCM alone for the evaluation and screening tool for the corrosion behaviour of carbon supports [170]. EQCM is able to monitor the change in mass of the electrode, which for carbon blacks was observed to increase $>0.25 \mathrm{~V}_{\mathrm{NHE}}$ typically peaking at $\geq 1.0 \mathrm{~V}_{\mathrm{NHE}}$ whereby the decrease is attributed to the oxidation of the carbon black. By combining EQCM with DEMS the change in the mass of the carbon black may be monitored alongside the formation of $\mathrm{CO}_{2}$ and $\mathrm{CO}$ which may bring further insights into the COR processes.

Although there is much that can be done, in order to study the COR processes at more PEMFC relevant potential, either an increase in the DEMS instrument detection limit or an increasing the complete $\mathrm{COR}$ rate is required. The detection limit of the DEMS instrument may be improved by enhancing the DEMS cell collection efficiency, although if we consider that it is typically $\sim 0.3$ at reasonable electrolyte flow rates $\left(\sim 5 \mu \mathrm{L} \mathrm{s}^{-1}\right)$, only modest gains are possible. A more likely successful alternative would be to further optimise the QMS to $\mathrm{m} / \mathrm{z}=44$ in order to enhance the mass ion current signal to noise ratio. In this study both $\mathrm{m} / \mathrm{z}=44$ and $\mathrm{m} / \mathrm{z}=32$ were measured simultaneously, although on the pure carbon blacks $\mathrm{O}_{2}$ is of perhaps less interest below $1.7 \mathrm{~V}_{\mathrm{RHE}}$ where the OER does not to occur on the polycrystalline Au WE substrate. It may therefore be useful to measure a 
single mass ion channel using the QMS, which would not suffer from the data loss that arise when using multiple mass ion channels, along with finite time required for the operational amplifier to rise and fall responds to stepped changes in the mass ion current (as the mass ion channels are switched - see Chapter 3 section 3.7.1.2.1). There are, of course, other possibilities to increase the QMS sensitivity by increasing the SEM voltage (although in this study it was at the upper limit of the linear range i.e. $1600 \mathrm{~V}$ ) or by being more liberal with the QMS resolution parameter so that a lower amplification range on the EP 422 unit may be used.

In terms of increasing the quantity of $\mathrm{CO}_{2}$ generated, the most conceivable option would be to increase the loading of carbon material on the WE substrate. In the current DEMS cell the WE substrate was a $5 \mathrm{~mm} \emptyset$ disc which, at the loading density of ca. $50 \mu \mathrm{g} \mathrm{cm}^{-2}$ supports $10 \mu \mathrm{g}$ of carbon. By increasing the WE substrate size to a ca. $10 \mathrm{~mm} \emptyset$ disc supporting $50 \mu \mathrm{g}$ of carbon material, a factor of five could be gained in the $\mathrm{CO}_{2}$ produced via to the complete $\mathrm{COR}$. An increase in the WE substrate size, however, will be difficult to implement in the current dual thin-layer flow cell design, however, if we consider that the flow of electrolyte is not critical in COR measurements, it may be beneficial to employ a thin-layer flow cell under static electrolyte conditions which would allow the easier implementation of a large WE electrode and higher DEMS cell collection efficiencies (see Chapter 2 section 2.2.1.2 for more details). It may also be easier to study the COR using chronoamperometry under static electrolyte conditions because the $\mathrm{CO}_{2}$ background is perhaps even more stable than when using the precision syringe pump and can be more reliably subtracted. Static electrolyte may, however, introduce certain practical difficulties such as the complete purging of the DEMS cell, along with the possible formation of gas bubbles at high complete COR or OER rates forming at the WE leading to loss in WE potential control. If the combined increase in the $\mathrm{CO}_{2}$ concentration, and with the enhancement of the QMS sensitivity can achieve a factor of ten in improved detect-ability, however, then the potential region of study for the COR of the carbon blacks can in principle be decreased by $\sim 200 \mathrm{mV}$ in potential (based on the Tafel-slopes in section 6.4.3.1) . 
Overall, the electrochemical oxidation of HSAC supports is dynamic and rather complex, requiring a number of complementary techniques and DEMS can certainly contribute significantly toward future understanding.

\subsection{Conclusions}

The purpose of this study was to utilise DEMS to investigate and compare the electrochemical COR processes of carbon blacks. The approach was to compare the electrochemical oxidation current using $C V$ up to $2.05 V_{R H E}$, and elucidate the contribution arising from the partial and complete COR, and OER on either the polycrystalline Au substrate or supported Pt particles using DEMS. As part of the study, a method was developed to quantify the loading of the HSAC support on the WE substrate in-situ by measuring the electrode capacitance using $\mathrm{CV}$ and comparing this with a pre-determined gravimetric capacitance. Furthermore, a polycrystalline Au was favoured as WE substrate rather than the more common GC substrate to avoid an ill-defined $I_{\mathrm{CO} 2}$ background.

By quantifying the $\mathrm{CO}_{2}$ produced during the electrochemical oxidation of a pristine EC300, and five heat-treated carbon black samples $\left(2100\right.$ to $\left.3200{ }^{\circ} \mathrm{C}\right)$ using DEMS, it was deduced that the complete COR occurs in combination with another reaction process. In this study, the remaining current was attributed to the partial COR which may proceed to either $\mathrm{CO}$ or stable oxygen-containing surface functional groups, which contributed up to $\sim 70 \%$ of the current on the EC300 and in general $\sim 50 \%$ for the heat-treated carbon blacks during the first potential excursion to $2.05 \mathrm{~V}_{\mathrm{RHE}}$. The rate of complete COR during the initial potential excursion was found to be greatest for the EC300 carbon, and less for the heat-treated samples, with a trend for decreasing rates with increasing heattreatment temperature. The difference between the EC300 and heat-treated Sample $4\left(2100{ }^{\circ} \mathrm{C}\right)$ normalised to BET area, however, was less significant. The potential at which $\mathrm{CO}_{2}$ was first observed above the detection limit of this DEMS instrument during the initial potential excursion were $1.35 V_{\text {RHE }}$ for EC300, $1.68 \mathrm{~V}_{\mathrm{RHE}}$ for Sample $4\left(2100{ }^{\circ} \mathrm{C}\right)$ and $\sim 1.88 \mathrm{~V}_{\mathrm{RHE}}$ for the remaining samples $\left(\geq 2450{ }^{\circ} \mathrm{C}\right.$ ), equivalent to a carbon rate loss of $2.754 \mathrm{ug}_{\mathrm{c}} \mathrm{hr}^{-1}$ evolved via the 


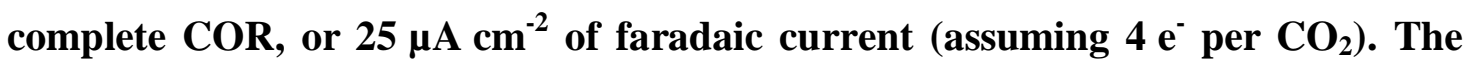
Tafel-slopes extracted from the observed $I_{\mathrm{CO} 2}$ meanwhile, were in the range of $200 \pm 30 \mathrm{mV} \mathrm{Dec}{ }^{-1}$ above $1.8 \mathrm{~V}_{\mathrm{RHE}}$, although shallower slopes of 400 and $300 \mathrm{mV} \mathrm{Dec}^{-1}$ were observed on the EC300 and mildly graphitised HSAC in the potential region below $1.8 \mathrm{~V}_{\mathrm{RHE}}$, corresponding to electron transfer coefficients $\left(\alpha^{*} n\right)$ values of between 0.143 and 0.354 . In order to fully rationalise the differences in the initial COR rates, however, greater characterisation of the bulk and surface properties of the pristine carbon blacks is required.

The behaviour of the COR tendencies of the carbon samples during repeated potential excursions between 1.05 and $2.05 \mathrm{~V}_{\mathrm{RHE}}$ (phase (ii)) and repeated potential excursions with decreasing electrode potential limit (down to $0.05 \mathrm{~V}_{\mathrm{RHE}}$ ) were revealed to be rather dynamic exhibiting three trends. In phase (ii) the COR processes either: (i) stopped abruptly (EC300), (ii) progressively increased and then decreased $\left(2100\right.$ and $\left.2450{ }^{\circ} \mathrm{C}\right)$, or (iii) progressively increased for each subsequent $\mathrm{CV}\left(\geq 2800{ }^{\circ} \mathrm{C}\right)$. Three different trends were also observed in phase (iii) of the electrochemical oxidation when the lower electrode potential limit was decrease to $\leq \mathbf{0 . 2 4} \mathrm{V}_{\mathrm{RHE}}$, either the COR processes were: (i) reinitiated (EC300), (ii) enhanced (Samples $\leq 2450{ }^{\circ} \mathrm{C}$ ), or (iii) unaffected, in the subsequent high potential excursions $\left(\geq 2800{ }^{\circ} \mathrm{C}\right)$. Although there is no established COR mechanism to explain all the experimental observations, these three trends were rationalised by considering the relative stability of the oxygen-containing surface functional groups arising from the partial COR and their influence on the EOSA of the carbon. Although not the only determining factor, the stable nonelectrochemically oxidisable functional $\operatorname{group}(s)$ may be considered to decrease the EOSA, whereas the formation of $\mathrm{CO}_{2}$ and $\mathrm{CO}$ regenerates at least one new free site, maintaining or increasing the EOSA (unless, of course, the carbon is completely consumed). By reducing the non-electrochemically oxidising oxygen containing functional groups, however, the complete COR can be reinitiated (EC300) or enhanced $\left(\leq 2450{ }^{\circ} \mathrm{C}\right)$ during the subsequent oxidation (i.e. the EOSA is increased by reducing the carbon). The study of the COR processes of carbon blacks is overall complex, and given the difficulties in characterising the pristine carbon black materials ex-situ (let alone in-situ) to try to fundamentally understand the influence of electrochemical oxidation, it is only possible to 
speculate at this stage on what is happening to the structure and surface of the carbon materials.

The study was then briefly extended to EC300 HSAC supported Pt catalysts, which essentially exhibited the same trends observed on the pure EC300, although a small additional peak was observed at $0.8 \mathrm{~V}_{\mathrm{RHE}}$ attributed to the oxidation of $\mathrm{CO}$ by $\mathrm{Pt}$; however, it was not necessarily concluded that $\mathrm{Pt}$ catalyses the corrosion of the carbon support (in contrast to previous DEMS studies) because CO can already be considered a corrosion product from the partial COR (although this is not confirmed in this study). Finally, the potential application of DEMS towards correlating the HSAC support weight loss with the loss in ECSA of supported Pt particles was briefly demonstrated which could have particular relevance to PEMFC development.

Overall, this study revealed clear and surprising trends in the COR tendencies of the HSAC support materials, demonstrating that DEMS is very useful tool in the elucidation of the electrochemical COR processes of carbon blacks. 


\section{Summary}

The aim of this thesis was to design, construct and demonstrate the research application(s) of a DEMS instrument. The approach to this problem began with the analysis of previous DEMS design solutions before embarking on the design and construction of the DEMS instrument created in this thesis. In doing so, the intended research application of the DEMS instrument was always kept in mind: that is, to perform fundamental research studies that approach problems which are relevant to the immediate development concerns of PEMFCs.

The setup of the DEMS instrument first required the design and construction of three crucial parts: the electrochemical half-cell, membrane interface and the vacuum system of a commercial QMS. The electrochemical half-cell constructed in this thesis was of similar design to the dual-cyclone thin-layer flow cell reported previously, whereby the WE is separated from the membrane interface and placed in two separate thin-layer compartments. The cell construction produced in this work, however, is considered to be rather modular and intuitive to the cell development process. Similarly to all DEMS instruments, the electrochemical cell was interfaced with the vacuum of the QMS via microporous PTFE membrane which possessed a geometric area of $0.95 \mathrm{~cm}^{2}$ and a total gas flux of 0.12 mbar $1 \mathrm{~s}^{-1} \mathrm{~cm}^{-2}$ when the membrane is exposed to the DEMS vacuum chamber. The DEMS vacuum system constructed here was of a 3-stage differentially pumped design, in contrast to the two-stage design more typically employed in DEMS. The sensitivity of the QMS was enhanced using a tubular aperture to direct the gas from the $1^{\text {st }}$ pumping stage at $10^{-2} \mathrm{mbar}$, perpendicularly into the $\mathrm{CB}$-ion source of the QMS in the $2^{\text {nd }}$ pumping stage. A second aperture, and addition vacuum pumping at the back of the QMS allowed a pressure of $\sim 2 * 10^{-5} \mathrm{mbar}$ in the $\mathrm{CB}$-ion source chamber whilst maintaining the necessary operating pressure of the SEM $<5 * 10^{-6}$ mbar. Once a working DEMS instrument had been established, the next step was to assemble and develop the instrument control hardware and software necessary to improve the instrument functionality and usability. This was primarily achieved using a combination a NI DAQ cards, and custom programmed Labview software. By incorporating the required DEMS functionality into an already powerful existing custom Labview 
software developed in our laboratory for RDE and MWE techniques, the resulting software not only allowed meticulous control of all experimental parameters, but also performs the acquisition, synchronisation, display and logging of both the electrochemical and QMS mass ion currents in real-time. Furthermore, additional software were also developed to allow easy calibration of the QMS, and to perform common DEMS data treatment and analysis tasks.

Before performing experimentation, it was crucial to understand and overcome some of the more practical aspects of the DEMS instrument, in order to optimise both the instrument and experimental parameters, understand its limitations and develop instrument calibration methods. For example, the large cell resistances inherent to thin-layer electrochemical flow cells combined with a small solution resistance between the WE and effective Luggin-capillary resulted potentiostat oscillation. In this DEMS instrument setup. These oscillations were overcome by adding a stabilising resistor to the WE connection which increased the effective solution resistance. IR-compensation of the stabilising resistor was then achieved using a NID allowing an acceptable uncompensated solution resistance of $\sim 10 \Omega$ with stable WE potential control. Furthermore, the performance of the DEMS electrochemical flow cell was found to be highly dependent on the electrolyte flow rate which influenced the instrument sensitivity, QMS response time and the correlation between electrochemical processes at the WE and the relevant mass ion currents observed. Optimum flow rates were found to be between 4 and $10 \mu \mathrm{l} \mathrm{s}{ }^{-1}$, with potential scan rates of $\leq 20 \mathrm{mV} \mathrm{s}$.

The research capabilities of the DEMS instrument were first demonstrated by re-visiting the MOR on a HSAC Pt and PtRu catalyst using CV, chronoamperometry and for the first time in combination with DEMS: three-dimensional voltammetry. An initial finding concerning ECSA determination was that contrary to previous reports, the CO-Stripping charge of the PtRu catalyst was not overestimated if the background CV in Argon saturated solution was subtracted prior to the peak integration. On the pure HSAC supported Pt catalyst an extremely close correlation between the faradaic MOR current, $I_{\mathrm{CO} 2}$, the conversion of methanol to $\mathrm{CO}_{2}$ and the ability of $\mathrm{Pt}$ to oxidise $\mathrm{CO}_{\text {ads }}$ was observed using all electroanalytical techniques, in agreement 
with the well-established understanding that the oxidation of $\mathrm{CO}_{\text {ads }}$ is the rds of the MOR. This close relationship was, however, was not mirrored by the MOR on the HSAC supported PtRu catalyst. Instead, the conversion of methanol to $\mathrm{CO}_{2}$ was found to be inversely correlated to the faradaic MOR current, which was not as closely dependent on the ability of the PtRu catalyst to oxidise $\mathrm{CO}_{\text {ads }}$. Consequently, the rds for the MOR on PtRu was concluded to be the initial dissociative adsorption of methanol on $\mathrm{Pt}$, although the poisoning $\mathrm{CO}_{\text {ads }}$ species can still be attributed to the significant electrode potential $>0.32 \mathrm{~V}_{\mathrm{RHE}}$ required for the MOR to proceed under these experimental conditions. The contrasting potential dependent conversion for the MOR and the Pt and PtRu catalysts was rationalised by considering that the role of $\mathrm{Ru}$ is to not only adsorb $\mathrm{OH}_{\mathrm{ads}}$ and facilitate the oxidation of $\mathrm{CO}_{\text {ads }}$ on $\mathrm{Pt}$, but to also oxidise desorbed partial MOR products that are produced via the incomplete MOR on the Pt portion of the surface ( $\mathrm{Ru}$ itself is understood not to adsorb methanol at ambient temperatures ca. $\left.<40{ }^{\circ} \mathrm{C}\right)$. Overall, the PtRu catalyst possessed a significantly higher mass activity of 72.6 compared to $1.6 \mathrm{~mA} \mathrm{mgmetal}^{-1}$ for Pt at $0.55 \mathrm{~V}_{\text {RHE }}$ using chronoamperometry, however, the comparison of these activities to other electrochemical half-cell MOR studies is not entirely meaningful because there is no commonly employed methodology, a situation that is not intuitive to DMFC electrocatalyst development, particularly where claims of novel materials with 'high' MOR activity are made.

In a second study, the application of DEMS toward fundamental study on the electrochemical oxidation of HSAC supports (a.k.a. carbon blacks) was demonstrated by comparing the electrochemical oxidation tendencies of a pristine EC300, and five graphitised carbon black samples (heat-treated at various temperatures between 2100 and $3200{ }^{\circ} \mathrm{C}$ ). As part of the study, a method was developed to quantify the loading of the carbon black on the WE substrate in-situ by measuring the sample capacitance and comparing this with a pre-determined gravimetric capacitance. Furthermore, a polycrystalline Au was employed as WE substrate rather than the more common GC which was found to electrochemically oxidise to $\mathrm{CO}_{2}$ at potentials $>1.4 \mathrm{~V}_{\mathrm{RHE}}$. By quantifying the $\mathrm{CO}_{2}$ produced during the high potential excursion using DEMS, it was observed that the faradaic current contains additional current contributions attributed to the 
partial COR of the carbon surface to either $\mathrm{CO}$ or oxygen containing surface functional groups. The electrochemical oxidation tendencies of the carbon black were compared in three experimental phases. In the initial potential excursion to 2.05 $\mathrm{V}_{\mathrm{RHE}}$ (phase (i)), the COR rates were observed to follow in the order EC300 $>$ mildly graphitised $\left(2100>2450{ }^{\circ} \mathrm{C}\right)>$ heavily graphitised carbons $\left(\geq 2800{ }^{\circ} \mathrm{C}\right)$. During repeated potential excursions between 1.05 and 2.05 $\mathrm{V}_{\mathrm{RHE}}$ (in phase (ii)), and repeated potential excursions with a progressively decreasing electrode potential limit down to $0.05 \mathrm{~V}_{\mathrm{RHE}}$ (in phase (iii)), the COR processes were revealed to be rather dynamic exhibiting three trends. In phase (ii) the COR processes either: (i) stopped abruptly (EC300), (ii) progressively increased and then decreased $\left(2100\right.$ and $\left.2450{ }^{\circ} \mathrm{C}\right)$, or (iii) progressively increased for each subsequent $\mathrm{CV}\left(\geq 2800^{\circ} \mathrm{C}\right)$, whilst in phase (iii) the COR processes were either: (i) reinitiated (EC300), (ii) enhanced (Samples $\leq 2450{ }^{\circ} \mathrm{C}$ ), or (iii) unaffected $\left(\geq 2800^{\circ} \mathrm{C}\right)$ in subsequent high potential excursions after decreasing the lower electrode potential limit to $\leq \mathbf{0 . 2 4} \mathrm{V}_{\mathrm{RHE}}$. These trends were rationalised by considering the relative stability of the oxygen containing surface functional groups and the consequence of the partial and complete COR on the EOSA of the carbon black. The overall COR charge evolved for each carbon followed in the order: mildly graphitised > heavily graphitised > EC300, in stark contrast to the initial COR rates. The study was then briefly extended to the electrochemical oxidation of EC300 HSAC Pt catalysts, which was dominated by the OER on the supported Pt particles. Nonetheless, the EC300 support exhibiting the same trend as the pure EC300 carbon, although an additional feature at $0.8 \mathrm{~V}_{\mathrm{RHE}}$ was observed during phase (iii) of the experiment which was attributed to the oxidation of $\mathrm{CO}$ by the supported Pt particles. Finally, the potential application of DEMS towards correlating the HSAC support weight loss, with the loss of ECSA of the supported Pt particles was briefly demonstrated, and could be of particular interest for industrial electrocatalyst development.

Overall, despite that the MOR and electrochemical COR of carbon blacks have long since been studied using a variety of experimental techniques, the DEMS instrument constructed in this thesis could, and can continue to bring new insights into both reaction processes. 


\section{References}

1. Boyle, R., et al., GLOBAL TRENDS IN SUSTAINABLE ENERGY INVESTMENT 2008. 2008, United Nations Environment Programme.

2. Commision, E., EUR 20719 EN - Hydrogen Energy and Fuel Cells - A vision of our future. 2003.

3. Ro, S.T. and J.L. Sohn, Some issues on performance analysis of fuel cells in thermodynamic point of view. Journal of Power Sources, 2007. 167(2): p. 295-301.

4. Haas, H.R. and M.T. Davis, Electrode and Catalyst Durability Requirements in Automotive PEM Applications: Technology Status of a Recent MEA Design and Next Generation Challenges. ECS Transactions, 2009. 25(1): p. 1623-1631.

5. Schmittinger, W. and A. Vahidi, A review of the main parameters influencing long-term performance and durability of PEM fuel cells. Journal of Power Sources, 2008. 180(1): p. 1-14.

6. Gasteiger, H.A. and N.M. Marković, Just a Dream-or Future Reality? Science, 2009. 324(5923): p. 48-49.

7. Gasteiger, H.A., et al., Activity benchmarks and requirements for Pt, Pt-alloy, and non-Pt oxygen reduction catalysts for PEMFCs. Applied Catalysis B: Environmental, 2005. 56(1-2): p. 9-35.

8. Lindermeir, A., et al., On the question of MEA preparation for DMFCs. Journal of Power Sources, 2004. 129(2): p. 180-187.

9. Gasteiger, H. and M. Mathias. FUNDAMENTAL RESEARCH AND DEVELOPMENT CHALLENGES IN POLYMER ELECTROLYTE FUEL CELL TECHNOLOGY. in 202nd Meeting of the ECS. 2002. Salt Lake City.

10. Wiberg, G.K.H., K.J.J. Mayrhofer, and M. Arenz, Investigation of the Oxygen Reduction Activity on Silver - A Rotating Disc Electrode Study. Fuel Cells, 2010. 10(4): p. 575-581.

11. Gasteiger, H.A., et al., Methanol electrooxidation on well-characterized platinum-ruthenium bulk alloys. The Journal of Physical Chemistry, 1993. 97(46): p. 12020-12029.

12. Markovic, N.M., et al., STRUCTURAL EFFECTS IN ELECTROCATALYSIS - OXYGEN REDUCTION ON PLATINUM LOWINDEX SINGLE-CRYSTAL SURFACES IN PERCHLORIC-ACID SOLUTIONS. Journal of Electroanalytical Chemistry, 1994. 377(1-2): p. 249-259. 
13. Stamenkovic, V.R., et al., Trends in electrocatalysis on extended and nanoscale Pt-bimetallic alloy surfaces. Nature Materials, 2007. 6(3): p. 241247.

14. Markovic, N.M., et al., ELECTROOXIDATION MECHANISMS OF METHANOL AND FORMIC-ACID ON PT-RU ALLOY SURFACES. Electrochimica Acta, 1995. 40(1): p. 91-98.

15. Gasteiger, H.A., et al., TEMPERATURE-DEPENDENT METHANOL ELECTROOXIDATION ON WELL-CHARACTERIZED PT-RU ALLOYS. Journal of the Electrochemical Society, 1994. 141(7): p. 1795-1803.

16. Gasteiger, H.A., et al., ELECTROOXIDATION OF SMALL ORGANICMOLECULES ON WELL-CHARACTERIZED PT-RU ALLOYS. Electrochimica Acta, 1994. 39(11-12): p. 1825-1832.

17. Koper, M.T.M., Combining experiment and theory for understanding electrocatalysis. Journal of Electroanalytical Chemistry, 2005. 574(2): p. 375-386.

18. Markovic, N.M. and P.N. Ross, Surface science studies of model fuel cell electrocatalysts. Surface Science Reports, 2002. 45(4-6): p. 117-229.

19. Markovic, N.M. and P.N. Ross, Electrocatalysts by design: from the tailored surface to a commercial catalyst. Electrochimica Acta, 2000. 45(25-26): p. 4101-4115.

20. Mayrhofer, K.J.J., et al., Measurement of oxygen reduction activities via the rotating disc electrode method: From Pt model surfaces to carbon-supported high surface area catalysts. Electrochimica Acta, 2008. 53(7): p. 3181-3188.

21. Paulus, U.A., et al., Oxygen reduction on high surface area Pt-based alloy catalysts in comparison to well defined smooth bulk alloy electrodes. Electrochimica Acta, 2002. 47(22-23): p. 3787-3798.

22. Paulus, U.A., et al., Oxygen reduction on a high-surface area Pt/Vulcan carbon catalyst: a thin-film rotating ring-disk electrode study. Journal of Electroanalytical Chemistry, 2001. 495(2): p. 134-145.

23. Schmidt, T.J., et al., Characterization of high-surface area electrocatalysts using a rotating disk electrode configuration. Journal of the Electrochemical Society, 1998. 145(7): p. 2354-2358.

24. Mayrhofer, K.J.J., et al., Fuel cell catalyst degradation on the nanoscale. Electrochemistry Communications, 2008. 10(8): p. 1144-1147.

25. Mayrhofer, K.J.J., et al., Non-destructive transmission electron microscopy study of catalyst degradation under electrochemical treatment. Journal of Power Sources, 2008. 185(2): p. 734-739. 
26. Wolter, O. and J. Heitbaum, Differential Electrochemical MassSpectroscopy (Dems) - a New Method for the Study of Electrode Processes. Berichte Der Bunsen-Gesellschaft-Physical Chemistry Chemical Physics, 1984. 88(1): p. 2-6.

27. Bruckenstein, S. and R.R. Gadde, Use of a porous electrode for in situ mass spectrometric determination of volatile electrode reaction products. Journal of the American Chemical Society, 1971. 93(3): p. 793-794.

28. Jusys, Z., H. Massong, and H. Baltruschat, $A$ new approach for simultaneous DEMS and EQCM: Electro-oxidation of adsorbed CO on Pt and Pt-Ru. Journal of the Electrochemical Society, 1999. 146(3): p. 10931098.

29. Hoch, G. and B. Kok, A mass spectrometer inlet system for sampling gases dissolved in liquid phases. Archives of Biochemistry and Biophysics, 1963. 101(1): p. 160-170.

30. Willsau, J. and J. Heitbaum, THE INFLUENCE OF PT-ACTIVATION ON THE CORROSION OF CARBON IN GAS-DIFFUSION ELECTRODES - A DEMS STUDY. Journal of Electroanalytical Chemistry, 1984. 161(1): p. 93-101.

31. Schmiemann, U. and H. Baltruschat, THE ADSORPTION OF ETHENE AT PT SINGLE-CRYSTAL ELECTRODES - DESORPTION PRODUCTS AND OBSERVATION OF MULTIPLE ADSORPTION STATES BY DEMS. Journal of Electroanalytical Chemistry, 1992. 340(1-2): p. 357-363.

32. Jusys, Z., J. Kaiser, and R.J. Behm, Composition and activity of high surface area PtRu catalysts towards adsorbed $\mathrm{CO}$ and methanol electrooxidation - A DEMS study. Electrochimica Acta, 2002. 47(22-23): p. 3693-3706.

33. Seiler, T., et al., Poisoning of PtRu/C catalysts in the anode of a direct methanol fuel cell: a DEMS study. Electrochimica Acta, 2004. 49(22-23): p. 3927-3936.

34. Baltruschat, H., Differential electrochemical mass spectrometry. Journal of the American Society for Mass Spectrometry, 2004. 15(12): p. 1693-1706.

35. Wieckowski, A., Interfacial Electrochemistry: Theory: Experiment, and Applications. 1999, New York: Marcel Dekker.

36. Fujihira, $M$. and T. Noguchi, A NOVEL DIFFERENTIAL ELECTROCHEMICAL MASS-SPECTROMETER (DEMS) WITH A STATIONARY GAS-PERMEABLE ELECTRODE IN A ROTATIONAL FLOW PRODUCED BY A ROTATING ROD. Journal of Electroanalytical Chemistry, 1993. 347(1-2): p. 457-463.

37. Mayrhofer, K.J.J., et al., An Electrochemical Cell Configuration Incorporating an Ion Conducting Membrane Separator between Reference 
and Working Electrode. International Journal of Electrochemical Science, 2009. 4(1): p. 1-8.

38. Lanz, M. and P. Novak, DEMS study of gas evolution at thick graphite electrodes for lithium-ion batteries: the effect of gamma-butyrolactone. Journal of Power Sources, 2001. 102(1-2): p. 277-282.

39. Novak, P., et al., Advanced in situ methods for the characterization of practical electrodes in lithium-ion batteries. Journal of Power Sources, 2000. 90(1): p. 52-58.

40. Hartung, T. and H. Baltruschat, Differential Electrochemical MassSpectrometry Using Smooth Electrodes - Adsorption and H/D-Exchange Reactions of Benzene on Pt. Langmuir, 1990. 6(5): p. 953-957.

41. Loffler, T. and $H$. Baltruschat, Temperature dependent formation of multiple adsorption states from ethene at polycrystalline Pt and Pt(111) electrodes studied by differential electrochemical mass spectrometry. Journal of Electroanalytical Chemistry, 2003. 554: p. 333-344.

42. Sanabria-Chinchilla, J., et al., A DEMS study of the electrocatalytic hydrogenation and oxidation of p-dihydroxybenzene at polycrystalline and monocrystalline platinum electrodes. Journal of Applied Electrochemistry, 2006. 36(11): p. 1253-1260.

43. Sanabria-Chinchilla, J., et al., Electrocatalytic hydrogenation and oxidation of aromatic compounds studied by DEMS: Benzene and p-dihydroxybenzene at ultrathin Pd films electrodeposited on $A u(h k l)$ surfaces. Journal of Colloid and Interface Science, 2007. 314(1): p. 152-159.

44. Heinen, M., et al., In situ ATR-FTIRS coupled with on-line DEMS under controlled mass transport conditions - A novel tool for electrocatalytic reaction studies. Electrochimica Acta, 2007. 52(18): p. 5634-5643.

45. Vielstich, W., H. Gasteiger, and H. Yokokawa, Handbook of Fuel Cells: Advanced in Electrocatalysis, Materials, Diagnostics and Durability. Vol. 5. 2009: Wiley.

46. Jusys, Z. and R.J. Behm, Methanol Oxidation on a Carbon-Supported Pt Fuel Cell CatalystA Kinetic and Mechanistic Study by Differential Electrochemical Mass Spectrometry. The Journal of Physical Chemistry B, 2001. 105(44): p. 10874-10883.

47. Holzbecher, E. Thin Layer Flow Cell Modelling. in COMSOL Users Conference. 2006. Frankfurt.

48. Colmenares, L.C., et al., Model study on the stability of carbon support materials under polymer electrolyte fuel cell cathode operation conditions. Journal of Power Sources, 2009. 190(1): p. 14-24. 
49. Ming-fang, L. and et al., Oxidation of Carbon Supports at Fuel Cell Cathodes: Differential Electrochemical Mass Spectrometric Study. Chinese Journal of Chemical Physics, 2010. 23(4): p. 442.

50. Gao, Y.Z., et al., NEW ONLINE MASS-SPECTROMETER SYSTEM DESIGNED FOR PLATINUM SINGLE-CRYSTAL ELECTRODE AND ELECTROREDUCTION OF ACETYLENE. Journal of Electroanalytical Chemistry, 1994. 372(1-2): p. 195-200.

51. Wonders, A.H., et al., On-line mass spectrometry system for measurements at single-crystal electrodes in hanging meniscus configuration. Journal of Applied Electrochemistry, 2006. 36(11): p. 1215-1221.

52. Jambunathan, K., S. Jayaraman, and A.C. Hillier, A Multielectrode Electrochemical and Scanning Differential Electrochemical Mass Spectrometry Study of Methanol Oxidation on Electrodeposited PtxRuy. Langmuir, 2004. 20(5): p. 1856-1863.

53. Jambunathan, K. and A.C. Hillier, Measuring Electrocatalytic Activity on a Local Scale with Scanning Differential Electrochemical Mass Spectrometry. Journal of the Electrochemical Society, 2003. 150(6): p. E312-E320.

54. Urtiaga, A.M., et al., Parallelism and differences of pervaporation and vacuum membrane distillation in the removal of VOCs from aqueous streams. Separation and Purification Technology, 2001. 22-23: p. 327-337.

55. Drioli, E., V. Calabro, and Y. Wu, MICROPOROUS MEMBRANES IN MEMBRANE DISTILLATION. Pure and Applied Chemistry, 1986. 58(12): p. 1657-\&.

56. Jusys, Z., J. Kaiser, and R.J. Behm, Methanol Electrooxidation over Pt/C Fuel Cell Catalysts: Dependence of Product Yields on Catalyst Loading. Langmuir, 2003. 19(17): p. 6759-6769.

57. Wang, H., Z. Jusys, and R.J. Behm, Ethanol electro-oxidation on carbonsupported Pt, PtRu and Pt3Sn catalysts: A quantitative DEMS study. Journal of Power Sources, 2006. 154(2): p. 351-359.

58. Schmidt, T.J., et al., On the CO tolerance of novel colloidal PdAu/carbon electrocatalysts. Journal of Electroanalytical Chemistry, 2001. 501(1-2): p. 132-140.

59. Smith, S.P.E., E. Casado-Rivera, and H.D. Abruna, Application of differential electrochemical mass spectrometry to the electrocatalytic oxidation of formic acid at a modified Bi/Pt electrode surface. Journal of Solid State Electrochemistry, 2003. 7(9): p. 582-587.

60. Pastor, E. and V.M. Schmidt, ELECTROCHEMICAL REACTIONS OF ETHENE ON POLYCRYSTALLINE AU ELECTRODES IN ACIDSOLUTION STUDIED BY DIFFERENTIAL ELECTROCHEMICAL 
MASS-SPECTROMETRY AND ISOTOPE LABELING. Journal of Electroanalytical Chemistry, 1995. 383(1-2): p. 175-180.

61. Rosca, V., G.L. Beltramo, and M.T.M. Koper, Hydroxylamine electrochemistry at polycrystalline platinum in acidic media: a voltammetric, DEMS and FTIR study. Journal of Electroanalytical Chemistry, 2004. 566(1): p. 53-62.

62. Willsau, J., O. Wolter, and J. Heitbaum, ON THE NATURE OF THE ADSORBATE DURING METHANOL OXIDATION AT PLATINUM - A DEMS STUDY. Journal of Electroanalytical Chemistry, 1985. 185(1): p. 163-170.

63. Jusys, Z., J. Liaukonis, and A. Vaskelis, THE CATALYTIC-OXIDATION OF HYPOPHOSPHITE ON NICKEL STUDIED BY ELECTROCHEMICAL MASS-SPECTROMETRY. Journal of Electroanalytical Chemistry, 1991. 307(1-2): p. 87-97.

64. Eggert, G. and J. Heitbaum, ELECTROCHEMICAL REACTIONS OF PROPYLENECARBONATE AND ELECTROLYTES SOLVED THEREIN A DEMS STUDY. Electrochimica Acta, 1986. 31(11): p. 1443-1448.

65. Hibbert, D.B. and C.R. Churchill, Kinetics of the electrochemical evolution of isotopically enriched gases. Part 3.-Hydrogen and deuterium evolution on platinum and platinised tungsten trioxide. Journal of the Chemical Society, Faraday Transactions 1: Physical Chemistry in Condensed Phases, 1984. 80(7): p. 1977-1984.

66. Diehl, G. and U. Karst, On-line electrochemistry - MS and related techniques. Analytical and Bioanalytical Chemistry, 2002. 373(6): p. 390398.

67. Deng, H. and G.J.V. Berkel, A Thin-Layer Electrochemical Flow Cell Coupled On-Line with Electrospray-Mass Spectrometry for the Study of Biological Redox Reactions. Electroanalysis, 1999. 11(12): p. 857-865.

68. Bökman, C.F., C. Zettersten, and L. Nyholm, A Setup for the Coupling of a Thin-Layer Electrochemical Flow Cell to Electrospray Mass Spectrometry. Analytical Chemistry, 2004. 76(7): p. 2017-2024.

69. Zhou, F., Electrochemistry combined on-line with atomic mass spectrometry and related techniques for trace-metal analysis and electrode-reaction studies. TrAC Trends in Analytical Chemistry, 2005. 24(3): p. 218-227.

70. Wasmus, S., S.R. Samms, and R.F. Savinell, MULTIPURPOSE ELECTROCHEMICAL MASS-SPECTROMETRY - A NEW POWERFUL EXTENSION OF DIFFERENTIAL ELECTROCHEMICAL MASSSPECTROMETRY. Journal of the Electrochemical Society, 1995. 142(4): p. 1183-1189. 
71. Willsau, J., O. Wolter, and J. Heitbaum, DOES THE OXIDE LAYER TAKE PART IN THE OXYGEN EVOLUTION REACTION ON PLATINUM - A DEMS STUDY. Journal of Electroanalytical Chemistry, 1985. 195(2): p. 299-306.

72. Bansch, B., et al., REDUCTION AND OXIDATION OF ADSORBED ACETONE AT PLATINUM-ELECTRODES STUDIED BY DEMS. Journal of Electroanalytical Chemistry, 1989. 259(1-2): p. 207-215.

73. Loffler, T., et al., Adsorption and desorption reactions of bicyclic aromatic compounds at polycrystalline and Pt(111) studied by DEMS. Journal of Electroanalytical Chemistry, 2003. 550: p. 81-92.

74. Vrestal, J., et al., Mass spectrometric study of desorption and hydrogenation of biphenyl, naphthalene and t-butylbenzene on poly-and monocrystalline platinum electrodes. Journal of Electroanalytical Chemistry, 1999. 461(1-2): p. 90-93.

75. Jusys, Z., et al., Activity of PtRuMeOx (Me =W, Mo or V) catalysts towards methanol oxidation and their characterization. Journal of Power Sources, 2002. 105(2): p. 297-304.

76. Wang, H. and H. Baltruschat, DEMS Study on Methanol Oxidation at Polyand Monocrystalline Platinum Electrodes: The Effect of Anion, Temperature, Surface Structure, Ru Adatom, and Potential. The Journal of Physical Chemistry C, 2007. 111(19): p. 7038-7048.

77. Fujiwara, N., K.A. Friedrich, and U. Stimming, Ethanol oxidation on PtRu electrodes studied by differential electrochemical mass spectrometry. Journal of Electroanalytical Chemistry, 1999. 472(2): p. 120-125.

78. Jiang, L., et al., Ethanol electrooxidation on novel carbon supported Pt/SnOx/C catalysts with varied Pt : Sn ratio. Electrochimica Acta, 2007. 53(2): p. 377-389.

79. Colmenares, L., et al., Ethanol oxidation on novel, carbon supported Pt alloy catalysts - Model studies under defined diffusion conditions. Electrochimica Acta, 2006. 52(1): p. 221-233.

80. Bagotzky, V.S., Y.B. Vassiliev, and O.A. Khazova, GENERALIZED SCHEME OF CHEMISORPTION, ELECTROOXIDATION AND ELECTROREDUCTION OF SIMPLE ORGANIC-COMPOUNDS ON PLATINUM GROUP METALS. Journal of Electroanalytical Chemistry, 1977. 81(2): p. 229-238.

81. Wang, H., T. Loffler, and H. Baltruschat, Formation of intermediates during methanol oxidation: A quantitative DEMS study. Journal of Applied Electrochemistry, 2001. 31(7): p. 759-765.

82. Anastasijevic, N.A., H. Baltruschat, and J. Heitbaum, DEMS AS A TOOL FOR THE INVESTIGATION OF DYNAMIC PROCESSES - 
GALVANOSTATIC FORMIC-ACID OXIDATION ON A PT ELECTRODE. Journal of Electroanalytical Chemistry, 1989. 272(1-2): p. 89-100.

83. Holzbecher, E., et al. Modellierung von Dünnschichtzellen (Thin Layer FLow Modelling), in COMSOL Users Conference. 2006. Frankfurt.

84. Fuhrmann, J., et al., Numerical calculation of the limiting current for a cylindrical thin layer flow cell. Electrochimica Acta, 2009. 55(2): p. 430-438.

85. Operating Instructions, Quadrupole Mass Spectrometer System, QMG 422. Vol. BG 805983 BE: Pfeiffer Vacuum GmbH.

86. Homan, F., Design for an inexpensive externally operable variable aperture to be used in UHV. Journal of Vacuum Science and Technology, 1980. 17(2): p. 664-664.

87. Velghe, M., D.A.G. Deacon, and J.M. Ortega, Realization of a variable aperture diaphragm working in ultra high vacuum. Applied Optics, 1984. 23(21).

88. Furman, S.A., et al., Improving the detection limit of a quadrupole mass spectrometer. Journal of Vacuum Science \& Technology A: Vacuum, Surfaces, and Films, 2001. 19(3): p. 1032-1033.

89. Gerlach, W., PPM analysis by using quadrupole mass spectrometers with closed ion source. Vacuum, 1996. 47(4): p. 371-374.

90. Wiberg, G.K.H., The devlopment of a state-of-the-art experimental setup demonstrated by the investigation of fuel cell reactions in alkaline electrolyte, in Lehrstühl für Physicalische Chemie. 2010, Technische Universität München: München.

91. KD Scientific Model 200 Series Manual: KD Scientific Inc.

92. Britz, D., $i R$ elimination in electrochemical cells. Journal of Electroanalytical Chemistry, 1978. 88(3): p. 309-352.

93. Oelßner, W., F. Berthold, and U. Guth, The iR drop-well-known but often underestimated in electrochemical polarization measurements and corrosion testing. Materials and Corrosion, 2006. 57(6): p. 455-466.

94. Lamy, C. and C.C. Herrmann, A NEW METHOD FOR OHMIC-DROP COMPENSATION IN POTENTIOSTATIC CIRCUITS - STABILITY AND BANDPASS ANALYSIS, INCLUDING EFFECT OF FARADAIC IMPEDANCE. Journal of Electroanalytical Chemistry, 1975. 59(2): p. 113135.

95. Quadstar 32-bit, Application Program for Quadrupole Mass Spectrometers QMS 422 and QMS 200.: Pfeiffer Vacuum GmbH. 
96. Operating Instructions, Compact Capacitance Gauge: Pfeiffer Vacuum GmbH.

97. Operating Instructions, Compact FullRange BA Gauge: Pfeiffer Vacuum GmbH.

98. Operating Instructions, Compact Cold Cathode Gauge: Pfeiffer Vacuum GmbH.

99. Operating manual, TPG 256 A, Vacuum measurement and control unit for Compact Gauges, MaxiGauge.

100. Operating Instructions: TRIVAC B Rotary Vane Vacuum Pump D 16 B / D25 B: Leybold Vacuum.

101. Lukindo, A. LabVIEW Queued State Machine Architecture. 2007 01.10.2007; Available from: http://expressionflow.com/2007/10/01/labviewqueued-state-machine-architecture/.

102. Technical Note 200: Getting to Know Your Potentiostat: Potentiostat Stability Considerations. 2010: Princeton Applied Research.

103. Electrochemistry - Application note $n^{\circ} 4$ : Potentiostat stability mystery explained. 2010: BioLogic Science Instruments.

104. Brett, C.M.A. and A.M.O. Brett, ELECTROCHEMISTRY: Principles, Methods, and Applications. 1993: Oxford University Press.

105. Operating Instructions, Quadrupole Analyzer QMA 125: Pfeiffer Vacuum GmbH.

106. Kendall, B.R.F., Apparatus for measuring the internal volumes of vacuum systems. Journal of Vacuum Science and Technology, 1974. 11(3): p. 610611.

107. Dobrozemsky, R., Calibration of vacuum systems by gas quantities. Vacuum, 1990. 41(7-9): p. 2109-2111.

108. Dillon, R., et al., International activities in DMFC R\&D: status of technologies and potential applications. Journal of Power Sources, 2004. 127(1-2): p. 112-126.

109. Wasmus, S. and A. Küver, Methanol oxidation and direct methanol fuel cells: a selective review. Journal of Electroanalytical Chemistry, 1999. 461(1-2): p. 14-31.

110. Wang, K., et al., On the reaction pathway for methanol and carbon monoxide electrooxidation on Pt-Sn alloy versus Pt-Ru alloy surfaces. Electrochimica Acta, 1996. 41(16): p. 2587-2593. 
111. Gasteiger, H.A., et al., Carbon monoxide electrooxidation on wellcharacterized platinum-ruthenium alloys. The Journal of Physical Chemistry, 1994. 98(2): p. 617-625.

112. Serov, A. and C. Kwak, Review of non-platinum anode catalysts for DMFC and PEMFC application. Applied Catalysis B: Environmental, 2009. 90(34): p. 313-320.

113. Lai, S., et al., Mechanisms of Carbon Monoxide and Methanol Oxidation at Single-crystal Electrodes. Topics in Catalysis, 2007. 46(3): p. 320-333.

114. Gojkovic, S.L., Electrochemical oxidation of methanol on $\mathrm{Pt}_{3}$ Co bulk alloy. Journal of the Serbian Chemical Society, 2003. 68(11): p. 11.

115. Wang, H., et al., Methanol oxidation on Pt, PtRu, and colloidal Pt electrocatalysts: a DEMS study of product formation. Journal of Electroanalytical Chemistry, 2001. 509(2): p. 163-169.

116. Christensen, P.A., et al., An in situ FTIR study of the electrochemical oxidation of methanol at small platinum particles. Journal of Electroanalytical Chemistry, 1994. 370(1-2): p. 251-258.

117. Korzeniewski, C. and C.L. Childers, Formaldehyde Yields from Methanol Electrochemical Oxidation on Platinum. The Journal of Physical Chemistry B, 1998. 102(3): p. 489-492.

118. Islam, M., R. Basnayake, and C. Korzeniewski, A study of formaldehyde formation during methanol oxidation over PtRu bulk alloys and nanometer scale catalyst. Journal of Electroanalytical Chemistry, 2007. 599(1): p. 3140.

119. Batista, E.A. and T. Iwasita, Adsorbed Intermediates of Formaldehyde Oxidation and Their Role in the Reaction Mechanism. Langmuir, 2006. 22(18): p. 7912-7916.

120. de Lima, R.B., et al., Catalysis of formaldehyde oxidation by electrodeposits of PtRu. Journal of Electroanalytical Chemistry, 2007. 603(1): p. 142-148.

121. Kucernak, A.R., et al., Anodic oxidation of methyl formate and its relationship to the reactions of methanol and formic acid. Electrochimica Acta, 1998. 43(12-13): p. 1705-1714.

122. Wang, H.S., et al., Methanol oxidation on Pt, PtRu, and colloidal Pt electrocatalysts: a DEMS study of product formation. Journal of Electroanalytical Chemistry, 2001. 509(2): p. 163-169.

123. Lin, W.F., et al., Electrochemical versus Gas-Phase Oxidation of Ru SingleCrystal Surfaces. The Journal of Physical Chemistry B, 2000. 104(25): p. 6040-6048. 
124. Tripkovic, A.V., et al., Methanol electrooxidation on supported Pt and PtRu catalysts in acid and alkaline solutions. Electrochimica Acta, 2002. 47(2223): p. 3707-3714.

125. Tripkovic, A.V., et al., Methanol oxidation at platinum electrodes in acid solution : comparison between model and real catalysts. Vol. 71. 2006, Belgrade, SERBIE: Serbian Chemical Society. 11.

126. Schmidt, T.J., H.A. Gasteiger, and R.J. Behm, Methanol electrooxidation on a colloidal PtRu-alloy fuel-cell catalyst. Electrochemistry Communications, 1999. 1(1): p. 1-4.

127. Teng, Z.-H., et al., High activity Pt/C catalyst for methanol and adsorbed CO electro-oxidation. Journal of Power Sources, 2007. 164(1): p. 105-110.

128. Li, L. and Y. Xing, Methanol Electro-Oxidation on Pt-Ru Alloy Nanoparticles Supported on Carbon Nanotubes. Energies, 2009. 2(3): p. 789-804.

129. Bergamaski, K., et al., Nanoparticle Size Effects on Methanol Electrochemical Oxidation on Carbon Supported Platinum Catalysts. The Journal of Physical Chemistry B, 2006. 110(39): p. 19271-19279.

130. Qiu, L., et al., Fabrication of ionic liquid-functionalized polypyrrole nanotubes decorated with platinum nanoparticles and their electrocatalytic oxidation of methanol. Chemical Communications, 2011. 47(10): p. 29342936.

131. García, G., et al., Methanol electrooxidation at mesoporous Pt and Pt-Ru electrodes: A comparative study with carbon supported materials. Journal of Power Sources, 2011. 196(6): p. 2979-2986.

132. Jeon, M.K. and P.J. McGinn, Improvement of methanol electro-oxidation activity of PtRu/C and PtNiCr/C catalysts by anodic treatment. Journal of Power Sources, 2009. 188(2): p. 427-432.

133. Gojkovic, S.L., Mass transfer effect in electrochemical oxidation of methanol at platinum electrocatalysts. Journal of Electroanalytical Chemistry, 2004. 573(2): p. 271-276.

134. Papadopoulos, N., et al., Three-dimensional electrochemistry: Utilization of i-E-t curves for elucidation of electrochemical reactions. Journal of Electroanalytical Chemistry, 1991. 308(1-2): p. 83-96.

135. Yang, R., et al., Monodispersed hard carbon spherules as a catalyst support for the electrooxidation of methanol. Carbon, 2005. 43(1): p. 11-16.

136. Maillard, F., E.R. Savinova, and U. Stimming, $C O$ monolayer oxidation on Pt nanoparticles: Further insights into the particle size effects. Journal of Electroanalytical Chemistry, 2007. 599(2): p. 221-232. 
137. Sarma, L.S., et al., Investigations of direct methanol fuel cell (DMFC) fading mechanisms. Journal of Power Sources, 2007. 167(2): p. 358-365.

138. Gojkovic, S.L., T.R. Vidakovic, and D.R. Durovic, Kinetic study of methanol oxidation on carbon-supported PtRu electrocatalyst. Electrochimica Acta, 2003. 48(24): p. 3607-3614.

139. Meli, G., et al., DIRECT ELECTROOXIDATION OF METHANOL ON HIGHLY DISPERSED PLATINUM-BASED CATALYST ELECTRODES TEMPERATURE EFFECT. Journal of Applied Electrochemistry, 1993. 23(3): p. 197-202.

140. Noel, M. and K.I. Vasu, Cyclic Voltammetry and the Frontiers of Electrochemistry. 1990: South Asia Books.

141. Maiyalagan, T. and B. Viswanathan, Catalytic activity of platinum/tungsten oxide nanorod electrodes towards electro-oxidation of methanol. Journal of Power Sources, 2008. 175(2): p. 789-793.

142. Wang, M., D.-j. Guo, and H.-l. Li, High activity of novel Pd/TiO2 nanotube catalysts for methanol electro-oxidation. Journal of Solid State Chemistry, 2005. 178(6): p. 1996-2000.

143. Lee, K.-S., et al., PtRu overlayers on Au nanoparticles for methanol electrooxidation. Catalysis Today, 2009. 146(1-2): p. 20-24.

144. Barranco, J. and A.R. Pierna, Bifunctional amorphous alloys more tolerant to carbon monoxide. Journal of Power Sources, 2007. 169(1): p. 71-76.

145. Park, I.-S., et al., Electrocatalytic activity of carbon-supported Pt-Au nanoparticles for methanol electro-oxidation. Electrochimica Acta, 2007. 52(18): p. 5599-5605.

146. Hamnett, A., et al., Long-Term Poisoning of Methanol Anodes. Berichte der Bunsengesellschaft für physikalische Chemie, 1990. 94(9): p. 1014-1020.

147. Watanabe, M., M. Uchida, and S. Motoo, Preparation of highly dispersed Pt $+R u$ alloy clusters and the activity for the electrooxidation of methanol. Journal of Electroanalytical Chemistry and Interfacial Electrochemistry, 1987. 229(1-2): p. 395-406.

148. Lin, M.-L., M.-Y. Lo, and C.-Y. Mou, PtRuP nanoparticles supported on mesoporous carbon thin film as highly active anode materials for direct methanol fuel cell. Catalysis Today, 2011. 160(1): p. 109-115.

149. Wiberg, G.K., K.J. Mayrhofer, and M. Arenz, Investigation of the Oxygen Reduction Activity of Non-platinum Catalysts: A RDE Methodology. ECS Meeting Abstracts, 2009. 902(6): p. 349.

150. Gasteiger, H.A., N.M. Markovic, and P.N. Ross, $\mathrm{H} 2$ and $\mathrm{CO}$ Electrooxidation on Well-Characterized Pt, Ru, and Pt-Ru. 1. Rotating Disk 
Electrode Studies of the Pure Gases Including Temperature Effects. The Journal of Physical Chemistry, 1995. 99(20): p. 8290-8301.

151. Zhang, S., et al., A review of platinum-based catalyst layer degradation in proton exchange membrane fuel cells. Journal of Power Sources, 2009. 194(2): p. 588-600.

152. Meyers, J.P. and R.M. Darling, Model of Carbon Corrosion in PEM Fuel Cells. Journal of the Electrochemical Society, 2006. 153(8): p. A1432A1442.

153. Reiser, C.A., et al., A Reverse-Current Decay Mechanism for Fuel Cells. Electrochemical and Solid-State Letters, 2005. 8(6): p. A273-A276.

154. Kim, J., J. Lee, and Y. Tak, Relationship between carbon corrosion and positive electrode potential in a proton-exchange membrane fuel cell during start/stop operation. Journal of Power Sources, 2009. 192(2): p. 674-678.

155. Tang, H., et al., PEM fuel cell cathode carbon corrosion due to the formation of air/fuel boundary at the anode. Journal of Power Sources, 2006. 158(2): p. 1306-1312.

156. Yu, P.T., et al., The Impact of Carbon Stability on PEM Fuel Cell Startup and Shutdown Voltage Degradation. ECS Transactions, 2006. 3(1): p. 797809.

157. Baumgartner, W.R., et al., Polarization study of a PEMFC with four reference electrodes at hydrogen starvation conditions. Journal of Power Sources, 2008. 182(2): p. 413-421.

158. Borup, R., et al., Scientific Aspects of Polymer Electrolyte Fuel Cell Durability and Degradation. Chemical Reviews, 2007. 107(10): p. 3904-3951.

159. Yoda, T., H. Uchida, and M. Watanabe, Effects of operating potential and temperature on degradation of electrocatalyst layer for PEFCs. Electrochimica Acta, 2007. 52(19): p. 5997-6005.

160. Gasteiger, H.A., et al., Catalyst Degradation Mechanisms in PEM and Direct Methanol Fuel Cells, in Mini-Micro Fuel Cells, S. Kakaç, A. Pramuanjaroenkij, and L. Vasiliev, Editors. 2008, Springer Netherlands. p. 225-233.

161. Patterson, T.W. and R.M. Darling, Damage to the Cathode Catalyst of a PEM Fuel Cell Caused by Localized Fuel Starvation. Electrochemical and Solid-State Letters, 2006. 9(4): p. A183-A185.

162. Chen, S., et al., Platinum-Alloy Cathode Catalyst Degradation in Proton Exchange Membrane Fuel Cells: Nanometer-Scale Compositional and Morphological Changes. Journal of the Electrochemical Society, 2010. 157(1): p. A82-A97. 
163. Zhang, J., et al., Effect of Hydrogen and Oxygen Partial Pressure on Pt Precipitation within the Membrane of PEMFCs. Journal of the Electrochemical Society, 2007. 154(10): p. B1006-B1011.

164. Maass, S., et al., Carbon support oxidation in PEM fuel cell cathodes. Journal of Power Sources, 2008. 176(2): p. 444-451.

165. CAI, M., et al., Investigation of thermal and electrochemical degradation of fuel cell catalysts. Vol. 160. 2006, Amsterdam, PAYS-BAS: Elsevier. 10.

166. Nose, M., et al., Electrochemical Oxidation of Highly Oriented Pyrolytic Graphite in Sulphuric Acid Solution under Potential Pulse Condition. Fuel Cells, 2009. 9(3): p. 284-290.

167. Kinumoto, T., et al., Stability of Pt-catalyzed highly oriented pyrolytic graphite against hydrogen peroxide in acid solution. Journal of the Electrochemical Society, 2006. 153(1): p. A58-A63.

168. Ball, S.C., et al., An investigation into factors affecting the stability of carbons and carbon supported platinum and platinum/cobalt alloy catalysts during $1.2 \mathrm{~V}$ potentiostatic hold regimes at a range of temperatures. Journal of Power Sources, 2007. 171(1): p. 18-25.

169. Antonucci, P.L., et al., A comparative analysis of structural and surface effects in the electrochemical corrosion of carbons. Materials Chemistry and Physics, 1989. 21(5): p. 495-506.

170. Hung, C.-C., et al., Corrosion of carbon support for PEM fuel cells by electrochemical quartz crystal microbalance. Journal of Power Sources, 2011. 196(1): p. 140-146.

171. Choo, H.S., et al., Electrochemical oxidation of highly oriented pyrolytic graphite during potential cycling in sulfuric acid solution. Journal of Power Sources, 2008. 185(2): p. 740-746.

172. Avasarala, B., R. Moore, and P. Haldar, Surface oxidation of carbon supports due to potential cycling under PEM fuel cell conditions. Electrochimica Acta, 2010. 55(16): p. 4765-4771.

173. Choo, H.-S., et al., Mechanism for Electrochemical Oxidation of Highly Oriented Pyrolytic Graphite in Sulfuric Acid Solution. Journal of the Electrochemical Society, 2007. 154(10): p. B1017-B1023.

174. Kodera, F., et al., Electrochemical corrosion of platinum electrode in concentrated sulfuric acid. Journal of Power Sources, 2007. 172(2): p. 698703.

175. Kinoshita, K., Chapter 2 Section 2.1 Crystallographic Structure, in Carbon Electrochemical and Physiochemical Properties. 1987, Wiley. p. 20-85. 
176. Kinoshita, K., Chapter 6 Section 6.2 Electrochemical Oxidation of Carbon Blacks and Graphites, in Carbon - Electrochemical and Physiochemical Properties. 1987, Wiley. p. 316-351.

177. Yu, P.T., et al., Carbon-Support Requirements for Highly Durable Fuel Cell Operation, in Polymer Electrolyte Fuel Cell Durability, F.N. Büchi, M. Inaba, and T.J. Schmidt, Editors. 2009.

178. Kinoshita, K., Carbon - Electrochemical and Physiochemical Properties. 1987: Wiley.

179. Kinoshita, K., Chapter 3 Section 3.1.1 Carbon-Oxygen Complexes, in Carbon - Electrochemical and Physiochemical Properties. 1987, Wiley. p. 87-88.

180. Kinoshita, K., Table 3.5 Effect of Heat Treatment on the Distribution of Surface Groups on Carbon Blacks, in Carbon - Electrochemical and Physiochemical Properties. 1987, Wiley. p. 99.

181. Trasatti, S. and O.A. Petrii, Real surface area measurements in electrochemistry. Journal of Electroanalytical Chemistry, 1992. 327(1-2): p. 353-376.

182. Kinoshita, K., Chapter 6 Section 6.1.1 Double-Layer Capacitance, in Carbon - Electrochemical and Physiochemical Properties. 1987, Wiley. p. 293-302.

183. Kinoshita, K., Table 6.1 Double-Layer Capacitance of Carbon and Graphite, in Carbon - Electrochemical and Physiochemical Properties. 1987, Wiley. p. 293-302.

184. Barbieri, O., et al., Capacitance limits of high surface area activated carbons for double layer capacitors. Carbon, 2005. 43(6): p. 1303-1310.

185. Taylor, R.J. and A.A. Humffray, Electrochemical studies on glassy carbon electrodes: I. Electron transfer kinetics. Journal of Electroanalytical Chemistry and Interfacial Electrochemistry, 1973. 42(3): p. 347-354.

186. Kinoshita, K., Chapter 6 Section 6.4.1 Oxygen Redcution/ Evolution, in Carbon - Electrochemical and Physiochemical Properties. 1987, Wiley. p. 369.

187. Kinoshita, K., Fig. 2.20 Affect of heat-treatment $(2 \mathrm{~h})$ on crystallite size and $002 d$-spacing of Vulcan XC-72, in Carbon - Electrochemical and Physiochemical Properties. 1987, Wiley. p. 20-85.

188. Kinoshita, K., Chapter 3 Section 3.2.2 Spectroscopic Analysis, in Carbon Electrochemical and Physiochemical Properties. 1987, Wiley. p. 105-132.

189. Kinoshita, K., Fig. 6.15: Effect of heat-treatment temperature on the electrochemical oxidation rate of various carbons after 1000 min at $1.0 \mathrm{~V}$ in 
$\mathrm{H}_{3} \mathrm{PO}_{4}$, in Carbon - Electrochemical and Physiochemical Properties. 1987, Wiley.

190. Kinoshita, K., Fig. 6.13: Variation in $Q \mathrm{QCO}_{2} / Q_{t}$ with time for the electrochemical oxidation of carbon blacks at $1.2 \mathrm{~V}$ in $96 \% \mathrm{H}_{3} \mathrm{PO}_{4}, 135{ }^{\circ} \mathrm{C}$, in Carbon - Electrochemical and Physiochemical Properties. 1987, Wiley.

191. van Veen, J.A.R., J.F. van Baar, and K.J. Kroese, Effect of heat treatment on the performance of carbon-supported transition-metal chelates in the electrochemical reduction of oxygen. Journal of the Chemical Society, Faraday Transactions 1: Physical Chemistry in Condensed Phases, 1981. 77(11): p. 2827-2843.

192. Kinoshita, K., Table. 2.6: Morphology Parameters and Physicsal Proprty of Carbon Blacks, in Carbon - Electrochemical and Physiochemical Properties. 1987, Wiley.

193. Li, W. and A.M. Lane, Investigation of Pt catalytic effects on carbon support corrosion of the cathode catalyst in PEM fuel cells using DEMS spectra. Electrochemistry Communications, 2009. 11(6): p. 1187-1190.

194. Kinoshita, K., Fig. 6.17: Tafel plots for the electrochemical oxidation of Vulcan $\mathrm{XC}-72$ and Vulcan-2700 in $96 \% \mathrm{H}_{3} \mathrm{PO}_{4}$ at 100 min after start of experiment., in Carbon - Electrochemical and Physiochemical Properties. 1987, Wiley.

195. Kinoshita, K., Fig. 6.16: Effect of heat-treatment temperature and the $002 d$ spacing on the Tafel slopes of the electrochemcial oxidation of Volcan XC-72 in $102 \% \mathrm{H}_{3} \mathrm{PO}_{4}$ at $180^{\circ} \mathrm{C}$., in Carbon - Electrochemical and Physiochemical Properties. 1987, Wiley.

196. Laine, N.R., F.J. FVastola, and P.L. Walker Jr, J. Phys. Chem., 1963(67).

197. Ahmed, S. and M.H. Back, Carbon, 1985. 23(5).

198. Kinoshita, K., Fig. 4.4: Schematic representation of the microstructural changes to carbon-black particles during oxidation., in Carbon Electrochemical and Physiochemical Properties. 1987, Wiley. p. 185.

199. Janssen, L.J.J. and J.G. Hoogland, Mechanism of bromine evolution at a graphite electrode. Electrochimica Acta, 1970. 15(10): p. 1677-1683.

200. Pittman, C.U., et al., Chemical modification of carbon fiber surfaces by nitric acid oxidation followed by reaction with tetraethylenepentamine. Carbon, 1997. 35(3): p. 317-331.

201. Stonehart, P., Carbon substrates for phosphoric acid fuel cell cathodes. Carbon, 1984. 22(4-5): p. 423-431. 
202. Torii, S. and H. Tanaka, Chapter 14 - Carboxylic Acids, in Organic Electrochemistry, 4th Edition, H. Lund and O. Hammerich, Editors. 2001. p. 499-544.

203. Grimshaw, J., Chapter 8 - Oxidation of Alcohols, Amines and Carboxylic Acids, in Electrochemical Reactions and Mechanisms in Organic Chemistry

2000, ELSEVIER.

204. Hammerich, O., Chapter 13 - Anodic Oxidation of Hydrocarbons, in Organic Electrochemistry, 4th Edition, H. Lund and O. Hammerich, Editors. 2001. p. 471-498.

205. Wagenknecht, J.H., L. Eberson, and J.H.P. Utley, Chapter 12 - Carboxylic Acids and Derivatives, in Organic Electrochemistry, 4th Edition, H. Lund and O. Hammerich, Editors. 2001. p. 453-470.

206. Lund, H. and O. Hammerich, Organic Electrochemistry, 4th Edition. Organic Electrochemistry, 4th Edition. 2001: Marcel Dekker, Inc.

207. Grimshaw, J., Electrochemical Reactions and Mechanisms in Organic Chemistry. 2000: ELSEVIER.

208. Kinoshita, K., Chapter 6 Section 6.1.3 Potentiodynamic Invetigations of Surface Groups, in Carbon - Electrochemical and Physiochemical Properties. 1987, Wiley. p. 293-302.

209. Shao, Y., et al., Comparative investigation of the resistance to electrochemical oxidation of carbon black and carbon nanotubes in aqueous sulfuric acid solution. Electrochimica Acta, 2006. 51(26): p. 5853-5857. 


\section{Appendix}

\section{$9.1 A$}
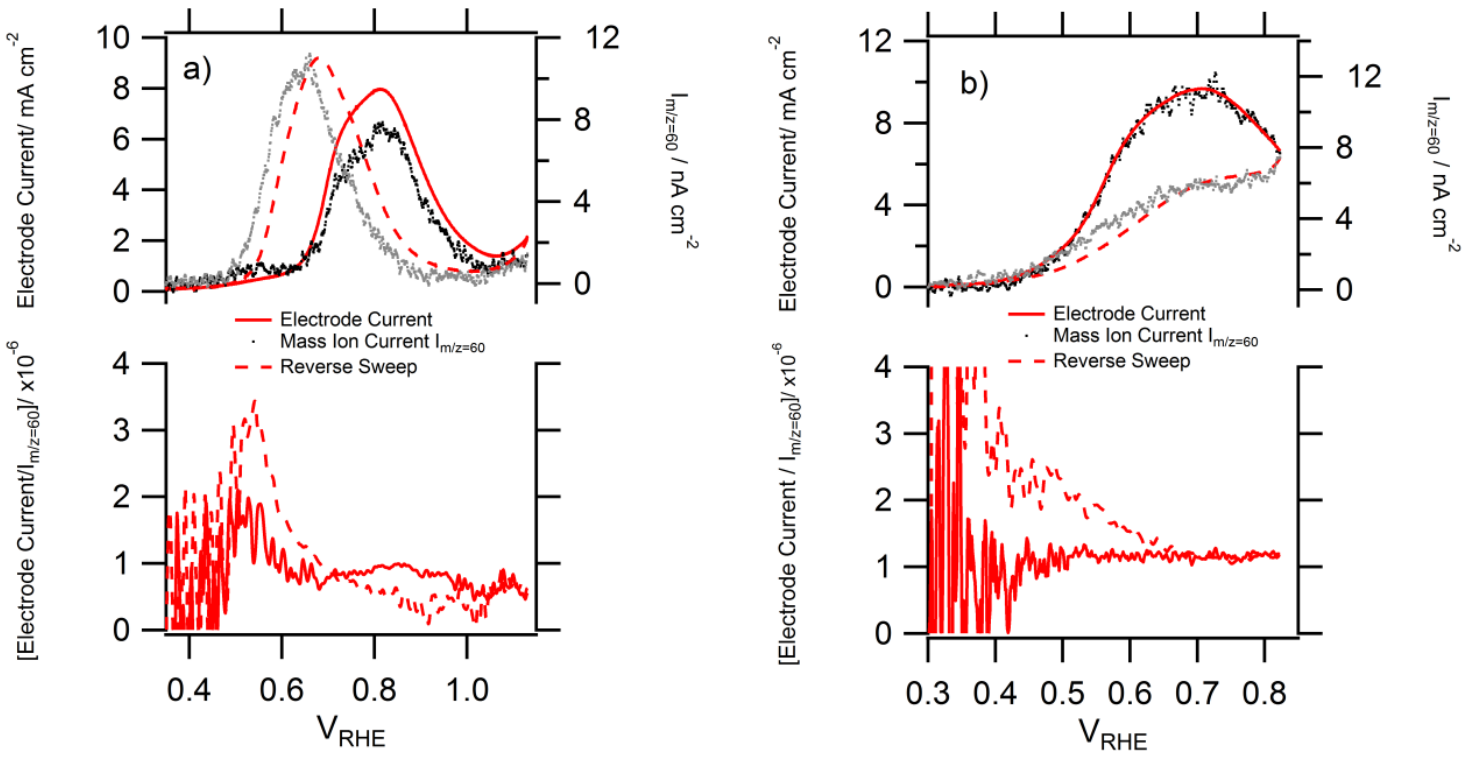

Figure 9-1: CV of the MOR on Pt (a) and PtRu (b) catalyst indicating the methylformate produced, and the ratio with the electrode current.
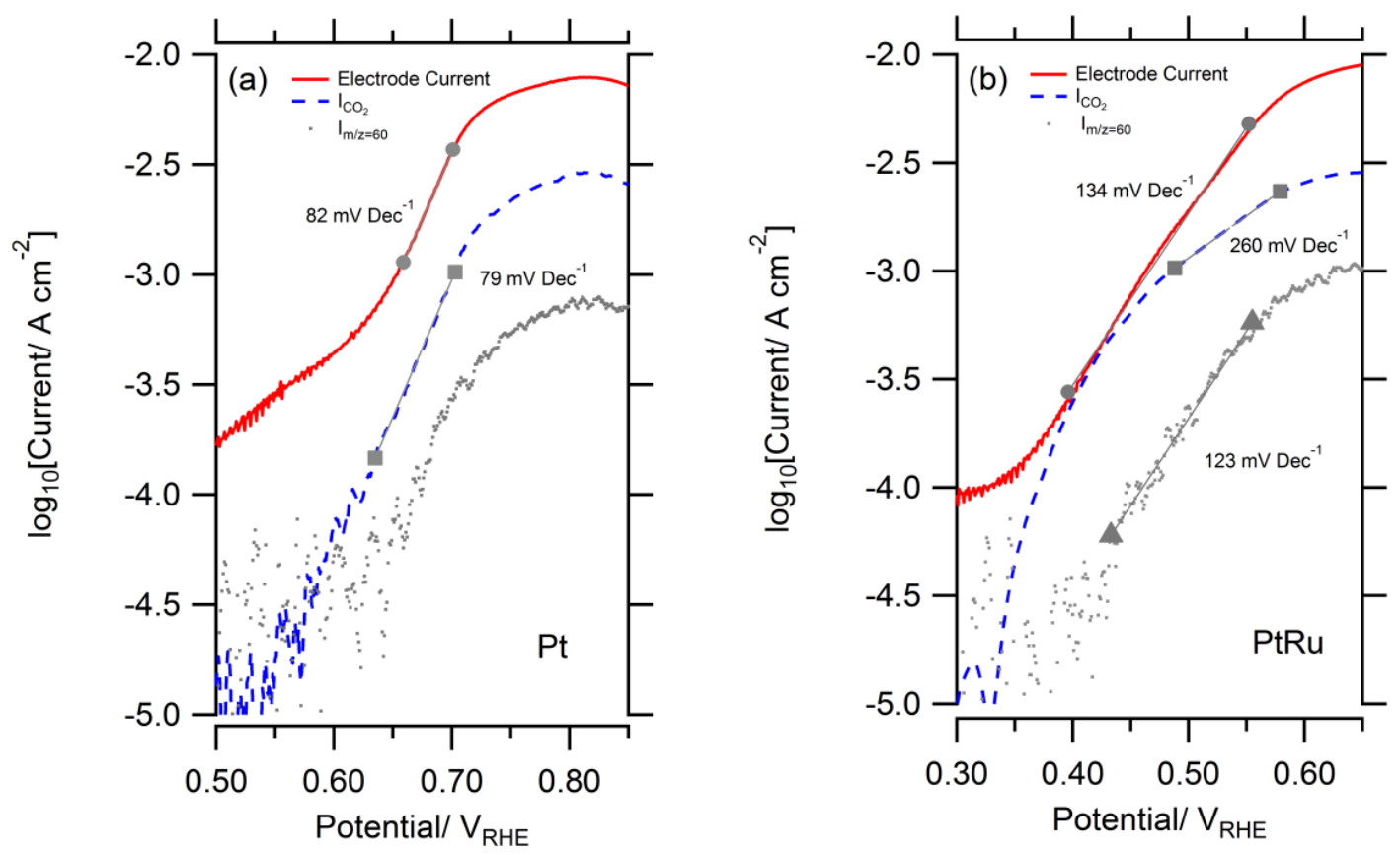

Figure 9-2: Tafel plot of the MOR on the Pt (a) and PtRu (b) catalysts. The Tafel-slope for methylformate does not correspond for the Pt catalysts, but does for the PtRu catalysts in contrast to $I_{\mathrm{CO} 2}$. 


\section{Approach to Determining MOR Activities}

Not all guidelines given in ref. [149] for the ORR activity determination (some of which are specific to the RDE technique) are entirely applicable to the MOR, however, those that are more general are given as follows:

i) The number of active sites $\left(\mathrm{N}_{\mathrm{ECAS}}\right)$ must be measured

ii) Capacitive current must be subtracted in the evaluation of activity

iii) Compensation schemes for the solution resistance must be used

iv) The observed reaction rate and the determined $\mathbf{N}_{\text {ECAS }}$ must depend linearly on the applied loading

v) Normalised activities, i.e. mass activity, specific activity and/or turn over frequency (TOF) should be evaluated in a fuel cell relevant potential window vs. RHE

In principle, applying these guidelines to the determination of the MOR activities should facilitate more meaningful and fuel cell relevant comparison between prospective catalyst materials. There are, however, a number of complications present for the MOR which are less clear cut than in the ORR, particularly concerning guidelines (i), (iv) and (v).

Although the total surface area of Pt and PtRu based catalyst can be rather straightforwardly measured using CO-Stripping, in the case of PtRu this does not necessarily correspond to the number of active sites (i), which is furthermore $y$ dependent on the temperature of the reaction. At $25^{\circ} \mathrm{C}$ the $\mathrm{Ru}$ component of the surface is not active to the adsorption of methanol and therefore only the $\mathrm{Pt}$ surface area possesses active sites. This is, of course, further complicated if the Ru component then contributes to the oxidation of desorbed partial MOR products. At $60{ }^{\circ} \mathrm{C}$ the situation changes and the $\mathrm{Ru}$ possesses some activity towards the MOR, so in such cases normalisation to the CO-Stripping charge is then of greater importance. The intended application could therefore impose different requirements on the normalisation of the catalyst material. 
The number of active sites then leads us directly to guideline (v), the normalisation of activity to either mass, specific activity or TOF at a fuel cell relevant potential with reference to the RHE. The next issue is, of course, how to normalise the observed electrode current. The usefulness of the mass activity is clearly the most technologically important, particular in terms of normalising to the overwhelmingly most expensive component of a catalyst (i.e. Pt). The normalisation to the surface area determined via CO-Stripping may not be entirely useful to the development of the PtRu electrocatalyst if we consider that at ambient temperatures the $\mathrm{Ru}$ component is not active to the adsorption of methanol and cannot be considered an active site. This is, however, perhaps less important for measurements performed at $60{ }^{\circ} \mathrm{C}$ where the $\mathrm{Ru}$ component possesses some activity within the potential region of interest. Unfortunately, TOF cannot be used because in general complete conversion of the MOR to $\mathrm{CO}_{2}$ is not achieved. The definition of the fuel cell relevant potential window is also an added complication, which depends upon the operating conditions of the DMFC. For instance, the anode catalyst of a DMFC operating at $>60^{\circ} \mathrm{C}$ will be unlikely to exceed $0.5 \mathrm{~V}_{\mathrm{RHE}}$, however, the anode of a portable DMFC operating at ambient temperature (the most viable commercial application [108]) would operate at potentials more in line with those observed in the Tafel region of the MOR in the electrochemical half-cell measurements (i.e. $<0.6 \mathrm{~V}_{\text {RHE}}$ ). The definition of fuel cell relevant potential region is therefore dependent on the intended DMFC operating conditions. The benefits of referencing electrode potentials to the RHE meanwhile should be clear enough, but as we observed in table 5-3 potentials are often referenced to the $\mathrm{SCE}$ or $\mathrm{Ag} / \mathrm{AgCl}$ reference electrodes. Moreover, there is also the added problem that there is no good model catalyst to which activites of prospective materials can be benchmarked too; polycrystalline Pt (which is useful for the ORR) is hardly a good MOR activity benchmark for PtRu catalysts.

Finally, the choice of conditions and electroanalytical technique also warrants some discussion: some of my own opinions on the appropriate approach are presented in Appendix A table 9-1. 
Table 9-1: List of experimental variables that must be considered when performing MOR activity measurements in an electrochemical half-cell using an RDE in acid electrolyte.

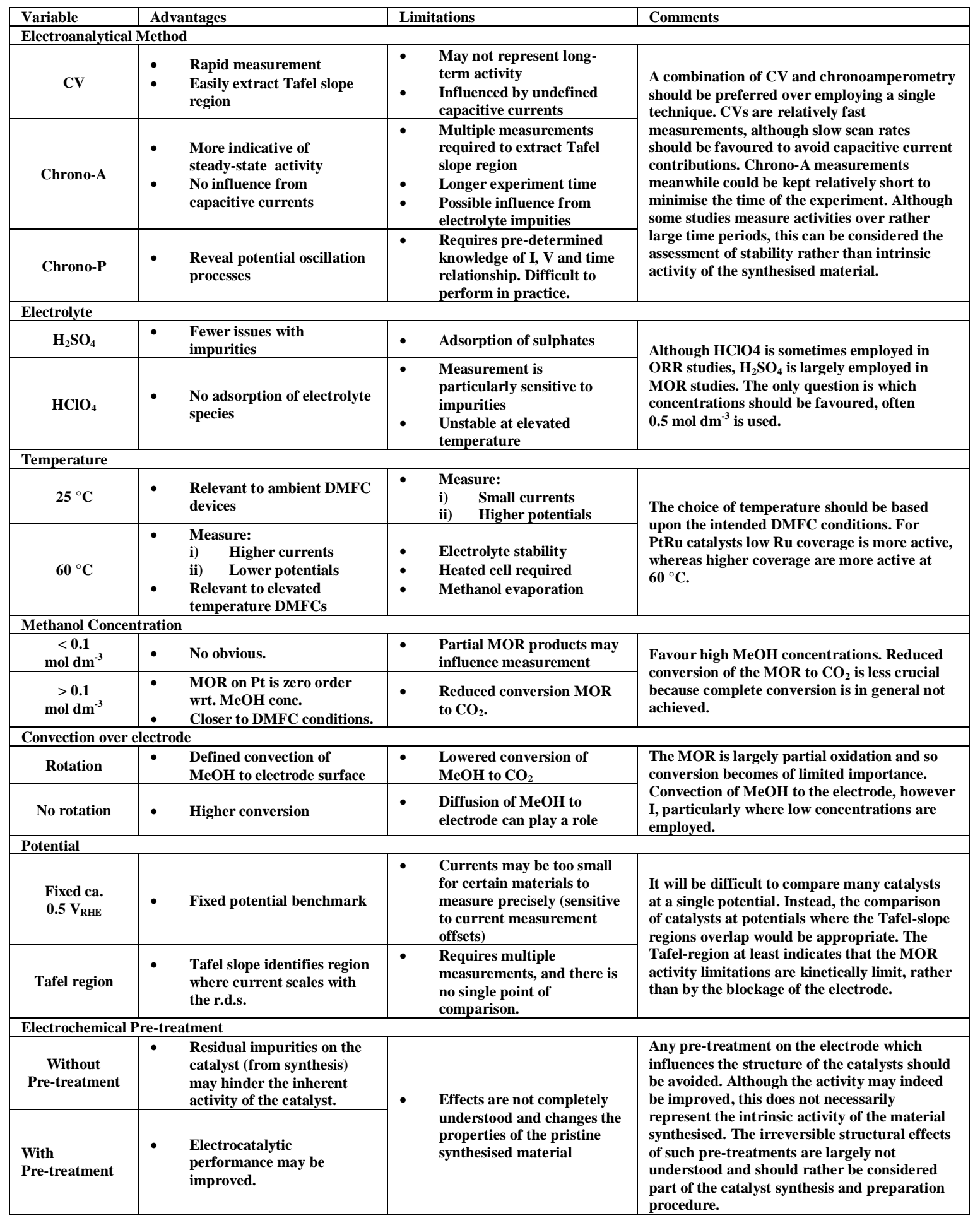

\section{The purpose of this discussion on the activity of the electrocatalysts was not to try}

to explain how to measure but was to rather highlight the problem, with the intention of opening the discussion and to offer some of my own thoughts on the 
matter; however, the development and agreement of a methodology will require discussion between different experts in the field. A documented aid to determine the activities would then be beneficial, clarifying the various techniques that can be employed and experimental considerations: such an informative guide could not make the situation any worse than it currently is.

\section{$9.2 B$}
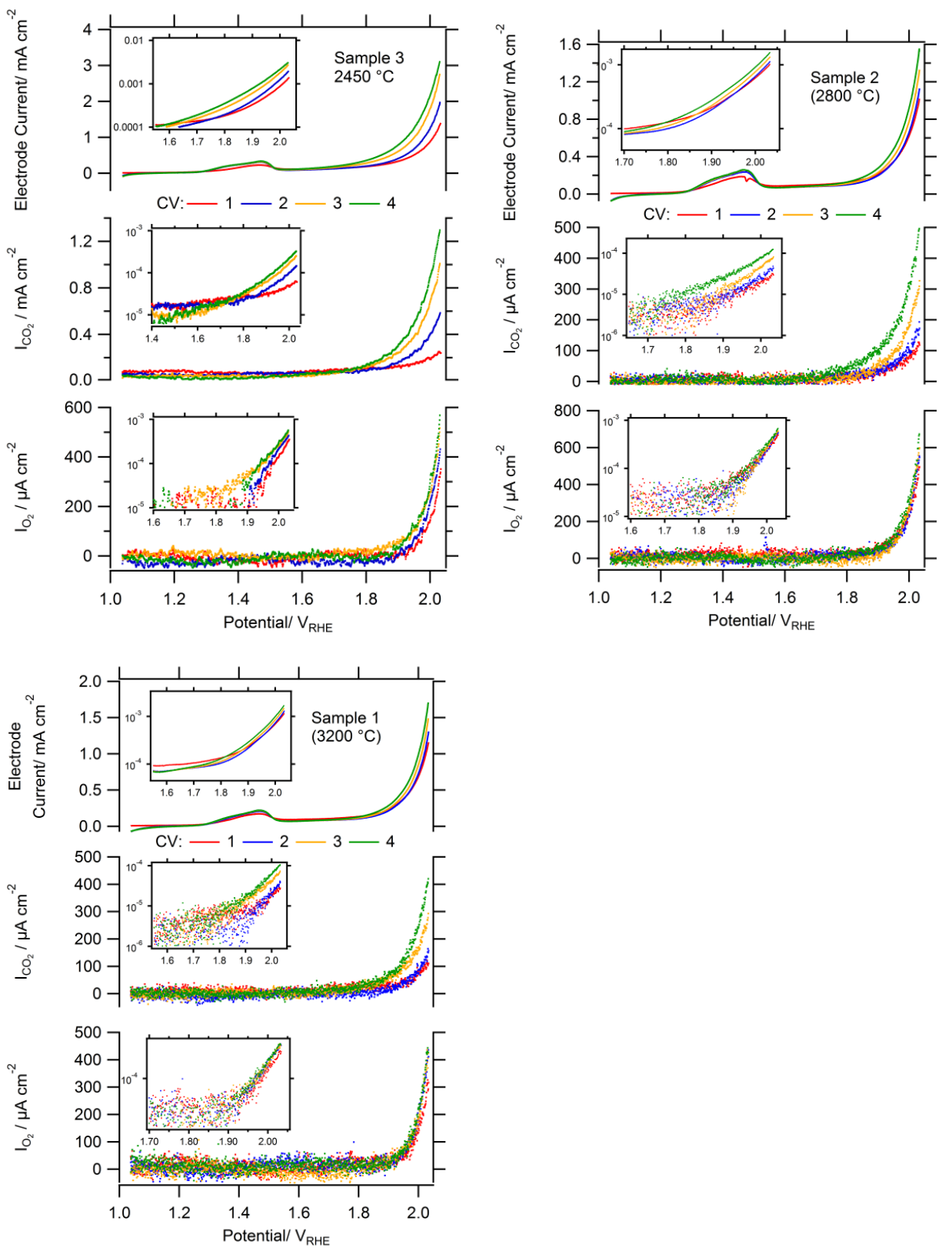

Figure 9-3: First four LSVs of the electrochemical COR of a heat treated carbon black Samples 3, 2 and $1 \mathrm{in} 0.5 \mathrm{~mol} \mathrm{dm}{ }^{-3} \mathrm{H}_{2} \mathrm{SO}_{4}$. Carbon black sample loadings of $85 \mathrm{ug}_{\mathrm{c}} \mathrm{cm}^{-2}$ on polycrystalline Au WE substrate. 

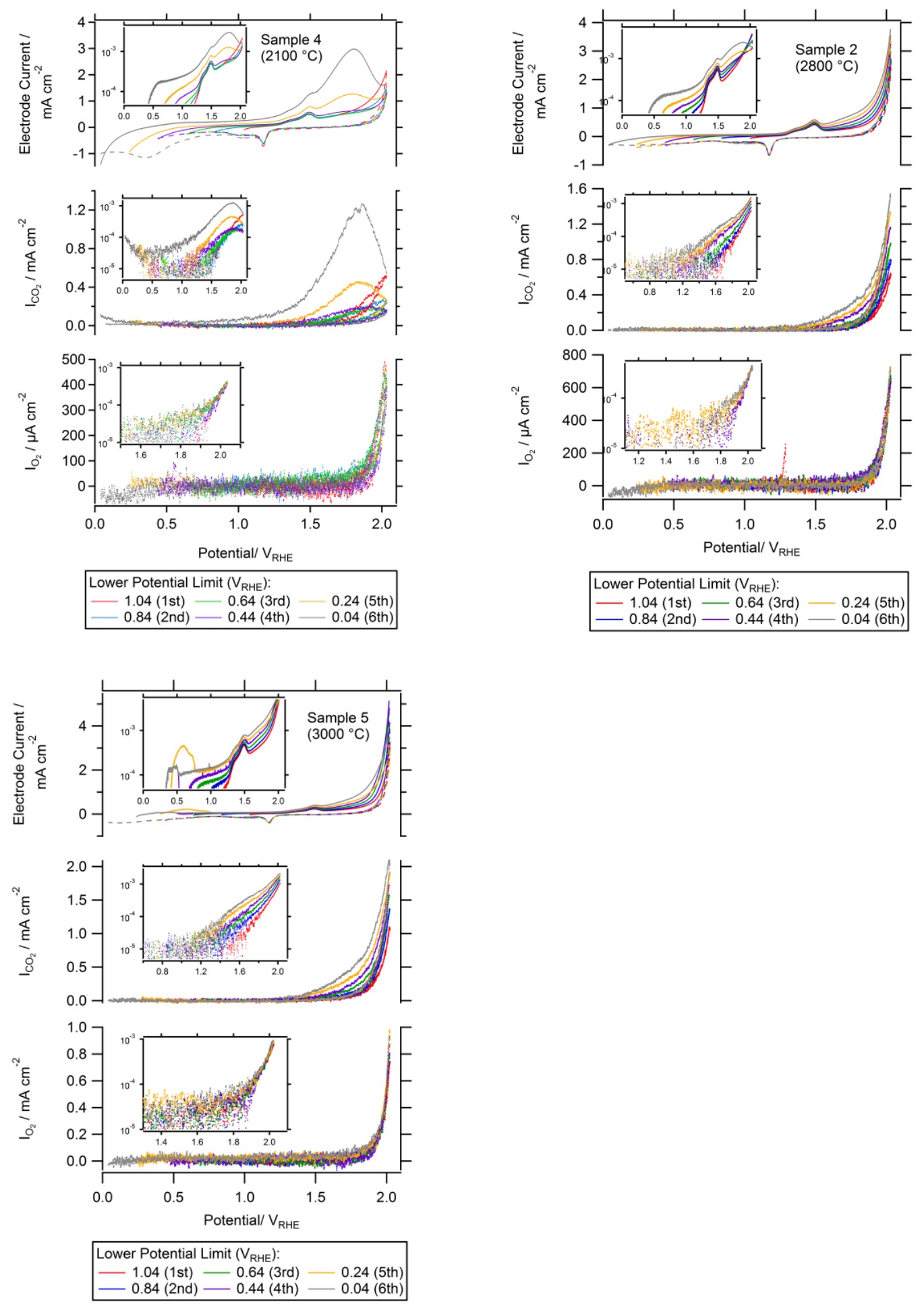

Figure 9-4: Effect of the lower potential limit on the electrochemical COR of the graphitised (and electrochemically oxidised in phase (ii) of the electrochemical treatment) Samples 2,4 and 5 in $0.5 \mathrm{~mol} \mathrm{dm}{ }^{-3} \mathrm{H}_{2} \mathrm{SO}_{4}$. Sample loading of $85 \mathrm{ug}_{\mathrm{c}} \mathrm{cm}^{-2}$ on a polycrystalline Au WE substrate. 

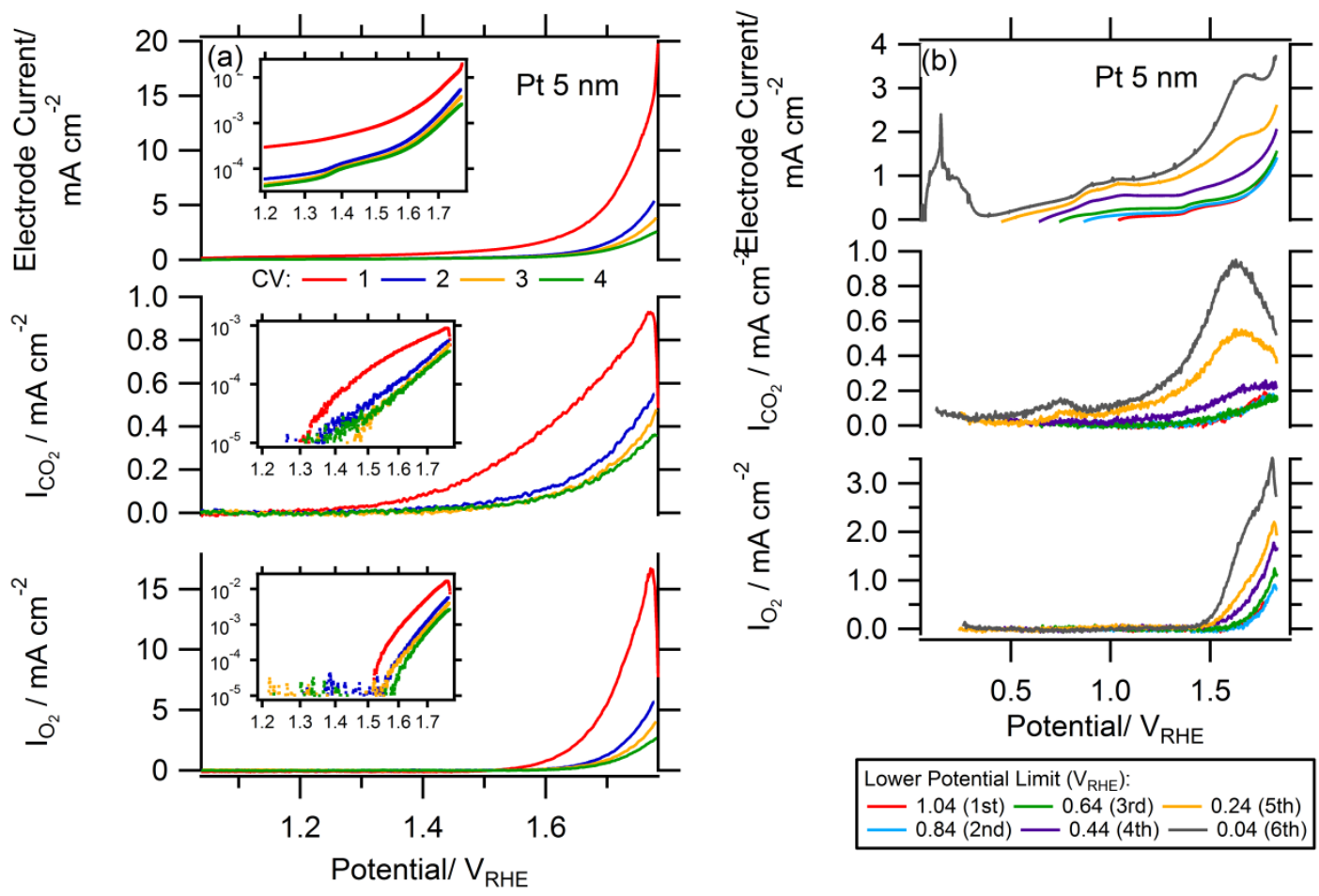

Figure 9-5: LSVs of repeated high potential excursion to $1.85 \mathrm{~V}_{\mathrm{RHE}}$ on the electrochemical oxidation of the HSAC supported Pt $5 \mathrm{~nm}$ catalyst (a) and influence of the lower electrode potential limit following electrochemical oxidation (b).
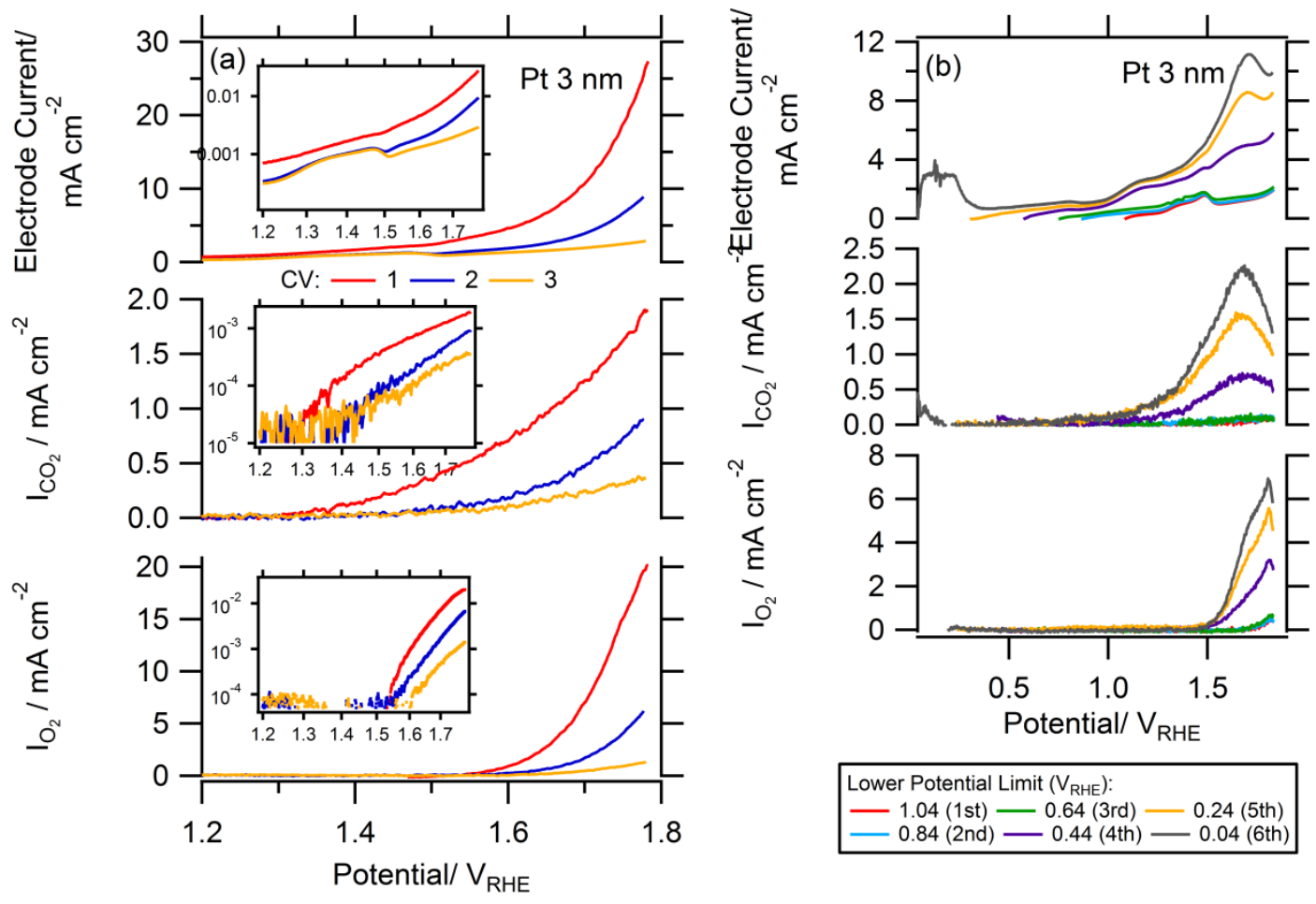

Figure 9-6: LSVs of repeated high potential excursion to $1.85 \mathrm{~V}_{\mathrm{RHE}}$ on the electrochemical oxidation of the HSAC supported Pt $3 \mathrm{~nm}$ catalyst (a) and influence of the lower electrode potential limit following electrochemical oxidation (b). 